\section{Y-12}

NATIONAL SECURITY COMPLEX
March 2003

Prepared by

Elvado Environmental LLC

Under Subcontract No. 4300021559

for the

Environmental Compliance Department Environment, Safety, and Health Organization Y-12 National Security Complex

Oak Ridge, Tennessee 37831

Managed by

BWXT Y-12, L.L.C.

for the U.S. Department of Energy

Under Contract No. DE-AC05-00OR22800 


\section{DISCLAIMER}

This report was prepared as an account of work sponsored by an agency of the United States Government. Neither the United States Government nor any agency thereof, nor any of their employees, makes any warranty, express or implied, or assumes any legal liability or responsibility for the accuracy, completeness, or usefulness of any information, apparatus, product, or process disclosed, or represents that its use would not infringe privately owned rights. Reference herein to any specific commercial product, process, or service by trade name, trademark, manufacturer, or otherwise, does not necessarily constitute or imply its endorsement, recommendation, or favoring of the United States Government or any agency thereof. The views and opinions of authors expressed herein do not necessarily state or reflect those of the United States Government or any agency thereof. 
CALENDAR YEAR 2002

GROUNDWATER MONITORING REPORT,

U.S. DEPARTMENT OF ENERGY

Y-12 NATIONAL SECURITY COMPLEX,

OAK RIDGE, TENNESSEE

March 2003

Prepared by

Elvado Environmental LLC

Under Subcontract No. 4300021559

for the

Environmental Compliance Department

Environment, Safety, and Health Organization

Y-12 National Security Complex

Oak Ridge, Tennessee 37831

\section{Managed by}

BWXT Y-12, L.L.C.

for the U.S. Department of Energy

Under Contract No. DE-AC05-00OR22800 


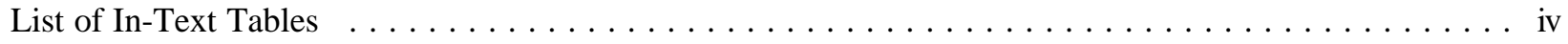

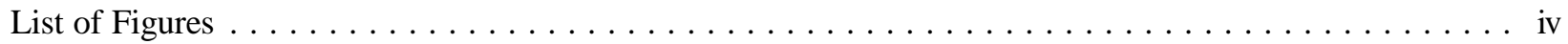

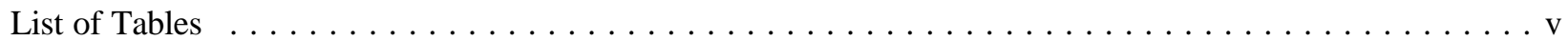

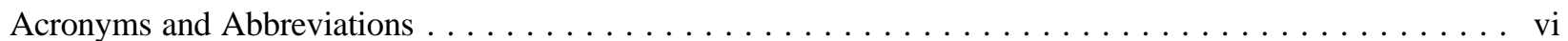

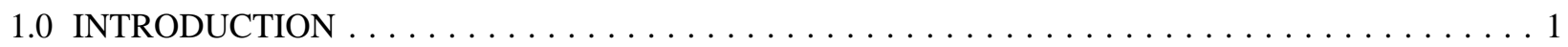

2.0 MONITORING PROGRAMS $\ldots \ldots \ldots \ldots \ldots \ldots \ldots \ldots \ldots \ldots \ldots \ldots \ldots \ldots \ldots \ldots$

3.0 SAMPLING LOCATIONS AND FREQUENCY $\ldots \ldots \ldots \ldots \ldots \ldots \ldots \ldots \ldots \ldots \ldots \ldots$

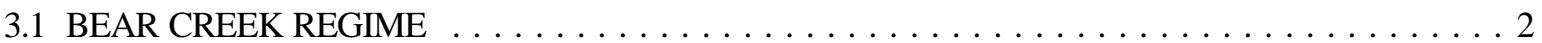

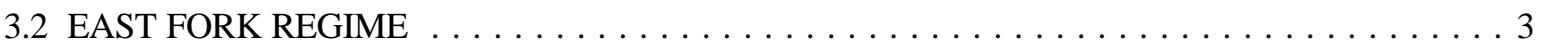

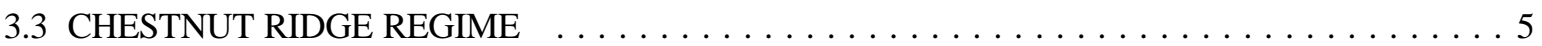

3.4 QUALITY ASSURANCE/QUALITY CONTROL SAMPLING $\ldots \ldots \ldots \ldots \ldots \ldots \ldots \ldots$

4.0 SAMPLE COLLECTION AND HANDLING $\ldots \ldots \ldots \ldots \ldots \ldots \ldots \ldots \ldots \ldots \ldots$

5.0 FIELD MEASUREMENTS AND LABORATORY ANALYTES $\ldots \ldots \ldots \ldots \ldots \ldots \ldots \ldots \ldots$

6.0 DATA MANAGEMENT AND DQO EVALUATION $\ldots \ldots \ldots \ldots \ldots \ldots \ldots \ldots \ldots$

7.0 GROUNDWATER ELEVATION MONITORING $\ldots \ldots \ldots \ldots \ldots \ldots \ldots \ldots \ldots$

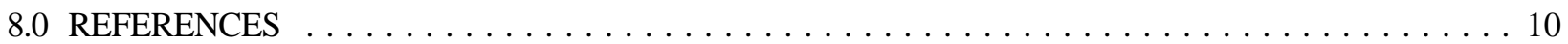

APPENDICES:

A FIGURES

B TABLES

C MONITORING WELL CONSTRUCTION DETAILS

D CY 2002 MONITORING DATA FOR THE BEAR CREEK HYDROGEOLOGIC REGIME

D.1 Field Measurements, Miscellaneous Analytes, Major Ions, and Trace Metals

D.2 Volatile Organic Compounds

D.3 Radiological Analytes

E CY 2002 MONITORING DATA FOR THE UPPER EAST FORK POPLAR CREEK HYDROGEOLOGIC REGIME

F.1 Field Measurements, Miscellaneous Analytes, Major Ions, and Trace Metals

F.2 Volatile Organic Compounds

F.3 Radiological Analytes

F CY 2002 MONITORING DATA FOR THE CHESTNUT RIDGE HYDROGEOLOGIC REGIME

E.1 Field Measurements, Miscellaneous Analytes, Major Ions, and Trace Metals

E.2 Volatile Organic Compounds

E.3 Radiological Analytes

G CY 2002 QUALITY ASSURANCE/QUALITY CONTROL DATA 


\section{List of In-Text Tables}

$\underline{\text { Table }}$

$\underline{\text { Page }}$

1. CY 2002 sampling locations in the Bear Creek Regime $\ldots \ldots \ldots \ldots \ldots \ldots \ldots \ldots \ldots \ldots \ldots$

2. CY 2002 sampling locations in the East Fork Regime, north of Pine Ridge, and in Union Valley . . . . . . 4

3. CY 2002 sampling locations in the Chestnut Ridge Regime $\ldots \ldots \ldots \ldots \ldots \ldots \ldots \ldots \ldots$

4. QA/QC samples analyzed in CY 2002 for the $\mathrm{Y}-12 \mathrm{GWPP} \ldots \ldots \ldots \ldots \ldots \ldots \ldots \ldots \ldots \ldots \ldots \ldots$

5. Summary of CY 2002 groundwater elevation monitoring in the Bear Creek, East Fork, and Chestnut Ridge Regimes . . . . . . . . . . . . . . . . . . . . . . . . . . . . . . . . 9

\section{List of Figures}

Figure $\quad \underline{\text { Page }}$

A.1 Hydrogeologic regimes at the Y-12 National Security Complex $\ldots \ldots \ldots \ldots \ldots \ldots \ldots$ A-1

A.2 CY 2002 surface water sampling locations in the Bear Creek Hydrogeologic Regime . . . . . . A A-2

A.3 CY 2002 sampling locations in the Upper East Fork Poplar Creek

Hydrogeologic Regime and in Union Valley . . . . . . . . . . . . . . . . A-3

A.4 CY 2002 sampling locations north of Pine Ridge $\ldots \ldots \ldots \ldots \ldots \ldots \ldots \ldots \ldots \ldots \ldots$ A-4

A.5 Westbay ${ }^{\mathrm{TM}}$ monitoring system sampling port depths in well GW-722 $\ldots \ldots \ldots \ldots \ldots \ldots$ A-5

A.6 CY 2002 sampling locations in the Chestnut Ridge Hydrogeologic Regime . . . . . . . . . . A-6

A.7 Seasonal groundwater elevations in the Bear Creek Hydrogeologic Regime, $2002 \ldots \ldots$. . . A A-7

A.8 Seasonal groundwater elevations in the Upper East Fork Poplar Creek

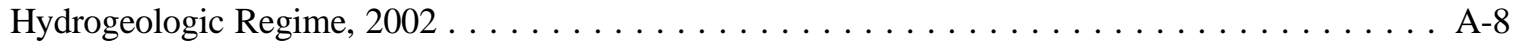

A.9 Seasonal groundwater elevations in the Chestnut Ridge Hydrogeologic Regime, 2002 . . . . . . A-9 


\section{List of Tables}

$\underline{\text { Table }}$

$\underline{\text { Page }}$

B.1 Summary of CY 2002 sampling and analysis plan addenda $\ldots \ldots \ldots \ldots \ldots \ldots \ldots \ldots$

B.2 CY 2002 groundwater and surface water sampling dates in the Bear Creek Hydrogeologic Regime $\ldots \ldots \ldots \ldots \ldots \ldots \ldots \ldots \ldots \ldots \ldots \ldots$

B.3 CY 2002 groundwater and surface water sampling dates in the Upper East Fork Poplar Creek Hydrogeologic Regime . . . . . . . . . . . . . . . . B-7

B.4 CY 2002 groundwater and surface water sampling dates in the Chestnut Ridge Hydrogeologic Regime $\ldots \ldots \ldots \ldots \ldots \ldots \ldots \ldots \ldots \ldots \ldots \ldots \ldots$

B.5 Field measurements and laboratory analytes for CY 2002 groundwater and

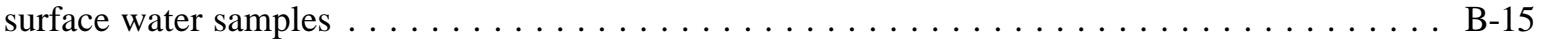

B.6 Depth-to-water measurements and groundwater elevations in the Bear Creek Hydrogeologic Regime, April and September $2002 \ldots \ldots \ldots \ldots \ldots$. . . . . . . . . . . . . .

B.7 Depth-to-water measurements and groundwater elevations in the Upper East Fork Poplar Creek Hydrogeologic Regime, April and September 2002

B.8 Depth-to-water measurements and groundwater elevations in the Chestnut Ridge Hydrogeologic Regime, April and September 2002 


\section{Acronyms and Abbreviations}

$\mathrm{ACO}$

BCK

$\mathrm{BCV}$

Bear Creek Regime

BJC

BWXT

CDL

CERCLA

Chestnut Ridge Regime

CRSDB

CRSP

CY

DOE

DQO

East Fork Regime

EMWMF

$\mathrm{ft}$

GWPP

IL

LMES

NT

OF

ORR

POC

QA/QC

RCRA

REDOX

ROD

SAP

SCR

SS

SWDF

TDEC

UEFPC

VOC

WRRP

Y-12
Analytical Chemistry Organization

Bear Creek kilometer

Bear Creek Valley

Bear Creek Hydrogeologic Regime

Bechtel Jacobs Company LLC

BWXT Y-12, L.L.C.

Construction/Demolition Landfill

Comprehensive Environmental Response, Compensation, and Liability Act

Chestnut Ridge Hydrogeologic Regime

Chestnut Ridge Sediment Disposal Basin

Chestnut Ridge Security Pits

calendar year

U.S. Department of Energy

data quality objective

Upper East Fork Poplar Creek Hydrogeologic Regime

Environmental Management Waste Management Facility

feet

Groundwater Protection Program

Industrial Landfill

Lockheed Martin Energy Systems, Inc.

northern tributary (of Bear Creek)

outfall (subsurface drains in Y-12)

Oak Ridge Reservation

point-of-compliance

quality assurance/quality control

Resource Conservation and Recovery Act

oxidation-reduction potential

record of decision (CERCLA)

sampling and analysis plan

south Chestnut Ridge

south side (of Bear Creek)

Solid Waste Disposal Facility

Tennessee Department of Environment and Conservation

Upper East Fork Poplar Creek

volatile organic compound

Water Resources Restoration Program

Y-12 National Security Complex 


\subsection{INTRODUCTION}

This report contains the groundwater and surface water monitoring data that were obtained during calendar year (CY) 2002 at the U.S. Department of Energy (DOE) Y-12 National Security Complex (hereafter referenced as Y-12) on the DOE Oak Ridge Reservation (ORR) in Oak Ridge, Tennessee. The CY 2002 monitoring data were obtained from groundwater and surface water sampling locations in three hydrogeologic regimes at Y-12 (Figure A.1). The Bear Creek Hydrogeologic Regime (Bear Creek Regime) encompasses a section of Bear Creek Valley (BCV) between the west end of Y-12 and the west end of the Bear Creek Watershed (directions are in reference to the Y-12 grid system). The Upper East Fork Poplar Creek Hydrogeologic Regime (East Fork Regime) encompasses the Y-12 industrial facilities and support structures in BCV. The Chestnut Ridge Hydrogeologic Regime (Chestnut Ridge Regime) encompasses a section of Chestnut Ridge south of Y-12.

The CY 2002 monitoring data were obtained under the Y-12 Groundwater Protection Program (GWPP) managed by BWXT Y-12, L.L.C. (BWXT) and several monitoring programs managed by Bechtel Jacobs Company LLC (BJC). Data contained in this report meet applicable requirements of DOE Order 5400.1 (General Environmental Protection Program). As described in the Environmental Monitoring Plan for the Oak Ridge Reservation (DOE 2001), DOE Order 5400.1 requires groundwater and surface water quality monitoring: (1) in areas which are, or could be, affected by operations at Y-12 (DOE Order 5400.1 site surveillance monitoring) and (2) in areas where contaminants from Y-12 are most likely to migrate beyond the boundaries of the ORR (DOE Order 5400.1 exit pathway/perimeter monitoring).

The following sections of this report provide details regarding the CY 2002 groundwater and surface water monitoring activities in the Bear Creek, East Fork, and Chestnut Ridge Regimes. Section 2 describes the monitoring programs implemented by the Y-12 GWPP and BJC during CY 2002. Section 3 identifies the sampling locations in each hydrogeologic regime and the corresponding sampling frequency during CY 2002, along with the associated quality assurance/quality control (QA/QC) sampling. Section 4 describes groundwater and surface water sample collection and Section 5 identifies the field measurements and laboratory analytes for each sampling location. Section 6 outlines the data management protocols and data quality objectives (DQOs). Section 7 describes the groundwater elevation monitoring in each regime during CY 2002 and Section 8 lists the documents cited for more detailed operational, regulatory, and technical information.

The narrative sections of the report reference several appendices. Figures (maps and diagrams) and tables (excluding data summary tables presented in the narrative sections) are in Appendix A and Appendix B, respectively. Monitoring well construction details are in Appendix C. Results of field measurements and laboratory analyses of the groundwater and surface water samples collected during CY 2002 are in

Appendix D (Bear Creek Regime), Appendix E (East Fork Regime and surrounding areas), and Appendix F (Chestnut Ridge Regime). Appendix G contains data for QA/QC samples associated with monitoring performed by the Y-12 GWPP.

\subsection{MONITORING PROGRAMS}

Groundwater and surface water sampling and analysis activities performed during CY 2002 specifically for the purposes of DOE Order 5400.1 were implemented in accordance with the Y-12 GWPP sampling and analysis plan (SAP) for CY 2002 (BWXT 2001a), and modified by applicable addenda (Table B.1). These sampling and analysis activities are hereafter referenced as DOE Order 5400.1 monitoring. 
Groundwater and surface water sampling and analysis activities managed by BJC during CY 2002 were performed under the ORR Water Resources Restoration Program (WRRP), the ORR Landfill Operations for Solid Waste Disposal, and operation of the Environmental Management Waste Management Facility (EMWMF). Monitoring activities were performed in accordance with the WRRP SAP for fiscal years 2002 (BJC 2001) and 2003 (BJC 2002a) for the following purposes: (1) Resource Conservation and Recovery Act (RCRA) post-closure detection monitoring and RCRA post-closure corrective action monitoring (collectively referenced as RCRA monitoring), as specified in applicable RCRA post-closure permits issued by the Tennessee Department of Environment and Conservation (TDEC); (2) Comprehensive Environmental Response, Compensation, and Liability Act (CERCLA) remediation effectiveness monitoring (CERCLA monitoring), as specified in the applicable record of decision (ROD) or decision documents pending final approval (referenced as CERCLA ROD monitoring), and CERCLA pre-remediation baseline water quality monitoring (referenced as CERCLA baseline monitoring). Detection monitoring for several nonhazardous solid waste disposal facilities (SWDFs) located in the Chestnut Ridge Regime (referenced as SWDF detection monitoring) was performed in accordance with the SAP (Duratek Federal Services 2002) and to comply with site-specific operating permits issued by the TDEC. Quarterly CERCLA monitoring at the EMWMF, located in the Bear Creek Regime, was performed in accordance with the site-specific requirements of an environmental monitoring plan for the site (DOE 2002).

\subsection{SAMPLING LOCATIONS AND FREQUENCY}

The CY 2002 groundwater and surface water quality monitoring addressed in this report includes a total of 203 sampling locations. Samples were collected from 159 monitoring wells (complete construction details for each well are provided in Appendix C), 12 springs, and 32 surface water stations. Cooperation between the Y-12 GWPP and BJC monitoring programs (i.e., preparing SAPs, coordinating sample collection, and sharing data) ensures that the CY 2002 monitoring results fulfill requirements of applicable monitoring drivers (DOE Order 5400.1, RCRA, CERCLA, and SWDF) with minimal duplication of sampling and analysis activities. Although the monitoring data from all of the CY 2002 sampling locations serve DOE Order 5400.1 monitoring purposes, the following sections identify the specific monitoring driver under which samples were collected in each regime.

\subsection{BEAR CREEK REGIME}

As shown below (Table 1), 67 monitoring wells, six springs, and 17 surface water stations in the Bear Creek Regime were sampled during CY 2002 for the purposes of DOE Order 5400.1 monitoring, RCRA monitoring, and CERCLA monitoring.

Table 1. CY 2002 sampling locations in the Bear Creek Regime

\begin{tabular}{|c|c|c|c|}
\hline \multicolumn{1}{|c|}{ Monitoring Driver } & Monitoring Wells & Springs & Surface Water Stations \\
\hline DOE Order 5400.1 & 39 & 3 & 6 \\
RCRA & 8 & 0 & 0 \\
CERCLA & 20 & 3 & 11 \\
\hline Totals: & $\mathbf{6 7}$ & $\mathbf{6}$ & $\mathbf{1 7}$ \\
\hline
\end{tabular}


Samples were collected semiannually during CY 2002 from most of the sampling locations. Two wells, one spring, and eight surface water stations were sampled during only the first quarter of CY 2002, and 14 wells associated with the EMWMF were sampled during the third and fourth quarters of the year (Table B.2).

Thirty-nine monitoring wells in the Bear Creek Regime were sampled for the specific purposes of DOE Order 5400.1 site surveillance monitoring during CY 2002 (Table B.2 and Figure A.2). Twenty-four of these wells are located near waste management facilities in $\mathrm{BCV}$, including the primary sources of groundwater contamination in the regime (S-3 Site, Oil Landfarm Waste Management Area, and Bear Creek Burial Grounds). The remaining wells are components of three Exit Pathway Pickets in the regime: Picket A (two wells) is located about 1,600 feet (ft) west of the Bear Creek Burial Grounds; Picket B (five wells) is located about 2,000 ft west of the Oil Landfarm; and Picket C (eight wells) is located about 3,000 ft west of the S-3 Site (Figure A.2). The wells in each Exit Pathway Picket are completed at various depths along strike-normal transects across the Maynardville Limestone, which is the primary contaminant migration pathway in the Bear Creek Regime.

Samples were collected from three springs and six surface water sampling stations in the Bear Creek Regime for the purposes of DOE Order 5400.1 exit pathway/perimeter monitoring during CY 2002 (Table B.2). The springs, which discharge into Bear Creek from the south side (SS) of the creek channel (Figure A.2), are located southwest (hydraulically downgradient) of the S-3 Site (SS-1), the Oil Landfarm (SS-4), and the Bear Creek Burial Grounds (SS-5). The surface water stations, as designated by the Bear Creek kilometer (BCK) value measured upstream from the confluence of Bear Creek and East Fork Poplar Creek, are located north of Pine Ridge (BCK-00.63); near the west end of BCV (BCK-04.55); about 6,200 ft (BCK-07.87) and $1,600 \mathrm{ft}$ (BCK-09.40) downstream of the Bear Creek Burial Grounds; about 3,600 ft downstream of the S-3 Site (BCK-11.97); and in a northern tributary (NT) of Bear Creek (NT-01) about 1,500 ft west of the S-3 Site (Figure A.2).

Eight monitoring wells in the Bear Creek Regime were sampled for the specific purposes of RCRA postclosure corrective action monitoring during CY 2002 (Table B.2). These wells include a background well located hydraulically upgradient of contaminated groundwater in the regime (GW-115); point-of-compliance (POC) wells located downgradient of the S-3 Site (GW-276), the Oil Landfarm (GW-008), and the Bear Creek Burial Grounds (GW-046); and four plume boundary wells that comprise Exit Pathway Picket W, which is located about 6,500 ft west of the Bear Creek Burial Grounds (Figure A.2).

Twenty monitoring wells, three springs, and 11 surface water stations in the Bear Creek Regime were sampled for CERCLA monitoring purposes during CY 2002 (Table B.2 and Figure A.2). Fourteen monitoring wells located at the EMWMF were sampled quarterly (August and November 2002) after waste disposal at the site began (June 2002). Six monitoring wells, three springs, and seven surface water stations were sampled for the purposes of CERCLA ROD monitoring; the monitoring wells are located along contaminant migration pathways hydraulically downgradient of the S-3 Site (GW-526 and GW-835) and the Bear Creek Burial Grounds (GW-077, GW-078, GW-079, and GW-080). Four surface water stations located near the Bear Creek Burial Grounds (NT-07, NT-08, NT-8E, and NT-8W) were sampled for CERCLA baseline monitoring purposes.

\subsection{EAST FORK REGIME}

As shown below in Table 2, a total of 52 monitoring wells, two springs, and ten surface water stations in the East Fork Regime (and surrounding areas) were sampled during CY 2002 for the purposes of DOE Order 5400.1 monitoring, RCRA monitoring, and CERCLA monitoring. 
Table 2. CY 2002 sampling locations in the East Fork Regime, north of Pine Ridge, and in Union Valley

\begin{tabular}{|c|c|c|c|}
\hline Monitoring Driver & Monitoring Wells & Springs & Surface Water Stations \\
\hline DOE Order 5400.1 & 32 & 0 & 6 \\
RCRA & 5 & 0 & 4 \\
CERCLA & 16 & 2 & $\mathbf{1 0}$ \\
\hline \multicolumn{2}{|c|}{ Totals: } & $\mathbf{5 2}$ & $\mathbf{2}$ \\
\hline \\
Note: Samples were collected from one well (GW-722) for DOE Order 5400.1 (February and July) and CERCLA (May and \\
November) monitoring purposes (see Table B.3).
\end{tabular}

Fifty-one of these sampling locations lie within the boundaries of the East Fork Regime (Figure A.3), which is divided into the three major areas for the purposes of this report: the western Y-12 area between Old Bear Creek Road and grid coordinate easting 55,000; the central Y-12 area between grid coordinate eastings 55,000 and 62,000; and the eastern Y-12 area between grid coordinate easting 62,000 and Scarboro Road. The other thirteen CY 2002 sampling locations lie outside the boundaries of the regime, including six wells and two springs located in Union Valley east the ORR boundary at Scarboro Road (Figure A.3), and five surface water stations located in drainage features along the ORR boundary on the north side of Pine Ridge (Figure A.4). Samples were collected at least semiannually from each CY 2002 sampling location; four of the monitoring wells were sampled quarterly and three wells were sampled only once during the year (Table B.3).

A total of 32 monitoring wells and six surface water stations were sampled for DOE Order 5400.1 monitoring purposes in the East Fork Regime during CY 2002. Groundwater samples from 23 monitoring wells were collected for the specific purposes of DOE Order 5400.1 site surveillance monitoring (Table B.3). Seven of these wells are located in the western Y-12 area, 11 wells are in the central Y-12 area, and five wells are in the eastern Y-12 area (Figure A.3). Nine monitoring wells and six surface water locations were sampled for the purposes of DOE Order 5400.1 exit pathway/perimeter monitoring in the East Fork Regime (Table B.3). Three of these monitoring wells are located next to Upper East Fork Poplar Creek (UEFPC) in the gap through Pine Ridge northeast of Y-12 (Figure A.3) and the other six wells are located between UEFPC and Scarboro Road at the east end of Y-12. One of these wells is equipped with a dedicated Westbay ${ }^{\mathrm{TM}}$ multiport sampling apparatus (Westbay well GW-722), with sampling ports set at ten discrete depths intervals in the well (Figure A.5). Surface water samples were collected from the exit point of the New Hope Pond Distribution Channel underdrain (LRSPW on Figure A.3) and from five tributaries located north of Pine Ridge (Figure A.4).

Five wells were sampled for the specific purposes of RCRA post-closure corrective action monitoring in the East Fork Regime during CY 2002. These wells include one POC well (GW-108) which is located in the western Y-12 area about $800 \mathrm{ft}$ southeast of the S-3 Site, and four plume delineation wells (GW-193, GW-605, GW-606, and GW-733) which are located several thousand ft east-southeast of the S-3 Site (Figure A.3). A background well (GW-115) which is located about $500 \mathrm{ft}$ north (upgradient) of the S-3 Site in the Bear Creek Regime (Section 3.1) also serves RCRA monitoring purposes in the East Fork Regime.

Sixteen monitoring wells, two springs, and four surface water stations were sampled during CY 2002 specifically for CERCLA monitoring purposes (Table B.3). Six monitoring wells and two springs located in Union Valley east of the ORR boundary along Scarboro Road, and eight monitoring wells (GW-151, GW-154, GW-223, GW-380, GW-382, GW-722, GW-762, and GW-832) in the eastern Y-12 area were sampled for CERCLA ROD monitoring purposes (Figure A.3). Sampling locations used for CERCLA baseline monitoring 
include two monitoring wells located in the western Y-12 area (GW-253 and GW-618) and four surface water stations (Table B.3): Outfall (OF) 51, OF 200, and Station 8 located in the central Y-12 area, and Station 17 located in the eastern Y-12 area (Figure A.3).

\subsection{CHESTNUT RIDGE REGIME}

As shown below in Table 3, a total of 40 monitoring wells, four springs, and five surface water stations in the Chestnut Ridge Regime were sampled during CY 2002 for the purposes of DOE Order 5400.1 monitoring, SWDF detection monitoring, RCRA monitoring, and CERCLA monitoring.

Table 3. CY 2002 sampling locations in the Chestnut Ridge Regime

\begin{tabular}{|c|c|c|c|}
\hline Monitoring Driver & Monitoring Wells & Springs & Surface Water Stations \\
\hline DOE Order 5400.1 & 0 & 1 & 3 \\
SWDF & 22 & 1 & 0 \\
RCRA & 14 & 0 & 2 \\
CERCLA & 6 & 2 & $\mathbf{5}$ \\
\hline \multicolumn{2}{|c|}{ Totals: } & $\mathbf{4 0}$ & $\mathbf{4}$ \\
\hline \\
Note: Two wells (GW-562 and GW-798) were sampled for SWDF and RCRA monitoring purposes during \\
January and July (see Table B.4).
\end{tabular}

Groundwater samples were collected semiannually during CY 2002 from all of the monitoring locations except for one well (GW-305) that was sampled during each quarter of the year (Table B.4). Samples were collected from the springs and surface water stations during seasonally wet (January-February) and seasonally dry (July-September) flow conditions (Table B.4).

Three surface water stations and one spring were sampled during CY 2002 for the specific purposes of DOE Order 5400.1 exit pathway/perimeter monitoring in the Chestnut Ridge Regime (Table B.4). Tributaries on south Chestnut Ridge (SCR) are numbered in ascending order from west to east (SCR1 through SCR5), and the surface water sampling stations (SCR1.5SW, SCR2.2SW, and SCR4.4SW) are located in main channels along Bethel Valley Road where surface water exits the Chestnut Ridge Regime (Figure A.6). The spring (SCR5.2SP) is located about $200 \mathrm{ft}$ west of Kerr Hollow Quarry (Figure A.6).

Twenty-two monitoring wells and one spring in the Chestnut Ridge Regime were sampled during CY 2002 for the purposes of SWDF detection monitoring (Table B.4). The monitoring wells are located at five SWDFs: four wells at Industrial Landfill (IL) II; five wells at IL IV; five wells at IL V; four wells at Construction/Demolition Landfill (CDL) VI; and four wells at CDL VII (Figure A.6). A spring (SCR4.3SP) was sampled for the purposes of SWDF detection monitoring at IL V and is located about 2,400 ft southeast of the site (Figure A.6). At the request of the TDEC, samples were collected quarterly from well GW-305 at IL IV during CY 2002 because the nickel concentration reported for the sample collected in July 1999 exceeded the Groundwater Protection Standard defined in the operating permit for the site (TDEC 1999).

A total of 14 monitoring wells were sampled for RCRA monitoring purposes during CY 2002 in the Chestnut Ridge Regime: five wells for RCRA post-closure corrective action monitoring and nine wells for RCRA postclosure detective monitoring (Table B.4). RCRA post-closure corrective action monitoring at the Chestnut Ridge Security Pits (CRSP) included one POC well (GW-177) located at the west end of the site and four 
plume delineation wells: one at the former Chestnut Ridge Borrow Area Waste Pile (GW-301) about 3,000 $\mathrm{ft}$ east of the site; two at the CDL VII about 1,800 ft (GW-798) and 3,200 ft (GW-562) southeast of the site; and one at the Filled Coal Ash Pond (GW-831) about 2,000 ft southwest of the site (Figure A.6). Note that the SWDF detection monitoring results for one background well (GW-521) at IL IV and three plume delineation wells (GW-557, GW-799, and GW-801) at IL V (Figure A.6) also serve the purposes of RCRA post-closure corrective action monitoring at the CRSP. RCRA post-closure detection monitoring included four wells at the Chestnut Ridge Sediment Disposal Basin (CRSDB) and five wells at Kerr Hollow Quarry (Table B.4). The RCRA monitoring well network at the CRSDB includes one well (GW-159) located hydraulically upgradient (northwest) of the site and three POC wells (GW-156, GW-731, and GW-732) to the east-southeast (hydraulically downgradient) of the site (Figure A.6). Two upgradient/background wells (GW142 and GW-231) and three downgradient POC wells (GW-143, GW-144, and GW-145) comprise the RCRA monitoring well network at Kerr Hollow Quarry (Figure A.6).

Samples were collected from six monitoring wells, two springs, and two surface water stations for the specific purposes of CERCLA monitoring in the Chestnut Ridge Regime during CY 2002. The wells, located at the United Nuclear Corporation Site (Figure A.6), were sampled for CERCLA ROD monitoring purposes (Table B.4). Two surface water stations (MCK 2.0 and MCK 2.05) and one spring (SCR3.5 SP) located in McCoy Branch and one spring (SCR1.25SP) in the southwestern portion of the regime (Figure A.6) were sampled for CERCLA baseline monitoring purposes (Table B.4).

\subsection{QUALITY ASSURANCE/QUALITY CONTROL SAMPLING}

The following discussion pertains to the QA/QC sampling activities managed by the Y-12 GWPP during CY 2002. Comparable QA/QC protocols were performed by monitoring programs managed by BJC (BJC 1999, Duratek Federal Services 2002). As shown below in Table 4, the QA/QC samples associated with the groundwater and surface water sampling performed under the Y-12 GWPP during CY 2002 include a total of 90 trip blanks, 54 method (laboratory) blanks, four field blanks, three equipment rinsate samples, and 22 duplicate groundwater and surface water samples.

Table 4. QA/QC samples analyzed in CY 2002 for the Y-12 GWPP

\begin{tabular}{|c|c|c|c|c|c|}
\hline \multirow{2}{*}{ Sample Type } & \multicolumn{4}{|c|}{ Total Number of Samples per Quarter of CY 2002} & \multirow{2}{*}{$\begin{array}{c}\text { Annua } \\
\text { Total }\end{array}$} \\
\hline & First & Second & Third & Fourth & \\
\hline Trip Blank Samples & 28 & 17 & 28 & 17 & 90 \\
\hline Method Blank Samples & 17 & 11 & 15 & 11 & 54 \\
\hline Field Blank Samples & 1 & 1 & 1 & 1 & 4 \\
\hline Equipment Rinsate Samples & 1 & 0 & 1 & 1 & 3 \\
\hline Duplicate Groundwater/Surface Water Samples & 7 & 4 & 7 & 4 & 22 \\
\hline
\end{tabular}

The blanks and equipment rinsate samples were prepared and analyzed as specified in the Quality Assurance Plan for the Analytical Chemistry Organization (BWXT 2002a). Analytical results for the blank samples help assess the environmental conditions in the field and laboratory under which associated groundwater and surface water samples were collected, transported, stored, and analyzed. Trip blanks were samples of deionized water prepared in the laboratory and transported to the field and then to the laboratory in coolers containing groundwater and surface water samples. Field blanks were samples of deionized water that were transported to the field in a sealed glass container and transferred to sample bottles at monitoring wells GW-225 (first and third quarters) and GW-383 (second and fourth quarters) and then transported to the 
laboratory in the cooler with other samples from the well. Method blanks were samples of deionized water that were analyzed along with one or more associated groundwater or surface water samples. Equipment rinsates were samples of the deionized water from the final rinse of the decontaminated portable sampling equipment after sampling was completed at Westbay well GW-722 (first and third quarters) and at well GW-689 (fourth quarter).

Method blanks, trip blanks, field blanks, and equipment rinsate samples were analyzed for volatile organic compounds (VOCs); equipment rinsates also were analyzed for miscellaneous analytes (e.g., suspended solids), major ions, trace metals, and radioanalytes. Analytical results for the QA/QC blanks and equipment rinsate samples are summarized in Appendix G.

A total of 22 field duplicate samples were collected for QA/QC purposes from sampling locations monitored under management of the Y-12 GWPP during CY 2002. These sampling locations include eight wells, one surface water station, and one spring in the Bear Creek Regime (Table B.2); six wells and one surface water station in the East Fork Regime and two surface water stations located north of Pine Ridge (Table B.3); and two surface water stations in the Chestnut Ridge Regime (Table B.4). The duplicate samples were analyzed for the same constituents and parameters specified for the sampling location from which they were collected; analytical results are presented with the regular sample results in Appendices D, E, and F.

\subsection{SAMPLE COLLECTION AND HANDLING}

The following discussion pertains to the groundwater and surface water sampling activities managed by the Y-12 GWPP during CY 2002. Personnel from the Sampling and Environmental Support Department of the Y-12 Analytical Chemistry Organization (ACO) were responsible for collection, transportation, and chain-ofcustody control of the groundwater and surface water samples. Sampling was performed in accordance with the most recent version of the technical procedures approved by the Y-12 GWPP Manager (Lockheed Martin Energy Systems, Inc. [LMES] 1999a and BWXT 2000a). All samples were collected in appropriate containers, preserved as required, labeled, logged, placed in ice-filled coolers, and transported to the designated ACO laboratory in accordance with chain-of-custody control requirements. Similar protocols were followed under the monitoring programs managed by BJC during CY 2002.

Unfiltered samples were collected from the monitoring wells, springs, and surface water stations in each hydrogeologic regime during CY 2002. Groundwater samples were obtained from most monitoring wells with dedicated bladder pumps (Well Wizard ${ }^{\mathrm{TM}}$ ). However, samples were obtained from three wells in the East Fork Regime (GW-686, GW-687, and GW-689) with a portable Bennett pump and from one well equipped with a dedicated Westbay ${ }^{\mathrm{TM}}$ multi-port sampling apparatus (Westbay well GW-722).

Samples were collected from the wells equipped with dedicated bladder pumps using the low-flow minimal drawdown sampling method. Under this method, representative groundwater samples are obtained from discrete depth intervals without including stagnant water in the well casing. The well is pumped at a flow rate which is low enough ( $<300$ milliliters per minute) to minimize drawdown of the water level in the well $(<0.1$ $\mathrm{ft}$ per quarter-hour). At five-minute intervals after the water-level drawdown has stabilized, field personnel record measurements of the $\mathrm{pH}$, conductivity, temperature, oxidation-reduction potential (REDOX), and dissolved oxygen of the groundwater pumped from the well. Samples of the groundwater in the well are

collected once the field measurements for each parameter show minimal variation over four consecutive readings. 
Samples were collected from wells GW-686, GW-687, and GW-689 (with a portable Bennett pump) using the "conventional" sampling method, which was used to collect groundwater samples before the Y-12 GWPP began using the low-flow sampling method (October 1997). The conventional sampling method involves purging at least three well volumes of groundwater (or until the well is dry) at a much higher pumping rate (1.0 - 1.8 gallons per minute) before collecting samples.

Groundwater samples were collected from Westbay well GW-722 in accordance with the most recent and approved version of the operating procedures for the multi-port sampling equipment (BWXT 2002b and 2002c). One or more 250-milliliter non-vented stainless steel sample collection bottles were used to obtain groundwater samples from the sampling ports. The sample collection bottles were lowered to the designated sampling port, the sampling port valve was opened, and the bottles were allowed to fill with groundwater. The filled bottles were retrieved to the surface and the contents were poured into the appropriate laboratory sample bottle(s). The sample collection bottles were lowered, filled, and retrieved as many times as needed to completely fill the laboratory sample bottles. Groundwater in the first sample collection bottle retrieved from each sampling port was used as a "formation rinse" to obtain field measurements and to condition the sample collection bottle.

\subsection{FIELD MEASUREMENTS AND LABORATORY ANALYTES}

The following discussion pertains to the field measurements and laboratory analytes associated with the CY 2002 groundwater and surface water sampling activities in the Bear Creek, East Fork, and Chestnut Ridge hydrogeologic regimes that were performed by the Y-12 GWPP. Functionally equivalent field measurements and laboratory analyses were performed under the monitoring programs managed by BJC during CY 2002 (BJC 2001, BJC 2002a, and Duratek Federal Services 2002).

Field personnel measured the depth to the static water surface before sampling groundwater in each monitoring well, and recorded field measurements of $\mathrm{pH}$, temperature, conductivity, dissolved oxygen, and REDOX for each groundwater and surface water sampling location (Table B.5). Note that the depth to water and REDOX were not recorded for Westbay well GW-722 (not applicable when a well is equipped with a multiport sampling apparatus). Field measurements were obtained in accordance with the most recent and approved technical procedures (LMES 1999b, BWXT 2000b, and BWXT 2001b). The field measurements recorded for the sampling locations in each regime are presented in Appendices D.1, E.1, and F.1.

All of the CY 2002 groundwater samples and surface water samples were analyzed for: (1) miscellaneous laboratory analytes $-\mathrm{pH}$, conductivity, turbidity, total suspended solids, and total dissolved solids; (2) major ions and trace metals; (3) VOCs; and (4) gross alpha and gross beta activity (Table B.5). Laboratory analyses of the samples were performed by the Y-12 ACO laboratories in accordance with the analytical methods and procedures listed in Table B.5. Analytical results are presented in Appendix D (Bear Creek Regime), Appendix E (East Fork Regime), and Appendix F (Chestnut Ridge Regime). Note that each data appendix contains the analytical results only for laboratory analytes that were detected in at least one sample from the CY 2002 sampling locations. 


\subsection{DATA MANAGEMENT AND DQO EVALUATION}

The ACO laboratories provided electronic files and hardcopy printouts of the analytical results and field measurements for the groundwater and surface water samples collected during CY 2002 under management of the Y-12 GWPP. The GWPP groundwater database management subcontractor downloaded the electronic files directly into $\mathrm{SAS}^{\circledR}$ files and verified the data in accordance with the Y-12 Plant Groundwater Protection Program Data Management Plan (LMES 2000). Appropriate ACO staff and the groundwater database management subcontractor worked to resolve any incomplete data transfers, irregular parameter names or reporting units, and discrepancies between electronic and hardcopy versions of the data.

Analytical results and field measurements associated with monitoring programs managed by BJC (see Section 2) were extracted from the project database by the GWPP groundwater database management subcontractor and formatted as $\mathrm{SAS}^{\circledR}$ files for presentation in this report. The BJC data management process (BJC 2002b) is similar to the process described above for the Y-12 GWPP.

The CY 2002 groundwater and surface water monitoring data presented in this report have been evaluated with respect to the DQO criteria defined in the Y-12 Plant Groundwater Protection Program Data Management Plan (LMES 2000) and the functionally equivalent DQO criteria for the monitoring programs managed by BJC (BJC 2002b). Specific DQO criteria apply to analytical results for major ions, trace metals, VOCs, radiological analytes (gross alpha, gross beta, and radionuclides), and miscellaneous laboratory analytes (e.g., total suspended solids). Only a few of the monitoring results (three charge balances and three sets of duplicate lead results) obtained under the Y-12 GWPP do not meet applicable DQOs; these results are flagged with an "R" qualifier in the applicable data appendices (Appendix D.1 and Appendix E.1).

\subsection{GROUNDWATER ELEVATION MONITORING}

As shown below in Table 5, respective networks of selected monitoring wells in the Bear Creek, East Fork, and Chestnut Ridge hydrogeologic regimes were used to monitor representative seasonal groundwater elevations during CY 2002.

Table 5. Summary of CY 2002 groundwater elevation monitoring in the Bear Creek, East Fork, and Chestnut Ridge Regimes

\begin{tabular}{|c|c|c|c|c|c|c|}
\hline \multirow{3}{*}{ REGIME } & \multicolumn{4}{|c|}{ DEPTH-TO-WATER MEASUREMENTS } & \multirow{2}{*}{\multicolumn{2}{|c|}{$\begin{array}{l}\text { GROUNDWATER } \\
\text { ELEVATIONS }\end{array}$}} \\
\hline & \multicolumn{2}{|c|}{ Seasonally High Flow } & \multicolumn{2}{|c|}{ Seasonally Low Flow } & & \\
\hline & $\begin{array}{l}\text { No. of } \\
\text { Wells }\end{array}$ & Dates & $\begin{array}{l}\text { No. of } \\
\text { Wells }\end{array}$ & Dates & Data & $\begin{array}{c}\text { Contour } \\
\text { Map }\end{array}$ \\
\hline Bear Creek & 64 & April 8-12, 2002 & 56 & September 17-30, 2002 & Table B.6 & Figure A.7 \\
\hline East Fork & 61 & April 8-11, 2002 & 58 & September 18-26, 2002 & Table B.7 & Figure A.8 \\
\hline Chestnut Ridge & 77 & April 8-10, 2002 & 74 & September 17-26, 2002 & Table B.8 & Figure A.9 \\
\hline
\end{tabular}

Field personnel with the Y-12 GWPP measured the depth to the static water surface in each well during seasonally high flow conditions (April 2002) and field personnel subcontracted by the WRRP measured the depth to the static water surface in each well during seasonally low flow conditions (September 2002). These depth-to-water measurements were performed in accordance with the respective operating procedures for the Y-12 GWPP (LMES 1999b) and the WRRP (MDM Services Corporation 2000). 


\subsection{REFERENCES}

Bechtel Jacobs Company LLC. 1999. Quality Assurance Project Plan for the Integrated Water Quality Program for, U.S. Department of Energy, Oak Ridge Reservation, Oak Ridge, Tennessee. Prepared by Science Applications International Corporation (BJC/OR-235).

Bechtel Jacobs Company LLC. 2001. Sampling and Analysis Plan for the Water Resources Restoration Program for Fiscal Year 2002, Oak Ridge Reservation, Oak Ridge, Tennessee. Prepared by Science Applications International Corporation (BJC/OR-960).

Bechtel Jacobs Company LLC. 2002a. Sampling and Analysis Plan for the Water Resources Restoration Program for Fiscal Year 2003 Oak Ridge Reservation Oak Ridge, Tennessee. Prepared by Science Applications International Corporation (BJC/OR-1173).

Bechtel Jacobs Company LLC. 2002b. Work Plan for the Technical Support Services, Water Resources Restoration Program, U.S. Department of Energy Oak Ridge Reservation, Oak Ridge, Tennessee. Prepared by Science Applications International Corporation (BJC/OR-361, Rev.1).

BWXT Y-12, L.L.C. 2000a. Liquid Grab Sampling. BWXT Y-12, L.L.C. management requirement prepared by the Environment, Safety, and Health Organization (Y50-71-005).

BWXT Y-12, L.L.C. 2000b. Field Measurements of Physical and Chemical Characteristics. BWXT Y-12, L.L.C. management requirement prepared by the Environment, Safety, and Health Organization (Y50-71-001).

BWXT Y-12, L.L.C. 2001a. Y-12 Groundwater Protection Program Groundwater and Surface Water Sampling and Analysis Plan for Calendar Year 2002. Prepared by AJA Technical Services, Inc. (Y/SUB/01-006512/6).

BWXT Y-12, L.L.C. 2001b. Redox Meter Calibration and Operation. BWXT Y-12, L.L.C. management requirement prepared by the Analytical Chemistry Organization (Y/P65-9156).

BWXT Y-12, L.L.C. 2002a. Quality Assurance Plan for the Analytical Chemistry Organization. Prepared by the Analytical Chemistry Organization (Y/P65-9006, Rev. K).

BWXT Y-12, L.L.C. 2002b. Groundwater Sampling of Westbay ${ }^{\mathrm{TM}}$ Monitoring System Instrumented Wells. Oak Ridge Y-12 Plant Procedure prepared by the Environment, Safety, and Health Organization (Y50-71-018).

BWXT Y-12, L.L.C. 2002c. Pressure Profiling of Wells Equipped with Westbay ${ }^{\mathrm{TM}}$ Monitoring System Instrumentation. Oak Ridge Y-12 Plant Procedure prepared by the Environment, Safety, and Health Organization (Y50-71-019).

Duratek Federal Services, Inc. 2002. Oak Ridge Reservation Landfill Operations for Solid Waste Disposal, Sampling and Analysis Plan, Revision 5. 
Lockheed Martin Energy Systems, Inc. 1998. Updated Subsurface Data Base for Bear Creek Valley, Chestnut Ridge, and Parts of Bethel Valley on the U.S. Department of Energy Oak Ridge Reservation. Lockheed Martin Energy Systems, Inc. (Y/TS-881/R4). (referenced in Appendix C).

Lockheed Martin Energy Systems, Inc. 1999a. Groundwater Sampling. Oak Ridge Y-12 Plant Procedure prepared by the Environment, Safety, and Health Organization (Y50-71-016).

Lockheed Martin Energy Systems, Inc. 1999b. Measurement of Static Water Level Elevation. Oak Ridge Y-12 Plant Procedure prepared by the Environment, Safety, and Health Organization (Y50-71-015).

Lockheed Martin Energy Systems, Inc. 2000. Y-12 Plant Groundwater Protection ProgramGroundwater Monitoring Program Data Management Plan. (Y/SUB/00-KFX63/C/1).

MDM Services Corporation. 2000. Environmental and Sampling Services Control Procedures.

Tennessee Department of Environment and Conservation. 1999. Cook, L.F. December 2, 1999. TDEC letter to D.W. McCune Bechtel Jacobs Company LLC; subject: "Groundwater Monitoring-Industrial Landfill IV: IDL 01-103-0075."

U.S. Department of Energy. 2001. Environmental Monitoring Plan for the Oak Ridge Reservation. U.S. Department of Energy Oak Ridge Field Office (DOE/OR-1066/R4).

U.S. Department of Energy. 2002. Attachment to Appendix B-IFC Environment, Safety, and Health Plan: Environmental Monitoring Plan Addendum to the Remedial Design Report for the Disposal of Oak Ridge Reservation Comprehensive Environmental Response, Compensation, and Liability Act of 1980 Waste, Oak Ridge, Tennessee. Prepared by Duratek Federal Services, Inc. (DOE/OR/01-1873\&D2/A1/R2).

U.S. Environmental Protection Agency. 1983. Methods for Chemical Analysis of Water and Wastes. (referenced in Table B.5).

U.S. Environmental Protection Agency. 1996. Test Methods for Evaluating Solid Waste Physical/Chemical Methods. (referenced in Table B.5). 
APPENDIX A

\section{FIGURES}




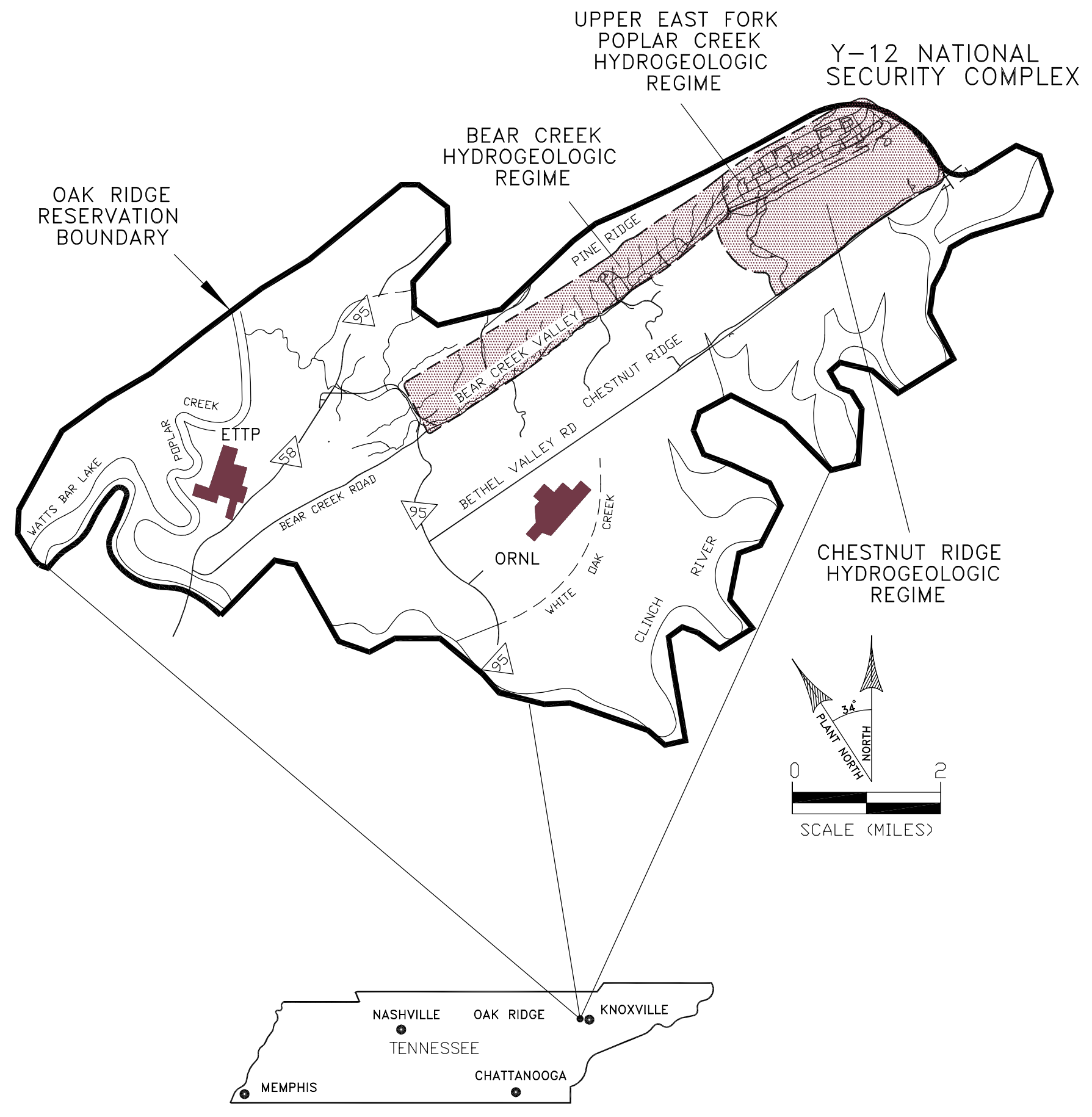

Fig. A.1. Hydrogeologic regimes at the Y-12 National Security Complex. 


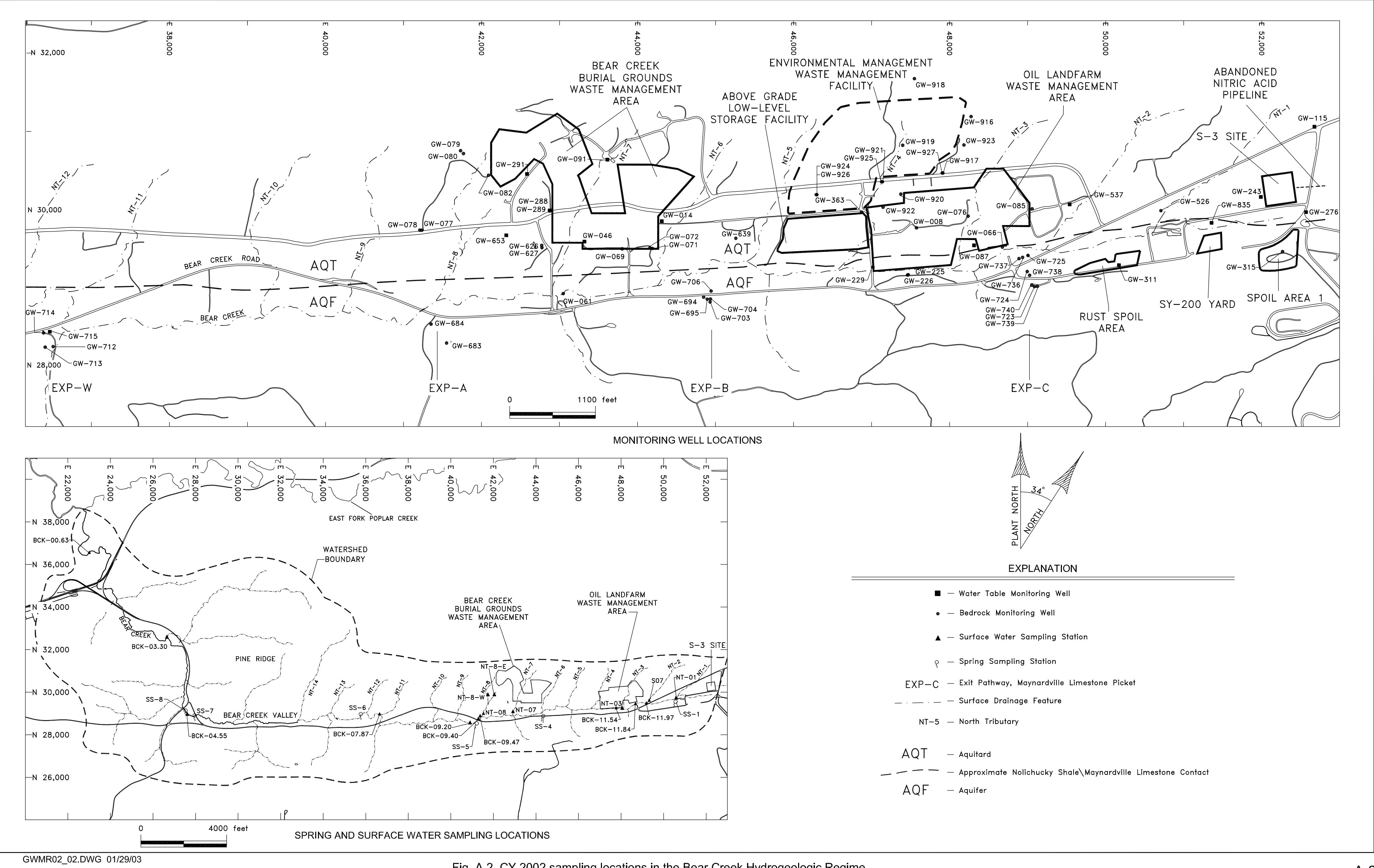

Fig. A.2. CY 2002 sampling locations in the Bear Creek Hydrogeologic Regime. 


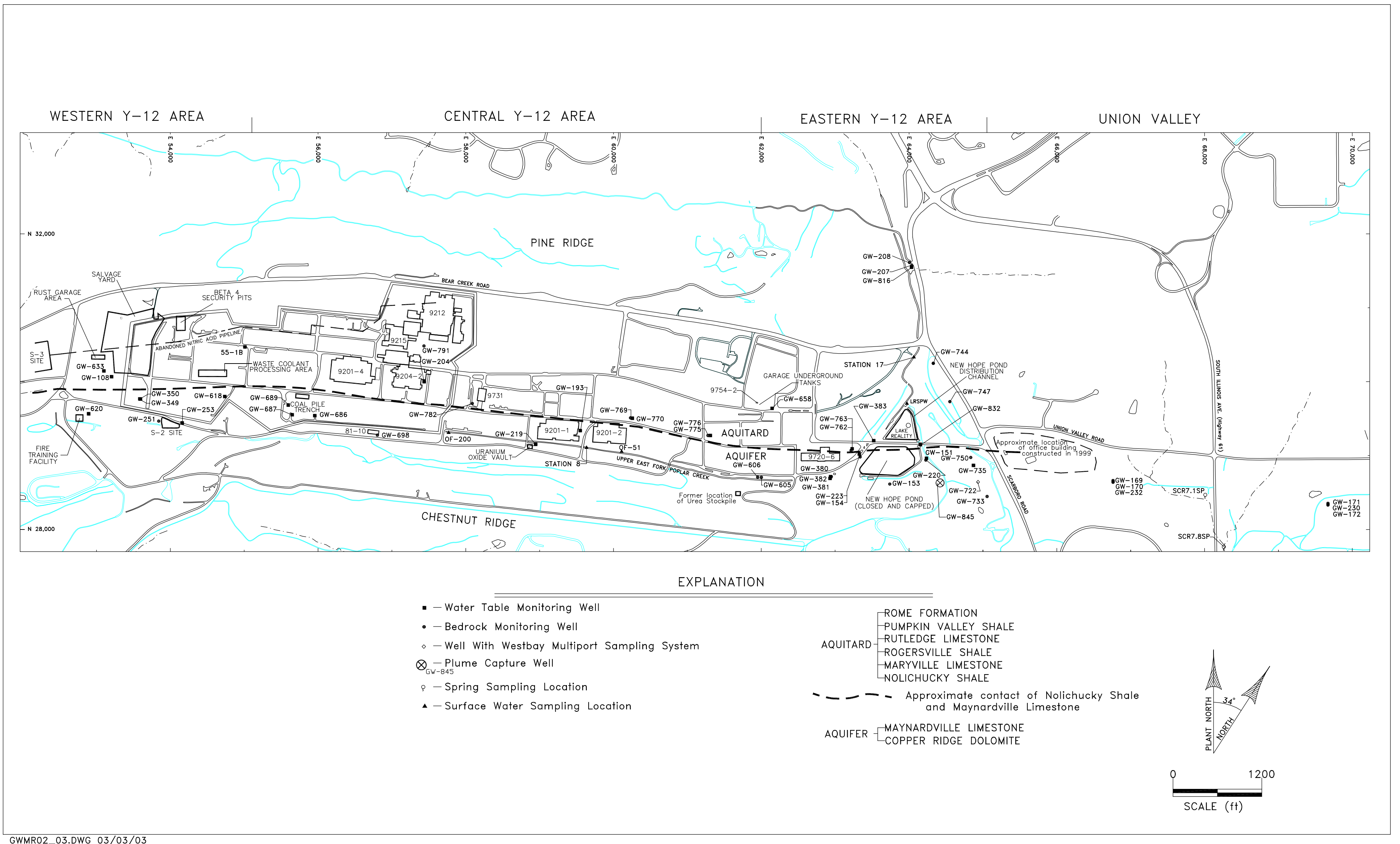




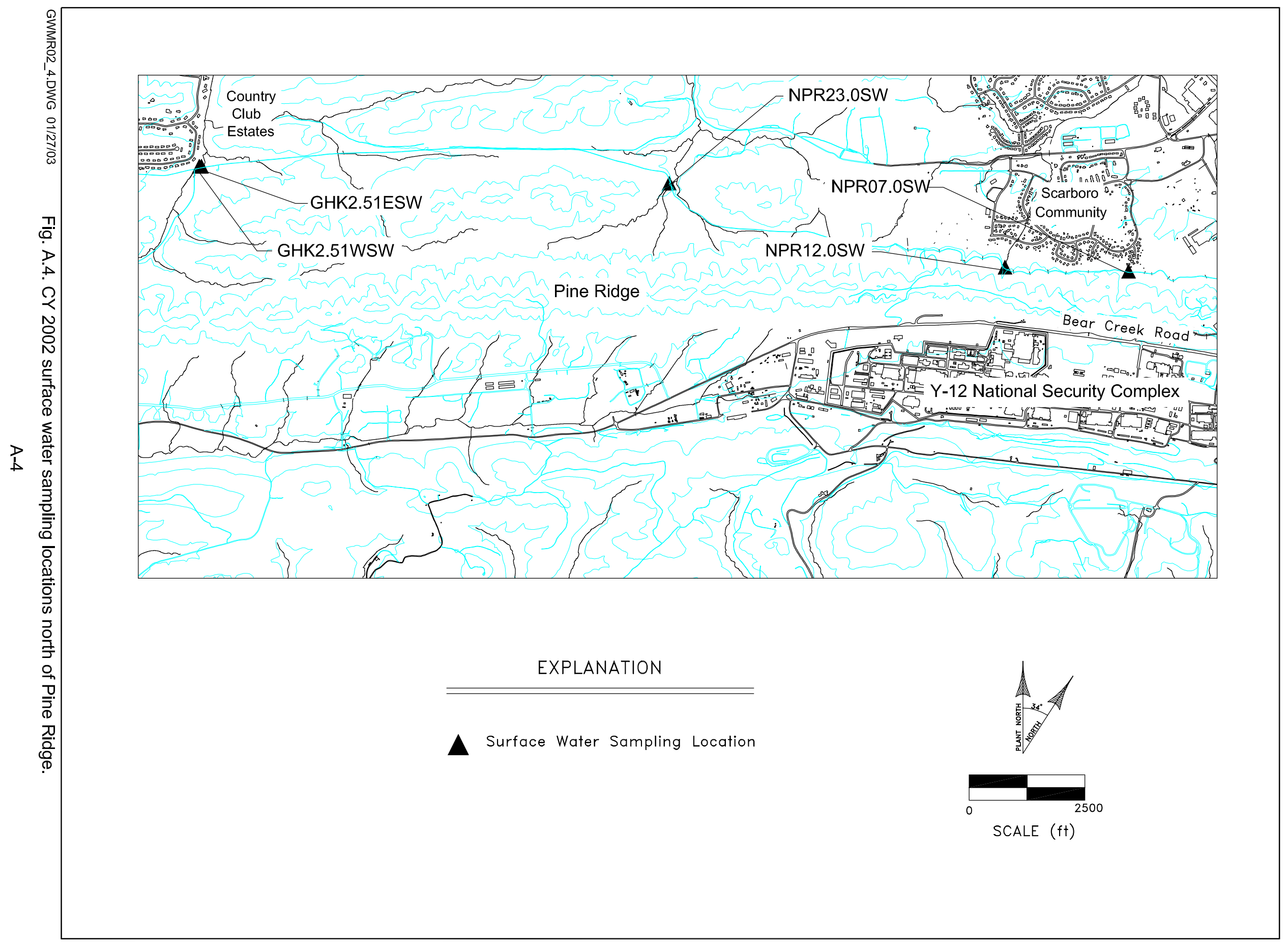


Elevation

(ft msl)

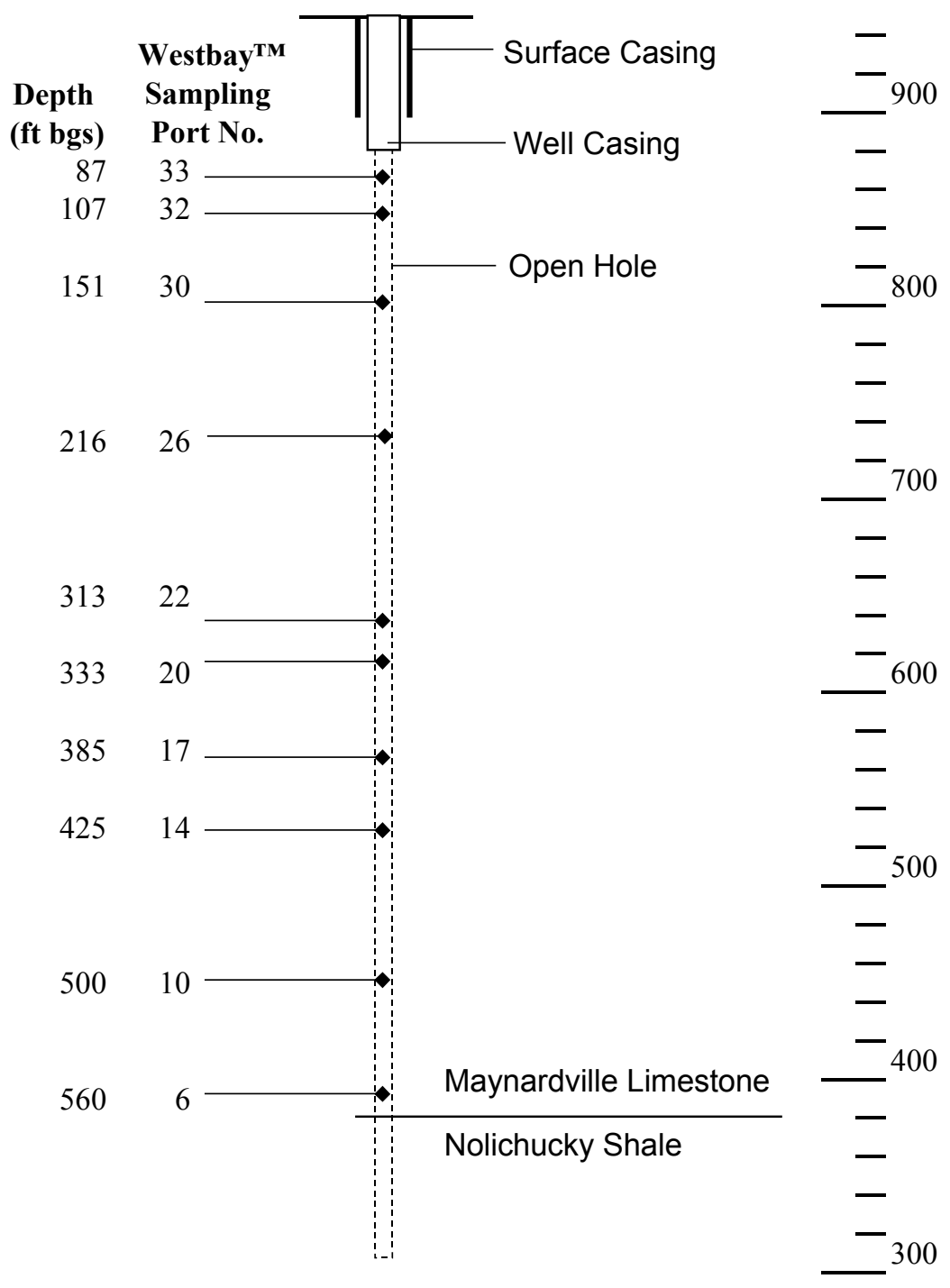




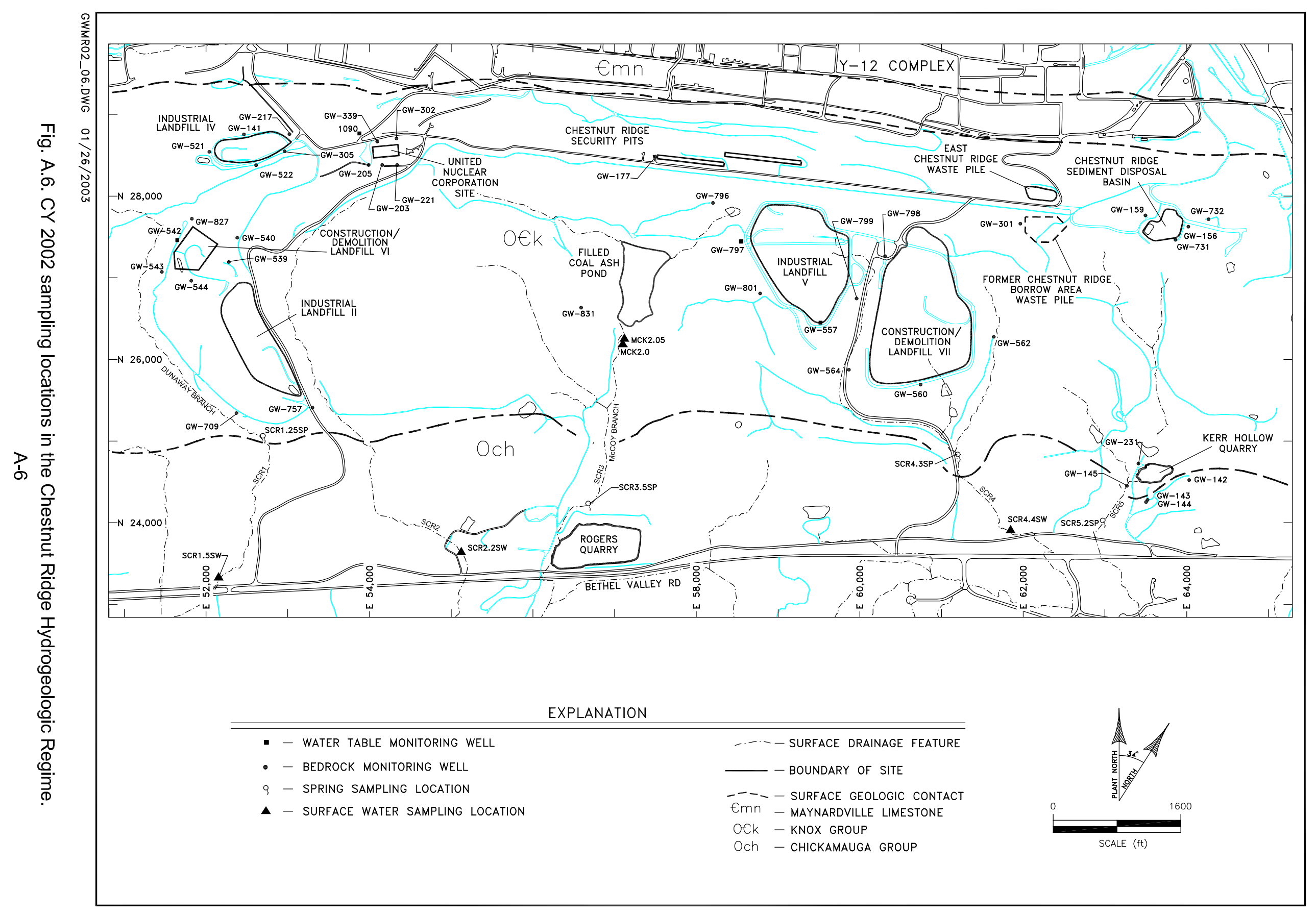



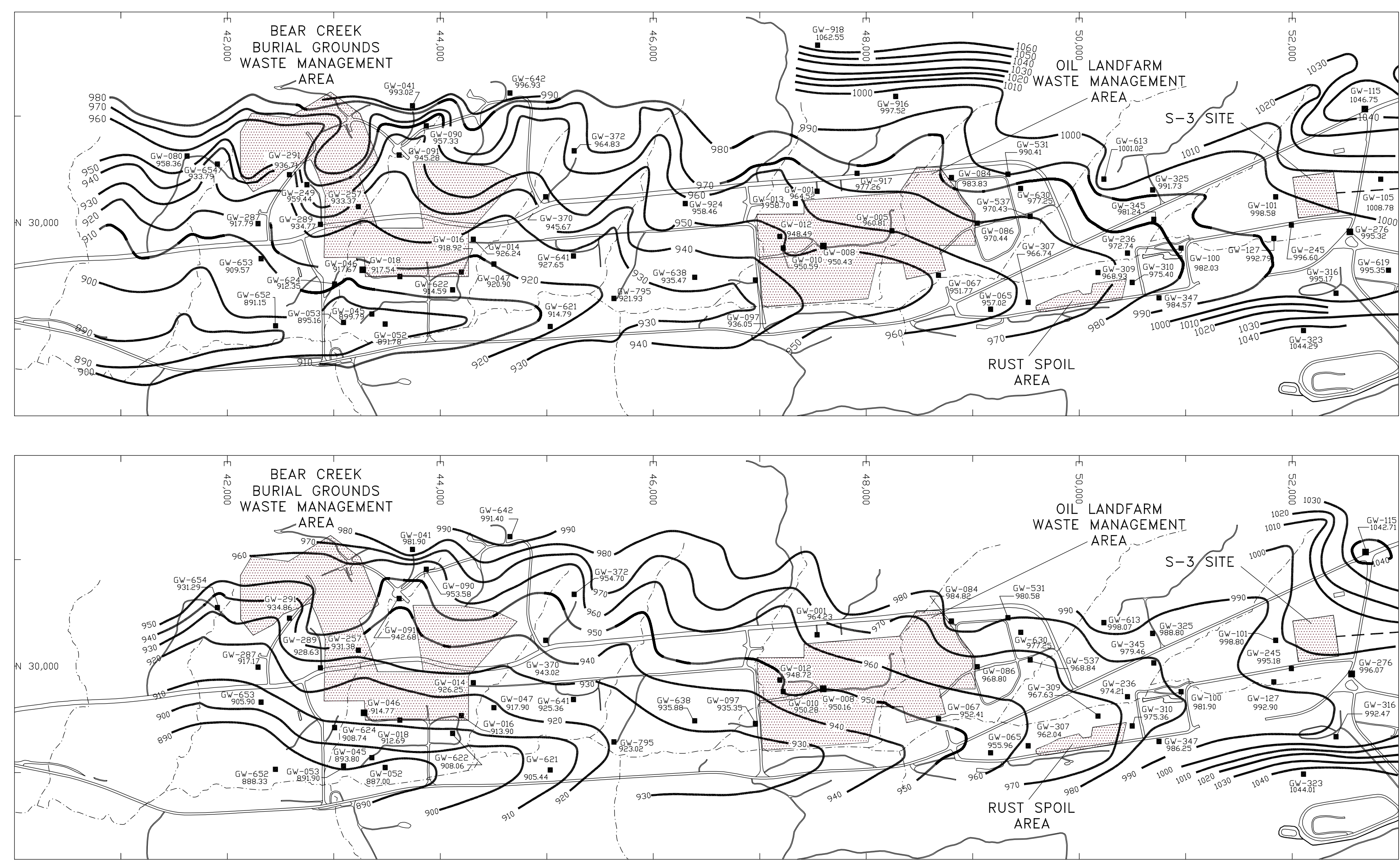

EXPLANATION

- WATER TABLE INTERVAL MONITORING WELL

VVEL ISOPLETH (ft ms) 

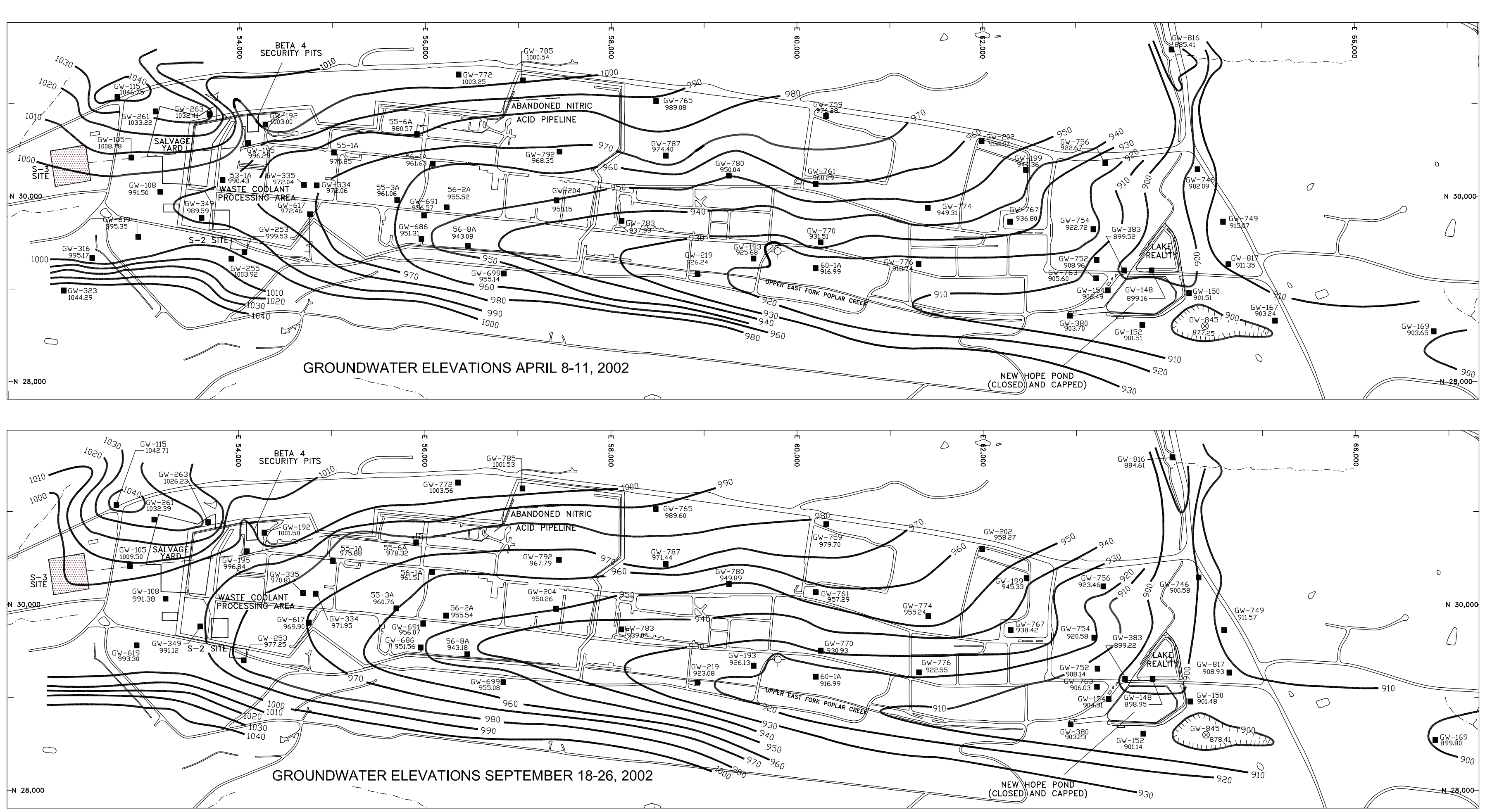

EXPLANATION

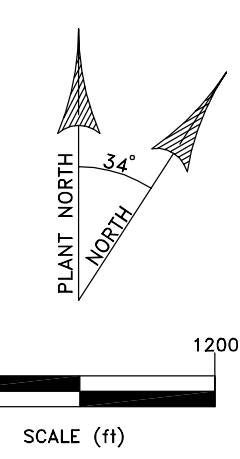

- WATER TABLE INTERVAL MONITORING WELL

GW-845 - EXTRACTION WELL AND APPROXIMATE ZONE OF INFLUENCE

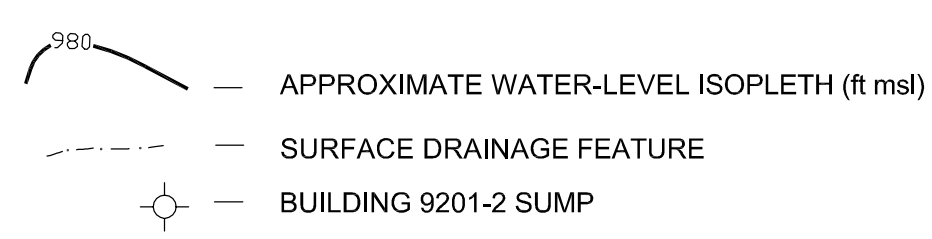



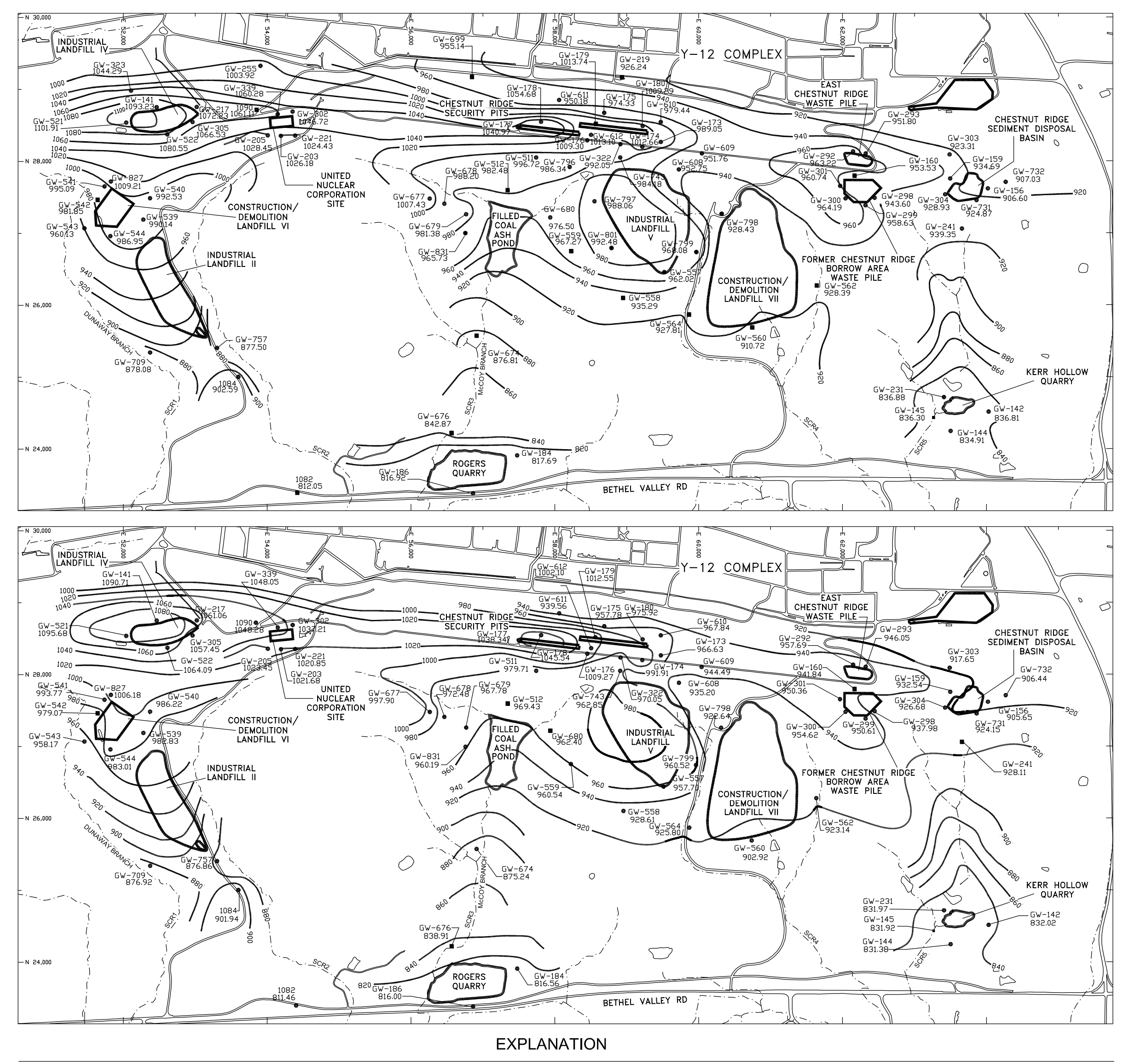

EXPLANATION

WATER TABLE INTERVAL MONITORING WELL
BEDROCK INTERVAL MONITORING WELL

$\begin{array}{cc}920 & \text { WATER-LEVEL ISOPLETH (ft mSI) } \\ -\cdots & \text { SURFAE DRAINAGE FEATURE }\end{array}$

GROUNDWATER ELEVATIONS
APRIL 8-10, 2002

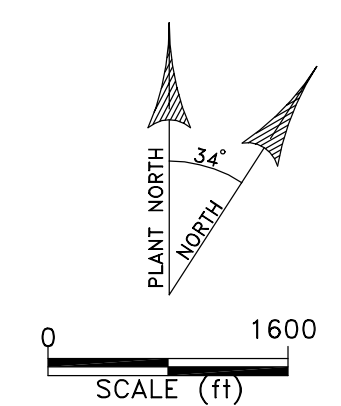

GROUNDWATER ELEVATIONS
SEPTEMBER 17-26. 2002 
APPENDIX B

TABLES 
Table B.1. Summary of CY 2002 sampling and analysis plan addenda

\begin{tabular}{|c|c|l|}
\hline $\begin{array}{c}\text { Addendum } \\
\text { No. }\end{array}$ & $\begin{array}{c}\text { Effective } \\
\text { Date }\end{array}$ & \multicolumn{1}{|c|}{ Modification to the CY 2002 Sampling and Analysis Plan ${ }^{1}$} \\
\hline $2002-01$ & $01 / 01 / 02$ & $\begin{array}{l}\text { Removed well GW-688 (sample group EF-2) from CY 2002 monitoring schedules because } \\
\text { the well was destroyed. }\end{array}$ \\
\hline $2002-02$ & $01 / 01 / 02$ & Added Bottle Lists for STD, RAD(2,3) and STD, RAD(12) to Appendix C of the SAP. \\
\hline $2002-03$ & $06 / 12 / 02$ & $\begin{array}{l}\text { Resampled two wells (GW-763 and GW-381) in sample group EF-1 during the second } \\
\text { quarter for volatile organics analyses. }\end{array}$ \\
\hline $2002-04$ & $08 / 09 / 02$ & $\begin{array}{l}\text { Removed wells GW-076 and GW-087 (sample group BC-5) from the third quarter sampling } \\
\text { monitoring schedule because they were plugged and abandoned. }\end{array}$ \\
\hline $2002-05$ & $11 / 14 / 02$ & Added wells GW-686, GW-687, and GW-689 to the fourth quarter monitoring schedule. \\
\hline
\end{tabular}

Note:

1 Modification to the Y-12 Plant Groundwater Protection Program Groundwater and Surface Water Sampling and Analysis Plan for Calendar Year 2002 (BWXT 2001a). 
B-2 
Table B.2. CY 2002 groundwater and surface water sampling dates in the Bear Creek Hydrogeologic Regime

\begin{tabular}{|c|c|c|c|c|c|c|c|c|c|}
\hline \multirow{2}{*}{\multicolumn{2}{|c|}{ BJC $^{1}$}} & \multicolumn{8}{|c|}{ CERCLA ROD (! ), Quarterly (Q), and Baseline (" ) Monitoring } \\
\hline & & \multicolumn{8}{|c|}{ RCRA Post-Closure Corrective Action Monitoring } \\
\hline \multirow{2}{*}{\multicolumn{2}{|c|}{ GWPP $^{2}$}} & \multicolumn{7}{|c|}{ DOE Order 5400.1 Exit Pathway/Perimeter Monitoring } & \\
\hline & & \multicolumn{8}{|c|}{ DOE Order 5400.1 Surveillance Monitoring } \\
\hline \multirow{2}{*}{$\begin{array}{c}\text { Sampling } \\
\text { Point }^{3}\end{array}$} & \multirow{2}{*}{$\begin{array}{l}\text { Sampling } \\
\text { Location }^{4}\end{array}$} & \multicolumn{4}{|c|}{ CY 2002 Sampling Date ${ }^{5}$} & & & & \\
\hline & & 1st Quarter & 2nd Quarter & 3rd Quarter & 4th Quarter & & & & \\
\hline BCK-00.63 & EXP-SW & $01 / 08 / 02$ & . & $07 / 10 / 02$ & . & & $!$ & & \\
\hline BCK-03.30 & EXP-SW & $03 / 12 / 02$ & . & $09 / 09 / 02$ & . & & & & $!$ \\
\hline BCK-04.55 & EXP-SW & $01 / 09 / 02$ & . & $07 / 10 / 02$ & . & & $!$ & & \\
\hline BCK-07.87 & EXP-SW & $01 / 08 / 02$ & . & $07 / 10 / 02$ & . & & $!$ & & \\
\hline BCK-09.20 & EXP-SW & $03 / 11 / 02$ & . & $09 / 09 / 02$ & . & & & & $!$ \\
\hline BCK-09.40 & EXP-SW & $01 / 09 / 02$ & 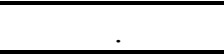 & 07/11/02 D & - & & $!$ & & \\
\hline BCK-09.47 & EXP-SW & $03 / 11 / 02$ & . & Dry & . & & & & $!$ \\
\hline BCK-11.54 & EXP-SW & $03 / 11 / 02$ & . & $09 / 09 / 02$ & . & & & & $!$ \\
\hline BCK-11.84 & EXP-SW & $03 / 11 / 02$ & . & Dry &. & & & & $!$ \\
\hline BCK-11.97 & EXP-SW & $01 / 09 / 02$ & . & $07 / 11 / 02$ & . & & $!$ & & \\
\hline GW-008 & OLF & $01 / 07 / 02$ & . & $07 / 02 / 02$ & . & & & $!$ & \\
\hline GW-014 & $\mathrm{BG}$ & 03/14/02 D & . & $08 / 12 / 02$ & . & $!$ & & & \\
\hline GW-046 & $\mathrm{BG}$ & $01 / 07 / 02$ & . & $07 / 02 / 02$ & . & & & $!$ & \\
\hline GW-061 & $\mathrm{BG}$ & $03 / 12 / 02$ & . & $08 / 05 / 02$ & . & $!$ & & & \\
\hline GW-066 & OLF & $03 / 25 / 02$ & . & 08/13/02 D & . & $!$ & & & \\
\hline GW-069 & $\mathrm{BG}$ & $03 / 11 / 02$ & . & $08 / 05 / 02$ & . & $!$ & & & \\
\hline GW-071 & $\mathrm{BG}$ & $03 / 12 / 02$ & . & 08/06/02 D & . & $!$ & & & \\
\hline GW-072 & $\mathrm{BG}$ & $03 / 11 / 02$ & . & $08 / 06 / 02$ & . & $!$ & & & \\
\hline GW-076 & OLF & $03 / 19 / 02$ & . & . & . & $!$ & & & \\
\hline GW-077 & $\mathrm{BG}$ & $02 / 26 / 02$ & . & $08 / 08 / 02$ & . & & & & $!$ \\
\hline GW-078 & $\mathrm{BG}$ & $02 / 26 / 02$ & . & $08 / 08 / 02$ & . & & & & $!$ \\
\hline GW-079 & $\mathrm{BG}$ & $02 / 26 / 02 \mathrm{D}$ & . & 08/07/02 D & . & & & & $!$ \\
\hline GW-080 & $\mathrm{BG}$ & $02 / 26 / 02$ & $\therefore$ & $08 / 07 / 02$ & . & & & & $!$ \\
\hline GW-082 & $\mathrm{BG}$ & $02 / 12 / 02$ & . & $07 / 29 / 02$ & . & $!$ & & & \\
\hline GW-085 & OLF & $02 / 18 / 02$ & . & $07 / 31 / 02$ & . & $!$ & & & \\
\hline GW-087 & OLF & $03 / 25 / 02$ & . & . & . & $!$ & & & \\
\hline GW-091 & $\mathrm{BG}$ & $03 / 14 / 02$ & . & $08 / 08 / 02$ & . & $!$ & & & \\
\hline GW-115 & S3 & $01 / 07 / 02$ & . & $07 / 08 / 02$ & . & & & $!$ & \\
\hline GW-225 & OLF & $02 / 19 / 02$ & . & $08 / 01 / 02$ & . & $!$ & & & \\
\hline GW-226 & OLF & $02 / 19 / 02$ & $\dot{0}$ & $08 / 01 / 02$ & . & $!$ & & & \\
\hline GW-229 & OLF & 03/19/02 D & . & $08 / 12 / 02$ & . & $!$ & & & \\
\hline GW-243 & S3 & $03 / 21 / 02$ & $x$ & $08 / 13 / 02$ & . & $!$ & & & \\
\hline GW-276 & $\mathrm{S3}$ & $01 / 07 / 02$ & - & $07 / 08 / 02$ & - & 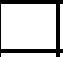 & & $!$ & \\
\hline GW-288 & $\mathrm{BG}$ & $03 / 13 / 02$ & 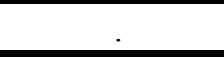 & $08 / 07 / 02$ & . & $!$ & & & \\
\hline
\end{tabular}


Table B.2 (continued)

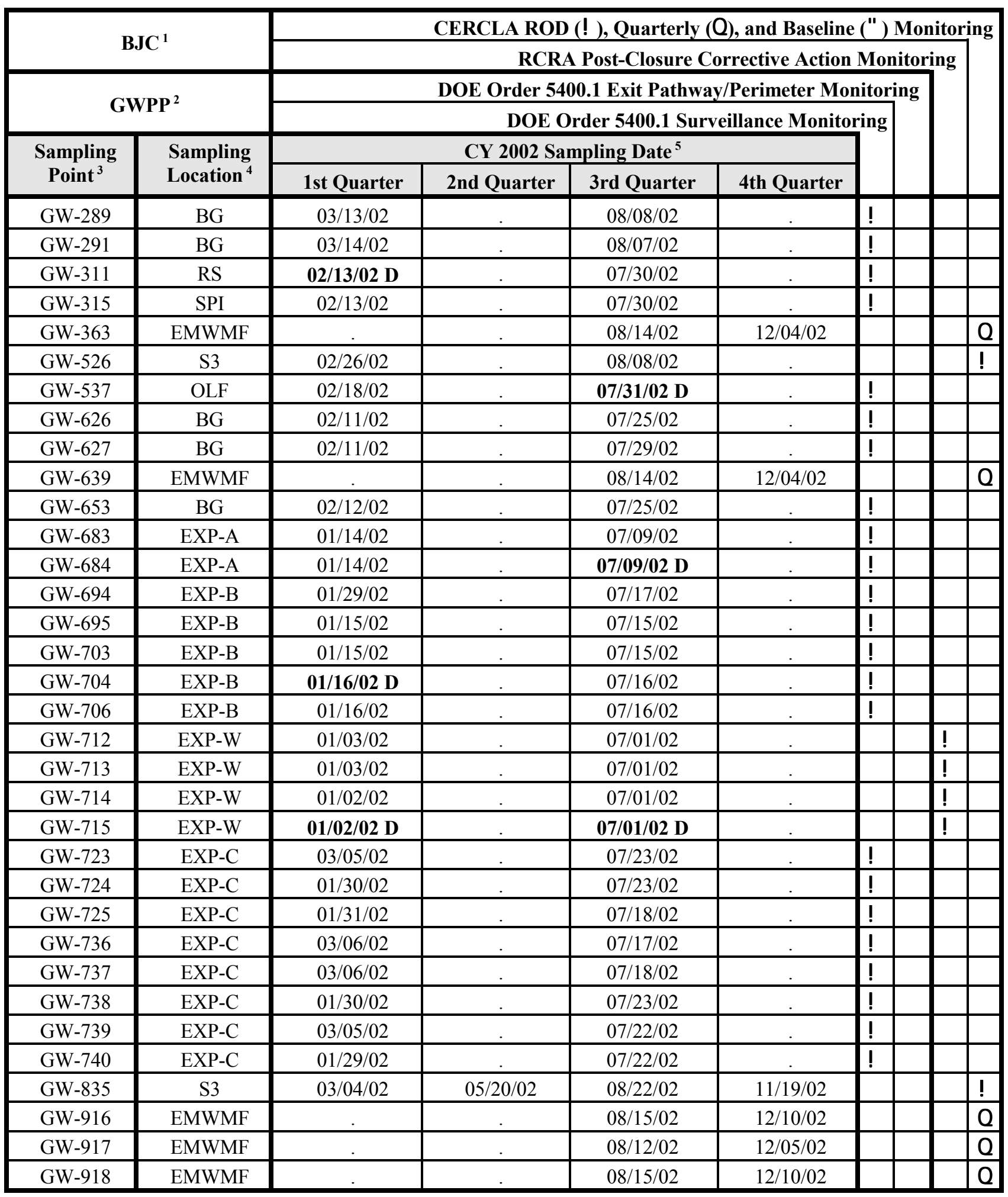


Table B.2 (continued)

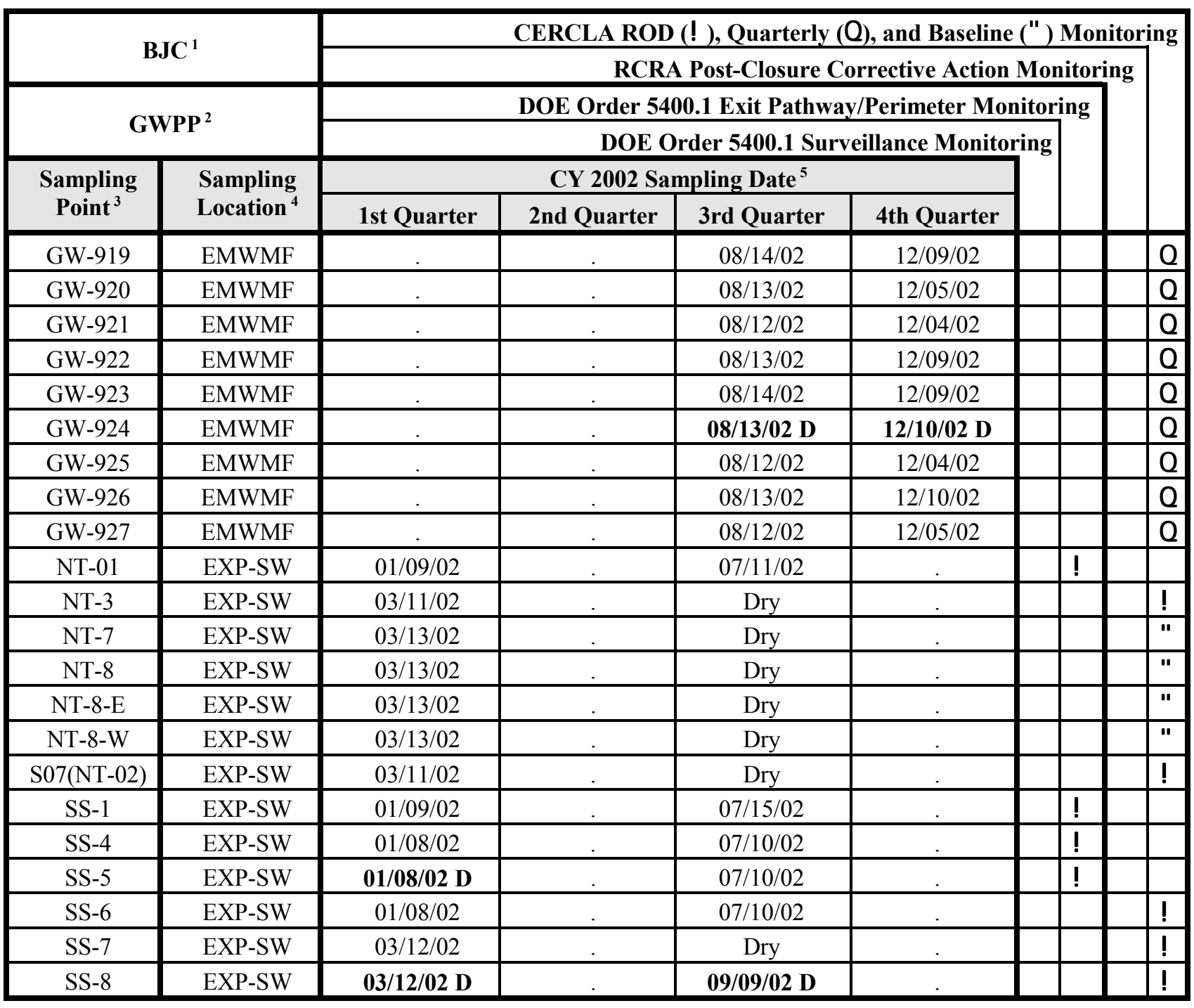

Notes:

1 Groundwater and surface water sampling performed for monitoring programs managed by Bechtel Jacobs Company LLC (BJC).

2 Groundwater and surface water sampling performed for the Y-12 Groundwater Protection Program (GWPP), managed by BWXT Y-12, L.L.C.

3 BCK - Bear Creek Kilometer

GW - Groundwater Monitoring Well

NT - Northern Tributary (to Bear Creek)

S07 - Surface water location in NT-2

SS - Spring sampling location (south side of Bear Creek) 


\section{Table B.2 (continued)}

Notes: (continued)

4

BG - Bear Creek Burial Grounds Waste Management Area

EMWMF - Environmental Management Waste Management Facility

EXP-A - Exit Pathway (Maynardville Limestone) Picket A

EXP-B - Exit Pathway Picket B

EXP-C - Exit Pathway Picket C

EXP-W - Exit Pathway Picket W

EXP-SW - Exit Pathway (Bear Creek) Surface Water

OLF - Oil Landfarm Waste Management Area

RS - Rust Spoil Area

SPI - Spoil Area I

S3 - S-3 Site

5

- - Not sampled

D - Duplicate sample collected on specified date (shown in bold typeface) 
Table B.3. CY 2002 groundwater and surface water sampling dates in the Upper East Fork Poplar Creek Hydrogeologic Regime

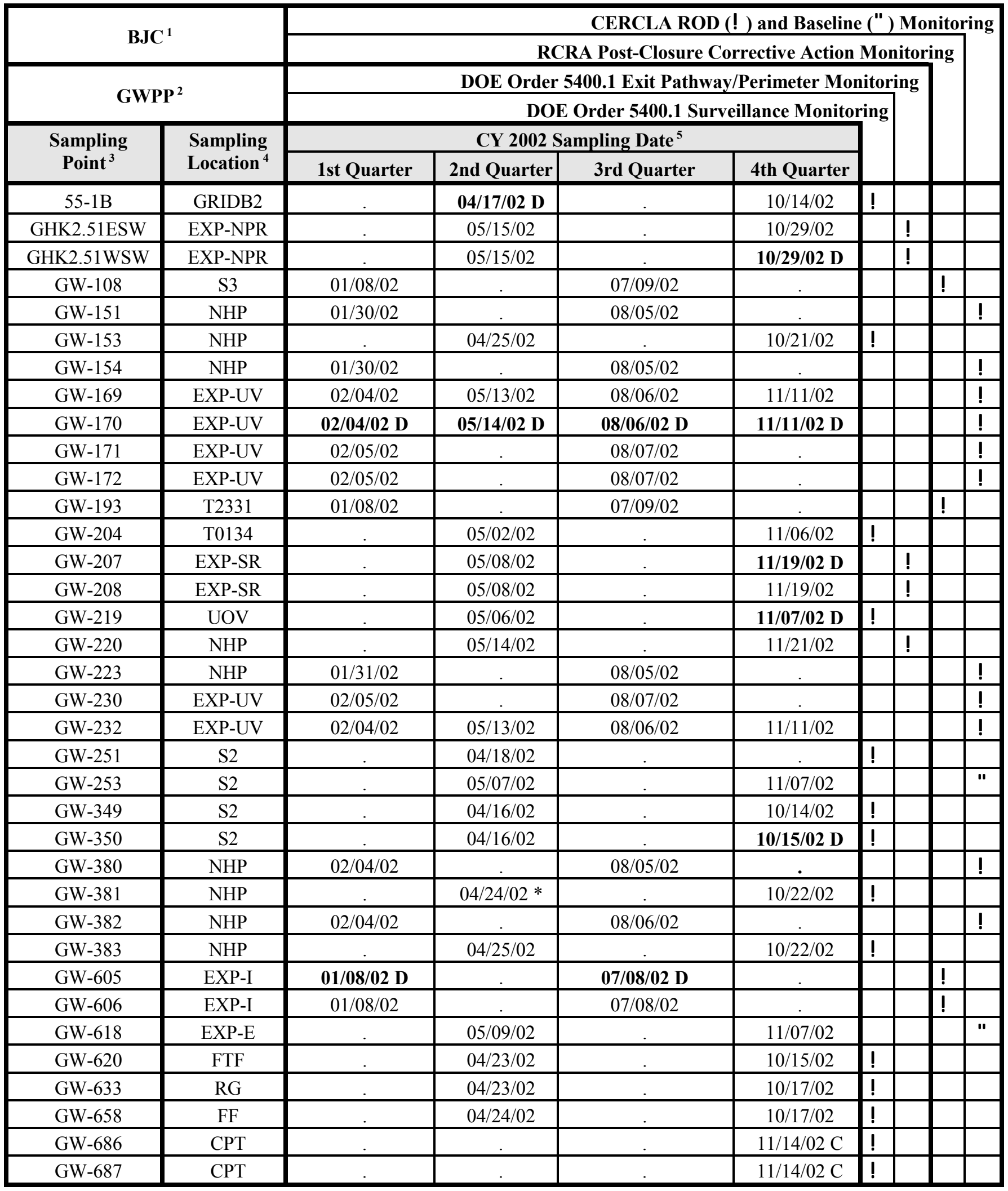


Table B.3 (continued)

\begin{tabular}{|c|c|c|c|c|c|c|c|c|c|}
\hline \multirow{2}{*}{\multicolumn{2}{|c|}{ BJC $^{1}$}} & & & \multicolumn{6}{|c|}{ CERCLA ROD (! ) and Baseline (" ) Monitoring } \\
\hline & & \multicolumn{8}{|c|}{ RCRA Post-Closure Corrective Action Monitoring } \\
\hline \multirow{2}{*}{\multicolumn{2}{|c|}{ GWPP $^{2}$}} & \multicolumn{7}{|c|}{ DOE Order 5400.1 Exit Pathway/Perimeter Monitoring } & \\
\hline & & \multicolumn{7}{|c|}{ DOE Order 5400.1 Surveillance Monitoring } & \\
\hline \multirow{2}{*}{$\begin{array}{c}\text { Sampling } \\
\text { Point }^{3}\end{array}$} & \multirow{2}{*}{$\begin{array}{l}\text { Sampling } \\
\text { Location }^{4}\end{array}$} & \multicolumn{4}{|c|}{ CY 2002 Sampling Date ${ }^{5}$} & & & & \\
\hline & & 1st Quarter & 2nd Quarter & 3rd Quarter & 4th Quarter & & & & \\
\hline GW-689 & CPT & & & & $11 / 14 / 02 \mathrm{C}$ & $!$ & & & \\
\hline GW-698 & $\mathrm{B} 8110$ & & 05/02/02 D & & $11 / 07 / 02$ & $!$ & & & \\
\hline GW-722-06 & EXP-J & $02 / 04 / 02$ & & $07 / 15 / 02$ & . & & $!$ & & \\
\hline GW-722-06 & EXP-J & . & $05 / 23 / 02$ & & $11 / 07 / 02$ & & & & $!$ \\
\hline GW-722-10 & EXP-J & $02 / 06 / 02$ & & $07 / 16 / 02$ & . & & $!$ & & \\
\hline GW-722-10 & EXP-J & & $05 / 29 / 02$ & & $11 / 12 / 02$ & & & & $!$ \\
\hline GW-722-14 & EXP-J & $02 / 08 / 02$ & & $07 / 17 / 02$ & . & & $!$ & & \\
\hline GW-722-14 & EXP-J & & $05 / 30 / 02$ & & $11 / 13 / 02$ & & & & $!$ \\
\hline GW-722-17 & EXP-J & $02 / 08 / 02$ & & $07 / 17 / 02$ & $\dot{0}$ & & $!$ & & \\
\hline GW-722-17 & EXP-J &. & $06 / 03 / 02$ & & $11 / 13 / 02$ & & & & $!$ \\
\hline GW-722-20 & EXP-J & . & & 07/17/02 D & . & & $!$ & & \\
\hline GW-722-20 & EXP-J & . & $05 / 30 / 02$ & 5 & $11 / 12 / 02$ & & & & $!$ \\
\hline GW-722-22 & EXP-J & 02/07/02 D & & & . & & $!$ & & \\
\hline GW-722-22 & EXP-J & . & $05 / 30 / 02$ & & $11 / 12 / 02$ & & & & $!$ \\
\hline GW-722-26 & EXP-J & $02 / 05 / 02$ & & $07 / 15 / 02$ & & & $!$ & & \\
\hline GW-722-26 & EXP-J & . & 05/28/02 D & & 11/11/02 D & & & & $!$ \\
\hline GW-722-30 & EXP-J & $02 / 05 / 02$ & & $07 / 15 / 02$ & - & & $!$ & & \\
\hline GW-722-30 & EXP-J & . & $05 / 28 / 02$ & - & $11 / 11 / 02$ & & & & $!$ \\
\hline GW-722-32 & EXP-J & $02 / 05 / 02$ & & $07 / 15 / 02$ & . & & $!$ & & \\
\hline GW-722-32 & EXP-J & 5 & $05 / 28 / 02$ & & $11 / 12 / 02$ & & & & $!$ \\
\hline GW-722-33 & EXP-J & $02 / 06 / 02$ & & $07 / 16 / 02$ & . & & $!$ & & \\
\hline GW-722-33 & EXP-J & . & $05 / 29 / 02$ & & $11 / 12 / 02$ & & & & $!$ \\
\hline GW-733 & EXP-J & $01 / 08 / 02$ & & $07 / 09 / 02$ & & & & $!$ & \\
\hline GW-735 & EXP-J & - & $05 / 14 / 02$ & $\cdot$ & $11 / 21 / 02$ & & $!$ & & \\
\hline GW-744 & GRIDK1 & . & $05 / 07 / 02$ & . & $11 / 18 / 02$ & & $!$ & & \\
\hline GW-747 & GRIDK2 & . & $05 / 09 / 02$ & & $11 / 20 / 02$ & & $!$ & & \\
\hline GW-750 & EXP-J & . & $05 / 05 / 02$ & & $11 / 20 / 02$ & & $!$ & & \\
\hline GW-762 & GRIDJ3 & 01/31/02 D & & 07/31/02 D & & & & & $!$ \\
\hline GW-763 & GRIDJ3 & - & $04 / 18 / 02 *$ & & $10 / 21 / 02$ & $!$ & & & \\
\hline GW-769 & GRIDG3 & . & $04 / 29 / 02$ & & $10 / 30 / 02$ & $!$ & & & \\
\hline GW-770 & GRIDG3 & . & $04 / 29 / 02$ & & $10 / 30 / 02$ & $!$ & & & \\
\hline GW-775 & GRIDH3 & . & $04 / 30 / 02$ & & $10 / 31 / 02$ & $!$ & & & \\
\hline GW-776 & GRIDH3 & . & $04 / 30 / 02$ & . & $10 / 31 / 02$ & $!$ & & & \\
\hline GW-782 & GRIDE3 & . & $05 / 01 / 02$ & & $11 / 04 / 02$ & $!$ & & & \\
\hline GW-791 & GRIDD2 & . & $05 / 01 / 02$ & & $11 / 06 / 02$ & $!$ & & & \\
\hline GW-816 & EXP-SR & & $05 / 07 / 02$ & & $11 / 18 / 02$ & & $!$ & & \\
\hline GW-832 & NHP & $01 / 31 / 02$ & & $08 / 05 / 02$ & & & & & $!$ \\
\hline
\end{tabular}


Table B.3 (continued)

\begin{tabular}{|c|c|c|c|c|c|c|c|c|}
\hline \multirow{2}{*}{\multicolumn{2}{|c|}{$\mathbf{B J C}^{1}$}} & \multicolumn{7}{|c|}{ CERCLA ROD (! ) and Baseline (") Monitoring } \\
\hline & & \multicolumn{7}{|c|}{ RCRA Post-Closure Corrective Action Monitoring } \\
\hline \multirow{2}{*}{\multicolumn{2}{|c|}{ GWPP $^{2}$}} & \multicolumn{7}{|c|}{ DOE Order 5400.1 Exit Pathway/Perimeter Monitoring } \\
\hline & & \multicolumn{7}{|c|}{ DOE Order 5400.1 Surveillance Monitoring } \\
\hline \multirow{2}{*}{$\begin{array}{l}\text { Sampling } \\
\text { Point }^{3}\end{array}$} & \multirow{2}{*}{$\begin{array}{l}\text { Sampling } \\
\text { Location }^{4}\end{array}$} & \multicolumn{4}{|c|}{ CY 2002 Sampling Date ${ }^{5}$} & & & \\
\hline & & 1st Quarter & 2nd Quarter & 3rd Quarter & 4th Quarter & & & \\
\hline LRSPW & EXP-SW & & 05/06/02 D & & $11 / 19 / 02$ & & & \\
\hline NPR07.0SW & EXP-NPR & . & $05 / 15 / 02$ & & $10 / 29 / 02$ & & & \\
\hline NPR12.0SW & EXP-NPR & . & 05/15/02 D & . & $10 / 29 / 02$ & & & \\
\hline NPR23.0SW & EXP-NPR & . & $05 / 15 / 02$ & . & $10 / 29 / 02$ & & & \\
\hline OF 51 & EXP-SW & $02 / 14 / 02$ & - & $09 / 03 / 02$ & . & & & 11 \\
\hline OF 200 & EXP-SW & $02 / 01 / 02 \mathrm{~S}$ & - & $08 / 20 / 02 \mathrm{~S}$ & . & & & $"$ \\
\hline SCR7.1SP & EXP-UV & $02 / 11 / 02$ & . & $07 / 11 / 02$ & . & & & $!$ \\
\hline SCR7.8SP & EXP-UV & $02 / 11 / 02$ & & $07 / 11 / 02$ & - & & & $!$ \\
\hline STATION 17 & EXP-SW & $02 / 01 / 02 \mathrm{~S}$ & & $08 / 20 / 02 \mathrm{~S}$ & . & & & " \\
\hline STATION 8 & EXP-SW & $02 / 01 / 02 \mathrm{~S}$ & & $08 / 20 / 02 \mathrm{~S}$ & . & & & $"$ \\
\hline
\end{tabular}

Notes:

1 Groundwater and surface water sampling performed for monitoring programs managed by Bechtel Jacobs Company LLC (BJC).

2 Groundwater and surface water sampling performed for the Y-12 Groundwater Protection Program (GWPP), managed by BWXT Y-12, L.L.C.

GHK - Gum Hollow Branch Kilometer

GW - Groundwater Monitoring Well (also 55-1B)

LRSPW - Outfall of the New Hope Pond distribution channel underdrain

NPR - North of Pine Ridge near the Scarboro Community

$\mathrm{OF}$ - Storm drain outfall

SCR - Spring sampling location in Union Valley

SP - Spring sampling location (suffix)

STATION - Surface water sampling location in Upper East Fork Poplar Creek

SW - Surface water sampling location (suffix)

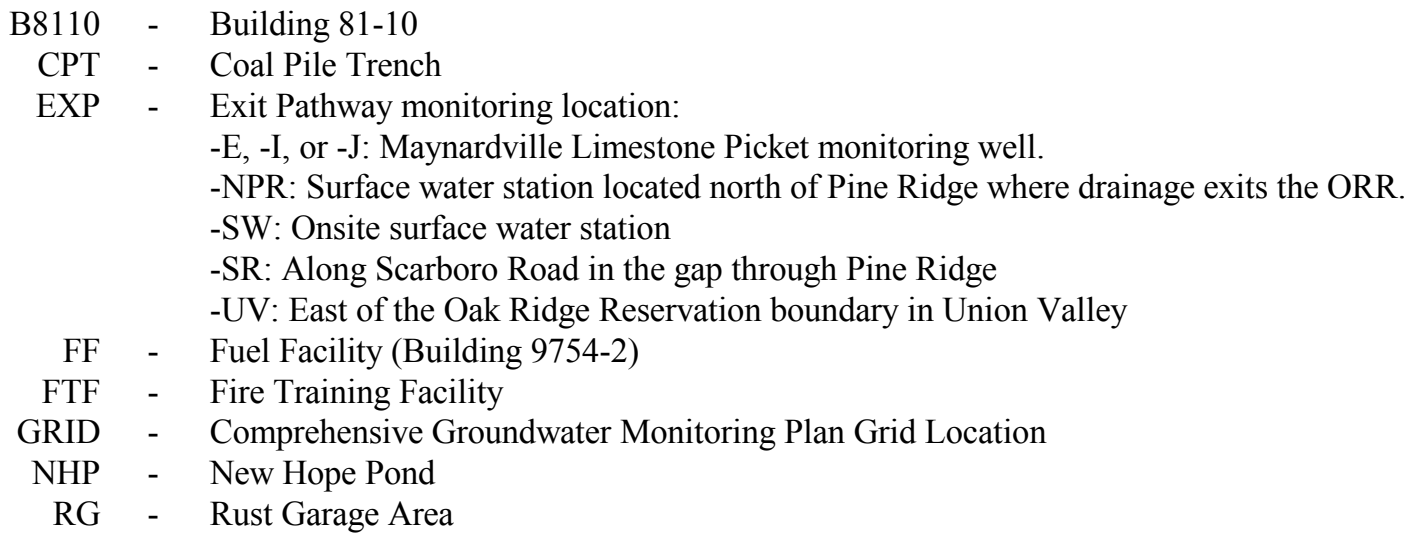




\section{Table B.3 (continued)}

Notes: (continued)

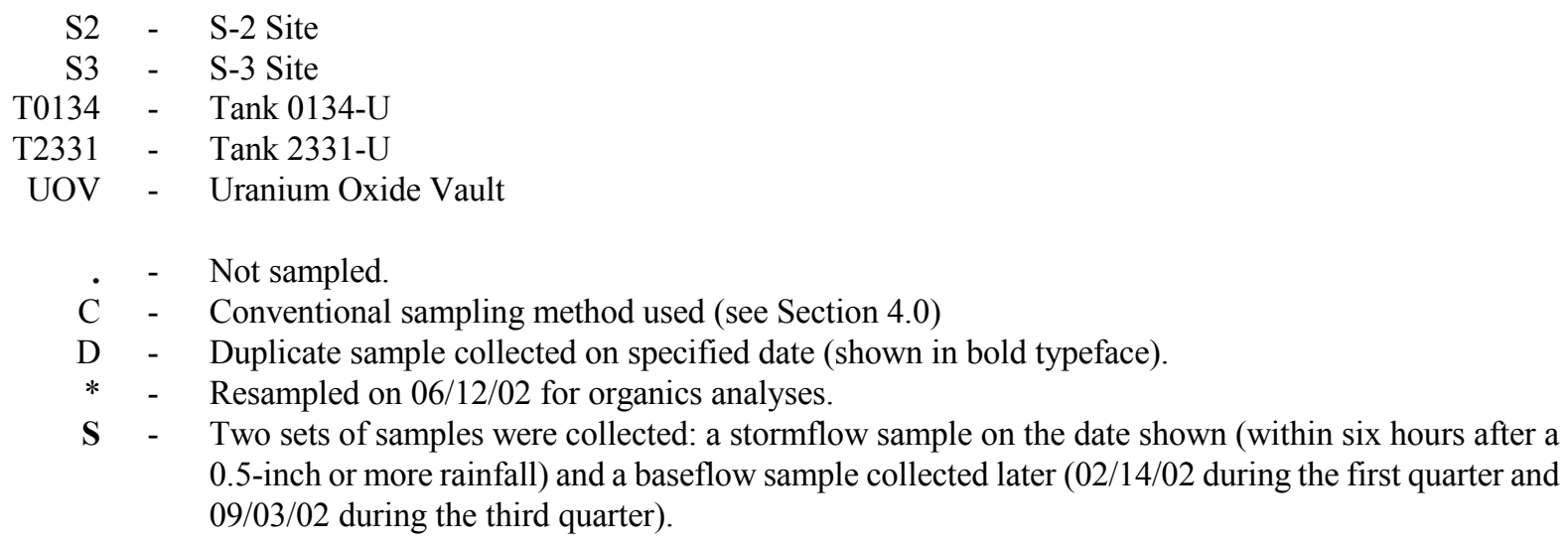

- - Not sampled.

C - Conventional sampling method used (see Section 4.0)

D - Duplicate sample collected on specified date (shown in bold typeface).

* - Resampled on 06/12/02 for organics analyses.

S - Two sets of samples were collected: a stormflow sample on the date shown (within six hours after a 0.5 -inch or more rainfall) and a baseflow sample collected later (02/14/02 during the first quarter and $09 / 03 / 02$ during the third quarter). 
Table B.4. CY 2002 groundwater and surface water sampling dates in the Chestnut Ridge Hydrogeologic Regime

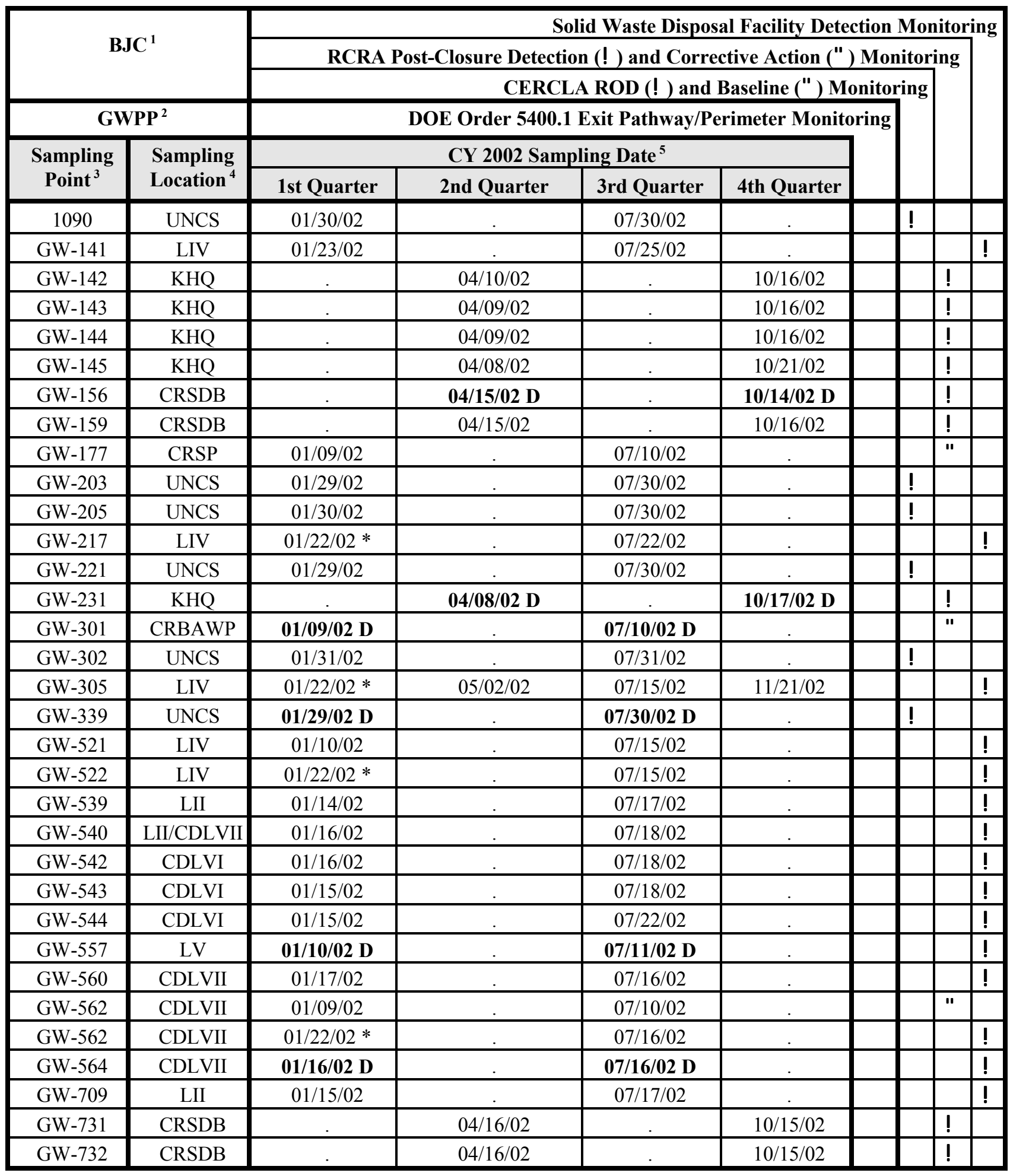


Table B.4 (continued)

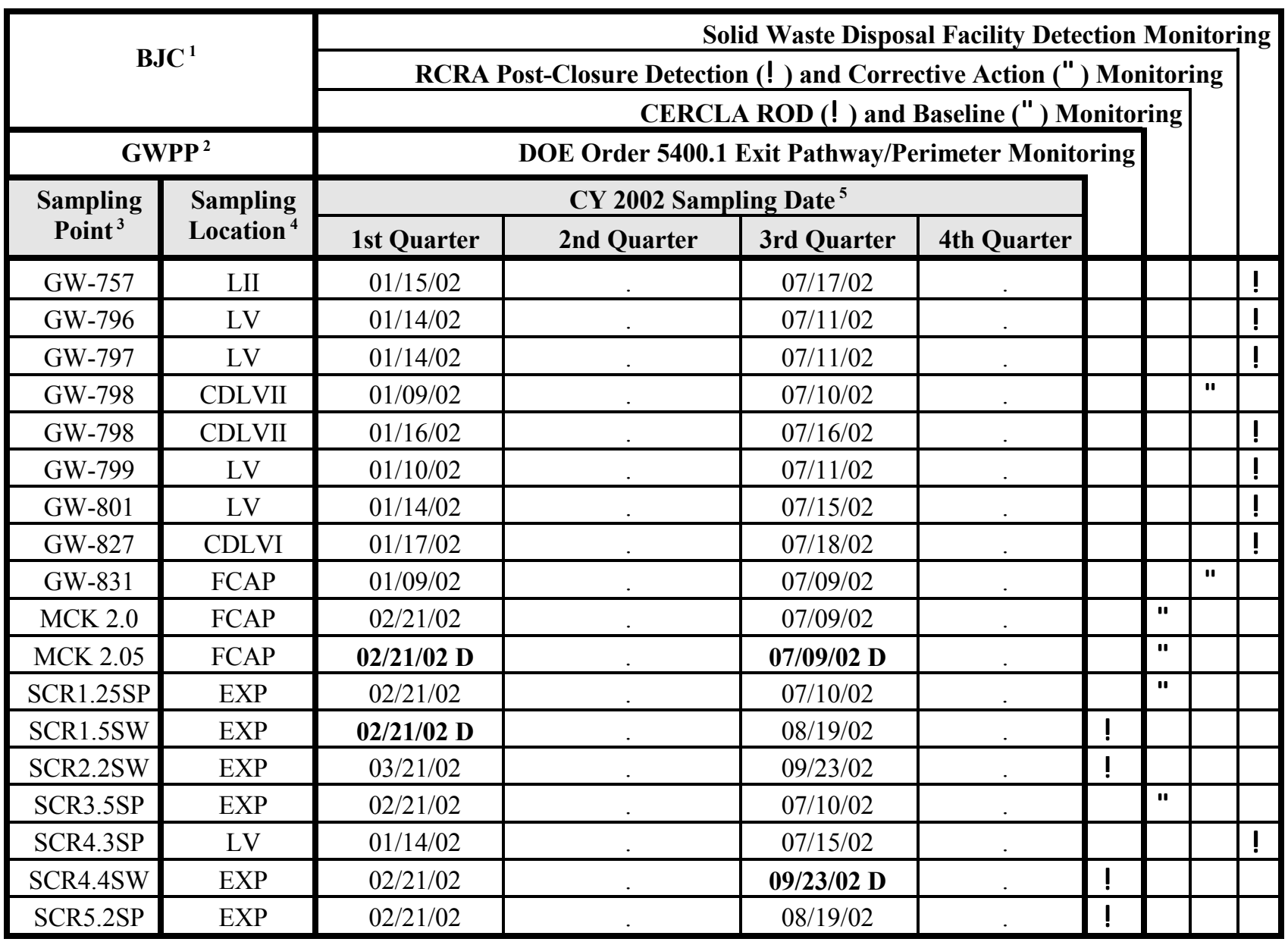

Notes:

1 Groundwater and surface water sampling performed under monitoring programs managed by Bechtel Jacobs Company LLC (BJC).

2 Surface water sampling performed under the Y-12 Groundwater Protection Program (GWPP) managed by BWXT Y-12, L.L.C.

$3 \quad$ GW - Groundwater monitoring well (also 1090)

MCK - McCoy Branch Kilometer

SCR - South Chestnut Ridge (tributary prefix)

SP - Spring sampling location (suffix)

SW - Surface water sampling location (suffix) 


\section{Table B.4 (continued)}

Notes: (continued)

4

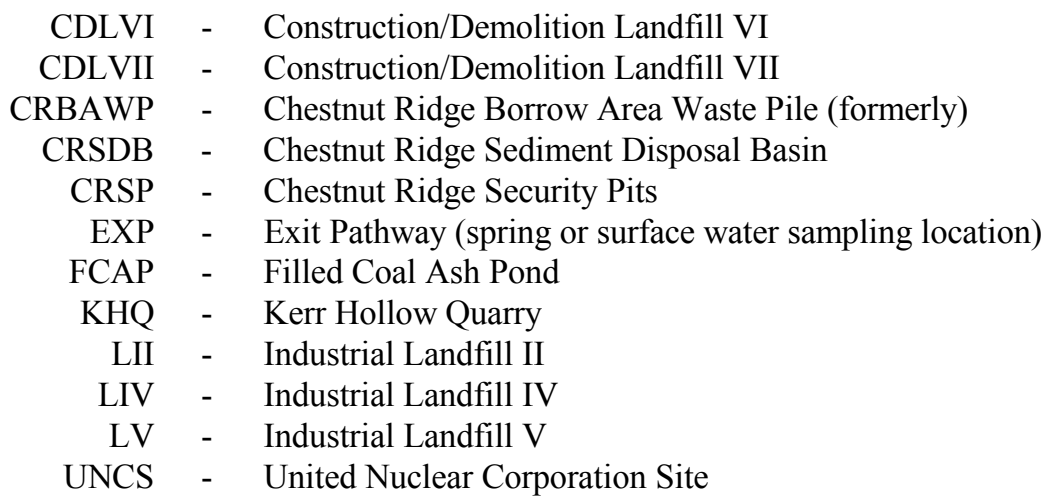

- - Not Sampled.

D - Duplicate sample collected on specified date (shown in bold typeface).

* - Groundwater samples were collected for organics analyses from wells GW-217, GW-305, GW522, and GW-562 on January 28, 2002. 
B-14 
Table B.5. Field measurements and laboratory analytes for CY 2002 groundwater and surface water samples

\begin{tabular}{|c|c|c|c|}
\hline Field Measurements & Analytical Method $^{1}$ & Reporting Limit $^{2}$ & Units $^{3}$ \\
\hline Depth to Water & Y50-71-015 & NA & $\mathrm{ft}$ \\
\hline Water Temperature & Y50-71-001 & NA & centigrade \\
\hline $\mathrm{pH}$ & Y50-71-001 & NA & $\mathrm{pH}$ units \\
\hline Conductivity & Y50-71-001 & NA & $\mu \mathrm{mho} / \mathrm{cm}$ \\
\hline Dissolved Oxygen & Y50-71-001 & $\mathrm{NA}$ & $\mathrm{ppm}$ \\
\hline Oxidation-Reduction Potential & Y/P65-9156 & NA & $\mathrm{mV}$ \\
\hline \multicolumn{4}{|l|}{ Miscellaneous Laboratory Analytes } \\
\hline $\mathrm{pH}$ & SW846-9040 & NA & $\mathrm{pH}$ units \\
\hline Conductivity & SW846-9050 & $\mathrm{NA}$ & $\mu \mathrm{mho} / \mathrm{cm}$ \\
\hline Total Dissolved Solids & EPA-160.1 & 1 & $\mathrm{mg} / \mathrm{L}$ \\
\hline Total Suspended Solids & EPA-160.2 & 1 & $\mathrm{mg} / \mathrm{L}$ \\
\hline Turbidity & EPA-180.1 & 0.1 & NTU \\
\hline \multicolumn{4}{|l|}{ Anions } \\
\hline Alkalinity - HCO3 & EPA-310.1 & 1.0 & $\mathrm{mg} / \mathrm{L}$ \\
\hline Alkalinity - $\mathrm{CO} 3$ & EPA-310.1 & 1.0 & $\mathrm{mg} / \mathrm{L}$ \\
\hline Chloride & EPA-300.0 & 0.2 & $\mathrm{mg} / \mathrm{L}$ \\
\hline Fluoride & EPA-340.2 & 0.1 & $\mathrm{mg} / \mathrm{L}$ \\
\hline Nitrate (as Nitrogen) & EPA-300.0 & 0.028 & $\mathrm{mg} / \mathrm{L}$ \\
\hline Sulfate & EPA-300.0 & 0.25 & $\mathrm{mg} / \mathrm{L}$ \\
\hline \multicolumn{4}{|l|}{ Metals/Cations } \\
\hline Aluminum & SW846-6010B & 0.2 & $\mathrm{mg} / \mathrm{L}$ \\
\hline Antimony & EPA-200.8 & 0.0025 & $\mathrm{mg} / \mathrm{L}$ \\
\hline Arsenic & EPA-200.8 & 0.005 & $\mathrm{mg} / \mathrm{L}$ \\
\hline Barium & SW846-6010B & 0.004 & $\mathrm{mg} / \mathrm{L}$ \\
\hline Beryllium & SW846-6010B & 0.0005 & $\mathrm{mg} / \mathrm{L}$ \\
\hline Boron & SW846-6010B & 0.1 & $\mathrm{mg} / \mathrm{L}$ \\
\hline Cadmium & EPA-200.8 & 0.0005 & $\mathrm{mg} / \mathrm{L}$ \\
\hline Calcium & SW846-6010B & 0.2 & $\mathrm{mg} / \mathrm{L}$ \\
\hline Chromium & EPA-200.8 & 0.0025 & $\mathrm{mg} / \mathrm{L}$ \\
\hline Cobalt & SW846-6010B & 0.02 & $\mathrm{mg} / \mathrm{L}$ \\
\hline Copper & SW846-6010B & 0.02 & $\mathrm{mg} / \mathrm{L}$ \\
\hline Iron & SW846-6010B & 0.05 & $\mathrm{mg} / \mathrm{L}$ \\
\hline Lead & EPA-200.8 & 0.0005 & $\mathrm{mg} / \mathrm{L}$ \\
\hline Lithium & SW846-6010B & 0.01 & $\mathrm{mg} / \mathrm{L}$ \\
\hline Magnesium & SW846-6010B & 0.2 & $\mathrm{mg} / \mathrm{L}$ \\
\hline Manganese & SW846-6010B & 0.005 & $\mathrm{mg} / \mathrm{L}$ \\
\hline Mercury & SW846-7470 & 0.0002 & $\mathrm{mg} / \mathrm{L}$ \\
\hline Molybdenum & SW846-6010B & 0.05 & $\mathrm{mg} / \mathrm{L}$ \\
\hline Nickel & EPA-200.8 & 0.005 & $\mathrm{mg} / \mathrm{L}$ \\
\hline
\end{tabular}


Table B.5 (continued)

\begin{tabular}{|c|c|c|c|}
\hline Metals/Cations (continued) & Analytical Method ${ }^{1}$ & Reporting Limit $^{2}$ & Units $^{3}$ \\
\hline Potassium & SW846-6010B & 2 & $\mathrm{mg} / \mathrm{L}$ \\
\hline Selenium & EPA-200.8 & 0.01 & $\mathrm{mg} / \mathrm{L}$ \\
\hline Silver & SW846-6010B & 0.02 & $\mathrm{mg} / \mathrm{L}$ \\
\hline Sodium & SW846-6010B & 0.2 & $\mathrm{mg} / \mathrm{L}$ \\
\hline Strontium & SW846-6010B & 0.005 & $\mathrm{mg} / \mathrm{L}$ \\
\hline Thallium & EPA-200.8 & 0.0005 & $\mathrm{mg} / \mathrm{L}$ \\
\hline Thorium & SW846-6010B & 0.2 & $\mathrm{mg} / \mathrm{L}$ \\
\hline Uranium & EPA-200.8 & 0.0005 & $\mathrm{mg} / \mathrm{L}$ \\
\hline Vanadium & SW846-6010B & 0.02 & $\mathrm{mg} / \mathrm{L}$ \\
\hline Zinc & SW846-6010B & 0.05 & $\mathrm{mg} / \mathrm{L}$ \\
\hline Volatile Organic Compounds & & $\mathrm{CRQL}^{4}$ & \\
\hline Acetone & SW846-8260B UP & 10 & $\mu \mathrm{g} / \mathrm{L}$ \\
\hline Acrolein & SW846-8260B UP & 10 & $\mu \mathrm{g} / \mathrm{L}$ \\
\hline Acrylonitrile & SW846-8260B UP & 5 & $\mu \mathrm{g} / \mathrm{L}$ \\
\hline Benzene & SW846-8260B UP & 5 & $\mu \mathrm{g} / \mathrm{L}$ \\
\hline Bromochloromethane & SW846-8260B UP & 5 & $\mu \mathrm{g} / \mathrm{L}$ \\
\hline Bromodichloromethane & SW846-8260B UP & 5 & $\mu \mathrm{g} / \mathrm{L}$ \\
\hline Bromoform & SW846-8260B UP & 5 & $\mu \mathrm{g} / \mathrm{L}$ \\
\hline Bromomethane & SW846-8260B UP & 5 & $\mu \mathrm{g} / \mathrm{L}$ \\
\hline 2-Butanone & SW846-8260B UP & 5 & $\mu \mathrm{g} / \mathrm{L}$ \\
\hline Carbon disulfide & SW846-8260B UP & 5 & $\mu \mathrm{g} / \mathrm{L}$ \\
\hline Carbon tetrachloride & SW846-8260B UP & 5 & $\mu \mathrm{g} / \mathrm{L}$ \\
\hline Chlorobenzene & SW846-8260B UP & 5 & $\mu \mathrm{g} / \mathrm{L}$ \\
\hline Chloroethane & SW846-8260B UP & 5 & $\mu \mathrm{g} / \mathrm{L}$ \\
\hline 2-Chloroethyl vinyl ether & SW846-8260B UP & 10 & $\mu \mathrm{g} / \mathrm{L}$ \\
\hline Chloroform & SW846-8260B UP & 5 & $\mu \mathrm{g} / \mathrm{L}$ \\
\hline Chloromethane & SW846-8260B UP & 5 & $\mu \mathrm{g} / \mathrm{L}$ \\
\hline Dibromochloromethane & SW846-8260B UP & 5 & $\mu \mathrm{g} / \mathrm{L}$ \\
\hline 1,2-Dibromo-3-chloropropane & SW846-8260B UP & 10 & $\mu \mathrm{g} / \mathrm{L}$ \\
\hline 1,2-Dibromoethane & SW846-8260B UP & 5 & $\mu \mathrm{g} / \mathrm{L}$ \\
\hline Dibromomethane & SW846-8260B UP & 5 & $\mu \mathrm{g} / \mathrm{L}$ \\
\hline 1,2-Dichlorobenzene & SW846-8260B UP & 5 & $\mu \mathrm{g} / \mathrm{L}$ \\
\hline 1,4-Dichlorobenzene & SW846-8260B UP & 5 & $\mu \mathrm{g} / \mathrm{L}$ \\
\hline 1,4-Dichloro-2-butene & SW846-8260B UP & 5 & $\mu \mathrm{g} / \mathrm{L}$ \\
\hline trans-1,4-Dichloro-2-butene & SW846-8260B UP & 5 & $\mu \mathrm{g} / \mathrm{L}$ \\
\hline Dichlorodifluoromethane & SW846-8260B UP & 5 & $\mu \mathrm{g} / \mathrm{L}$ \\
\hline 1,1-Dichloroethane & SW846-8260B UP & 5 & $\mu \mathrm{g} / \mathrm{L}$ \\
\hline 1,2-Dichloroethane & SW846-8260B UP & 5 & $\mu \mathrm{g} / \mathrm{L}$ \\
\hline 1,1-Dichloroethene & SW846-8260B UP & 5 & $\mu \mathrm{g} / \mathrm{L}$ \\
\hline cis-1,2-Dichloroethene & SW846-8260B UP & 5 & $\mu \mathrm{g} / \mathrm{L}$ \\
\hline trans-1,2-Dichloroethene & SW846-8260B UP & 5 & $\mu \mathrm{g} / \mathrm{L}$ \\
\hline 1,2-Dichloropropane & SW846-8260B UP & 5 & $\mu \mathrm{g} / \mathrm{L}$ \\
\hline
\end{tabular}


Table B.5 (continued)

\begin{tabular}{|c|c|c|c|}
\hline Volatile Organic Compounds (cont'd) & Analytical Method ${ }^{1}$ & $\mathrm{CRQL}^{4}$ & Units $^{3}$ \\
\hline cis-1,3-Dichloropropene & SW846-8260B UP & 5 & $\mu \mathrm{g} / \mathrm{L}$ \\
\hline trans-1,3-Dichloropropene & SW846-8260B UP & 5 & $\mu \mathrm{g} / \mathrm{L}$ \\
\hline Dimethylbenzene & SW846-8260B UP & 5 & $\mu \mathrm{g} / \mathrm{L}$ \\
\hline Ethanol & SW846-8260B UP & 200 & $\mu \mathrm{g} / \mathrm{L}$ \\
\hline Ethylbenzene & SW846-8260B UP & 5 & $\mu \mathrm{g} / \mathrm{L}$ \\
\hline Ethyl methacrylate & SW846-8260B UP & 5 & $\mu \mathrm{g} / \mathrm{L}$ \\
\hline 2-Hexanone & SW846-8260B UP & 5 & $\mu \mathrm{g} / \mathrm{L}$ \\
\hline Iodomethane & SW846-8260B UP & 5 & $\mu \mathrm{g} / \mathrm{L}$ \\
\hline 4-Methyl-2-pentanone & SW846-8260B UP & 5 & $\mu \mathrm{g} / \mathrm{L}$ \\
\hline Methylene chloride & SW846-8260B UP & 5 & $\mu \mathrm{g} / \mathrm{L}$ \\
\hline Styrene & SW846-8260B UP & 5 & $\mu \mathrm{g} / \mathrm{L}$ \\
\hline 1,1,1,2-Tetrachloroethane & SW846-8260B UP & 5 & $\mu \mathrm{g} / \mathrm{L}$ \\
\hline 1,1,2,2-Tetrachloroethane & SW846-8260B UP & 5 & $\mu \mathrm{g} / \mathrm{L}$ \\
\hline Tetrachloroethene & SW846-8260B UP & 5 & $\mu \mathrm{g} / \mathrm{L}$ \\
\hline Toluene & SW846-8260B UP & 5 & $\mu \mathrm{g} / \mathrm{L}$ \\
\hline 1,1,1-Trichloroethane & SW846-8260B UP & 5 & $\mu \mathrm{g} / \mathrm{L}$ \\
\hline 1,1,2-Trichloroethane & SW846-8260B UP & 5 & $\mu \mathrm{g} / \mathrm{L}$ \\
\hline Trichloroethene & SW846-8260B UP & 5 & $\mu \mathrm{g} / \mathrm{L}$ \\
\hline Trichlorofluoromethane & SW846-8260B UP & 5 & $\mu \mathrm{g} / \mathrm{L}$ \\
\hline 1,2,3-Trichloropropane & SW846-8260B UP & 10 & $\mu \mathrm{g} / \mathrm{L}$ \\
\hline Vinyl acetate & SW846-8260B UP & 10 & $\mu \mathrm{g} / \mathrm{L}$ \\
\hline Vinyl chloride & SW846-8260B UP & 2 & $\mu \mathrm{g} / \mathrm{L}$ \\
\hline Radiological Analytes & & 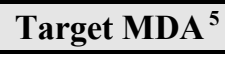 & \\
\hline Gross Alpha Activity & EPA-900.0 & 3.5 & $\mathrm{pCi} / \mathrm{L}$ \\
\hline Gross Beta Activity & EPA-900.0 & 7.0 & $\mathrm{pCi} / \mathrm{L}$ \\
\hline Americium-241 & $\mathrm{Y} / \mathrm{P} 65-7226$ & 0.4 & $\mathrm{pCi} / \mathrm{L}$ \\
\hline Iodine-129 & EPA-901.1 & 3.0 & $\mathrm{pCi} / \mathrm{L}$ \\
\hline Neptunium-237 & Y/P65-7206 & 0.4 & $\mathrm{pCi} / \mathrm{L}$ \\
\hline Plutonium-238 \& -239/240 & Y/P65-7226 & 0.4 & $\mathrm{pCi} / \mathrm{L}$ \\
\hline Radium-223/224/226 & EPA-903.0 / 904.0 & 0.5 & $\mathrm{pCi} / \mathrm{L}$ \\
\hline Strontium- $89 / 90$ & Y/P65-7037 & 4.0 & $\mathrm{pCi} / \mathrm{L}$ \\
\hline Technetium-99 & $\mathrm{Y} / \mathrm{P} 65-7060$ & 10 & $\mathrm{pCi} / \mathrm{L}$ \\
\hline Thorium-228, 230, 232, \& 234 & Y/P65-7052 & 0.4 & $\mathrm{pCi} / \mathrm{L}$ \\
\hline Tritium & EPA-906.0 & 300 & $\mathrm{pCi} / \mathrm{L}$ \\
\hline Uranium-234, 235, \& 238 & Y/P65-7061 & 0.4 & $\mathrm{pCi} / \mathrm{L}$ \\
\hline $\begin{array}{r}\text { Total Uranium and } \\
\text { weight \% Uranium-234, 235, 236, \& } 238\end{array}$ & Y/P65-8044 & 0.002 & $\mathrm{mg} / \mathrm{L}$ \\
\hline
\end{tabular}


Table B.5 (continued)

Notes:

1 Analytical/field methods/procedures from:

! Y-12 System Operation Procedures (BWXT 2000b, BWXT 2001b, and LMES 1999b)

! Test Methods for Evaluating Solid Waste Physical/Chemical Methods (U.S. Environmental Protection Agency 1996)

! Methods for Chemical Analysis of Water and Wastes

(U.S. Environmental Protection Agency 1983)

! BWXT Y-12 Analytical Chemistry Organization Control Procedures: (Y/P65-7037, Y/P65-7052, Y/P65-7060, Y/P65-7061, Y/P65-7206, Y/P65-7226, and $\mathrm{Y} / \mathrm{P} 65-8044)$

2 The lowest concentration reported.

NA - not applicable

$3 \quad \mathrm{ft}-$ feet

$\mu \mathrm{g} / \mathrm{L}$ - micrograms per liter

$\mu \mathrm{mho} / \mathrm{cm}$ - micromhos per centimeter

$\mathrm{mg} / \mathrm{L}$ - milligrams per liter

$\mathrm{mV}$ - millivolts

NTU - nephelometric turbidity units

ppm - parts per million

$\mathrm{pCi} / \mathrm{L}$ - picoCuries per liter

4 CRQL - contract-required quantitation limit; estimated values are reported below this level and above the instrument detection limit. Results below the instrument detection limit are reported as not detected at the CRQL.

5 MDA - minimum detectable activity. The target MDA may be obtained under optimal analytical conditions; actual MDAs are sample-specific and, in some cases, may vary significantly from the target value. 
Table B.6. Depth-to-water measurements and groundwater elevations in the Bear Creek Hydrogeologic Regime, April and September 2002

\begin{tabular}{|c|c|c|c|c|c|c|c|c|c|}
\hline \multirow{2}{*}{\multicolumn{4}{|c|}{$\begin{array}{r}\text { Groundwater Elevation } \\
\text { (ft above mean sea level) }\end{array}$}} & \multicolumn{6}{|c|}{ September 17-30, 2002} \\
\hline & & & & \multicolumn{5}{|c|}{ April 8-12, 2002} & \\
\hline \multirow{3}{*}{\multicolumn{4}{|c|}{$\begin{array}{r}\text { Depth-to-Water } \\
\text { (ft below measuring point) }\end{array}$}} & \multicolumn{4}{|c|}{ Seasonal Fluctuation $(+/-\mathbf{f t})$} & & \\
\hline & & & & \multirow{4}{*}{\multicolumn{2}{|c|}{\begin{tabular}{|c|} 
September 17 \\
\multicolumn{2}{|c|}{ April 8-12, 2002} \\
$\begin{array}{c}\text { Measuring Pt. } \\
\text { Elevation }^{3}\end{array}$ \\
\end{tabular}}} & \multirow[t]{4}{*}{30,2002} & & & \\
\hline & & & & & & & & & \\
\hline \multirow{2}{*}{$\begin{array}{l}\text { Well } \\
\text { Number }\end{array}$} & \multirow{2}{*}{ Location $^{1}$} & \multicolumn{2}{|c|}{ Hydrogeologic Unit ${ }^{2}$} & \multirow{2}{*}{$\begin{array}{c}\text { Measuring Pt. } \\
\text { Elevation }^{3}\end{array}$} & & & & & \\
\hline & & Aquifer & Aquitard & & & & & & \\
\hline GW-001 & OLF & & q & 981.00 & 16.48 & 16.77 & -0.29 & 964.52 & 964.23 \\
\hline GW-005 & OLF & & q & 967.81 & 7.00 & $\mathrm{NM}^{4}$ & $\mathrm{NA}^{5}$ & 960.81 & $\mathrm{NA}$ \\
\hline GW-008 & OLF & & q & 965.39 & 14.96 & 15.23 & -0.27 & 950.43 & 950.16 \\
\hline GW-010 & OLF & & q & 952.70 & 2.11 & 2.42 & -0.31 & 950.59 & 950.28 \\
\hline GW-012 & OLF & & q & 955.57 & 7.08 & 6.85 & 0.23 & 948.49 & 948.72 \\
\hline GW-013 & OLF & & q & 965.12 & 6.42 & NM & NA & 958.70 & NA \\
\hline GW-014 & $\mathrm{BG}$ & & q & 934.50 & 8.26 & 8.25 & 0.01 & 926.24 & 926.25 \\
\hline GW-016 & $\mathrm{BG}$ & & 9 & 928.80 & 9.88 & 14.90 & -5.02 & 918.92 & 913.90 \\
\hline GW-018 & BG & & $q$ & 924.49 & 6.95 & 11.80 & -4.85 & 917.54 & 912.69 \\
\hline GW-041 & BG & & $q$ & 1008.10 & 15.08 & 26.20 & -11.12 & 993.02 & 981.90 \\
\hline GW-045 & $\mathrm{BG}$ & $M$ & & 910.30 & 10.51 & 16.50 & -5.99 & 899.79 & 893.80 \\
\hline GW-046 & $\mathrm{BG}$ & & $q$ & 921.17 & 3.50 & 6.40 & -2.90 & 917.67 & 914.77 \\
\hline GW-047 & $\mathrm{BG}$ & & 9 & 929.00 & 8.10 & 11.10 & -3.00 & 920.90 & 917.90 \\
\hline GW-052 & $\mathrm{BG}$ & $M$ & & 905.70 & 13.92 & 18.70 & -4.78 & 891.78 & 887.00 \\
\hline GW-053 & $\mathrm{BG}$ & $q$ & & 903.40 & 8.24 & 11.50 & -3.26 & 895.16 & 891.90 \\
\hline GW-065 & OLF & $q$ & & 982.50 & 25.48 & 26.54 & -1.06 & 957.02 & 955.96 \\
\hline GW-067 & OLF & $q$ & & 961.60 & 9.83 & 9.19 & 0.64 & 951.77 & 952.41 \\
\hline GW-080 & $\mathrm{BG}$ & & $q$ & 981.00 & 22.64 & NM & NA & 958.36 & NA \\
\hline GW-084 & OLF & & $q$ & 997.20 & 13.37 & 12.38 & 0.99 & 983.83 & 984.82 \\
\hline GW-086 & OLF & & $q$ & 982.80 & 12.36 & 14.00 & -1.64 & 970.44 & 968.80 \\
\hline GW-090 & BG & & $q$ & 961.88 & 4.55 & 8.30 & -3.75 & 957.33 & 953.58 \\
\hline GW-091 & $\mathrm{BG}$ & & $q$ & 953.48 & 8.20 & 10.80 & -2.60 & 945.28 & 942.68 \\
\hline GW-097 & OLF & & $q$ & 945.30 & 9.25 & 9.95 & -0.70 & 936.05 & 935.35 \\
\hline GW-100 & S3 & $q$ & & 987.40 & 5.37 & 5.50 & -0.13 & 982.03 & 981.90 \\
\hline GW-101 & S3 & & $q$ & 1008.00 & 9.42 & 9.20 & 0.22 & 998.58 & 998.80 \\
\hline GW-115 & S3 & & $q$ & 1055.01 & 8.26 & 12.30 & -4.04 & 1046.75 & 1042.71 \\
\hline GW-127 & S3 & & $q$ & 1005.90 & 13.11 & 13.00 & 0.11 & 992.79 & 992.90 \\
\hline GW-236 & S3 & $q$ & & 983.21 & 10.47 & 9.00 & 1.47 & 972.74 & 974.21 \\
\hline GW-245 & S3 & & $q$ & 1009.08 & 12.48 & 13.90 & -1.42 & 996.60 & 995.18 \\
\hline GW-249 & $\mathrm{BG}$ & & q & 991.15 & 31.71 & NM & NA & 959.44 & NA \\
\hline GW-257 & BG & & $q$ & 961.68 & 28.31 & 30.30 & -1.99 & 933.37 & 931.38 \\
\hline GW-276 & S3 & & q & 1001.57 & 6.25 & 5.50 & 0.75 & 995.32 & 996.07 \\
\hline GW-287 & BG & & $q$ & 927.07 & 9.28 & 9.90 & -0.62 & 917.79 & 917.17 \\
\hline GW-289 & $\mathrm{BG}$ & & $q$ & 948.73 & 13.96 & 20.10 & -6.14 & 934.77 & 928.63 \\
\hline GW-291 & $\mathrm{BG}$ & & $q$ & 948.56 & 11.85 & 13.70 & -1.85 & 936.71 & 934.86 \\
\hline
\end{tabular}


Table B.6 (continued)

\begin{tabular}{|c|c|c|c|c|c|c|c|c|c|}
\hline \multirow{2}{*}{\multicolumn{4}{|c|}{$\begin{array}{r}\text { Groundwater Elevation } \\
\text { (ft above mean sea level) }\end{array}$}} & & & & \multicolumn{3}{|c|}{ September 17-30, 2002} \\
\hline & & & & \multirow{2}{*}{\multicolumn{4}{|c|}{$\frac{\text { April 8 }}{\text { Seasonal Fluctuation }(+/-\mathrm{ft})}$}} & & \\
\hline \multirow{5}{*}{$\begin{array}{l}\text { Well } \\
\text { Number }\end{array}$} & & & & & & & & & \\
\hline & \multirow{2}{*}{\multicolumn{3}{|c|}{$\begin{array}{r}\text { Depth-to-Water } \\
\text { (ft below measuring point) }\end{array}$}} & \multirow{4}{*}{\multicolumn{2}{|c|}{\begin{tabular}{|c|}
\multicolumn{2}{|c|}{ September 17} \\
\multicolumn{2}{|c|}{ April 8-12, 2002} \\
$\begin{array}{c}\text { Measuring Pt. } \\
\text { Elevation }^{3}\end{array}$ \\
\end{tabular}}} & \multirow[t]{4}{*}{30,2002} & \multirow{4}{*}{$+/-11)$} & & \\
\hline & & & & & & & & & \\
\hline & \multirow{2}{*}{ Location $^{1}$} & \multicolumn{2}{|c|}{ Hydrogeologic Unit ${ }^{2}$} & \multirow{2}{*}{$\begin{array}{c}\text { Measuring Pt. } \\
\text { Elevation }^{3}\end{array}$} & & & & & \\
\hline & & Aquifer & Aquitard & & & & & & \\
\hline GW-307 & $\mathrm{RS}$ & $\mathrm{q}$ & & 993.14 & 26.40 & 31.10 & -4.70 & 966.74 & 962.04 \\
\hline GW-309 & RS & q & & 988.17 & 19.24 & 20.54 & -1.30 & 968.93 & 967.63 \\
\hline GW-310 & $\mathrm{RS}$ & $\mathrm{q}$ & & 995.35 & 19.95 & 19.99 & -0.04 & 975.40 & 975.36 \\
\hline GW-316 & SPI & q & & 1047.17 & 52.00 & 54.70 & -2.70 & 995.17 & 992.47 \\
\hline GW-323 & SPI & $q$ & & 1130.11 & 85.82 & 86.10 & -0.28 & 1044.29 & 1044.01 \\
\hline GW-325 & S3 & & $q$ & 1003.00 & 11.27 & 14.20 & -2.93 & 991.73 & 988.80 \\
\hline GW-345 & S3 & & q & 999.66 & 18.42 & 20.20 & -1.78 & 981.24 & 979.46 \\
\hline GW-347 & S3 & $M$ & & 1001.05 & 16.48 & 14.80 & 1.68 & 984.57 & 986.25 \\
\hline GW-370 & $\mathrm{BG}$ & & q & 960.82 & 15.15 & 17.80 & -2.65 & 945.67 & 943.02 \\
\hline GW-372 & $\mathrm{BG}$ & & 9 & 983.20 & 18.37 & 28.50 & -10.13 & 964.83 & 954.70 \\
\hline GW-531 & LD & & $q$ & 1004.61 & 14.20 & 24.03 & -9.83 & 990.41 & 980.58 \\
\hline GW-537 & OLF & & $q$ & 976.44 & 6.01 & 7.60 & -1.59 & 970.43 & 968.84 \\
\hline GW-613 & $\mathrm{S} 3$ & & $q$ & 1013.57 & 12.55 & 15.50 & -2.95 & 1001.02 & 998.07 \\
\hline GW-621 & EXP-B & 9 & & 925.44 & 10.65 & 20.00 & -9.35 & 914.79 & 905.44 \\
\hline GW-622 & $\mathrm{BG}$ & & 9 & 924.16 & 9.57 & 16.10 & -6.53 & 914.59 & 908.06 \\
\hline GW-624 & $\mathrm{BG}$ & & $q$ & 922.14 & 9.79 & 13.40 & -3.61 & 912.35 & 908.74 \\
\hline GW-630 & LD & & 9 & 986.65 & 9.40 & 9.40 & 0.00 & 977.25 & 977.25 \\
\hline GW-638 & OLF & & 9 & 941.77 & 6.30 & 5.89 & 0.41 & 935.47 & 935.88 \\
\hline GW-641 & $\mathrm{BG}$ & & q & 946.66 & 19.01 & 21.30 & -2.29 & 927.65 & 925.36 \\
\hline GW-642 & $\mathrm{BG}$ & & 9 & 1014.90 & 17.97 & 23.50 & -5.53 & 996.93 & 991.40 \\
\hline GW-648 & $\mathrm{RS}$ & $M$ & & 1029.20 & 65.22 & 69.83 & -4.61 & 963.98 & 959.37 \\
\hline GW-652 & $\mathrm{BG}$ & $M$ & & 900.83 & 9.68 & 12.50 & -2.82 & 891.15 & 888.33 \\
\hline GW-653 & $\mathrm{BG}$ & & $q$ & 931.80 & 22.23 & 25.90 & -3.67 & 909.57 & 905.90 \\
\hline GW-654 & $\mathrm{BG}$ & & $q$ & 940.79 & 7.00 & 9.50 & -2.50 & 933.79 & 931.29 \\
\hline GW-795 & AGLLSF & & $q$ & 925.98 & 4.05 & 2.96 & 1.09 & 921.93 & 923.02 \\
\hline GW-916 & EMWMF & & $q$ & 1002.85 & 5.33 & NM & NA & 997.52 & NA \\
\hline GW-917 & EMWMF & & $q$ & 997.10 & 19.84 & NM & NA & 977.26 & NA \\
\hline GW-918 & EMWMF & & $q$ & 1067.96 & 5.41 & NM & NA & 1062.55 & NA \\
\hline GW-924 & EMWMF & & $q$ & 968.90 & 10.44 & NM & NA & 958.46 & NA \\
\hline
\end{tabular}




\section{Table B.6 (continued)}

Notes:

1 AGLLSF - Above Grade Low-Level Storage Facility

BG - Bear Creek Burial Grounds Waste Management Area

EMWMF - Environmental Management Waste Management Facility

EXP-B - Exit Pathway (Maynardville Limestone) Picket B

LD - Lysimeter Demonstration Site

OLF - Oil Landfarm Waste Management Area

RS - Rust Spoil Area

SPI - Spoil Area I

S3 - S-3 Site

2 Aquifer - Completed in the upper Conasauga Group (Maynardville Limestone) or the Knox Group Aquitard - Completed in the lower Conasauga Group (Nolichucky Shale, Maryville Limestone, Rogersville Shale, Rutledge Limestone, or Pumpkin Valley Shale)

3 Measuring point elevation in feet above mean sea level. The measuring point is either the top of the innermost well casing or the top of dedicated sampling equipment mounted on the casing.

$4 \quad \mathrm{NM} \quad-$ Not Measured

$5 \quad$ NA - Not Applicable 
B-22 
Table B.7. Depth-to-water measurements and groundwater elevations in the Upper East Fork Poplar Creek Hydrogeologic Regime, April and September 2002

\begin{tabular}{|c|c|c|c|c|c|c|c|c|c|}
\hline \multirow{2}{*}{\multicolumn{3}{|c|}{$\begin{array}{r}\text { Groundwater Elevation } \\
\text { (ft above mean sea level) }\end{array}$}} & & & & \multicolumn{4}{|c|}{ September 18-26, 2002} \\
\hline & & & & & & \multicolumn{3}{|c|}{ April 8-11, 2002} & \\
\hline & & & & \multicolumn{4}{|c|}{ Seasonal Fluctuation $(+/-\mathbf{f t})$} & & \\
\hline \multirow{2}{*}{\multicolumn{3}{|c|}{$\begin{array}{r}\text { Depth-to-Water } \\
\text { (ft below measuring point) }\end{array}$}} & & \multicolumn{3}{|c|}{ September 18-26, 2002} & & & \\
\hline & & & & \multicolumn{2}{|c|}{ April 8-11, 2002} & & & & \\
\hline \multirow{2}{*}{$\begin{array}{c}\text { Well } \\
\text { Number }\end{array}$} & \multirow{2}{*}{ Location $^{1}$} & \multicolumn{2}{|c|}{ Hydrogeologic Unit $^{2}$} & \multirow{2}{*}{$\begin{array}{l}\text { Measuring Point } \\
\text { Elevation }^{3}\end{array}$} & & & & & \\
\hline & & Aquifer & Aquitard & & & & & & \\
\hline $53-1 \mathrm{~A}$ & $\mathrm{Y} 12$ & & $q$ & 993.65 & 3.22 & $\mathrm{NM}^{4}$ & $\mathrm{NA}^{5}$ & 990.43 & NA \\
\hline $55-1 \mathrm{~A}$ & Y12 & & q & 986.67 & 10.82 & 10.79 & 0.03 & 975.85 & 975.88 \\
\hline $55-3 \mathrm{~A}$ & $\mathrm{Y} 12$ & & q & 972.46 & 11.40 & 11.70 & -0.30 & 961.06 & 960.76 \\
\hline $55-6 \mathrm{~A}$ & Y12 & & q & 989.04 & 8.47 & 10.72 & -2.25 & 980.57 & 978.32 \\
\hline $56-1 \mathrm{~A}$ & Y12 & & q & 969.25 & 7.62 & 7.74 & -0.12 & 961.63 & 961.51 \\
\hline $56-2 \mathrm{~A}$ & $\mathrm{Y} 12$ & & q & 963.30 & 7.78 & 7.76 & 0.02 & 955.52 & 955.54 \\
\hline $56-8 \mathrm{~A}$ & $\mathrm{Y} 12$ & $q$ & & 962.46 & 19.38 & 19.28 & 0.10 & 943.08 & 943.18 \\
\hline $60-1 \mathrm{~A}$ & Y12 & q & & 929.66 & 12.67 & 12.67 & 0.00 & 916.99 & 916.99 \\
\hline GW-105 & S3 & & $q$ & 1018.20 & 9.42 & 8.70 & 0.72 & 1008.78 & 1009.50 \\
\hline GW-108 & S3 & & $q$ & 999.00 & 7.50 & 7.50 & 0.00 & 991.50 & 991.50 \\
\hline GW-115 & S3 & & q & 1055.01 & 8.26 & 12.3 & -4.04 & 1046.75 & 1042.71 \\
\hline GW-148 & NHP & $q$ & & 907.56 & 8.40 & 8.61 & -0.21 & 899.16 & 898.95 \\
\hline GW-150 & NHP & q & & 915.56 & 14.05 & 14.08 & -0.03 & 901.51 & 901.48 \\
\hline GW-152 & NHP & q & & 921.18 & 19.67 & 20.04 & -0.37 & 901.51 & 901.14 \\
\hline GW-154 & NHP & q & & 911.50 & 9.01 & 7.19 & 1.82 & 902.49 & 904.31 \\
\hline GW-167 & EXP & q & & 931.97 & 28.73 & NM & NA & 903.24 & $\mathrm{NA}$ \\
\hline GW-169 & EXP-UV & $q$ & & 932.12 & 28.47 & 32.32 & -3.85 & 903.65 & 899.80 \\
\hline GW-191 & B4 & & $q$ & 1011.27 & 3.65 & 6.07 & -2.42 & 1007.62 & 1005.20 \\
\hline GW-192 & B4 & & 9 & 1008.83 & 5.83 & 7.25 & -1.42 & 1003.00 & 1001.58 \\
\hline GW-193 & $\mathrm{T} 2331$ & $q$ & & 934.17 & 8.49 & 8.04 & 0.45 & 925.68 & 926.13 \\
\hline GW-195 & B4 & & $q$ & 1002.90 & 6.61 & 6.06 & 0.55 & 996.29 & 996.84 \\
\hline GW-199 & GRIDI1 & & $q$ & 961.08 & 16.72 & 15.75 & 0.97 & 944.36 & 945.33 \\
\hline GW-202 & RDS & & $q$ & 968.02 & 9.95 & 9.75 & 0.20 & 958.07 & 958.27 \\
\hline GW-204 & T0134 & & 9 & 958.77 & 8.62 & 8.51 & 0.11 & 950.15 & 950.26 \\
\hline GW-219 & UOV & $q$ & & 935.84 & 9.60 & 12.76 & -3.16 & 926.24 & 923.08 \\
\hline GW-253 & S2 & $q$ & & 1004.24 & 4.71 & 26.99 & -22.28 & 999.53 & 977.25 \\
\hline GW-255 & S2 & $q$ & & 1027.13 & 23.21 & NM & NA & 1003.92 & NA \\
\hline GW-261 & SY & & $q$ & 1049.99 & 16.77 & 17.60 & -0.83 & 1033.22 & 1032.39 \\
\hline GW-263 & SY & & 9 & 1057.73 & 25.32 & 31.50 & -6.18 & 1032.41 & 1026.23 \\
\hline GW-334 & WC & & q & 983.73 & 11.67 & 11.78 & -0.11 & 972.06 & 971.95 \\
\hline GW-335 & WC & & $q$ & 981.88 & 9.84 & 11.07 & -1.23 & 972.04 & 970.81 \\
\hline GW-349 & S2 & $q$ & & 993.50 & 3.91 & 2.38 & 1.53 & 989.59 & 991.12 \\
\hline GW-380 & NHP & q & & 913.55 & 9.85 & 10.32 & -0.47 & 903.70 & 903.23 \\
\hline GW-383 & NHP & & $q$ & 908.77 & 9.25 & 9.55 & -0.30 & 899.52 & 899.22 \\
\hline GW-384 & NHP & & $q$ & 909.23 & 10.96 & 11.20 & -0.24 & 898.27 & 898.03 \\
\hline
\end{tabular}


Table B.7 (continued)

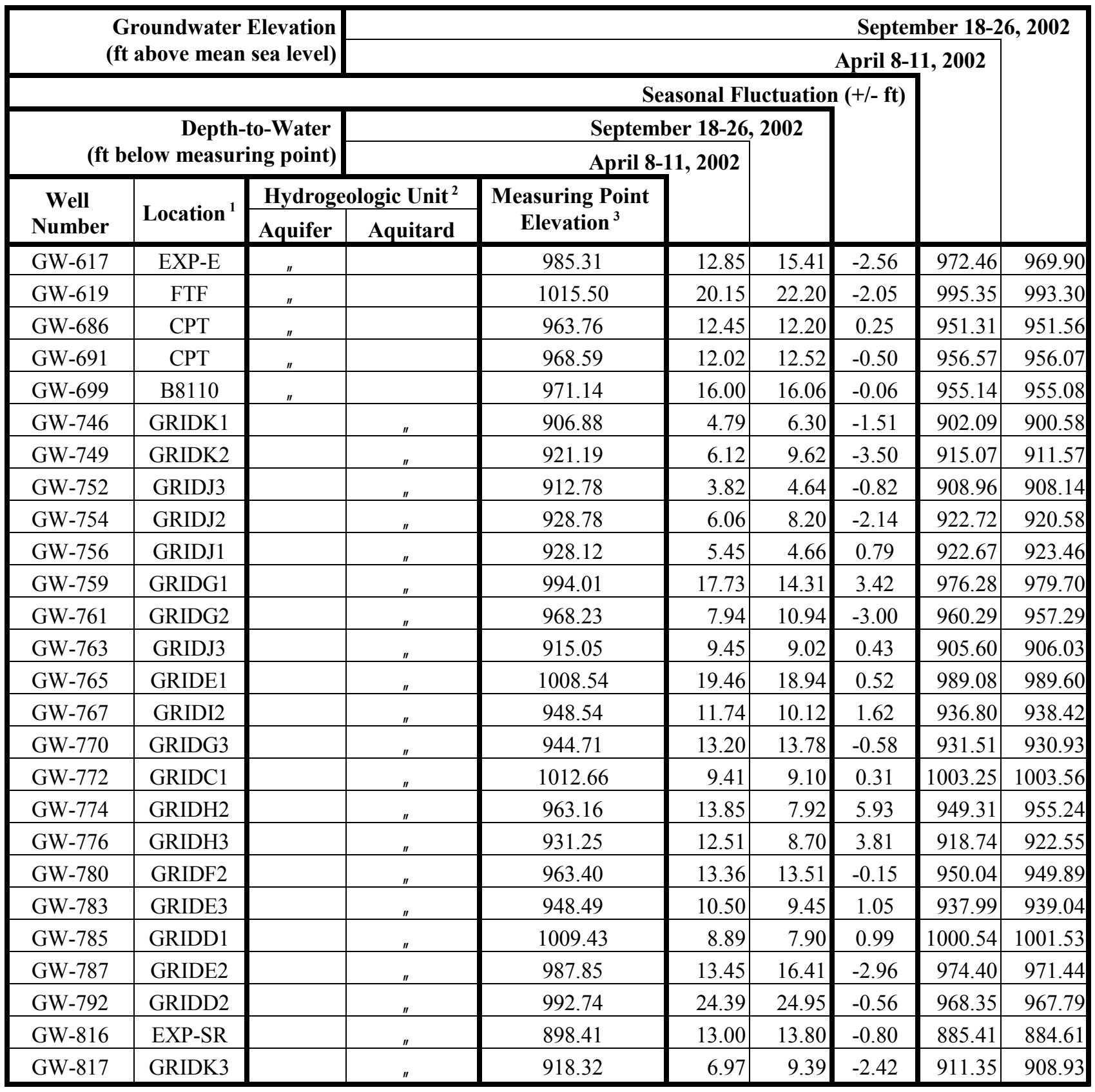




\section{Table B.7 (continued)}

Notes:

$1 \quad$ B4 - Beta-4 Security Pits

B8110 - Building 81-10

CPT - Coal Pile Trench

EXP - Exit Pathway (Maynardville Limestone) monitoring well

! -E: Maynardville Limestone Picket

! -UV: Offsite in Union Valley

! -SR: Along Scarboro Road in the gap through Pine Ridge

FTF - Fire Training Facility

GRID - Comprehensive Groundwater Monitoring Plan Grid Location

NHP - New Hope Pond

RDS - Ravine Disposal Site

RG - Rust Garage Area

S2 - S-2 Site

S3 - S-3 Site

SY - Y-12 Plant Salvage Yard

T0134 - Tank 0134-U

T2331 - Tank 2331-U

UOV - Uranium Oxide Vault

WC - Waste Coolant Processing Area

Y12 - Y-12 Complex

2 Aquifer - Completed in the upper Conasauga Group (Maynardville Limestone) or the Knox Group

Aquitard - Completed in the lower Conasauga Group (Nolichucky Shale, Maryville Limestone, Rogersville Shale, Rutledge Limestone, or Pumpkin Valley Shale)

3 Measuring point elevation is in feet above mean sea level. The measuring point is either the top of the innermost well casing or the top of dedicated sampling equipment.

$4 \quad$ NM - Not measured or unusable

$5 \quad$ NA - Not applicable 
B-26 
Table B.8. Depth-to-water measurements and groundwater elevations in the Chestnut Ridge Hydrogeologic Regime, April and September 2002

\begin{tabular}{|c|c|c|c|c|c|c|c|}
\hline \multirow{2}{*}{\multicolumn{2}{|c|}{$\begin{array}{l}\text { Groundwater Elevation } \\
\text { (ft above mean sea level) }\end{array}$}} & & & & \multicolumn{3}{|c|}{ September 17-26, 2002} \\
\hline & & \multirow{2}{*}{\multicolumn{4}{|c|}{$\begin{array}{c}\text { April } \\
\text { Seasonal Fluctuation }(+/-\mathbf{f t})\end{array}$}} & & \\
\hline & & & & & & & \\
\hline \multirow{2}{*}{\multicolumn{2}{|c|}{$\begin{array}{r}\text { Depth-to-Water } \\
\text { (ft below measuring point) }\end{array}$}} & \multicolumn{3}{|c|}{ September 17-26, 2002} & & & \\
\hline & & \multicolumn{2}{|c|}{ April 8-10, 2002} & & & & \\
\hline Well Number & Location $^{1}$ & $\begin{array}{l}\text { Measuring Point } \\
\text { Elevation }^{2}\end{array}$ & & & & & \\
\hline 1082 & ORSF & 837.28 & 25.23 & 25.82 & -0.59 & 812.05 & 811.46 \\
\hline 1084 & ORSF & 965.40 & 62.81 & 63.46 & -0.65 & 902.59 & 901.94 \\
\hline 1090 & UNCS & 1104.48 & 43.37 & 56.20 & -12.83 & 1061.11 & 1048.28 \\
\hline GW-141 & LIV & 1186.23 & 93.00 & 95.52 & -2.52 & 1093.23 & 1090.71 \\
\hline GW-142 & KHQ & 971.15 & 134.34 & 139.13 & -4.79 & 836.81 & 832.02 \\
\hline GW-144 & KHQ & 913.54 & 78.63 & 82.16 & -3.53 & 834.91 & 831.38 \\
\hline GW-145 & KHQ & 840.24 & 3.94 & 8.32 & -4.38 & 836.30 & 831.92 \\
\hline GW-156 & CRSDB & 1049.30 & 142.70 & 143.65 & -0.95 & 906.60 & 905.65 \\
\hline GW-159 & CRSDB & 1051.40 & 116.71 & 118.86 & -2.15 & 934.69 & 932.54 \\
\hline GW-160 & CRBAWP & 1093.09 & 139.56 & 151.25 & -11.69 & 953.53 & 941.84 \\
\hline GW-173 & CRSP & 1115.00 & 125.95 & 148.37 & -22.42 & 989.05 & 966.63 \\
\hline GW-174 & CRSP & 1116.66 & 104.00 & 124.75 & -20.75 & 1012.66 & 991.91 \\
\hline GW-175 & CRSP & 1084.19 & 109.86 & 126.41 & -16.55 & 974.33 & 957.78 \\
\hline GW-176 & CRSP & 1125.30 & 116.00 & 116.03 & -0.03 & 1009.30 & 1009.27 \\
\hline GW-177 & CRSP & 1158.20 & 117.23 & 119.86 & -2.63 & 1040.97 & 1038.34 \\
\hline GW-178 & CRSP & 1143.49 & 88.81 & 97.95 & -9.14 & 1054.68 & 1045.54 \\
\hline GW-179 & CRSP & 1128.00 & 114.26 & 115.45 & -1.19 & 1013.74 & 1012.55 \\
\hline GW-180 & CRSP & 1104.14 & 94.85 & 128.22 & -33.37 & 1009.29 & 975.92 \\
\hline GW-184 & RQ & 927.63 & 109.94 & 111.07 & -1.13 & 817.69 & 816.56 \\
\hline GW-186 & RQ & 831.32 & 14.40 & 15.32 & -0.92 & 816.92 & 816.00 \\
\hline GW-203 & UNCS & 1105.45 & 79.27 & 83.77 & -4.50 & 1026.18 & 1021.68 \\
\hline GW-205 & UNCS & 1104.14 & 75.69 & 80.69 & -5.00 & 1028.45 & 1023.45 \\
\hline GW-217 & LIV & 1177.06 & 104.83 & 116.00 & -11.17 & 1072.23 & 1061.06 \\
\hline GW-221 & UNCS & 1106.16 & 81.73 & 85.31 & -3.58 & 1024.43 & 1020.85 \\
\hline GW-231 & KHQ & 849.67 & 12.79 & 17.70 & -4.91 & 836.88 & 831.97 \\
\hline GW-241 & CRSDB & 982.84 & 43.49 & 54.73 & -11.24 & 939.35 & 928.11 \\
\hline GW-292 & ECRWP & 1073.00 & 109.78 & 115.31 & -5.53 & 963.22 & 957.69 \\
\hline GW-293 & ECRWP & 1063.90 & 112.10 & 117.85 & -5.75 & 951.80 & 946.05 \\
\hline GW-298 & CRBAWP & 1049.01 & 105.41 & 111.03 & -5.62 & 943.60 & 937.98 \\
\hline GW-299 & CRBAWP & 1053.86 & 95.23 & 103.25 & -8.02 & 958.63 & 950.61 \\
\hline GW-300 & CRBAWP & 1073.12 & 108.93 & 118.50 & -9.57 & 964.19 & 954.62 \\
\hline
\end{tabular}


Table B.8 (continued)

\begin{tabular}{|c|c|c|c|c|c|c|c|}
\hline \multirow{2}{*}{\multicolumn{2}{|c|}{$\begin{array}{r}\text { Groundwater Elevation } \\
\text { (ft above mean sea level) }\end{array}$}} & & & & \multicolumn{3}{|c|}{ September 17-26, 2002} \\
\hline & & \multirow{2}{*}{\multicolumn{4}{|c|}{$\begin{array}{l}\text { April } 8 \\
\text { Seasonal Fluctuation }(+/-\mathrm{ft})\end{array}$}} & & \\
\hline & & & & & & & \\
\hline \multirow{2}{*}{\multicolumn{2}{|c|}{$\begin{array}{r}\text { Depth-to-Water } \\
\text { (ft below measuring point) }\end{array}$}} & \multicolumn{3}{|c|}{ September 17-26, 2002} & & & \\
\hline & & \multicolumn{2}{|c|}{ April 8-10, 2002} & & & & \\
\hline Well Number & Location $^{1}$ & $\begin{array}{l}\text { Measuring Point } \\
\text { Elevation }^{2}\end{array}$ & & & & & \\
\hline GW-301 & CRBAWP & 1086.55 & 125.81 & 136.19 & -10.38 & 960.74 & 950.36 \\
\hline GW-302 & UNCS & 1141.84 & 95.12 & 104.63 & -9.51 & 1046.72 & 1037.21 \\
\hline GW-303 & CRSDB & 1007.16 & 83.85 & 89.51 & -5.66 & 923.31 & 917.65 \\
\hline GW-304 & CRSDB & 1045.49 & 116.56 & 118.81 & -2.25 & 928.93 & 926.68 \\
\hline GW-305 & LIV & 1183.75 & 117.22 & 126.30 & -9.08 & 1066.53 & 1057.45 \\
\hline GW-322 & CRSP & 1135.05 & 143.00 & 165.00 & -22.00 & 992.05 & 970.05 \\
\hline GW-339 & UNCS & 1124.83 & 64.55 & 76.78 & -12.23 & 1060.28 & 1048.05 \\
\hline GW-511 & CRSP & 1093.21 & 96.49 & 113.50 & -17.01 & 996.72 & 979.71 \\
\hline GW-512 & FCAP & 1001.54 & 19.06 & 32.11 & -13.05 & 982.48 & 969.43 \\
\hline GW-521 & LIV & 1182.88 & 80.97 & 87.20 & -6.23 & 1101.91 & 1095.68 \\
\hline GW-522 & LIV & 1175.49 & 94.94 & 111.40 & -16.46 & 1080.55 & 1064.09 \\
\hline GW-539 & LII & 1093.00 & 102.86 & 110.17 & -7.31 & 990.14 & 982.83 \\
\hline GW-540 & CDLVI & 1072.32 & 79.79 & 86.10 & -6.31 & 992.53 & 986.22 \\
\hline GW-541 & CDLVI & 1058.40 & 63.31 & 64.63 & -1.32 & 995.09 & 993.77 \\
\hline GW-542 & CDLVI & 1051.77 & 69.92 & 72.70 & -2.78 & 981.85 & 979.07 \\
\hline GW-543 & CDLVI & 1023.98 & 63.85 & 65.81 & -1.96 & 960.13 & 958.17 \\
\hline GW-544 & CDLVI & 1045.20 & 58.25 & 62.19 & -3.94 & 986.95 & 983.01 \\
\hline GW-557 & LV & 1081.36 & 119.34 & 123.66 & -4.32 & 962.02 & 957.70 \\
\hline GW-558 & SSCR & 981.42 & 46.13 & 52.81 & -6.68 & 935.29 & 928.61 \\
\hline GW-559 & SSCR & 1102.79 & 135.52 & 142.25 & -6.73 & 967.27 & 960.54 \\
\hline GW-560 & CDLVII & 949.05 & 38.33 & 46.13 & -7.80 & 910.72 & 902.92 \\
\hline GW-562 & CDLVII & 934.69 & 6.30 & 11.55 & -5.25 & 928.39 & 923.14 \\
\hline GW-564 & CDLVII & 937.97 & 10.16 & 12.17 & -2.01 & 927.81 & 925.80 \\
\hline GW-608 & CRSP & 1074.75 & 122.00 & 139.55 & -17.55 & 952.75 & 935.20 \\
\hline GW-609 & CRSP & 1112.31 & 160.55 & 167.82 & -7.27 & 951.76 & 944.49 \\
\hline GW-610 & CRSP & 1059.44 & 80.00 & 91.60 & -11.60 & 979.44 & 967.84 \\
\hline GW-611 & CRSP & 1048.38 & 98.20 & 108.82 & -10.62 & 950.18 & 939.56 \\
\hline GW-612 & CRSP & 1131.03 & 117.93 & 128.93 & -11.00 & 1013.10 & 1002.10 \\
\hline GW-674 & FCAP & 883.79 & 6.98 & 8.55 & -1.57 & 876.81 & 875.24 \\
\hline GW-676 & FCAP & 846.50 & 3.63 & 7.59 & -3.96 & 842.87 & 838.91 \\
\hline GW-677 & FCAP & 1030.40 & 22.97 & 32.50 & -9.53 & 1007.43 & 997.90 \\
\hline GW-678 & FCAP & 1000.70 & 12.50 & 28.22 & -15.72 & 988.20 & 972.48 \\
\hline GW-679 & FCAP & 1026.90 & 45.52 & 59.12 & -13.60 & 981.38 & 967.78 \\
\hline GW-680 & FCAP & 1001.50 & 25.00 & 39.10 & -14.10 & 976.50 & 962.40 \\
\hline GW-709 & LII & 906.78 & 28.70 & 29.86 & -1.16 & 878.08 & 876.92 \\
\hline
\end{tabular}


Table B.8 (continued)

\begin{tabular}{|c|c|c|c|c|c|c|c|}
\hline \multirow{2}{*}{\multicolumn{2}{|c|}{$\begin{array}{r}\text { Groundwater Elevation } \\
\text { (ft above mean sea level) }\end{array}$}} & & & & \multicolumn{3}{|c|}{ September 17-26, 2002} \\
\hline & & & & & \multicolumn{2}{|c|}{ April 8-10, 2002} & \\
\hline & & \multicolumn{4}{|c|}{ Seasonal Fluctuation (+/- ft) } & & \\
\hline \multirow{2}{*}{\multicolumn{2}{|c|}{$\begin{array}{r}\text { Depth-to-Water } \\
\text { (ft below measuring point) }\end{array}$}} & \multicolumn{3}{|c|}{ September 17-26, 2002} & & & \\
\hline & & \multicolumn{2}{|c|}{ April 8-10, 2002} & & & & \\
\hline Well Number & Location $^{1}$ & $\begin{array}{l}\text { Measuring Point } \\
\text { Elevation }^{2}\end{array}$ & & & & & \\
\hline GW-731 & CRSDB & 1049.38 & 124.51 & 125.23 & -0.72 & 924.87 & 924.15 \\
\hline GW-732 & CRSDB & 1064.29 & 157.26 & 157.85 & -0.59 & 907.03 & 906.44 \\
\hline GW-743 & CRSP & 1100.36 & 116.18 & 137.51 & -21.33 & 984.18 & 962.85 \\
\hline GW-757 & LII & 961.61 & 84.11 & 84.75 & -0.64 & 877.50 & 876.86 \\
\hline GW-796 & LV & 1052.62 & 66.28 & $\mathrm{NM}^{3}$ & $\mathrm{NA}^{4}$ & 986.34 & NA \\
\hline GW-797 & LV & 1060.00 & 71.94 & NM & NA & 988.06 & NA \\
\hline GW-798 & CDLVII & 1006.00 & 77.57 & 83.36 & -5.79 & 928.43 & 922.64 \\
\hline GW-799 & LV & 981.29 & 13.21 & 20.77 & -7.56 & 968.08 & 960.52 \\
\hline GW-801 & LV & 1097.16 & 104.68 & NM & NA & 992.48 & NA \\
\hline GW-827 & CDLVI & 1051.58 & 42.37 & 45.40 & -3.03 & 1009.21 & 1006.18 \\
\hline GW-831 & FCAP & 1091.29 & 125.56 & 131.10 & -5.54 & 965.73 & 960.19 \\
\hline
\end{tabular}

Notes:

1 CDLVI - Construction/Demolition Landfill VI

CDLVII - Construction/Demolition Landfill VII

CRBAWP - Chestnut Ridge Borrow Area Waste Pile

CRSDB - Chestnut Ridge Sediment Disposal Basin

CRSP - Chestnut Ridge Security Pits

ECRWP - East Chestnut Ridge Waste Pile

FCAP - Filled Coal Ash Pond

KHQ - Kerr Hollow Quarry

LII - Industrial Landfill II

LIV - Industrial Landfill IV

LV - Industrial Landfill V

ORSF - Oak Ridge Sludge Farm

RQ - Rogers Quarry

SSCR - South Side Chestnut Ridge

UNCS - United Nuclear Corporation Site

2 Measuring point elevation is in feet above mean sea level. The measuring point is either the top of the innermost well casing or the top of dedicated sampling equipment mounted on the casing.

3 NM - Not measured or unusable

4 NA - Not applicable 
APPENDIX C

\section{MONITORING WELL CONSTRUCTION DETAILS}




\section{EXPLANATION}

Hydrogeologic Regime:

$\begin{array}{lll}\text { BC } & - & \text { Bear Creek Hydrogeologic Regime } \\ \mathrm{CR} & - & \text { Chestnut Ridge Hydrogeologic Regime } \\ \mathrm{EF} & - & \text { Upper East Fork Poplar Creek Hydrogeologic Regime }\end{array}$

Location:

$\begin{array}{rll}\text { B8110 } & - & \text { Building 81-10 } \\ \text { BG } & - & \text { Bear Creek Burial Grounds WMA } \\ \text { CDLVI } & - & \text { Construction/Demolition Landfill VI } \\ \text { CDLVII } & - & \text { Construction/Demolition Landfill VII } \\ \text { CPT } & - & \text { Coal Pile Trench } \\ \text { CRBAWP } & - & \text { Chestnut Ridge Borrow Area Waste Pile (former site) } \\ \text { CRSDB } & - & \text { Chestnut Ridge Sediment Disposal Basin } \\ \text { CRSP } & - & \text { Chestnut Ridge Security Pits } \\ \text { EMWMF } & - & \text { Environmental Management Waste Management Facility } \\ \text { EXP } & - & \text { Exit Pathway Monitoring Location: } \\ & & \quad \text { Maynardville Limestone Picket (-A, -B, -C, -E, -I, -J, and -W) } \\ & & \quad \text { Along Scarboro Road in the gap through Pine Ridge (-SR) } \\ & & \quad \text { East of Scarboro Road in Union Valley (-UV) } \\ \text { FCAP } & - & \text { Filled Coal Ash Pond } \\ \text { FF } & - & \text { Fuel Facility (Building 9754-2) } \\ \text { FTF } & - & \text { Fire Training Facility } \\ \text { GRID } & - & \text { Comprehensive Groundwater Monitoring Plan Grid Location } \\ \text { KHQ } & - & \text { Kerr Hollow Quarry } \\ \text { LII } & - & \text { Industrial Landfill II } \\ \text { LIV } & - & \text { Industrial Landfill IV } \\ \text { LV } & - & \text { Industrial Landfill V } \\ \text { NHP } & - & \text { New Hope Pond } \\ \text { OLF } & - & \text { Oil Landfarm WMA } \\ \text { RG } & - & \text { Rust Garage Area } \\ \text { RS } & - & \text { Rust Spoil Area } \\ \text { S2 } & - & \text { S-2 Site } \\ \text { S3 } & - & \text { S-3 Site } \\ \text { SPI } & - & \text { Spoil Area I } \\ \text { T0134 } & - & \text { Tank 0134-U } \\ \text { T2331 } & - & \text { Tank 2331-U, near Building 9201-1 } \\ \text { UNCS } & - & \text { United Nuclear Corporation Site } \\ \text { UOV } & - & \text { Uranium Oxide Vault } \\ & & \end{array}$




\section{EXPLANATION (continued)}

\section{General Information:}

Depth - Feet below ground surface (rounded to nearest $0.1 \mathrm{ft}$ )

Coordinates - Y-12 grid system (rounded to nearest foot)

Measuring Point - Top of well casing or Well Wizard ${ }^{\mathrm{TM}}$

Elevation - Feet above mean sea level (rounded to nearest $0.01 \mathrm{ft}$ )

- Not Applicable or not available

\section{Geologic Information (regarding the monitored interval):}

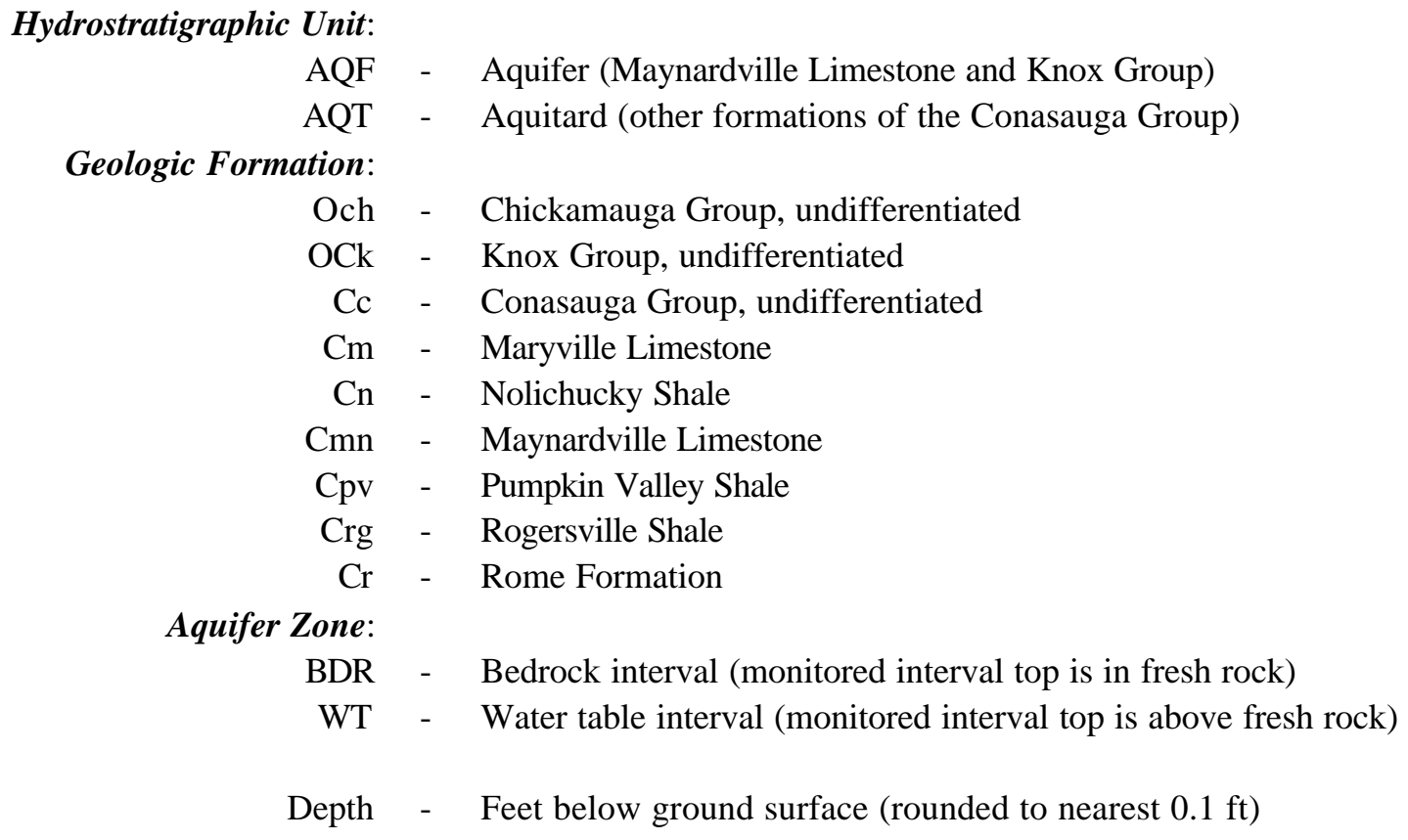

\section{Conductor (Surface) Casing and Well Casing:}

Depth - Feet below ground surface (rounded to nearest $0.1 \mathrm{ft}$ )

Diameter - Outside dimensions, in inches (nominal)

PVC40 - Polyvinyl chloride, schedule 40

SS304 - Stainless steel, schedule 304

STL - Carbon steel

SF25/SJ55 - Steel; American Petroleum Institute Grade

\section{Monitored Interval:}

Top - Depth to top of filter pack or open-hole (feet below ground surface)

Bottom - Depth to bottom of filter pack or open-hole (feet below ground surface)

Screen Material:

$\mathrm{PVC} / \mathrm{sl}$ - PVC/slotted

$\mathrm{PVC} / \mathrm{sw}$ - PVC, spiral wound

SS/sw - Stainless steel, spiral wound

SS/ppk - Stainless steel prepack screen, spiral wound

Slot Size - size of screen openings, in inches 


\title{
EXPLANATION (continued)
}

\section{Dedicated Pump:}

\author{
Screen Midpoint - Depth (feet below the top of casing [TOC]) \\ Pump Intake - Depth to the intake of the Well Wizard ${ }^{\mathrm{TM}}$ pump (feet below the TOC)
}

\section{NOTE:}

Data compiled from the Updated Subsurface Data Base for Bear Creek Valley, Chestnut Ridge, and parts of Bethel Valley on the U.S. Department of Energy Oak Ridge Reservation (LMES 1998). 
APPENDIX C: MONITORING WELL CONSTRUCTION DETAILS, 2002

\begin{tabular}{|c|c|c|c|c|c|c|c|c|c|c|}
\hline $\begin{array}{r}\text { Well Number } \\
\text { Hydrogeologic Regime } \\
\text { Location }\end{array}$ & $\begin{array}{c}1090 \\
\text { CR } \\
\text { UNCS }\end{array}$ & $\begin{array}{l}55-1 B \\
\text { PC } \\
\text { Y12 }\end{array}$ & $\begin{array}{c}\text { GW-008 } \\
\text { BC } \\
\text { OLF }\end{array}$ & $\begin{array}{c}\text { GW-014 } \\
\text { BC } \\
\text { BG }\end{array}$ & $\begin{array}{c}\text { GW-046 } \\
\text { BC } \\
\text { BG }\end{array}$ & $\begin{array}{l}W-061 \\
B C \\
B G\end{array}$ & $\begin{array}{c}\text { GW-066 } \\
\text { BC } \\
\text { OLF }\end{array}$ & $\begin{array}{c}\text { GW-069 } \\
\text { BC } \\
\text { BG }\end{array}$ & $\begin{array}{c}\text { GW-071 } \\
\text { BC } \\
\text { BG }\end{array}$ & $\begin{array}{c}\text { GW-072 } \\
\text { BC } \\
\text { BG }\end{array}$ \\
\hline $\begin{array}{r}\text { General Information } \\
\text { Date Installed } \\
\text { Total Depth Drilled } \\
\text { East Coordinate } \\
\text { North Coordinate } \\
\text { Measuring Point Elevation } \\
\text { Well Wizard Elevation } \\
\text { Top of Casing Elevation } \\
\text { Ground Surface Elevation }\end{array}$ & $\begin{array}{r}96.7 \\
53,853 \\
28,718 \\
1,104.48 \\
1,104.48 \\
1,103.88 \\
1,101.58 \\
\end{array}$ & $\begin{array}{r}09 / 12 / 83 \\
38.8 \\
55,010 \\
30,469 \\
986.26 \\
. \\
986.26 \\
986.05 \\
\end{array}$ & $\begin{array}{r}09 / 21 / 83 \\
25.5 \\
47,596 \\
29,783 \\
965.39 \\
965.39 \\
964.79 \\
962.11 \\
\end{array}$ & $\begin{array}{r}09 / 29 / 83 \\
13.2 \\
44,308 \\
29,848 \\
934.50 \\
934.50 \\
933.90 \\
931.50 \\
\end{array}$ & $\begin{array}{r}10 / 27 / 83 \\
20.5 \\
43,284 \\
29,562 \\
921.17 \\
921.17 \\
920.57 \\
918.13 \\
\end{array}$ & $\begin{array}{r}03 / 21 / 84 \\
25.0 \\
43,049 \\
28,916 \\
904.60 \\
. \\
904.60 \\
901.00 \\
\end{array}$ & $\begin{array}{r}03 / 24 / 84 \\
55.8 \\
48,677 \\
29,513 \\
961.60 \\
. \\
961.60 \\
960.52 \\
\end{array}$ & $\begin{array}{r}03 / 26 / 84 \\
100.0 \\
43,802 \\
29,489 \\
927.60 \\
927.60 \\
927.00 \\
924.20 \\
\end{array}$ & $\begin{array}{r}03 / 25 / 84 \\
220.6 \\
44,191 \\
29,495 \\
928.90 \\
928.90 \\
928.30 \\
925.40 \\
\end{array}$ & $\begin{array}{r}03 / 30 / 84 \\
101.4 \\
44,159 \\
29,502 \\
930.50 \\
930.51 \\
929.90 \\
926.30 \\
\end{array}$ \\
\hline $\begin{array}{r}\text { Hydrostratigra } \\
\text { Geologic F } \\
\text { Aqu } \\
\text { Weathered Ro } \\
\text { Fresh Ro }\end{array}$ & $\begin{array}{r}\text { AQF } \\
\text { OCk } \\
\text { WT }\end{array}$ & $\begin{array}{r}\mathrm{AQT} \\
\mathrm{Cn} \\
\mathrm{WT} \\
8.0\end{array}$ & $\begin{array}{r}\mathrm{AQT} \\
\mathrm{Cn} \\
\mathrm{WT} \\
0.6\end{array}$ & $\begin{array}{r}\mathrm{AQT} \\
\mathrm{Cn} \\
\mathrm{WT} \\
4.0\end{array}$ & $\begin{array}{r}\mathrm{AQT} \\
\mathrm{Cn} \\
\mathrm{WT} \\
7.7\end{array}$ & $\begin{array}{r}\text { AQF } \\
\text { Cmn } \\
\text { BDR } \\
. \\
15.7\end{array}$ & $\begin{array}{l}\text { AQF } \\
\text { Cmn } \\
\text { BDR } \\
16.2\end{array}$ & $\begin{array}{r}\text { AQT } \\
\mathrm{Cn} \\
\mathrm{BDR} \\
3.0 \\
24.0\end{array}$ & $\begin{array}{r}\text { AQT } \\
\text { Cn } \\
\text { BDR } \\
5.5 \\
16.0\end{array}$ & $\begin{array}{r}\text { AQT } \\
\mathrm{Cn} \\
\mathrm{BDR} \\
5.0 \\
34.0\end{array}$ \\
\hline $\begin{array}{l}\text { onductor Casing } \\
\text { Casing Depth } \\
\text { Casing Diameter } \\
\text { Casing Material }\end{array}$ & & & & & & 15.7 & & $\begin{array}{r}19.0 \\
10.75 \\
\text { PVC }\end{array}$ & $\begin{array}{r}16.0 \\
10.63 \\
\text { PVC40 }\end{array}$ & $\begin{array}{r}13.2 \\
10.63 \\
\text { PVC40 } \\
\end{array}$ \\
\hline $\begin{array}{r}\text { Borehole } \\
\text { Borehole Di} \\
\text { Casing } \\
\text { Casing Di } \\
\text { Casing } \mathrm{N}\end{array}$ & $\begin{array}{r}96.7 \\
8 \\
. \\
6.5 \\
\text { PVC } 40\end{array}$ & $\begin{array}{r}38.8 \\
6 \\
33.8 \\
4.5 \\
\text { PVC40 }\end{array}$ & $\begin{array}{r}25.5 \\
4.5 \\
15.7 \\
2.37 \\
\text { SS304 }\end{array}$ & $\begin{array}{r}13.2 \\
6 \\
10.0 \\
2.37 \\
S S 304 \\
\end{array}$ & $\begin{array}{r}20.5 \\
6 \\
8.1 \\
2.37 \\
\text { SS304 }\end{array}$ & $\begin{array}{r}25.0 \\
4.75 \\
19.6 \\
2.37 \\
\text { SS } 304 \\
\end{array}$ & $\begin{array}{r}55.8 \\
4 \\
52.9 \\
2.37 \\
\text { SS304 }\end{array}$ & $\begin{array}{r}100.0 \\
7.88 \\
89.0 \\
2.37 \\
\text { SS304 }\end{array}$ & $\begin{array}{r}220.6 \\
8.75 \\
198.4 \\
2.37 \\
\text { SS } 304\end{array}$ & $\begin{array}{r}101.4 \\
8.75 \\
87.8 \\
2.37 \\
\text { SS304 }\end{array}$ \\
\hline $\begin{array}{r}\text { onitored Interval } \\
\text { Top-Depth } \\
\text { Midpoint-Depth } \\
\text { Bottom of Screen-Depth } \\
\text { Bottom-Depth } \\
\text { Top-Elevation } \\
\text { Midpoint-Elevation } \\
\text { Bottom-Elevation } \\
\text { Screen Length } \\
\text { Screen Material } \\
\text { Slot Size } \\
\text { Open-Hole Length } \\
\text { Open-Hole Diameter }\end{array}$ & $\mathrm{PVC} / \mathrm{sl}$ & $\begin{array}{r}30.8 \\
34.8 \\
38.8 \\
38.8 \\
955.25 \\
951.25 \\
947.25 \\
5 \\
\mathrm{SS} / \mathrm{sw} \\
0.01\end{array}$ & $\begin{array}{r}13.0 \\
19.3 \\
20.7 \\
25.5 \\
949.11 \\
942.86 \\
936.61 \\
5 \\
\mathrm{SS} / \mathrm{sw} \\
0.01\end{array}$ & $\begin{array}{r}5.0 \\
9.1 \\
12.0 \\
13.2 \\
926.50 \\
922.40 \\
918.30 \\
2 \\
\mathrm{SS} / \mathrm{sw} \\
0.01\end{array}$ & $\begin{array}{r}5.0 \\
12.7 \\
18.1 \\
20.3 \\
913.13 \\
905.48 \\
897.83 \\
10 \\
\mathrm{SS} / \mathrm{sw} \\
0.01\end{array}$ & $\begin{array}{r}17.5 \\
21.1 \\
24.6 \\
24.6 \\
883.50 \\
879.95 \\
876.40 \\
5 \\
\text { SS/sw } \\
0.01\end{array}$ & $\begin{array}{r}50.0 \\
52.5 \\
54.9 \\
54.9 \\
910.52 \\
908.07 \\
905.62 \\
2 \\
\mathrm{SS} / \mathrm{sw} \\
0.01\end{array}$ & $\begin{array}{r}79.0 \\
89.1 \\
99.2 \\
99.2 \\
845.20 \\
835.10 \\
825.00 \\
10.2 \\
\mathrm{SS} / \mathrm{sw} \\
0.01\end{array}$ & $\begin{array}{r}195.1 \\
207.1 \\
219.0 \\
219.0 \\
730.30 \\
718.35 \\
706.40 \\
20.6 \\
\mathrm{SS} / \mathrm{sw} \\
0.01\end{array}$ & $\begin{array}{r}84.5 \\
91.5 \\
98.4 \\
98.4 \\
841.80 \\
834.85 \\
827.90 \\
10.6 \\
S S / s w \\
0.01\end{array}$ \\
\hline $\begin{array}{r}\text { Screen Midpoint-(TOC) } \\
\text { Pump Intake-(TOC) } \\
\text { Intake-Elevation }\end{array}$ & & $\begin{array}{r}36.5 \\
36.5 \\
949.8\end{array}$ & $\begin{array}{r}20.9 \\
21.0 \\
943.8\end{array}$ & $\begin{array}{r}13.4 \\
12.0 \\
921.9\end{array}$ & $\begin{array}{r}15.5 \\
15.0 \\
905.6\end{array}$ & $\begin{array}{r}25.7 \\
25.0 \\
879.6\end{array}$ & $\begin{array}{r}55.0 \\
58.0 \\
903.6\end{array}$ & $\begin{array}{r}96.9 \\
96.0 \\
831.0\end{array}$ & $\begin{array}{l}211.6 \\
212.0 \\
716.3\end{array}$ & $\begin{array}{r}96.7 \\
98.0 \\
831.9\end{array}$ \\
\hline
\end{tabular}


APPENDIX C: MONITORING WELL CONSTRUCTION DETAILS, 2002

\begin{tabular}{|c|c|c|c|c|c|c|c|c|c|c|}
\hline $\begin{array}{r}\text { Well Number } \\
\text { Hydrogeologic Regime } \\
\text { Location }\end{array}$ & $\begin{array}{c}\text { GW-076 } \\
\text { BC } \\
\text { OLF }\end{array}$ & $\begin{array}{c}\text { GW-077 } \\
\text { BC } \\
\text { BG }\end{array}$ & $\begin{array}{c}\text { GW-078 } \\
\text { BC } \\
\text { BG }\end{array}$ & $\begin{array}{c}\text { GW-079 } \\
\text { BC } \\
\text { BG }\end{array}$ & $\begin{array}{c}\text { GW-080 } \\
\text { BC } \\
\text { BG }\end{array}$ & $\begin{array}{c}\text { GW-082 } \\
\text { BC } \\
\text { BG }\end{array}$ & $\begin{array}{c}\text { GW-085 } \\
\text { BC } \\
\text { OLF }\end{array}$ & $\begin{array}{c}\text { GW-087 } \\
\text { BC } \\
\text { OLF }\end{array}$ & $\begin{array}{c}\text { GW-091 } \\
\text { BC } \\
\text { BG }\end{array}$ & $\begin{array}{c}\text { GW-108 } \\
\text { PC } \\
\text { S3 }\end{array}$ \\
\hline General Information & & & & & & & & & & \\
\hline Date Installed & $03 / 28 / 84$ & $03 / 29 / 84$ & $03 / 30 / 84$ & $03 / 23 / 84$ & $03 / 24 / 84$ & $03 / 17 / 84$ & $03 / 22 / 84$ & $03 / 23 / 84$ & & $09 / 26 / 84$ \\
\hline Total Depth Drilled & 81.0 & 100.5 & 21.1 & 65.0 & 30.0 & 35.0 & 62.0 & 19.0 & 25.0 & 58.6 \\
\hline East Coordinate & 48,238 & 41,234 & 41,209 & 41,616 & 41,621 & 42,090 & 49,058 & 48,313 & 43,621 & 53,207 \\
\hline North Coordinate & 29,909 & 29,729 & 29,730 & 30,630 & 30,622 & 30,434 & 30,003 & 29,534 & 30,621 & 30,070 \\
\hline Measuring Point Elevation & 967.06 & 919.30 & 918.10 & 981.20 & 981.00 & 964.00 & 983.50 & 961.20 & 953.48 & 999.00 \\
\hline Well Wizard Elevation & & 919.30 & 918.10 & 981.20 & 981.00 & 964.00 & 983.57 & 961.18 & & 999.00 \\
\hline Top of Casing Elevation & 967.06 & 918.70 & 917.50 & 980.60 & 980.40 & 963.40 & 982.90 & 960.60 & 952.62 & 998.80 \\
\hline Ground Surface Elevation & 964.01 & 914.70 & 914.50 & 977.20 & 977.10 & 960.52 & 979.80 & 957.30 & 949.82 & 995.80 \\
\hline Geologic Information & & & & & & & & & & \\
\hline Hydrostratigraphic Unit & AQT & AQT & AQT & AQT & $\mathrm{AQT}$ & $\mathrm{AQT}$ & AQT & $\mathrm{AQT}$ & AQT & AQT \\
\hline Geologic Formation & $\mathrm{Cn}$ & $\mathrm{Cn}$ & $\mathrm{Cn}$ & $\mathrm{Crg}$ & $\mathrm{Crg}$ & $\mathrm{Cm}$ & $\mathrm{Cn}$ & $\mathrm{Cn}$ & $\mathrm{Crg}$ & $\mathrm{Cn}$ \\
\hline Aquifer Zone & $\mathrm{BDR}$ & BDR & $\mathrm{BDR}$ & BDR & WT & $\mathrm{BDR}$ & BDR & WT & WT & WT \\
\hline Weathered Rock-Depth & 12.0 & 7.0 & 6.5 & 4.0 & 3.5 & 7.0 & 2.0 & 19.0 & & 4.0 \\
\hline Fresh Rock-Depth & 48.0 & 13.0 & 8.5 & 26.5 & 23.5 & 23.0 & 40.0 & & & \\
\hline Conductor Casing & & & & & & & & & & \\
\hline Casing Depth & 19.0 & 35.0 & & & & 25.0 & & & & 20.7 \\
\hline Casing Diameter & 10.63 & 4.5 & & & & 6.5 & & & & 10.63 \\
\hline Casing Material & PVC40 & STL & & & & STL & & & & PVC40 \\
\hline Well Casing & & & & & & & & & & \\
\hline Borehole Depth & 81.0 & 100.5 & 21.1 & 65.0 & 30.0 & 35.0 & 62.0 & 19.0 & 25.0 & 58.6 \\
\hline Borehole Diameter & 8.75 & 3.88 & 6.5 & 6.5 & 6.5 & 4 & 4 & 6.5 & 6.5 & 9 \\
\hline Casing Depth & 69.7 & 90.3 & 16.1 & 59.9 & 24.7 & 29.4 & 53.8 & 9.0 & 23.0 & 46.7 \\
\hline Casing Diameter & 2.37 & 2.37 & 2.37 & 2.37 & 2.37 & 2.37 & 2.37 & 2.37 & 2.37 & 4.5 \\
\hline Casing Material & SS304 & SS304 & SS304 & SS304 & SS304 & SS304 & SS304 & SS304 & SS304 & PVC40 \\
\hline Monito & & & & & & & & & & \\
\hline Top-Depth & 67.8 & 87.4 & 11.7 & 49.9 & 20.8 & 24.1 & 48.4 & 7.5 & 20.0 & 41.0 \\
\hline Midpoint-Depth & 74.1 & 93.9 & 16.4 & 57.4 & 25.3 & 29.3 & 53.6 & 13.3 & 22.5 & 49.8 \\
\hline Bottom of Screen-Depth & 80.3 & 100.3 & 21.1 & 64.9 & 29.7 & 34.4 & 58.8 & 19.0 & 25.0 & 55.7 \\
\hline Bottom-Depth & 80.3 & 100.3 & 21.1 & 64.9 & 29.7 & 34.4 & 58.8 & 19.0 & 25.0 & 58.6 \\
\hline Top-Elevation & 896.21 & 827.30 & 902.80 & 927.30 & 956.30 & 936.42 & 931.40 & 949.80 & 929.82 & 954.80 \\
\hline Midpoint-Elevation & 889.96 & 820.85 & 898.10 & 919.80 & 951.85 & 931.27 & 926.20 & 944.05 & 927.32 & 946.00 \\
\hline Bottom-Elevation & 883.71 & 814.40 & 893.40 & 912.30 & 947.40 & 926.12 & 921.00 & 938.30 & 924.82 & 937.20 \\
\hline Screen Length & 10.6 & 10 & & & & & 5 & 10 & 2 & 9 \\
\hline Screen Material & SS/sw & $\mathrm{SS} / \mathrm{sw}$ & $\mathrm{SS} / \mathrm{sw}$ & SS/sw & $\mathrm{SS} / \mathrm{sw}$ & SS/sw & $\mathrm{SS} / \mathrm{sw}$ & SS/sw & $\mathrm{SS} / \mathrm{sw}$ & $\mathrm{PVC} / \mathrm{sl}$ \\
\hline $\begin{array}{r}\text { Slot Size } \\
\text { Open-Hole Length } \\
\text { Open-Hole Diameter }\end{array}$ & 0.01 & 0.01 & 0.01 & 0.01 & 0.01 & 0.01 & 0.01 & 0.01 & 0.01 & 0.01 \\
\hline Dedicated Pump & & & & & & & & & & \\
\hline Screen Midpoint-(TOC) & 78.1 & 99.3 & 21.6 & 65.8 & 30.5 & 34.8 & 59.4 & 17.3 & 26.8 & 54.2 \\
\hline Pump Intake-(TOC) & 79.0 & & & & & 35.0 & 55.0 & 18.0 & 26.5 & 53.0 \\
\hline Intake-Elevation & 888.1 & & & & & 928.4 & 927.9 & 942.6 & 926.1 & 945.8 \\
\hline
\end{tabular}


APPENDIX C: MONITORING WELL CONSTRUCTION DETAILS, 2002

\begin{tabular}{|c|c|c|c|c|c|c|c|c|c|c|}
\hline $\begin{array}{r}\text { Well Number } \\
\text { Hydrogeologic Regime } \\
\text { Location }\end{array}$ & $\begin{array}{c}\text { GW-115 } \\
\text { BC } \\
\text { S3 }\end{array}$ & $\begin{array}{c}\text { GW-141 } \\
\text { CR } \\
\text { LIV }\end{array}$ & $\begin{array}{c}\text { GW-142 } \\
\text { CR } \\
\text { KHQ }\end{array}$ & $\begin{array}{c}\text { GW-143 } \\
\text { CR } \\
\text { KHQ }\end{array}$ & $\begin{array}{c}\text { GW-144 } \\
\text { CR } \\
\text { KHQ }\end{array}$ & $\begin{array}{c}\text { GW-145 } \\
\text { CR } \\
\text { KHQ }\end{array}$ & $\begin{array}{c}\text { GW-151 } \\
\text { PC } \\
\text { NHP }\end{array}$ & $\begin{array}{c}\text { GW-153 } \\
\text { PC } \\
\text { NHP }\end{array}$ & $\begin{array}{c}\text { GW-154 } \\
\text { PC } \\
\text { NHP }\end{array}$ & $\begin{array}{c}\text { GW-156 } \\
\text { CR } \\
\text { CRSDB }\end{array}$ \\
\hline \multicolumn{11}{|l|}{ General Information } \\
\hline Date Installed & & 09/04/87 & $10 / 03 / 85$ & $10 / 24 / 85$ & $10 / 24 / 85$ & $10 / 14 / 85$ & $08 / 14 / 85$ & $10 / 31 / 85$ & $07 / 30 / 85$ & $10 / 18 / 85$ \\
\hline Total Depth Drilled & 53.0 & 156.0 & 295.0 & 253.0 & 195.0 & 110.0 & 96.5 & 60.0 & 11.2 & 157.6 \\
\hline East Coordinate & 52,685 & 52,463 & 64,030 & 63,522 & 63,502 & 63,266 & 64,232 & 63,728 & 63,346 & 64,020 \\
\hline North Coordinate & 31,073 & 28,755 & 24,524 & 24,257 & 24,255 & 24,441 & 28,958 & 28,613 & 28,987 & 27,626 \\
\hline Measuring Point Elevation & $1,055.01$ & $1,186.23$ & 971.15 & 913.98 & 913.54 & 840.24 & 916.17 & 921.64 & 911.70 & $1,049.30$ \\
\hline Well Wizard Elevation & $1,055.01$ & $1,186.23$ & 971.15 & 913.98 & 913.54 & 840.24 & 916.17 & 921.68 & 911.70 & $1,049.28$ \\
\hline Top of Casing Elevation & $1,054.41$ & $1,186.06$ & 970.35 & 913.18 & 913.34 & 840.04 & 915.97 & 921.44 & 911.50 & $1,049.10$ \\
\hline Ground Surface Elev & $1,051.92$ & $1,183.45$ & 968.29 & 911.04 & 910.48 & 837.29 & 913.06 & 918.53 & 908.60 & $1,046.94$ \\
\hline \multicolumn{11}{|l|}{ Geologic Information } \\
\hline Geologic Formation & $\mathrm{Cm}$ & OCk & OCk & ock & Ock/Och & OCk & $\mathrm{Cmn}$ & $\mathrm{Cmn}$ & $\mathrm{Cmn}$ & OCk \\
\hline Aquifer Zone & WT & BDR & $\mathrm{BDR}$ & $\mathrm{BDR}$ & BDR & WT & $\mathrm{BDR}$ & BDR & WT & BDR \\
\hline Weathered Rock-Depth & 20.0 & & & & & & & & 11.2 & 84.0 \\
\hline Fresh Rock- & & 57.0 & & 18.0 & 40.0 & 12.0 & 12.0 & 14.0 & & 93.0 \\
\hline \multicolumn{11}{|l|}{ Conductor Casing } \\
\hline Casing & & 65.0 & 20.0 & 20.0 & 40.0 & 12.0 & 12.0 & 29.0 & & 94.0 \\
\hline Casing Diameter & & 10.75 & 12.5 & 10.63 & 12.5 & 12.5 & 12.5 & 10.63 & & 10.75 \\
\hline Casing $\mathrm{Ma}$ & & SF25 & PVC40 & PVC40 & PVC40 & PVC40 & PVC40 & PVC40 & & SF25 \\
\hline \multicolumn{11}{|l|}{ Well Casing } \\
\hline Borehole & 53.0 & 156.0 & 250.0 & 205.0 & 195.0 & 110.0 & 96.5 & 60.0 & 11.2 & 157.0 \\
\hline Borehole Diameter & & & 11 & 10 & 11 & 11 & 11 & 11 & 8 & 8.5 \\
\hline Casing Depth & 42.0 & 144.5 & 248.5 & 205.0 & 150.0 & 88.5 & 86.0 & 49.5 & 5.7 & 147.0 \\
\hline Casing Diameter & 2.37 & 4.5 & 6.62 & 6.62 & 4.5 & 4.5 & 4.5 & 4.5 & 4.5 & 4.5 \\
\hline Casing Material & SS304 & SS304 & SF25 & SF25 & PVC40 & PVC40 & PVC40 & PVC40 & PVC40 & PVC40 \\
\hline \multicolumn{11}{|l|}{ Monitored Interval } \\
\hline Top & 37.6 & 141.0 & 248.5 & 205.0 & 148.0 & 86.0 & 85.0 & 45.0 & 4.7 & 145.0 \\
\hline Midpoint- & 45.3 & 148.5 & 271.8 & 229.0 & 171.5 & 98.0 & 90.8 & 52.5 & 8.0 & 151.3 \\
\hline of Screen- & 52.0 & 155.2 & & & 190.0 & 108.5 & 96.0 & 59.5 & 10.7 & 157.0 \\
\hline Bottom-Depth & 53.0 & 156.0 & 295.0 & 253.0 & 195.0 & 110.0 & 96.5 & 60.0 & 11.2 & 157.6 \\
\hline Top-Elevation & 1014.32 & 1042.45 & 719.79 & 706.04 & 762.48 & 751.29 & 828.06 & 873.53 & 903.90 & 901.94 \\
\hline Midpoint-Elevation & 1006.62 & 1034.95 & 696.54 & 682.04 & 738.98 & 739.29 & 822.31 & 866.03 & 900.65 & 895.64 \\
\hline Bottom-Elevation & 998.92 & 1027.45 & 673.29 & 658.04 & 715.48 & 727.29 & 816.56 & 858.53 & 897.40 & 889.34 \\
\hline Screen Length & & 10.7 & & & & & & & & \\
\hline Screen Material & SS/sw & SS/sw & & & PVC/sw & PVC/sw & PVC/sw & PVC/sw & PVC/sw & $\mathrm{PVC} / \mathrm{sw}$ \\
\hline Slot Size & 0.01 & 0.01 & & & 0.01 & 0.01 & 0.01 & 0.01 & 0.01 & 0.01 \\
\hline Open-Hole Length & & & 46.5 & 48 & & & & & & \\
\hline Open-Hole Diameter & & & 6 & 6 & & & & & & \\
\hline \multicolumn{11}{|l|}{ Dedicated Pump } \\
\hline Screen Midpoint-(TOC) & 49.5 & 152.5 & & & 172.9 & 101.3 & 93.9 & 57.4 & 11.1 & 154.2 \\
\hline Pump Intake-(TOC) & 50.0 & 150.5 & 275.0 & 229.0 & 174.0 & 103.0 & & 56.0 & & 153.0 \\
\hline Intake-Elevation & 1004.4 & 1035.6 & 695.4 & 684.2 & 739.3 & 737.0 & & 865.4 & & 896.1 \\
\hline
\end{tabular}


APPENDIX C: MONITORING WELL CONSTRUCTION DETAILS, 2002

\begin{tabular}{|c|c|c|c|c|c|c|c|c|c|c|}
\hline $\begin{array}{r}\text { Well Number } \\
\text { Hydrogeologic Regime } \\
\text { Location }\end{array}$ & $\begin{array}{c}\text { GW-159 } \\
\text { CR } \\
\text { CRSDB }\end{array}$ & $\begin{array}{c}\text { GW-169 } \\
\text { PC } \\
\text { EXP-UV }\end{array}$ & $\begin{array}{l}\text { GW-170 } \\
\text { PC } \\
\text { EXP-UV }\end{array}$ & $\begin{array}{l}\text { GW-171 } \\
\text { PC } \\
\text { EXP-UV }\end{array}$ & $\begin{array}{l}\text { GW-172 } \\
\text { PC } \\
\text { EXP-UV }\end{array}$ & $\begin{array}{c}\text { GW-177 } \\
\text { CR } \\
\text { CRSP }\end{array}$ & $\begin{array}{l}\text { GW-193 } \\
\text { PC } \\
\text { T2331 }\end{array}$ & $\begin{array}{c}\text { GW-203 } \\
\text { CR } \\
\text { UNCS }\end{array}$ & $\begin{array}{l}\text { GW-204 } \\
\text { PC } \\
\text { T0134 }\end{array}$ & $\begin{array}{c}\text { GW-205 } \\
\text { CR } \\
\text { UNCS }\end{array}$ \\
\hline $\begin{array}{r}\text { General Information } \\
\text { Date Installed } \\
\text { Total Depth Drilled } \\
\text { East Coordinate } \\
\text { North Coordinate } \\
\text { Measuring Point Elevation } \\
\text { Well Wizard Elevation } \\
\text { Top of Casing Elevation } \\
\text { Ground Surface Elevation }\end{array}$ & $\begin{array}{r}10 / 18 / 85 \\
157.0 \\
63,496 \\
27,764 \\
1,051.40 \\
1,051.38 \\
1,051.20 \\
1,048.79 \\
\end{array}$ & $\begin{array}{r}09 / 16 / 86 \\
34.8 \\
66,854 \\
28,545 \\
932.12 \\
932.12 \\
931.50 \\
929.95 \\
\end{array}$ & $\begin{array}{r}04 / 01 / 86 \\
156.9 \\
66,843 \\
28,545 \\
932.63 \\
932.64 \\
932.44 \\
930.70 \\
\end{array}$ & $\begin{array}{r}02 / 26 / 86 \\
31.2 \\
69,654 \\
28,403 \\
920.72 \\
920.72 \\
920.10 \\
918.55 \\
\end{array}$ & $\begin{array}{r}05 / 05 / 86 \\
133.9 \\
69,579 \\
28,358 \\
926.57 \\
926.69 \\
926.37 \\
922.85 \\
\end{array}$ & $\begin{array}{r}10 / 24 / 85 \\
145.0 \\
57,497 \\
28,483 \\
1,158.20 \\
1,158.20 \\
1,158.00 \\
1,155.52 \\
\end{array}$ & $\begin{array}{r}08 / 04 / 89 \\
18.5 \\
59,536 \\
29,344 \\
934.17 \\
934.17 \\
934.00 \\
931.11\end{array}$ & $\begin{array}{r}10 / 24 / 85 \\
156.0 \\
54,190 \\
28,356 \\
1,105.45 \\
1,105.45 \\
1,105.26 \\
1,102.34 \\
\end{array}$ & $\begin{array}{r}08 / 30 / 89 \\
17.5 \\
57,411 \\
29,956 \\
958.77 \\
958.74 \\
958.57 \\
955.47 \\
\end{array}$ & $\begin{array}{r}10 / 25 / 85 \\
164.0 \\
54,008 \\
28,363 \\
1,104.14 \\
1,104.14 \\
1,103.97 \\
1,101.46 \\
\end{array}$ \\
\hline $\begin{array}{r}\text { eologic Information } \\
\text { Hydrostratigraphic Unit } \\
\text { Geologic Formation } \\
\text { Aquifer Zone } \\
\text { Weathered Rock-Depth } \\
\text { Fresh Rock-Depth }\end{array}$ & $\begin{array}{r}\text { AQF } \\
\text { OCk } \\
\text { BDR } \\
. \\
100.0\end{array}$ & $\begin{array}{r}\mathrm{AQF} \\
\mathrm{Cmn} \\
\mathrm{WT}\end{array}$ & $\begin{array}{r}\text { AQF } \\
\text { Cmn } \\
\text { BDR } \\
. \\
30.0\end{array}$ & $\begin{array}{r}\mathrm{AQF} \\
\mathrm{Cmn} \\
\mathrm{WT}\end{array}$ & $\begin{array}{l}\text { AQF } \\
\text { Cmn } \\
\text { BDR } \\
15.0 \\
19.0\end{array}$ & $\begin{array}{c}\text { AQF } \\
\text { OCk } \\
\text { BDR } \\
62.0 \\
98.0\end{array}$ & $\begin{array}{r}\text { AQF } \\
\text { Cmn } \\
\text { WT } \\
2.5\end{array}$ & $\begin{array}{c}\text { AQF } \\
\text { OCk } \\
\text { BDR } \\
86.0 \\
93.0\end{array}$ & $\begin{array}{r}A Q T \\
C c \\
W T \\
10.0\end{array}$ & $\begin{array}{r}\text { AQF } \\
\text { OCk } \\
\text { BDR } \\
100.0 \\
146.0\end{array}$ \\
\hline $\begin{array}{r}\text { onductor Casing } \\
\text { Casing Depth } \\
\text { Casing Diameter } \\
\text { Casing Material }\end{array}$ & $\begin{array}{l}123.0 \\
10.75 \\
\text { SF25 }\end{array}$ & & $\begin{array}{r}30.0 \\
8.63 \\
\text { PVC40 } \\
\end{array}$ & & $\begin{array}{r}35.0 \\
8.63 \\
\text { SF25 }\end{array}$ & $\begin{array}{r}82.0 \\
10.75 \\
\text { SF25 }\end{array}$ & $\begin{array}{r}5.0 \\
9.63 \\
\text { STL } \\
\end{array}$ & $\begin{array}{r}94.0 \\
10.75 \\
\text { SF25 }\end{array}$ & & $\begin{array}{l}154.0 \\
10.75 \\
\text { SF25 } \\
\end{array}$ \\
\hline \begin{tabular}{|r|} 
Well Casing \\
Borehole Depth \\
Borehole Diameter \\
Casing Depth \\
Casing Diameter \\
Casing Material \\
\end{tabular} & $\begin{array}{r}157.0 \\
8.5 \\
147.0 \\
4.5 \\
\text { PVC40 } \\
\end{array}$ & $\begin{array}{r}42.0 \\
8 \\
29.7 \\
2.37 \\
\text { PVC40 }\end{array}$ & $\begin{array}{r}104.0 \\
6.62 \\
104.0 \\
4.38 \\
\text { STL } \\
\end{array}$ & $\begin{array}{r}31.2 \\
8 \\
26.8 \\
2.37 \\
\text { PVC40 } \\
\end{array}$ & $\begin{array}{r}105.0 \\
6.62 \\
105.0 \\
4.38 \\
\text { STL }\end{array}$ & $\begin{array}{r}145.0 \\
8 \\
133.0 \\
4.5 \\
\text { PVC40 } \\
\end{array}$ & $\begin{array}{r}18.5 \\
8 \\
8.2 \\
4.5 \\
\text { SS304 } \\
\end{array}$ & $\begin{array}{r}156.0 \\
8.5 \\
146.0 \\
4.5 \\
\text { PVC40 } \\
\end{array}$ & $\begin{array}{r}17.5 \\
6 \\
7.3 \\
4.5 \\
\text { SS304 } \\
\end{array}$ & $\begin{array}{r}164.0 \\
10 \\
154.0 \\
4.5 \\
\text { PVC40 } \\
\end{array}$ \\
\hline $\begin{array}{r}\text { onitored Interval } \\
\text { Top-Depth } \\
\text { Midpoint-Depth } \\
\text { Bottom of Screen-Depth } \\
\text { Bottom-Depth } \\
\text { Top-Elevation } \\
\text { Midpoint-Elevation } \\
\text { Bottom-Elevation } \\
\text { Screen Length } \\
\text { Screen Material } \\
\text { Slot Size } \\
\text { Open-Hole Length } \\
\text { Open-Hole Diameter }\end{array}$ & $\begin{array}{r}145.0 \\
151.0 \\
157.0 \\
157.0 \\
903.79 \\
897.79 \\
891.79 \\
10 \\
\text { PVC/sw } \\
0.01\end{array}$ & $\begin{array}{r}28.7 \\
31.8 \\
34.7 \\
34.8 \\
901.25 \\
898.20 \\
895.15 \\
5 \\
\mathrm{PVC} / \mathrm{sl} \\
0.01\end{array}$ & $\begin{array}{r}104.0 \\
130.5 \\
156.9 \\
826.70 \\
800.25 \\
773.80 \\
. \\
. \\
52.9 \\
3.88 \\
\end{array}$ & $\begin{array}{r}25.8 \\
28.5 \\
31.2 \\
31.2 \\
892.75 \\
890.05 \\
887.35 \\
4.4 \\
\mathrm{PVC} / \mathrm{sl} \\
0.01\end{array}$ & $\begin{array}{r}105.0 \\
119.4 \\
\\
133.8 \\
817.85 \\
803.45 \\
789.05\end{array}$ & $\begin{array}{r}130.0 \\
137.5 \\
143.0 \\
145.0 \\
1025.52 \\
1018.02 \\
1010.52 \\
10 \\
\mathrm{PVC} / \mathrm{sl} \\
0.01\end{array}$ & $\begin{array}{r}5.5 \\
12.0 \\
18.5 \\
18.5 \\
925.61 \\
919.14 \\
912.66 \\
10.3 \\
\mathrm{SS} / \mathrm{sw} \\
0.01\end{array}$ & $\begin{array}{r}144.0 \\
150.0 \\
156.0 \\
156.0 \\
958.34 \\
952.34 \\
946.34 \\
10 \\
P V C / \mathrm{sl} \\
0.01\end{array}$ & $\begin{array}{r}6.5 \\
11.9 \\
17.3 \\
17.3 \\
948.97 \\
943.57 \\
938.17 \\
10 \\
\mathrm{SS} / \mathrm{sw} \\
0.01\end{array}$ & $\begin{array}{r}152.0 \\
158.0 \\
164.0 \\
164.0 \\
949.46 \\
943.46 \\
937.46 \\
10 \\
\mathrm{PVC} / \mathrm{sl} \\
0.01\end{array}$ \\
\hline \begin{tabular}{|} 
Dedicated Pump \\
Screen Midpoint-(TOC) \\
Pump Intake-(TOC) \\
Intake-Elevation
\end{tabular} & $\begin{array}{l}154.4 \\
150.8 \\
900.4\end{array}$ & 3.8 & & 30.6 & & 140.5 & $\begin{array}{r}16.2 \\
17.0 \\
917.0\end{array}$ & 153.9 & $\begin{array}{r}15.4 \\
15.0 \\
943.6\end{array}$ & 161.5 \\
\hline
\end{tabular}


APPENDIX C: MONITORING WELL CONSTRUCTION DETAILS, 2002

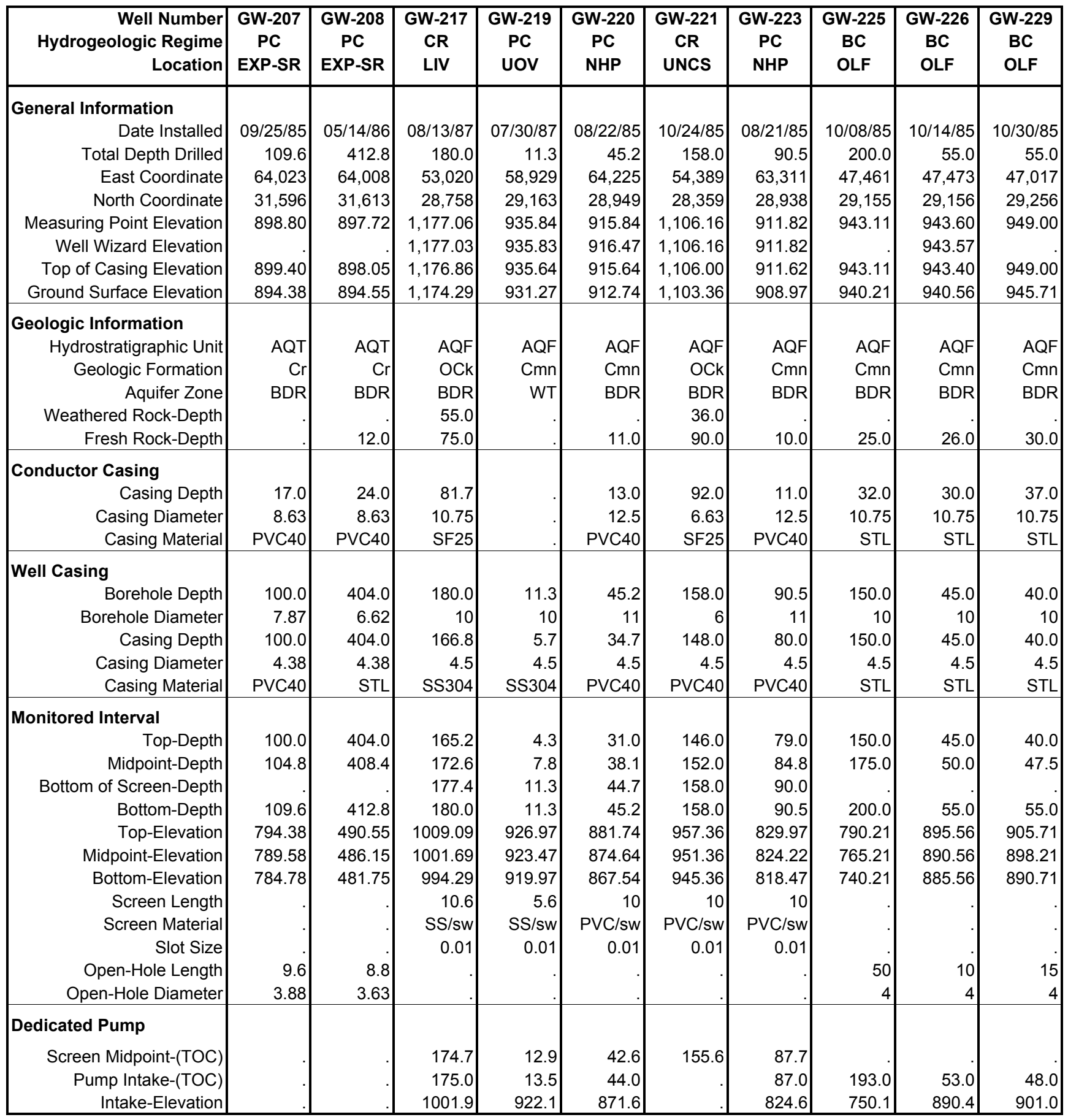


APPENDIX C: MONITORING WELL CONSTRUCTION DETAILS, 2002

\begin{tabular}{|c|c|c|c|c|c|c|c|c|c|c|}
\hline $\begin{array}{r}\text { Well Number } \\
\text { Hydrogeologic Regime } \\
\text { Location }\end{array}$ & $\begin{array}{l}\text { GW-230 } \\
\text { PC } \\
\text { EXP-UV }\end{array}$ & $\begin{array}{c}\text { GW-231 } \\
\text { CR } \\
\text { KHQ }\end{array}$ & $\begin{array}{l}\text { GW-232 } \\
\text { PC } \\
\text { EXP-UV }\end{array}$ & $\begin{array}{c}\text { GW-243 } \\
\text { BC } \\
\text { S3 }\end{array}$ & $\begin{array}{l}\text { W-251 } \\
\text { PC } \\
\text { S2 }\end{array}$ & \begin{tabular}{|l|} 
W-253 \\
PC \\
S2
\end{tabular} & $\begin{array}{l}\text { GW-276 } \\
\text { BC } \\
\text { S3 }\end{array}$ & $\begin{array}{c}\text { GW-288 } \\
\text { BC } \\
\text { BG }\end{array}$ & $\begin{array}{c}\text { GW-289 } \\
\text { BC } \\
\text { BG }\end{array}$ & $\begin{array}{c}\text { GW-291 } \\
\text { BC } \\
\text { BG }\end{array}$ \\
\hline $\begin{array}{r}\text { General Information } \\
\text { Date Installed } \\
\text { Total Depth Drilled } \\
\text { East Coordinate } \\
\text { North Coordinate } \\
\text { Measuring Point Elevation } \\
\text { Well Wizard Elevation } \\
\text { Top of Casing Elevation } \\
\text { Ground Surface Elevation }\end{array}$ & $\begin{array}{r}5 / 12 / 86 \\
406.4 \\
69,617 \\
28,388 \\
923.14 \\
923.11 \\
922.86 \\
919.57 \\
\end{array}$ & $\begin{array}{r}10 / 02 / 85 \\
35.0 \\
63,410 \\
24,725 \\
849.67 \\
849.67 \\
849.47 \\
846.90 \\
\end{array}$ & $\begin{array}{r}03 / 27 / 86 \\
411.7 \\
66,863 \\
28,546 \\
931.42 \\
931.39 \\
931.22 \\
929.52 \\
\end{array}$ & $\begin{array}{r}03 / 10 / 86 \\
77.0 \\
51,990 \\
30,155 \\
1,011.75 \\
1,011.75 \\
1,011.55 \\
1,008.58 \\
\end{array}$ & $\begin{array}{r}04 / 08 / 86 \\
51.0 \\
53,843 \\
29,467 \\
1,003.80 \\
1,003.80 \\
1,003.60 \\
1,001.60 \\
\end{array}$ & $\begin{array}{r}04 / 11 / 86 \\
50.0 \\
54,057 \\
29,404 \\
1,004.24 \\
1,004.24 \\
1,003.99 \\
1,001.60 \\
\end{array}$ & $\begin{array}{r}07 / 15 / 86 \\
18.5 \\
52,557 \\
29,926 \\
1,001.57 \\
1,001.57 \\
1,001.27 \\
998.70 \\
\end{array}$ & $\begin{array}{r}11 / 13 / 86 \\
60.0 \\
42,874 \\
29,975 \\
948.36 \\
. \\
948.36 \\
946.07 \\
\end{array}$ & $\begin{array}{r}11 / 20 / 86 \\
40.8 \\
42,875 \\
29,982 \\
948.73 \\
948.73 \\
948.47 \\
946.32 \\
\end{array}$ & $\begin{array}{r}11 / 14 / 86 \\
14.2 \\
42,583 \\
30,449 \\
948.56 \\
948.56 \\
948.36 \\
945.12 \\
\end{array}$ \\
\hline $\begin{array}{r}\text { Hydrostratigr } \\
\text { Geologic } \\
A q \\
\text { Weathered } R \\
\text { Fresh } R\end{array}$ & $\begin{array}{l}\text { AQF } \\
\text { Cmn } \\
\text { BDR } \\
19.0 \\
38.0\end{array}$ & $\begin{array}{r}\text { AQF } \\
\text { OCk } \\
\text { BDR } \\
. \\
10.5\end{array}$ & $\begin{array}{l}\text { AQF } \\
\text { Cmn } \\
\text { BDR }\end{array}$ & $\begin{array}{r}\mathrm{AQT} \\
\mathrm{Cn} \\
\mathrm{WT} \\
20.0\end{array}$ & $\begin{array}{l}\text { AQF } \\
\text { Cmn } \\
\text { BDR } \\
32.5\end{array}$ & $\begin{array}{r}\mathrm{AQF} \\
\mathrm{Cmn} \\
\mathrm{WT}\end{array}$ & $\begin{array}{r}\mathrm{AQT} \\
\mathrm{Cn} \\
\mathrm{WT} \\
18.5\end{array}$ & $\begin{array}{r}\text { AQT } \\
\mathrm{Cm} \\
\mathrm{BDR} \\
25.0 \\
42.0\end{array}$ & $\begin{array}{r}\mathrm{AQT} \\
\mathrm{Cm} \\
\mathrm{WT} \\
24.0 \\
38.0\end{array}$ & $\begin{array}{r}\mathrm{AQT} \\
\mathrm{Cm} \\
\mathrm{WT} \\
8.0\end{array}$ \\
\hline $\begin{array}{r}\text { onductor Casing } \\
\text { Casing Depth } \\
\text { Casing Diameter } \\
\text { Casing Material }\end{array}$ & $\begin{array}{l}31.0 \\
8.63 \\
\text { STL }\end{array}$ & $\begin{array}{r}11.0 \\
10.63 \\
\text { PVC40 }\end{array}$ & $\begin{array}{r}33.0 \\
8.63 \\
\text { PVC40 }\end{array}$ & $\begin{array}{r}37.8 \\
12.5 \\
\text { PVC40 } \\
\end{array}$ & & & & $\begin{array}{r}43.0 \\
10.75 \\
\text { STL }\end{array}$ & & \\
\hline $\begin{array}{r}\text { Bore } \\
\text { Boreho } \\
\text { Ca } \\
\text { Casin } \\
\text { Cas }\end{array}$ & $\begin{array}{r}341.0 \\
5.5 \\
341.0 \\
4.38 \\
\text { STL }\end{array}$ & $\begin{array}{r}35.0 \\
11 \\
24.5 \\
4.5 \\
\text { PVC } 40\end{array}$ & $\begin{array}{r}401.0 \\
6.62 \\
401.0 \\
4.38 \\
\text { STL }\end{array}$ & $\begin{array}{r}77.0 \\
11 \\
45.1 \\
6.5 \\
\text { PVC40 }\end{array}$ & $\begin{array}{r}51.0 \\
8.25 \\
37.5 \\
4.5 \\
\text { PVC40 }\end{array}$ & $\begin{array}{r}50.0 \\
8.25 \\
37.0 \\
4.5 \\
\text { PVC40 }\end{array}$ & $\begin{array}{r}18.5 \\
8 \\
13.0 \\
4.5 \\
\text { SS304 }\end{array}$ & $\begin{array}{r}60.0 \\
9 \\
49.1 \\
4.5 \\
\text { SS304 }\end{array}$ & $\begin{array}{r}40.8 \\
9 \\
30.6 \\
4.5 \\
\text { SS304 }\end{array}$ & $\begin{array}{r}17.0 \\
9 \\
8.7 \\
4.5 \\
\text { SS304 }\end{array}$ \\
\hline $\begin{array}{r}\text { onitored Interval } \\
\text { Top-Depth } \\
\text { Midpoint-Depth } \\
\text { Bottom of Screen-Depth } \\
\text { Bottom-Depth } \\
\text { Top-Elevation } \\
\text { Midpoint-Elevation } \\
\text { Bottom-Elevation } \\
\text { Screen Length } \\
\text { Screen Material } \\
\text { Slot Size } \\
\text { Open-Hole Length } \\
\text { Open-Hole Diameter }\end{array}$ & $\begin{array}{r}341.0 \\
373.7 \\
406.4 \\
578.57 \\
545.87 \\
513.17\end{array}$ & $\begin{array}{r}22.8 \\
28.9 \\
34.5 \\
35.0 \\
824.10 \\
818.00 \\
811.90 \\
10 \\
\mathrm{PVC} / \mathrm{sw} \\
0.01\end{array}$ & $\begin{array}{r}411.7 \\
528.52 \\
523.17 \\
517.82\end{array}$ & $\begin{array}{r}43.2 \\
60.1 \\
72.9 \\
77.0 \\
965.38 \\
948.48 \\
931.58 \\
27.8 \\
\text { PVC/sw } \\
0.03\end{array}$ & $\begin{array}{r}35.0 \\
43.0 \\
47.1 \\
51.0 \\
966.60 \\
958.60 \\
950.60 \\
9.6 \\
\mathrm{PVC} / \mathrm{sl} \\
0.01\end{array}$ & $\begin{array}{r}36.2 \\
43.1 \\
46.7 \\
50.0 \\
965.40 \\
958.50 \\
951.60 \\
9.7 \\
\mathrm{PVC} / \mathrm{sl} \\
0.01\end{array}$ & $\begin{array}{r}11.3 \\
14.9 \\
18.3 \\
18.5 \\
987.40 \\
983.80 \\
980.20 \\
5.3 \\
\mathrm{SS} / \mathrm{sw} \\
0.01\end{array}$ & $\begin{array}{r}46.0 \\
53.0 \\
59.1 \\
60.0 \\
900.07 \\
893.07 \\
886.07 \\
10 \\
\mathrm{SS} / \mathrm{sw} \\
0.01\end{array}$ & $\begin{array}{r}28.9 \\
34.9 \\
40.6 \\
40.8 \\
917.42 \\
911.47 \\
905.52 \\
10 \\
\mathrm{SS} / \mathrm{sw} \\
0.01\end{array}$ & $\begin{array}{r}6.7 \\
10.5 \\
13.7 \\
14.2 \\
938.42 \\
934.67 \\
930.92 \\
5 \\
\mathrm{SS} / \mathrm{sw} \\
0.01\end{array}$ \\
\hline $\begin{array}{r}\text { dicated Pump } \\
\text { Screen Midpoint-(TOC) } \\
\text { Pump Intake-(TOC) } \\
\text { Intake-Elevation }\end{array}$ & & $\begin{array}{r}32.1 \\
32.4 \\
817.1\end{array}$ & & $\begin{array}{r}62.0 \\
70.0 \\
941.6\end{array}$ & $\begin{array}{r}44.3 \\
45.0 \\
958.6\end{array}$ & 44.2 & $\begin{array}{r}18.2 \\
17.0 \\
984.3\end{array}$ & $\begin{array}{r}56.4 \\
58.0 \\
890.4\end{array}$ & $\begin{array}{r}37.8 \\
38.0 \\
910.5\end{array}$ & $\begin{array}{r}14.4 \\
17.8 \\
930.6\end{array}$ \\
\hline
\end{tabular}


APPENDIX C: MONITORING WELL CONSTRUCTION DETAILS, 2002

\begin{tabular}{|c|c|c|c|c|c|c|c|c|c|c|}
\hline $\begin{array}{r}\text { Well Number } \\
\text { Hydrogeologic Regime } \\
\text { Location }\end{array}$ & $\begin{array}{c}\text { GW-301 } \\
\text { CR } \\
\text { CRBAWP }\end{array}$ & $\begin{array}{c}\text { GW-302 } \\
\text { CR } \\
\text { UNCS }\end{array}$ & $\begin{array}{c}\text { GW-305 } \\
\text { CR } \\
\text { LIV }\end{array}$ & $\begin{array}{c}\text { GW-311 } \\
\text { BC } \\
\text { RS }\end{array}$ & $\begin{array}{c}\text { GW-315 } \\
\text { BC } \\
\text { SPI }\end{array}$ & $\begin{array}{c}\text { GW-339 } \\
\text { CR } \\
\text { UNCS }\end{array}$ & $\begin{array}{c}\text { GW-349 } \\
\text { PC } \\
\text { S2 }\end{array}$ & $\begin{array}{c}\text { GW-350 } \\
\text { PC } \\
\text { S2 }\end{array}$ & $\begin{array}{c}\text { GW-363 } \\
\text { BC } \\
\text { EMWMF }\end{array}$ & $\begin{array}{c}\text { GW-380 } \\
\text { PC } \\
\text { NHP }\end{array}$ \\
\hline $\begin{array}{r}\text { General Information } \\
\text { Date Installed } \\
\text { Total Depth Drilled } \\
\text { East Coordinate } \\
\text { North Coordinate } \\
\text { Measuring Point Elevation } \\
\text { Well Wizard Elevation } \\
\text { Top of Casing Elevation } \\
\text { Ground Surface Elevation }\end{array}$ & $\begin{array}{r}07 / 02 / 87 \\
182.0 \\
61,964 \\
27,662 \\
1,086.55 \\
1,086.55 \\
1,086.38 \\
1,083.94 \\
\end{array}$ & $\begin{array}{r}11 / 10 / 89 \\
136.0 \\
54,353 \\
28,694 \\
1,141.84 \\
1,141.84 \\
1,141.67 \\
1,139.59 \\
\end{array}$ & $\begin{array}{r}08 / 25 / 87 \\
179.6 \\
52,962 \\
28,548 \\
1,183.75 \\
1,183.72 \\
1,183.55 \\
1,181.07 \\
\end{array}$ & $\begin{array}{r}07 / 15 / 87 \\
40.3 \\
50,126 \\
29,267 \\
999.65 \\
999.52 \\
999.35 \\
996.43 \\
\end{array}$ & $\begin{array}{r}09 / 25 / 87 \\
104.0 \\
52,268 \\
29,455 \\
1,047.48 \\
1,047.45 \\
1,047.28 \\
1,044.84 \\
\end{array}$ & $\begin{array}{r}12 / 04 / 89 \\
114.0 \\
54,147 \\
28,659 \\
1,124.83 \\
1,124.83 \\
1,124.59 \\
1,122.18 \\
\end{array}$ & $\begin{array}{r}05 / 31 / 88 \\
25.5 \\
53,588 \\
29,766 \\
993.50 \\
. \\
993.50 \\
990.98 \\
\end{array}$ & $\begin{array}{r}05 / 27 / 88 \\
46.0 \\
53,595 \\
29,764 \\
993.51 \\
\\
993.51 \\
991.00 \\
\end{array}$ & $\begin{array}{r}03 / 16 / 88 \\
75.0 \\
46,872 \\
29,961 \\
958.71 \\
958.71 \\
957.91 \\
955.41 \\
\end{array}$ & $\begin{array}{r}08 / 19 / 88 \\
15.5 \\
62,938 \\
28,714 \\
913.75 \\
913.75 \\
913.55 \\
913.66 \\
\end{array}$ \\
\hline $\begin{array}{r}\text { Hydrostratig } \\
\text { Geologic } \\
\text { Ac } \\
\text { Weathered R } \\
\text { Fresh R }\end{array}$ & $\begin{array}{r}\text { AQF } \\
\text { OCk } \\
\text { BDR } \\
94.0 \\
136.0\end{array}$ & $\begin{array}{r}\text { AQF } \\
\text { OCk } \\
\text { BDR } \\
63.0 \\
102.0\end{array}$ & $\begin{array}{c}\text { AQF } \\
\text { OCk } \\
\text { BDR } \\
53.0 \\
84.0\end{array}$ & $\begin{array}{r}\text { AQF } \\
\text { Cmn } \\
\text { WT } \\
40.3\end{array}$ & $\begin{array}{c}\text { AQF } \\
\text { Cmn } \\
\text { BDR } \\
54.0 \\
71.0\end{array}$ & $\begin{array}{c}\text { AQF } \\
\text { OCk } \\
\text { BDR } \\
45.0 \\
91.0\end{array}$ & $\begin{array}{r}\text { AQF } \\
\text { Cmn } \\
\text { WT } \\
25.5\end{array}$ & $\begin{array}{r}\mathrm{AQF} \\
\mathrm{Cmn} \\
\mathrm{WT} \\
18.0\end{array}$ & $\begin{array}{r}\mathrm{AQT} \\
\mathrm{Cn} \\
\mathrm{BDR} \\
9.0 \\
21.0\end{array}$ & $\begin{array}{r}\text { AQF } \\
\text { Cmn } \\
\text { WT } \\
15.5\end{array}$ \\
\hline $\begin{array}{r}\text { onductor Casing } \\
\text { Casing Depth } \\
\text { Casing Diameter } \\
\text { Casing Material } \\
\end{array}$ & $\begin{array}{l}105.0 \\
10.75 \\
\text { SF25 }\end{array}$ & $\begin{array}{r}63.8 \\
10.75 \\
\text { STL }\end{array}$ & $\begin{array}{r}64.0 \\
10.75 \\
\text { SF25 }\end{array}$ & & $\begin{array}{r}84.4 \\
10.75 \\
\text { SF25 }\end{array}$ & $\begin{array}{r}91.0 \\
10.75 \\
\text { STL }\end{array}$ & & $\begin{array}{r}18.0 \\
10.75 \\
\text { STL }\end{array}$ & $\begin{array}{r}36.0 \\
10.75 \\
\text { STL }\end{array}$ & \\
\hline $\begin{array}{r}\text { Bore } \\
\text { Borehol } \\
\text { Casin } \\
\text { Casi }\end{array}$ & $\begin{array}{r}163.5 \\
10 \\
151.0 \\
4.5 \\
\text { SS304 }\end{array}$ & $\begin{array}{r}136.0 \\
9.5 \\
124.5 \\
4.5 \\
\text { SS304 }\end{array}$ & $\begin{array}{r}179.6 \\
10 \\
168.9 \\
4.5 \\
\text { SS304 }\end{array}$ & $\begin{array}{r}40.3 \\
10 \\
29.7 \\
4.5 \\
\text { SS304 }\end{array}$ & $\begin{array}{r}104.0 \\
10 \\
93.3 \\
4.5 \\
\text { SS304 }\end{array}$ & $\begin{array}{r}114.0 \\
9.5 \\
103.7 \\
4.5 \\
\text { SS304 }\end{array}$ & $\begin{array}{r}25.5 \\
8 \\
9.0 \\
4.5 \\
\text { SS304 }\end{array}$ & $\begin{array}{r}46.0 \\
9.5 \\
33.4 \\
4.5 \\
\text { SS304 }\end{array}$ & $\begin{array}{r}50.0 \\
9.5 \\
48.3 \\
6.62 \\
\text { SF25 }\end{array}$ & $\begin{array}{r}15.5 \\
10 \\
4.8 \\
4.5 \\
\text { SS304 }\end{array}$ \\
\hline $\begin{array}{r}\text { onitored Interval } \\
\text { Top-Depth } \\
\text { Midpoint-Depth } \\
\text { Bottom of Screen-Depth } \\
\text { Bottom-Depth } \\
\text { Top-Elevation } \\
\text { Midpoint-Elevation } \\
\text { Bottom-Elevation } \\
\text { Screen Length } \\
\text { Screen Material } \\
\text { Slot Size } \\
\text { Open-Hole Length } \\
\text { Open-Hole Diameter }\end{array}$ & $\begin{array}{r}148.5 \\
156.0 \\
161.0 \\
163.5 \\
935.44 \\
927.94 \\
920.44 \\
10 \\
\mathrm{SS} / \mathrm{sw} \\
0.01\end{array}$ & $\begin{array}{r}121.5 \\
128.2 \\
134.8 \\
134.8 \\
1018.09 \\
1011.44 \\
1004.79 \\
10.3 \\
\mathrm{SS} / \mathrm{sW} \\
0.01\end{array}$ & $\begin{array}{r}165.3 \\
172.5 \\
179.6 \\
179.6 \\
1015.77 \\
1008.62 \\
1001.47 \\
10.7 \\
\mathrm{SS} / \mathrm{sw} \\
0.01\end{array}$ & $\begin{array}{r}25.6 \\
33.0 \\
40.3 \\
40.3 \\
970.83 \\
963.48 \\
956.13 \\
10.6 \\
\mathrm{SS} / \mathrm{sw} \\
0.01\end{array}$ & $\begin{array}{r}90.0 \\
97.0 \\
103.3 \\
104.0 \\
954.84 \\
947.84 \\
940.84 \\
10 \\
\mathrm{SS} / \mathrm{sw} \\
0.01\end{array}$ & $\begin{array}{r}101.0 \\
107.5 \\
114.0 \\
114.0 \\
1021.18 \\
1014.68 \\
1008.18 \\
10.3 \\
\text { SS/sw } \\
0.01\end{array}$ & $\begin{array}{r}5.0 \\
14.7 \\
24.0 \\
24.3 \\
985.98 \\
976.33 \\
966.68 \\
15 \\
\mathrm{SS} / \mathrm{sw} \\
0.01\end{array}$ & $\begin{array}{r}28.0 \\
35.9 \\
43.4 \\
43.7 \\
963.00 \\
955.15 \\
947.30 \\
10 \\
\mathrm{SS} / \mathrm{sw} \\
0.01\end{array}$ & $\begin{array}{r}50.0 \\
62.5 \\
. \\
75.0 \\
905.41 \\
892.91 \\
880.41\end{array}$ & $\begin{array}{r}2.8 \\
9.2 \\
15.2 \\
15.5 \\
910.86 \\
904.51 \\
898.16 \\
10.4 \\
\mathrm{SS} / \mathrm{sw} \\
0.01\end{array}$ \\
\hline $\begin{array}{r}\text { Screen Midpoint-(TOC) } \\
\text { Pump Intake-(TOC) } \\
\text { Intake-Elevation }\end{array}$ & $\begin{array}{l}158.4 \\
160.5 \\
925.9\end{array}$ & 1.7 & $\begin{array}{r}176.7 \\
176.0 \\
1007.6\end{array}$ & $\begin{array}{r}37.9 \\
40.0 \\
959.4\end{array}$ & $\begin{array}{l}100.7 \\
100.0 \\
947.3\end{array}$ & 11.3 & $\begin{array}{r}19.0 \\
23.0 \\
970.5\end{array}$ & $\begin{array}{r}40.9 \\
41.0 \\
952.5\end{array}$ & & $\begin{array}{r}9.9 \\
12.5 \\
901.1\end{array}$ \\
\hline
\end{tabular}


APPENDIX C: MONITORING WELL CONSTRUCTION DETAILS, 2002

\begin{tabular}{|c|c|c|c|c|c|c|c|c|c|c|}
\hline $\begin{array}{r}\text { Well Number } \\
\text { Hydrogeologic Regime } \\
\text { Location }\end{array}$ & $\begin{array}{c}\text { GW-381 } \\
\text { PC } \\
\text { NHP }\end{array}$ & $\begin{array}{c}\text { GW-382 } \\
\text { PC } \\
\text { NHP }\end{array}$ & $\begin{array}{c}\text { GW-383 } \\
\text { PC } \\
\text { NHP }\end{array}$ & $\begin{array}{c}\text { GW-521 } \\
\text { CR } \\
\text { LIV }\end{array}$ & $\begin{array}{c}\text { GW-522 } \\
\text { CR } \\
\text { LIV }\end{array}$ & $\begin{array}{l}\mathrm{W}-526 \\
\text { BC } \\
\text { S3 }\end{array}$ & $\begin{array}{c}\text { GW-537 } \\
\text { BC } \\
\text { OLF }\end{array}$ & $\begin{array}{c}\text { GW-539 } \\
\text { CR } \\
\text { LII }\end{array}$ & $\begin{array}{c}\text { GW-540 } \\
\text { CR } \\
\text { LII }\end{array}$ & $\begin{array}{c}\text { GW-542 } \\
\text { CR } \\
\text { CDLVI }\end{array}$ \\
\hline $\begin{array}{r}\text { General Information } \\
\text { Date Installed } \\
\text { Total Depth Drilled } \\
\text { East Coordinate } \\
\text { North Coordinate } \\
\text { Measuring Point Elevation } \\
\text { Well Wizard Elevation } \\
\text { Top of Casing Elevation } \\
\text { Ground Surface Elevation }\end{array}$ & $\begin{array}{r}4 / 25 / 88 \\
60.4 \\
62,948 \\
28,715 \\
913.36 \\
. \\
913.36 \\
913.44\end{array}$ & $\begin{array}{r}04 / 11 / 88 \\
173.0 \\
62,956 \\
28,716 \\
913.17 \\
. \\
913.17 \\
913.16\end{array}$ & $\begin{array}{r}04 / 04 / 88 \\
24.1 \\
63,522 \\
29,201 \\
908.77 \\
908.77 \\
908.50 \\
906.00\end{array}$ & $\begin{array}{r}09 / 14 / 88 \\
136.0 \\
52,040 \\
28,541 \\
1,182.88 \\
1,182.88 \\
1,182.68 \\
1,179.46\end{array}$ & $\begin{array}{r}09 / 20 / 88 \\
195.5 \\
52,612 \\
28,377 \\
1,175.49 \\
1,175.48 \\
1,175.31 \\
1,172.04\end{array}$ & $\begin{array}{r}06 / 13 / 88 \\
123.0 \\
50,708 \\
30,033 \\
998.25 \\
998.25 \\
997.45 \\
995.34\end{array}$ & $\begin{array}{r}09 / 14 / 88 \\
24.5 \\
49,539 \\
30,057 \\
976.44 \\
976.41 \\
976.24 \\
974.19\end{array}$ & $\begin{array}{r}05 / 11 / 89 \\
156.0 \\
52,278 \\
27,193 \\
1,093.22 \\
1,093.20 \\
1,093.00 \\
1,090.39\end{array}$ & $\begin{array}{r}06 / 02 / 89 \\
171.5 \\
52,371 \\
27,489 \\
1,072.32 \\
1,072.31 \\
1,072.12 \\
1,069.38\end{array}$ & $\begin{array}{r}05 / 18 / 89 \\
77.5 \\
51,642 \\
27,466 \\
1,051.77 \\
1,051.81 \\
1,051.60 \\
1,049.03\end{array}$ \\
\hline $\begin{array}{r}\text { Hydrostratig } \\
\text { Geologic } \\
\text { Weathered } \\
\text { Fresh }\end{array}$ & $\begin{array}{l}\text { AQF } \\
\text { Cmn } \\
\text { BDR } \\
13.5 \\
26.0\end{array}$ & $\begin{array}{l}\text { AQF } \\
\text { Cmn } \\
\text { BDR } \\
12.7 \\
17.0\end{array}$ & $\begin{array}{r}\mathrm{AQT} \\
\mathrm{Cn} \\
\mathrm{WT} \\
11.5\end{array}$ & $\begin{array}{l}\text { AQF } \\
\text { OCk } \\
\text { BDR }\end{array}$ & $\begin{array}{r}\text { AQF } \\
\text { OCk } \\
\text { BDR } \\
85.0 \\
130.0\end{array}$ & $\begin{array}{r}\mathrm{AQT} \\
\mathrm{Cn} \\
\mathrm{BDR} \\
3.5 \\
23.6\end{array}$ & $\begin{array}{r}\mathrm{AQT} \\
\mathrm{Cn} \\
\mathrm{WT} \\
14.9\end{array}$ & $\begin{array}{l}\mathrm{AQF} \\
\mathrm{OCk} \\
\mathrm{BDR}\end{array}$ & $\begin{array}{r}\text { AQF } \\
\text { OCk } \\
\text { BDR } \\
110.0 \\
150.0\end{array}$ & $\begin{array}{c}\text { AQF } \\
\text { OCk } \\
\text { WT }\end{array}$ \\
\hline $\begin{array}{r}\text { onductor Casing } \\
\text { Casing Depth } \\
\text { Casing Diameter } \\
\text { Casing Material }\end{array}$ & $\begin{array}{r}17.3 \\
10.75 \\
\text { STL }\end{array}$ & $\begin{array}{r}12.7 \\
10.75 \\
\text { STL }\end{array}$ & $\begin{array}{r}5.0 \\
10.75 \\
\text { STL }\end{array}$ & $\begin{array}{r}60.5 \\
10.75 \\
\text { STL }\end{array}$ & $\begin{array}{r}90.0 \\
10.75 \\
\text { STL }\end{array}$ & $\begin{array}{r}23.6 \\
10.75 \\
\text { STL }\end{array}$ & & $\begin{array}{r}79.0 \\
10.75 \\
\text { STL }\end{array}$ & $\begin{array}{c}154.0 \\
10.75 \\
\text { STL }\end{array}$ & \\
\hline $\begin{array}{r}\text { Bore } \\
\text { Boreho } \\
\text { Ca } \\
\text { Casin } \\
\text { Cas }\end{array}$ & $\begin{array}{r}49.3 \\
9.5 \\
47.8 \\
6.62 \\
\text { SF25 }\end{array}$ & $\begin{array}{r}125.0 \\
9.5 \\
123.2 \\
6.62 \\
\text { SF25 }\end{array}$ & $\begin{array}{r}24.1 \\
8.75 \\
18.1 \\
4.5 \\
\text { SS304 }\end{array}$ & $\begin{array}{r}136.0 \\
9.5 \\
124.9 \\
4.5 \\
\text { SS304 }\end{array}$ & $\begin{array}{r}195.5 \\
9.5 \\
184.6 \\
4.5 \\
\text { SS304 }\end{array}$ & $\begin{array}{r}101.0 \\
9.5 \\
99.7 \\
6.62 \\
\text { SF25 }\end{array}$ & $\begin{array}{r}24.5 \\
8.75 \\
8.0 \\
4.5 \\
\text { SS304 }\end{array}$ & $\begin{array}{r}156.0 \\
9.25 \\
139.8 \\
4.5 \\
\text { SS304 }\end{array}$ & $\begin{array}{r}171.5 \\
9.25 \\
161.2 \\
4.5 \\
\text { SS304 }\end{array}$ & $\begin{array}{r}76.5 \\
9.25 \\
60.8 \\
4.5 \\
\text { SS304 }\end{array}$ \\
\hline $\begin{array}{r}\text { onitored Interval } \\
\text { Top-Depth } \\
\text { Midpoint-Depth } \\
\text { Bottom of Screen-Depth } \\
\text { Bottom-Depth } \\
\text { Top-Elevation } \\
\text { Midpoint-Elevation } \\
\text { Bottom-Elevation } \\
\text { Screen Length } \\
\text { Screen Material } \\
\text { Slot Size } \\
\text { Open-Hole Length } \\
\text { Open-Hole Diameter }\end{array}$ & $\begin{array}{r}60.4 \\
864.14 \\
858.59 \\
853.04\end{array}$ & $\begin{array}{r}173.0 \\
788.16 \\
764.16 \\
740.16 \\
. \\
. \\
. \\
48 \\
6.13\end{array}$ & $\begin{array}{r}16.6 \\
20.1 \\
23.1 \\
23.6 \\
889.40 \\
885.90 \\
882.40 \\
5 \\
\mathrm{SS} / \mathrm{sw} \\
0.01\end{array}$ & $\begin{array}{r}123.2 \\
129.6 \\
135.2 \\
136.0 \\
1056.26 \\
1049.86 \\
1043.46 \\
10.3 \\
\mathrm{SS} / \mathrm{sw} \\
0.01\end{array}$ & $\begin{array}{r}183.0 \\
189.2 \\
195.0 \\
195.3 \\
989.04 \\
982.89 \\
976.74 \\
10.4 \\
\mathrm{SS} / \mathrm{sw} \\
0.01\end{array}$ & $\begin{array}{r}101.0 \\
112.0 \\
123.0 \\
894.34 \\
883.34 \\
872.34\end{array}$ & $\begin{array}{r}4.8 \\
14.1 \\
23.0 \\
23.3 \\
969.39 \\
960.14 \\
950.89 \\
15 \\
\mathrm{SS} / \mathrm{sw} \\
0.01\end{array}$ & $\begin{array}{r}136.4 \\
146.2 \\
155.7 \\
156.0 \\
953.99 \\
944.19 \\
934.39 \\
15.9 \\
\mathrm{SS} / \mathrm{sl} \\
0.01\end{array}$ & $\begin{array}{r}158.5 \\
165.0 \\
171.5 \\
171.5 \\
910.88 \\
904.38 \\
897.88 \\
10.3 \\
\mathrm{SS} / \mathrm{sl} \\
0.01\end{array}$ & $\begin{array}{r}59.0 \\
67.8 \\
76.5 \\
76.5 \\
990.03 \\
981.28 \\
972.53 \\
15.7 \\
\mathrm{SS} / \mathrm{sl} \\
0.01\end{array}$ \\
\hline $\begin{array}{r}\text { Screen Midpoint-(TOC) } \\
\text { Pump Intake-(TOC) } \\
\text { Intake-Elevation }\end{array}$ & $\begin{array}{r}55.4 \\
858.0\end{array}$ & & $\begin{array}{r}23.1 \\
23.3 \\
885.2\end{array}$ & $\begin{array}{r}133.3 \\
132.4 \\
1050.3\end{array}$ & $\begin{array}{l}193.1 \\
191.0 \\
984.3\end{array}$ & $\begin{array}{l}115.0 \\
882.5\end{array}$ & $\begin{array}{r}17.6 \\
25.0 \\
951.2\end{array}$ & $\begin{array}{l}150.4 \\
150.0 \\
943.0\end{array}$ & $\begin{array}{l}169.1 \\
169.0 \\
903.1\end{array}$ & $\begin{array}{r}71.2 \\
78.0 \\
973.6\end{array}$ \\
\hline
\end{tabular}


APPENDIX C: MONITORING WELL CONSTRUCTION DETAILS, 2002

\begin{tabular}{|c|c|c|c|c|c|c|c|c|c|c|}
\hline $\begin{array}{r}\text { Well Number } \\
\text { Hydrogeologic Regime } \\
\text { Location }\end{array}$ & $\begin{array}{c}\text { GW-543 } \\
\text { CR } \\
\text { CDLVI }\end{array}$ & $\begin{array}{c}\text { GW-544 } \\
\text { CR } \\
\text { CDLVI }\end{array}$ & $\begin{array}{c}\text { GW-557 } \\
\text { CR } \\
\text { LV }\end{array}$ & $\begin{array}{c}\text { GW-560 } \\
\text { CR } \\
\text { CDLVII }\end{array}$ & $\begin{array}{c}\text { GW-562 } \\
\text { CR } \\
\text { CDLVII }\end{array}$ & $\begin{array}{c}\text { GW-564 } \\
\text { CR } \\
\text { CDLVII }\end{array}$ & $\begin{array}{c}\text { GW-605 } \\
\text { PC } \\
\text { EXP-I }\end{array}$ & $\begin{array}{c}\text { GW-606 } \\
\text { PC } \\
\text { EXP-I }\end{array}$ & $\begin{array}{c}\text { GW-618 } \\
\text { PC } \\
\text { EXP-E }\end{array}$ & $\begin{array}{c}\text { GW-620 } \\
\text { PC } \\
\text { FTF }\end{array}$ \\
\hline $\begin{array}{r}\text { General Information } \\
\text { Date Installed } \\
\text { Total Depth Drilled } \\
\text { East Coordinate } \\
\text { North Coordinate } \\
\text { Measuring Point Elevation } \\
\text { Well Wizard Elevation } \\
\text { Top of Casing Elevation } \\
\text { Ground Surface Elevation }\end{array}$ & $\begin{array}{r}06 / 02 / 89 \\
94.0 \\
51,458 \\
27,072 \\
1,023.98 \\
1,024.01 \\
1,023.80 \\
1,021.19 \\
\end{array}$ & $\begin{array}{r}05 / 30 / 89 \\
110.0 \\
51,820 \\
26,963 \\
1,045.20 \\
1,045.19 \\
1,044.99 \\
1,042.53 \\
\end{array}$ & $\begin{array}{r}12 / 02 / 88 \\
139.0 \\
59,520 \\
26,450 \\
1,081.36 \\
1,081.36 \\
1,081.16 \\
1,078.63 \\
\end{array}$ & $\begin{array}{r}12 / 30 / 88 \\
117.0 \\
60,743 \\
25,692 \\
949.05 \\
949.05 \\
948.85 \\
945.76 \\
\end{array}$ & $\begin{array}{r}01 / 13 / 89 \\
133.0 \\
61,640 \\
26,276 \\
934.69 \\
934.69 \\
934.49 \\
931.86 \\
\end{array}$ & $\begin{array}{r}01 / 27 / 89 \\
88.0 \\
59,865 \\
25,873 \\
937.97 \\
938.07 \\
937.77 \\
935.12 \\
\end{array}$ & $\begin{array}{r}03 / 19 / 91 \\
40.5 \\
62,002 \\
28,707 \\
919.06 \\
919.06 \\
918.88 \\
916.97 \\
\end{array}$ & $\begin{array}{r}03 / 20 / 91 \\
175.0 \\
61,951 \\
28,708 \\
919.59 \\
919.59 \\
919.39 \\
916.98 \\
\end{array}$ & $\begin{array}{r}03 / 15 / 90 \\
37.0 \\
54,738 \\
29,798 \\
985.14 \\
985.14 \\
984.94 \\
982.64 \\
\end{array}$ & $\begin{array}{r}03 / 27 / 90 \\
75.0 \\
52,895 \\
29,565 \\
1,015.54 \\
1,015.57 \\
1,015.34 \\
1,012.84 \\
\end{array}$ \\
\hline $\begin{array}{r}\text { Hydrostratigr } \\
\text { Geologic } \\
A q \\
\text { Weathered } R \\
\text { Fresh } R\end{array}$ & $\begin{array}{c}\text { AQF } \\
\text { OCk } \\
\text { BDR } \\
16.0 \\
37.0\end{array}$ & $\begin{array}{c}\text { AQF } \\
\text { OCk } \\
\text { BDR } \\
47.0 \\
52.5\end{array}$ & $\begin{array}{r}\text { AQF } \\
\text { OCk } \\
W T \\
113.8 \\
134.0\end{array}$ & $\begin{array}{c}\text { AQF } \\
\text { OCk } \\
\text { WT } \\
92.0\end{array}$ & $\begin{array}{c}\text { AQF } \\
\text { OCk } \\
\text { WT }\end{array}$ & $\begin{array}{r}\text { AQF } \\
\text { OCk } \\
\text { WT } \\
72.0\end{array}$ & $\begin{array}{l}\text { AQF } \\
\text { Cmn } \\
\text { BDR }\end{array}$ & $\begin{array}{l}\text { AQF } \\
\text { Cmn } \\
\text { BDR }\end{array}$ & $\begin{array}{r}\text { AQF } \\
\text { Cmn } \\
\text { WT } \\
25.0 \\
27.0\end{array}$ & $\begin{array}{r}\text { AQF } \\
\text { Cmn } \\
\text { WT } \\
41.0 \\
70.0\end{array}$ \\
\hline $\begin{array}{r}\text { onductor Casing } \\
\text { Casing Depth } \\
\text { Casing Diameter } \\
\text { Casing Material } \\
\end{array}$ & $\begin{array}{r}29.3 \\
10.75 \\
\text { STL } \\
\end{array}$ & $\begin{array}{r}54.5 \\
10.75 \\
\text { STL }\end{array}$ & $\begin{array}{r}85.0 \\
10.75 \\
\text { STL }\end{array}$ & & & & $\begin{array}{r}9.5 \\
11.75 \\
\text { SJ55 }\end{array}$ & $\begin{array}{r}64.7 \\
7 \\
\text { SJ55 }\end{array}$ & $\begin{array}{r}27.5 \\
10.75 \\
\text { SJ55 }\end{array}$ & $\begin{array}{r}42.5 \\
10.75 \\
\text { SJ55 }\end{array}$ \\
\hline $\begin{array}{r}\text { Borehole Depth } \\
\text { Borehole Diameter } \\
\text { Casing Depth } \\
\text { Casing Diameter } \\
\text { Casing Material }\end{array}$ & $\begin{array}{r}93.6 \\
9.25 \\
78.0 \\
4.5 \\
\text { SS304 }\end{array}$ & $\begin{array}{r}109.3 \\
9.25 \\
93.4 \\
4.5 \\
\text { SS304 }\end{array}$ & $\begin{array}{r}138.0 \\
9.5 \\
115.8 \\
4.5 \\
\text { SS304 }\end{array}$ & $\begin{array}{r}117.0 \\
9.5 \\
49.0 \\
4.5 \\
\text { SS304 }\end{array}$ & $\begin{array}{r}60.0 \\
9.5 \\
38.0 \\
4.5 \\
\text { SS304 }\end{array}$ & $\begin{array}{r}81.0 \\
9.5 \\
55.3 \\
4.5 \\
\text { SS304 }\end{array}$ & $\begin{array}{r}40.5 \\
10.6 \\
29.7 \\
4.25 \\
\text { SS304 }\end{array}$ & $\begin{array}{r}175.0 \\
9.63 \\
161.0 \\
4.25 \\
\text { SS304 }\end{array}$ & $\begin{array}{r}37.0 \\
9.5 \\
26.7 \\
4.5 \\
\text { SS304 }\end{array}$ & $\begin{array}{r}75.0 \\
9.5 \\
64.2 \\
4.5 \\
\text { SS304 }\end{array}$ \\
\hline $\begin{array}{r}\text { onitored Interval } \\
\text { Top-Depth } \\
\text { Midpoint-Depth } \\
\text { Bottom of Screen-Depth } \\
\text { Bottom-Depth } \\
\text { Top-Elevation } \\
\text { Midpoint-Elevation } \\
\text { Bottom-Elevation } \\
\text { Screen Length } \\
\text { Screen Material } \\
\text { Slot Size } \\
\text { Open-Hole Length } \\
\text { Open-Hole Diameter }\end{array}$ & $\begin{array}{r}76.2 \\
84.9 \\
93.6 \\
93.6 \\
944.99 \\
936.29 \\
927.59 \\
15.65 \\
\mathrm{SS} / \mathrm{sl} \\
0.01\end{array}$ & $\begin{array}{r}91.0 \\
100.2 \\
109.3 \\
109.3 \\
951.53 \\
942.38 \\
933.23 \\
15.9 \\
\mathrm{SS} / \mathrm{sl} \\
0.01\end{array}$ & $\begin{array}{r}112.9 \\
125.5 \\
135.8 \\
138.0 \\
965.73 \\
953.18 \\
940.63 \\
20 \\
\mathrm{SS} / \mathrm{sw} \\
0.01\end{array}$ & $\begin{array}{r}45.2 \\
57.1 \\
69.0 \\
69.0 \\
900.56 \\
888.66 \\
876.76 \\
20 \\
\mathrm{SS} / \mathrm{sw} \\
0.01\end{array}$ & $\begin{array}{r}36.0 \\
48.0 \\
58.0 \\
60.0 \\
895.86 \\
883.86 \\
871.86 \\
20 \\
\mathrm{SS} / \mathrm{sw} \\
0.01\end{array}$ & $\begin{array}{r}52.0 \\
66.5 \\
75.3 \\
81.0 \\
883.12 \\
868.62 \\
854.12 \\
20 \\
\mathrm{SS} / \mathrm{sw} \\
0.01\end{array}$ & $\begin{array}{r}28.2 \\
34.1 \\
39.7 \\
39.9 \\
888.77 \\
882.92 \\
877.07 \\
10 \\
\mathrm{SS} / \mathrm{sw} \\
0.01\end{array}$ & $\begin{array}{r}155.0 \\
163.0 \\
171.0 \\
171.0 \\
761.98 \\
753.98 \\
745.98 \\
10 \\
\text { SS/ppk } \\
0.01\end{array}$ & $\begin{array}{r}26.0 \\
31.5 \\
37.0 \\
37.0 \\
956.64 \\
951.14 \\
945.64 \\
10.3 \\
\mathrm{SS} / \mathrm{sw} \\
0.01\end{array}$ & $\begin{array}{r}61.7 \\
68.4 \\
75.0 \\
75.0 \\
951.14 \\
944.49 \\
937.84 \\
10.8 \\
S S / s w \\
0.01\end{array}$ \\
\hline $\begin{array}{r}\text { Screen Midpoint-(TOC) } \\
\text { Pump Intake-(TOC) } \\
\text { Intake-Elevation }\end{array}$ & $\begin{array}{r}88.4 \\
88.4 \\
935.4\end{array}$ & $\begin{array}{l}103.8 \\
103.5 \\
941.5\end{array}$ & $\begin{array}{l}128.3 \\
126.3 \\
954.9\end{array}$ & 2.1 & 0.6 & 67.9 & $\begin{array}{r}36.6 \\
36.0 \\
882.9\end{array}$ & $\begin{array}{l}168.4 \\
169.0 \\
750.4\end{array}$ & $\begin{array}{r}34.2 \\
35.0 \\
949.9\end{array}$ & $\begin{array}{r}72.1 \\
72.0 \\
943.3\end{array}$ \\
\hline
\end{tabular}


APPENDIX C: MONITORING WELL CONSTRUCTION DETAILS, 2002

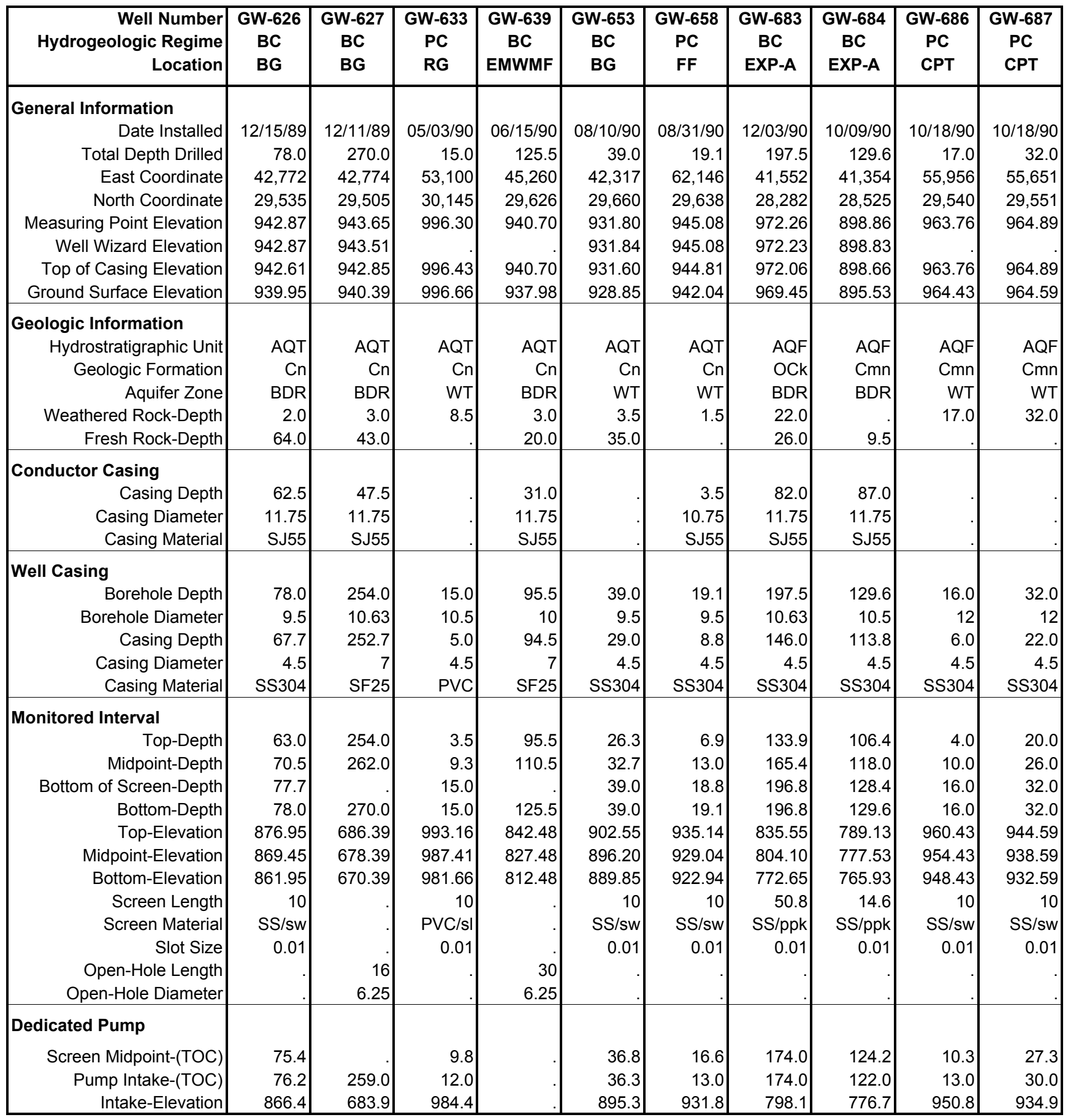


APPENDIX C: MONITORING WELL CONSTRUCTION DETAILS, 2002

\begin{tabular}{|c|c|c|c|c|c|c|c|c|c|c|}
\hline $\begin{array}{r}\text { Well Number } \\
\text { Hydrogeologic Regime } \\
\text { Location }\end{array}$ & $\begin{array}{c}\text { GW-689 } \\
\text { PC } \\
\text { CPT }\end{array}$ & $\begin{array}{c}\text { GW-694 } \\
\text { BC } \\
\text { EXP-B }\end{array}$ & $\begin{array}{c}\text { GW-695 } \\
\text { BC } \\
\text { EXP-B }\end{array}$ & $\begin{array}{c}\text { GW-698 } \\
\text { PC } \\
\text { B8110 }\end{array}$ & $\begin{array}{c}\text { GW-703 } \\
\text { BC } \\
\text { EXP-B }\end{array}$ & $\begin{array}{c}\text { GW-704 } \\
\text { BC } \\
\text { EXP-B }\end{array}$ & $\begin{array}{c}\text { GW-706 } \\
\text { BC } \\
\text { EXP-B }\end{array}$ & $\begin{array}{c}\text { GW-709 } \\
\text { CR } \\
\text { LII }\end{array}$ & $\begin{array}{c}\text { GW-712 } \\
\text { BC } \\
\text { EXP-W }\end{array}$ & $\begin{array}{c}\text { GW-713 } \\
\text { BC } \\
\text { EXP-W }\end{array}$ \\
\hline $\begin{array}{r}\text { General Information } \\
\text { Date Installed } \\
\text { Total Depth Drilled } \\
\text { East Coordinate } \\
\text { North Coordinate } \\
\text { Measuring Point Elevation } \\
\text { Well Wizard Elevation } \\
\text { Top of Casing Elevation } \\
\text { Ground Surface Elevation }\end{array}$ & $\begin{array}{r}10 / 22 / 90 \\
20.0 \\
55,597 \\
29,683 \\
967.00 \\
. \\
967.00 \\
967.34\end{array}$ & $\begin{array}{r}02 / 07 / 91 \\
204.5 \\
44,893 \\
28,845 \\
942.24 \\
941.98 \\
941.38 \\
938.58\end{array}$ & $\begin{array}{r}02 / 21 / 91 \\
62.6 \\
44,868 \\
28,845 \\
939.57 \\
939.54 \\
939.37 \\
937.22\end{array}$ & $\begin{array}{r}11 / 02 / 90 \\
75.0 \\
56,804 \\
29,277 \\
970.29 \\
970.29 \\
970.09 \\
970.09\end{array}$ & $\begin{array}{r}12 / 07 / 90 \\
182.0 \\
44,931 \\
28,806 \\
955.49 \\
955.29 \\
954.69 \\
951.80\end{array}$ & $\begin{array}{r}12 / 20 / 90 \\
256.0 \\
44,935 \\
28,845 \\
945.53 \\
945.33 \\
944.73 \\
941.99\end{array}$ & $\begin{array}{r}01 / 27 / 91 \\
182.5 \\
44,944 \\
28,946 \\
929.47 \\
929.47 \\
928.67 \\
925.78\end{array}$ & $\begin{array}{r}04 / 05 / 91 \\
80.6 \\
52,372 \\
25,344 \\
906.78 \\
906.81 \\
906.60 \\
903.84\end{array}$ & $\begin{array}{r}06 / 20 / 91 \\
457.5 \\
36,507 \\
28,233 \\
877.89 \\
877.89 \\
877.09 \\
873.61\end{array}$ & $\begin{array}{r}01 / 13 / 92 \\
315.2 \\
36,434 \\
28,236 \\
881.43 \\
881.43 \\
880.63 \\
877.83\end{array}$ \\
\hline $\begin{array}{r}\text { Hydrostratig } \\
\text { Geologic } \\
\text { Weathered } \mathrm{A} \\
\text { Fresh } \mathrm{F}\end{array}$ & $\begin{array}{r}\text { AQF } \\
\text { Cmn } \\
\text { WT } \\
20.0\end{array}$ & $\begin{array}{l}\text { AQF } \\
\text { Cmn } \\
\text { BDR } \\
11.0 \\
21.0\end{array}$ & $\begin{array}{r}\text { AQF } \\
\text { OCk } \\
\text { BDR } \\
6.0 \\
18.0\end{array}$ & $\begin{array}{l}\text { AQF } \\
\text { Cmn } \\
\text { BDR } \\
42.0\end{array}$ & $\begin{array}{r}\text { AQF } \\
\text { Cmn } \\
\text { BDR } \\
7.0 \\
10.0\end{array}$ & $\begin{array}{l}\text { AQF } \\
\text { Cmn } \\
\text { BDR } \\
16.0 \\
23.0\end{array}$ & $\begin{array}{l}\text { AQF } \\
\text { Cmn } \\
\text { BDR } \\
17.0 \\
27.0\end{array}$ & $\begin{array}{c}\text { AQF } \\
\text { OCk } \\
\text { BDR } \\
39.0 \\
43.0\end{array}$ & $\begin{array}{c}\text { AQF } \\
\text { OCk } \\
\text { BDR } \\
12.0 \\
66.0\end{array}$ & $\begin{array}{l}\text { AQF } \\
\text { Cmn } \\
\text { BDR } \\
26.8 \\
63.8\end{array}$ \\
\hline $\begin{array}{r}\mathrm{C} \\
\text { Casir } \\
\text { Cas }\end{array}$ & & $\begin{array}{r}25.8 \\
11.75 \\
\text { STL }\end{array}$ & $\begin{array}{r}22.5 \\
11.75 \\
\text { SJ55 } \\
\end{array}$ & $\begin{array}{r}42.0 \\
10.5 \\
\text { PVC40 } \\
\end{array}$ & & $\begin{array}{r}21.0 \\
11.75 \\
\text { SJ55 }\end{array}$ & $\begin{array}{r}40.3 \\
11.75 \\
\text { SJ55 }\end{array}$ & $\begin{array}{r}50.0 \\
11.75 \\
\text { SJ55 }\end{array}$ & $\begin{array}{r}44.8 \\
11.75 \\
\text { SJ55 }\end{array}$ & $\begin{array}{r}80.2 \\
\text { SJ55 } \\
\end{array}$ \\
\hline $\begin{array}{r}\text { Bore } \\
\text { Boreho } \\
\text { C } \\
\text { Casir } \\
\text { Cas }\end{array}$ & $\begin{array}{r}20.0 \\
12 \\
10.0 \\
4.5 \\
\text { SS304 }\end{array}$ & $\begin{array}{r}154.0 \\
10.6 \\
152.0 \\
7 \\
\text { SF25 }\end{array}$ & $\begin{array}{r}62.6 \\
9.88 \\
52.4 \\
4.5 \\
\text { SS304 }\end{array}$ & $\begin{array}{r}75.0 \\
8.5 \\
65.0 \\
4.5 \\
\text { SS304 }\end{array}$ & $\begin{array}{r}135.0 \\
10.63 \\
132.8 \\
7 \\
\text { SF25 }\end{array}$ & $\begin{array}{r}246.0 \\
10.63 \\
243.5 \\
7 \\
\text { SF25 }\end{array}$ & $\begin{array}{r}157.0 \\
10.6 \\
155.1 \\
7 \\
\text { SF25 }\end{array}$ & $\begin{array}{r}80.6 \\
10.6 \\
70.4 \\
4.25 \\
\text { SS304 }\end{array}$ & $\begin{array}{r}441.5 \\
10.6 \\
440.2 \\
7 \\
\text { SF25 }\end{array}$ & $\begin{array}{r}305.0 \\
10.6 \\
303.7 \\
7 \\
\text { SF25 }\end{array}$ \\
\hline $\begin{array}{r}\text { onitored Interval } \\
\text { Top-Depth } \\
\text { Midpoint-Depth } \\
\text { Bottom of Screen-Depth } \\
\text { Bottom-Depth } \\
\text { Top-Elevation } \\
\text { Midpoint-Elevation } \\
\text { Bottom-Elevation } \\
\text { Screen Length } \\
\text { Screen Material } \\
\text { Slot Size } \\
\text { Open-Hole Length } \\
\text { Open-Hole Diameter }\end{array}$ & $\begin{array}{r}8.0 \\
14.0 \\
20.0 \\
20.0 \\
959.34 \\
953.34 \\
947.34 \\
10 \\
\mathrm{SS} / \mathrm{sw} \\
0.01\end{array}$ & $\begin{array}{r}153.0 \\
178.8 \\
204.5 \\
785.58 \\
759.83 \\
734.08 \\
. \\
. \\
51.5 \\
6.25\end{array}$ & $\begin{array}{r}50.6 \\
56.6 \\
62.4 \\
62.6 \\
886.62 \\
880.62 \\
874.62 \\
10 \\
\mathrm{SS} / \mathrm{sw} \\
0.01\end{array}$ & $\begin{array}{r}63.0 \\
69.0 \\
75.0 \\
75.0 \\
907.09 \\
901.09 \\
895.09 \\
10 \\
\mathrm{SS} / \mathrm{sw} \\
0.01\end{array}$ & $\begin{array}{r}182.0 \\
818.00 \\
793.90 \\
769.80\end{array}$ & $\begin{array}{r}256.0 \\
697.49 \\
691.74 \\
685.99\end{array}$ & $\begin{array}{r}182.5 \\
769.68 \\
756.48 \\
743.28\end{array}$ & $\begin{array}{r}68.7 \\
74.7 \\
80.4 \\
80.6 \\
835.14 \\
829.19 \\
823.24 \\
10 \\
\mathrm{SS} / \mathrm{sw} \\
0.01\end{array}$ & $\begin{array}{r}457.5 \\
432.11 \\
424.11 \\
416.11\end{array}$ & $\begin{array}{r}315.2 \\
572.83 \\
567.73 \\
562.63\end{array}$ \\
\hline $\begin{array}{r}\text { Screen Midpoint-(TOC) } \\
\text { Pump Intake-(TOC) } \\
\text { Intake-Elevation }\end{array}$ & 14.7 & $\begin{array}{l}183.0 \\
758.4\end{array}$ & $\begin{array}{r}59.6 \\
60.0 \\
879.4\end{array}$ & $\begin{array}{r}70.0 \\
20.0 \\
950.1\end{array}$ & $\begin{array}{l}162.0 \\
792.7\end{array}$ & $\begin{array}{l}254.5 \\
690.2\end{array}$ & $\begin{array}{l}178.5 \\
750.2\end{array}$ & $\begin{array}{r}78.2 \\
76.0 \\
830.6\end{array}$ & $\begin{array}{l}450.5 \\
426.6\end{array}$ & $\begin{array}{l}311.0 \\
569.6\end{array}$ \\
\hline
\end{tabular}


APPENDIX C: MONITORING WELL CONSTRUCTION DETAILS, 2002

\begin{tabular}{|c|c|c|c|c|c|c|c|c|c|c|}
\hline $\begin{array}{r}\text { Well Number } \\
\text { Hydrogeologic Regime } \\
\text { Location }\end{array}$ & $\begin{array}{c}\text { GW-714 } \\
\text { BC } \\
\text { EXP-W }\end{array}$ & $\begin{array}{c}\text { GW-715 } \\
\text { BC } \\
\text { EXP-W }\end{array}$ & $\begin{array}{c}\text { GW-722 } \\
\text { PC } \\
\text { NHP }\end{array}$ & $\begin{array}{c}\text { GW-723 } \\
\text { BC } \\
\text { EXP-C }\end{array}$ & $\begin{array}{c}\text { GW-724 } \\
\text { BC } \\
\text { EXP-C }\end{array}$ & $\begin{array}{c}\text { GW-725 } \\
\text { BC } \\
\text { EXP-C }\end{array}$ & $\begin{array}{c}\text { GW-731 } \\
\text { CR } \\
\text { CRSDB }\end{array}$ & $\begin{array}{c}\text { GW-732 } \\
\text { CR } \\
\text { CRSDB }\end{array}$ & $\begin{array}{c}\text { GW-733 } \\
\text { PC } \\
\text { EXP-J }\end{array}$ & $\begin{array}{c}\text { GW-735 } \\
\text { PC } \\
\text { EXP-J }\end{array}$ \\
\hline $\begin{array}{r}\text { General Information } \\
\text { Date Installed } \\
\text { Total Depth Drilled } \\
\text { East Coordinate } \\
\text { North Coordinate } \\
\text { Measuring Point Elevation } \\
\text { Well Wizard Elevation } \\
\text { Top of Casing Elevation } \\
\text { Ground Surface Elevation }\end{array}$ & $\begin{array}{r}1 / 24 / 92 \\
145.0 \\
36,435 \\
28,422 \\
875.88 \\
875.88 \\
875.08 \\
872.30 \\
\end{array}$ & $\begin{array}{r}01 / 29 / 92 \\
44.6 \\
36,453 \\
28,425 \\
874.92 \\
874.92 \\
874.72 \\
872.17 \\
\end{array}$ & $\begin{array}{r}08 / 09 / 91 \\
644.3 \\
64,926 \\
28,532 \\
953.71\end{array}$ & $\begin{array}{r}08 / 15 / 91 \\
444.5 \\
49,089 \\
29,006 \\
1,022.23 \\
. \\
1,022.23 \\
1,019.31 \\
\end{array}$ & $\begin{array}{r}08 / 12 / 91 \\
301.6 \\
48,995 \\
29,198 \\
979.27 \\
979.75 \\
979.27 \\
976.62 \\
\end{array}$ & $\begin{array}{r}08 / 27 / 91 \\
142.5 \\
48,989 \\
29,405 \\
961.05 \\
961.63 \\
961.05 \\
958.26 \\
\end{array}$ & $\begin{array}{r}09 / 12 / 91 \\
180.4 \\
63,863 \\
27,464 \\
1,049.38 \\
1,049.29 \\
1,049.18 \\
1,045.75 \\
\end{array}$ & $\begin{array}{r}09 / 11 / 91 \\
190.6 \\
64,268 \\
27,717 \\
1,064.29 \\
1,064.29 \\
1,064.09 \\
1,060.65 \\
\end{array}$ & $\begin{array}{r}10 / 02 / 91 \\
256.5 \\
65,067 \\
28,447 \\
959.84 \\
959.84 \\
959.04 \\
955.69 \\
\end{array}$ & $\begin{array}{r}10 / 30 / 91 \\
83.0 \\
64,872 \\
28,867 \\
924.46 \\
924.46 \\
924.28 \\
921.34 \\
\end{array}$ \\
\hline $\begin{array}{r}\text { Hydrostratic } \\
\text { Geologic } \\
\text { Weathered } \\
\text { Fresh }\end{array}$ & $\begin{array}{l}\text { AQF } \\
\text { Cmn } \\
\text { BDR } \\
27.0 \\
35.0\end{array}$ & $\begin{array}{r}\text { AQF } \\
\text { Cmn } \\
\text { WT } \\
34.0\end{array}$ & $\begin{array}{l}\text { AQF } \\
\text { Cmn } \\
\text { BDR } \\
54.0 \\
73.0\end{array}$ & $\begin{array}{l}\text { AQF } \\
\text { Cmn } \\
\text { BDR } \\
26.7 \\
29.7\end{array}$ & $\begin{array}{l}\text { AQF } \\
\text { Cmn } \\
\text { BDR } \\
33.5 \\
40.0\end{array}$ & $\begin{array}{l}\text { AQF } \\
\text { Cmn } \\
\text { BDR } \\
14.0 \\
17.5\end{array}$ & $\begin{array}{r}\text { AQF } \\
\text { OCk } \\
\text { BDR } \\
95.4 \\
129.4\end{array}$ & $\begin{array}{c}\text { AQF } \\
\text { OCk } \\
\text { BDR } \\
85.0 \\
96.0\end{array}$ & $\begin{array}{l}\text { AQF } \\
\text { Cmn } \\
\text { BDR } \\
42.5 \\
47.1\end{array}$ & $\begin{array}{r}A Q T \\
C n \\
W T \\
19.0 \\
77.5\end{array}$ \\
\hline $\begin{array}{r}\text { onductor Casing } \\
\text { Casing Depth } \\
\text { Casing Diameter } \\
\text { Casing Material }\end{array}$ & $\begin{array}{r}40.5 \\
11.75 \\
\text { SJ55 }\end{array}$ & & $\begin{array}{r}56.2 \\
10.75 \\
\text { SJ55 }\end{array}$ & $\begin{array}{r}39.0 \\
11.75 \\
\text { SJ55 }\end{array}$ & $\begin{array}{r}40.0 \\
11.75 \\
\text { SJ55 }\end{array}$ & $\begin{array}{r}21.0 \\
11.75 \\
\text { SJ55 }\end{array}$ & $\begin{array}{l}122.0 \\
11.75 \\
\text { SJ55 }\end{array}$ & $\begin{array}{l}100.7 \\
11.75 \\
\text { SJ55 }\end{array}$ & $\begin{array}{r}51.8 \\
11.75 \\
\text { SJ55 }\end{array}$ & $\begin{array}{r}25.5 \\
11.75 \\
\text { SJ55 }\end{array}$ \\
\hline $\begin{array}{r}\text { Bore } \\
\text { Boreho } \\
\text { C } \\
\text { Casir } \\
\text { Cas }\end{array}$ & $\begin{array}{r}115.1 \\
10.6 \\
113.8 \\
7 \\
\text { SF25 }\end{array}$ & $\begin{array}{r}44.6 \\
10.6 \\
33.1 \\
4.25 \\
\text { SS304 }\end{array}$ & $\begin{array}{r}75.0 \\
6 \\
74.5 \\
4.5 \\
\text { SJ55 }\end{array}$ & $\begin{array}{r}340.6 \\
10.6 \\
339.3 \\
7 \\
\text { SF25 }\end{array}$ & $\begin{array}{r}289.6 \\
10.6 \\
288.3 \\
7 \\
\text { SF25 }\end{array}$ & $\begin{array}{r}132.5 \\
10.6 \\
131.2 \\
7 \\
\text { SF25 }\end{array}$ & $\begin{array}{r}175.4 \\
10.6 \\
165.2 \\
4.5 \\
\text { SS304 }\end{array}$ & $\begin{array}{r}189.5 \\
10.6 \\
179.3 \\
4.5 \\
\text { SS304 }\end{array}$ & $\begin{array}{r}240.1 \\
10.6 \\
238.8 \\
7 \\
\text { SF25 }\end{array}$ & $\begin{array}{r}83.0 \\
10.6 \\
67.9 \\
4.5 \\
S 304\end{array}$ \\
\hline $\begin{array}{r}\text { onitored Interval } \\
\text { Top-Depth } \\
\text { Midpoint-Depth } \\
\text { Bottom of Screen-Depth } \\
\text { Bottom-Depth } \\
\text { Top-Elevation } \\
\text { Midpoint-Elevation } \\
\text { Bottom-Elevation } \\
\text { Screen Length } \\
\text { Screen Material } \\
\text { Slot Size } \\
\text { Open-Hole Length } \\
\text { Open-Hole Diameter }\end{array}$ & $\begin{array}{r}115.1 \\
130.1 \\
145.0 \\
757.20 \\
742.25 \\
727.30 \\
. \\
. \\
. \\
29.9 \\
6.25\end{array}$ & $\begin{array}{r}32.0 \\
38.0 \\
43.1 \\
44.0 \\
840.17 \\
834.17 \\
828.17 \\
10 \\
\mathrm{SS} / \mathrm{sw} \\
0.01\end{array}$ & $\begin{array}{r}644.3 \\
876.54 \\
591.64 \\
306.74 \\
. \\
. \\
. \\
569.8 \\
3.5\end{array}$ & $\begin{array}{r}444.5 \\
678.71 \\
626.76 \\
574.81\end{array}$ & $\begin{array}{r}301.6 \\
687.02 \\
681.02 \\
675.02\end{array}$ & $\begin{array}{r}132.5 \\
137.5 \\
. \\
142.5 \\
825.76 \\
820.76 \\
815.76\end{array}$ & $\begin{array}{r}164.0 \\
171.4 \\
175.2 \\
178.7 \\
881.75 \\
874.40 \\
867.05 \\
10 \\
\mathrm{SS} / \mathrm{sw} \\
0.01\end{array}$ & $\begin{array}{r}178.3 \\
184.2 \\
189.3 \\
190.0 \\
882.35 \\
876.50 \\
870.65 \\
10 \\
\mathrm{SS} / \mathrm{sw} \\
0.01\end{array}$ & $\begin{array}{r}256.5 \\
715.59 \\
707.39 \\
699.19\end{array}$ & $\begin{array}{r}67.5 \\
73.4 \\
77.9 \\
79.2 \\
853.84 \\
847.99 \\
842.14 \\
10 \\
\text { SS/sw } \\
0.01\end{array}$ \\
\hline $\begin{array}{r}\text { Screen Midpoint-(TOC) } \\
\text { Pump Intake-(TOC) } \\
\text { Intake-Elevation }\end{array}$ & $\begin{array}{l}142.0 \\
733.1\end{array}$ & $\begin{array}{r}40.7 \\
40.0 \\
834.7\end{array}$ & & $\begin{array}{l}437.0 \\
585.2\end{array}$ & $\begin{array}{l}297.5 \\
681.8\end{array}$ & $\begin{array}{l}140.5 \\
820.6\end{array}$ & $\begin{array}{l}173.6 \\
173.5 \\
875.7\end{array}$ & $\begin{array}{l}187.7 \\
188.0 \\
876.1\end{array}$ & $\begin{array}{l}253.0 \\
706.0\end{array}$ & $\begin{array}{r}75.8 \\
76.7 \\
847.6\end{array}$ \\
\hline
\end{tabular}


APPENDIX C: MONITORING WELL CONSTRUCTION DETAILS, 2002

\begin{tabular}{|c|c|c|c|c|c|c|c|c|c|c|}
\hline $\begin{array}{r}\text { Well Number } \\
\text { Hydrogeologic Regime } \\
\text { Location }\end{array}$ & $\begin{array}{c}\text { GW-736 } \\
\text { BC } \\
\text { EXP-C }\end{array}$ & $\begin{array}{c}\text { GW-737 } \\
\text { BC } \\
\text { EXP-C }\end{array}$ & $\begin{array}{c}\text { GW-738 } \\
\text { BC } \\
\text { EXP-C }\end{array}$ & $\begin{array}{c}\text { GW-739 } \\
\text { BC } \\
\text { EXP-C }\end{array}$ & $\begin{array}{c}\text { GW-740 } \\
\text { BC } \\
\text { EXP-C }\end{array}$ & $\begin{array}{l}\text { GW-744 } \\
\text { PC } \\
\text { GRIDK1 }\end{array}$ & $\begin{array}{l}\text { GW-747 } \\
\text { PC } \\
\text { GRIDK2 }\end{array}$ & $\begin{array}{c}\text { GW-750 } \\
\text { PC } \\
\text { EXP-J }\end{array}$ & $\begin{array}{c}\text { GW-757 } \\
\text { CR } \\
\text { LII }\end{array}$ & $\begin{array}{l}\text { GW-762 } \\
\text { PC } \\
\text { GRIDJ3 }\end{array}$ \\
\hline $\begin{array}{r}\text { General Information } \\
\text { Date Installed } \\
\text { Total Depth Drilled } \\
\text { East Coordinate } \\
\text { North Coordinate } \\
\text { Measuring Point Elevation } \\
\text { Well Wizard Elevation } \\
\text { Top of Casing Elevation } \\
\text { Ground Surface Elevation }\end{array}$ & $\begin{array}{r}10 / 25 / 91 \\
105.0 \\
48,936 \\
29,381 \\
960.12 \\
960.12 \\
960.12 \\
957.55\end{array}$ & $\begin{array}{r}11 / 07 / 91 \\
89.5 \\
48,890 \\
29,365 \\
959.91 \\
960.07 \\
959.91 \\
957.50\end{array}$ & $\begin{array}{r}11 / 21 / 91 \\
90.1 \\
49,026 \\
29,150 \\
983.08\end{array}$ & $\begin{array}{r}11 / 26 / 91 \\
320.0 \\
49,126 \\
29,010 \\
1,023.74 \\
1,023.74 \\
1,020.66\end{array}$ & $\begin{array}{r}12 / 20 / 91 \\
190.0 \\
49,055 \\
29,027 \\
1,020.43 \\
1,020.33 \\
1,019.63 \\
1,016.95\end{array}$ & $\begin{array}{r}01 / 08 / 92 \\
69.5 \\
64,324 \\
30,282 \\
907.62 \\
907.60 \\
907.43 \\
905.05\end{array}$ & $\begin{array}{r}01 / 28 / 92 \\
79.9 \\
64,570 \\
29,730 \\
921.13 \\
921.14 \\
920.96 \\
918.33\end{array}$ & $\begin{array}{r}02 / 06 / 92 \\
72.8 \\
64,835 \\
28,975 \\
919.03 \\
919.03 \\
918.86 \\
915.96\end{array}$ & $\begin{array}{r}04 / 24 / 92 \\
166.5 \\
53,303 \\
25,410 \\
961.61 \\
961.64 \\
961.43 \\
958.65\end{array}$ & $\begin{array}{r}05 / 15 / 92 \\
60.2 \\
63,193 \\
29,115 \\
915.34 \\
915.56 \\
915.34 \\
911.85\end{array}$ \\
\hline $\begin{array}{r}\text { Hydrostratig } \\
\text { Geologic } \\
\text { Weathered } R \\
\text { Fresh } R\end{array}$ & $\begin{array}{r}\text { AQF } \\
\text { Cmn } \\
\text { BDR } \\
7.5 \\
12.0\end{array}$ & $\begin{array}{l}\text { AQF } \\
\text { Cmn } \\
\text { BDR }\end{array}$ & $\begin{array}{r}\text { AQF } \\
\text { Cmn } \\
\text { BDR } \\
12.0 \\
15.1\end{array}$ & $\begin{array}{l}\text { AQF } \\
\text { Cmn } \\
\text { BDR } \\
34.0 \\
42.0\end{array}$ & $\begin{array}{l}\text { AQF } \\
\text { Cmn } \\
\text { BDR } \\
38.1 \\
45.1\end{array}$ & $\begin{array}{r}\text { AQT } \\
\text { Cpv } \\
\text { BDR } \\
9.6 \\
14.6\end{array}$ & $\begin{array}{r}\text { AQT } \\
\text { Cm } \\
\text { BDR } \\
10.5 \\
12.0\end{array}$ & $\begin{array}{r}\mathrm{AQT} \\
\mathrm{Cn} \\
\mathrm{BDR} \\
18.5 \\
24.8\end{array}$ & $\begin{array}{c}\text { AQF } \\
\text { OCk } \\
\text { BDR } \\
29.5 \\
48.0\end{array}$ & $\begin{array}{r}\text { AQT } \\
\text { Cn } \\
\text { BDR } \\
12.0 \\
14.5\end{array}$ \\
\hline $\begin{array}{r}\text { onductor Casing } \\
\text { Casing Depth } \\
\text { Casing Diameter } \\
\text { Casing Material }\end{array}$ & $\begin{array}{r}21.5 \\
11.75 \\
\text { SJ55 }\end{array}$ & $\begin{array}{r}22.0 \\
11.75 \\
\text { SJ55 }\end{array}$ & $\begin{array}{r}16.5 \\
11.75 \\
\text { SJ55 }\end{array}$ & $\begin{array}{r}60.3 \\
11.75 \\
\text { SJ55 }\end{array}$ & $\begin{array}{r}46.9 \\
11.75 \\
\text { SJ55 }\end{array}$ & $\begin{array}{r}27.6 \\
10.75 \\
\text { SJ55 } \\
\end{array}$ & $\begin{array}{r}23.8 \\
10.75 \\
\text { SJ55 }\end{array}$ & $\begin{array}{r}21.7 \\
11.75 \\
\text { SJ55 }\end{array}$ & $\begin{array}{r}46.8 \\
10.75 \\
\text { SJ55 }\end{array}$ & $\begin{array}{r}19.4 \\
11.75 \\
\text { SJ55 } \\
\end{array}$ \\
\hline $\begin{array}{r}\text { Bor } \\
\text { Boreho } \\
\text { Casir } \\
\text { Cas }\end{array}$ & $\begin{array}{r}102.5 \\
10.6 \\
92.4 \\
4.5 \\
\text { SS304 }\end{array}$ & $\begin{array}{r}89.5 \\
10.6 \\
79.4 \\
4.5 \\
\text { SS304 }\end{array}$ & $\begin{array}{r}90.1 \\
10.6 \\
67.3 \\
4.5 \\
\text { SS304 }\end{array}$ & $\begin{array}{r}289.2 \\
10.6 \\
287.9 \\
7 \\
\text { SF25 }\end{array}$ & $\begin{array}{r}165.6 \\
10.6 \\
164.3 \\
7 \\
\text { SF25 }\end{array}$ & $\begin{array}{r}69.5 \\
9.87 \\
57.0 \\
4.5 \\
\text { SS304 }\end{array}$ & $\begin{array}{r}79.9 \\
9.87 \\
69.2 \\
4.5 \\
\text { SS304 }\end{array}$ & $\begin{array}{r}72.8 \\
10.6 \\
62.4 \\
4.5 \\
\text { SS304 }\end{array}$ & $\begin{array}{r}166.5 \\
9.62 \\
135.5 \\
4.5 \\
\text { SS304 }\end{array}$ & $\begin{array}{r}60.2 \\
9.87 \\
48.2 \\
4.5 \\
5304\end{array}$ \\
\hline $\begin{array}{r}\text { onitored Interval } \\
\text { Top-Depth } \\
\text { Midpoint-Depth } \\
\text { Bottom of Screen-Depth } \\
\text { Bottom-Depth } \\
\text { Top-Elevation } \\
\text { Midpoint-Elevation } \\
\text { Bottom-Elevation } \\
\text { Screen Length } \\
\text { Screen Material } \\
\text { Slot Size } \\
\text { Open-Hole Length } \\
\text { Open-Hole Diameter }\end{array}$ & $\begin{array}{r}92.0 \\
98.5 \\
102.4 \\
105.0 \\
865.55 \\
859.05 \\
852.55 \\
10 \\
\mathrm{SS} / \mathrm{sw} \\
0.01\end{array}$ & $\begin{array}{r}79.0 \\
84.3 \\
89.4 \\
89.5 \\
878.50 \\
873.25 \\
868.00 \\
10 \\
\mathrm{SS} / \mathrm{sw} \\
0.01\end{array}$ & $\begin{array}{r}63.5 \\
75.8 \\
87.3 \\
88.0 \\
916.86 \\
904.61 \\
892.36 \\
20 \\
\mathrm{SS} / \mathrm{sw} \\
0.01\end{array}$ & $\begin{array}{r}289.2 \\
304.6 \\
320.0 \\
731.46 \\
716.06 \\
700.66\end{array}$ & $\begin{array}{r}165.6 \\
177.8 \\
190.0 \\
851.35 \\
839.15 \\
826.95\end{array}$ & $\begin{array}{r}55.0 \\
62.3 \\
66.9 \\
69.5 \\
850.05 \\
842.80 \\
835.55 \\
9.9 \\
\mathrm{SS} / \mathrm{sw} \\
0.01\end{array}$ & $\begin{array}{r}67.4 \\
73.5 \\
79.1 \\
79.6 \\
850.93 \\
844.83 \\
838.73 \\
9.9 \\
\mathrm{SS} / \mathrm{sw} \\
0.01\end{array}$ & $\begin{array}{r}61.2 \\
67.0 \\
72.3 \\
72.7 \\
854.76 \\
849.01 \\
843.26 \\
9.9 \\
\text { SS/sw } \\
0.01\end{array}$ & $\begin{array}{r}134.0 \\
150.3 \\
165.5 \\
166.5 \\
824.65 \\
808.40 \\
792.15 \\
30 \\
\mathrm{SS} / \mathrm{sw} \\
0.01\end{array}$ & $\begin{array}{r}46.4 \\
52.6 \\
58.1 \\
58.7 \\
865.45 \\
859.30 \\
853.15 \\
9.9 \\
\text { SS/sw } \\
0.01\end{array}$ \\
\hline $\begin{array}{r}\text { Screen Midpoint-(TOC) } \\
\text { Pump Intake-(TOC) } \\
\text { Intake-Elevation }\end{array}$ & $\begin{array}{r}100.0 \\
99.0 \\
861.1\end{array}$ & $\begin{array}{r}86.8 \\
87.0 \\
872.9\end{array}$ & $\begin{array}{r}80.0 \\
81.5 \\
901.6\end{array}$ & $\begin{array}{l}317.0 \\
706.7\end{array}$ & $\begin{array}{l}187.0 \\
832.6\end{array}$ & $\begin{array}{r}64.3 \\
67.5 \\
839.9\end{array}$ & $\begin{array}{r}76.8 \\
78.0 \\
843.0\end{array}$ & $\begin{array}{r}70.3 \\
70.7 \\
848.2\end{array}$ & $\begin{array}{l}153.3 \\
158.0 \\
803.4\end{array}$ & 56.6 \\
\hline
\end{tabular}


APPENDIX C: MONITORING WELL CONSTRUCTION DETAILS, 2002

\begin{tabular}{|c|c|c|c|c|c|c|c|c|c|c|}
\hline $\begin{array}{r}\text { Well Number } \\
\text { Hydrogeologic Regime } \\
\text { Location }\end{array}$ & $\begin{array}{c}\text { GW-763 } \\
\text { PC } \\
\text { GRIDJ3 }\end{array}$ & $\begin{array}{c}\text { GW-769 } \\
\text { PC } \\
\text { GRIDG3 }\end{array}$ & $\begin{array}{l}\text { GW-770 } \\
\text { PC } \\
\text { GRIDG3 }\end{array}$ & $\begin{array}{c}\text { GW-775 } \\
\text { PC } \\
\text { GRIDH3 }\end{array}$ & $\begin{array}{c}\text { GW-776 } \\
\text { PC } \\
\text { GRIDH3 }\end{array}$ & $\begin{array}{l}\text { GW-782 } \\
\text { PC } \\
\text { GRIDE3 }\end{array}$ & $\begin{array}{l}\text { GW-791 } \\
\text { PC } \\
\text { GRIDD2 }\end{array}$ & $\begin{array}{c}\text { GW-796 } \\
\text { CR } \\
\text { LV }\end{array}$ & $\begin{array}{c}\text { GW-797 } \\
\text { CR } \\
\text { LV }\end{array}$ & $\begin{array}{c}\text { GW-798 } \\
\text { CR } \\
\text { CDLVII }\end{array}$ \\
\hline $\begin{array}{r}\text { General Information } \\
\text { Date Installed } \\
\text { Total Depth Drilled } \\
\text { East Coordinate } \\
\text { North Coordinate } \\
\text { Measuring Point Elevation } \\
\text { Well Wizard Elevation } \\
\text { Top of Casing Elevation } \\
\text { Ground Surface Elevation }\end{array}$ & $\begin{array}{r}5 / 13 / 92 \\
17.0 \\
63,220 \\
29,117 \\
915.05 \\
915.03 \\
914.85 \\
911.38\end{array}$ & $\begin{array}{r}06 / 04 / 92 \\
61.4 \\
60,230 \\
29,510 \\
944.44 \\
944.43 \\
944.26 \\
941.53\end{array}$ & $\begin{array}{r}06 / 04 / 92 \\
20.0 \\
60,255 \\
29,505 \\
944.71 \\
944.72 \\
944.55 \\
941.67\end{array}$ & $\begin{array}{r}07 / 16 / 92 \\
60.5 \\
61,278 \\
29,272 \\
931.35\end{array}$ & $\begin{array}{r}07 / 21 / 92 \\
24.0 \\
61,309 \\
29,271 \\
931.25 \\
. \\
931.25 \\
931.44\end{array}$ & $\begin{array}{r}08 / 12 / 92 \\
36.0 \\
58,099 \\
29,719 \\
947.76 \\
947.73 \\
947.56 \\
944.48\end{array}$ & $\begin{array}{r}09 / 21 / 92 \\
70.6 \\
57,423 \\
30,483 \\
992.16 \\
992.13 \\
991.96 \\
988.51\end{array}$ & $\begin{array}{r}03 / 04 / 93 \\
139.7 \\
58,206 \\
27,924 \\
1,052.62 \\
1,052.62 \\
1,052.42 \\
1,048.80\end{array}$ & $\begin{array}{r}03 / 16 / 93 \\
134.1 \\
58,550 \\
27,447 \\
1,060.00 \\
1,060.00 \\
1,059.80 \\
1,056.10\end{array}$ & $\begin{array}{r}03 / 18 / 93 \\
135.5 \\
60,310 \\
27,265 \\
1,006.00 \\
1,006.00 \\
1,005.80 \\
1,002.42\end{array}$ \\
\hline $\begin{array}{r}\text { Hydrostratigr } \\
\text { Geologic } \\
\text { Aqu } \\
\text { Weathered Rc } \\
\text { Fresh Rc }\end{array}$ & $\begin{array}{r}A Q T \\
C n \\
W T \\
17.0\end{array}$ & $\begin{array}{r}\mathrm{AQT} \\
\mathrm{Cn} \\
\mathrm{BDR} \\
14.2\end{array}$ & $\begin{array}{r}\text { AQT } \\
\mathrm{Cn} \\
\mathrm{WT} \\
12.0 \\
16.5\end{array}$ & $\begin{array}{r}\mathrm{AQT} \\
\mathrm{Cn} \\
\mathrm{BDR}\end{array}$ & $\begin{array}{r}\mathrm{AQT} \\
\mathrm{Cn} \\
\mathrm{WT} \\
14.5 \\
19.3\end{array}$ & $\begin{array}{r}\mathrm{AQT} \\
\mathrm{Cn} \\
\mathrm{BDR} \\
1.0 \\
7.5\end{array}$ & $\begin{array}{r}\text { AQT } \\
\text { Cm } \\
\text { BDR } \\
14.7 \\
26.0\end{array}$ & $\begin{array}{r}\text { AQF } \\
\text { OCk } \\
\text { BDR } \\
102.0 \\
103.0\end{array}$ & $\begin{array}{c}\text { AQF } \\
\text { OCk } \\
\text { BDR } \\
67.1 \\
89.0\end{array}$ & $\begin{array}{l}\text { AQF } \\
\text { OCk } \\
\text { BDR } \\
94.4 \\
95.8\end{array}$ \\
\hline $\begin{array}{l}\text { onductor Casing } \\
\text { Casing Depth } \\
\text { Casing Diameter } \\
\text { Casing Material }\end{array}$ & & $\begin{array}{r}17.2 \\
11.75 \\
\text { SJ55 }\end{array}$ & & $\begin{array}{r}16.7 \\
11.75 \\
\text { SJ55 }\end{array}$ & & & $\begin{array}{r}31.5 \\
10.75 \\
\text { SJ55 }\end{array}$ & $\begin{array}{l}107.6 \\
10.75 \\
\text { SJ55 }\end{array}$ & $\begin{array}{r}95.0 \\
10.75 \\
\text { SJ55 }\end{array}$ & $\begin{array}{r}99.7 \\
10.75 \\
\text { SJ55 } \\
\end{array}$ \\
\hline $\begin{array}{r}\text { Bore } \\
\text { Boreho } \\
\text { Casin } \\
\text { Cas }\end{array}$ & $\begin{array}{r}17.0 \\
8 \\
5.2 \\
4.5 \\
\text { SS304 }\end{array}$ & $\begin{array}{r}61.4 \\
10.62 \\
49.4 \\
4.5 \\
\text { SS304 }\end{array}$ & $\begin{array}{r}20.0 \\
10.62 \\
8.5 \\
4.5 \\
\text { SS304 }\end{array}$ & $\begin{array}{r}60.5 \\
10.62 \\
46.3 \\
4.5 \\
\text { SS304 }\end{array}$ & $\begin{array}{r}24.0 \\
9.87 \\
12.3 \\
4.5 \\
\text { SS304 }\end{array}$ & $\begin{array}{r}36.0 \\
9.87 \\
25.0 \\
4.5 \\
\text { SS304 }\end{array}$ & $\begin{array}{r}70.6 \\
9.87 \\
59.0 \\
4.5 \\
\text { SS304 }\end{array}$ & $\begin{array}{r}139.7 \\
9.5 \\
126.5 \\
4.5 \\
\text { SS304 }\end{array}$ & $\begin{array}{r}134.1 \\
9.5 \\
123.5 \\
4.5 \\
\text { SS304 }\end{array}$ & $\begin{array}{r}135.5 \\
9.5 \\
124.5 \\
4.5 \\
\text { SS304 }\end{array}$ \\
\hline $\begin{array}{r}\text { lonitored Interval } \\
\text { Top-Depth } \\
\text { Midpoint-Depth } \\
\text { Bottom of Screen-Depth } \\
\text { Bottom-Depth } \\
\text { Top-Elevation } \\
\text { Midpoint-Elevation } \\
\text { Bottom-Elevation } \\
\text { Screen Length } \\
\text { Screen Material } \\
\text { Slot Size } \\
\text { Open-Hole Length } \\
\text { Open-Hole Diameter }\end{array}$ & $\begin{array}{r}4.0 \\
10.0 \\
15.2 \\
16.0 \\
907.38 \\
901.38 \\
895.38 \\
10 \\
\mathrm{SS} / \mathrm{sw} \\
0.01\end{array}$ & $\begin{array}{r}48.2 \\
54.3 \\
59.3 \\
60.3 \\
893.33 \\
887.28 \\
881.23 \\
9.9 \\
\mathrm{SS} / \mathrm{sw} \\
0.01\end{array}$ & $\begin{array}{r}7.5 \\
13.3 \\
18.4 \\
19.0 \\
934.17 \\
928.42 \\
922.67 \\
9.9 \\
\mathrm{SS} / \mathrm{sw} \\
0.01\end{array}$ & $\begin{array}{r}45.0 \\
50.7 \\
56.2 \\
56.4 \\
886.48 \\
880.78 \\
875.08 \\
9.9 \\
\mathrm{SS} / \mathrm{sw} \\
0.01\end{array}$ & $\begin{array}{r}10.6 \\
16.8 \\
22.2 \\
23.0 \\
920.84 \\
914.64 \\
908.44 \\
9.9 \\
\mathrm{SS} / \mathrm{sw} \\
0.01\end{array}$ & $\begin{array}{r}23.8 \\
29.9 \\
34.9 \\
35.9 \\
920.68 \\
914.63 \\
908.58 \\
9.9 \\
\mathrm{SS} / \mathrm{sw} \\
0.01\end{array}$ & $\begin{array}{r}57.5 \\
64.1 \\
68.9 \\
70.6 \\
931.01 \\
924.46 \\
917.91 \\
9.9 \\
\mathrm{SS} / \mathrm{sw} \\
0.01\end{array}$ & $\begin{array}{r}122.9 \\
129.7 \\
136.4 \\
136.5 \\
925.90 \\
919.10 \\
912.30 \\
9.9 \\
\mathrm{SS} / \mathrm{sw} \\
0.01\end{array}$ & $\begin{array}{r}118.0 \\
126.1 \\
133.4 \\
134.1 \\
938.10 \\
930.05 \\
922.00 \\
9.9 \\
\mathrm{SS} / \mathrm{sw} \\
0.01\end{array}$ & $\begin{array}{r}122.0 \\
128.7 \\
134.4 \\
135.4 \\
880.42 \\
873.72 \\
867.02 \\
9.9 \\
\mathrm{SS} / \mathrm{sW} \\
0.01\end{array}$ \\
\hline $\begin{array}{r}\text { Screen Midpoint-(TOC) } \\
\text { Pump Intake-(TOC) } \\
\text { Intake-Elevation }\end{array}$ & $\begin{array}{r}13.6 \\
15.4 \\
899.5\end{array}$ & $\begin{array}{r}57.1 \\
57.8 \\
886.5\end{array}$ & $\begin{array}{r}16.3 \\
16.7 \\
927.9\end{array}$ & $\begin{array}{r}51.1 \\
51.0 \\
880.4\end{array}$ & $\begin{array}{r}17.1 \\
17.0 \\
914.3\end{array}$ & $\begin{array}{r}33.0 \\
33.0 \\
914.6\end{array}$ & $\begin{array}{r}67.4 \\
67.4 \\
924.6\end{array}$ & $\begin{array}{l}135.1 \\
135.0 \\
917.4\end{array}$ & $\begin{array}{l}132.2 \\
132.2 \\
927.6\end{array}$ & 32.8 \\
\hline
\end{tabular}


APPENDIX C: MONITORING WELL CONSTRUCTION DETAILS, 2002

\begin{tabular}{|c|c|c|c|c|c|c|c|c|c|c|}
\hline $\begin{array}{r}\text { Well Number } \\
\text { Hydrogeologic Regime } \\
\text { Location }\end{array}$ & $\begin{array}{c}\text { GW-799 } \\
\text { CR } \\
\text { LV }\end{array}$ & $\begin{array}{c}\text { GW-801 } \\
\text { CR } \\
\text { LV }\end{array}$ & $\begin{array}{l}\text { GW-816 } \\
\text { PC } \\
\text { EXP-SR }\end{array}$ & $\begin{array}{l}\text { GW-827 } \\
\text { CR } \\
\text { CDLVI }\end{array}$ & $\begin{array}{c}\text { GW-831 } \\
\text { CR } \\
\text { FCAP }\end{array}$ & $\begin{array}{c}\text { GW-832 } \\
\text { PC } \\
\text { NHP }\end{array}$ & $\begin{array}{c}\text { GW-835 } \\
\text { BC } \\
\text { S3 }\end{array}$ & $\begin{array}{c}\text { GW-916 } \\
\text { BC } \\
\text { EMWMF }\end{array}$ & $\begin{array}{c}\text { GW-917 } \\
\text { BC } \\
\text { EMWMF }\end{array}$ & $\begin{array}{c}\text { GW-918 } \\
\text { BC } \\
\text { EMWMF }\end{array}$ \\
\hline \begin{tabular}{|r|} 
General Information \\
Date Installed \\
Total Depth Drilled \\
East Coordinate \\
North Coordinate \\
Measuring Point Elevation \\
Well Wizard Elevation \\
Top of Casing Elevation \\
Ground Surface Elevation \\
\end{tabular} & $\begin{array}{r}03 / 25 / 93 \\
92.0 \\
59,961 \\
26,746 \\
981.29 \\
981.29 \\
981.09 \\
978.10 \\
\end{array}$ & $\begin{array}{r}07 / 01 / 93 \\
188.9 \\
58,780 \\
26,808 \\
1,097.16 \\
1,097.16 \\
1,096.96 \\
1,093.82 \\
\end{array}$ & $\begin{array}{r}06 / 02 / 94 \\
16.1 \\
64,031 \\
31,582 \\
898.41 \\
898.42 \\
898.21 \\
894.56\end{array}$ & $\begin{array}{r}01 / 24 / 95 \\
135.0 \\
51,826 \\
27,721 \\
1,051.58 \\
1,051.60 \\
1,051.39 \\
1,048.13\end{array}$ & $\begin{array}{r}07 / 30 / 96 \\
200.0 \\
56,593 \\
26,654 \\
1,091.29 \\
1,091.29 \\
1,091.09 \\
1,088.04 \\
\end{array}$ & $\begin{array}{r}05 / 09 / 96 \\
11.9 \\
64,134 \\
29,142 \\
906.18 \\
906.18 \\
906.83 \\
\end{array}$ & $\begin{array}{r}19.2 \\
51,358 \\
29,822 \\
1,000.91 \\
1,000.91 \\
998.04\end{array}$ & $\begin{array}{r}01 / 29 / 01 \\
36.0 \\
48,276 \\
31,186 \\
1,002.85 \\
1,002.85\end{array}$ & $\begin{array}{r}01 / 22 / 01 \\
51.0 \\
47,914 \\
30,463 \\
997.10 \\
997.10\end{array}$ & $\begin{array}{r}02 / 02 / 01 \\
75.0 \\
47,549 \\
31,672 \\
1,067.96 \\
1,067.96\end{array}$ \\
\hline \begin{tabular}{|} 
Geologic Information \\
Hydrostratigraphic Unit \\
Geologic Formation \\
Aquifer Zone \\
Weathered Rock-Depth \\
Fresh Rock-Depth
\end{tabular} & $\begin{array}{c}\text { AQF } \\
\text { OCk } \\
\text { BDR } \\
60.8 \\
62.8\end{array}$ & $\begin{array}{r}\mathrm{AQF} \\
\mathrm{OCk} \\
\mathrm{BDR} \\
112.5 \\
113.4\end{array}$ & $\begin{array}{r}\mathrm{AQT} \\
\mathrm{Cr} \\
\mathrm{WT}\end{array}$ & $\begin{array}{r}\text { AQF } \\
\text { OCk } \\
\text { BDR } \\
\\
40.5\end{array}$ & $\begin{array}{r}\mathrm{AQF} \\
\mathrm{OCk} \\
\mathrm{BDR} \\
134.8 \\
140.8\end{array}$ & $\begin{array}{r}\mathrm{AQF} \\
\mathrm{Cmn} \\
\mathrm{WT}\end{array}$ & $\begin{array}{c}\mathrm{WT} \\
19.2\end{array}$ & $\begin{array}{r}A Q T \\
C C \\
W T \\
10.0 \\
15.0\end{array}$ & $\begin{array}{r}A Q T \\
C C \\
W T \\
21.0 \\
27.0\end{array}$ & $\begin{array}{r}\text { AQT } \\
\mathrm{CC} \\
\mathrm{WT} \\
. \\
30.0\end{array}$ \\
\hline $\begin{array}{r}\text { Conductor Casing } \\
\text { Casing Depth } \\
\text { Casing Diameter } \\
\text { Casing Material }\end{array}$ & $\begin{array}{r}65.0 \\
10.75 \\
\text { SJ55 } \\
\end{array}$ & $\begin{array}{l}115.4 \\
10.75 \\
\text { SJ55 }\end{array}$ & & $\begin{array}{r}43.4 \\
10.75 \\
\text { STL }\end{array}$ & $\begin{array}{c}138.3 \\
10.75 \\
\text { STL }\end{array}$ & & & & & \\
\hline $\begin{array}{l}\text { ell Casing } \\
\text { Borehole Depth } \\
\text { Borehole Diameter } \\
\text { Casing Depth } \\
\text { Casing Diameter } \\
\text { Casing Material }\end{array}$ & $\begin{array}{r}92.0 \\
9.5 \\
81.0 \\
4.5 \\
\text { SS304 }\end{array}$ & $\begin{array}{r}188.9 \\
9.87 \\
178.1 \\
4.5 \\
\text { SS } 304\end{array}$ & $\begin{array}{r}15.8 \\
10 \\
4.2 \\
4.500 \\
\text { SS304 }\end{array}$ & $\begin{array}{r}135.0 \\
9.87 \\
124.1 \\
4.500 \\
\text { SS304 }\end{array}$ & $\begin{array}{r}200.0 \\
9.87 \\
183.2 \\
4.500 \\
\text { SLS }\end{array}$ & $\begin{array}{r}11.9 \\
12 \\
5.9 \\
6.630 \\
\text { PVC }\end{array}$ & $\begin{array}{r}17.2 \\
4.500 \\
\mathrm{PVC}\end{array}$ & & $\begin{array}{r}20.0 \\
2.370 \\
S S 304\end{array}$ & $\begin{array}{r}20.0 \\
\text { SS304 }\end{array}$ \\
\hline $\begin{array}{r}\text { Ionitored Interval } \\
\text { Top-Depth } \\
\text { Midpoint-Depth } \\
\text { Bottom of Screen-Depth } \\
\text { Bottom-Depth } \\
\text { Top-Elevation } \\
\text { Midpoint-Elevation } \\
\text { Bottom-Elevation } \\
\text { Screen Length } \\
\text { Screen Material } \\
\text { Slot Size } \\
\text { Open-Hole Length } \\
\text { Open-Hole Diameter }\end{array}$ & $\begin{array}{r}78.7 \\
85.4 \\
90.9 \\
92.0 \\
899.40 \\
892.75 \\
886.10 \\
9.9 \\
\mathrm{SS} / \mathrm{sw} \\
0.01\end{array}$ & $\begin{array}{r}175.8 \\
182.4 \\
188.0 \\
188.9 \\
918.02 \\
911.47 \\
904.92 \\
9.9 \\
\mathrm{SS} / \mathrm{sw} \\
0.01\end{array}$ & $\begin{array}{r}2.9 \\
9.4 \\
13.6 \\
15.8 \\
891.66 \\
885.21 \\
878.76 \\
9.4 \\
\mathrm{SS} / \mathrm{sw} \\
0.01\end{array}$ & $\begin{array}{r}122.1 \\
128.5 \\
134.1 \\
134.8 \\
926.03 \\
919.68 \\
913.33 \\
10 \\
\mathrm{SS} / \mathrm{sw} \\
0.01\end{array}$ & $\begin{array}{r}182.0 \\
190.8 \\
193.6 \\
199.6 \\
906.04 \\
897.24 \\
888.44 \\
10.4 \\
S S / s w \\
0.01\end{array}$ & $\begin{array}{r}4.0 \\
7.9 \\
10.9 \\
11.8 \\
902.83 \\
898.93 \\
895.03 \\
5 \\
\mathrm{PVC} / \mathrm{sl} \\
0.02\end{array}$ & $\begin{array}{r}16.2 \\
17.7 \\
19.2 \\
19.2 \\
981.84 \\
980.34 \\
978.84 \\
2 \\
\mathrm{PVC} / \mathrm{sl}\end{array}$ & $\begin{array}{r}20 \\
\mathrm{SS} / \mathrm{sw} \\
0.01\end{array}$ & $\begin{array}{l}18.0 \\
34.5 \\
50.0 \\
51.0\end{array}$ & $\begin{array}{l}18.0 \\
25.5 \\
30.0 \\
33.0\end{array}$ \\
\hline $\begin{array}{r}\text { Dedicated Pump } \\
\text { Screen Midpoint-(TOC) } \\
\text { Pump Intake-(TOC) } \\
\text { Intake-Elevation }\end{array}$ & $\begin{array}{r}88.9 \\
89.0 \\
892.1\end{array}$ & $\begin{array}{l}186.2 \\
181.0 \\
916.0\end{array}$ & $\begin{array}{r}12.6 \\
15.0 \\
883.2\end{array}$ & $\begin{array}{l}132.4 \\
132.2 \\
919.2\end{array}$ & $\begin{array}{l}191.5 \\
192.0 \\
899.1\end{array}$ & & & & & \\
\hline
\end{tabular}


APPENDIX C: MONITORING WELL CONSTRUCTION DETAILS, 2002

\begin{tabular}{|c|c|c|c|c|c|c|c|c|c|}
\hline $\begin{array}{r}\text { Well Number } \\
\text { Hydrogeologic Regime } \\
\text { Location }\end{array}$ & $\begin{array}{c}\text { GW-919 } \\
\text { BC } \\
\text { EMWMF }\end{array}$ & $\begin{array}{c}\text { GW-920 } \\
\text { BC } \\
\text { EMWMF }\end{array}$ & $\begin{array}{l}\text { GW-921 } \\
\text { BC } \\
\text { EMWMF }\end{array}$ & $\begin{array}{c}\text { GW-922 } \\
\text { BC } \\
\text { EMWMF }\end{array}$ & $\begin{array}{c}\text { GW-923 } \\
\text { BC } \\
\text { EMWMF }\end{array}$ & $\begin{array}{c}\text { GW-924 } \\
\text { BC } \\
\text { EMWMF }\end{array}$ & $\begin{array}{c}\text { GW-925 } \\
\text { BC } \\
\text { EMWMF }\end{array}$ & $\begin{array}{c}\text { GW-926 } \\
\text { BC } \\
\text { EMWMF }\end{array}$ & $\begin{array}{c}\text { GW-927 } \\
\text { BC } \\
\text { EMWMF }\end{array}$ \\
\hline \multicolumn{10}{|l|}{ General Information } \\
\hline Date Installed & $08 / 21 / 01$ & $01 / 16 / 01$ & 01/31/01 & $01 / 17 / 01$ & 02/01/01 & $01 / 29 / 01$ & $02 / 05 / 01$ & $02 / 01 / 01$ & 02/01/01 \\
\hline Total Depth Drilled & 32.0 & 55.0 & 50.0 & 46.0 & 102.0 & 54.0 & 170.0 & 145.0 & 172.0 \\
\hline East Coordinate & 47,326 & 47,375 & 47,139 & 47,147 & 48,184 & 46,300 & 47,128 & 46,290 & 47,906 \\
\hline North Coordinate & 30,739 & 30,193 & 30,350 & 30,024 & 30,822 & 30,185 & 30,349 & 30,185 & 30,463 \\
\hline $\begin{array}{l}\text { Measuring Point Elevation } \\
\text { Well Wizard Elevation }\end{array}$ & 990.52 & 967.43 & 971.29 & 956.91 & $1,016.73$ & 968.90 & 971.14 & 968.94 & 997.19 \\
\hline $\begin{array}{l}\text { Top of Casing Elevation } \\
\text { Ground Surface Elevation }\end{array}$ & $\begin{array}{l}990.52 \\
987.50 \\
\end{array}$ & 967.43 & 971.29 & 956.91 & $1,016.73$ & 968.90 & 971.14 & 968.94 & 997.19 \\
\hline \multicolumn{10}{|l|}{ Geologic Information } \\
\hline Geologic Formation & $\mathrm{cC}$ & $\mathrm{cc}$ & $\mathrm{CC}$ & $\mathrm{CC}$ & $\mathrm{CC}$ & $\mathrm{CC}$ & $\mathrm{cC}$ & $\mathrm{CC}$ & $\mathrm{CC}$ \\
\hline Aquifer Zone & WT & $\mathrm{BDR}$ & BDR & $\mathrm{BDR}$ & WT & WT & $\mathrm{BDR}$ & $\mathrm{BDR}$ & BDR \\
\hline Weathered Rock-Depth & 12.0 & & & 10.0 & & 21.8 & & 15.0 & 25.0 \\
\hline Fresh Rock-Depth & 22.0 & 12.0 & 13.0 & 13.0 & 62.0 & 22.0 & 15.0 & 18.0 & 30.0 \\
\hline \multicolumn{10}{|l|}{$\begin{array}{r}\text { Conductor Casing } \\
\text { Casing Depth } \\
\text { Casing Diameter } \\
\text { Casing Material } \\
\end{array}$} \\
\hline \multicolumn{10}{|l|}{$\begin{array}{r}\text { Well Casing } \\
\text { Borehole Depth } \\
\text { Borehole Diameter }\end{array}$} \\
\hline Casing Depth & 16.5 & 24.0 & 18.0 & 25.0 & 40.0 & 23.0 & 97.0 & 113.0 & 60.0 \\
\hline Casing Diameter & 2.380 & 2.370 & 2.370 & 2.370 & 2.370 & 2.370 & 2.370 & 2.370 & 2.370 \\
\hline Casing Material & SS304 & SS304 & SS304 & SS304 & SS304 & SS304 & SS304 & SS304 & SS304 \\
\hline \multicolumn{10}{|l|}{ Monitored Interval } \\
\hline Top-Depth & 11.6 & 22.0 & 16.0 & 23.0 & 36.0 & 21.0 & 92.0 & 103.0 & 57.0 \\
\hline Midpoint-Depth & 21.8 & 38.5 & 33.0 & 34.5 & 55.5 & 37.5 & 120.0 & 124.0 & 74.5 \\
\hline Bottom of Screen-Depth & 31.5 & 54.0 & 48.0 & 45.0 & 70.0 & 53.0 & 147.0 & 143.0 & 90.0 \\
\hline Bottom-Depth & 32.0 & 55.0 & 50.0 & 46.0 & 75.0 & 54.0 & 148.0 & 145.0 & 92.0 \\
\hline Top-Elevation & 975.90 & & & & & & & & \\
\hline Midpoint-Elevation & 965.70 & & & & & & & & \\
\hline Bottom-Elevation & 955.50 & & & & & & & & \\
\hline Screen Length & 15 & 30 & 30 & 20 & 30 & 30 & 50 & 30 & 30 \\
\hline Screen Material & SS/sw & SS/sw & SS/sw & SS/sw & SS/sw & SS/sw & SS/sw & SS/sw & SS/sw \\
\hline $\begin{array}{r}\text { Slot Size } \\
\text { Open-Hole Length } \\
\text { Open-Hole Diameter }\end{array}$ & 0.01 & 0.01 & 0.01 & 0.01 & 0.01 & 0.01 & 0.01 & 0.01 & 0.01 \\
\hline \multicolumn{10}{|l|}{ Dedicated Pump } \\
\hline Screen Midpoint-(TOC) & & & & & & & & & \\
\hline $\begin{array}{r}\text { Pump Intake-(TOC) } \\
\text { Intake-Elevation }\end{array}$ & & & & & & & & & \\
\hline
\end{tabular}


APPENDIX D

CY 2002 MONITORING DATA FOR THE

BEAR CREEK HYDROGEOLOGIC REGIME 


\section{EXPLANATION}

\section{Sampling Point:}

BCK - Bear Creek Kilometer

GW - Monitoring Well

NT - Northern Tributary (Bear Creek)

SS - South Side (of Bear Creek, spring sampling station)

\section{Location:}

BG - Bear Creek Burial Grounds

EMWMF - Environmental Management Waste Management Facility

EXP - Exit Pathway Monitoring Location:

Maynardville Limestone Picket (-A, -B, -C, -W)

Spring or Surface Water Location (-SW)

OLF - Oil Landfarm

RS - Rust Spoil Area

S3 - S-3 Site

SPI - Spoil Area I

\section{Monitoring Program:}

BJC - managed by Bechtel Jacobs Company LLC

GWPP - managed by Y-12 Groundwater Protection Program

Sample Type:

Dup - Field Duplicate Sample

Units:

$\mathrm{ft}$ - feet (elevations are above mean sea level and depths are below grade)

$\mu \mathrm{g} / \mathrm{L}-$ micrograms per liter

$\mathrm{mg} / \mathrm{L}$ - milligrams per liter

$\mathrm{mV}$ - millivolts

$\mu \mathrm{mho} / \mathrm{cm}$ - micromhos per centimeter

NTU - Nephelometric Turbidity Units

$\mathrm{pCi} / \mathrm{L}$ - picoCuries per liter

ppm - parts per million 


\section{EXPLANATION (continued)}

Only the analytes that were detected above the program reporting limits in at least one sample are included in this appendix. Additionally, results that are below the reporting limits are replaced with missing values (e.g., " < ") to emphasize the detected results. The following sections describe the reporting limits and data qualifiers for each sub-appendix. A comprehensive list of the GWPP analytes, analytical methods, and reporting limits is provided in Appendix B, Table B.5.

\section{D.1 Field Measurements, Miscellaneous Analytes, Major Ions, and Trace Metals:}

Results for all of the field measurements, miscellaneous analytes, and major ions are included in this appendix. The reporting limits for the major ions are shown in the following summary.

\begin{tabular}{|c|c|c|c|c|c|}
\hline \multirow{2}{*}{ Analyte } & \multicolumn{2}{|c|}{ Reporting Limit (mg/L) } & \multirow{2}{*}{ Analyte } & \multicolumn{2}{|c|}{ Reporting Limit (mg/L) } \\
\hline & GWPP & BJC & & GWPP & BJC \\
\hline $\begin{array}{r}\text { Calcium } \\
\text { Magnesium } \\
\text { Potassium } \\
\text { Sodium }\end{array}$ & $\begin{array}{l}0.2 \\
0.2 \\
2.0 \\
0.2\end{array}$ & $\begin{array}{l}0.25 \\
0.05 \\
0.25 \\
0.25\end{array}$ & $\begin{array}{r}\text { Anions } \\
\text { Alkalinity - HCO3 } \\
\text { Alkalinity - CO3 } \\
\text { Chloride } \\
\text { Fluoride } \\
\text { Nitrate (as Nitrogen) } \\
\text { Sulfate }\end{array}$ & $\begin{array}{c}1.0 \\
1.0 \\
0.2 \\
0.1 \\
0.028 \\
0.25\end{array}$ & $\begin{array}{c}\text { NA } \\
\text { NA } \\
0.1 \\
0.05 \\
0.1 \\
0.1\end{array}$ \\
\hline
\end{tabular}

The major ion results for the January 2002 sample from NT-01 are qualitative because the ion charge balance (relative percent difference) exceeds $20 \%$.

The Y-12 GWPP SAP (BWXT 2001a) specifies reporting limits for trace metals that are appropriate for DOE Order 5400.1 monitoring. The laboratories subcontracted by monitoring programs managed by BJC may use reporting limits (sometimes reporting estimated values) that are lower than the GWPP reporting limits for the metals. To retain the highest quality data for DOE Order 5400.1 monitoring purposes and to standardize reporting limits for trace metal results obtained from all sources, the GWPP reporting limits were given precedence over the BJC reporting limits (BJC 2001 and 2002a) shown below. The trace metals shown in bold typeface below were detected in at least one groundwater or surface water sample collected during CY 2002 and are presented in Appendix D.1. 
EXPLANATION (continued)

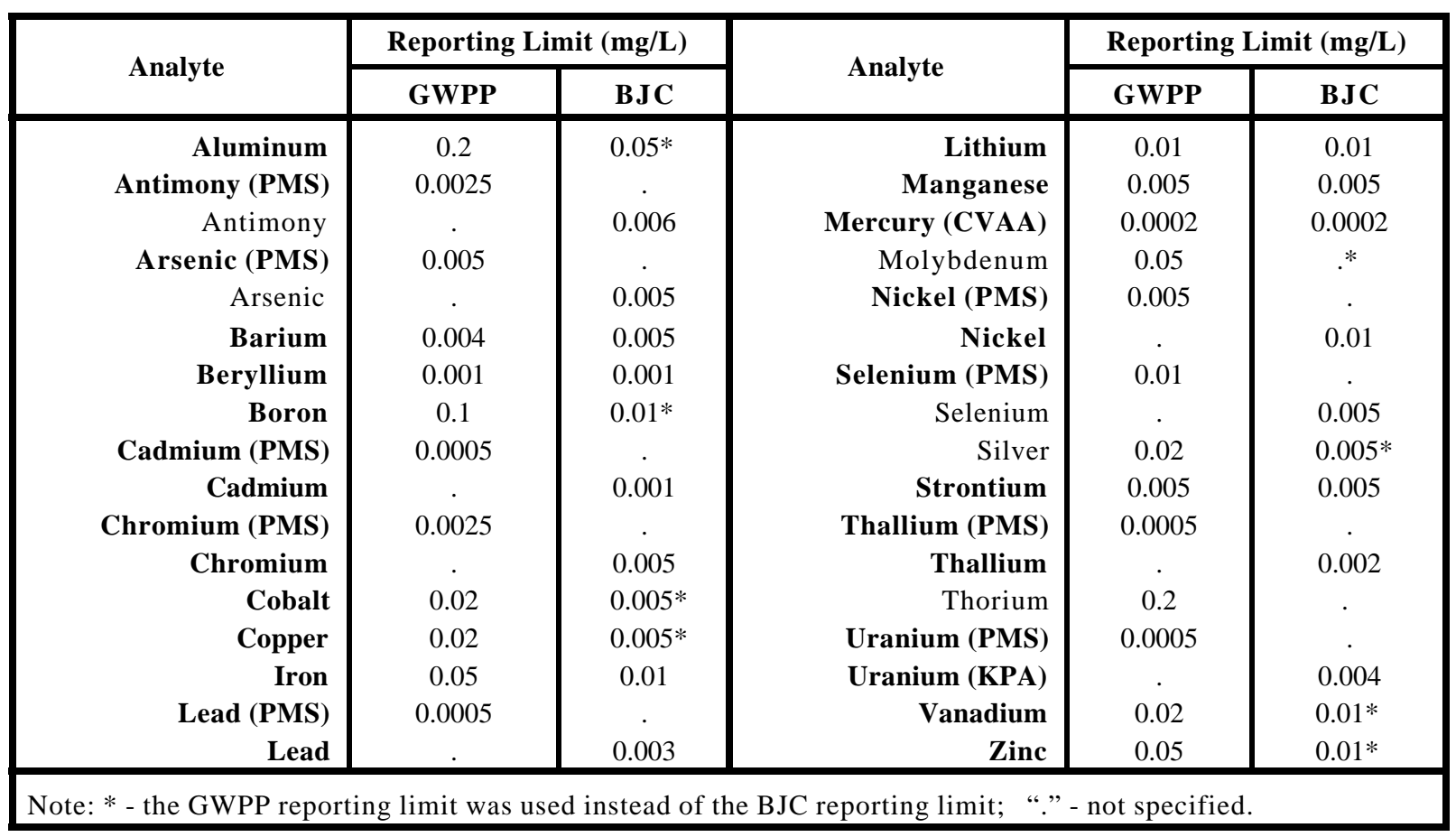

Metals analyses were performed using the inductively coupled plasma (ICP) spectroscopy method (SW8466010B) unless otherwise noted.

$\begin{array}{rll}\text { CVAA } & - & \text { Cold Vapor Atomic Absorption (EPA-7470) } \\ \text { KPA } & - & \text { Kinetic Phosphorescent Analysis (ASTM-D5174-M) } \\ \text { PMS } & - & \text { Plasma Mass Spectroscopy (EPA-200.8) }\end{array}$

Groundwater samples collected from the following wells for metals analysis by the ICP method during CY 2002 were diluted before analysis to obtain an optimum matrix. The detected results are valid, but some metals may be present at concentrations below the elevated reporting limits.

\begin{tabular}{|c|c|c|c|c|c|}
\hline Sampling Location & $\begin{array}{c}\text { Date } \\
\text { Sampled }\end{array}$ & Dilution Factor & $\begin{array}{c}\text { Sampling } \\
\text { Location }\end{array}$ & $\begin{array}{c}\text { Date } \\
\text { Sampled }\end{array}$ & $\begin{array}{c}\text { Dilution } \\
\text { Factor }\end{array}$ \\
\hline GW-091 & $08 / 08 / 02$ & $2 \mathrm{X}$ & $\mathrm{GW}-537$ & $02 / 18 / 02$ & $2 \mathrm{X}$ \\
GW-243 & $03 / 21 / 02$ & $100 \mathrm{X}$ & $\mathrm{GW}-537$ & $07 / 31 / 02$ & $2 \mathrm{X}$ \\
GW-243 & $08 / 13 / 02$ & $50 \mathrm{X}$ & $\mathrm{NT}-01$ & $07 / 11 / 02$ & $10 \mathrm{X}$ \\
\hline
\end{tabular}

The following symbols and data qualifiers are used in Appendix D.1:

\footnotetext{
- $\quad$ Not analyzed or not applicable

$<\quad-\quad$ Analyzed but not detected at the project reporting level

$\mathrm{R}$ - Result does not meet data quality objectives (charge balance for NT-01 and duplicate lead results for GW-311 that differ by at least an order of magnitude).
} 


\section{EXPLANATION (continued)}

\section{D.2 Volatile Organic Compounds:}

The Y-12 GWPP reporting limits for volatile organic compounds (Table B.5) and those used for monitoring programs managed by BJC are contract-required quantitation limits. Results below the quantitation limit and above the instrument detection limit are reported as estimated quantities. Therefore, non-detected results are assumed to equal zero for all compounds.

As summarized below, 27 compounds were detected (of the 53 compounds requested) in the CY 2002 groundwater and surface water samples collected in the Bear Creek Regime. Results for these compounds are grouped by similar chemical composition (e.g., chloroethenes) in Appendix D.2.

\begin{tabular}{|c|c|c|c|c|c|}
\hline Compound & $\begin{array}{c}\text { No. } \\
\text { Detected }\end{array}$ & $\begin{array}{c}\text { Maximum } \\
(\mu \mathrm{g} / \mathrm{L})\end{array}$ & Compound & $\begin{array}{c}\text { No. } \\
\text { Detected }\end{array}$ & $\begin{array}{c}\text { Maximum } \\
(\mu \mathrm{g} / \mathrm{L})\end{array}$ \\
\hline Trichloroethene & 71 & 710 & Toluene & 5 & 10 \\
\hline cis-1,2-Dichloroethene & 71 & 7,200 & 1,2-Dichloroethane & 4 & 32 \\
\hline Tetrachloroethene & 49 & 4,400 & Dichlorodifluoromethane & 4 & 12 \\
\hline 1,1-Dichloroethene & 33 & 120 & Chlorobenzene & 3 & 8 \\
\hline 1,1-Dichloroethane & 26 & 1,780 & Chloromethane & 3 & $3 \mathrm{~J}$ \\
\hline Vinyl chloride & 24 & 1,200 & Dimethylbenzene & 3 & 9 \\
\hline Benzene & 16 & 820 & Trichlorofluoromethane & 3 & 5 \\
\hline $1,1,1$-Trichloroethane & 15 & 120 & 2-Butanone & 2 & 25 \\
\hline Chloroethane & 12 & 25 & Bromoform & 2 & $3 \mathrm{~J}$ \\
\hline Chloroform & 12 & 100 & Ethylbenzene & 2 & $2 \mathrm{~J}$ \\
\hline trans-1,2-Dichloroethene & 12 & 13 & 1,1,2-Trichloroethane & 1 & $2 \mathrm{~J}$ \\
\hline Acetone & 6 & 700 & 1,2-Dichloropropane & 1 & $1 \mathrm{~J}$ \\
\hline Carbon tetrachloride & 5 & 8 & 1,4-Dichlorobenzene & 1 & $3 \mathrm{~J}$ \\
\hline Methylene chloride & 5 & 120 & & & \\
\hline
\end{tabular}

The following symbols and data qualifiers are used in Appendix D.2.

. $\quad$ - Not analyzed

$<\quad$ - Analyzed but not detected (also false-positive results for data provided by the WRRP)

J - Positively identified; estimated concentration 


\section{EXPLANATION (continued)}

\section{D.3 Radiological Analytes:}

Reporting limits for radiological analytes are sample-specific and analyte-specific minimum detectable activities that are reported with each result. The following summary shows the radiological analytes reported for at least one groundwater sample collected during CY 2002 in the Bear Creek Regime.

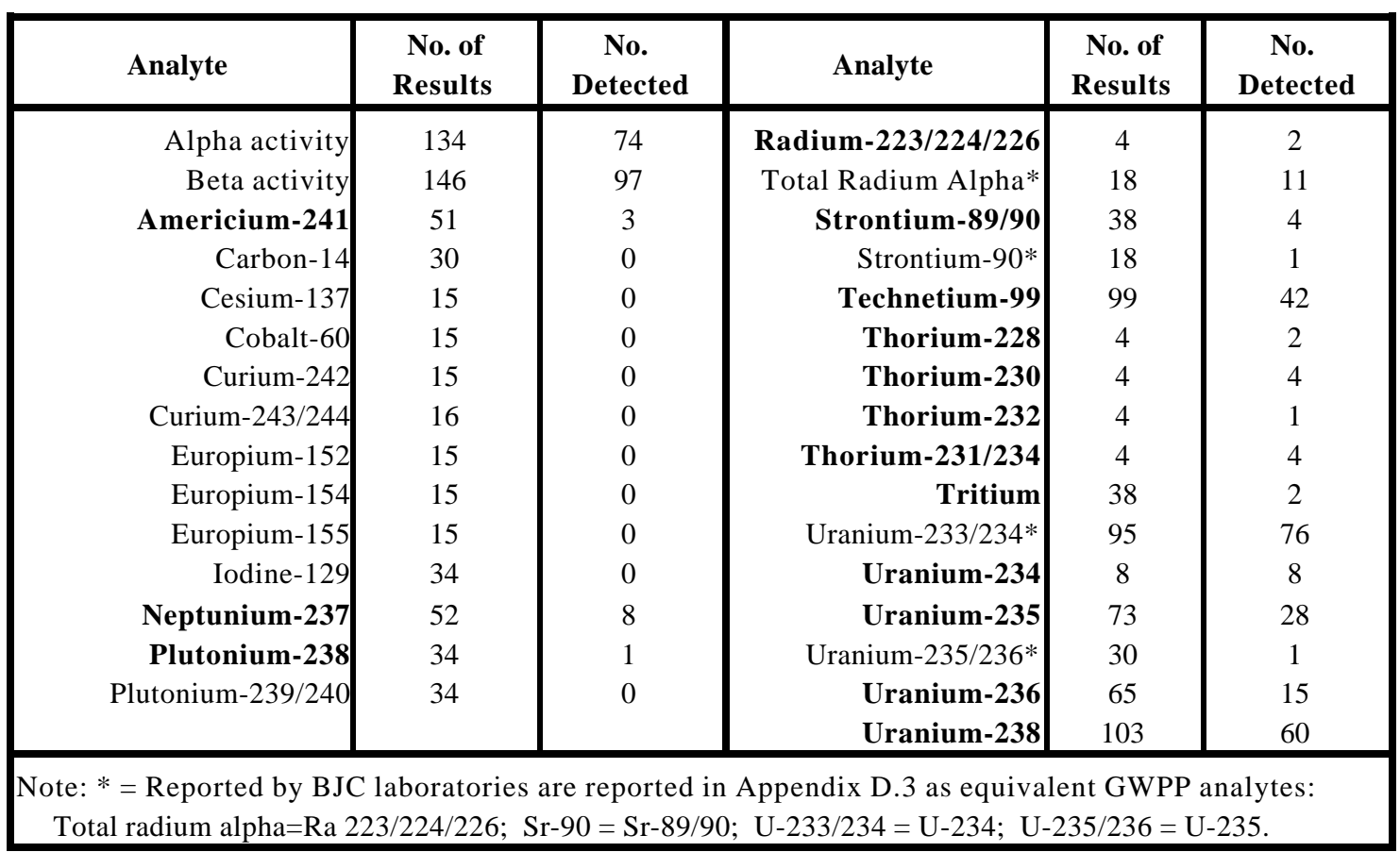

All of the results for gross alpha and gross beta are presented in the first three pages of Appendix D.3, followed by results for the isotopes detected in at least one sample (shown in bold typeface above) on pages D.3-5 through D.3-24.

The following notes and qualifiers apply to Appendix D.3:

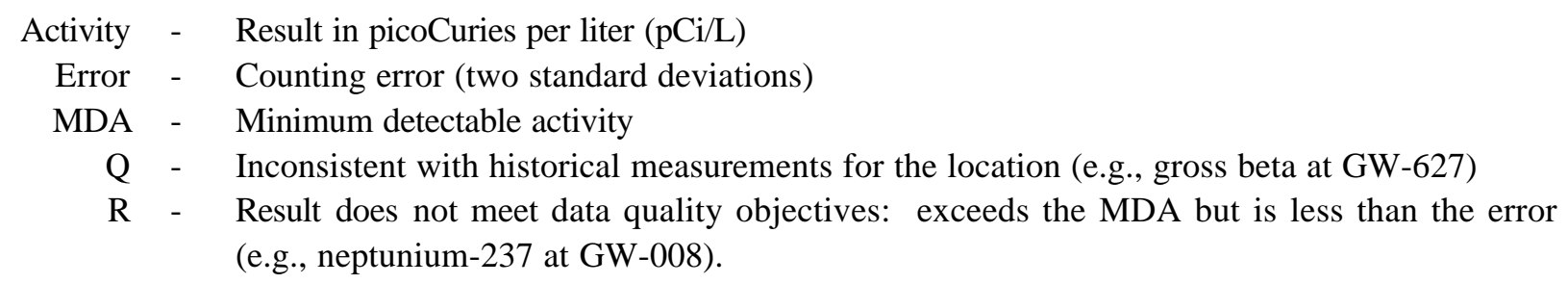




\section{EXPLANATION (continued)}

\section{Additional Analytes Not Presented in Appendix D tables:}

The Y-12 GWPP requested determination of the weight percent of uranium isotopes for the samples from the following sampling locations.

\begin{tabular}{|c|c|c|c|c|c|c|}
\hline \multirow{2}{*}{ Sampling Location } & \multirow{2}{*}{$\begin{array}{c}\text { Date } \\
\text { Sampled }\end{array}$} & \multirow{2}{*}{$\begin{array}{c}\text { Total Uranium } \\
(\mathbf{m g} / \mathbf{L})\end{array}$} & \multicolumn{4}{|c|}{ Weight Percent } \\
\cline { 4 - 7 } & & $\mathbf{U - 2 3 4}$ & $\mathbf{U - 2 3 5}$ & $\mathbf{U - 2 3 6}$ & U-238 \\
\hline BCK-11.97 & $01 / 09 / 02$ & 0.148 & 0.0025 & 0.386 & 0.023 & 99.59 \\
BCK-11.97 & $07 / 11 / 02$ & 0.14 & 0.002 & 0.414 & 0.004 & 99.58 \\
GW-243 & $03 / 21 / 02$ & 0.82 & 0.003 & 0.325 & 0.002 & 99.67 \\
GW-243 & $08 / 13 / 02$ & 0.76 & 0.002 & 0.317 & 0.006 & 99.68 \\
\hline
\end{tabular}

The WRRP also requested field measurement of iron and manganese metal species at well GW-835, associated with remedial actions at the S-3 Site.

\begin{tabular}{|c|c|c|c|}
\hline $\begin{array}{c}\text { Well } \\
\text { Number }\end{array}$ & $\begin{array}{c}\text { Date } \\
\text { Sampled }\end{array}$ & $\begin{array}{c}\text { Manganese ++ } \\
(\mathbf{m g} / \mathbf{L})\end{array}$ & $\begin{array}{c}\text { Iron ++ } \\
(\mathbf{m g} / \mathbf{L})\end{array}$ \\
\hline GW-835 & $03 / 04 / 02$ & 1.8 & 0.01 \\
& $05 / 20 / 02$ & 1.7 & 0.01 \\
& $08 / 22 / 02$ & 1.8 & 0 \\
& $11 / 19 / 02$ & 1.5 & 0.01 \\
\hline
\end{tabular}


APPENDIX D.1

FIELD MEASUREMENTS, MISCELLANEOUS ANALYTES, MAJOR IONS, AND TRACE METALS 
APPENDIX D.1: CY 2002 MONITORING DATA FOR THE BEAR CREEK HYDROGEOLOGIC REGIME

Field Measurements, Miscellaneous Analytes, Major Ions, and Trace Metals

\begin{tabular}{|c|c|c|c|c|c|c|c|c|c|}
\hline \multicolumn{2}{|l|}{ Sampling Point } & \multicolumn{2}{|c|}{ BCK-00.63 } & \multicolumn{2}{|c|}{ BCK-03.30 } & \multicolumn{2}{|c|}{ BCK-04.55 } & \multicolumn{2}{|c|}{ BCK-07.87 } \\
\hline Location & & \multicolumn{2}{|c|}{ EXP-SW } & \multicolumn{2}{|c|}{ EXP-SW } & \multicolumn{2}{|c|}{ EXP-SW } & \multicolumn{2}{|c|}{ EXP-SW } \\
\hline Date Sampled & & $01 / 08 / 02$ & $07 / 10 / 02$ & $03 / 12 / 02$ & 09/09/02 & 01/09/02 & $07 / 10 / 02$ & $01 / 08 / 02$ & 07/10/02 \\
\hline Program & & GWPP & GWPP & BJC & BJC & GWPP & GWPP & GWPP & GWPP \\
\hline \multicolumn{10}{|l|}{ Sample Type } \\
\hline Field Measurements & & & & & & & & & \\
\hline $\begin{array}{r}\text { Time Sampled } \\
\text { Measuring Point Elev. (ft) } \\
\text { Depth to Water (ft) } \\
\text { Groundwater Elevation (ft) }\end{array}$ & & $9: 30$ & $8: 15$ & $9: 56$ & $10: 41$ & $9: 45$ & $8: 45$ & $10: 00$ & 9:05 \\
\hline Conductivity $(\mu \mathrm{mho} / \mathrm{cm})$ & & 625 & 519 & 157 & 181 & 640 & 364 & 768 & 512 \\
\hline Dissolved Oxygen (ppm) & & 9.44 & 3.22 & 14.1 & 9.43 & 7.21 & 4.24 & 9.31 & 3.52 \\
\hline Oxidation/Reduction (mV) & & 249 & 220 & 155 & 170 & 249 & 177 & 239 & 166 \\
\hline Temperature (degrees C) & & 2.4 & 21.9 & 10.5 & 19.1 & 4.9 & 17.6 & 1.1 & 22.7 \\
\hline Turbidity (NTU) & & & & 8 & 28 & & & & \\
\hline $\mathrm{pH}$ & & 7.36 & 7.58 & 7.89 & 7.42 & 7.51 & 7.46 & 7.69 & 7.9 \\
\hline Miscellaneous Analytes & & & & & & & & & \\
\hline Conductivity $(\mu \mathrm{mho} / \mathrm{cm})$ & & 348 & 381 & & & 420 & 339 & 589 & 474 \\
\hline Dissolved Solids (mg/L) & & 208 & 255 & 184 & 214 & 230 & 190 & 367 & 295 \\
\hline Suspended Solids (mg/L) & & $<$ & $<$ & $<$ & $<$ & $<$ & 4 & 6 & $<$ \\
\hline Turbidity (NTU) & & 2.81 & 1.86 & & & 3.79 & 7.26 & 2.17 & 3.93 \\
\hline $\mathrm{pH}$ & & 8.02 & 8.02 & & & 7.96 & 7.8 & 8.03 & 8.22 \\
\hline Major lons (mg/L) & & & & & & & & & \\
\hline Calcium & & 52 & 49.3 & 44.6 & 47 & 61.9 & 42.4 & 90.6 & 61.2 \\
\hline Magnesium & & 13.3 & 17.5 & 11.8 & 17.2 & 13.7 & 18.4 & 16.3 & 19.9 \\
\hline Potassium & & $<$ & $<$ & 1.2 & 1.41 & $<$ & $<$ & $<$ & $<$ \\
\hline Sodium & & 4.8 & 3.25 & 4.33 & 3.01 & 6.34 & 2.05 & 11.1 & 7.4 \\
\hline Alkalinity as $\mathrm{CO} 3$ & & $<$ & $<$ & $<$ & $<$ & $<$ & $<$ & $<$ & $<$ \\
\hline Alkalinity as $\mathrm{HCO} 3$ & & 137 & 153 & 138 & 177 & 165 & 171 & 198 & 193 \\
\hline Chloride & & 6.26 & 3.17 & 7.8 & 5.6 & 9.52 & 3.55 & 17.9 & 15.1 \\
\hline Fluoride & & $<$ & 0.118 & $<$ & $<$ & 0.121 & $<$ & 0.24 & 0.172 \\
\hline Nitrate as $\mathrm{N}$ & & 2.82 & 0.176 & 4.1 & 0.44 & 6.69 & 0.234 & 17 & 3.52 \\
\hline Sulfate & & 23.9 & 39.3 & 10 & 7.4 & 13.5 & 4.11 & 20.9 & 13.4 \\
\hline Charge balance & (RPD) & 3.8 & 0.7 & -0.9 & 0.6 & 1.8 & 1.3 & 1.8 & 1.9 \\
\hline Trace Metals (mg/L) & & & & & & & & & \\
\hline Aluminum & & $<$ & $<$ & $<$ & 0.289 & 0.231 & 0.645 & $<$ & 0.319 \\
\hline Antimony & PMS & $<$ & $<$ & & & $<$ & $<$ & $<$ & $<$ \\
\hline Arsenic & PMS & $<$ & $<$ & & & $<$ & $<$ & $<$ & $<$ \\
\hline Barium & & 0.0577 & 0.065 & 0.0569 & 0.0661 & 0.067 & 0.0636 & 0.0971 & 0.0882 \\
\hline Beryllium & & $<$ & $<$ & $<$ & $<$ & $<$ & $<$ & $<$ & $<$ \\
\hline Boron & & $<$ & $<$ & $<$ & $<$ & $<$ & $<$ & 0.175 & 0.1 \\
\hline Cadmium & PMS & $<$ & $<$ & & & $<$ & $<$ & $<$ & $<$ \\
\hline Cadmium & & & & $<$ & $<$ & 1 & & & \\
\hline Chromium & PMS & $<$ & $<$ & & & $<$ & $<$ & $<$ & $<$ \\
\hline Chromium & & & & $<$ & $<$ & 1 & & & \\
\hline Cobalt & & $<$ & $<$ & $<$ & $<$ & $<$ & $<$ & $<$ & $<$ \\
\hline Copper & & $<$ & & $<$ & $<$ & $<$ & $<$ & $<$ & $<$ \\
\hline Iron & & 0.203 & 0.128 & 0.203 & 0.341 & 0.182 & 0.4 & 0.112 & 0.175 \\
\hline Lead & PMS & $<$ & 0.00111 & & & $<$ & 0.00259 & 0.00106 & 0.00128 \\
\hline Lead & & & & $<$ & $<$ & & & & \\
\hline Lithium & & $<$ & $<$ & $<$ & $<$ & 0.014 & $<$ & 0.0372 & 0.0144 \\
\hline Manganese & & 0.0295 & 0.0407 & 0.0169 & 0.0324 & 0.0168 & 0.0621 & 0.0248 & 0.0251 \\
\hline Mercury & CVAA & $<$ & $<$ & $<$ & $<$ & $<$ & $<$ & $<$ & $<$ \\
\hline Nickel & PMS & $<$ & $<$ & & & $<$ & $<$ & $<$ & $<$ \\
\hline Nickel & & & & $<$ & $<$ & & & & \\
\hline Selenium & PMS & $<$ & $<$ & & & $<$ & $<$ & $<$ & $<$ \\
\hline Strontium & & 0.138 & 0.175 & 0.084 & 0.0745 & 0.117 & 0.0518 & 0.208 & 0.138 \\
\hline Thallium & PMS & $<$ & $<$ & & & $<$ & $<$ & $<$ & $<$ \\
\hline Thallium & & & & $<$ & $<$ & & & & \\
\hline Uranium & PMS & 0.0242 & 0.0118 & & & 0.0568 & 0.00916 & 0.153 & 0.0693 \\
\hline Uranium & KPA & & & 0.0345 & 0.0173 & & & & \\
\hline Vanadium & & $<$ & $<$ & $<$ & $<$ & $<$ & $<$ & $<$ & $<$ \\
\hline Zinc & & $<$ & $<$ & $<$ & $<$ & $<$ & $<$ & $<$ & $<$ \\
\hline
\end{tabular}


APPENDIX D.1: CY 2002 MONITORING DATA FOR THE BEAR CREEK HYDROGEOLOGIC REGIME

Field Measurements, Miscellaneous Analytes, Major Ions, and Trace Metals

\begin{tabular}{|c|c|c|c|c|c|c|c|c|c|}
\hline \multicolumn{2}{|l|}{ Sampling Point } & \multicolumn{2}{|c|}{ BCK-09.20 } & \multicolumn{3}{|c|}{ BCK-09.40 } & \multirow{3}{*}{\begin{tabular}{|c|} 
BCK-09.47 \\
EXP-SW \\
$03 / 11 / 02$ \\
\end{tabular}} & \multicolumn{2}{|c|}{ BCK-11.54 } \\
\hline Location & & \multicolumn{2}{|c|}{ EXP-SW } & \multicolumn{3}{|c|}{ EXP-SW } & & \multicolumn{2}{|c|}{ EXP-SW } \\
\hline \multicolumn{2}{|l|}{ Date Sampled } & $03 / 11 / 02$ & $09 / 09 / 02$ & $01 / 09 / 02$ & \multicolumn{2}{|c|}{ 07/11/02 } & & $03 / 11 / 02$ & $09 / 09 / 02$ \\
\hline Program & & BJC & BJC & GWPP & GWPP & GWPP & BJC & BJC & BJC \\
\hline Sample Type & & & & & & Dup & & & \\
\hline Field Measurements & & & & & & & & & \\
\hline $\begin{array}{r}\text { Time Sampled } \\
\text { Measuring Point Elev. }(\mathrm{ft}) \\
\text { Depth to Water }(\mathrm{ft}) \\
\text { Groundwater Elevation }(\mathrm{ft})\end{array}$ & & $12: 59$ & $13: 14$ & $10: 15$ & $8: 30$ & $8: 30$ & $12: 44$ & $13: 12$ & $13: 42$ \\
\hline Conductivity $(\mu \mathrm{mho} / \mathrm{cm})$ & & 282 & 287 & 740 & 439 & 439 & 332 & 477 & 190 \\
\hline Dissolved Oxygen (ppm) & & 12.29 & 8.3 & 8.33 & 1.4 & 1.4 & 13.3 & 12.24 & 7.15 \\
\hline Oxidation/Reduction (mV) & & 155 & 155 & 230 & 215 & 215 & 165 & 155 & 115 \\
\hline Temperature (degrees C) & & 11.7 & 19 & 1.4 & 22.1 & 22.1 & 10 & 11.9 & 24.3 \\
\hline Turbidity (NTU) & & 2 & 29 & & & & 6 & 4 & 6 \\
\hline $\mathrm{pH}$ & & 8.34 & 6.38 & 7.48 & 7.27 & 7.27 & 8.5 & 8.11 & 7.33 \\
\hline $\begin{array}{r}\text { Miscellaneous Analytes } \\
\text { Conductivity ( } \mu \mathrm{mho} / \mathrm{cm}) \\
\text { Dissolved Solids (mg/L) } \\
\text { Suspended Solids (mg/L) } \\
\text { Turbidity (NTU) } \\
\mathrm{pH}\end{array}$ & & $\begin{array}{r}333 \\
<\end{array}$ & $\begin{array}{r}390 \\
<\end{array}$ & $\begin{array}{r}598 \\
376 \\
< \\
3.15 \\
8.03\end{array}$ & $\begin{array}{r}358 \\
226 \\
27 \\
34.8 \\
7.68 \\
\end{array}$ & $\begin{array}{r}357 \\
231 \\
26 \\
34 \\
7.75\end{array}$ & $\begin{array}{r}419 \\
<\end{array}$ & $\begin{array}{r}641 \\
<\end{array}$ & $\begin{array}{r}212 \\
6.4\end{array}$ \\
\hline Major lons (mg/L) & & & & & & & & & \\
\hline Calcium & & 77.8 & 74.3 & 93.1 & 55.7 & 56.5 & 87.2 & 140 & 42.7 \\
\hline Magnesium & & 17.2 & 20.2 & 14.4 & 7.01 & 7.02 & 15.5 & 19.8 & 24.6 \\
\hline Potassium & & 1.88 & 2.21 & 2.29 & 3.64 & 3.66 & 2.32 & 2.8 & 1.08 \\
\hline Sodium & & 9.78 & 9.09 & 11.1 & 5.38 & 5.36 & 12.3 & 18.3 & 0.68 \\
\hline Alkalinity as $\mathrm{CO} 3$ & & $<$ & $<$ & $<$ & $<$ & $<$ & $<$ & $<$ & $<$ \\
\hline Alkalinity as $\mathrm{HCO} 3$ & & 177 & 190 & 186 & 152 & 150 & 173 & 161 & 174 \\
\hline Chloride & & 19 & 19 & 19.7 & 16.2 & 15.7 & 25.5 & 32.1 & 1.6 \\
\hline Fluoride & & 0.14 & 0.18 & 0.282 & 0.237 & 0.239 & 0.17 & 0.32 & 0.18 \\
\hline Nitrate as $\mathrm{N}$ & & 15.9 & 9.9 & 19.4 & 0.042 & 0.0412 & 23.4 & 61.2 & 0.041 \\
\hline Sulfate & & 17.4 & 16.9 & 23.1 & 8.51 & 8.36 & 19.3 & 25.1 & 3.2 \\
\hline Charge balance & (RPD) & 1.7 & 3.7 & 1.7 & -0.1 & 1.3 & -0.3 & 2.4 & 7.8 \\
\hline Trace Metals (mg/L) & & & & & & & & & \\
\hline Aluminum & & $<$ & $<$ & $<$ & 2.97 & 3.72 & $<$ & $<$ & $<$ \\
\hline Antimony & PMS & & & $<$ & $<$ & $<$ & & & \\
\hline Arsenic & PMS & & & $<$ & $<$ & $<$ & & & \\
\hline Barium & & 0.101 & 0.103 & 0.104 & 0.107 & 0.111 & 0.116 & 0.219 & 0.148 \\
\hline Beryllium & & & $<$ & $<$ & $<$ & $<$ & $<$ & $<$ & $<$ \\
\hline Boron & & 0.152 & $<$ & 0.404 & 2.12 & 2.1 & 0.351 & $<$ & $<$ \\
\hline Cadmium & PMS & & & $<$ & $<$ & $<$ & & & \\
\hline Cadmium & & $<$ & $<$ & & & & $<$ & 0.0018 & $<$ \\
\hline Chromium & PMS & & & $<$ & $<$ & $<$ & & & \\
\hline Chromium & & $<$ & $<$ & & & & $<$ & $<$ & $<$ \\
\hline Cobalt & & $<$ & $<$ & $<$ & $<$ & $<$ & $<$ & $<$ & $<$ \\
\hline Copper & & & $<$ & & $<$ & $<$ & $<$ & $<$ & $<$ \\
\hline Iron & & 0.081 & 0.0906 & 0.157 & 1.6 & 2.04 & 0.145 & 0.0729 & 0.193 \\
\hline Lead & PMS & & & $<$ & 0.00136 & 0.00137 & & & \\
\hline Lead & & $<$ & $<$ & & & & $<$ & $<$ & $<$ \\
\hline Lithium & & 0.0282 & 0.0107 & 0.0676 & 0.235 & 0.235 & 0.062 & 0.0112 & $<$ \\
\hline Manganese & & 0.0221 & 0.0069 & 0.0825 & 0.52 & 0.577 & 0.0747 & 0.317 & 0.0512 \\
\hline Mercury & CVAA & $<$ & 0 & 0 & $<$ & $<$ & $0.01+$ & $<$ & 0.0012 \\
\hline Nickel & PMS & & & $<$ & $<$ & 0.00581 & & & \\
\hline Nickel & & $<$ & $<$ & & & & $<$ & 0.0107 & $<$ \\
\hline Selenium & PMS & & & & $<$ & $<$ & & & \\
\hline Strontium & & 0.182 & 0.16 & 0.226 & 0.142 & 0.142 & 0.235 & 0.38 & 0.0603 \\
\hline Thallium & PMS & & & & & $<$ & & & \\
\hline Thallium & & $<$ & $<$ & & & & $<$ & $<$ & $<$ \\
\hline Uranium & PMS & & & 0.204 & 0.154 & 0.18 & & & \\
\hline Uranium & KPA & 0.121 & 0.0625 & & & & 0.205 & 0.145 & 0.0146 \\
\hline Vanadium & & $<$ & $<$ & $<$ & $<$ & $<$ & $<$ & $<$ & $<$ \\
\hline Zinc & & $<$ & $<$ & $<$ & $<$ & $<$ & $<$ & $<$ & $<$ \\
\hline
\end{tabular}


APPENDIX D.1: CY 2002 MONITORING DATA FOR THE BEAR CREEK HYDROGEOLOGIC REGIME

Field Measurements, Miscellaneous Analytes, Major lons, and Trace Metals

\begin{tabular}{|c|c|c|c|c|c|c|c|c|c|}
\hline Sampling Point & & BCK-11.84 & \multicolumn{2}{|c|}{ BCK-11.97 } & \multicolumn{2}{|c|}{ GW-008 } & \multicolumn{3}{|c|}{ GW-014 } \\
\hline Location & & EXP-SW & \multicolumn{2}{|c|}{ EXP-SW } & \multicolumn{2}{|c|}{ OLF } & \multicolumn{3}{|c|}{ BG } \\
\hline Date Sampled & & $03 / 11 / 02$ & 01/09/02 & $07 / 11 / 02$ & $01 / 07 / 02$ & $07 / 02 / 02$ & \multicolumn{2}{|c|}{ 03/14/02 } & $08 / 12 / 02$ \\
\hline Program & & BJC & GWPP & GWPP & BJC & BJC & GWPP & GWPP & GWPP \\
\hline Sample Type & & & & & & & & Dup & \\
\hline Field Measurements & & & & & & & & & \\
\hline Time Sampled & & $13: 45$ & $10: 40$ & $9: 00$ & $13: 20$ & $9: 55$ & $8: 35$ & $8: 35$ & $8: 30$ \\
\hline Measuring Point Elev. (ft) & & & & & 965.39 & 965.39 & 934.50 & 934.50 & 934.50 \\
\hline Depth to Water (ft) & & & & & 15.70 & 17.56 & 7.21 & 7.21 & 9.15 \\
\hline Groundwater Elevation (ft) & & & & & 949.69 & 947.83 & 927.29 & 927.29 & 925.35 \\
\hline Conductivity ( $\mu \mathrm{mho} / \mathrm{cm})$ & & 636 & 1,631 & 2,810 & 131 & 190 & 665 & 665 & 514 \\
\hline Dissolved Oxygen (ppm) & & 13.54 & 6.78 & 3.41 & 0.81 & 1.1 & 5.14 & 5.14 & 4.49 \\
\hline Oxidation/Reduction (mV) & & 105 & 236 & 203 & 51 & 145 & 218 & 218 & 157 \\
\hline Temperature (degrees C) & & 9.6 & 1.7 & 21.6 & 13.5 & 17.4 & 11.4 & 11.4 & 19.6 \\
\hline Turbidity (NTU) & & 7 & & & 16 & 16 & & & \\
\hline $\mathrm{pH}$ & & 8.03 & 7.79 & 7.76 & 5.71 & 5.26 & 7.2 & 7.2 & 7.43 \\
\hline Miscellaneous Analytes & & & & & & & & & \\
\hline Conductivity $(\mu \mathrm{mho} / \mathrm{cm})$ & & & 1,348 & 2,680 & & & 640 & 647 & 641 \\
\hline Dissolved Solids (mg/L) & & 994 & $6,380 \mathrm{Q}$ & 2,030 & 99 & 75.8 & 372 & 371 & 375 \\
\hline Suspended Solids (mg/L) & & $<$ & $<$ & 4 & $<$ & $<$ & $<$ & $<$ & $<$ \\
\hline Turbidity (NTU) & & & 0.609 & 2.74 & & & 0.896 & 0.886 & 1.04 \\
\hline $\mathrm{pH}$ & & & 8.02 & 7.92 & & & 7.28 & 7.26 & 7.68 \\
\hline Major lons (mg/L) & & & & & & & & & \\
\hline Calcium & & 165 & 206 & 359 & 12 & 9.54 & 98.3 & 99.3 & 101 \\
\hline Magnesium & & 22.8 & 25.3 & 48.7 & 6.95 & 5.59 & 16 & 16.3 & 15.6 \\
\hline Potassium & & 3.11 & 3.74 & 9.19 & 1.05 & 1.04 & $<$ & $<$ & $<$ \\
\hline Sodium & & 25.6 & 32.5 & 70.7 & 2.45 & 2.37 & 9.79 & 9.91 & 9.7 \\
\hline Alkalinity as $\mathrm{CO} 3$ & & $<$ & $<$ & $<$ & $<$ & $<$ & $<$ & $<$ & $<$ \\
\hline Alkalinity as $\mathrm{HCO} 3$ & & 181 & 258 & 153 & 40 & 37.6 & 302 & 316 & 308 \\
\hline Chloride & & 48.6 & 45.3 & 78.6 & 8.6 & 11.6 & 16.6 & 16.3 & 16.5 \\
\hline Fluoride & & 0.48 & 0.916 & 0.959 & $<$ & $<$ & $<$ & $<$ & $<$ \\
\hline Nitrate as $\mathrm{N}$ & & 88.8 & 83.8 & 257 & 0.11 & 0.89 & $<$ & $<$ & $<$ \\
\hline Sulfate & & 29.3 & 38.6 & 40.7 & 1.9 & 2.6 & 7.01 & 7.3 & 5.47 \\
\hline Charge balance & (RPD) & -2.9 & 2.2 & 1.4 & 9 & -5.5 & 0 & -1.5 & 0.1 \\
\hline Trace Metals (mg/L) & & & & & & & & & \\
\hline Aluminum & & $<$ & 0.27 & $<$ & $<$ & $<$ & $<$ & $<$ & $<$ \\
\hline Antimony & PMS & & $<$ & $<$ & & & $<$ & $<$ & $<$ \\
\hline Arsenic & PMS & & $<$ & $<$ & & & $<$ & $<$ & $<$ \\
\hline Barium & & 0.297 & 0.297 & 0.612 & 0.0754 & 0.0785 & 0.548 & 0.558 & 0.56 \\
\hline Beryllium & & $<$ & $<$ & $<$ & $<$ & $<$ & $<$ & $<$ & $<$ \\
\hline Boron & & $<$ & $<$ & $<$ & $<$ & $<$ & 3.8 & 3.86 & 3.96 \\
\hline Cadmium & PMS & & 0.012 & 0.000573 & & . & $<$ & $<$ & 0.000531 \\
\hline Cadmium & & 0.0049 & & & $<$ & $<$ & & & \\
\hline Chromium & PMS & & $<$ & $<$ & & . & 0.0359 & 0.0315 & $<$ \\
\hline Chromium & & $<$ & & & $<$ & $<$ & & & \\
\hline Cobalt & & $<$ & $<$ & $<$ & $<$ & $<$ & $<$ & $<$ & $<$ \\
\hline Copper & & $<$ & $<$ & $<$ & $<$ & $<$ & $<$ & $<$ & $<$ \\
\hline Iron & & 0.0556 & 0.0935 & 0.126 & 1.79 & 1.86 & 0.054 & $<$ & $<$ \\
\hline Lead & PMS & & $<$ & 0.00122 & & & 0.00355 & 0.00294 & 0.00158 \\
\hline Lead & & $<$ & & & $<$ & $<$ & & & \\
\hline Lithium & & $<$ & $<$ & $<$ & $<$ & $<$ & 0.0254 & 0.0269 & 0.0266 \\
\hline Manganese & & 0.874 & 2.09 & 0.0433 & 1.48 & 1.33 & 0.149 & 0.163 & 0.278 \\
\hline Mercury & CVAA & $<$ & $<$ & $<$ & $<$ & $<$ & $<$ & $<$ & $<$ \\
\hline Nickel & PMS & & 0.057 & 0.00985 & & & 0.0117 & 0.0101 & 0.0194 \\
\hline Nickel & & 0.023 & & & 0.0217 & 0.0237 & & & \\
\hline Selenium & PMS & & 0.015 & $<$ & & & $<$ & $<$ & $<$ \\
\hline Strontium & & 0.514 & 0.54 & 0.999 & 0.0257 & 0.0237 & 0.347 & 0.353 & 0.337 \\
\hline Thallium & PMS & & $<$ & & & & & $<$ & $<$ \\
\hline Thallium & & $<$ & & & $<$ & $<$ & & & \\
\hline Uranium & PMS & & 0.151 & 0.14 & & & 0.00384 & 0.00365 & 0.00247 \\
\hline Uranium & KPA & 0.109 & & & $<$ & $<$ & & & \\
\hline Vanadium & & $<$ & $<$ & $<$ & $<$ & $<$ & $<$ & $<$ & $<$ \\
\hline Zinc & & $<$ & $<$ & $<$ & $<$ & $<$ & $<$ & $<$ & $<$ \\
\hline
\end{tabular}


APPENDIX D.1: CY 2002 MONITORING DATA FOR THE BEAR CREEK HYDROGEOLOGIC REGIME

Field Measurements, Miscellaneous Analytes, Major lons, and Trace Metals

\begin{tabular}{|c|c|c|c|c|c|c|c|c|c|c|}
\hline \multicolumn{2}{|l|}{ Sampling Point } & \multicolumn{2}{|c|}{ GW-046 } & \multicolumn{2}{|c|}{ GW-061 } & \multicolumn{3}{|c|}{ GW-066 } & \multicolumn{2}{|c|}{ GW-069 } \\
\hline Location & & \multicolumn{2}{|c|}{ BG } & \multicolumn{2}{|c|}{ BG } & \multicolumn{3}{|c|}{ OLF } & \multicolumn{2}{|c|}{ BG } \\
\hline \multicolumn{2}{|l|}{ Date Sampled } & 01/07/02 & 07/02/02 & 03/12/02 & 08/05/02 & $03 / 25 / 02$ & \multicolumn{2}{|c|}{$08 / 13 / 02$} & 03/11/02 & $08 / 05 / 02$ \\
\hline Program & & BJC & BJC & GWPP & GWPP & GWPP & GWPP & GWPP & GWPP & GWPP \\
\hline Sample Type & & & & & & & & Dup & & \\
\hline Field Measurements & & & & & & & & & & \\
\hline Time Sampled & & $14: 40$ & $9: 30$ & $10: 45$ & $10: 20$ & $10: 10$ & $7: 50$ & $7: 50$ & $10: 15$ & 9:00 \\
\hline Measuring Point Elev. (ft) & & 921.17 & 921.17 & 904.60 & 904.60 & 961.60 & 961.60 & 961.60 & 927.60 & 927.60 \\
\hline Depth to Water (ft) & & 5.26 & 5.17 & 16.71 & 18.17 & 7.34 & 9.98 & 9.98 & 10.34 & 11.96 \\
\hline Groundwater Elevation (ft) & & 915.91 & 916.00 & 887.89 & 886.43 & 954.26 & 951.62 & 951.62 & 917.26 & 915.64 \\
\hline Conductivity $(\mu \mathrm{mho} / \mathrm{cm})$ & & 197 & 217 & 702 & 666 & 864 & 749 & 749 & 477 & 465 \\
\hline Dissolved Oxygen (ppm) & & 0.67 & 2.12 & 1.95 & 0.31 & 2.26 & 1.19 & 1.19 & 0.45 & 1.69 \\
\hline Oxidation/Reduction (mV) & & 143 & 223 & 104 & 130 & 55 & -49 & -49 & -233 & -268 \\
\hline Temperature (degrees C) & & 11.5 & 18.3 & 12 & 14.2 & 14.8 & 16.4 & 16.4 & 13.1 & 18.4 \\
\hline Turbidity (NTU) & & 39 & 34 & & & & & & & \\
\hline $\mathrm{pH}$ & & 5.43 & 5.39 & 7.02 & 7.08 & 7.31 & 7.38 & 7.38 & 9.53 & 9.07 \\
\hline Miscellaneous Analytes & & & & & & & & & & \\
\hline Conductivity $(\mu \mathrm{mho} / \mathrm{cm})$ & & & & 640 & 634 & 864 & 754 & 764 & 434 & 446 \\
\hline Dissolved Solids (mg/L) & & 126 & 132 & 400 & 385 & 515 & 480 & 516 & 238 & 251 \\
\hline Suspended Solids (mg/L) & & $<$ & $<$ & 42 & 248 & $<$ & $<$ & $<$ & $<$ & $<$ \\
\hline Turbidity (NTU) & & & & 70 & 271 & 3.3 & 1.97 & 1.98 & 1.28 & 0.923 \\
\hline $\mathrm{pH}$ & & & & 7.22 & 7.73 & 7.18 & 7.28 & 7.16 & 8.87 & 8.86 \\
\hline Major lons (mg/L) & & & & & & & & & & \\
\hline Calcium & & 18.5 & 18.6 & 95.8 & 104 & 128 & 97.8 & 92 & 8.19 & 9.38 \\
\hline Magnesium & & 5.35 & 4.74 & 16.2 & 19.3 & 28.8 & 30.1 & 30.9 & 6.48 & 6.44 \\
\hline Potassium & & 3.99 & 3.15 & 3.62 & 6.96 & 3.25 & 5.01 & 5.2 & 7.67 & 6.93 \\
\hline Sodium & & 4.65 & 5.63 & 9.08 & 9 & 11 & 8.63 & 9.11 & 74.3 & 73.6 \\
\hline Alkalinity as $\mathrm{CO} 3$ & & $<$ & $<$ & $<$ & $<$ & $<$ & $<$ & $<$ & 17.2 & 19.9 \\
\hline Alkalinity as $\mathrm{HCO} 3$ & & 40 & 45.5 & 224 & 240 & 306 & 270 & 280 & 121 & 126 \\
\hline Chloride & & 22.1 & 20.4 & 19.6 & 18.8 & 9.79 & 7.93 & 8.15 & 51.5 & 48.2 \\
\hline Fluoride & & $<$ & $<$ & 0.129 & 0.155 & $<$ & $<$ & $<$ & 0.107 & $<$ \\
\hline Nitrate as $\mathrm{N}$ & & 0.034 & 1.2 & 13.2 & 8.73 & 2.77 & 2.47 & 2.71 & $<$ & 0.0305 \\
\hline Sulfate & & 11.9 & 6.4 & 28.5 & 37.2 & 120 & 108 & 103 & 1.95 & 1.99 \\
\hline Charge balance & (RPD) & -0.1 & -1.7 & 0.2 & 4.4 & 1.2 & -1.2 & -3.2 & 0.9 & 0.5 \\
\hline Trace Metals (mg/L) & & & & & & & & & & \\
\hline Aluminum & & $<$ & $<$ & 7.16 & 22.3 & 0.292 & $<$ & $<$ & $<$ & $<$ \\
\hline Antimony & PMS & & & $<$ & $<$ & $<$ & $<$ & $<$ & $<$ & $<$ \\
\hline Arsenic & PMS & & & $<$ & $<$ & $<$ & $<$ & $<$ & 0.00654 & $<$ \\
\hline Barium & & 0.164 & 0.167 & 0.117 & 0.218 & 0.14 & 0.11 & 0.111 & 0.392 & 0.386 \\
\hline Beryllium & & $<$ & $<$ & $<$ & 0.00107 & $<$ & $<$ & $<$ & $<$ & $<$ \\
\hline Boron & & $<$ & $<$ & $<$ & $<$ & $<$ & $<$ & $<$ & 0.11 & 0.11 \\
\hline Cadmium & PMS & & & $<$ & $<$ & $<$ & $<$ & $<$ & 0.00297 & 0.00063 \\
\hline Cadmium & & $<$ & $<$ & & & d & 1 & & & \\
\hline Chromium & PMS & & & 0.00364 & 0.00478 & $<$ & $<$ & $<$ & 0.0117 & 0.00358 \\
\hline Chromium & & $<$ & $<$ & & & & & & & \\
\hline Cobalt & & 0.0239 & $<$ & $<$ & $<$ & $<$ & $<$ & $<$ & $<$ & $<$ \\
\hline Copper & & $<$ & $<$ & $<$ & $<$ & $<$ & $<$ & $<$ & $<$ & $<$ \\
\hline Iron & & 3.14 & 0.423 & 4.27 & 17.8 & 0.119 & 0.0973 & 0.161 & 0.0666 & 0.0769 \\
\hline Lead & PMS & & & 0.00969 & 0.0137 & 0.00268 & 0.000586 & 0.000963 & 0.000965 & 0.00117 \\
\hline Lead & & & $<$ & & & & & & & \\
\hline Lithium & & 0.0528 & 0.137 & 0.011 & 0.0273 & 0.012 & 0.012 & 0.0117 & 0.0439 & 0.0427 \\
\hline Manganese & & 1.67 & 1.22 & 0.326 & 1.55 & 0.0136 & 0.00766 & 0.00901 & $<$ & $<$ \\
\hline Mercury & CVAA & $<$ & $<$ & $<$ & $<$ & $<$ & $<$ & $<$ & $<$ & $<$ \\
\hline Nickel & PMS & & & 0.00748 & 0.0164 & 0.0119 & 0.0211 & 0.0304 & 0.00608 & $<$ \\
\hline Nickel & & 0.0174 & 0.0153 & & & & & & & \\
\hline Selenium & PMS & & & $<$ & $<$ & $<$ & $<$ & $<$ & 0.0266 & 0.0143 \\
\hline Strontium & & 0.0704 & 0.07 & 0.187 & 0.199 & 0.298 & 0.296 & 0.315 & 0.995 & 0.967 \\
\hline Thallium & PMS & & & $<$ & $<$ & $<$ & 0.00106 & 0.00116 & $<$ & $<$ \\
\hline Thallium & & $<$ & $<$ & & & & & & & \\
\hline Uranium & PMS & . & & 0.0642 & 0.0581 & 0.00719 & 0.00969 & 0.0109 & $<$ & $<$ \\
\hline Uranium & KPA & $<$ & $<$ & & & & & & & \\
\hline Vanadium & & $<$ & $<$ & $<$ & 0.0254 & $<$ & $<$ & $<$ & $<$ & $<$ \\
\hline Zinc & & $<$ & $<$ & $<$ & 0.0575 & $<$ & $<$ & $<$ & $<$ & 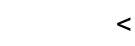 \\
\hline
\end{tabular}


APPENDIX D.1: CY 2002 MONITORING DATA FOR THE BEAR CREEK HYDROGEOLOGIC REGIME

Field Measurements, Miscellaneous Analytes, Major Ions, and Trace Metals

\begin{tabular}{|c|c|c|c|c|c|c|c|c|c|}
\hline \multicolumn{2}{|l|}{ Sampling Point } & \multicolumn{3}{|c|}{ GW-071 } & \multicolumn{2}{|c|}{ GW-072 } & \multirow{3}{*}{$\begin{array}{c}\text { GW-076 } \\
\text { OLF } \\
03 / 19 / 02\end{array}$} & \multicolumn{2}{|c|}{ GW-077 } \\
\hline Location & & \multicolumn{3}{|c|}{ BG } & \multicolumn{2}{|c|}{ BG } & & \multicolumn{2}{|c|}{ BG } \\
\hline Date Sampled & & $03 / 12 / 02$ & \multicolumn{2}{|c|}{$08 / 06 / 02$} & $03 / 11 / 02$ & $08 / 06 / 02$ & & $02 / 26 / 02$ & 08/08/02 \\
\hline Program & & GWPP & GWPP & GWPP & GWPP & GWPP & GWPP & BJC & BJC \\
\hline Sample Type & & & & Dup & & & & & \\
\hline Field Measurements & & & & & & & & & \\
\hline Time Sampled & & $9: 10$ & $10: 00$ & $10: 00$ & $8: 40$ & $8: 25$ & $9: 20$ & $13: 10$ & $11: 15$ \\
\hline Measuring Point Elev. (ft) & & 928.90 & 928.90 & 928.90 & 930.51 & 930.51 & 967.06 & 919.30 & 919.30 \\
\hline Depth to Water (ft) & & 8.42 & 10.12 & 10.12 & 11.48 & 13.61 & 4.06 & 9.35 & 11.97 \\
\hline Groundwater Elevation (ft) & & 920.48 & 918.78 & 918.78 & 919.03 & 916.90 & 963.00 & 909.95 & 907.33 \\
\hline Conductivity $(\mu \mathrm{mho} / \mathrm{cm})$ & & 2,210 & 2,070 & 2,070 & 640 & 601 & 414 & 353 & 411 \\
\hline Dissolved Oxygen (ppm) & & 0.13 & 0.78 & 0.78 & 0.77 & 1.38 & 0.31 & 1.25 & 3.11 \\
\hline Oxidation/Reduction (mV) & & -373 & -385 & -385 & -328 & -285 & -242 & 52 & -20 \\
\hline Temperature (degrees C) & & 11.4 & 19.4 & 19.4 & 11.8 & 18.5 & 14.1 & 11.3 & 17.6 \\
\hline Turbidity (NTU) & & & & & & & & 28 & 8 \\
\hline $\mathrm{pH}$ & & 11.4 & 11.13 & 11.13 & 9.98 & 9.62 & 8.47 & 7.48 & 6.84 \\
\hline Miscellaneous Analytes & & & & & & & & & \\
\hline Conductivity $(\mu \mathrm{mho} / \mathrm{cm})$ & & 1,997 & 1,951 & 1,953 & 561 & 556 & 393 & & \\
\hline Dissolved Solids (mg/L) & & 969 & 970 & 973 & 299 & 309 & 224 & 214 & 220 \\
\hline Suspended Solids (mg/L) & & 4 & 4 & 2 & $<$ & $<$ & 2 & $<$ & 6.9 \\
\hline Turbidity (NTU) & & 4.52 & 5.65 & 5.5 & 2.2 & 5.05 & 0.795 & & \\
\hline $\mathrm{pH}$ & & 11.21 & 11.13 & 11.1 & 9.99 & 9.83 & 8.16 & & \\
\hline Major lons (mg/L) & & & & & & & & & \\
\hline Calcium & & 3.82 & 2.49 & 3.52 & 1.56 & 2.24 & 21.2 & 49.7 & 51.2 \\
\hline Magnesium & & $<$ & $<$ & $<$ & 0.527 & 0.971 & 10.8 & 11.7 & 11.5 \\
\hline Potassium & & 2.32 & 2.38 & 2.14 & 2.58 & 2.18 & 6.84 & 2.37 & 2.45 \\
\hline Sodium & & 403 & 401 & 387 & 122 & 119 & 42.2 & 6.75 & 6.38 \\
\hline Alkalinity as $\mathrm{CO} 3$ & & 712 & 784 & 776 & 138 & 115 & $<$ & $<$ & $<$ \\
\hline Alkalinity as $\mathrm{HCO} 3$ & & $<$ & $<$ & $<$ & 90 & 121 & 174 & 173 & 175 \\
\hline Chloride & & 21.3 & 37.3 & 35.7 & 24.3 & 52.9 & 13.5 & 1.4 & 1.3 \\
\hline Fluoride & & 6.02 & 6 & 6.08 & 0.336 & 0.354 & 0.254 & $<$ & 0.13 \\
\hline Nitrate as $\mathrm{N}$ & & 0.0347 & $<$ & $<$ & $<$ & 0.0439 & $<$ & 0.058 & 0.18 \\
\hline Sulfate & & 12.5 & 10.9 & 10.1 & 3.88 & 1.74 & 5.25 & 11.4 & 10.9 \\
\hline Charge balance & (RPD) & -0.4 & -2.7 & -3.7 & 0.5 & -7.5 & -0.3 & 0.8 & 0.8 \\
\hline Trace Metals (mg/L) & & & & & & & & & \\
\hline Aluminum & & 1.54 & 1.37 & 1.32 & $<$ & 0.463 & $<$ & $<$ & $<$ \\
\hline Antimony & PMS & $<$ & $<$ & $<$ & $<$ & $<$ & $<$ & 1 & \\
\hline Arsenic & PMS & 0.0139 & 0.0103 & 0.0108 & $<$ & $<$ & $<$ & & \\
\hline Barium & & 0.0687 & 0.068 & 0.0718 & 0.176 & 0.208 & 0.329 & 0.415 & 0.438 \\
\hline Beryllium & & & & $<$ & $<$ & $<$ & $<$ & $<$ & $<$ \\
\hline Boron & & 0.819 & 0.813 & 0.786 & 0.293 & 0.294 & $<$ & $<$ & $<$ \\
\hline Cadmium & PMS & $<$ & $<$ & $<$ & $<$ & 0.00136 & $<$ & t & \\
\hline Cadmium & & & & & & & & $<$ & $<$ \\
\hline Chromium & PMS & $<$ & $<$ & $<$ & $<$ & $<$ & $<$ &. & \\
\hline Chromium & & & & & & & & $<$ & $<$ \\
\hline Cobalt & & $<$ & $<$ & $<$ & $<$ & $<$ & $<$ & $<$ & $<$ \\
\hline Copper & & $<$ & $<$ & $<$ & $<$ & $<$ & $<$ & $<$ & $<$ \\
\hline Iron & & $<$ & $<$ & $<$ & $<$ & 0.346 & $<$ & 0.105 & 0.104 \\
\hline Lead & PMS & 0.00141 & 0.000703 & 0.00103 & 0.000562 & 0.00694 & 0.00102 & & \\
\hline Lead & & & & & & & & $<$ & $<$ \\
\hline Lithium & & 0.109 & 0.116 & 0.111 & 0.0606 & 0.0622 & 0.0316 & 0.0106 & 0.0116 \\
\hline Manganese & & $<$ & $<$ & $<$ & $<$ & 0.0129 & $<$ & 0.063 & 0.0721 \\
\hline Mercury & CVAA & $<$ & $<$ & $<$ & $<$ & $<$ & $<$ & & \\
\hline Nickel & PMS & $<$ & $<$ & $<$ & $<$ & $<$ & $<$ & . & \\
\hline Nickel & & & & & & & & $<$ & $<$ \\
\hline Selenium & PMS & 0.0158 & $<$ & $<$ & 0.0188 & 0.0121 & $<$ & & \\
\hline Strontium & & 0.248 & 0.221 & 0.237 & 0.321 & 0.347 & 0.866 & 1.24 & 1.24 \\
\hline Thallium & PMS & $<$ & $<$ & $<$ & $<$ & $<$ & $<$ & . & \\
\hline Thallium & & & & & & & & $<$ & $<$ \\
\hline Uranium & PMS & $<$ & $<$ & $<$ & $<$ & $<$ & $<$ &. & . \\
\hline Uranium & KPA & & & & & & & $<$ & $<$ \\
\hline Vanadium & & $<$ & $<$ & $<$ & $<$ & $<$ & $<$ & $<$ & $<$ \\
\hline Zinc & & $<$ & $<$ & $<$ & $<$ & $<$ & $<$ & $<$ & $<$ \\
\hline
\end{tabular}


APPENDIX D.1: CY 2002 MONITORING DATA FOR THE BEAR CREEK HYDROGEOLOGIC REGIME

Field Measurements, Miscellaneous Analytes, Major Ions, and Trace Metals

\begin{tabular}{|c|c|c|c|c|c|c|c|c|c|}
\hline Sampling Point & & GW & 078 & GW & 079 & & GW & & \\
\hline Location & & B & & B & & & B & & \\
\hline Date Sampled & & $02 / 26 / 02$ & $08 / 08 / 02$ & $02 / 26 / 02$ & $08 / 07 / 02$ & $02 / 2$ & & $08 / 0$ & \\
\hline Program & & BJC & BJC & BJC & BJC & BJC & BJC & BJC & BJC \\
\hline Sample Type & & & & & & & Dup & & Dup \\
\hline Field Measurements & & & & & & & & & \\
\hline Time Sampled & & $12: 41$ & $9: 21$ & $14: 35$ & $13: 15$ & $13: 42$ & & $9: 15$ & \\
\hline Measuring Point Elev. (ft) & & 918.10 & 918.10 & 981.20 & 981.20 & 981.00 & & 981.00 & \\
\hline Depth to Water (ft) & & 8.21 & 10.42 & 21.30 & 24.16 & 23.60 & & & \\
\hline Groundwater Elevation (ft) & & 909.89 & 907.68 & 959.90 & 957.04 & 957.40 & & & \\
\hline Conductivity $(\mu \mathrm{mho} / \mathrm{cm})$ & & 373 & 408 & 232 & 345 & 182 & & 236 & \\
\hline Dissolved Oxygen (ppm) & & 3.01 & 1.17 & 1.23 & 1.5 & 6.21 & & 0.5 & \\
\hline Oxidation/Reduction (mV) & & 229 & 189 & -61 & -112 & 69 & & -54 & \\
\hline Temperature (degrees C) & & 13.6 & 15.4 & 12.5 & 20.9 & 12.4 & & 16.1 & \\
\hline Turbidity (NTU) & & 18 & 6 & 10 & 21 & 32 & & 28 & \\
\hline $\mathrm{pH}$ & & 8.05 & 6.79 & 7.25 & 7.19 & 8.21 & & 6.35 & \\
\hline $\begin{array}{r}\text { Miscellaneous Analytes } \\
\text { Conductivity }(\mu \mathrm{mho} / \mathrm{cm}) \\
\text { Dissolved Solids }(\mathrm{mg} / \mathrm{L}) \\
\text { Suspended Solids }(\mathrm{mg} / \mathrm{L}) \\
\text { Turbidity (NTU) } \\
\mathrm{pH}\end{array}$ & & $\begin{array}{r}249 \\
<\end{array}$ & $\begin{array}{r}239 \\
<\end{array}$ & $\begin{array}{r}151 \\
<\end{array}$ & $\begin{array}{r}199 \\
5.8\end{array}$ & $\begin{array}{r}117 \\
9\end{array}$ & $\begin{array}{r}97 \\
9\end{array}$ & $\begin{array}{r}102 \\
11.9\end{array}$ & $\begin{array}{r}100 \\
14.9\end{array}$ \\
\hline Major lons (mg/L) & & & & & & & & & \\
\hline Calcium & & 62.9 & 62.9 & 35.6 & 51.6 & 6.25 & 5.96 & 6.36 & 6.28 \\
\hline Magnesium & & 8.13 & 7.65 & 3.85 & 5.53 & 6.82 & 6.52 & 7.02 & 6.92 \\
\hline Potassium & & 1.24 & 1.36 & 1.12 & 2.65 & 1.49 & 1.43 & 1.84 & 1.67 \\
\hline Sodium & & 4.46 & 4.69 & 4.26 & 5.57 & 14.3 & 13.8 & 13 & 12.6 \\
\hline Alkalinity as $\mathrm{CO} 3$ & & $<$ & $<$ & $<$ & $<$ & $<$ & $<$ & $<$ & $<$ \\
\hline Alkalinity as $\mathrm{HCO} 3$ & & 185 & 178 & 106 & 174 & 73 & 74 & 73 & 70 \\
\hline Chloride & & 1.8 & 1.5 & 1 & 1.5 & 0.95 & 1 & 1.3 & 1.2 \\
\hline Fluoride & & $<$ & 0.16 & $<$ & $<$ & 0.13 & 0.13 & 0.17 & 0.16 \\
\hline Nitrate as $\mathrm{N}$ & & 0.071 & 0.25 & 0.04 & $<$ & 0.076 & 0.075 & 0.087 & 0.099 \\
\hline Sulfate & & 15.5 & 11.7 & 7.3 & 1.7 & 12.2 & 11.7 & 13.1 & 13.1 \\
\hline Charge balance & (RPD) & -0.5 & 1.7 & 0.1 & -3.1 & -6.7 & & -8.4 & \\
\hline Trace Metals (mg/L) & & & & & & & & & \\
\hline Aluminum & & $<$ & $<$ & $<$ & $<$ & $<$ & $<$ & $<$ & $<$ \\
\hline Antimony & PMS & & & & & & & & \\
\hline Arsenic & PMS & & & & & & & & \\
\hline Barium & & 0.165 & 0.171 & 0.209 & 0.47 & 0.0286 & 0.0276 & 0.0305 & 0.0295 \\
\hline Beryllium & & $<$ & $<$ & $<$ & $<$ & $<$ & $<$ & $<$ & $<$ \\
\hline Boron & & $<$ & $<$ & $<$ & $<$ & $<$ & $<$ & $<$ & $<$ \\
\hline Cadmium & PMS & & &. & $\cdot$ &. & 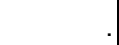 &. & . \\
\hline Cadmium & & $<$ & $<$ & $<$ & $<$ & $<$ & $<$ & $<$ & $<$ \\
\hline Chromium & PMS & & & .1 & & & & .1 & . \\
\hline Chromium & & $<$ & $<$ & $<$ & $<$ & $<$ & $<$ & $<$ & $<$ \\
\hline Cobalt & & $<$ & $<$ & $<$ & $<$ & $<$ & $<$ & $<$ & $<$ \\
\hline Copper & & & $<$ & & $<$ & $<$ & $<$ & $<$ & $<$ \\
\hline Iron & & 0.062 & 0.0608 & 0.199 & 1.58 & 5.88 & 6.2 & 7.15 & 7.05 \\
\hline Lead & PMS & & & & & & & & \\
\hline Lead & & $<$ & $<$ & $<$ & 0.0038 & $<$ & $<$ & 0.004 & $<$ \\
\hline Lithium & & 0.0109 & 0.0112 & $<$ & $<$ & 0.0121 & 0.0118 & 0.0112 & 0.0112 \\
\hline Manganese & & 0.009 & 0.0257 & 0.0824 & 0.232 & 0.239 & 0.239 & 0.262 & 0.259 \\
\hline Mercury & CVAA & & & & & & & & \\
\hline Nickel & PMS & & & & & & & & \\
\hline Nickel & & $<$ & $<$ & $<$ & $<$ & $<$ & $<$ & $<$ & $<$ \\
\hline Selenium & PMS & & & & & & & & \\
\hline Strontium & & 0.124 & 0.123 & 0.128 & 0.207 & 0.0301 & 0.0292 & 0.0307 & 0.0303 \\
\hline Thallium & PMS & & & & & & & & \\
\hline Thallium & & $<$ & $<$ & $<$ & $<$ & $<$ & $<$ & $<$ & $<$ \\
\hline Uranium & PMS & & & & & & & & \\
\hline Uranium & KPA & $<$ & $<$ & $<$ & $<$ & $<$ & $<$ & $<$ & $<$ \\
\hline Vanadium & & $<$ & $<$ & $<$ & $<$ & $<$ & $<$ & $<$ & $<$ \\
\hline Zinc & & $<$ & $<$ & $<$ & $<$ & $<$ & $<$ & $<$ & $<$ \\
\hline
\end{tabular}


APPENDIX D.1: CY 2002 MONITORING DATA FOR THE BEAR CREEK HYDROGEOLOGIC REGIME

Field Measurements, Miscellaneous Analytes, Major lons, and Trace Metals

\begin{tabular}{|c|c|c|c|c|c|c|c|c|c|c|}
\hline \multirow{2}{*}{\multicolumn{2}{|c|}{$\begin{array}{c}\text { Sampling Point } \\
\text { Location }\end{array}$}} & \multirow{2}{*}{\multicolumn{2}{|c|}{$\frac{\text { GW-082 }}{\text { BG }}$}} & \multirow{2}{*}{\multicolumn{2}{|c|}{$\begin{array}{c}\text { GW-085 } \\
\text { OLF }\end{array}$}} & \multirow{3}{*}{\begin{tabular}{c|} 
GW-087 \\
OLF \\
$03 / 25 / 02$
\end{tabular}} & \multicolumn{2}{|c|}{ GW-091 } & \multirow{2}{*}{\multicolumn{2}{|c|}{$\begin{array}{c}\text { GW-115 } \\
\text { S3 }\end{array}$}} \\
\hline & & & & & & & \multirow{2}{*}{\begin{tabular}{|c|} 
BG \\
$03 / 14 / 02$
\end{tabular}} & \multirow{2}{*}{\begin{tabular}{|c|} 
BG \\
$08 / 08 / 02$
\end{tabular}} & & \\
\hline Date Sampled & & 02/12/02 & $07 / 29 / 02$ & $02 / 18 / 02$ & 07/31/02 & & & & 01/07/02 & 07/08/02 \\
\hline Program & & GWPP & GWPP & GWPP & GWPP & GWPP & GWPP & GWPP & BJC & BJC \\
\hline \multicolumn{11}{|l|}{ Sample Type } \\
\hline \multicolumn{11}{|l|}{ Field Measurements } \\
\hline Time Sampled & & $10: 40$ & $9: 50$ & 9:10 & $8: 05$ & $11: 35$ & $11: 35$ & $10: 15$ & $12: 40$ & 9:40 \\
\hline Measuring Point Elev. (ft) & & 964.00 & 964.00 & 983.57 & 983.57 & 961.18 & 952.62 & 952.62 & $1,055.01$ & $1,055.01$ \\
\hline Depth to Water (ft) & & 21.31 & 21.71 & 13.87 & 15.79 & 8.09 & 7.91 & 9.73 & 12.96 & 14.23 \\
\hline Groundwater Elevation (ft) & & 942.69 & 942.29 & 969.70 & 967.78 & 953.09 & 944.71 & 942.89 & $1,042.05$ & $1,040.78$ \\
\hline Conductivity $(\mu \mathrm{mho} / \mathrm{cm})$ & & 1,121 & 941 & 2,560 & 2,430 & 469 & 179.1 & 471 & 610 & 502 \\
\hline Dissolved Oxygen (ppm) & & 0.02 & 1.01 & 0.66 & 1.27 & 0.42 & 0.43 & 0.76 & 0.65 & 3.46 \\
\hline Oxidation/Reduction (mV) & & -133 & -65 & 170 & 188 & -145 & 111 & -76 & -53 & -52 \\
\hline Temperature (degrees C) & & 13.2 & 16 & 15.1 & 16.9 & 15.3 & 12.2 & 17.2 & 14.7 & 18.8 \\
\hline Turbidity (NTU) & & & & & & & & & 17 & \\
\hline $\mathrm{pH}$ & & 7.16 & 6.96 & 6.97 & 6.72 & 6.41 & 5.9 & 6.05 & 7.31 & 7.56 \\
\hline \multicolumn{11}{|l|}{ Miscellaneous Analytes } \\
\hline Conductivity $(\mu \mathrm{mho} / \mathrm{cm})$ & & 1,084 & 916 & 2,310 & 2,310 & 377 & 168.5 & 442 & & \\
\hline Dissolved Solids (mg/L) & & 822 & 708 & 1,630 & 1,830 & 197 & 95 & 267 & 341 & 380 \\
\hline Suspended Solids (mg/L) & & $<$ & 2 & 8 & 16 & 5 & 8 & 7 & $<$ & $<$ \\
\hline Turbidity (NTU) & & 3.92 & 5.4 & 5.06 & 14.7 & 66.5 & 25.4 & 11.4 & & \\
\hline $\mathrm{pH}$ & & 6.97 & 7.16 & 7.02 & 6.9 & 6.36 & 6.03 & 6.33 & & \\
\hline \multicolumn{11}{|l|}{ Major lons (mg/L) } \\
\hline Calcium & & 172 & 147 & 398 & 392 & 47.5 & 17.1 & 56.5 & 95.9 & 88.9 \\
\hline Magnesium & & 18.5 & 17.4 & 25.9 & 25.9 & 7 & 5.27 & 14.2 & 16.6 & 16 \\
\hline Potassium & & $<$ & $<$ & 2.43 & 2.99 & 5.06 & $<$ & $<$ & 2.14 & 2.96 \\
\hline Sodium & & 9.48 & 8.57 & 15.4 & 15.8 & 13.4 & 1.68 & 7.99 & 12.1 & 12.7 \\
\hline Alkalinity as $\mathrm{CO} 3$ & & $<$ & $<$ & $<$ & $<$ & $<$ & $<$ & $<$ & $<$ & $<$ \\
\hline Alkalinity as $\mathrm{HCO} 3$ & & 302 & 302 & 206 & 218 & 148 & 55.6 & 195 & 233 & 248 \\
\hline Chloride & & 147 & 114 & 15.5 & 14.1 & 4.89 & 1.22 & 11.7 & 38.6 & 35.6 \\
\hline Fluoride & & & $<$ & $<$ & $<$ & $<$ & & $<$ & $<$ & $<$ \\
\hline Nitrate as $\mathrm{N}$ & & 0.0802 & $<$ & 244 & 233 & $<$ & 0.0281 & $<$ & $<$ & 0.1 \\
\hline Sulfate & & 4.86 & 6.28 & 6.87 & 7.28 & 19.5 & 21.2 & 23.4 & 16.2 & 16.9 \\
\hline Charge balance & (RPD) & 1.1 & -1.3 & 1.3 & 2.1 & 2.2 & -7.7 & -4.2 & 5.1 & 0.5 \\
\hline Trace Metals (mg/L) & & & & & & & & & & \\
\hline Aluminum & & $<$ & $<$ & 0.727 & 2.03 & $<$ & 1.59 & 1.26 & $<$ & $<$ \\
\hline Antimony & PMS & $<$ & $<1$ & $<$ & $<$ & $<$ & $<$ & $<$ & & \\
\hline Arsenic & PMS & $<$ & $<$ & $<$ & $<$ & $<$ & $<$ & $<$ & & \\
\hline Barium & & 1.2 & 0.925 & 1.08 & 1.08 & 0.115 & 0.116 & 0.173 & 0.231 & 0.229 \\
\hline Beryllium & & $<$ & $<$ & $<$ & $<$ & $<$ & $<$ & $<$ & $<$ & $<$ \\
\hline Boron & & 19.6 & 13.2 & $<$ & $<$ & 0.207 & $<$ & $<$ & $<$ & $<$ \\
\hline Cadmium & PMS & $<$ & $<$ & $<$ & $<$ & $<$ & $<$ & $<$ & $\cdot$ & . \\
\hline Cadmium & & & & & & & & & $<$ & $<$ \\
\hline Chromium & PMS & $<$ & 0.0147 & $<$ & $<$ & 0.0259 & $<$ & $<$ & . & ${ }^{\circ}$ \\
\hline Chromium & & & & & & & & & $<$ & $<$ \\
\hline Cobalt & & $<$ & $<1$ & $<$ & $<$ & $<$ & $<$ & $<$ & $<$ & $<$ \\
\hline Copper & & $<$ & $<$ & $<$ & $<$ & $<$ & $<$ & $<$ & $<$ & $<$ \\
\hline Iron & & 0.554 & 0.923 & 0.416 & 1.18 & 10.4 & 2.4 & 6.3 & 0.459 & 0.706 \\
\hline Lead & PMS & 0.126 & 0.00331 & 0.00111 & 0.0015 & 0.00691 & 0.00919 & 0.00172 & & \\
\hline Lead & & & & & & & & & $<$ & $<$ \\
\hline Lithium & & 0.508 & 0.236 & 0.0264 & 0.0256 & $<$ & $<$ & $<$ & 0.023 & 0.0241 \\
\hline Manganese & & 1.35 & 1.36 & 0.0158 & 0.0348 & 1.71 & 5.43 & 8.91 & 0.743 & 0.72 \\
\hline Mercury & CVAA & $<$ & $<$ & $<$ & $<$ & $<$ & $<$ & $<$ & $<$ & $<$ \\
\hline Nickel & PMS & 0.0282 & 0.0641 & 0.00561 & 0.00586 & 0.01 & 0.00625 & 0.00734 & & \\
\hline Nickel & & & & & & & & & $<$ & $<$ \\
\hline Selenium & PMS & $<$ & $<$ & $<$ & $<$ & $<$ & $<$ & $<$ & & \\
\hline Strontium & & 0.325 & 0.289 & 0.924 & 0.935 & 0.107 & 0.0632 & 0.275 & 0.165 & 0.16 \\
\hline Thallium & PMS & 0.000645 & $<$ & $<$ & $<$ & & & $<$ & & \\
\hline Thallium & & & & & & & & & $<$ & $<$ \\
\hline Uranium & PMS & $<$ & $<$ & 0.000616 & $<$ & 0.023 & 0.00331 & 0.0129 & & \\
\hline Uranium & KPA & . &. & & & & & & $<$ & $<$ \\
\hline Vanadium & & $<$ & $<$ & $<$ & $<$ & $<$ & $<$ & $<$ & $<$ & $<$ \\
\hline Zinc & & $<$ & $<$ & $<$ & $<$ & $<$ & $<$ & $<$ & $<$ & $<$ \\
\hline
\end{tabular}


APPENDIX D.1: CY 2002 MONITORING DATA FOR THE BEAR CREEK HYDROGEOLOGIC REGIME

Field Measurements, Miscellaneous Analytes, Major Ions, and Trace Metals

\begin{tabular}{|c|c|c|c|c|c|c|c|c|c|c|}
\hline \multicolumn{2}{|l|}{ Sampling Point } & \multicolumn{2}{|c|}{ GW-225 } & \multicolumn{2}{|c|}{ GW-226 } & \multicolumn{3}{|c|}{ GW-229 } & \multicolumn{2}{|c|}{ GW-243 } \\
\hline Location & & \multicolumn{2}{|c|}{ OLF } & \multicolumn{2}{|c|}{ OLF } & \multicolumn{3}{|c|}{ OLF } & \multicolumn{2}{|c|}{ S3 } \\
\hline \multicolumn{2}{|l|}{ Date Sampled } & $02 / 19 / 02$ & $08 / 01 / 02$ & $02 / 19 / 02$ & 08/01/02 & 03/19/02 & 03/19/02 & $08 / 12 / 02$ & $03 / 21 / 02$ & $08 / 13 / 02$ \\
\hline \multicolumn{2}{|l|}{ Program } & GWPP & GWPP & GWPP & GWPP & GWPP & GWPP & GWPP & GWPP & GWPP \\
\hline \multicolumn{2}{|l|}{ Sample Type } & & & & & & Dup & & & \\
\hline Field Measurements & & & & & & & & & & \\
\hline Time Sampled & & $10: 05$ & $10: 25$ & $8: 50$ & $9: 05$ & $13: 10$ & $13: 10$ & $10: 05$ & $8: 50$ & $9: 45$ \\
\hline Measuring Point Elev. (ft) & & 943.11 & 943.11 & 943.40 & 943.40 & 949.00 & 949.00 & 949.00 & $1,011.75$ & $1,011.75$ \\
\hline Depth to Water (ft) & & 13.05 & 18.45 & 14.00 & 18.78 & 14.22 & 14.22 & 28.51 & 15.94 & 17.60 \\
\hline Groundwater Elevation (ft) & & 930.06 & 924.66 & 929.40 & 924.62 & 934.78 & 934.78 & 920.49 & 995.81 & 994.15 \\
\hline Conductivity $(\mu \mathrm{mho} / \mathrm{cm})$ & & 1,134 & 1,032 & 1,182 & 802 & 1,486 & 1,486 & 1575 & 35,300 & 35,700 \\
\hline Dissolved Oxygen (ppm) & & 0.01 & 0.99 & 0.1 & 0.46 & 0.22 & 0.22 & 1.83 & 0.62 & 0.53 \\
\hline Oxidation/Reduction (mV) & & 147 & -8 & 193 & 7 & -108 & -108 & -127 & 271 & 300 \\
\hline Temperature (degrees C) & & 13.2 & 18.3 & 10.6 & 12.1 & 15.5 & 15.5 & 16.4 & 16.2 & 18.4 \\
\hline Turbidity (NTU) & & & & & & & & & & \\
\hline $\mathrm{pH}$ & & 7.53 & 6.46 & 6.93 & 7.35 & 6.65 & 6.65 & 6.55 & 5.6 & 5.42 \\
\hline Miscellaneous Analytes & & & & & & & & & & \\
\hline Conductivity $(\mu \mathrm{mho} / \mathrm{cm})$ & & 1,029 & 1,007 & 1,072 & 779 & 1,447 & 1,443 & 1430 & 46,000 & 47,000 \\
\hline Dissolved Solids (mg/L) & & 559 & 549 & 587 & 439 & 751 & 753 & 827 & 48300 & 51300 \\
\hline Suspended Solids (mg/L) & & $<$ & $<$ & $<$ & $<$ & 9 & 11 & 32 & 136 & 106 \\
\hline Turbidity (NTU) & & 1.17 & 2.1 & 9.18 & 3.62 & 217 & 214 & 255 & 150 & 11 \\
\hline $\mathrm{pH}$ & & 7.32 & 7.53 & 7.16 & 7.1 & 6.71 & 6.58 & 6.7 & 5.64 & 5.66 \\
\hline Major lons (mg/L) & & & & & & & & & & \\
\hline Calcium & & 107 & 104 & 137 & 84.6 & 154 & 157 & 162 & 9,010 & 9,270 \\
\hline Magnesium & & 45.6 & 44.9 & 42.1 & 25.9 & 29.8 & 29.9 & 29.8 & 1,230 & 1,160 \\
\hline Potassium & & 2.87 & 2.72 & 3.11 & 6.48 & 11.4 & 11.7 & 11.5 & $<$ & $<$ \\
\hline Sodium & & 23.2 & 22.9 & 23.8 & 20.8 & 65 & 65.4 & 69.9 & 658 & 656 \\
\hline Alkalinity as $\mathrm{CO} 3$ & & $<$ & $<$ & $<$ & $<$ & $<$ & $<$ & $<$ & $<$ & $<$ \\
\hline Alkalinity as $\mathrm{HCO} 3$ & & 214 & 210 & 396 & 280 & 542 & 522 & 520 & 774 & 728 \\
\hline Chloride & & 63.6 & 59.9 & 66.2 & 41.7 & 119 & 120 & 125 & 384 & 353 \\
\hline Fluoride & & 0.514 & 0.594 & $<$ & $<$ & 0.2 & 0.204 & 0.207 & 5.21 & 1.47 \\
\hline Nitrate as $\mathrm{N}$ & & 37.4 & 34.9 & 10.3 & 12.2 & $<$ & 0.219 & $<$ & 7,240 & 8,480 \\
\hline Sulfate & & 57.1 & 62.8 & 49.1 & 21.7 & 31.5 & 31.4 & 29.2 & 14.9 & 9.77 \\
\hline Charge balance & (RPD) & 1.1 & 1.2 & -0.5 & -4.3 & -5.7 & -3.8 & -2.3 & 3.2 & -3.6 \\
\hline Trace Metals (mg/L) & & & & & & & & & & \\
\hline Aluminum & & $<$ & $<$ & $<$ & $<$ & $<$ & $<$ & $<$ & $<$ & $<$ \\
\hline Antimony & PMS & $<$ & $<$ & $<$ & $<$ & $<$ & $<$ & $<$ & $<$ & $<$ \\
\hline Arsenic & PMS & $<$ & $<$ & $<$ & $<$ & 0.00801 & 0.00894 & 0.00866 & $<$ & $<$ \\
\hline Barium & & 0.15 & 0.15 & 0.199 & 0.128 & 1.16 & 1.17 & 1.17 & 54.9 & 55 \\
\hline Beryllium & & & $<$ & & & $<$ & $<$ & $<$ & $<$ & $<$ \\
\hline Boron & & 0.127 & 0.129 & 0.171 & 0.115 & 3.41 & 3.43 & 3.41 & $<$ & $<$ \\
\hline Cadmium & PMS & $<$ & $<$ & $<$ & $<$ & $<$ & $<$ & $<$ & 2.37 & 1.92 \\
\hline Cadmium & & & & & & & & & & \\
\hline Chromium & PMS & $<$ & $<$ & $<$ & $<$ & $<$ & $<$ & $<$ & 0.0113 & $<$ \\
\hline Chromium & & & & & & & & & & \\
\hline Cobalt & & $<$ & $<$ & $<$ & $<$ & $<$ & $<$ & $<$ & $<$ & $<$ \\
\hline Copper & & $<$ & $<$ & $<$ & $<$ & $<$ & $<$ & $<$ & $<$ & $<$ \\
\hline Iron & & 0.234 & 0.317 & 0.999 & 0.704 & 25.8 & 25.8 & 22 & 7.55 & $<$ \\
\hline Lead & PMS & 0.00123 & $<$ & 0.00163 & 0.000605 & $<$ & $<$ & 0.000978 & 0.00434 & 0.00219 \\
\hline Lead & & & & & & & & & & \\
\hline Lithium & & 0.0206 & 0.0192 & 0.0188 & 0.0142 & 0.139 & 0.14 & 0.148 & $<$ & $<$ \\
\hline Manganese & & $<$ & 0.0425 & 0.878 & 0.538 & 5 & 5 & 4.44 & 276 & 261 \\
\hline Mercury & CVAA & $<$ & $<$ & $<$ & $<$ & $<$ & $<$ & $<$ & 0.00272 & 0.00384 \\
\hline Nickel & PMS & $<$ & $<$ & 0.011 & 0.00728 & 0.0429 & 0.0439 & 0.0487 & 4.41 & 3.27 \\
\hline Nickel & & & & & & & & & & \\
\hline Selenium & PMS & $<$ & $<$ & $<$ & $<$ & $<$ & 0.0104 & $<$ & $<$ & $<$ \\
\hline Strontium & & 2.16 & 2.14 & 0.613 & 0.405 & 0.496 & 0.499 & 0.508 & 24.1 & 24.9 \\
\hline Thallium & PMS & 0.000805 & $<$ & 0.00109 & $<$ & $<$ & $<$ & $<$ & 0.001 & 0.00276 \\
\hline Thallium & & & & & & & & & & \\
\hline Uranium & PMS & 0.00372 & 0.00356 & 0.0189 & 0.00828 & 0.238 & 0.242 & 0.266 & 0.839 & 0.653 \\
\hline Uranium & KPA & & & & & & & & & \\
\hline Vanadium & & $<$ & $<$ & $<$ & $<$ & $<$ & $<$ & $<$ & $<$ & $<$ \\
\hline Zinc & & $<$ & $<$ & $<$ & $<$ & $<$ & $<$ & $<$ & $<$ & $<$ \\
\hline
\end{tabular}


APPENDIX D.1: CY 2002 MONITORING DATA FOR THE BEAR CREEK HYDROGEOLOGIC REGIME

Field Measurements, Miscellaneous Analytes, Major Ions, and Trace Metals

\begin{tabular}{|c|c|c|c|c|c|c|c|c|c|}
\hline \multicolumn{2}{|l|}{ Sampling Point } & \multicolumn{2}{|c|}{ GW-276 } & \multicolumn{2}{|c|}{ GW-288 } & \multicolumn{2}{|c|}{ GW-289 } & \multicolumn{2}{|c|}{ GW-291 } \\
\hline Location & & \multicolumn{2}{|c|}{ S3 } & \multicolumn{2}{|c|}{ BG } & \multicolumn{2}{|c|}{ BG } & \multicolumn{2}{|c|}{ BG } \\
\hline Date Sampled & & $01 / 07 / 02$ & $07 / 08 / 02$ & $03 / 13 / 02$ & 08/07/02 & $03 / 13 / 02$ & 08/08/02 & 03/14/02 & 08/07/02 \\
\hline Program & & BJC & BJC & GWPP & GWPP & GWPP & GWPP & GWPP & GWPP \\
\hline \multicolumn{10}{|l|}{ Sample Type } \\
\hline \multicolumn{10}{|l|}{ Field Measurements } \\
\hline Time Sampled & & $10: 15$ & $9: 35$ & 9:00 & $10: 25$ & $9: 50$ & $8: 45$ & $10: 15$ & $8: 50$ \\
\hline Measuring Point Elev. (ft) & & $1,001.57$ & $1,001.57$ & 948.36 & 948.36 & 948.73 & 948.73 & 948.56 & 948.56 \\
\hline Depth to Water (ft) & & 6.47 & 7.95 & 16.95 & 18.40 & 17.75 & 19.14 & 11.13 & 13.69 \\
\hline Groundwater Elevation (ft) & & 995.10 & 993.62 & 931.41 & 929.96 & 930.98 & 929.59 & 937.43 & 934.87 \\
\hline Conductivity $(\mu \mathrm{mho} / \mathrm{cm})$ & & 1,123 & 1,298 & 347 & 330 & 294 & 328 & 467 & 412 \\
\hline Dissolved Oxygen (ppm) & & 4.55 & 1.04 & 0.14 & 0.89 & 0.96 & 1.25 & 1.74 & 0.39 \\
\hline Oxidation/Reduction (mV) & & 265 & 212 & 53 & 25 & 158 & 176 & 275 & 237 \\
\hline Temperature (degrees C) & & 13.9 & 22.7 & 14.8 & 17.3 & 15.4 & 17.6 & 13.2 & 17.9 \\
\hline Turbidity (NTU) & & 15 & 14 & & & & & & \\
\hline $\mathrm{pH}$ & & 5.28 & 5.64 & 7.17 & 7.2 & 6.52 & 6.63 & 6.74 & 6.46 \\
\hline \multicolumn{10}{|l|}{ Miscellaneous Analytes } \\
\hline Conductivity $(\mu \mathrm{mho} / \mathrm{cm})$ & & & & 285 & 299 & 235 & 284 & 437 & 320 \\
\hline Dissolved Solids (mg/L) & & 705 & 1,030 & 183 & 183 & 148 & 205 & 256 & 176 \\
\hline Suspended Solids (mg/L) & & $<$ & $<$ & $<$ & $<$ & $<$ & $<$ & $<$ & $<$ \\
\hline Turbidity (NTU) & & & & 0.817 & 1.97 & 0.11 & 1.14 & 0.57 & 0.605 \\
\hline $\mathrm{pH}$ & & & & 7.46 & 7.18 & 6.85 & 6.73 & 6.94 & 6.54 \\
\hline \multicolumn{10}{|l|}{ Major lons (mg/L) } \\
\hline Calcium & & 86.4 & 109 & 50.4 & 50.5 & 37.1 & 45.6 & 61.2 & 48.3 \\
\hline Magnesium & & 14.8 & 17.4 & 5.05 & 4.83 & 5.03 & 5.78 & 11.1 & 9.02 \\
\hline Potassium & & 10.5 & 10.2 & $<$ & $<$ & $<$ & $<$ & $<$ & $<$ \\
\hline Sodium & & 76.2 & 67.6 & 4.23 & 4.22 & 5.05 & 5.36 & 10.4 & 8.58 \\
\hline Alkalinity as $\mathrm{CO} 3$ & & $<$ & $<$ & $<$ & $<$ & $<$ & $<$ & $<$ & $<$ \\
\hline Alkalinity as $\mathrm{HCO} 3$ & & 5 & 32.7 & 150 & 149 & 110 & 134 & 206 & 140 \\
\hline Chloride & & 173 & 181 & 3.35 & 3.53 & 2.95 & 3.23 & 3.95 & 5.03 \\
\hline Fluoride & & 1.9 & 1.5 & $<$ & $<$ & $<$ & $<$ & $<$ & $<$ \\
\hline Nitrate as $\mathrm{N}$ & & 51.3 & 73.7 & 0.0849 & $<$ & 0.307 & 0.221 & 0.234 & 0.242 \\
\hline Sulfate & & 42.9 & 53.8 & 6.03 & 6.27 & 6.9 & 6.86 & 18.2 & 17.2 \\
\hline Charge balance & $(\mathrm{RPD})$ & -2.8 & -9.6 & -1.7 & -1.7 & 0.8 & 1 & -2.3 & 3.1 \\
\hline Trace Metals (mg/L) & & & & & & & & & \\
\hline Aluminum & & 2.93 & 2.23 & $<$ & $<$ & $<$ & $<$ & $<$ & $<$ \\
\hline Antimony & PMS & & & $<$ & $<$ & $<$ & $<$ & $<$ & $<$ \\
\hline Arsenic & PMS & & & $<$ & & $<$ & $<$ & $<$ & $<$ \\
\hline Barium & & 0.204 & 0.202 & 0.28 & 0.278 & 0.0929 & 0.109 & 0.198 & 0.167 \\
\hline Beryllium & & 0.0041 & 0.0033 & $<$ & $<$ & $<$ & $<$ & $<$ & $<$ \\
\hline Boron & & $<$ & $<$ & $<$ & $<$ & $<$ & $<$ & 2.42 & 2.22 \\
\hline Cadmium & PMS & & & $<$ & $<$ & $<$ & $<$ & $<$ & $<$ \\
\hline Cadmium & & 0.0214 & 0.0168 & . & . & & & & \\
\hline Chromium & PMS & & & $<$ & $<$ & $<$ & $<$ & $<$ & $<$ \\
\hline Chromium & & & 0.0127 & 1 & & & & & \\
\hline Cobalt & & 0.0898 & 0.0542 & $<$ & $<$ & $<$ & $<$ & $<$ & $<$ \\
\hline Copper & & $<$ & & $<$ & $<$ & $<$ & $<$ & 0.152 & 0.0528 \\
\hline Iron & & $<$ & 0.0527 & 0.117 & 0.169 & $<$ & $<$ & $<$ & $<$ \\
\hline Lead & PMS & & & 0.00079 & 0.000788 & $<$ & $<$ & 0.00297 & 0.00245 \\
\hline Lead & & & & & & & & & \\
\hline Lithium & & 0.0209 & 0.0133 & $<$ & $<$ & $<$ & $<$ & 0.0144 & 0.0111 \\
\hline Manganese & & 4.26 & 3.67 & 0.025 & 0.0312 & $<$ & $<$ & $<$ & 0.0103 \\
\hline Mercury & CVAA & $<$ & $<$ & $<$ & $<$ & $<$ & $<$ & $<$ & $<$ \\
\hline Nickel & PMS & & & 0.0125 & $<$ & $<$ & $<$ & $<$ & $<$ \\
\hline Nickel & & 0.223 & 0.254 & & & & & & \\
\hline Selenium & PMS & & & $<$ & $<$ & $<$ & $<$ & $<$ & $<$ \\
\hline Strontium & & 0.202 & 0.253 & 0.104 & 0.103 & 0.0755 & 0.0896 & 0.13 & 0.104 \\
\hline Thallium & PMS & & & $<$ & $<$ & $<$ & $<$ & $<$ & $<$ \\
\hline Thallium & & & $<$ & & & & & & \\
\hline Uranium & PMS & & & $<$ & $<$ & $<$ & $<$ & $<$ & $<$ \\
\hline Uranium & KPA & 0.723 & 0.79 & $\cdot$ & & & & & \\
\hline Vanadium & & & $<$ & $<$ & $<$ & $<$ & $<$ & $<$ & $<$ \\
\hline Zinc & & 0.0599 & $<$ & $<$ & $<$ & $<$ & $<$ & $<$ & $<$ \\
\hline
\end{tabular}


APPENDIX D.1: CY 2002 MONITORING DATA FOR THE BEAR CREEK HYDROGEOLOGIC REGIME

Field Measurements, Miscellaneous Analytes, Major lons, and Trace Metals

\begin{tabular}{|c|c|c|c|c|c|c|c|c|c|c|}
\hline Sampling Point & & & GW-311 & & GW & 315 & GW & 363 & GW & 526 \\
\hline Location & & & RS & & S & & EMV & VMF & s & \\
\hline Date Sampled & & $02 / 1$ & $3 / 02$ & $07 / 30 / 02$ & $02 / 13 / 02$ & 07/30/02 & $08 / 14 / 02$ & $12 / 04 / 02$ & $02 / 26 / 02$ & 08/08/02 \\
\hline Program & & GWPP & GWPP & GWPP & GWPP & GWPP & BJC & BJC & BJC & BJC \\
\hline Sample Type & & & Dup & & & & & & & \\
\hline Field Measurements & & & & & & & & & & \\
\hline Time Sampled & & $8: 45$ & $8: 45$ & $10: 20$ & $10: 25$ & $8: 40$ & $10: 25$ & $13: 45$ & $9: 20$ & $9: 40$ \\
\hline Measuring Point Elev. (ft) & & 999.52 & 999.52 & 999.52 & $1,047.45$ & $1,047.45$ & 958.71 & 958.71 & 998.25 & 998.25 \\
\hline Depth to Water (ft) & & 33.55 & 33.55 & 39.47 & 53.45 & 59.90 & 7.78 & 4.77 & 14.52 & 14.25 \\
\hline Groundwater Elevation (ft) & & 965.97 & 965.97 & 960.05 & 994.00 & 987.55 & 950.93 & 953.94 & 983.73 & 984.00 \\
\hline Conductivity $(\mu \mathrm{mho} / \mathrm{cm})$ & & 485 & 485 & 495 & 809 & 809 & 491 & 504 & 6,220 & 7,250 \\
\hline Dissolved Oxygen (ppm) & & 4.73 & 4.73 & 1.87 & 0.09 & 1 & 1.29 & 2.13 & 1.38 & 0.92 \\
\hline Oxidation/Reduction (mV) & & 225 & 225 & 154 & 153 & 135 & 138 & 335 & 225 & 180 \\
\hline Temperature (degrees C) & & 10.3 & 10.3 & 19.3 & 14.2 & 17 & 22.4 & 7.6 & 10.4 & 19.5 \\
\hline Turbidity (NTU) & & & & & & & 15 & 12 & 24 & 6 \\
\hline $\mathrm{pH}$ & & 7.13 & 7.13 & 6.88 & 7.08 & 7.28 & 8.93 & 6.56 & 8.52 & 8.33 \\
\hline Miscellaneous Analytes & & & & & & & & & & \\
\hline Conductivity $(\mu \mathrm{mho} / \mathrm{cm})$ & & 431 & 431 & 451 & 733 & 746 & & & & \\
\hline Dissolved Solids (mg/L) & & 243 & 238 & 262 & 449 & 462 & & & 7,370 & 8,800 \\
\hline Suspended Solids (mg/L) & & $<$ & $<$ & $<$ & $<$ & $<$ & & & 8 & 9.1 \\
\hline Turbidity (NTU) & & 0.342 & 0.32 & 0.325 & 0.405 & 0.125 & & & & \\
\hline $\mathrm{pH}$ & & 7.44 & 7.29 & 7.38 & 7.35 & 7.32 & & & & \\
\hline Major lons (mg/L) & & & & & & & & & & \\
\hline Calcium & & 78.4 & 79.7 & 84 & 123 & 126 & & & 174 & 168 \\
\hline Magnesium & & 5.1 & 5.12 & 5.69 & 14.1 & 15.1 & & & 63.3 & 64.8 \\
\hline Potassium & & $<$ & $<$ & $<$ & 3.48 & 3.48 & & & 27.4 & 27.4 \\
\hline Sodium & & 3.17 & 3.23 & 3.19 & 7.4 & 8 & & & 1,450 & 1,780 \\
\hline Alkalinity as $\mathrm{CO} 3$ & & $<$ & $<$ & $<$ & $<$ & $<$ & & & $<$ & $<$ \\
\hline Alkalinity as $\mathrm{HCO} 3$ & & 216 & 212 & 242 & 270 & 300 & & & 41 & 44 \\
\hline Chloride & & 2.24 & 2.12 & 2.22 & 12.3 & 13 & & & 25.7 & 29.5 \\
\hline Fluoride & & $<$ & & $<$ & $<$ & $<$ & & & & $<$ \\
\hline Nitrate as $\mathrm{N}$ & & 0.276 & 0.274 & 0.331 & 7.84 & 7.77 & & & 1,260 & 1,300 \\
\hline Sulfate & & 2.85 & 2.63 & 3.34 & 64.9 & 65.6 & & & 2.7 & $<$ \\
\hline Charge balance & (RPD) & 0.1 & 1.9 & -2 & 0.4 & -1.9 & & & -8.2 & -1.4 \\
\hline Trace Metals (mg/L) & & & & & & & & & & \\
\hline Aluminum & & $<$ & $<$ & $<1$ & $<$ & $<$ & & & $<$ & $<$ \\
\hline Antimony & PMS & $<$ & $<$ & $<$ & $<$ & $<$ & & & & \\
\hline Arsenic & PMS & $<$ & $<$ & $<$ & $<$ & $<$ & & & & \\
\hline Barium & & 0.0185 & 0.0185 & 0.0199 & 0.0606 & 0.0637 & 0.067 & 0.069 & 14.6 & 15.3 \\
\hline Beryllium & & $<$ & $<$ & $<$ & $<$ & $<$ & & & & $<$ \\
\hline Boron & & $<$ & $<$ & $<$ & $<$ & $<$ & & & 0.215 & 0.225 \\
\hline Cadmium & PMS & $<$ & $<$ & $<$ & $<$ & $<$ & & & & \\
\hline Cadmium & & & & & & & & & $<$ & $<$ \\
\hline Chromium & PMS & 0.00565 & 0.00597 & 0.00403 & $<$ & $<$ & & & & \\
\hline Chromium & & & & & & & $<$ & $<1$ & $<$ & $<$ \\
\hline Cobalt & & $<$ & $<$ & $<$ & $<$ & $<$ & & & $<$ & $<$ \\
\hline Copper & & $<$ & $<$ & $<$ & $<$ & $<$ & & & $<$ & $<$ \\
\hline Iron & & $<$ & $<$ & $<$ & $<$ & 0.0563 & & & 0.197 & 0.859 \\
\hline Lead & PMS & $0.00585 \mathrm{R}$ & $0.0005 \mathrm{R}$ & $<$ & 0.00105 & 0.0066 & & & & \\
\hline Lead & & & & & & & $<$ & $<$ & $<$ & $<$ \\
\hline Lithium & & $<$ & $<$ & $<$ & $<$ & $<$ & & & 1.39 & 0.996 \\
\hline Manganese & & $<$ & $<$ & $<$ & 0.0124 & 0.11 & & & 0.0345 & 0.0408 \\
\hline Mercury & CVAA & $<$ & $<$ & $<$ & $<$ & $<$ & & & & \\
\hline Nickel & PMS & $<$ & $<$ & $<$ & $<$ & $<$ & & & & \\
\hline Nickel & & & & & & & & & $<$ & $<$ \\
\hline Selenium & PMS & $<$ & & $<$ & & $<$ & & & & \\
\hline Strontium & & 0.069 & 0.0691 & 0.0745 & 0.205 & 0.208 & 0.087 & 0.085 & 19 & 19.4 \\
\hline Thallium & PMS & $<$ & $<$ & $<$ & $<$ & $<$ & & & & \\
\hline Thallium & & & & & & & & & $<$ & $<$ \\
\hline Uranium & PMS & $<$ & $<$ & $<$ & 0.00259 & 0.00236 & & & & \\
\hline Uranium & KPA & 1 & & & & & & & $<$ & $<$ \\
\hline Vanadium & & $<$ & $<$ & $<$ & $<$ & $<$ & & & $<$ & $<$ \\
\hline Zinc & & $<$ & $<$ & $<$ & $<$ & $<$ & & & $<$ & $<$ \\
\hline
\end{tabular}


APPENDIX D.1: CY 2002 MONITORING DATA FOR THE BEAR CREEK HYDROGEOLOGIC REGIME

Field Measurements, Miscellaneous Analytes, Major lons, and Trace Metals

\begin{tabular}{|c|c|c|c|c|c|c|c|c|c|c|}
\hline \multicolumn{2}{|l|}{ Sampling Point } & \multicolumn{3}{|c|}{ GW-537 } & \multicolumn{2}{|c|}{ GW-626 } & \multicolumn{2}{|c|}{ GW-627 } & \multicolumn{2}{|c|}{ GW-639 } \\
\hline Location & & \multicolumn{3}{|c|}{ OLF } & \multicolumn{2}{|c|}{ BG } & \multicolumn{2}{|c|}{ BG } & \multicolumn{2}{|c|}{ EMWMF } \\
\hline Date Sampled & & 02/18/02 & \multicolumn{2}{|c|}{$07 / 31 / 02$} & 02/11/02 & 07/25/02 & 02/11/02 & 07/29/02 & 08/14/02 & $12 / 04 / 02$ \\
\hline Program & & GWPP & GWPP & GWPP & GWPP & GWPP & GWPP & GWPP & BJC & BJC \\
\hline Sample Type & & & & Dup & & & & & & \\
\hline Field Measurements & & & & & & & & & & \\
\hline Time Sampled & & $9: 55$ & 9:10 & 9:10 & $9: 45$ & $9: 55$ & $8: 45$ & $8: 40$ & $9: 55$ & $10: 15$ \\
\hline Measuring Point Elev. (ft) & & 976.41 & 976.41 & 976.41 & 942.87 & 942.87 & 943.51 & 943.51 & 940.70 & 940.70 \\
\hline Depth to Water (ft) & & 6.75 & 7.86 & 7.86 & 26.64 & 27.78 & 24.27 & 25.72 & 12.70 & 11.55 \\
\hline Groundwater Elevation (ft) & & 969.66 & 968.55 & 968.55 & 916.23 & 915.09 & 919.24 & 917.79 & 928.00 & 929.15 \\
\hline Conductivity $(\mu \mathrm{mho} / \mathrm{cm})$ & & 5,710 & 5,260 & 5,260 & 671 & 420 & 1,436 & 1,311 & 1,088 & 941 \\
\hline Dissolved Oxygen (ppm) & & 0.18 & 0.42 & 0.42 & 0.02 & 1.78 & 0 & 0.88 & 1.89 & 2.1 \\
\hline Oxidation/Reduction (mV) & & 171 & 176 & 176 & -175 & -16 & -307 & -285 & 186 & 183 \\
\hline Temperature (degrees $\mathrm{C}$ ) & & 14.9 & 15.9 & 15.9 & 14.1 & 16.1 & 13.8 & 15.8 & 20.9 & 10.2 \\
\hline Turbidity (NTU) & & & & & & & & & 10 & 10 \\
\hline $\mathrm{pH}$ & & 6.78 & 6.46 & 6.46 & 7.39 & 7.28 & 9.11 & 9.1 & 9.06 & 9.5 \\
\hline Miscellaneous Analytes & & & & & & & & & & \\
\hline Conductivity ( $\mu \mathrm{mho} / \mathrm{cm})$ & & 5,380 & 5,270 & 5,220 & 577 & 376 & 1,228 & 1,229 & & \\
\hline Dissolved Solids (mg/L) & & 4,150 & 4,390 & 4,370 & 349 & 238 & 721 & 722 & & \\
\hline Suspended Solids (mg/L) & & $<$ & $<$ & $<$ & $<$ & $<$ & $<$ & $<$ & & \\
\hline Turbidity (NTU) & & 1.16 & 1.77 & 1.74 & 0.313 & 0.452 & 0.934 & 0.707 & & \\
\hline $\mathrm{pH}$ & & 6.78 & 6.72 & 6.7 & 7.46 & 7.56 & 9.18 & 9.15 & & \\
\hline Major lons (mg/L) & & & & & & & & & & \\
\hline Calcium & & 973 & 944 & 921 & 104 & 68.2 & 1.27 & 1.21 & & \\
\hline Magnesium & & 71.6 & 68.7 & 68 & 6.9 & 4.33 & 0.243 & 0.237 & & \\
\hline Potassium & & $<$ & $<$ & $<$ & $<$ & $<$ & $<$ & $<$ & & \\
\hline Sodium & & 43 & 39.2 & 38.5 & 6.58 & 4.88 & 309 & 293 & & \\
\hline Alkalinity as $\mathrm{CO} 3$ & & $<$ & $<$ & $<$ & $<$ & $<$ & 123 & 110 & & \\
\hline Alkalinity as $\mathrm{HCO} 3$ & & 334 & 310 & 294 & 224 & 186 & 435 & 482 & & \\
\hline Chloride & & 34.3 & 29.9 & 29.8 & 43.5 & 10.3 & 47.5 & 51.1 & & \\
\hline Fluoride & & $<$ & $<$ & $<$ & $<$ & & 4.99 & 4.72 & & \\
\hline Nitrate as $\mathrm{N}$ & & 669 & 610 & 586 & $<$ & 0.0307 & $<$ & $<$ & & \\
\hline Sulfate & & 4.43 & 3.86 & 3.9 & 7.14 & 3.55 & 13.5 & 11.9 & & \\
\hline Charge balance & (RPD) & 0.7 & 3.6 & 4.5 & 1.6 & -1.4 & 1.8 & -3.6 & & \\
\hline Trace Metals (mg/L) & & & & & & & & & & \\
\hline Aluminum & & $<$ & $<$ & $<$ & $<$ & $<$ & $<$ & $<$ & & \\
\hline Antimony & PMS & $<$ & $<$ & $<$ & $<$ & $<$ & $<$ & $<$ & & \\
\hline Arsenic & PMS & $<$ & $<$ & $<$ & $<$ & $<$ & $<$ & $<$ & & \\
\hline Barium & & 2.38 & 2.25 & 2.21 & 0.33 & 0.205 & 0.0471 & 0.0453 & 0.08 & 0.084 \\
\hline Beryllium & & $<$ & $<$ & $<$ & $<$ & $<$ & $<$ & $<$ & & \\
\hline Boron & & $<$ & $<$ & $<$ & $<$ & $<$ & 0.499 & 0.48 & & \\
\hline Cadmium & PMS & $<$ & $<$ & $<$ & $<$ & $<$ & $<$ & $<$ & & \\
\hline Cadmium & & & & & & & & & & \\
\hline Chromium & PMS & $<$ & $<$ & $<$ & $<$ & $<$ & $<$ & $<$ & & ${ }^{\circ}$ \\
\hline Chromium & & & & & & & & & $<$ & $<$ \\
\hline Cobalt & & $<$ & $<$ & $<$ & $<$ & $<$ & $<$ & $<$ & & \\
\hline Copper & & $<$ & $<$ & $<$ & $<$ & $<$ & $<$ & $<$ & & \\
\hline Iron & & $<$ & $<$ & 0.125 & 0.0778 & $<$ & 0.105 & 0.146 & & \\
\hline Lead & PMS & 0.00086 & 0.00122 & $<$ & $<$ & $<$ & $<$ & 0.0191 & & \\
\hline Lead & & & & & & & & & $<$ & $<$ \\
\hline Lithium & & 0.0417 & 0.0356 & 0.0362 & 0.0164 & 0.0112 & 0.0925 & 0.0885 & & \\
\hline Manganese & & $<$ & $<$ & $<$ & $<$ & $<$ & 0.0152 & 0.0148 & & \\
\hline Mercury & CVAA & $<$ & $<$ & $<$ & $<$ & $<$ & $<$ & $<$ & & \\
\hline Nickel & PMS & 0.0142 & 0.0141 & 0.0143 & 0.119 & 0.0288 & $<$ & $<$ & & \\
\hline Nickel & & & & & & & & & & \\
\hline Selenium & PMS & $<$ & $<$ & $<$ & $<$ & $<$ & $<$ & $<$ & & \\
\hline Strontium & & 2.71 & 2.59 & 2.56 & 0.222 & 0.14 & 0.0888 & 0.0855 & 0.14 & 0.12 \\
\hline Thallium & PMS & 0.00116 & $<$ & $<$ & $<$ & $<$ & $<$ & $<$ & & \\
\hline Thallium & & & & & & & & & & \\
\hline Uranium & PMS & 0.00156 & 0.0011 & 0.00111 & $<$ & $<$ & $<$ & $<$ & & \\
\hline Uranium & KPA & & & & &. & . & & & \\
\hline Vanadium & & $<$ & $<$ & $<$ & $<$ & $<$ & $<$ & $<$ & & \\
\hline Zinc & & $<$ & $<$ & $<$ & $<$ & $<$ & $<$ & $<$ & & \\
\hline
\end{tabular}


APPENDIX D.1: CY 2002 MONITORING DATA FOR THE BEAR CREEK HYDROGEOLOGIC REGIME

Field Measurements, Miscellaneous Analytes, Major lons, and Trace Metals

\begin{tabular}{|c|c|c|c|c|c|c|c|c|c|c|}
\hline \multicolumn{2}{|l|}{ Sampling Point } & \multicolumn{2}{|c|}{ GW-653 } & \multicolumn{2}{|c|}{ GW-683 } & \multicolumn{3}{|c|}{ GW-684 } & \multicolumn{2}{|c|}{ GW-694 } \\
\hline Location & & \multicolumn{2}{|c|}{ BG } & \multicolumn{2}{|c|}{ EXP-A } & \multicolumn{3}{|c|}{ EXP-A } & \multicolumn{2}{|c|}{ EXP-B } \\
\hline \multicolumn{2}{|l|}{ Date Sampled } & 02/12/02 & $07 / 25 / 02$ & $01 / 14 / 02$ & 07/09/02 & $01 / 14 / 02$ & \multicolumn{2}{|c|}{ 07/09/02 } & 01/29/02 & 07/17/02 \\
\hline Program & & GWPP & GWPP & GWPP & GWPP & GWPP & GWPP & GWPP & GWPP & GWPP \\
\hline Sample Type & & & & & & & & Dup & & \\
\hline Field Measurements & & & & & & & & & & \\
\hline Time Sampled & & $8: 45$ & $8: 40$ & $11: 05$ & $8: 05$ & $10: 05$ & 9:20 & 9:20 & $9: 20$ & $9: 20$ \\
\hline Measuring Point Elev. (ft) & & 931.84 & 931.84 & 972.23 & 972.23 & 898.83 & 898.83 & 898.83 & 941.98 & 941.98 \\
\hline Depth to Water (ft) & & 22.69 & 24.86 & 88.95 & 89.12 & 15.72 & 15.91 & 15.91 & 23.28 & 31.56 \\
\hline Groundwater Elevation (ft) & & 909.15 & 906.98 & 883.28 & 883.11 & 883.11 & 882.92 & 882.92 & 918.70 & 910.42 \\
\hline Conductivity $(\mu \mathrm{mho} / \mathrm{cm})$ & & 41.4 & 40.6 & 659 & 524 & 642 & 579 & 579 & 499 & 456 \\
\hline Dissolved Oxygen (ppm) & & 5.65 & 2.24 & 0.87 & 2.32 & 0.22 & 1.34 & 1.34 & 0.1 & 0.19 \\
\hline Oxidation/Reduction (mV) & & 291 & 306 & 173 & 189 & 14 & 161 & 161 & 54 & -29 \\
\hline Temperature (degrees $\mathrm{C}$ ) & & 12.6 & 14.4 & 12.6 & 13.2 & 13.2 & 14.1 & 14.1 & 13.3 & 14.4 \\
\hline Turbidity (NTU) & & & & & & & & & & \\
\hline $\mathrm{pH}$ & & 5.4 & 4.79 & 7.63 & 7.58 & 7.49 & 7.57 & 7.57 & 7.62 & 7.54 \\
\hline Miscellaneous Analytes & & & & & & & & & & \\
\hline Conductivity $(\mu \mathrm{mho} / \mathrm{cm})$ & & 32.3 & 31.8 & 581 & 423 & 575 & 472 & 474 & 443 & 439 \\
\hline Dissolved Solids (mg/L) & & 40 & 60 & 315 & 256 & 311 & 288 & 288 & 240 & 260 \\
\hline Suspended Solids (mg/L) & & $<$ & $<$ & $<$ & 2 & $<$ & $<$ & $<$ & $<$ & $<$ \\
\hline Turbidity (NTU) & & 0.407 & 0.641 & 1.95 & 10.7 & 1.22 & 0.807 & 0.819 & 6.85 & 6.15 \\
\hline $\mathrm{pH}$ & & 5.67 & 5.64 & 7.6 & 7.95 & 7.53 & 7.95 & 7.96 & 7.86 & 7.99 \\
\hline Major lons (mg/L) & & & & & & & & & & \\
\hline Calcium & & 1.94 & 1.9 & 79.7 & 53.2 & 78.9 & 60.9 & 61.8 & 59.2 & 54.4 \\
\hline Magnesium & & 1.07 & 1.05 & 23.8 & 20.7 & 19.3 & 19.1 & 19.6 & 15.4 & 15.1 \\
\hline Potassium & & $<$ & $<$ & $<$ & $<$ & 6.65 & 4.82 & 4.94 & 3.26 & 3.64 \\
\hline Sodium & & 2.17 & 2.13 & 7.92 & 2.99 & 8.67 & 5.9 & 6.06 & 9.28 & 8.68 \\
\hline Alkalinity as $\mathrm{CO} 3$ & & $<$ & $<$ & $<$ & $<$ & $<$ & $<$ & $<$ & $<$ & $<$ \\
\hline Alkalinity as $\mathrm{HCO} 3$ & & 12.4 & 13.4 & 226 & 191 & 222 & 197 & 200 & 174 & 157 \\
\hline Chloride & & 1.04 & 1.1 & 19.2 & 5.41 & 24.2 & 13.1 & 13.3 & 16.3 & 15.3 \\
\hline Fluoride & & $<$ & $<$ & 0.116 & 0.112 & 0.17 & 0.17 & 0.168 & 0.298 & 0.289 \\
\hline Nitrate as $\mathrm{N}$ & & $<$ & $<$ & 13.3 & 2.65 & 9.95 & 4.36 & 4.36 & 4.79 & 3.69 \\
\hline Sulfate & & 1.84 & 1.32 & 29.4 & 17.2 & 24.4 & 10.3 & 11.3 & 19.4 & 17.8 \\
\hline Charge balance & (RPD) & -5.4 & -5.9 & -2.7 & -0.4 & -2.2 & 1.5 & 1.6 & 0.1 & 2.4 \\
\hline Trace Metals (mg/L) & & & & & & & & & & \\
\hline Aluminum & & $<$ & $<$ & $<$ & 0.609 & $<$ & $<$ & $<$ & $<$ & $<$ \\
\hline Antimony & PMS & $<$ & $<1$ & $<$ & $<$ & $<$ & $<$ & $<1$ & $<$ & $<$ \\
\hline Arsenic & PMS & $<$ & $<$ & $<$ & $<$ & $<$ & $<$ & $<$ & $<$ & $<$ \\
\hline Barium & & 0.0335 & 0.0314 & 0.134 & 0.111 & 0.12 & 0.0826 & 0.0842 & 0.0856 & 0.0805 \\
\hline Beryllium & & $<$ & $<$ & $<$ & $<$ & $<$ & $<$ & $<$ & $<$ & $<$ \\
\hline Boron & & $<$ & $<$ & $<$ & $<$ & $<$ & $<$ & $<$ & $<$ & $<$ \\
\hline Cadmium & PMS & $<$ & $<$ & $<$ & $<$ & $<$ & $<$ & $<$ & $<$ & $<$ \\
\hline Cadmium & & & & & & 1 & 1 & & & \\
\hline $\begin{array}{l}\text { Chromium } \\
\text { Chromium }\end{array}$ & PMS & 0.00294 & 0.00271 & 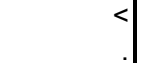 & 0.00645 & $<$ & $<$ & 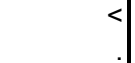 & $<$ & $<$ \\
\hline Cobalt & & $<$ & $<$ & $<$ & $<$ & $<$ & $<1$ & $<$ & $<$ & $<$ \\
\hline Copper & & $<$ & $<$ & $<$ & $<$ & $<$ & $<$ & $<$ & $<$ & $<$ \\
\hline Iron & & $<$ & $<$ & 0.143 & 0.49 & 0.0904 & $<$ & $<$ & 0.841 & 0.424 \\
\hline Lead & PMS & $<$ & 0.000717 & $<$ & 0.00094 & $<$ & $<$ & $<$ & $<$ & $<$ \\
\hline Lead & & & & & & & & & & \\
\hline Lithium & & $<$ & $<$ & $<$ & $<$ & 0.0326 & 0.0252 & 0.0248 & 0.0134 & 0.0124 \\
\hline Manganese & & $<$ & $<$ & $<$ & 0.0116 & 0.0976 & 0.017 & 0.0174 & 0.121 & 0.0718 \\
\hline Mercury & CVAA & $<$ & $<1$ & $<$ & $<$ & $<$ & $<$ & $<$ & $<$ & $<$ \\
\hline Nickel & PMS & $<$ & $<$ & 0.0141 & 0.00941 & $<$ & $<$ & $<$ & $<$ & $<$ \\
\hline Nickel & & & & & & & & & & \\
\hline Selenium & PMS & $<$ & $<$ & $<$ & $<$ & $<$ & $<$ & $<$ & $<$ & $<$ \\
\hline Strontium & & 0.0177 & 0.0172 & 0.171 & 0.137 & 0.166 & 0.124 & 0.127 & 0.146 & 0.135 \\
\hline Thallium & PMS & $<$ & $<$ & $<$ & $<$ & $<$ & $<$ & $<$ & $<$ & $<$ \\
\hline Thallium & & & & & & & & & & \\
\hline Uranium & PMS & $<$ & $<$ & 0.0485 & 0.0231 & 0.0454 & 0.0269 & 0.0257 & 0.0393 & 0.0355 \\
\hline Uranium & KPA & . & & & & & & & & \\
\hline Vanadium & & $<$ & $<$ & $<$ & $<$ & $<$ & $<$ & $<$ & $<$ & $<$ \\
\hline Zinc & & $<$ & $<$ & $<$ & $<$ & $<$ & $<$ & $<$ & $<$ & $<$ \\
\hline
\end{tabular}


APPENDIX D.1: CY 2002 MONITORING DATA FOR THE BEAR CREEK HYDROGEOLOGIC REGIME

Field Measurements, Miscellaneous Analytes, Major lons, and Trace Metals

\begin{tabular}{|c|c|c|c|c|c|c|c|c|c|c|}
\hline \multicolumn{2}{|l|}{ Sampling Point } & \multicolumn{2}{|c|}{ GW-695 } & \multicolumn{2}{|c|}{ GW-703 } & \multicolumn{3}{|c|}{ GW-704 } & \multicolumn{2}{|c|}{ GW-706 } \\
\hline Location & & \multicolumn{2}{|c|}{ EXP-B } & \multicolumn{2}{|c|}{ EXP-B } & \multicolumn{3}{|c|}{ EXP-B } & \multicolumn{2}{|c|}{ EXP-B } \\
\hline Date Sampled & & $01 / 15 / 02$ & $07 / 15 / 02$ & $01 / 15 / 02$ & 07/15/02 & \multicolumn{2}{|c|}{ 01/16/02 } & 07/16/02 & $01 / 16 / 02$ & $07 / 16 / 02$ \\
\hline Program & & GWPP & GWPP & GWPP & GWPP & GWPP & GWPP & GWPP & GWPP & GWPP \\
\hline Sample Type & & & & & & & Dup & & & \\
\hline Field Measurements & & & & & & & & & & \\
\hline Time Sampled & & $10: 00$ & 9:25 & $8: 50$ & $8: 20$ & $10: 05$ & $10: 05$ & $8: 25$ & $11: 40$ & $9: 40$ \\
\hline Measuring Point Elev. (ft) & & 939.54 & 939.54 & 954.69 & 954.69 & 944.73 & 944.73 & 944.73 & 929.47 & 929.47 \\
\hline Depth to Water (ft) & & 30.21 & 28.22 & 44.05 & 44.94 & 36.56 & 36.56 & 34.80 & 18.54 & 17.69 \\
\hline Groundwater Elevation (ft) & & 909.33 & 911.32 & 910.64 & 909.75 & 908.17 & 908.17 & 909.93 & 910.93 & 911.78 \\
\hline Conductivity ( $\mu \mathrm{mho} / \mathrm{cm})$ & & 633 & 577 & 798 & 779 & 664 & 664 & 645 & 1,072 & 845 \\
\hline Dissolved Oxygen (ppm) & & 3.81 & 1.95 & 0.13 & 0.6 & 0.08 & 0.08 & 1.42 & 0 & 0.94 \\
\hline Oxidation/Reduction (mV) & & 178 & 182 & 207 & 139 & 23 & 23 & 41 & 87 & 37 \\
\hline Temperature (degrees C) & & 11.5 & 16.7 & 11.1 & 15.7 & 12.7 & 12.7 & 14.2 & 13.3 & 15.3 \\
\hline Turbidity (NTU) & & & & & & & & & & \\
\hline $\mathrm{pH}$ & & 7.34 & 7.42 & 7.31 & 7.33 & 7.76 & 7.76 & 7.45 & 7.65 & 7.13 \\
\hline Miscellaneous Analytes & & & & & & & & & & \\
\hline Conductivity $(\mu \mathrm{mho} / \mathrm{cm})$ & & 543 & 531 & 707 & 727 & 629 & 626 & 591 & 956 & 795 \\
\hline Dissolved Solids (mg/L) & & 288 & 326 & 375 & 434 & 335 & 332 & 333 & 562 & 454 \\
\hline Suspended Solids (mg/L) & & $<$ & 2 & $<$ & $<$ & 3 & $<$ & $<$ & $<$ & $<$ \\
\hline Turbidity (NTU) & & 0.774 & 6.37 & 3.38 & 5.8 & 28.8 & 28.2 & 3.88 & 0.606 & 3.7 \\
\hline $\mathrm{pH}$ & & 7.7 & 7.77 & 7.65 & 7.65 & 7.64 & 7.71 & 7.68 & 7.45 & 7.36 \\
\hline Major lons (mg/L) & & & & & & & & & & \\
\hline Calcium & & 65 & 58.9 & 79 & 79.8 & 64.5 & 63.3 & 61.2 & 136 & 109 \\
\hline Magnesium & & 27.1 & 25.1 & 33.1 & 32 & 29.9 & 30.3 & 27.9 & 25.3 & 23.7 \\
\hline Potassium & & 2.16 & 3.65 & 4.08 & 3.82 & 5.19 & 5.82 & 3.89 & 7 & 5.31 \\
\hline Sodium & & 6.87 & 8.23 & 13.2 & 13.1 & 13.5 & 13.6 & 11.7 & 20.7 & 14.9 \\
\hline Alkalinity as $\mathrm{CO} 3$ & & $<$ & $<$ & $<$ & $<$ & $<$ & $<$ & $<$ & $<$ & $<$ \\
\hline Alkalinity as $\mathrm{HCO} 3$ & & 210 & 200 & 224 & 226 & 206 & 214 & 198 & 254 & 270 \\
\hline Chloride & & 14.6 & 13 & 25 & 25.4 & 25.9 & 26.4 & 24 & 45 & 32.2 \\
\hline Fluoride & & $<$ & 0.109 & 0.182 & 0.172 & 0.174 & 0.175 & 0.183 & 0.292 & 0.25 \\
\hline Nitrate as $\mathrm{N}$ & & 12 & 10.8 & 20.8 & 20.7 & 14.5 & 14.1 & 12 & 36.8 & 15.7 \\
\hline Sulfate & & 15.8 & 13 & 29.6 & 26.6 & 23.7 & 26.6 & 21 & 33.9 & 21.9 \\
\hline Charge balance & (RPD) & 0.3 & 0.4 & 0.3 & 0.1 & 0.1 & -1.5 & 0.2 & 1.3 & 1.7 \\
\hline Trace Metals (mg/L) & & & & & & & & & & \\
\hline Aluminum & & $<$ & $<1$ & $<$ & $<$ & $<$ & $<$ & $<1$ & $<$ & $<$ \\
\hline Antimony & PMS & $<$ & $<$ & $<$ & $<$ & $<$ & $<$ & $<$ & $<$ & $<$ \\
\hline Arsenic & PMS & $<$ & $<$ & $<$ & $<$ & $<$ & $<$ & $<$ & $<$ & $<$ \\
\hline Barium & & 0.0471 & 0.0443 & 0.0925 & 0.0959 & 0.105 & 0.104 & 0.0939 & 0.181 & 0.153 \\
\hline Beryllium & & $<$ & $<$ & $<$ & $<$ & $<$ & $<$ & $<$ & $<$ & $<$ \\
\hline Boron & & $<$ & $<$ & $<$ & $<$ & $<$ & $<$ & $<$ & 0.137 & 0.145 \\
\hline Cadmium & PMS & $<$ & 0.000617 & $<$ & $<$ & $<$ & $<$ & $<$ & $<$ & $<$ \\
\hline Cadmium & & & & & & & & & & \\
\hline Chromium & PMS & $<$ & 0.014 & $<$ & $<$ & $<$ & $<$ & $<$ & $<$ & $<$ \\
\hline Chromium & & & & & & & & & & \\
\hline Cobalt & & $<$ & $<$ & $<$ & $<$ & $<$ & $<$ & $<$ & $<$ & $<$ \\
\hline Copper & & $<$ & $<$ & $<$ & $<$ & $<$ & $<$ & $<$ & $<$ & $<$ \\
\hline Iron & & $<$ & 0.725 & 0.472 & 0.384 & 0.414 & 0.508 & 0.469 & 0.324 & 0.684 \\
\hline Lead & PMS & 0.00068 & 0.000922 & 0.000551 & 0.000558 & $<$ & $<$ & 0.000574 & $<$ & 0.000523 \\
\hline Lead & & & & & & & & & & \\
\hline Lithium & & $<$ & 0.011 & 0.0201 & 0.0185 & 0.0189 & 0.0188 & 0.0152 & 0.0246 & 0.0198 \\
\hline Manganese & & $<$ & 0.00537 & 0.0816 & 0.0381 & 0.0127 & 0.0145 & 0.00755 & 0.0126 & 0.00663 \\
\hline Mercury & CVAA & $<$ & $<$ & 0 & $<$ & $<$ & $<$ & $<$ & $<$ & 0.0000 \\
\hline Nickel & PMS & $<$ & $<$ & $<$ & 0.00691 & $<$ & $<$ & $<$ & $<$ & $<$ \\
\hline Nickel & & & & & & & & & & \\
\hline Selenium & PMS & & & $<$ & $<$ & $<$ & $<$ & $<$ & $<$ & $<$ \\
\hline Strontium & & 0.0954 & 0.092 & 0.274 & 0.27 & 0.264 & 0.26 & 0.249 & 0.433 & 0.312 \\
\hline Thallium & PMS & $<$ & $<$ & $<$ & 0.000554 & $<$ & $<$ & $<$ & $<$ & $<$ \\
\hline Thallium & & & & & & & & & & \\
\hline Uranium & PMS & 0.00666 & 0.00589 & 0.00669 & 0.00718 & 0.0104 & 0.0101 & 0.0127 & 0.132 & 0.106 \\
\hline Uranium & $\mathrm{KPA}$ & & & & & & & & & \\
\hline Vanadium & & $<$ & $<$ & $<$ & $<$ & $<$ & $<$ & $<$ & $<$ & $<$ \\
\hline Zinc & & $<$ & $<$ & $<$ & $<$ & $<$ & $<$ & $<$ & $<$ & 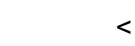 \\
\hline
\end{tabular}


APPENDIX D.1: CY 2002 MONITORING DATA FOR THE BEAR CREEK HYDROGEOLOGIC REGIME

Field Measurements, Miscellaneous Analytes, Major Ions, and Trace Metals

\begin{tabular}{|c|c|c|c|c|c|c|c|c|c|}
\hline Sampling Point & & GW & 712 & GW & 713 & GW & 714 & GW & \\
\hline Location & & EXF & $-W$ & EXI & & EXF & $-W$ & EXF & \\
\hline Date Sampled & & $01 / 03 / 02$ & 07/01/02 & $01 / 03 / 02$ & $07 / 01 / 02$ & $01 / 02 / 02$ & 07/01/02 & $01 / 0$ & \\
\hline Program & & BJC & BJC & BJC & BJC & BJC & BJC & BJC & BJC \\
\hline Sample Type & & & & & & & & & Dup \\
\hline Field Measurements & & & & & & & & & \\
\hline Time Sampled & & $14: 05$ & $13: 50$ & $10: 05$ & $13: 50$ & $10: 05$ & $10: 05$ & $13: 15$ & \\
\hline Measuring Point Elev. (ft) & & 877.89 & 877.89 & 881.43 & 881.43 & 875.88 & 875.88 & 874.92 & \\
\hline Depth to Water (ft) & & 35.05 & 35.77 & 38.24 & 39.03 & 30.68 & 31.27 & 28.95 & \\
\hline Groundwater Elevation (ft) & & 842.84 & 842.12 & 843.19 & 842.40 & 845.20 & 844.61 & 845.97 & \\
\hline Conductivity $(\mu \mathrm{mho} / \mathrm{cm})$ & & 478 & 483 & 558 & 523 & 493 & 530 & 512 & \\
\hline Dissolved Oxygen (ppm) & & 1.15 & 2.56 & 0.8 & 2.31 & 1.06 & 2.62 & 5.65 & \\
\hline Oxidation/Reduction (mV) & & -77 & -139 & -167 & -26 & 218 & 198 & 144 & \\
\hline Temperature (degrees C) & & 7.3 & 17.5 & 6.4 & 23.5 & 3 & 21.1 & 9.4 & \\
\hline Turbidity (NTU) & & 30 & 25 & 44 & 29 & 38 & 60 & 25 & \\
\hline $\mathrm{pH}$ & & 8.25 & 7.89 & 7.92 & 7.87 & 8.07 & 8.05 & 7.2 & \\
\hline $\begin{array}{r}\text { Miscellaneous Analytes } \\
\text { Conductivity ( } \mu \mathrm{mho} / \mathrm{cm}) \\
\text { Dissolved Solids }(\mathrm{mg} / \mathrm{L}) \\
\text { Suspended Solids (mg/L) } \\
\text { Turbidity (NTU) } \\
\mathrm{pH}\end{array}$ & & $\begin{array}{r}292 \\
<\end{array}$ & $\begin{array}{r}318 \\
<\end{array}$ & $\begin{array}{r}340 \\
<\end{array}$ & $\begin{array}{r}367 \\
6.4\end{array}$ & $\begin{array}{r}263 \\
<\end{array}$ & $\begin{array}{r}295 \\
10.3\end{array}$ & $\begin{array}{r}277 \\
<\end{array}$ & 272 \\
\hline Major lons (mg/L) & & & & & & & & & \\
\hline Calcium & & 57.3 & 50 & 62.1 & 56.9 & 60.8 & 58.4 & 69.1 & 78 \\
\hline Magnesium & & 33.2 & 32.4 & 32.8 & 32.3 & 24.5 & 24.1 & 11.1 & 12.5 \\
\hline Potassium & & 2.13 & 2.3 & 2.8 & 3.03 & 1.56 & 2 & 1.39 & 1.55 \\
\hline Sodium & & 8.31 & 8.74 & 12.1 & 12.4 & 5.08 & 5.29 & 16.3 & 18.2 \\
\hline Alkalinity as $\mathrm{CO} 3$ & & $<$ & $<$ & $<$ & $<$ & $<$ & $<$ & $<$ & $<$ \\
\hline Alkalinity as $\mathrm{HCO} 3$ & & 164 & 178 & 157 & 179 & 188 & 190 & 182 & 183 \\
\hline Chloride & & 9.7 & 10.2 & 16.1 & 14.6 & 12.4 & 11 & 24.5 & 24.4 \\
\hline Fluoride & & 0.27 & 0.32 & 0.39 & 0.44 & 0.26 & 0.44 & $<$ & $<$ \\
\hline Nitrate as $\mathrm{N}$ & & $<$ & 0.034 & $<$ & $<$ & 1.6 & 1.7 & 1.3 & 1.2 \\
\hline Sulfate & & 79.2 & 70.7 & 90.6 & 88.9 & 38.8 & 33.9 & 13.9 & 13.9 \\
\hline Charge balance & (RPD) & 7 & 2.4 & 7.5 & 2.1 & 2.6 & 2.2 & 4 & \\
\hline Trace Metals (mg/L) & & & & & & & & & \\
\hline Aluminum & & $<$ & $<$ & $<$ & $<$ & $<$ & $<$ & $<$ & $<$ \\
\hline Antimony & PMS & & & & & & & & \\
\hline Arsenic & PMS & & & & & & & & \\
\hline Barium & & 0.0371 & 0.034 & 0.0403 & 0.0396 & 0.0699 & 0.0724 & 0.0636 & 0.0716 \\
\hline Beryllium & & $<$ & $<$ & $<$ & $<$ & $<$ & $<$ & $<$ & $<$ \\
\hline Boron & & $<$ & $<$ & $<$ & $<$ & $<$ & $<$ & $<$ & $<$ \\
\hline Cadmium & PMS & & & & & . & & . & \\
\hline Cadmium & & $<$ & $<$ & $<$ & $<$ & $<$ & $<$ & $<$ & $<$ \\
\hline Chromium & PMS & & & & & & & & \\
\hline Chromium & & $<$ & $<$ & $<$ & $<$ & $<$ & $<$ & 0.0576 & 0.0534 \\
\hline Cobalt & & $<$ & $<$ & $<$ & $<$ & $<$ & $<$ & $<$ & $<$ \\
\hline Copper & & $<$ & $<$ & $<$ & $<$ & $<$ & $<$ & $<$ & $<$ \\
\hline Iron & & 1.38 & 2.52 & 3.75 & 3.41 & 3.43 & 6.21 & 0.425 & 0.39 \\
\hline Lead & PMS & & & & & & & & \\
\hline Lead & & $<$ & $<$ & $<$ & $<$ & $<$ & $<$ & $<$ & $<$ \\
\hline Lithium & & 0.0114 & 0.0117 & 0.0152 & 0.0154 & $<$ & $<$ & $<$ & $<$ \\
\hline Manganese & & 0.17 & 0.18 & 0.141 & 0.147 & 0.0141 & 0.0609 & 0.0152 & 0.0146 \\
\hline Mercury & CVAA & $<$ & $<$ & $<$ & $<$ & $<$ & $<$ & $<$ & $<$ \\
\hline Nickel & PMS & & & & & & & & \\
\hline Nickel & & $<$ & $<$ & $<$ & $<$ & $<$ & $<$ & 0.134 & 0.159 \\
\hline Selenium & PMS & & & & & & & & \\
\hline Strontium & & 0.693 & 0.593 & 1.27 & 1.21 & 0.264 & 0.256 & 0.0717 & 0.0811 \\
\hline Thallium & PMS & & & & & & & & \\
\hline Thallium & & $<$ & $<$ & $<$ & $<$ & $<$ & $<$ & $<$ & $<$ \\
\hline Uranium & PMS & & & & & & & & \\
\hline Uranium & KPA & $<$ & $<$ & $<$ & $<$ & $<$ & $<$ & 0.0145 & 0.0139 \\
\hline Vanadium & & $<$ & $<$ & $<$ & $<$ & $<$ & $<$ & $<$ & $<$ \\
\hline Zinc & & $<$ & $<$ & $<$ & $<$ & $<$ & $<$ & $<$ & $<$ \\
\hline
\end{tabular}


APPENDIX D.1: CY 2002 MONITORING DATA FOR THE BEAR CREEK HYDROGEOLOGIC REGIME

Field Measurements, Miscellaneous Analytes, Major Ions, and Trace Metals

\begin{tabular}{|c|c|c|c|c|c|c|c|c|c|}
\hline \multicolumn{2}{|l|}{ Sampling Point } & \multicolumn{2}{|c|}{ GW-715 } & \multicolumn{2}{|c|}{ GW-723 } & \multicolumn{2}{|c|}{ GW-724 } & \multicolumn{2}{|c|}{ GW-725 } \\
\hline Location & & \multicolumn{2}{|c|}{ EXP-W } & \multicolumn{2}{|c|}{ EXP-C } & \multicolumn{2}{|c|}{ EXP-C } & \multicolumn{2}{|c|}{ EXP-C } \\
\hline Date Sampled & & \multicolumn{2}{|c|}{$07 / 01 / 02$} & $03 / 05 / 02$ & $07 / 23 / 02$ & $01 / 30 / 02$ & $07 / 23 / 02$ & $01 / 31 / 02$ & 07/18/02 \\
\hline Program & & BJC & BJC & GWPP & GWPP & GWPP & GWPP & GWPP & GWPP \\
\hline Sample Type & & & Dup & & & & & & \\
\hline Field Measurements & & & & & & & & & \\
\hline Time Sampled & & $9: 55$ & & $9: 40$ & $8: 35$ & $10: 55$ & $9: 35$ & $9: 05$ & $8: 35$ \\
\hline Measuring Point Elev. (ft) & & 874.92 & & $1,022.23$ & $1,022.23$ & 979.27 & 979.27 & 961.05 & 961.05 \\
\hline Depth to Water (ft) & & 29.09 & & 68.74 & 70.73 & 25.14 & 31.66 & 7.26 & 13.00 \\
\hline Groundwater Elevation (ft) & & 845.83 & & 953.49 & 951.50 & 954.13 & 947.61 & 953.79 & 948.05 \\
\hline Conductivity $(\mu \mathrm{mho} / \mathrm{cm})$ & & 469 & & 390 & 374 & 1,006 & 783 & 1,321 & 1,140 \\
\hline Dissolved Oxygen (ppm) & & 3.38 & & 0.14 & 1.19 & 1.27 & 0.26 & 0.1 & 0.94 \\
\hline Oxidation/Reduction (mV) & & 170 & & -235 & -278 & 113 & -76 & 64 & 72 \\
\hline Temperature (degrees C) & & 18 & & 11.5 & 14.5 & 14.4 & 15.6 & 14.1 & 15.6 \\
\hline Turbidity (NTU) & & & & & & & & & \\
\hline $\mathrm{pH}$ & & 7.15 & & 8.17 & 8.16 & 7.3 & 7.62 & 6.96 & 6.76 \\
\hline Miscellaneous Analytes & & & & & & & & & \\
\hline Conductivity $(\mu \mathrm{mho} / \mathrm{cm})$ & & & & 347 & 347 & 920 & 747 & 1,210 & 1,095 \\
\hline Dissolved Solids (mg/L) & & 268 & 282 & 170 & 195 & 490 & 427 & 719 & 704 \\
\hline Suspended Solids (mg/L) & & $<$ & $<$ & 4 & $<$ & $<$ & $<$ & 2 & $<$ \\
\hline Turbidity (NTU) & & & & 21.2 & 10.8 & 2 & 6.59 & 5.46 & 3.96 \\
\hline $\mathrm{pH}$ & & & & 8.08 & 8.11 & 7.54 & 7.74 & 7.14 & 7.08 \\
\hline Major lons (mg/L) & & & & & & & & & \\
\hline Calcium & & 63.3 & 63.2 & 16.2 & 15.4 & 97 & 57.6 & 179 & 155 \\
\hline Magnesium & & 16.4 & 16.2 & 28.6 & 27.7 & 40.8 & 36.1 & 35.5 & 23.3 \\
\hline Potassium & & 1.63 & 1.64 & 2.55 & 2.5 & 2.22 & 4.05 & 2.91 & 2.97 \\
\hline Sodium & & 7.68 & 7.58 & 7.18 & 7.04 & 31.2 & 28 & 23.3 & 29.4 \\
\hline Alkalinity as $\mathrm{CO} 3$ & & $<$ & $<$ & $<$ & $<$ & $<$ & $<$ & $<$ & $<$ \\
\hline Alkalinity as $\mathrm{HCO} 3$ & & 188 & 190 & 131 & 140 & 234 & 186 & 286 & 318 \\
\hline Chloride & & 14.9 & 15.3 & 10 & 9.21 & 75.8 & 71.1 & 75.2 & 73.3 \\
\hline Fluoride & & $<$ & 0.11 & 0.406 & 0.34 & 0.216 & 0.189 & 0.103 & 0.237 \\
\hline Nitrate as $\mathrm{N}$ & & 4.2 & 4.2 & $<$ & $<$ & 25.8 & 12 & 48.2 & 18.5 \\
\hline Sulfate & & 13.4 & 13.5 & 35.6 & 33 & 30.1 & 29.7 & 40.2 & 39.5 \\
\hline Charge balance & (RPD) & 1.3 & & -1.7 & -4.8 & 1.7 & -0.3 & 3.3 & 2 \\
\hline Trace Metals (mg/L) & & & & & & & & & \\
\hline Aluminum & & $<$ & $<$ & $<$ & $<$ & $<$ & $<$ & $<$ & $<$ \\
\hline Antimony & PMS & & & $<$ & 0.00663 & $<$ & $<$ & $<$ & $<$ \\
\hline Arsenic & PMS & & & $<$ & & $<$ & $<$ & $<$ & $<$ \\
\hline Barium & & 0.0571 & 0.0573 & 0.0444 & 0.0436 & 0.183 & 0.144 & 0.296 & 0.218 \\
\hline Beryllium & & $<$ & $<$ & $<$ & $<$ & $<$ & $<$ & $<$ & $<$ \\
\hline Boron & & $<$ & $<$ & $<$ & $<$ & $<$ & $<$ & $<$ & $<$ \\
\hline Cadmium & PMS & & & $<$ & $<$ & $<$ & $<$ & $<$ & $<$ \\
\hline Cadmium & & $<$ & $<$ & . & & & & . & . \\
\hline Chromium & PMS & & & $<$ & $<$ & $<$ & $<$ & $<$ & $<$ \\
\hline Chromium & & 0.019 & 0.0211 &. & & & & . & . \\
\hline Cobalt & & & & $<$ & $<$ & $<$ & $<$ & $<$ & $<$ \\
\hline Copper & & & & $<$ & $<$ & $<$ & $<$ & $<$ & $<$ \\
\hline Iron & & 0.223 & 0.231 & 3.18 & 2 & 0.536 & 1.55 & 0.391 & 0.269 \\
\hline Lead & PMS & & & 0.000693 & $<$ & $<$ & $<$ & $<$ & $<$ \\
\hline Lead & & & & & & & & & \\
\hline Lithium & & 0.0108 & 0.0105 & 0.0142 & 0.0136 & 0.0189 & 0.0179 & $<$ & $<$ \\
\hline Manganese & & 0.0204 & 0.0202 & 0.213 & 0.203 & 0.00997 & 0.0262 & 0.159 & 0.768 \\
\hline Mercury & CVAA & $<$ & $<$ & $<$ & $<$ & $<$ & $<$ & $<$ & $<$ \\
\hline Nickel & PMS & & & $<$ & $<$ & $<$ & $<$ & $<$ & $<$ \\
\hline Nickel & & 0.0281 & 0.0291 & & & & & 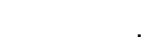 & \\
\hline Selenium & PMS & & & $<$ & $<$ & $<$ & $<$ & $<$ & $<$ \\
\hline Strontium & & 0.123 & 0.123 & 0.368 & 0.358 & 1.32 & 0.789 & 0.763 & 0.386 \\
\hline Thallium & PMS & & & 0.00273 & $<$ & $<$ & 0.000803 & $<$ & 0.000706 \\
\hline Thallium & & $<$ & $<$ & & & & & & \\
\hline Uranium & PMS & & & $<$ & $<$ & 0.000619 & $<$ & 0.00446 & 0.0105 \\
\hline Uranium & KPA & 0.043 & 0.0396 & & & & & & \\
\hline Vanadium & & $<$ & $<$ & $<$ & $<$ & $<$ & $<$ & $<$ & $<$ \\
\hline Zinc & & $<$ & $<$ & $<$ & $<$ & $<$ & $<$ & $<$ & $<$ \\
\hline
\end{tabular}


APPENDIX D.1: CY 2002 MONITORING DATA FOR THE BEAR CREEK HYDROGEOLOGIC REGIME

Field Measurements, Miscellaneous Analytes, Major Ions, and Trace Metals

\begin{tabular}{|c|c|c|c|c|c|c|c|c|c|}
\hline \multicolumn{2}{|l|}{ Sampling Point } & \multicolumn{2}{|c|}{ GW-736 } & \multicolumn{2}{|c|}{ GW-737 } & \multicolumn{2}{|c|}{ GW-738 } & \multicolumn{2}{|c|}{ GW-739 } \\
\hline Location & & \multicolumn{2}{|c|}{ EXP-C } & \multicolumn{2}{|c|}{ EXP-C } & \multicolumn{2}{|c|}{ EXP-C } & \multicolumn{2}{|c|}{ EXP-C } \\
\hline Date Sampled & & 03/06/02 & $07 / 17 / 02$ & $03 / 06 / 02$ & $07 / 18 / 02$ & $01 / 30 / 02$ & $07 / 23 / 02$ & 03/05/02 & 07/22/02 \\
\hline Program & & GWPP & GWPP & GWPP & GWPP & GWPP & GWPP & GWPP & GWPP \\
\hline \multicolumn{10}{|l|}{ Sample Type } \\
\hline Field Measurements & & & & & & & & & \\
\hline Time Sampled & & $8: 55$ & $10: 25$ & $9: 50$ & $9: 35$ & $9: 35$ & $10: 35$ & $10: 45$ & $9: 50$ \\
\hline Measuring Point Elev. (ft) & & 960.12 & 960.12 & 959.91 & 959.91 & 983.08 & 983.08 & $1,023.74$ & $1,023.74$ \\
\hline Depth to Water (ft) & & 10.19 & 11.47 & 10.08 & 11.47 & 26.41 & 30.58 & 75.13 & 75.83 \\
\hline Groundwater Elevation (ft) & & 949.93 & 948.65 & 949.83 & 948.44 & 956.67 & 952.50 & 948.61 & 947.91 \\
\hline Conductivity $(\mu \mathrm{mho} / \mathrm{cm})$ & & 1,239 & 1,159 & 1,242 & 1,152 & 949 & 893 & 556 & 562 \\
\hline Dissolved Oxygen (ppm) & & 0 & 2.48 & 0.06 & 2.27 & 1 & 1.35 & 0.27 & 2.22 \\
\hline Oxidation/Reduction (mV) & & 191 & 203 & 159 & 122 & 218 & 84 & -6 & 157 \\
\hline Temperature (degrees C) & & 14 & 17.5 & 14.1 & 16 & 14.3 & 15.9 & 12.3 & 14.6 \\
\hline \multicolumn{10}{|l|}{ Turbidity (NTU) } \\
\hline $\mathrm{pH}$ & & 7.12 & 6.72 & 7.29 & 6.85 & 6.89 & 6.9 & 7.69 & 7.5 \\
\hline \multicolumn{10}{|l|}{ Miscellaneous Analytes } \\
\hline Conductivity $(\mu \mathrm{mho} / \mathrm{cm})$ & & 1,173 & 1,111 & 1,157 & 1,111 & 862 & 861 & 506 & 520 \\
\hline Dissolved Solids (mg/L) & & 956 & 776 & 666 & 670 & 459 & 498 & 270 & 303 \\
\hline Suspended Solids (mg/L) & & 91 & 8 & $<$ & $<$ & $<$ & $<$ & $<$ & 3 \\
\hline Turbidity (NTU) & & 184 & 44.4 & 0.689 & 1.55 & 0.322 & 0.916 & 3.96 & 27.4 \\
\hline $\mathrm{pH}$ & & 7.06 & 7.7 & 7.4 & 7.08 & 7.1 & 7.16 & 7.77 & 7.83 \\
\hline \multicolumn{10}{|l|}{ Major lons (mg/L) } \\
\hline Calcium & & 173 & 164 & 157 & 157 & 131 & 134 & 52.9 & 56.7 \\
\hline Magnesium & & 26 & 23 & 22.7 & 22.2 & 29.1 & 25.5 & 32 & 31.6 \\
\hline Potassium & & 10.5 & 5.39 & 3.33 & 3.29 & 2.25 & 2.43 & 2.56 & 2.55 \\
\hline Sodium & & 35.3 & 35.1 & 40.2 & 35.9 & 9.03 & 8.6 & 2.2 & 2.23 \\
\hline Alkalinity as $\mathrm{CO} 3$ & & $<$ & $<$ & $<$ & $<$ & $<$ & $<$ & $<$ & $<$ \\
\hline Alkalinity as $\mathrm{HCO} 3$ & & 360 & 344 & 344 & 364 & 334 & 350 & 238 & 238 \\
\hline Chloride & & 95.7 & 87.8 & 96.3 & 79.5 & 22.7 & 18.9 & 8.51 & 7.61 \\
\hline Fluoride & & 0.357 & 0.344 & 0.142 & 0.154 & $<$ & $<$ & 0.356 & 0.3 \\
\hline Nitrate as $\mathrm{N}$ & & 17.8 & 15.3 & 14.2 & 11.6 & 17.2 & 15.1 & 1.33 & 1.7 \\
\hline Sulfate & & 43.5 & 40.9 & 40.6 & 40.3 & 30.1 & 30.6 & 19.5 & 20.5 \\
\hline Charge balance & (RPD) & 2 & 1.9 & 0.3 & 0.5 & 1.1 & -0.1 & -0.8 & 0.5 \\
\hline Trace Metals (mg/L) & & & & & & & & & \\
\hline Aluminum & & 30.7 & 9.35 & $<$ & $<$ & $<$ & $<$ & $<$ & $<$ \\
\hline Antimony & PMS & $<$ & $<$ & $<$ & $<$ & $<$ & $<$ & $<$ & $<$ \\
\hline Arsenic & PMS & 0.00764 & $<$ & $<$ & $<$ & $<$ & $<$ & $<$ & $<$ \\
\hline Barium & & 0.327 & 0.229 & 0.194 & 0.186 & 0.0542 & 0.0546 & 0.0326 & 0.0335 \\
\hline Beryllium & & 0.0009 & $<$ & $<$ & $<$ & $<$ & $<$ & $<$ & $<$ \\
\hline Boron & & $<$ & $<$ & $<$ & $<$ & $<$ & $<$ & $<$ & $<$ \\
\hline Cadmium & PMS & $<$ & $<$ & $<$ & $<$ & $<$ & $<$ & $<$ & $<$ \\
\hline Cadmium & & & & & & & & & \\
\hline Chromium & PMS & 0.0182 & $<$ & $<$ & $<$ & $<$ & $<$ & $<$ & $<$ \\
\hline Chromium & & & & & & & & & \\
\hline Cobalt & & $<$ & $<$ & $<$ & $<$ & $<$ & $<$ & $<$ & $<$ \\
\hline Copper & & 0.0488 & $<$ & $<$ & $<$ & $<$ & $<$ & $<$ & $<$ \\
\hline Iron & & 20.7 & 5.14 & $<$ & $<$ & $<$ & 0.0513 & 0.273 & 2.19 \\
\hline Lead & PMS & 0.0282 & 0.00484 & 0.00293 & $<$ & 0.012 & $<$ & 0.0199 & $<$ \\
\hline Lead & & & & & & & & & \\
\hline Lithium & & 0.0355 & 0.0109 & $<$ & $<$ & $<$ & $<$ & 0.017 & 0.017 \\
\hline Manganese & & 1.1 & 0.772 & 0.926 & 0.925 & $<$ & $<$ & 0.0218 & 0.0236 \\
\hline Mercury & CVAA & $<$ & $<$ & $<$ & $<$ & $<$ & $<$ & $<$ & $<$ \\
\hline Nickel & PMS & 0.0292 & 0.0112 & 0.0101 & 0.00592 & $<$ & $<$ & $<$ & $<$ \\
\hline Nickel & & & & & & & & & \\
\hline Selenium & PMS & & $<$ & $<$ & $<$ & $<$ & $<$ & $<$ & $<$ \\
\hline Strontium & & 0.389 & 0.364 & 0.324 & 0.32 & 0.129 & 0.135 & 0.108 & 0.108 \\
\hline Thallium & PMS & 0.00735 & $<$ & 0.00129 & $<$ & $<$ & $<$ & $<$ & $<$ \\
\hline Thallium & & & & & & & & & \\
\hline Uranium & PMS & 0.0298 & 0.0256 & 0.0162 & 0.0141 & 0.00177 & 0.00228 & 0.000729 & 0.000503 \\
\hline Uranium & KPA & & & & & & & & \\
\hline Vanadium & & 0.0253 & $<$ & $<$ & $<$ & $<$ & $<$ & $<$ & $<$ \\
\hline Zinc & & 0.0823 & $<$ & $<$ & $<$ & $<$ & $<$ & $<$ & $<$ \\
\hline
\end{tabular}


APPENDIX D.1: CY 2002 MONITORING DATA FOR THE BEAR CREEK HYDROGEOLOGIC REGIME

Field Measurements, Miscellaneous Analytes, Major Ions, and Trace Metals

\begin{tabular}{|c|c|c|c|c|c|c|c|c|c|}
\hline Sampling Point & & \multicolumn{2}{|c|}{ GW-740 } & \multicolumn{4}{|c|}{ GW-835 } & \multicolumn{2}{|c|}{ GW-916 } \\
\hline Location & & \multicolumn{2}{|c|}{ EXP-C } & \multicolumn{4}{|c|}{ S3 } & \multicolumn{2}{|c|}{ EMWMF } \\
\hline Date Sampled & & $01 / 29 / 02$ & $07 / 22 / 02$ & $03 / 04 / 02$ & $05 / 20 / 02$ & $08 / 22 / 02$ & $11 / 19 / 02$ & $08 / 15 / 02$ & $12 / 10 / 02$ \\
\hline Program & & GWPP & GWPP & BJC & BJC & BJC & BJC & BJC & BJC \\
\hline \multicolumn{10}{|l|}{ Sample Type } \\
\hline Field Measurements & & & & & & & & & \\
\hline Time Sampled & & $10: 30$ & $11: 00$ & $10: 46$ & $13: 14$ & $13: 49$ & $14: 10$ & $10: 00$ & $10: 13$ \\
\hline Measuring Point Elev. (ft) & & $1,019.63$ & $1,019.63$ & $1,000.91$ & $1,000.91$ & $1,000.91$ & $1,000.91$ & $1,002.85$ & $1,002.85$ \\
\hline Depth to Water (ft) & & 65.99 & 72.43 & 15.31 & 15.04 & 15.52 & 14.80 & 7.60 & 4.85 \\
\hline Groundwater Elevation (ft) & & 953.64 & 947.20 & 985.60 & 985.87 & 985.39 & 986.11 & 995.25 & 998.00 \\
\hline Conductivity $(\mu \mathrm{mho} / \mathrm{cm})$ & & 614 & 599 & 923 & 900 & 932 & 915 & 444 & 384 \\
\hline Dissolved Oxygen (ppm) & & 1.86 & 1.19 & 3.18 & 2.4 & 2.85 & 7.45 & 3.1 & 5.25 \\
\hline Oxidation/Reduction (mV) & & 207 & 71 & 177 & 160 & 213 & 185 & 171 & 203 \\
\hline Temperature (degrees C) & & 13.5 & 15 & 15.2 & 16.3 & 21 & 19.5 & 17.2 & 13.6 \\
\hline Turbidity (NTU) & & & & 5 & 20 & 8 & 1 & 18 & 18 \\
\hline $\mathrm{pH}$ & & 7.29 & 7.14 & 6.63 & 6.72 & 6.48 & 6.77 & 7.32 & 7.32 \\
\hline \multicolumn{10}{|l|}{ Miscellaneous Analytes } \\
\hline Conductivity $(\mu \mathrm{mho} / \mathrm{cm})$ & & 548 & 558 & & & & & & \\
\hline Dissolved Solids (mg/L) & & 290 & 317 & & & & & & \\
\hline Suspended Solids (mg/L) & & $<$ & $<$ & & & & & & \\
\hline Turbidity (NTU) & & 1.03 & 3.44 & & & & & & \\
\hline $\mathrm{pH}$ & & 7.57 & 7.59 & & & & & & \\
\hline \multicolumn{10}{|l|}{ Major lons (mg/L) } \\
\hline Calcium & & 69.3 & 67.1 & 147 & 146 & $<$ & 130 & & \\
\hline Magnesium & & 32.8 & 30.4 & 23.9 & 24.3 & 24.8 & 23.8 & & \\
\hline Potassium & & $<$ & $<$ & 4.59 & 5.87 & 5.93 & $<$ & & \\
\hline Sodium & & 2.11 & 1.85 & 17.8 & 21.3 & 18.2 & $<$ & & \\
\hline Alkalinity as $\mathrm{CO} 3$ & & $<$ & $<$ & $<$ & $<$ & $<$ & $<$ & & \\
\hline Alkalinity as $\mathrm{HCO} 3$ & & 278 & 274 & 316 & 313 & 328 & 362 & & \\
\hline Chloride & & 7.1 & 5.93 & 19.1 & 30.5 & 24.1 & 16.1 & & \\
\hline Fluoride & & 0.177 & 0.189 & 0.52 & 0.5 & 0.64 & 0.7 & & \\
\hline Nitrate as $\mathrm{N}$ & & 2.44 & 2.29 & 19.9 & 11.5 & 15 & 6.5 & & \\
\hline Sulfate & & 12.7 & 11.8 & 228 & 83 & 99.1 & 91 & & \\
\hline Charge balance & (RPD) & 0.4 & -1.1 & -12.3 & 3.3 & 0.6 & -2.3 & & \\
\hline \multicolumn{10}{|l|}{ Trace Metals (mg/L) } \\
\hline Aluminum & & $<$ & $<$ & $<$ & $<$ & $<$ & $<$ & & \\
\hline Antimony & PMS & $<$ & $<$ & & & & & & \\
\hline Arsenic & PMS & & $<$ & & & & & & \\
\hline Barium & & 0.0925 & 0.086 & 0.0732 & 0.0751 & 0.0717 & 0.074 & 0.25 & 0.22 \\
\hline Beryllium & & $<$ & $<$ & $<$ & $<$ & $<$ & $<$ & & \\
\hline Boron & & $<$ & $<$ & $<$ & $<$ & $<$ & $<$ & & \\
\hline Cadmium & PMS & $<$ & $<$ & & & & & & \\
\hline Cadmium & & & & 0.0054 & 0.0043 & 0.0069 & 0.0021 & & \\
\hline Chromium & PMS & $<$ & $<$ & & & & & & \\
\hline Chromium & & & & $<$ & $<$ & $<$ & $<$ & $<$ & $<$ \\
\hline Cobalt & & $<$ & $<$ & $<$ & $<$ & $<$ & $<$ & & \\
\hline Copper & & $<$ & $<$ & $<$ & $<$ & $<$ & $<$ & & \\
\hline Iron & & 0.65 & 0.402 & $<$ & $<$ & $<$ & $<$ & & \\
\hline Lead & PMS & $<$ & 0.000672 & & & 1 & & & \\
\hline Lead & & & & $<$ & $<$ & $<$ & $<$ & $<$ & $<$ \\
\hline Lithium & & 0.0154 & 0.0143 & 0.0261 & 0.025 & 0.0236 & 0.04 & & \\
\hline Manganese & & $<$ & $<$ & 1.81 & 2.18 & 2.3 & 1.6 & & \\
\hline Mercury & CVAA & $<$ & $<$ & & & & $<$ & & \\
\hline Nickel & PMS & $<$ & $<$ & & & & & & \\
\hline Nickel & & & & $<$ & $<$ & $<$ & $<$ & & \\
\hline Selenium & PMS & $<$ & $<$ & & & & & & \\
\hline Strontium & & 0.0574 & 0.0534 & 0.41 & 0.402 & 0.419 & 0.41 & 0.86 & 0.79 \\
\hline Thallium & PMS & $<$ & $<$ & & & & & & \\
\hline Thallium & & & & $<$ & $<$ & $<$ & 0.002 & & \\
\hline Uranium & PMS & $<$ & 0.000522 & & & & & & \\
\hline Uranium & KPA & . & & 1.21 & 1.37 & 1.45 & 1.31 & & \\
\hline Vanadium & & $<$ & $<$ & $<$ & $<$ & $<$ & $<$ & & \\
\hline Zinc & & $<$ & $<$ & $<$ & $<$ & $<$ & $<$ & & \\
\hline
\end{tabular}


APPENDIX D.1: CY 2002 MONITORING DATA FOR THE BEAR CREEK HYDROGEOLOGIC REGIME

Field Measurements, Miscellaneous Analytes, Major Ions, and Trace Metals

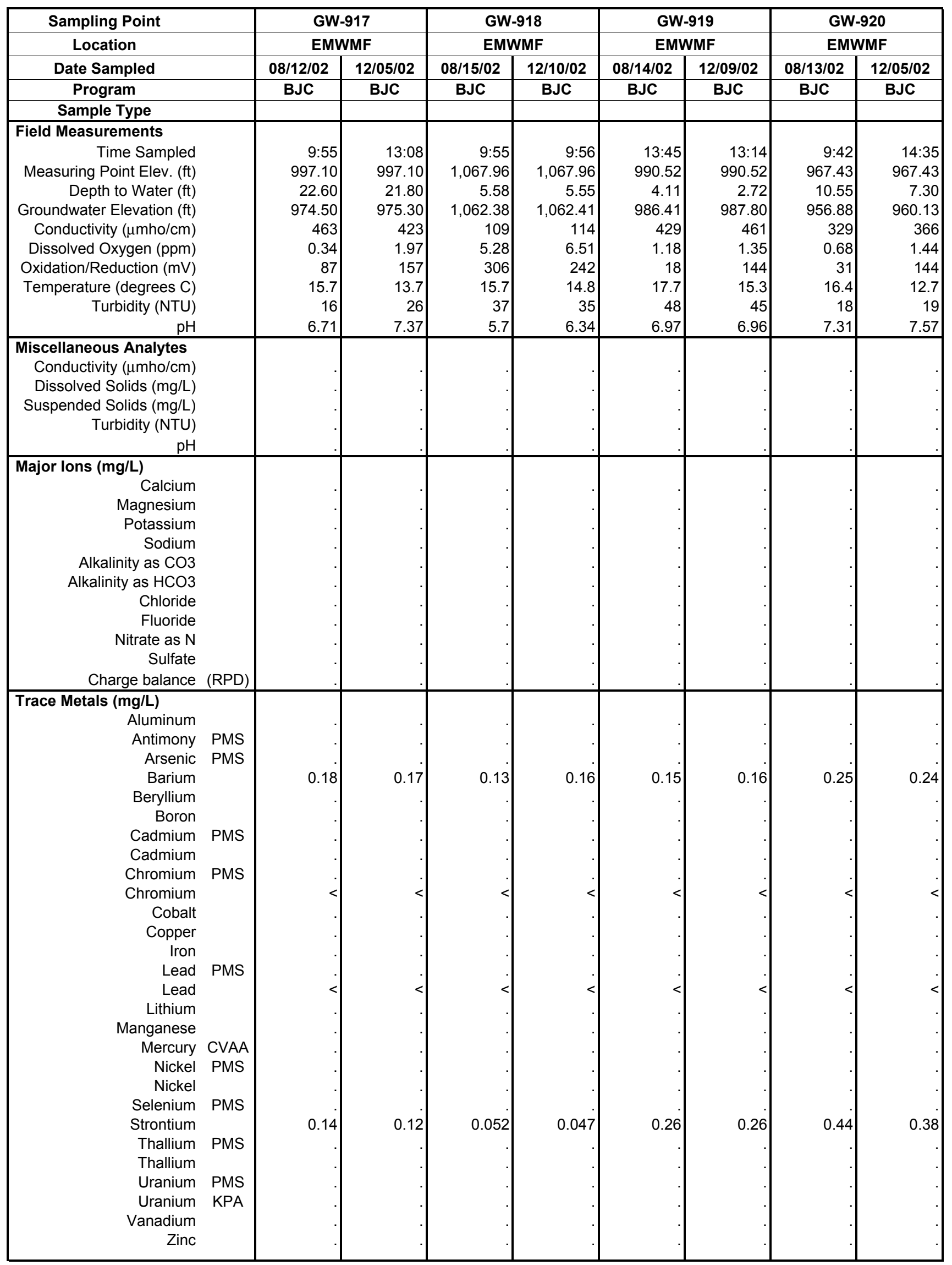


APPENDIX D.1: CY 2002 MONITORING DATA FOR THE BEAR CREEK HYDROGEOLOGIC REGIME

Field Measurements, Miscellaneous Analytes, Major Ions, and Trace Metals

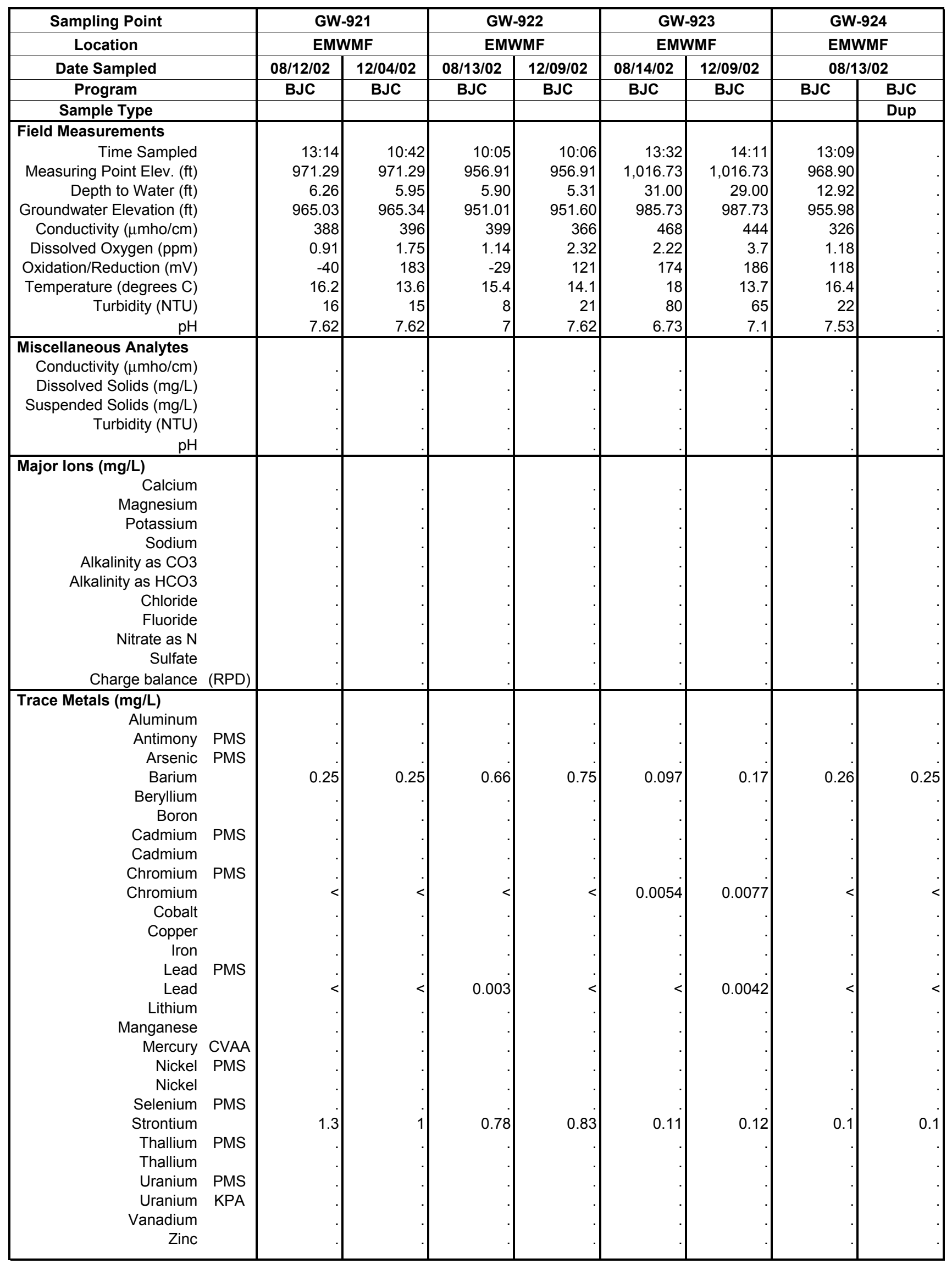


APPENDIX D.1: CY 2002 MONITORING DATA FOR THE BEAR CREEK HYDROGEOLOGIC REGIME

Field Measurements, Miscellaneous Analytes, Major Ions, and Trace Metals

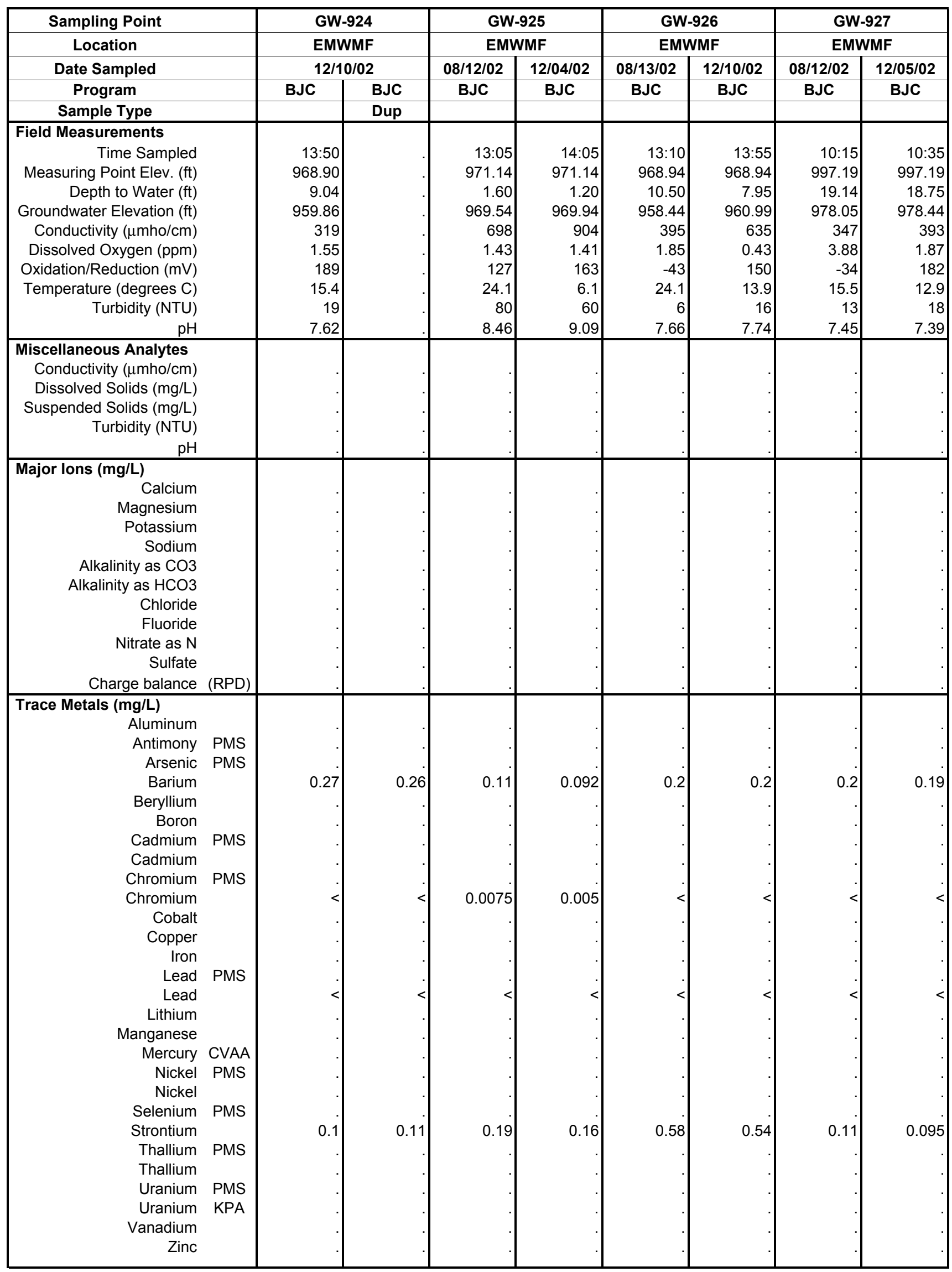


APPENDIX D.1: CY 2002 MONITORING DATA FOR THE BEAR CREEK HYDROGEOLOGIC REGIME

Field Measurements, Miscellaneous Analytes, Major Ions, and Trace Metals

\begin{tabular}{|c|c|c|c|c|c|c|c|c|c|}
\hline Sampling Point & & \multicolumn{2}{|c|}{ NT-01 } & NT-03 & NT-07 & NT-08 & NT-8-E & NT-8-W & S07 \\
\hline Location & & \multicolumn{2}{|c|}{ EXP-SW } & EXP-SW & EXP-SW & EXP-SW & EXP-SW & EXP-SW & EXP-SW \\
\hline Date Sampled & & $01 / 09 / 02$ & 07/11/02 & 03/11/02 & 03/13/02 & 03/13/02 & 03/13/02 & 03/13/02 & 03/11/02 \\
\hline Program & & GWPP & GWPP & BJC & BJC & BJC & BJC & BJC & BJC \\
\hline \multicolumn{10}{|l|}{ Sample Type } \\
\hline \multicolumn{10}{|l|}{ Field Measurements } \\
\hline Time Sampled & & $11: 10$ & $9: 30$ & $13: 26$ & $13: 16$ & $12: 49$ & $13: 32$ & $13: 48$ & $14: 00$ \\
\hline Measuring Point Elev. (ft) & & & & & & & & & \\
\hline Depth to Water (ft) & & & & & & & & & \\
\hline Groundwater Elevation (ft) & & & & & & & & & \\
\hline Conductivity $(\mu \mathrm{mho} / \mathrm{cm})$ & & 2,650 & 9,130 & 200 & 164 & 131 & 56 & 156 & 373 \\
\hline Dissolved Oxygen (ppm) & & 7.15 & 2.68 & 12.75 & 12.94 & 11.97 & 12.09 & 12.71 & 13.9 \\
\hline Oxidation/Reduction (mV) & & 250 & 209 & 110 & 115 & 135 & -30 & 20 & 150 \\
\hline Temperature (degrees C) & & 2 & 22.8 & 11.2 & 10.3 & 11.9 & 11.7 & 11.1 & 9.5 \\
\hline Turbidity (NTU) & & & & 3 & 13 & 19 & 3 & 19 & 3 \\
\hline $\mathrm{pH}$ & & 6.57 & 6.56 & 8.31 & 8.14 & 8.9 & 8.17 & 7.79 & 8.13 \\
\hline \multicolumn{10}{|l|}{ Miscellaneous Analytes } \\
\hline Conductivity $(\mu \mathrm{mho} / \mathrm{cm})$ & & 2,300 & 9,440 & & & & & & \\
\hline Dissolved Solids (mg/L) & & 1,630 & 7,540 & & 197 & 161 & 209 & 83 & \\
\hline Suspended Solids (mg/L) & & 4 & 3 & & $<$ & $<$ & 7.2 & 9.1 & \\
\hline Turbidity (NTU) & & 7.2 & 5.79 & & & & & & \\
\hline $\mathrm{pH}$ & & 6.58 & 6.8 & & & & & & \\
\hline \multicolumn{10}{|l|}{ Major lons (mg/L) } \\
\hline Calcium & & 341 & 1,450 & & 50.9 & 41.4 & 52.9 & 14.9 & \\
\hline Magnesium & & 43.3 & 186 & & 8.17 & 4.42 & 5.64 & 3.06 & \\
\hline Potassium & & 4.97 & 27 & . & 2.39 & 1.79 & 3.1 & 1.59 & \\
\hline Sodium & & 43.7 & 212 & & 4.94 & 3.59 & 2.69 & 4.1 & \\
\hline Alkalinity as $\mathrm{CO} 3$ & & $<$ & $<$ & $<$ & $<$ & $<$ & $<$ & $<$ & $<$ \\
\hline Alkalinity as $\mathrm{HCO} 3$ & & 185 & 238 & 144 & 128 & 110 & 136 & 39.4 & 71.9 \\
\hline Chloride & & 28.8 & 111 & 2.9 & 18.9 & 8 & 6.3 & 8.4 & 6.3 \\
\hline Fluoride & & 1.71 & 4.16 & 0.79 & $<$ & $<$ & $<$ & 0.2 & $<$ \\
\hline Nitrate as $\mathrm{N}$ & & 479 & 1,180 & 0.02 & 0.11 & 0.045 & 0.13 & 0.029 & 61.2 \\
\hline Sulfate & & 26.4 & 55.2 & 50.7 & 16.8 & 9.5 & 14.6 & 6.5 & 12.8 \\
\hline Charge balance & (RPD) & $-27 \mathrm{R}$ & 2.1 & & 0.5 & 0 & 1.4 & 1.7 & \\
\hline \multicolumn{10}{|l|}{ Trace Metals (mg/L) } \\
\hline Aluminum & & 1.72 & $<$ & & $<$ & 0.204 & 0.443 & $<$ & \\
\hline Antimony & PMS & $<$ & $<$ & & & & & & \\
\hline Arsenic & PMS & $<$ & $<$ & & & & & & \\
\hline Barium & & 0.755 & 3.7 & & 0.041 & 0.063 & 0.0672 & 0.0383 & \\
\hline Beryllium & & 0.00086 & $<$ & & $<$ & $<$ & $<$ & $<$ & \\
\hline Boron & & & $<$ & & 0.308 & 0.97 & 1.95 & 1.35 & \\
\hline Cadmium & PMS & 0.0493 & 0.24 & & & & & & \\
\hline Cadmium & & & & & $<$ & $<$ & $<$ & $<$ & \\
\hline Chromium & PMS & $<$ & $<$ & & & . & . & . & \\
\hline Chromium & & & & & $<$ & $<$ & $<$ & $<$ & \\
\hline Cobalt & & 0.0227 & $<$ & & $<$ & $<$ & $<$ & $<$ & \\
\hline Copper & & $<$ & $<$ & & $<$ & $<$ & $<$ & $<$ & \\
\hline Iron & & 0.0642 & $<$ & & 0.248 & 0.636 & 1.13 & 1.02 & \\
\hline Lead & PMS & $<$ & $<$ & & & & & & \\
\hline Lead & & & & & $<$ & $<$ & $<$ & $<$ & \\
\hline Lithium & & $<$ & $<$ & & 0.0693 & 0.178 & 0.566 & 0.0898 & \\
\hline Manganese & & 9.32 & 46.1 & & 0.0103 & 0.212 & 0.177 & 0.242 & \\
\hline Mercury & CVAA & $<$ & $<$ & & & & & & \\
\hline Nickel & PMS & 0.225 & 0.997 & & & & & & \\
\hline Nickel & & & & & $<$ & $<$ & $<$ & $<$ & \\
\hline Selenium & PMS & $<$ & $<$ & & & & & & \\
\hline Strontium & & 0.878 & 3.68 & & 0.115 & 0.0964 & 0.0966 & 0.0535 & \\
\hline Thallium & PMS & $<$ & $<$ & & & & & & \\
\hline Thallium & & & & & $<$ & $<$ & $<$ & $<$ & \\
\hline Uranium & PMS & 0.0286 & 0.132 & & & & & & \\
\hline Uranium & KPA & & & & 0.0154 & 0.361 & $1.16 \mathrm{Q}$ & 0.016 & \\
\hline Vanadium & & $<$ & $<$ & & $<$ & $<$ & $<$ & $<$ & \\
\hline Zinc & & $<$ & $<$ & & & $<$ & $<$ & $<$ & \\
\hline
\end{tabular}


APPENDIX D.1: CY 2002 MONITORING DATA FOR THE BEAR CREEK HYDROGEOLOGIC REGIME

Field Measurements, Miscellaneous Analytes, Major Ions, and Trace Metals

\begin{tabular}{|c|c|c|c|c|c|c|c|c|}
\hline Sampling Point & & \multicolumn{2}{|c|}{ SS-1 } & \multicolumn{2}{|c|}{ SS-4 } & \multicolumn{3}{|c|}{ SS-5 } \\
\hline Location & & \multicolumn{2}{|c|}{ EXP-SW } & \multicolumn{2}{|c|}{ EXP-SW } & \multicolumn{3}{|c|}{ EXP-SW } \\
\hline Date Sampled & & $01 / 09 / 02$ & $07 / 15 / 02$ & $01 / 08 / 02$ & $07 / 10 / 02$ & \multicolumn{2}{|c|}{$01 / 08 / 02$} & $07 / 10 / 02$ \\
\hline Program & & GWPP & GWPP & GWPP & GWPP & GWPP & GWPP & GWPP \\
\hline Sample Type & & & & & & & Dup & \\
\hline Field Measurements & & & & & & & & \\
\hline $\begin{array}{r}\text { Time Sampled } \\
\text { Measuring Point Elev. }(\mathrm{ft}) \\
\text { Depth to Water (ft) } \\
\text { Groundwater Elevation }(\mathrm{ft})\end{array}$ & & $10: 55$ & $7: 20$ & $11: 00$ & $9: 45$ & $10: 30$ & $10: 30$ & $9: 20$ \\
\hline Conductivity $(\mu \mathrm{mho} / \mathrm{cm})$ & & 1,560 & 1,401 & 1,154 & 787 & 849 & 849 & 558 \\
\hline Dissolved Oxygen (ppm) & & 7.54 & 3.65 & 5.73 & 1.16 & 5.41 & 5.41 & 3.63 \\
\hline Oxidation/Reduction (mV) & & 216 & 208 & 222 & 164 & 233 & 233 & 168 \\
\hline $\begin{array}{r}\text { Temperature (degrees C) } \\
\text { Turbidity (NTU) }\end{array}$ & & 7 & 18.9 & 7.9 & 15.3 & 10.1 & 10.1 & 15.9 \\
\hline $\mathrm{pH}$ & & 7.57 & 7.06 & 7.01 & 6.77 & 7.21 & 7.21 & 7.06 \\
\hline Miscellaneous Analytes & & & & & & & & \\
\hline Conductivity $(\mu \mathrm{mho} / \mathrm{cm})$ & & 1,290 & 1,314 & 874 & 737 & 639 & 635 & 525 \\
\hline Dissolved Solids (mg/L) & & 827 & 869 & 642 & 455 & 387 & 386 & 325 \\
\hline Suspended Solids (mg/L) & & $<$ & 9 & $<$ & 2 & $<$ & $<$ & $<$ \\
\hline Turbidity (NTU) & & 1.9 & 10.7 & 1.34 & 2.17 & 1.8 & 1.83 & 3.12 \\
\hline $\mathrm{pH}$ & & 7.66 & 7.48 & 7.26 & 7.26 & 7.38 & 7.39 & 7.59 \\
\hline Major lons (mg/L) & & & & & & & & \\
\hline Calcium & & 215 & 199 & 133 & 97.7 & 96.9 & 94 & 70.5 \\
\hline Magnesium & & 20.2 & 19.1 & 20.3 & 23.9 & 19.7 & 19.3 & 20.5 \\
\hline Potassium & & 4.19 & 4.02 & 2.75 & 2.79 & 2.14 & $<$ & $<$ \\
\hline Sodium & & 39.8 & 38.1 & 17.1 & 14.1 & 11.6 & 11.4 & 7.39 \\
\hline Alkalinity as $\mathrm{CO} 3$ & & $<$ & $<$ & $<$ & $<$ & $<$ & $<$ & $<$ \\
\hline Alkalinity as $\mathrm{HCO} 3$ & & 376 & 366 & 210 & 288 & 218 & 218 & 218 \\
\hline Chloride & & 120 & 130 & 24.3 & 31 & 19.2 & 18.8 & 16.2 \\
\hline Fluoride & & 0.279 & 0.271 & 0.502 & 0.241 & 0.241 & 0.245 & 0.156 \\
\hline Nitrate as $\mathrm{N}$ & & 18.5 & 17.4 & 43.7 & 8.53 & 16.6 & 16.5 & 4.48 \\
\hline Sulfate & & 69.9 & 54.4 & 31.2 & 20.4 & 22.6 & 23.7 & 13.6 \\
\hline Charge balance & (RPD) & 1.9 & -0.4 & 2.5 & -1 & 3.3 & 1.5 & 0.9 \\
\hline Trace Metals (mg/L) & & & & & & & & \\
\hline Aluminum & & $<$ & 1.38 & $<$ & $<$ & $<$ & $<$ & 0.272 \\
\hline Antimony & PMS & $<$ & $<$ & $<$ & $<$ & $<$ & $<$ & $<$ \\
\hline Arsenic & PMS & $<$ & $<$ & $<$ & $<$ & $<$ & $<$ & $<$ \\
\hline Barium & & 0.1 & 0.103 & 0.165 & 0.154 & 0.106 & 0.104 & 0.0945 \\
\hline Beryllium & & $<$ & $<$ & $<$ & $<$ & $<$ & $<$ & $<$ \\
\hline Boron & & $<$ & $<$ & $<$ & 0.185 & $<$ & $<$ & $<$ \\
\hline Cadmium & PMS & $<$ & $<$ & $<$ & $<$ & $<$ & $<$ & $<$ \\
\hline Cadmium & & . & 1 & & & . & & \\
\hline $\begin{array}{l}\text { Chromium } \\
\text { Chromium }\end{array}$ & PMS & $<$ & $<$ & $<$ & $<$ & $<$ & $<$ & $<$ \\
\hline Cobalt & & $<$ & $<$ & $<$ & $<$ & $<$ & $<$ & $<$ \\
\hline Copper & & $<$ & $<$ & $<$ & $<$ & $<$ & $<$ & $<$ \\
\hline Iron & & 0.116 & 0.934 & 0.135 & 0.153 & 0.0621 & 0.0736 & 0.156 \\
\hline Lead & PMS & 0.000689 & 0.00216 & $<$ & $<$ & $<$ & $<$ & 0.000512 \\
\hline Lead & & & & & & & & \\
\hline Lithium & & $<$ & $<$ & 0.0167 & 0.0208 & 0.0117 & 0.0118 & $<$ \\
\hline Manganese & & 0.018 & 0.16 & 0.0461 & 0.0267 & $<$ & $<$ & 0.0105 \\
\hline Mercury & CVAA & $<$ & $<$ & $<$ & $<$ & $<$ & $<$ & $<$ \\
\hline Nickel & PMS & $<$ & $<$ & 0.00563 & 0.00572 & $<$ & $<$ & $<$ \\
\hline Nickel & & & & & & & & \\
\hline Selenium & PMS & $<$ & $<$ & $<$ & $<$ & $<$ & $<$ & $<$ \\
\hline Strontium & & 0.647 & 0.616 & 0.354 & 0.241 & 0.209 & 0.206 & 0.137 \\
\hline Thallium & PMS & $<$ & $<$ & $<$ & $<$ & $<$ & $<$ & $<$ \\
\hline Thallium & & & & & & & & \\
\hline Uranium & PMS & 0.0415 & 0.0373 & 0.182 & 0.0945 & 0.109 & 0.106 & 0.0476 \\
\hline Uranium & KPA & & & & & & & \\
\hline Vanadium & & $<$ & $<$ & $<$ & $<$ & $<$ & $<$ & $<$ \\
\hline Zinc & & $<$ & $<$ & $<$ & $<$ & $<$ & $<$ & $<$ \\
\hline
\end{tabular}


APPENDIX D.1: CY 2002 MONITORING DATA FOR THE BEAR CREEK HYDROGEOLOGIC REGIME Field Measurements, Miscellaneous Analytes, Major lons, and Trace Metals

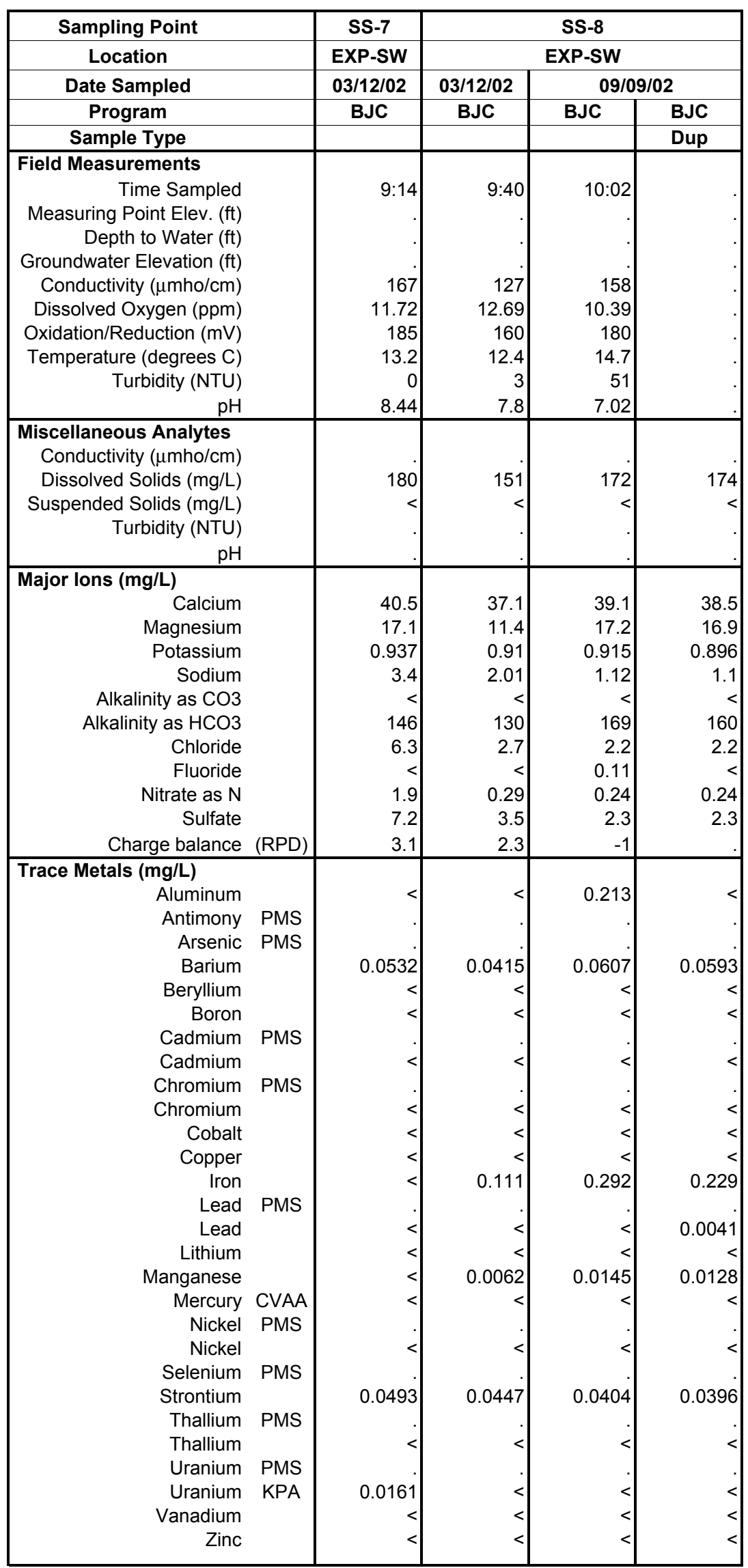


APPENDIX D.2

VOLATILE ORGANIC COMPOUNDS 
APPENDIX D.2: CY 2002 MONITORING DATA FOR THE BEAR CREEK HYDROGEOLOGIC REGIME Volatile Organic Compounds

\begin{tabular}{|c|c|c|c|c|c|c|c|c|c|c|}
\hline Sampling Point & \multicolumn{2}{|c|}{ BCK-00.63 } & \multicolumn{2}{|c|}{ BCK-03.30 } & \multicolumn{2}{|c|}{ BCK-04.55 } & \multicolumn{2}{|c|}{ BCK-07.87 } & \multicolumn{2}{|c|}{ BCK-09.20 } \\
\hline Location & \multicolumn{2}{|c|}{ EXP-SW } & \multicolumn{2}{|c|}{ EXP-SW } & \multicolumn{2}{|c|}{ EXP-SW } & \multicolumn{2}{|c|}{ EXP-SW } & \multicolumn{2}{|c|}{ EXP-SW } \\
\hline Date Sampled & 01/08/02 & 07/10/02 & 03/12/02 & 09/09/02 & $01 / 09 / 02$ & 07/10/02 & 01/08/02 & $07 / 10 / 02$ & $03 / 11 / 02$ & 09/09/02 \\
\hline Program & GWPP & GWPP & BJC & BJC & GWPP & GWPP & GWPP & GWPP & BJC & BJC \\
\hline \multicolumn{11}{|l|}{ Sample Type } \\
\hline Chloroethenes ( $\mu \mathrm{g} / \mathrm{L}$ ) & & & & & & & & & & \\
\hline Tetrachloroethene & $<$ & $<$ & $<$ & $<$ & $<$ & $<$ & $<$ & $<$ & $<$ & $<$ \\
\hline Trichloroethene & $<$ & $<$ & $<$ & $<$ & $<$ & $<$ & $<$ & $<$ & $<$ & $<$ \\
\hline cis-1,2-Dichloroethene & $<$ & $<$ & $<$ & $<$ & $<$ & $<$ & $<$ & $<$ & $2 \mathrm{~J}$ & $<$ \\
\hline trans-1,2-Dichloroethene & $<$ & $<$ & $<$ & $<$ & $<$ & $<$ & $<$ & $<$ & $<$ & $<$ \\
\hline 1,1-Dichloroethene & $<$ & $<$ & $<$ & $<$ & $<$ & $<$ & $<$ & $<$ & $<$ & $<$ \\
\hline Vinyl chloride & $<$ & $<$ & $<$ & $<$ & $<$ & $<$ & $<$ & $<$ & $<$ & $<$ \\
\hline \multicolumn{11}{|l|}{ Chloroethanes ( $\mu \mathrm{g} / \mathrm{L})$} \\
\hline 1,1,1-Trichloroethane & $<$ & $<$ & $<$ & $<$ & $<$ & $<$ & $<$ & $<$ & $<$ & $<$ \\
\hline 1,1,2-Trichloroethane & $<$ & $<$ & $<$ & $<$ & $<$ & $<$ & $<$ & $<$ & $<$ & $<$ \\
\hline 1,2-Dichloroethane & $<$ & $<$ & $<$ & $<$ & $<$ & $<$ & $<$ & $<$ & $<$ & $<$ \\
\hline 1,1-Dichloroethane & $<$ & $<$ & $<$ & $<$ & $<$ & $<$ & $<$ & $<$ & $<$ & $<$ \\
\hline Chloroethane & $<$ & $<$ & $<$ & $<$ & $<$ & $<$ & $<$ & $<$ & $<$ & $<$ \\
\hline \multicolumn{11}{|l|}{ Chloromethanes $(\mu \mathrm{g} / \mathrm{L})$} \\
\hline Carbon tetrachloride & $<$ & $<$ & $<$ & $<$ & $<$ & $<$ & $<$ & $<$ & $<$ & $<$ \\
\hline Chloroform & $<$ & $<$ & $<$ & $<$ & $<$ & $<$ & $<$ & $<$ & $<$ & $<$ \\
\hline Methylene chloride & $<$ & $<$ & $<$ & $<$ & $<$ & $<$ & $<$ & $<$ & $<$ & $<$ \\
\hline Chloromethane & $<$ & $<$ & $<$ & $<$ & $<$ & $<$ & $<$ & $<$ & $<$ & $<$ \\
\hline \multicolumn{11}{|l|}{ Petrol. Hydrocarb. ( $\mu \mathrm{g} / \mathrm{L})$} \\
\hline Benzene & $<$ & $<$ & $<$ & $<$ & $<$ & $<$ & $<$ & $<$ & $<$ & $<$ \\
\hline Dimethylbenzene & $<$ & $<$ & $<$ & $<$ & $<$ & $<$ & $<$ & $<$ & $<$ & $<$ \\
\hline Ethylbenzene & $<$ & $<$ & $<$ & $<$ & $<$ & $<$ & $<$ & $<$ & $<$ & $<$ \\
\hline Toluene & $<$ & $<$ & $<$ & $<$ & $<$ & $<$ & $<$ & $<$ & $<$ & $<$ \\
\hline \multicolumn{11}{|l|}{ Miscellaneous ( $\mu \mathrm{g} / \mathrm{L})$} \\
\hline 1,2-Dichloropropane & $<$ & $<$ & $<$ & $<$ & $<$ & $<$ & $<$ & $<$ & $<$ & $<$ \\
\hline 1,4-Dichlorobenzene & $<$ & $<$ & & & $<$ & $<$ & $<$ & $<$ & & \\
\hline 2-Butanone & $<$ & $<$ & $<$ & $<$ & $<$ & $<$ & $<$ & $<$ & $<$ & $<$ \\
\hline Acetone & $<$ & $<$ & $<$ & $<$ & $<$ & $<$ & $<$ & $<$ & $<$ & $<$ \\
\hline Bromoform & $<$ & $<$ & $<$ & $<$ & $<$ & $<$ & $<$ & $<$ & $<$ & $<$ \\
\hline Chlorobenzene & $<$ & $<$ & $<$ & $<$ & $<$ & $<$ & $<$ & $<$ & $<$ & $<$ \\
\hline Dichlorodifluoromethane & $<$ & $<$ & & & $<$ & $<$ & $<$ & $<$ & & \\
\hline Trichlorofluoromethane & $<$ & $<$ & & & $<$ & $<$ & $<$ & $<$ & & \\
\hline
\end{tabular}


APPENDIX D.2: CY 2002 MONITORING DATA FOR THE BEAR CREEK HYDROGEOLOGIC REGIME

Volatile Organic Compounds

\begin{tabular}{|c|c|c|c|c|c|c|c|c|c|c|c|}
\hline Sampling Point & \multicolumn{3}{|c|}{ BCK-09.40 } & BCK-09.47 & \multicolumn{2}{|c|}{ BCK-11.54 } & BCK-11.84 & \multicolumn{2}{|c|}{ BCK-11.97 } & \multicolumn{2}{|c|}{ GW-008 } \\
\hline Location & \multicolumn{3}{|c|}{ EXP-SW } & EXP-SW & \multicolumn{2}{|c|}{ EXP-SW } & EXP-SW & \multicolumn{2}{|c|}{ EXP-SW } & \multicolumn{2}{|c|}{ OLF } \\
\hline Date Sampled & 01/09/02 & \multicolumn{2}{|c|}{ 07/11/02 } & 03/11/02 & 03/11/02 & 09/09/02 & 03/11/02 & 01/09/02 & 07/11/02 & 01/07/02 & 07/02/02 \\
\hline Program & GWPP & GWPP & GWPP & BJC & BJC & BJC & BJC & GWPP & GWPP & BJC & BJC \\
\hline Sample Type & & & Dup & & & & & & & & \\
\hline Chloroethenes ( $\mu \mathrm{g} / \mathrm{L}$ ) & & & & & & & & & & & \\
\hline Tetrachloroethene & $2 \mathrm{~J}$ & $<$ & $<$ & $2 \mathrm{~J}$ & $<$ & $<$ & $<$ & $<$ & $<$ & 53 & 89 \\
\hline trans-1,2-Dichloroethene & $<$ & $<$ & $<$ & $<$ & $<$ & $<$ & $<$ & $<$ & $<$ & $<$ & $<$ \\
\hline 1,1-Dichloroethene & $<$ & $<$ & $<$ & $<$ & $<$ & $<$ & $<$ & $<$ & $<$ & 6 & 7 \\
\hline Vinyl chloride & $<$ & $<$ & $<$ & $<$ & $<$ & $<$ & $<$ & $<$ & $<$ & $<$ & $<$ \\
\hline Chloroethanes ( $\mu \mathrm{g} / \mathrm{L}$ ) & & & & & & & & & & & \\
\hline 1,1,1-Trichloroethane & $<$ & $<$ & $<$ & $<$ & $<$ & $<$ & $<$ & $<$ & $<$ & $<$ & $<$ \\
\hline Chloromethanes ( $\mu \mathrm{g} / \mathrm{L})$ & & & & & & & & & & & \\
\hline Carbon tetrachloride & $<$ & $<$ & $<$ & $<$ & $<$ & $<$ & $<$ & $<$ & $<$ & $<$ & $<$ \\
\hline Chloroform & $<$ & $<$ & $<$ & $<$ & $<$ & $<$ & $<$ & $<$ & $<$ & $<$ & $<$ \\
\hline Methylene chloride & $<$ & $<$ & $<$ & $<$ & $<$ & $<$ & $<$ & $<$ & $<$ & $<$ & $<$ \\
\hline Chloromethane & $<$ & $<$ & $<$ & $2 \mathrm{~J}$ & $<$ & $<$ & $<$ & $<$ & $<$ & $<$ & $<$ \\
\hline Petrol. Hydrocarb. ( $\mu \mathrm{g} / \mathrm{L})$ & & & & & & & & & & & \\
\hline Benzene & $<$ & $<$ & $<$ & $<$ & $<$ & $<$ & $<$ & $<$ & $<$ & $1 \mathrm{~J}$ & $1 \mathrm{~J}$ \\
\hline Dimethylbenzene & $<$ & $<$ & $<$ & $<$ & $<$ & $<$ & $<$ & $<$ & $<$ & $<$ & $<$ \\
\hline Ethylbenzene & $<$ & $<$ & $<$ & $<$ & $<$ & $<$ & $<$ & $<$ & $<$ & $<$ & $<$ \\
\hline Toluene & $<$ & $<$ & $<$ & $<$ & $<$ & $<$ & $<$ & $<$ & $<$ & $<$ & $<$ \\
\hline Miscellaneous ( $\mu \mathrm{g} / \mathrm{L}$ ) & & & & & & & & & & & \\
\hline
\end{tabular}


APPENDIX D.2: CY 2002 MONITORING DATA FOR THE BEAR CREEK HYDROGEOLOGIC REGIME Volatile Organic Compounds

\begin{tabular}{|c|c|c|c|c|c|c|c|c|c|c|}
\hline Sampling Point & \multicolumn{3}{|c|}{ GW-014 } & \multicolumn{2}{|c|}{ GW-046 } & \multicolumn{2}{|c|}{ GW-061 } & \multicolumn{3}{|c|}{ GW-066 } \\
\hline Location & \multicolumn{3}{|c|}{ BG } & \multicolumn{2}{|c|}{ BG } & \multicolumn{2}{|c|}{ BG } & \multicolumn{3}{|c|}{ OLF } \\
\hline Date Sampled & 03/14/02 & 03/14/02 & 08/12/02 & $01 / 07 / 02$ & 07/02/02 & $03 / 12 / 02$ & 08/05/02 & $03 / 25 / 02$ & \multicolumn{2}{|c|}{ 08/13/02 } \\
\hline Program & GWPP & GWPP & GWPP & BJC & BJC & GWPP & GWPP & GWPP & GWPP & GWPP \\
\hline Sample Type & & Dup & & & & & & & & Dup \\
\hline Chloroethenes ( $\mu \mathrm{g} / \mathrm{L}$ ) & & & & & & & & & & \\
\hline Tetrachloroethene & 21 & 19 & 18 & 500 & 990 & $<$ & $<$ & 8 & 6 & 6 \\
\hline Trichloroethene & 160 & 170 & 140 & 1,000 & 980 & $<$ & $<$ & $5 \mathrm{~J}$ & $4 \mathrm{~J}$ & $4 \mathrm{~J}$ \\
\hline cis-1,2-Dichloroethene & 720 & 860 & 970 & 2,600 & 2,000 & $<$ & $<$ & $2 \mathrm{~J}$ & $<$ & $<$ \\
\hline trans-1,2-Dichloroethene & $2 \mathrm{~J}$ & $2 \mathrm{~J}$ & $2 \mathrm{~J}$ & 13 & 11 & $<$ & $<$ & $<$ & $<$ & $<$ \\
\hline 1,1-Dichloroethene & 37 & 40 & 25 & 83 & 40 & $<$ & $<$ & $<$ & $<$ & $<$ \\
\hline Vinyl chloride & 75 & 84 & 87 & 230 & 260 & $<$ & $<$ & $<$ & $<$ & $<$ \\
\hline Chloroethanes ( $\mu \mathrm{g} / \mathrm{L}$ ) & & & & & & & & & & \\
\hline 1,1,1-Trichloroethane & $<$ & $<$ & $<$ & 77 & 32 & $<$ & $<$ & $<$ & $<$ & $<$ \\
\hline 1,1,2-Trichloroethane & $<$ & $<$ & $<$ & $<$ & $<$ & $<$ & $<$ & $<$ & $<$ & $<$ \\
\hline 1,2-Dichloroethane & $<$ & $<$ & $<$ & $2 \mathrm{~J}$ & $2 \mathrm{~J}$ & $<$ & $<$ & $<$ & $<$ & $<$ \\
\hline 1,1-Dichloroethane & 200 & 250 & 170 & 140 & 73 & $<$ & $<$ & $<$ & $<$ & $<$ \\
\hline Chloroethane & 7 & 8 & 8 & $5 \mathrm{~J}$ & $<$ & $<$ & $<$ & $<$ & $<$ & $<$ \\
\hline Chloromethanes ( $\mu \mathrm{g} / \mathrm{L})$ & & & & & & & & & & \\
\hline Carbon tetrachloride & $<$ & $<$ & $<$ & $<$ & $<$ & $<$ & $<$ & $<$ & $<$ & $<$ \\
\hline Chloroform & $<$ & $<$ & $<$ & $2 \mathrm{~J}$ & $1 \mathrm{~J}$ & $<$ & $<$ & $<$ & $<$ & $<$ \\
\hline Methylene chloride & $<$ & $<$ & $<$ & $<$ & $<$ & $<$ & $<$ & $<$ & $<$ & $<$ \\
\hline Chloromethane & $<$ & $<$ & $<$ & $<$ & $<$ & $<$ & $<$ & $<$ & $<$ & $<$ \\
\hline Petrol. Hydrocarb. ( $\mu \mathrm{g} / \mathrm{L})$ & & & & & & & & & & \\
\hline Benzene & $<$ & $<$ & $2 \mathrm{~J}$ & 10 & 9 & $<$ & $<$ & $<$ & $<$ & $<$ \\
\hline Dimethylbenzene & $<$ & $<$ & $<$ & $2 \mathrm{~J}$ & $<$ & $<$ & $<$ & $<$ & $<$ & $<$ \\
\hline Ethylbenzene & $<$ & $<$ & $<$ & $<$ & $<$ & $<$ & $<$ & $<$ & $<$ & $<$ \\
\hline Toluene & $<$ & $<$ & $<$ & $<$ & $<$ & $<$ & $<$ & $<$ & $<$ & $<$ \\
\hline Miscellaneous ( $\mu \mathrm{g} / \mathrm{L})$ & & & & & & & & & & \\
\hline 1,2-Dichloropropane & $<$ & $<$ & $<$ & $<$ & $<$ & $<$ & $<$ & $<$ & $<$ & $<$ \\
\hline 1,4-Dichlorobenzene & $<$ & $<$ & $<$ & & & $<$ & $<$ & $<$ & $<$ & $<$ \\
\hline 2-Butanone & $<$ & $<$ & $<$ & $<$ & $<$ & $<$ & $<$ & $<$ & $<$ & $<$ \\
\hline Acetone & $<$ & $<$ & $<$ & $<$ & $<$ & $<$ & $<$ & $<$ & $<$ & $<$ \\
\hline Bromoform & $<$ & $<$ & $<$ & $<$ & $<$ & $<$ & $<$ & $<$ & $<$ & $<$ \\
\hline Chlorobenzene & $<$ & $<$ & $<$ & $<$ & $<$ & $<$ & $<$ & $<$ & $<$ & $<$ \\
\hline Dichlorodifluoromethane & $<$ & $<$ & $<$ & & & $<$ & $<$ & $<$ & $<$ & $<$ \\
\hline Trichlorofluoromethane & $<$ & $<$ & $<$ & & & $<$ & $<$ & $<$ & $<$ & $<$ \\
\hline
\end{tabular}


APPENDIX D.2: CY 2002 MONITORING DATA FOR THE BEAR CREEK HYDROGEOLOGIC REGIME Volatile Organic Compounds

\begin{tabular}{|c|c|c|c|c|c|c|c|c|c|c|}
\hline Sampling Point & \multicolumn{2}{|c|}{ GW-069 } & \multicolumn{3}{|c|}{ GW-071 } & \multicolumn{2}{|c|}{ GW-072 } & GW-076 & \multicolumn{2}{|c|}{ GW-077 } \\
\hline Location & \multicolumn{2}{|c|}{ BG } & \multicolumn{3}{|c|}{ BG } & \multicolumn{2}{|c|}{ BG } & OLF & \multicolumn{2}{|c|}{ BG } \\
\hline Date Sampled & $03 / 11 / 02$ & 08/05/02 & $03 / 12 / 02$ & \multicolumn{2}{|c|}{ 08/06/02 } & 03/11/02 & 08/06/02 & $03 / 19 / 02$ & $02 / 26 / 02$ & 08/08/02 \\
\hline Program & GWPP & GWPP & GWPP & GWPP & GWPP & GWPP & GWPP & GWPP & BJC & BJC \\
\hline Sample Type & & & & & Dup & & & & & \\
\hline Chloroethenes ( $\mu \mathrm{g} / \mathrm{L}$ ) & & & & & & & & & & \\
\hline Tetrachloroethene & $<$ & $<$ & 320 & 390 & 410 & $<$ & $<$ & $<$ & $<$ & $<$ \\
\hline Trichloroethene & $<$ & $<$ & 110 & 99 & 64 & $<$ & $<$ & $<$ & $<$ & $<$ \\
\hline cis-1,2-Dichloroethene & $<$ & $<$ & 35 & 25 & 23 & $<$ & $2 \mathrm{~J}$ & $<$ & $<$ & $<$ \\
\hline trans-1,2-Dichloroethene & $<$ & $<$ & 9 & 6 & $4 \mathrm{~J}$ & $<$ & $<$ & $<$ & $<$ & $<$ \\
\hline 1,1-Dichloroethene & $<$ & $<$ & 38 & 40 & 32 & $2 \mathrm{~J}$ & 6 & $<$ & $<$ & $<$ \\
\hline Vinyl chloride & $<$ & $2 \mathrm{~J}$ & $2 \mathrm{~J}$ & $2 \mathrm{~J}$ & $2 \mathrm{~J}$ & $<$ & $<$ & $<$ & $<$ & $<$ \\
\hline Chloroethanes ( $\mu \mathrm{g} / \mathrm{L})$ & & & & & & & & & & \\
\hline 1,1,1-Trichloroethane & $<$ & $<$ & 14 & 120 & 80 & $<$ & $<$ & $<$ & $<$ & $<$ \\
\hline 1,1,2-Trichloroethane & $<$ & $<$ & $<$ & $<$ & $<$ & $<$ & $<$ & $<$ & $<$ & $<$ \\
\hline 1,2-Dichloroethane & $<$ & $<$ & $<$ & $<$ & $<$ & $<$ & $<$ & $<$ & $<$ & $<$ \\
\hline 1,1-Dichloroethane & $<$ & $<$ & 1,780 & 1,600 & 1,600 & 64 & 130 & $<$ & $<$ & $<$ \\
\hline Chloroethane & $<$ & $<$ & 9 & 13 & 11 & $<$ & $4 \mathrm{~J}$ & $<$ & $<$ & $<$ \\
\hline Chloromethanes ( $\mu \mathrm{g} / \mathrm{L})$ & & & & & & & & & & \\
\hline Carbon tetrachloride & $<$ & $<$ & $<$ & $<$ & $<$ & $<$ & $<$ & $<$ & $<$ & $<$ \\
\hline Chloroform & $<$ & $<$ & $<$ & $<$ & $<$ & $<$ & $<$ & $<$ & $<$ & $<$ \\
\hline Methylene chloride & $<$ & $<$ & $<$ & $<$ & $<$ & $<$ & $<$ & $<$ & $<$ & $<$ \\
\hline Chloromethane & $<$ & $<$ & $<$ & $<$ & $<$ & $<$ & $<$ & $<$ & $<$ & $<$ \\
\hline Petrol. Hydrocarb. ( $\mu \mathrm{g} / \mathrm{L})$ & & & & & & & & & & \\
\hline Benzene & $<$ & $<$ & 820 & 750 & 790 & $<$ & $<$ & $<$ & $<$ & $<$ \\
\hline Dimethylbenzene & $<$ & $<$ & 7 & 9 & $<$ & $<$ & $<$ & $<$ & $<$ & $<$ \\
\hline Ethylbenzene & $<$ & $<$ & $2 \mathrm{~J}$ & $2 \mathrm{~J}$ & $<$ & $<$ & $<$ & $<$ & $<$ & $<$ \\
\hline Toluene & $<$ & $<$ & 10 & 10 & 6 & $<$ & $<$ & $<$ & $<$ & $<$ \\
\hline Miscellaneous ( $\mu \mathrm{g} / \mathrm{L})$ & & & & & & & & & & \\
\hline 1,2-Dichloropropane & $<$ & $<$ & $<$ & $<$ & $<$ & $<$ & $<$ & $<$ & $<$ & $<$ \\
\hline 1,4-Dichlorobenzene & $<$ & $<$ & $<$ & $<$ & $<$ & $<$ & $<$ & $<$ & & \\
\hline 2-Butanone & $<$ & $<$ & $<$ & $<$ & $<$ & $<$ & $<$ & $<$ & $<$ & $<$ \\
\hline Acetone & $<$ & $<$ & 48 & $<$ & $<$ & $<$ & $<$ & $<$ & $<$ & $<$ \\
\hline Bromoform & $<$ & $<$ & $<$ & $<$ & $<$ & $<$ & $<$ & $<$ & $<$ & $<$ \\
\hline Chlorobenzene & $<$ & $<$ & $<$ & $<$ & $<$ & $<$ & $<$ & $<$ & $<$ & $<$ \\
\hline Dichlorodifluoromethane & $<$ & $<$ & $<$ & $3 \mathrm{~J}$ & $3 \mathrm{~J}$ & $<$ & $<$ & $<$ & & \\
\hline Trichlorofluoromethane & $<$ & $<$ & $<$ & $<$ & $<$ & $<$ & $<$ & $<$ & & \\
\hline
\end{tabular}


APPENDIX D.2: CY 2002 MONITORING DATA FOR THE BEAR CREEK HYDROGEOLOGIC REGIME Volatile Organic Compounds

\begin{tabular}{|c|c|c|c|c|c|c|c|c|c|c|}
\hline Sampling Point & \multicolumn{2}{|c|}{ GW-078 } & \multicolumn{2}{|c|}{ GW-079 } & \multicolumn{4}{|c|}{ GW-080 } & \multicolumn{2}{|c|}{ GW-082 } \\
\hline Location & \multicolumn{2}{|c|}{ BG } & \multicolumn{2}{|c|}{ BG } & \multicolumn{4}{|c|}{ BG } & \multicolumn{2}{|c|}{ BG } \\
\hline Date Sampled & $02 / 26 / 02$ & 08/08/02 & $02 / 26 / 02$ & $08 / 07 / 02$ & \multicolumn{2}{|c|}{ 02/26/02 } & \multicolumn{2}{|c|}{ 08/07/02 } & $02 / 12 / 02$ & 07/29/02 \\
\hline Program & BJC & BJC & BJC & BJC & BJC & BJC & BJC & BJC & GWPP & GWPP \\
\hline Sample Type & & & & & & Dup & & Dup & & \\
\hline Chloroethenes ( $\mu \mathrm{g} / \mathrm{L}$ ) & & & & & & & & & & \\
\hline Tetrachloroethene & $<$ & $<$ & $<$ & $<$ & $<$ & $<$ & $<$ & $<$ & $3 \mathrm{~J}$ & $<$ \\
\hline Trichloroethene & $<$ & $<$ & $<$ & $<$ & $<$ & $<$ & $<$ & $<$ & $<$ & $<$ \\
\hline cis-1,2-Dichloroethene & $<$ & $<$ & $<$ & $<$ & $<$ & $<$ & $<$ & $<$ & 1,100 & 820 \\
\hline trans-1,2-Dichloroethene & $<$ & $<$ & $<$ & $<$ & $<$ & $<$ & $<$ & $<$ & $<$ & $<$ \\
\hline 1,1-Dichloroethene & $<$ & $<$ & $<$ & $<$ & $<$ & $<$ & $<$ & $<$ & 43 & 22 \\
\hline Vinyl chloride & $<$ & $<$ & $<$ & $<$ & $<$ & $<$ & $<$ & $<$ & 290 & 200 \\
\hline Chloroethanes ( $\mu \mathrm{g} / \mathrm{L})$ & & & & & & & & & & \\
\hline 1,1,1-Trichloroethane & $<$ & $<$ & $<$ & $<$ & $<$ & $<$ & $<$ & $<$ & 6 & $4 \mathrm{~J}$ \\
\hline 1,1,2-Trichloroethane & $<$ & $<$ & $<$ & $<$ & $<$ & $<$ & $<$ & $<$ & $<$ & $<$ \\
\hline 1,2-Dichloroethane & $<$ & $<$ & $<$ & $<$ & $<$ & $<$ & $<$ & $<$ & $<$ & $<$ \\
\hline 1,1-Dichloroethane & $<$ & $<$ & $<$ & $<$ & $<$ & $<$ & $<$ & $<$ & 1,600 & 730 \\
\hline Chloroethane & $<$ & $<$ & $<$ & $<$ & $<$ & $<$ & $<$ & $<$ & 17 & 25 \\
\hline Chloromethanes ( $\mu \mathrm{g} / \mathrm{L})$ & & & & & & & & & & \\
\hline Carbon tetrachloride & $<$ & $<$ & $<$ & $<$ & $<$ & $<$ & $<$ & $<$ & $<$ & $<$ \\
\hline Chloroform & $<$ & $<$ & $<$ & $<$ & $<$ & $<$ & $<$ & $<$ & $<$ & $<$ \\
\hline Methylene chloride & $<$ & $<$ & $<$ & $<$ & $<$ & $<$ & $<$ & $<$ & $<$ & $<$ \\
\hline Chloromethane & $<$ & $<$ & $<$ & $<$ & $<$ & $<$ & $<$ & $<$ & $<$ & $<$ \\
\hline Petrol. Hydrocarb. ( $\mu \mathrm{g} / \mathrm{L})$ & & & & & & & & & & \\
\hline Benzene & $<$ & $<$ & $<$ & $<$ & $<$ & $<$ & $<$ & $<$ & 45 & 22 \\
\hline Dimethylbenzene & $<$ & $<$ & $<$ & $<$ & $<$ & $<$ & $<$ & $<$ & $<$ & $<$ \\
\hline Ethylbenzene & $<$ & $<$ & $<$ & $<$ & $<$ & $<$ & $<$ & $<$ & $<$ & $<$ \\
\hline Toluene & $<$ & $<$ & $<$ & $<$ & $<$ & $<$ & $<$ & $<$ & $<$ & $<$ \\
\hline Miscellaneous ( $\mu \mathrm{g} / \mathrm{L})$ & & & & & & & & & & \\
\hline 1,2-Dichloropropane & $<$ & $<$ & $<$ & $<$ & $<$ & $<$ & $<$ & $<$ & $<$ & $<$ \\
\hline 1,4-Dichlorobenzene & & & & & & & & & $<$ & $<$ \\
\hline 2-Butanone & $<$ & $<$ & $<$ & $<$ & $<$ & $<$ & $<$ & $<$ & $<$ & $<$ \\
\hline Acetone & $<$ & $<$ & $<$ & $<$ & $<$ & $<$ & $<$ & $<$ & $<$ & $<$ \\
\hline Bromoform & $<$ & $<$ & $<$ & $<$ & $<$ & $<$ & $<$ & $<$ & $<$ & $<$ \\
\hline Chlorobenzene & $<$ & $<$ & $<$ & $<$ & $<$ & $<$ & $<$ & $<$ & $<$ & $<$ \\
\hline Dichlorodifluoromethane & & & & & & & & & $<$ & $<$ \\
\hline Trichlorofluoromethane & & & & & & & & & $<$ & $<$ \\
\hline
\end{tabular}


APPENDIX D.2: CY 2002 MONITORING DATA FOR THE BEAR CREEK HYDROGEOLOGIC REGIME Volatile Organic Compounds

\begin{tabular}{|c|c|c|c|c|c|c|c|c|c|c|c|}
\hline Sampling Point & \multicolumn{2}{|c|}{ GW-085 } & GW-087 & \multicolumn{2}{|c|}{ GW-091 } & \multicolumn{2}{|c|}{ GW-115 } & \multicolumn{2}{|c|}{ GW-225 } & \multicolumn{2}{|c|}{ GW-226 } \\
\hline Location & \multicolumn{2}{|c|}{ OLF } & OLF & \multicolumn{2}{|c|}{ BG } & \multicolumn{2}{|c|}{ S3 } & \multicolumn{2}{|c|}{ OLF } & \multicolumn{2}{|c|}{ OLF } \\
\hline Date Sampled & $02 / 18 / 02$ & 07/31/02 & $03 / 25 / 02$ & 03/14/02 & 08/08/02 & 01/07/02 & 07/08/02 & 02/19/02 & 08/01/02 & 02/19/02 & $08 / 01 / 02$ \\
\hline Program & GWPP & GWPP & GWPP & GWPP & GWPP & BJC & BJC & GWPP & GWPP & GWPP & GWPP \\
\hline \multicolumn{12}{|l|}{ Sample Type } \\
\hline Chloroethenes $(\mu \mathrm{g} / \mathrm{L})$ & & & & & & & & & & & \\
\hline Tetrachloroethene & $<$ & $<$ & 9 & $<$ & $<$ & $<$ & $<$ & $<$ & $<$ & $<$ & $<$ \\
\hline Trichloroethene & $<$ & $<$ & 20 & $<$ & $<$ & $<$ & $<$ & 190 & 180 & 110 & 89 \\
\hline cis-1,2-Dichloroethene & $<$ & $<$ & 59 & $<$ & $<$ & $<$ & $<$ & $3 \mathrm{~J}$ & $2 \mathrm{~J}$ & 6 & $3 \mathrm{~J}$ \\
\hline trans-1,2-Dichloroethene & $<$ & $<$ & $<$ & $<$ & $<$ & $<$ & $<$ & $<$ & $<$ & $<$ & $<$ \\
\hline 1,1-Dichloroethene & $<$ & $<$ & $<$ & $<$ & $<$ & $<$ & $<$ & $2 \mathrm{~J}$ & $<$ & $<$ & $<$ \\
\hline Vinyl chloride & $<$ & $<$ & $<$ & $<$ & $<$ & $<$ & $<$ & $<$ & $<$ & $<$ & $<$ \\
\hline Chloroethanes ( $\mu \mathrm{g} / \mathrm{L})$ & & & & & & & & & & & \\
\hline 1,1,1-Trichloroethane & $<$ & $<$ & $<$ & $<$ & $<$ & $<$ & $<$ & $<$ & $<$ & $<$ & $<$ \\
\hline 1,1,2-Trichloroethane & $<$ & $<$ & $<$ & $<$ & $<$ & $<$ & $<$ & $<$ & $<$ & $<$ & $<$ \\
\hline 1,2-Dichloroethane & $<$ & $<$ & $<$ & $<$ & $<$ & $<$ & $<$ & $<$ & $<$ & $<$ & $<$ \\
\hline 1,1-Dichloroethane & $<$ & $<$ & $<$ & $<$ & $<$ & $<$ & $<$ & $<$ & $<$ & $<$ & $<$ \\
\hline Chloroethane & $<$ & $<$ & $<$ & $<$ & $<$ & $<$ & $<$ & $<$ & $<$ & $<$ & $<$ \\
\hline Chloromethanes $(\mu \mathrm{g} / \mathrm{L})$ & & & & & & & & & & & \\
\hline Carbon tetrachloride & $<$ & $<$ & $<$ & $<$ & $<$ & $<$ & $<$ & $4 \mathrm{~J}$ & $2 \mathrm{~J}$ & $<$ & $<$ \\
\hline Chloroform & $<$ & $<$ & $<$ & $<$ & $<$ & $<$ & $<$ & $<$ & $<$ & $<$ & $<$ \\
\hline Methylene chloride & $<$ & $<$ & $<$ & $<$ & $<$ & $<$ & $<$ & $<$ & $<$ & $<$ & $<$ \\
\hline Chloromethane & $<$ & $<$ & $3 \mathrm{~J}$ & $<$ & $<$ & $<$ & $<$ & $<$ & $<$ & $<$ & $<$ \\
\hline Petrol. Hydrocarb. ( $\mu \mathrm{g} / \mathrm{L})$ & & & & & & & & & & & \\
\hline Benzene & $<$ & $<$ & $2 \mathrm{~J}$ & $<$ & $<$ & $<$ & $<$ & $<$ & $<$ & $<$ & $<$ \\
\hline Dimethylbenzene & $<$ & $<$ & $<$ & $<$ & $<$ & $<$ & $<$ & $<$ & $<$ & $<$ & $<$ \\
\hline Ethylbenzene & $<$ & $<$ & $<$ & $<$ & $<$ & $<$ & $<$ & $<$ & $<$ & $<$ & $<$ \\
\hline Toluene & $<$ & $<$ & $<$ & $<$ & $<$ & $<$ & $<$ & $<$ & $<$ & $<$ & $<$ \\
\hline Miscellaneous ( $\mu \mathrm{g} / \mathrm{L})$ & & & & & & & & & & & \\
\hline 1,2-Dichloropropane & $<$ & $<$ & $<$ & $<$ & $<$ & $<$ & $<$ & $<$ & $<$ & $<$ & $<$ \\
\hline 1,4-Dichlorobenzene & $<$ & $<$ & $<$ & $<$ & $<$ & & & $<$ & $<$ & $<$ & $<$ \\
\hline 2-Butanone & $<$ & $<$ & $<$ & $<$ & $<$ & $<$ & $<$ & $<$ & $<$ & $<$ & $<$ \\
\hline Acetone & $<$ & $<$ & $7 \mathrm{~J}$ & $<$ & $<$ & $<$ & $<$ & $<$ & $<$ & $<$ & $<$ \\
\hline Bromoform & $<$ & $<$ & $<$ & $<$ & $<$ & $<$ & $<$ & $<$ & $<$ & $<$ & $<$ \\
\hline Chlorobenzene & $<$ & $<$ & $<$ & $<$ & $<$ & $<$ & $<$ & $<$ & $<$ & $<$ & $<$ \\
\hline Dichlorodifluoromethane & $<$ & $<$ & 12 & $<$ & $<$ & & & $<$ & $<$ & $<$ & $<$ \\
\hline Trichlorofluoromethane & $<$ & $<$ & $<$ & $<$ & $<$ & & & $<$ & $<$ & $<$ & $<$ \\
\hline
\end{tabular}


APPENDIX D.2: CY 2002 MONITORING DATA FOR THE BEAR CREEK HYDROGEOLOGIC REGIME

Volatile Organic Compounds

\begin{tabular}{|c|c|c|c|c|c|c|c|c|c|c|c|}
\hline Sampling Point & \multicolumn{3}{|c|}{ GW-229 } & \multicolumn{2}{|c|}{ GW-243 } & \multicolumn{2}{|c|}{ GW-276 } & \multicolumn{2}{|c|}{ GW-288 } & \multicolumn{2}{|c|}{ GW-289 } \\
\hline Location & \multicolumn{3}{|c|}{ OLF } & \multicolumn{2}{|c|}{ S3 } & \multicolumn{2}{|c|}{ S3 } & \multicolumn{2}{|c|}{ BG } & \multicolumn{2}{|c|}{ BG } \\
\hline Date Sampled & \multicolumn{2}{|c|}{ 03/19/02 } & $08 / 12 / 02$ & $03 / 21 / 02$ & $08 / 13 / 02$ & 01/07/02 & 07/08/02 & 03/13/02 & 08/07/02 & $03 / 13 / 02$ & 08/08/02 \\
\hline Program & GWPP & GWPP & GWPP & GWPP & GWPP & BJC & BJC & GWPP & GWPP & GWPP & GWPP \\
\hline Sample Type & & Dup & & & & & & & & & \\
\hline Chloroethenes ( $\mu \mathrm{g} / \mathrm{L})$ & & & & & & & & & & & \\
\hline Tetrachloroethene & $<$ & $<$ & $<$ & 4,400 & 3,600 & 8 & 10 & 180 & 250 & 670 & 690 \\
\hline Trichloroethene & $<$ & $<$ & $<$ & 20 & 17 & $<$ & $<$ & 11 & 14 & 13 & 14 \\
\hline cis-1,2-Dichloroethene & 110 & 100 & 100 & 19 & 12 & $<$ & $<$ & $3 \mathrm{~J}$ & $3 \mathrm{~J}$ & $2 \mathrm{~J}$ & $2 \mathrm{~J}$ \\
\hline trans-1,2-Dichloroethene & $<$ & $<$ & $<$ & $<$ & $<$ & $<$ & $<$ & $<$ & $<$ & $<$ & $<$ \\
\hline 1,1-Dichloroethene & 11 & 10 & 10 & 6 & $4 \mathrm{~J}$ & $<$ & $<$ & $<$ & $<$ & $<$ & $<$ \\
\hline Vinyl chloride & 37 & 32 & 40 & $<$ & $<$ & $<$ & $<$ & 3 & 4 & $2 \mathrm{~J}$ & 2 \\
\hline Chloroethanes ( $\mu \mathrm{g} / \mathrm{L})$ & & & & & & & & & & & \\
\hline 1,1,1-Trichloroethane & $<$ & $<$ & $<$ & 12 & 8 & $<$ & $<$ & $<$ & $<$ & $<$ & $<$ \\
\hline 1,1,2-Trichloroethane & $<$ & $<$ & $<$ & $<$ & $<$ & $<$ & $<$ & $<$ & $<$ & $<$ & $<$ \\
\hline 1,2-Dichloroethane & $<$ & $<$ & $<$ & $<$ & $<$ & $<$ & $<$ & $<$ & $<$ & $<$ & $<$ \\
\hline 1,1-Dichloroethane & 6 & 6 & 6 & $<$ & $<$ & $<$ & $<$ & $<$ & $<$ & $<$ & $<$ \\
\hline Chloroethane & $2 \mathrm{~J}$ & $<$ & $2 \mathrm{~J}$ & $<$ & $<$ & $<$ & $<$ & $<$ & $<$ & $<$ & $<$ \\
\hline Chloromethanes $(\mu \mathrm{g} / \mathrm{L})$ & & & & & & & & & & & \\
\hline Carbon tetrachloride & $<$ & $<$ & $<$ & $<$ & $<$ & $<$ & $<$ & $<$ & $<$ & $<$ & $<$ \\
\hline Chloroform & $<$ & $<$ & $<$ & 39 & 29 & $<$ & $2 \mathrm{~J}$ & $<$ & $<$ & $<$ & $<$ \\
\hline Methylene chloride & $<$ & $<$ & $<$ & 120 & 100 & $<$ & $<$ & $<$ & $<$ & $<$ & $<$ \\
\hline Chloromethane & $<$ & $<$ & $<$ & $<$ & $<$ & $<$ & $<$ & $<$ & $<$ & $<$ & $<$ \\
\hline Petrol. Hydrocarb. ( $\mu \mathrm{g} / \mathrm{L})$ & & & & & & & & & & & \\
\hline Benzene & 8 & 7 & 6 & $<$ & $<$ & $<$ & $<$ & $<$ & $<$ & $<$ & $<$ \\
\hline Dimethylbenzene & $<$ & $<$ & $<$ & $<$ & $<$ & $<$ & $<$ & $<$ & $<$ & $<$ & $<$ \\
\hline Ethylbenzene & $<$ & $<$ & $<$ & $<$ & $<$ & $<$ & $<$ & $<$ & $<$ & $<$ & $<$ \\
\hline Toluene & $<$ & $<$ & $<$ & $4 \mathrm{~J}$ & $4 \mathrm{~J}$ & $<$ & $<$ & $<$ & $<$ & $<$ & $<$ \\
\hline Miscellaneous ( $\mu \mathrm{g} / \mathrm{L})$ & & & & & & & & & & & \\
\hline 1,2-Dichloropropane & $<$ & $<$ & $<$ & $<$ & $<$ & $<$ & $<$ & $<$ & $<$ & $<$ & $<$ \\
\hline 1,4-Dichlorobenzene & $<$ & $<$ & $3 \mathrm{~J}$ & $<$ & $<$ & & & $<$ & $<$ & $<$ & $<$ \\
\hline 2-Butanone & $<$ & $<$ & $<$ & 25 & 15 & $<$ & $<$ & $<$ & $<$ & $<$ & $<$ \\
\hline Acetone & $<$ & $<$ & $4 \mathrm{~J}$ & 700 & 280 & $<$ & $<$ & $<$ & $<$ & $<$ & $<$ \\
\hline Bromoform & $<$ & $<$ & $<$ & $3 \mathrm{~J}$ & $3 \mathrm{~J}$ & $<$ & $<$ & $<$ & $<$ & $<$ & $<$ \\
\hline Chlorobenzene & 8 & 7 & 7 & $<$ & $<$ & $<$ & $<$ & $<$ & $<$ & $<$ & $<$ \\
\hline Dichlorodifluoromethane & $<$ & $<$ & $<$ & $<$ & $4 \mathrm{~J}$ & & & $<$ & $<$ & $<$ & $<$ \\
\hline Trichlorofluoromethane & $<$ & $<$ & $<$ & $<$ & $<$ & & & $<$ & $<$ & $<$ & $<$ \\
\hline
\end{tabular}


APPENDIX D.2: CY 2002 MONITORING DATA FOR THE BEAR CREEK HYDROGEOLOGIC REGIME

Volatile Organic Compounds

\begin{tabular}{|c|c|c|c|c|c|c|c|c|c|c|c|}
\hline Sampling Point & \multicolumn{2}{|c|}{ GW-291 } & \multicolumn{3}{|c|}{ GW-311 } & \multicolumn{2}{|c|}{ GW-315 } & \multicolumn{2}{|c|}{ GW-363 } & \multicolumn{2}{|c|}{ GW-526 } \\
\hline Location & \multicolumn{2}{|c|}{ BG } & \multicolumn{3}{|c|}{ RS } & \multicolumn{2}{|c|}{ SPI } & \multicolumn{2}{|c|}{ EMWMF } & \multicolumn{2}{|c|}{ S3 } \\
\hline Date Sampled & 03/14/02 & 08/07/02 & \multicolumn{2}{|c|}{ 02/13/02 } & 07/30/02 & $02 / 13 / 02$ & $07 / 30 / 02$ & $08 / 14 / 02$ & $12 / 04 / 02$ & $02 / 26 / 02$ & $08 / 08 / 02$ \\
\hline Program & GWPP & GWPP & GWPP & GWPP & GWPP & GWPP & GWPP & BJC & BJC & BJC & BJC \\
\hline Sample Type & & & & Dup & & & & & & & \\
\hline Chloroethenes ( $\mu \mathrm{g} / \mathrm{L}$ ) & & & & & & & & & & & \\
\hline Tetrachloroethene & 340 & 380 & $<$ & $<$ & $<$ & 9 & 11 & $<$ & $<$ & $<$ & $<$ \\
\hline Trichloroethene & 42 & 37 & $3 \mathrm{~J}$ & $3 \mathrm{~J}$ & $4 \mathrm{~J}$ & $5 \mathrm{~J}$ & $4 \mathrm{~J}$ & $<$ & $<$ & $<$ & $<$ \\
\hline cis-1,2-Dichloroethene & $3 \mathrm{~J}$ & $3 \mathrm{~J}$ & $<$ & $<$ & $<$ & $2 \mathrm{~J}$ & $2 \mathrm{~J}$ & & . & $<$ & $<$ \\
\hline trans-1,2-Dichloroethene & $<$ & $<$ & $<$ & $<$ & $<$ & $<$ & $<$ & & & $<$ & $<$ \\
\hline 1,1-Dichloroethene & $<$ & $<$ & $<$ & $<$ & $<$ & $<$ & $<$ & & & $<$ & $<$ \\
\hline Vinyl chloride & $<$ & $<$ & $<$ & $<$ & $<$ & $<$ & $<$ & & & $<$ & $<$ \\
\hline Chloroethanes $(\mu \mathrm{g} / \mathrm{L})$ & & & & & & & & & & & \\
\hline 1,1,1-Trichloroethane & $<$ & $<$ & $<$ & $<$ & $<$ & $<$ & $<$ & & & $<$ & 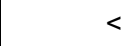 \\
\hline 1,1,2-Trichloroethane & $<$ & $<$ & $<$ & $<$ & $<$ & $<$ & $<$ & & & $<$ & $<$ \\
\hline 1,2-Dichloroethane & $<$ & $<$ & $<$ & $<$ & $<$ & $<$ & $<$ & & & $<$ & $<$ \\
\hline 1,1-Dichloroethane & $<$ & $2 \mathrm{~J}$ & $<$ & $<$ & $<$ & $<$ & $<$ & & & $<$ & $<$ \\
\hline Chloroethane & $<$ & $<$ & $<$ & $<$ & $<$ & $<$ & $<$ & & & $<$ & $<$ \\
\hline Chloromethanes $(\mu \mathrm{g} / \mathrm{L})$ & & & & & & & & & & & \\
\hline Carbon tetrachloride & $<$ & $<$ & $<$ & $<$ & $<$ & $<$ & $<$ & & $<$ & $<$ & - \\
\hline Chloroform & $<$ & $<$ & $<$ & $<$ & $<$ & $<$ & $<$ & & $<$ & $<$ & $<$ \\
\hline Methylene chloride & $<$ & $<$ & $<$ & $<$ & $<$ & $<$ & $<$ & & . & $<$ & $<$ \\
\hline Chloromethane & $<$ & $<$ & $<$ & $<$ & $<$ & $<$ & $<$ & & & $<$ & $<$ \\
\hline Petrol. Hydrocarb. ( $\mu \mathrm{g} / \mathrm{L})$ & & & & & & & & & & & \\
\hline Benzene & $<$ & $<$ & $<$ & $<$ & $<$ & $<$ & $<$ & & . & $<$ & - \\
\hline Dimethylbenzene & $<$ & $<$ & $<$ & $<$ & $<$ & $<$ & $<$ & & & $<$ & $<$ \\
\hline Ethylbenzene & $<$ & $<$ & $<$ & $<$ & $<$ & $<$ & $<$ & & & $<$ & $<$ \\
\hline Toluene & $<$ & $<$ & $<$ & $<$ & $<$ & $<$ & $<$ & $<$ & $<$ & $<$ & $<$ \\
\hline Miscellaneous $(\mu \mathrm{g} / \mathrm{L})$ & & & & & & & & & & & \\
\hline 1,2-Dichloropropane & $<$ & $<$ & $<$ & $<$ & $<$ & $<$ & $<$ & & & $<$ & $<$ \\
\hline 1,4-Dichlorobenzene & $<$ & $<$ & $<$ & $<$ & $<$ & $<$ & $<$ & & & & \\
\hline 2-Butanone & $<$ & $<$ & $<$ & $<$ & $<$ & $<$ & $<$ & & & $<$ & $<$ \\
\hline Acetone & $<$ & $<$ & $<$ & $<$ & $<$ & $<$ & $<$ & $<$ & $<$ & $<$ & $<$ \\
\hline Bromoform & $<$ & $<$ & $<$ & $<$ & $<$ & $<$ & $<$ & & & $<$ & $<$ \\
\hline Chlorobenzene & $<$ & $<$ & $<$ & $<$ & $<$ & $<$ & $<$ & & & $<$ & $<$ \\
\hline Dichlorodifluoromethane & $<$ & $<$ & $<$ & $<$ & $<$ & $<$ & $<$ & & & & \\
\hline Trichlorofluoromethane & $5 \mathrm{~J}$ & $5 \mathrm{~J}$ & $<$ & $<$ & $<$ & $<$ & $<$ & & & & \\
\hline
\end{tabular}


APPENDIX D.2: CY 2002 MONITORING DATA FOR THE BEAR CREEK HYDROGEOLOGIC REGIME Volatile Organic Compounds

\begin{tabular}{|c|c|c|c|c|c|c|c|c|c|c|c|}
\hline Sampling Point & \multicolumn{3}{|c|}{ GW-537 } & \multicolumn{2}{|c|}{ GW-626 } & \multicolumn{2}{|c|}{ GW-627 } & \multicolumn{2}{|c|}{ GW-639 } & \multicolumn{2}{|c|}{ GW-653 } \\
\hline Location & \multicolumn{3}{|c|}{ OLF } & \multicolumn{2}{|c|}{ BG } & \multicolumn{2}{|c|}{ BG } & \multicolumn{2}{|c|}{ EMWMF } & \multicolumn{2}{|c|}{ BG } \\
\hline Date Sampled & 02/18/02 & \multicolumn{2}{|c|}{ 07/31/02 } & $02 / 11 / 02$ & 07/25/02 & $02 / 11 / 02$ & 07/29/02 & $08 / 14 / 02$ & $12 / 04 / 02$ & $02 / 12 / 02$ & 07/25/02 \\
\hline Program & GWPP & GWPP & GWPP & GWPP & GWPP & GWPP & GWPP & BJC & BJC & GWPP & GWPP \\
\hline Sample Type & & & Dup & & & & & & & & \\
\hline Chloroethenes $(\mu \mathrm{g} / \mathrm{L})$ & & & & & & & & & & & \\
\hline Tetrachloroethene & $<$ & $<$ & $<$ & 920 & 320 & 550 & 530 & $<$ & $<$ & 11 & 10 \\
\hline Trichloroethene & $<$ & $<$ & $<$ & 710 & 190 & 160 & 190 & $<$ & $<$ & 8 & 6 \\
\hline cis-1,2-Dichloroethene & $<$ & $<$ & $<$ & 7,200 & 1,500 & 12 & 14 & & & 170 & 130 \\
\hline trans-1,2-Dichloroethene & $<$ & $<$ & $<$ & 13 & $2 \mathrm{~J}$ & $2 \mathrm{~J}$ & $2 \mathrm{~J}$ & & & $<$ & $<$ \\
\hline 1,1-Dichloroethene & $<$ & $<$ & $<$ & 120 & 38 & 19 & 23 & & & 5 & $4 \mathrm{~J}$ \\
\hline Vinyl chloride & $<$ & $<$ & $<$ & 1,200 & 42 & 18 & 20 & & & $<$ & $<$ \\
\hline Chloroethanes ( $\mu \mathrm{g} / \mathrm{L})$ & & & & & & & & & & & \\
\hline 1,1,1-Trichloroethane & $<$ & $<$ & $<$ & 44 & 13 & $<$ & $<$ & & & $2 \mathrm{~J}$ & $<$ \\
\hline 1,1,2-Trichloroethane & $<$ & $<$ & $<$ & $2 \mathrm{~J}$ & $<$ & $<$ & $<$ & & & $<$ & $<$ \\
\hline 1,2-Dichloroethane & $<$ & $<$ & $<$ & 32 & 7 & $<$ & $<$ & & & $<$ & $<$ \\
\hline 1,1-Dichloroethane & $<$ & $<$ & $<$ & 290 & 65 & 68 & 70 & & & 12 & 9 \\
\hline Chloroethane & $<$ & $<$ & $<$ & $<$ & $<$ & $<$ & $<$ & & & $<$ & $<$ \\
\hline Chloromethanes $(\mu \mathrm{g} / \mathrm{L})$ & & & & & & & & & & & \\
\hline Carbon tetrachloride & $<$ & $<$ & $<$ & 8 & $<$ & $<$ & $<$ & & $<$ & $<$ & $<$ \\
\hline Chloroform & $<$ & $2 \mathrm{~J}$ & $2 \mathrm{~J}$ & 100 & 24 & $<$ & $<$ & & $<$ & $<$ & $<$ \\
\hline Methylene chloride & $<$ & $<$ & $<$ & 6 & $<$ & $<$ & $<$ & & & $<$ & $<$ \\
\hline Chloromethane & $<$ & $<$ & $<$ & $<$ & $<$ & $<$ & $<$ & & & $<$ & $<$ \\
\hline Petrol. Hydrocarb. ( $\mu \mathrm{g} / \mathrm{L})$ & & & & & & & & & & & \\
\hline Benzene & $<$ & $<$ & $<$ & 190 & $4 \mathrm{~J}$ & $<$ & $<$ & & & $<$ & $<$ \\
\hline Dimethylbenzene & $<$ & $<$ & $<$ & $<$ & $<$ & $<$ & $<$ & & & $<$ & $<$ \\
\hline Ethylbenzene & $<$ & $<$ & $<$ & $<$ & $<$ & $<$ & $<$ & & & $<$ & $<$ \\
\hline Toluene & $<$ & $<$ & $<$ & $<$ & $<$ & $<$ & $<$ & $<$ & $<$ & $<$ & $<$ \\
\hline Miscellaneous ( $\mu \mathrm{g} / \mathrm{L})$ & & & & & & & & & & & \\
\hline 1,2-Dichloropropane & $<$ & $<$ & $<$ & $<$ & $<$ & $<$ & $<$ & & & $<$ & $<$ \\
\hline 1,4-Dichlorobenzene & $<$ & $<$ & $<$ & $<$ & $<$ & $<$ & $<$ & & & $<$ & $<$ \\
\hline 2-Butanone & $<$ & $<$ & $<$ & $<$ & $<$ & $<$ & $<$ & & & $<$ & $<$ \\
\hline Acetone & $<$ & $<$ & $<$ & $<$ & $<$ & $<$ & $<$ & $<$ & $<$ & $<$ & $<$ \\
\hline Bromoform & $<$ & $<$ & $<$ & $<$ & $<$ & $<$ & $<$ & & & $<$ & $<$ \\
\hline Chlorobenzene & $<$ & $<$ & $<$ & $<$ & $<$ & $<$ & $<$ & & & $<$ & $<$ \\
\hline Dichlorodifluoromethane & $<$ & $<$ & $<$ & $<$ & $<$ & $<$ & $<$ & & & $<$ & $<$ \\
\hline Trichlorofluoromethane & $<$ & $<$ & $<$ & $3 \mathrm{~J}$ & $<$ & $<$ & $<$ & & & $<$ & $<$ \\
\hline
\end{tabular}


APPENDIX D.2: CY 2002 MONITORING DATA FOR THE BEAR CREEK HYDROGEOLOGIC REGIME

Volatile Organic Compounds

\begin{tabular}{|c|c|c|c|c|c|c|c|c|c|c|c|}
\hline Sampling Point & \multicolumn{2}{|c|}{ GW-683 } & \multicolumn{3}{|c|}{ GW-684 } & \multicolumn{2}{|c|}{ GW-694 } & \multicolumn{2}{|c|}{ GW-695 } & \multicolumn{2}{|c|}{ GW-703 } \\
\hline Location & \multicolumn{2}{|c|}{ EXP-A } & \multicolumn{3}{|c|}{ EXP-A } & \multicolumn{2}{|c|}{ EXP-B } & \multicolumn{2}{|c|}{ EXP-B } & \multicolumn{2}{|c|}{ EXP-B } \\
\hline Date Sampled & 01/14/02 & 07/09/02 & 01/14/02 & $07 / 0$ & $/ 02$ & 01/29/02 & 07/17/02 & $01 / 15 / 02$ & 07/15/02 & $01 / 15 / 02$ & 07/15/02 \\
\hline Program & GWPP & GWPP & GWPP & GWPP & GWPP & GWPP & GWPP & GWPP & GWPP & GWPP & GWPP \\
\hline Sample Type & & & & & Dup & & & & & & \\
\hline Chloroethenes $(\mu \mathrm{g} / \mathrm{L})$ & & & & & & & & & & & \\
\hline Tetrachloroethene & $<$ & $<$ & $<$ & $<$ & $<$ & $<$ & $<$ & $<$ & $<$ & $<$ & $<$ \\
\hline Trichloroethene & $<$ & $<$ & $<$ & $<$ & $<$ & $<$ & $3 \mathrm{~J}$ & 5 & $5 \mathrm{~J}$ & 15 & 16 \\
\hline cis-1,2-Dichloroethene & $<$ & $<$ & $<$ & $<$ & $<$ & $<$ & $<$ & $3 \mathrm{~J}$ & $2 \mathrm{~J}$ & 6 & 6 \\
\hline trans-1,2-Dichloroethene & $<$ & $<$ & $<$ & $<$ & $<$ & $<$ & $<$ & $<$ & $<$ & $<$ & $<$ \\
\hline 1,1-Dichloroethene & $<$ & $<$ & $<$ & $<$ & $<$ & $<$ & $<$ & $<$ & $<$ & $<$ & $<$ \\
\hline Vinyl chloride & $<$ & $<$ & $<$ & $<$ & $<$ & $<$ & $<$ & $<$ & $<$ & $<$ & $<$ \\
\hline Chloroethanes ( $\mu \mathrm{g} / \mathrm{L})$ & & & & & & & & & & & \\
\hline 1,1,1-Trichloroethane & $<$ & $<$ & $<$ & $<$ & $<$ & $<$ & $<$ & $<$ & $<$ & $<$ & $<$ \\
\hline 1,1,2-Trichloroethane & $<$ & $<$ & $<$ & $<$ & $<$ & $<$ & $<$ & $<$ & $<$ & $<$ & $<$ \\
\hline 1,2-Dichloroethane & $<$ & $<$ & $<$ & $<$ & $<$ & $<$ & $<$ & $<$ & $<$ & $<$ & $<$ \\
\hline 1,1-Dichloroethane & $<$ & $<$ & $<$ & $<$ & $<$ & $<$ & $<$ & $<$ & $<$ & $<$ & $<$ \\
\hline Chloroethane & $<$ & $<$ & $<$ & $<$ & $<$ & $<$ & $<$ & $<$ & $<$ & $<$ & $<$ \\
\hline Chloromethanes $(\mu \mathrm{g} / \mathrm{L})$ & & & & & & & & & & & \\
\hline Carbon tetrachloride & $<$ & $<$ & $<$ & $<$ & $<$ & $<$ & $<$ & $<$ & $<$ & $<$ & $<$ \\
\hline Chloroform & $<$ & $<$ & $<$ & $<$ & $<$ & $<$ & $<$ & $<$ & $<$ & $<$ & $<$ \\
\hline Methylene chloride & $<$ & $<$ & $<$ & $<$ & $<$ & $<$ & $<$ & $<$ & $<$ & $<$ & $<$ \\
\hline Chloromethane & $<$ & $<$ & $<$ & $<$ & $<$ & $<$ & $<$ & $<$ & $<$ & $<$ & $<$ \\
\hline Petrol. Hydrocarb. ( $\mu \mathrm{g} / \mathrm{L})$ & & & & & & & & & & & \\
\hline Benzene & $<$ & $<$ & $<$ & $<$ & $<$ & $<$ & $<$ & $<$ & $<$ & $<$ & $<$ \\
\hline Dimethylbenzene & $<$ & $<$ & $<$ & $<$ & $<$ & $<$ & $<$ & $<$ & $<$ & $<$ & $<$ \\
\hline Ethylbenzene & $<$ & $<$ & $<$ & $<$ & $<$ & $<$ & $<$ & $<$ & $<$ & $<$ & $<$ \\
\hline Toluene & $<$ & $<$ & $<$ & $<$ & $<$ & $<$ & $<$ & $<$ & $<$ & $<$ & $<$ \\
\hline Miscellaneous ( $\mu \mathrm{g} / \mathrm{L})$ & & & & & & & & & & & \\
\hline 1,2-Dichloropropane & $<$ & $<$ & $<$ & $<$ & $<$ & $<$ & $<$ & $<$ & $<$ & $<$ & $<$ \\
\hline 1,4-Dichlorobenzene & $<$ & $<$ & $<$ & $<$ & $<$ & $<$ & $<$ & $<$ & $<$ & $<$ & $<$ \\
\hline 2-Butanone & $<$ & $<$ & $<$ & $<$ & $<$ & $<$ & $<$ & $<$ & $<$ & $<$ & $<$ \\
\hline Acetone & $<$ & $<$ & $<$ & $<$ & $<$ & $<$ & $<$ & $<$ & $<$ & $<$ & $<$ \\
\hline Bromoform & $<$ & $<$ & $<$ & $<$ & $<$ & $<$ & $<$ & $<$ & $<$ & $<$ & $<$ \\
\hline Chlorobenzene & $<$ & $<$ & $<$ & $<$ & $<$ & $<$ & $<$ & $<$ & $<$ & $<$ & $<$ \\
\hline Dichlorodifluoromethane & $<$ & $<$ & $<$ & $<$ & $<$ & $<$ & $<$ & $<$ & $<$ & $<$ & $<$ \\
\hline Trichlorofluoromethane & $<$ & $<$ & $<$ & $<$ & $<$ & $<$ & $<$ & $<$ & $<$ & $<$ & $<$ \\
\hline
\end{tabular}


APPENDIX D.2: CY 2002 MONITORING DATA FOR THE BEAR CREEK HYDROGEOLOGIC REGIME

Volatile Organic Compounds

\begin{tabular}{|c|c|c|c|c|c|c|c|c|c|c|c|}
\hline Sampling Point & \multicolumn{3}{|c|}{ GW-704 } & \multicolumn{2}{|c|}{ GW-706 } & \multicolumn{2}{|c|}{ GW-712 } & \multicolumn{2}{|c|}{ GW-713 } & \multicolumn{2}{|c|}{ GW-714 } \\
\hline Location & \multicolumn{3}{|c|}{ EXP-B } & \multicolumn{2}{|c|}{ EXP-B } & \multicolumn{2}{|c|}{ EXP-W } & \multicolumn{2}{|c|}{ EXP-W } & \multicolumn{2}{|c|}{ EXP-W } \\
\hline Date Sampled & \multicolumn{2}{|c|}{ 01/16/02 } & 07/16/02 & $01 / 16 / 02$ & 07/16/02 & 01/03/02 & 07/01/02 & $01 / 03 / 02$ & 07/01/02 & 01/02/02 & 07/01/02 \\
\hline Program & GWPP & GWPP & GWPP & GWPP & GWPP & BJC & BJC & BJC & BJC & BJC & BJC \\
\hline Sample Type & & Dup & & & & & & & & & \\
\hline Chloroethenes ( $\mu \mathrm{g} / \mathrm{L}$ ) & & & & & & & & & & & \\
\hline Tetrachloroethene & $<$ & $<$ & $<$ & $<$ & $<$ & $<$ & $<$ & $<$ & $<$ & $<$ & $<$ \\
\hline Trichloroethene & 45 & 44 & 41 & 17 & 16 & $<$ & $<$ & $<$ & $<$ & $<$ & $<$ \\
\hline cis-1,2-Dichloroethene & $4 \mathrm{~J}$ & $4 \mathrm{~J}$ & $4 \mathrm{~J}$ & 14 & 13 & $<$ & $<$ & $<$ & $<$ & $<$ & $<$ \\
\hline trans-1,2-Dichloroethene & $<$ & $<$ & $<$ & $<$ & $<$ & $<$ & $<$ & $<$ & $<$ & $<$ & $<$ \\
\hline 1,1-Dichloroethene & $4 \mathrm{~J}$ & $4 \mathrm{~J}$ & $3 \mathrm{~J}$ & $<$ & $<$ & $<$ & $<$ & $<$ & $<$ & $<$ & $<$ \\
\hline Vinyl chloride & $<$ & $<$ & $<$ & $<$ & $<$ & $<$ & $<$ & $<$ & $<$ & $<$ & $<$ \\
\hline Chloroethanes ( $\mu \mathrm{g} / \mathrm{L})$ & & & & & & & & & & & \\
\hline 1,1,1-Trichloroethane & $<$ & $<$ & $<$ & $<$ & $<$ & $<$ & $<$ & $<$ & $<$ & $<$ & 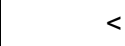 \\
\hline 1,1,2-Trichloroethane & $<$ & $<$ & $<$ & $<$ & $<$ & $<$ & $<$ & $<$ & $<$ & $<$ & $<$ \\
\hline 1,2-Dichloroethane & $<$ & $<$ & $<$ & $<$ & $<$ & $<$ & $<$ & $<$ & $<$ & $<$ & $<$ \\
\hline 1,1-Dichloroethane & $<$ & $<$ & $<$ & $<$ & $<$ & $<$ & $<$ & $<$ & $<$ & $<$ & $<$ \\
\hline Chloroethane & $<$ & $<$ & $<$ & $<$ & $<$ & $<$ & $<$ & $<$ & $<$ & $<$ & $<$ \\
\hline Chloromethanes $(\mu \mathrm{g} / \mathrm{L})$ & & & & & & & & & & & \\
\hline Carbon tetrachloride & $<$ & $<$ & $<$ & $<$ & $<$ & $<$ & $<$ & $<$ & $<$ & $<$ & 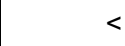 \\
\hline Chloroform & $<$ & $<$ & $<$ & $<$ & $<$ & $<$ & $<$ & $<$ & $<$ & $<$ & $<$ \\
\hline Methylene chloride & $<$ & $<$ & $<$ & $<$ & $<$ & $<$ & $<$ & $<$ & $<$ & $<$ & $<$ \\
\hline Chloromethane & $<$ & $<$ & $<$ & $<$ & $<$ & $<$ & $<$ & $<$ & $<$ & $<$ & $<$ \\
\hline Petrol. Hydrocarb. ( $\mu \mathrm{g} / \mathrm{L})$ & & & & & & & & & & & \\
\hline Benzene & $<$ & $<$ & $<$ & $<$ & $<$ & $<$ & $<$ & $<$ & $<$ & $<$ & - \\
\hline Dimethylbenzene & $<$ & $<$ & $<$ & $<$ & $<$ & $<$ & $<$ & $<$ & $<$ & $<$ & $<$ \\
\hline Ethylbenzene & $<$ & $<$ & $<$ & $<$ & $<$ & $<$ & $<$ & $<$ & $<$ & $<$ & $<$ \\
\hline Toluene & $<$ & $<$ & $<$ & $<$ & $<$ & $<$ & $<$ & $<$ & $<$ & $<$ & $<$ \\
\hline Miscellaneous $(\mu \mathrm{g} / \mathrm{L})$ & & & & & & & & & & & \\
\hline 1,2-Dichloropropane & $<$ & $<$ & $<$ & $<$ & $<$ & $<$ & $<$ & $<$ & $<$ & $<$ & $<$ \\
\hline 1,4-Dichlorobenzene & $<$ & $<$ & $<$ & $<$ & $<$ & & & & & & \\
\hline 2-Butanone & $<$ & $<$ & $<$ & $<$ & $<$ & $<$ & $<$ & $<$ & $<$ & $<$ & $<$ \\
\hline Acetone & $<$ & $<$ & $<$ & $<$ & $<$ & $<$ & $<$ & $<$ & $<$ & $<$ & $<$ \\
\hline Bromoform & $<$ & $<$ & $<$ & $<$ & $<$ & $<$ & $<$ & $<$ & $<$ & $<$ & $<$ \\
\hline Chlorobenzene & $<$ & $<$ & $<$ & $<$ & $<$ & $<$ & $<$ & $<$ & $<$ & $<$ & $<$ \\
\hline Dichlorodifluoromethane & $<$ & $<$ & $<$ & $<$ & $<$ & & & & & & \\
\hline Trichlorofluoromethane & $<$ & $<$ & $<$ & $<$ & $<$ & & & & & & \\
\hline
\end{tabular}


APPENDIX D.2: CY 2002 MONITORING DATA FOR THE BEAR CREEK HYDROGEOLOGIC REGIME Volatile Organic Compounds

\begin{tabular}{|c|c|c|c|c|c|c|c|c|c|c|}
\hline Sampling Point & \multicolumn{4}{|c|}{ GW-715 } & \multicolumn{2}{|c|}{ GW-723 } & \multicolumn{2}{|c|}{ GW-724 } & \multicolumn{2}{|c|}{ GW-725 } \\
\hline Location & \multicolumn{4}{|c|}{ EXP-W } & \multicolumn{2}{|c|}{ EXP-C } & \multicolumn{2}{|c|}{ EXP-C } & \multicolumn{2}{|c|}{ EXP-C } \\
\hline Date Sampled & \multicolumn{2}{|c|}{ 01/02/02 } & \multicolumn{2}{|c|}{ 07/01/02 } & $03 / 05 / 02$ & $07 / 23 / 02$ & 01/30/02 & 07/23/02 & $01 / 31 / 02$ & 07/18/02 \\
\hline Program & BJC & BJC & BJC & BJC & GWPP & GWPP & GWPP & GWPP & GWPP & GWPP \\
\hline Sample Type & & Dup & & Dup & & & & & & \\
\hline Chloroethenes ( $\mu \mathrm{g} / \mathrm{L}$ ) & & & & & & & & & & \\
\hline Tetrachloroethene & $<$ & $<$ & $<$ & $<$ & $<$ & $<$ & $3 \mathrm{~J}$ & $3 \mathrm{~J}$ & $<$ & $2 \mathrm{~J}$ \\
\hline Trichloroethene & $<$ & $<$ & $<$ & $<$ & 6 & 6 & 98 & 110 & 200 & 15 \\
\hline cis-1,2-Dichloroethene & $<$ & $<$ & $<$ & $<$ & $<$ & $<$ & $3 \mathrm{~J}$ & $3 \mathrm{~J}$ & $4 \mathrm{~J}$ & $2 \mathrm{~J}$ \\
\hline trans-1,2-Dichloroethene & $<$ & $<$ & $<$ & $<$ & $<$ & $<$ & $<$ & $<$ & $<$ & $<$ \\
\hline 1,1-Dichloroethene & $<$ & $<$ & $<$ & $<$ & $<$ & $<$ & $<$ & $<$ & 5 & $<$ \\
\hline Vinyl chloride & $<$ & $<$ & $<$ & $<$ & $<$ & $<$ & $<$ & $<$ & $<$ & $<$ \\
\hline Chloroethanes ( $\mu \mathrm{g} / \mathrm{L})$ & & & & & & & & & & \\
\hline 1,1,1-Trichloroethane & $<$ & $<$ & $<$ & $<$ & $<$ & $<$ & $<$ & $<$ & $3 \mathrm{~J}$ & $<$ \\
\hline 1,1,2-Trichloroethane & $<$ & $<$ & $<$ & $<$ & $<$ & $<$ & $<$ & $<$ & $<$ & $<$ \\
\hline 1,2-Dichloroethane & $<$ & $<$ & $<$ & $<$ & $<$ & $<$ & $<$ & $<$ & $<$ & $<$ \\
\hline 1,1-Dichloroethane & $<$ & $<$ & $<$ & $<$ & $<$ & $<$ & $<$ & $<$ & $<$ & $<$ \\
\hline Chloroethane & $<$ & $<$ & $<$ & $<$ & $<$ & $<$ & $<$ & $<$ & $<$ & $<$ \\
\hline Chloromethanes ( $\mu \mathrm{g} / \mathrm{L})$ & & & & & & & & & & \\
\hline Carbon tetrachloride & $<$ & $<$ & $<$ & $<$ & $<$ & $<$ & $2 \mathrm{~J}$ & $<$ & 7 & $<$ \\
\hline Chloroform & $<$ & $<$ & $<$ & $<$ & $<$ & $<$ & $<$ & $<$ & $2 \mathrm{~J}$ & $<$ \\
\hline Methylene chloride & $<$ & $<$ & $<$ & $<$ & $<$ & $<$ & $<$ & $<$ & $<$ & $<$ \\
\hline Chloromethane & $<$ & $<$ & $<$ & $<$ & $<$ & $<$ & $<$ & $<$ & $<$ & $<$ \\
\hline Petrol. Hydrocarb. ( $\mu \mathrm{g} / \mathrm{L})$ & & & & & & & & & & \\
\hline Benzene & $<$ & $<$ & $<$ & $<$ & $<$ & $<$ & $<$ & $<$ & $<$ & $<$ \\
\hline Dimethylbenzene & $<$ & $<$ & $<$ & $<$ & $<$ & $<$ & $<$ & $<$ & $<$ & $<$ \\
\hline Ethylbenzene & $<$ & $<$ & $<$ & $<$ & $<$ & $<$ & $<$ & $<$ & $<$ & $<$ \\
\hline Toluene & $<$ & $<$ & $<$ & $<$ & $<$ & $<$ & $<$ & $<$ & $<$ & $<$ \\
\hline Miscellaneous ( $\mu \mathrm{g} / \mathrm{L})$ & & & & & & & & & & \\
\hline 1,2-Dichloropropane & $<$ & $<$ & $<$ & $<$ & $<$ & $<$ & $<$ & $<$ & $<$ & $<$ \\
\hline 1,4-Dichlorobenzene & & & & & $<$ & $<$ & $<$ & $<$ & $<$ & $<$ \\
\hline 2-Butanone & $<$ & $<$ & $<$ & $<$ & $<$ & $<$ & $<$ & $<$ & $<$ & $<$ \\
\hline Acetone & $<$ & $<$ & $<$ & $<$ & $<$ & $<$ & $<$ & $<$ & $<$ & $<$ \\
\hline Bromoform & $<$ & $<$ & $<$ & $<$ & $<$ & $<$ & $<$ & $<$ & $<$ & $<$ \\
\hline Chlorobenzene & $<$ & $<$ & $<$ & $<$ & $<$ & $<$ & $<$ & $<$ & $<$ & $<$ \\
\hline Dichlorodifluoromethane & & & & & $<$ & $<$ & $<$ & $<$ & $<$ & $<$ \\
\hline Trichlorofluoromethane & & & & & $<$ & $<$ & $<$ & $<$ & $<$ & $<$ \\
\hline
\end{tabular}


APPENDIX D.2: CY 2002 MONITORING DATA FOR THE BEAR CREEK HYDROGEOLOGIC REGIME Volatile Organic Compounds

\begin{tabular}{|c|c|c|c|c|c|c|c|c|c|c|}
\hline Sampling Point & \multicolumn{2}{|c|}{ GW-736 } & \multicolumn{2}{|c|}{ GW-737 } & \multicolumn{2}{|c|}{ GW-738 } & \multicolumn{2}{|c|}{ GW-739 } & \multicolumn{2}{|c|}{ GW-740 } \\
\hline Location & \multicolumn{2}{|c|}{ EXP-C } & \multicolumn{2}{|c|}{ EXP-C } & \multicolumn{2}{|c|}{ EXP-C } & \multicolumn{2}{|c|}{ EXP-C } & \multicolumn{2}{|c|}{ EXP-C } \\
\hline Date Sampled & $03 / 06 / 02$ & 07/17/02 & 03/06/02 & $07 / 18 / 02$ & $01 / 30 / 02$ & $07 / 23 / 02$ & 03/05/02 & $07 / 22 / 02$ & $01 / 29 / 02$ & $07 / 22 / 02$ \\
\hline Program & GWPP & GWPP & GWPP & GWPP & GWPP & GWPP & GWPP & GWPP & GWPP & GWPP \\
\hline \multicolumn{11}{|l|}{ Sample Type } \\
\hline Chloroethenes ( $\mu \mathrm{g} / \mathrm{L}$ ) & & & & & & & & & & \\
\hline Tetrachloroethene & $<$ & $2 \mathrm{~J}$ & $3 \mathrm{~J}$ & $2 \mathrm{~J}$ & $<$ & $<$ & $<$ & $<$ & $<$ & $<$ \\
\hline Trichloroethene & 8 & 9 & 5 & 7 & 37 & 25 & 36 & 37 & 58 & 57 \\
\hline cis-1,2-Dichloroethene & $2 \mathrm{~J}$ & $3 \mathrm{~J}$ & $2 \mathrm{~J}$ & $<$ & $2 \mathrm{~J}$ & $<$ & $<$ & $<$ & $2 \mathrm{~J}$ & $2 \mathrm{~J}$ \\
\hline trans-1,2-Dichloroethene & $<$ & $<$ & $<$ & $<$ & $<$ & $<$ & $<$ & $<$ & $<$ & $<$ \\
\hline 1,1-Dichloroethene & $<$ & $<$ & $<$ & $<$ & $<$ & $<$ & $<$ & $<$ & $<$ & $<$ \\
\hline Vinyl chloride & $<$ & $<$ & $<$ & $<$ & $<$ & $<$ & $<$ & $<$ & $<$ & $<$ \\
\hline Chloroethanes ( $\mu \mathrm{g} / \mathrm{L})$ & & & & & & & & & & \\
\hline 1,1,1-Trichloroethane & $<$ & $<$ & $<$ & $<$ & $<$ & $<$ & $<$ & $<$ & $2 \mathrm{~J}$ & $<$ \\
\hline 1,1,2-Trichloroethane & $<$ & $<$ & $<$ & $<$ & $<$ & $<$ & $<$ & $<$ & $<$ & $<$ \\
\hline 1,2-Dichloroethane & $<$ & $<$ & $<$ & $<$ & $<$ & $<$ & $<$ & $<$ & $<$ & $<$ \\
\hline 1,1-Dichloroethane & $<$ & $<$ & $<$ & $<$ & $<$ & $<$ & $<$ & $<$ & $<$ & $<$ \\
\hline Chloroethane & $<$ & $<$ & $<$ & $<$ & $<$ & $<$ & $<$ & $<$ & $<$ & $<$ \\
\hline Chloromethanes ( $\mu \mathrm{g} / \mathrm{L})$ & & & & & & & & & & \\
\hline Carbon tetrachloride & $<$ & $<$ & $<$ & $<$ & $<$ & $<$ & $<$ & $<$ & $<$ & $<$ \\
\hline Chloroform & $<$ & $<$ & $<$ & $<$ & $<$ & $<$ & $<$ & $<$ & $<$ & $<$ \\
\hline Methylene chloride & $<$ & $<$ & $<$ & $<$ & $<$ & $<$ & $<$ & $<$ & $<$ & $<$ \\
\hline Chloromethane & $<$ & $<$ & $<$ & $<$ & $<$ & $<$ & $<$ & $<$ & $<$ & $<$ \\
\hline Petrol. Hydrocarb. ( $\mu \mathrm{g} / \mathrm{L})$ & & & & & & & & & & \\
\hline Benzene & $<$ & $<$ & $<$ & $<$ & $<$ & $<$ & $<$ & $<$ & $<$ & $<$ \\
\hline Dimethylbenzene & $<$ & $<$ & $<$ & $<$ & $<$ & $<$ & $<$ & $<$ & $<$ & $<$ \\
\hline Ethylbenzene & $<$ & $<$ & $<$ & $<$ & $<$ & $<$ & $<$ & $<$ & $<$ & $<$ \\
\hline Toluene & $<$ & $<$ & $<$ & $<$ & $<$ & $<$ & $<$ & $<$ & $<$ & $<$ \\
\hline Miscellaneous ( $\mu \mathrm{g} / \mathrm{L})$ & & & & & & & & & & \\
\hline 1,2-Dichloropropane & $<$ & $<$ & $<$ & $<$ & $<$ & $<$ & $<$ & $<$ & $<$ & $<$ \\
\hline 1,4-Dichlorobenzene & $<$ & $<$ & $<$ & $<$ & $<$ & $<$ & $<$ & $<$ & $<$ & $<$ \\
\hline 2-Butanone & $<$ & $<$ & $<$ & $<$ & $<$ & $<$ & $<$ & $<$ & $<$ & $<$ \\
\hline Acetone & $<$ & $<$ & $<$ & $<$ & $<$ & $6 \mathrm{~J}$ & $<$ & $<$ & $<$ & $<$ \\
\hline Bromoform & $<$ & $<$ & $<$ & $<$ & $<$ & $<$ & $<$ & $<$ & $<$ & $<$ \\
\hline Chlorobenzene & $<$ & $<$ & $<$ & $<$ & $<$ & $<$ & $<$ & $<$ & $<$ & $<$ \\
\hline Dichlorodifluoromethane & $<$ & $<$ & $<$ & $<$ & $<$ & $<$ & $<$ & $<$ & $<$ & $<$ \\
\hline Trichlorofluoromethane & $<$ & $<$ & $<$ & $<$ & $<$ & $<$ & $<$ & $<$ & $<$ & $<$ \\
\hline
\end{tabular}


APPENDIX D.2: CY 2002 MONITORING DATA FOR THE BEAR CREEK HYDROGEOLOGIC REGIME Volatile Organic Compounds

\begin{tabular}{|c|c|c|c|c|c|c|c|c|c|c|}
\hline Sampling Point & \multicolumn{4}{|c|}{ GW-835 } & \multicolumn{2}{|c|}{ GW-916 } & \multicolumn{2}{|c|}{ GW-917 } & \multicolumn{2}{|c|}{ GW-918 } \\
\hline Location & \multicolumn{4}{|c|}{ S3 } & \multicolumn{2}{|c|}{ EMWMF } & \multicolumn{2}{|c|}{ EMWMF } & \multicolumn{2}{|c|}{ EMWMF } \\
\hline Date Sampled & $03 / 04 / 02$ & $05 / 20 / 02$ & $08 / 22 / 02$ & $11 / 19 / 02$ & $08 / 15 / 02$ & $12 / 10 / 02$ & $08 / 12 / 02$ & $12 / 05 / 02$ & $08 / 15 / 02$ & $12 / 10 / 02$ \\
\hline Program & BJC & BJC & BJC & BJC & BJC & BJC & BJC & BJC & BJC & BJC \\
\hline \multicolumn{11}{|l|}{ Sample Type } \\
\hline Chloroethenes ( $\mu \mathrm{g} / \mathrm{L}$ ) & & & & & & & & & & \\
\hline Tetrachloroethene & $3 \mathrm{~J}$ & $3 \mathrm{~J}$ & $4 \mathrm{~J}$ & 4 & $<$ & $<$ & $<$ & $<$ & $<$ & $<$ \\
\hline Trichloroethene & $<$ & $<$ & $<$ & 1 & $<$ & $<$ & $<$ & $<$ & $<$ & $<$ \\
\hline cis-1,2-Dichloroethene & $<$ & $<$ & $<$ & 0.9 & & & & & & \\
\hline trans-1,2-Dichloroethene & $<$ & $<$ & $<$ & $<$ & & & & & & \\
\hline 1,1-Dichloroethene & $<$ & $<$ & $<$ & $<$ & 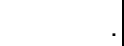 & & & & & \\
\hline Vinyl chloride & $<$ & $<$ & $<$ & $<$ & & & & & & \\
\hline \multirow{6}{*}{\multicolumn{11}{|c|}{$\begin{array}{r}\text { Chloroethanes }(\mu \mathrm{g} / \mathrm{L}) \\
1,1,1-\text { Trichloroethane } \\
1,1,2 \text {-Trichloroethane } \\
\text { 1,2-Dichloroethane } \\
\text { 1,1-Dichloroethane } \\
\text { Chloroethane }\end{array}$}} \\
\hline & & & & & & & & & & \\
\hline & & & & & & & & & & \\
\hline & & & & & & & & & & \\
\hline & & & & & & & & & & \\
\hline & & & & & & & & & & \\
\hline \multicolumn{11}{|l|}{ Chloromethanes $(\mu \mathrm{g} / \mathrm{L})$} \\
\hline Carbon tetrachloride & $<$ & $<$ & $<$ & $<$ & & $<$ & & $<$ & & $<$ \\
\hline Chloroform & $<$ & $<$ & $<$ & $<$ & & $<$ & & $<$ & & $<$ \\
\hline Methylene chloride & $<$ & $<$ & $<$ & $<$ & & & & & & \\
\hline Chloromethane & $<$ & $<$ & $<$ & $<$ & & & & & & \\
\hline \multicolumn{11}{|l|}{ Petrol. Hydrocarb. ( $\mu \mathrm{g} / \mathrm{L})$} \\
\hline Benzene & $<$ & $<$ & $<$ & $<$ & & & & & & \\
\hline Dimethylbenzene & $<$ & $<$ & $<$ & $<$ & & & & & & \\
\hline Ethylbenzene & $<$ & $<$ & $<$ & $<$ & & & & & & \\
\hline Toluene & $<$ & $<$ & $<$ & $<$ & $<$ & $<$ & $<$ & $<$ & $<$ & $<$ \\
\hline \multicolumn{11}{|l|}{ Miscellaneous $(\mu \mathrm{g} / \mathrm{L})$} \\
\hline 1,2-Dichloropropane & $<$ & $<$ & $<$ & $<$ & & & & & & \\
\hline 1,4-Dichlorobenzene & & & & & & & & & & \\
\hline 2-Butanone & $<$ & $<$ & $<$ & $<$ & & & & & & \\
\hline Acetone & $<$ & $<$ & $<$ & $<$ & $<$ & $<$ & $<$ & $<$ & $<$ & $<$ \\
\hline Bromoform & $<$ & $<$ & $<$ & $<$ & & & & & & \\
\hline Chlorobenzene & $<$ & $<$ & $<$ & $<$ & & & & & & \\
\hline Dichlorodifluoromethane & & & & & & & & & & \\
\hline Trichlorofluoromethane & & & & & & & & & & \\
\hline
\end{tabular}


APPENDIX D.2: CY 2002 MONITORING DATA FOR THE BEAR CREEK HYDROGEOLOGIC REGIME Volatile Organic Compounds

\begin{tabular}{|c|c|c|c|c|c|c|c|c|c|c|}
\hline Sampling Point & \multicolumn{2}{|c|}{ GW-919 } & \multicolumn{2}{|c|}{ GW-920 } & \multicolumn{2}{|c|}{ GW-921 } & \multicolumn{2}{|c|}{ GW-922 } & \multicolumn{2}{|c|}{ GW-923 } \\
\hline Location & \multicolumn{2}{|c|}{ EMWMF } & \multicolumn{2}{|c|}{ EMWMF } & \multicolumn{2}{|c|}{ EMWMF } & \multicolumn{2}{|c|}{ EMWMF } & \multicolumn{2}{|c|}{ EMWMF } \\
\hline Date Sampled & $08 / 14 / 02$ & $12 / 09 / 02$ & 08/13/02 & $12 / 05 / 02$ & 08/12/02 & $12 / 04 / 02$ & $08 / 13 / 02$ & $12 / 09 / 02$ & $08 / 14 / 02$ & $12 / 09 / 02$ \\
\hline Program & BJC & BJC & BJC & BJC & BJC & BJC & BJC & BJC & BJC & BJC \\
\hline Sample Type & & & & & & & & & & \\
\hline $\begin{array}{r}\text { Chloroethenes }(\mu \mathrm{g} / \mathrm{L}) \\
\text { Tetrachloroethene } \\
\text { Trichloroethene } \\
\text { cis-1,2-Dichloroethene } \\
\text { trans-1,2-Dichloroethene } \\
1,1 \text {-Dichloroethene } \\
\text { Vinyl chloride } \\
\end{array}$ & $<1$ & $\begin{array}{l}< \\
<\end{array}$ & $\begin{array}{l}< \\
<\end{array}$ & $\begin{array}{l}< \\
<\end{array}$ & $\begin{array}{l}< \\
<\end{array}$ & $\begin{array}{l}< \\
<\end{array}$ & $\begin{array}{l}< \\
<\end{array}$ & $\begin{array}{l}< \\
<\end{array}$ & $\begin{array}{l}< \\
<\end{array}$ & $\begin{array}{l}< \\
<\end{array}$ \\
\hline $\begin{array}{r}\text { Chloroethanes }(\boldsymbol{\mu g} / \mathrm{L}) \\
1,1,1-\text { Trichloroethane } \\
1,1,2 \text {-Trichloroethane } \\
1,2 \text {-Dichloroethane } \\
\text { 1,1-Dichloroethane } \\
\text { Chloroethane }\end{array}$ & & & & & & & & & & \\
\hline $\begin{array}{r}\text { Chloromethanes }(\mu \mathrm{g} / \mathrm{L}) \\
\text { Carbon tetrachloride } \\
\text { Chloroform } \\
\text { Methylene chloride } \\
\text { Chloromethane }\end{array}$ & & $\begin{array}{l}< \\
<\end{array}$ & & $\begin{array}{l}< \\
<\end{array}$ & & $\begin{array}{l}< \\
<\end{array}$ & & $\begin{array}{l}< \\
<\end{array}$ & & $\begin{array}{l}< \\
< \\
<\end{array}$ \\
\hline $\begin{array}{r}\text { Petrol. Hydrocarb. }(\boldsymbol{\mu g} / \mathbf{L}) \\
\text { Benzene } \\
\text { Dimethylbenzene } \\
\text { Ethylbenzene } \\
\text { Toluene }\end{array}$ & $<$ & $<$ & $<$ & $<$ & $<$ & $<$ & $<$ & $<$ & $<$ & $<$ \\
\hline $\begin{array}{r}\text { Miscellaneous ( } \boldsymbol{\mu g} / \mathbf{L}) \\
1,2-\text { Dichloropropane } \\
1,4 \text {-Dichlorobenzene } \\
\text { 2-Butanone } \\
\text { Acetone } \\
\text { Bromoform } \\
\text { Chlorobenzene } \\
\text { Dichlorodifluoromethane } \\
\text { Trichlorofluoromethane }\end{array}$ & $<1$ & $<1$ & $<1$ & $<$ & $<1$ & $<$ & $<$ & $<$ & $<1$ & $<$ \\
\hline
\end{tabular}


APPENDIX D.2: CY 2002 MONITORING DATA FOR THE BEAR CREEK HYDROGEOLOGIC REGIME Volatile Organic Compounds

\begin{tabular}{|c|c|c|c|c|c|c|c|c|c|c|}
\hline Sampling Point & \multicolumn{4}{|c|}{ GW-924 } & \multicolumn{2}{|c|}{ GW-925 } & \multicolumn{2}{|c|}{ GW-926 } & \multicolumn{2}{|c|}{ GW-927 } \\
\hline Location & \multicolumn{4}{|c|}{ EMWMF } & \multicolumn{2}{|c|}{ EMWMF } & \multicolumn{2}{|c|}{ EMWMF } & \multicolumn{2}{|c|}{ EMWMF } \\
\hline Date Sampled & $08 / 1$ & & $12 / 10$ & & 08/12/02 & $12 / 04 / 02$ & 08/13/02 & $12 / 10 / 02$ & $08 / 12 / 02$ & $12 / 05 / 02$ \\
\hline Program & BJC & BJC & BJC & BJC & BJC & BJC & BJC & BJC & BJC & BJC \\
\hline Sample Type & & Dup & & Dup & & & & & & \\
\hline $\begin{array}{r}\text { Chloroethenes }(\mu \mathrm{g} / \mathrm{L}) \\
\text { Tetrachloroethene } \\
\text { Trichloroethene } \\
\text { cis-1,2-Dichloroethene } \\
\text { trans-1,2-Dichloroethene } \\
1,1 \text {-Dichloroethene } \\
\text { Vinyl chloride } \\
\end{array}$ & $\begin{array}{l}< \\
<\end{array}$ & $<<$ & $\begin{array}{l}< \\
<\end{array}$ & $\begin{array}{l}< \\
<\end{array}$ & $\begin{array}{l}< \\
<\end{array}$ & $\begin{array}{l}< \\
<\end{array}$ & $\begin{array}{l}< \\
<\end{array}$ & $\begin{array}{l}< \\
<\end{array}$ & $\begin{array}{l}< \\
<\end{array}$ & $<$ \\
\hline $\begin{array}{r}\text { Chloroethanes }(\mu \mathrm{g} / \mathrm{L}) \\
1,1,1-\text { Trichloroethane } \\
1,1,2-\text { Trichloroethane } \\
1,2 \text {-Dichloroethane } \\
\text { 1,1-Dichloroethane } \\
\text { Chloroethane }\end{array}$ & & & & & & & & & & \\
\hline $\begin{array}{r}\text { Chloromethanes }(\mu \mathrm{g} / \mathrm{L}) \\
\text { Carbon tetrachloride } \\
\text { Chloroform } \\
\text { Methylene chloride } \\
\text { Chloromethane }\end{array}$ & & & $\begin{array}{l}< \\
<\end{array}$ & $\begin{array}{l}< \\
<\end{array}$ & & $\begin{array}{l}< \\
<\end{array}$ & & $\begin{array}{l}< \\
<\end{array}$ & & $<$ \\
\hline $\begin{array}{r}\text { Petrol. Hydrocarb. }(\boldsymbol{\mu g} / \mathbf{L}) \\
\text { Benzene } \\
\text { Dimethylbenzene } \\
\text { Ethylbenzene } \\
\text { Toluene }\end{array}$ & $<$ & $<1$ & $<$ & $<$ & $<$ & $<$ & $<$ & $<$ & $<$ & $<$ \\
\hline $\begin{array}{r}\text { Miscellaneous ( } \boldsymbol{\mu g} / \mathrm{L}) \\
1,2-\text {-Dichloropropane } \\
1,4 \text {-Dichlorobenzene } \\
2 \text {-Butanone } \\
\text { Acetone } \\
\text { Bromoform } \\
\text { Chlorobenzene } \\
\text { Dichlorodifluoromethane } \\
\text { Trichlorofluoromethane }\end{array}$ & $<$ & $<$ & $<$ & $<$ & $<$ & $<$ & $<$ & $<$ & $<1$ & $<$ \\
\hline
\end{tabular}


APPENDIX D.2: CY 2002 MONITORING DATA FOR THE BEAR CREEK HYDROGEOLOGIC REGIME Volatile Organic Compounds

\begin{tabular}{|c|c|c|c|c|c|c|c|c|c|c|}
\hline Sampling Point & \multicolumn{2}{|c|}{ NT-01 } & NT-03 & NT-07 & NT-08 & NT-8-E & NT-8-W & S07 & \multicolumn{2}{|c|}{ SS-1 } \\
\hline Location & \multicolumn{2}{|c|}{ EXP-SW } & EXP-SW & EXP-SW & EXP-SW & EXP-SW & EXP-SW & EXP-SW & \multicolumn{2}{|c|}{ EXP-SW } \\
\hline Date Sampled & $01 / 09 / 02$ & 07/11/02 & 03/11/02 & $03 / 13 / 02$ & $03 / 13 / 02$ & $03 / 13 / 02$ & $03 / 13 / 02$ & $03 / 11 / 02$ & $01 / 09 / 02$ & $07 / 15 / 02$ \\
\hline Program & GWPP & GWPP & BJC & BJC & BJC & BJC & BJC & BJC & GWPP & GWPP \\
\hline \multicolumn{11}{|l|}{ Sample Type } \\
\hline Chloroethenes ( $\mu \mathrm{g} / \mathrm{L}$ ) & & & & & & & & & & \\
\hline Tetrachloroethene & 62 & 58 & $<$ & 28 & 11 & $<$ & $<$ & $<$ & $<$ & $<$ \\
\hline Trichloroethene & $<$ & $<$ & $<$ & 23 & 6 & $<$ & $<$ & $<$ & $<$ & $<$ \\
\hline cis-1,2-Dichloroethene & $2 \mathrm{~J}$ & $2 \mathrm{~J}$ & $<$ & 85 & 66 & $2 \mathrm{~J}$ & $<$ & $<$ & $<$ & $<$ \\
\hline trans-1,2-Dichloroethene & $<$ & $<$ & $<$ & $<$ & $<$ & $<$ & $<$ & $<$ & $<$ & $<$ \\
\hline 1,1-Dichloroethene & $<$ & $<$ & $<$ & $3 \mathrm{~J}$ & $1 \mathrm{~J}$ & $<$ & $<$ & $<$ & $<$ & $<$ \\
\hline Vinyl chloride & $<$ & $<$ & $<$ & 3 & $1 \mathrm{~J}$ & $<$ & $<$ & $<$ & $<$ & $<$ \\
\hline \multicolumn{11}{|l|}{ Chloroethanes ( $\mu \mathrm{g} / \mathrm{L})$} \\
\hline 1,1,1-Trichloroethane & $<$ & $<$ & $<$ & $2 \mathrm{~J}$ & $<$ & $<$ & $<$ & $<$ & $<$ & $<$ \\
\hline 1,1,2-Trichloroethane & $<$ & $<$ & $<$ & $<$ & $<$ & $<$ & $<$ & $<$ & $<$ & $<$ \\
\hline 1,2-Dichloroethane & $<$ & $<$ & $<$ & $<$ & $<$ & $<$ & $<$ & $<$ & $<$ & $<$ \\
\hline 1,1-Dichloroethane & $<$ & $<$ & $<$ & 8 & $4 \mathrm{~J}$ & $<$ & $<$ & $<$ & $<$ & $<$ \\
\hline Chloroethane & $<$ & $<$ & $<$ & $<$ & $<$ & $<$ & $<$ & $<$ & $<$ & $<$ \\
\hline \multicolumn{11}{|l|}{ Chloromethanes $(\mu \mathrm{g} / \mathrm{L})$} \\
\hline Carbon tetrachloride & $<$ & $<$ & $<$ & $<$ & $<$ & $<$ & $<$ & $<$ & $<$ & $<$ \\
\hline Chloroform & $<$ & $2 \mathrm{~J}$ & $<$ & $1 \mathrm{~J}$ & $<$ & $<$ & $<$ & $<$ & $<$ & $<$ \\
\hline Methylene chloride & $4 \mathrm{~J}$ & $3 \mathrm{~J}$ & $<$ & $<$ & $<$ & $<$ & $<$ & $<$ & $<$ & $<$ \\
\hline Chloromethane & $<$ & $<$ & $<$ & $2 \mathrm{~J}$ & $<$ & $<$ & $<$ & $<$ & $<$ & $<$ \\
\hline \multicolumn{11}{|l|}{ Petrol. Hydrocarb. ( $\mu \mathrm{g} / \mathrm{L})$} \\
\hline Benzene & $<$ & $<$ & $<$ & $<$ & $<$ & $<$ & $<$ & $<$ & $<$ & $<$ \\
\hline Dimethylbenzene & $<$ & $<$ & $<$ & $<$ & $<$ & $<$ & $<$ & $<$ & $<$ & $<$ \\
\hline Ethylbenzene & $<$ & $<$ & $<$ & $<$ & $<$ & $<$ & $<$ & $<$ & $<$ & $<$ \\
\hline Toluene & $<$ & $<$ & $<$ & $<$ & $<$ & $<$ & $<$ & $<$ & $<$ & $<$ \\
\hline \multicolumn{11}{|l|}{ Miscellaneous ( $\mu \mathrm{g} / \mathrm{L})$} \\
\hline 1,2-Dichloropropane & $<$ & $<$ & $<$ & $<$ & $<$ & $<$ & $<$ & $<$ & $<$ & $<$ \\
\hline 1,4-Dichlorobenzene & $<$ & $<$ & & & & & & & $<$ & $<$ \\
\hline 2-Butanone & $<$ & $<$ & $<$ & $<$ & $<$ & $<$ & $<$ & $<$ & $<$ & $<$ \\
\hline Acetone & $<$ & $<$ & $<$ & $<$ & $<$ & $<$ & $<$ & $<$ & $<$ & $<$ \\
\hline Bromoform & $<$ & $<$ & $<$ & $<$ & $<$ & $<$ & $<$ & $<$ & $<$ & $<$ \\
\hline Chlorobenzene & $<$ & $<$ & $<$ & $<$ & $<$ & $<$ & $<$ & $<$ & $<$ & $<$ \\
\hline Dichlorodifluoromethane & $<$ & $<$ & & & & & & & $<$ & $<$ \\
\hline Trichlorofluoromethane & $<$ & $<$ & & & & & & & $<$ & $<$ \\
\hline
\end{tabular}


APPENDIX D.2: CY 2002 MONITORING DATA FOR THE BEAR CREEK HYDROGEOLOGIC REGIME Volatile Organic Compounds

\begin{tabular}{|c|c|c|c|c|c|c|c|c|c|}
\hline Sampling Point & \multicolumn{2}{|c|}{ SS-4 } & \multicolumn{3}{|c|}{ SS-5 } & SS-7 & \multicolumn{3}{|c|}{ SS-8 } \\
\hline Location & \multicolumn{2}{|c|}{ EXP-SW } & \multicolumn{3}{|c|}{ EXP-SW } & EXP-SW & \multicolumn{3}{|c|}{ EXP-SW } \\
\hline Date Sampled & \multirow{2}{*}{$\begin{array}{c}\text { 01/08/02 } \\
\text { GWPP }\end{array}$} & \multirow{2}{*}{\begin{tabular}{|c|} 
07/10/02 \\
GWPP \\
\end{tabular}} & \multicolumn{2}{|c|}{$01 / 08 / 02$} & \multirow{2}{*}{\begin{tabular}{|c|} 
07/10/02 \\
GWPP \\
\end{tabular}} & \multirow{2}{*}{\begin{tabular}{|c|}
$03 / 12 / 02$ \\
BJC \\
\end{tabular}} & \multirow{2}{*}{$\begin{array}{c}03 / 12 / 02 \\
\text { BJC }\end{array}$} & \multicolumn{2}{|c|}{ 09/09/02 } \\
\hline Program & & & GWPP & GWPP & & & & BJC & BJC \\
\hline Sample Type & & & & Dup & & & & & Dup \\
\hline Chloroethenes ( $\mu \mathrm{g} / \mathrm{L}$ ) & & & & & & & & & \\
\hline Tetrachloroethene & $<$ & $<$ & $<$ & $<$ & $<$ & $<$ & $<$ & $<$ & $<$ \\
\hline Trichloroethene & 8 & 20 & $<$ & $<$ & $2 \mathrm{~J}$ & $<$ & $<$ & $<$ & $<$ \\
\hline cis-1,2-Dichloroethene & $4 \mathrm{~J}$ & 17 & $2 \mathrm{~J}$ & $2 \mathrm{~J}$ & $3 \mathrm{~J}$ & $<$ & $<$ & $<$ & $<$ \\
\hline trans-1,2-Dichloroethene & $<$ & $<$ & $<$ & $<$ & $<$ & $<$ & $<$ & $<$ & $<$ \\
\hline 1,1-Dichloroethene & $<$ & $2 \mathrm{~J}$ & $<$ & $<$ & $<$ & $<$ & $<$ & $<$ & $<$ \\
\hline Vinyl chloride & $<$ & $<$ & $<$ & $<$ & $<$ & $<$ & $<$ & $<$ & $<$ \\
\hline Chloroethanes ( $\mu \mathrm{g} / \mathrm{L})$ & & & & & & & & & \\
\hline 1,1,1-Trichloroethane & $<$ & $<$ & $<$ & $<$ & $<$ & $<$ & $<$ & $<$ & $<$ \\
\hline 1,1,2-Trichloroethane & $<$ & $<$ & $<$ & $<$ & $<$ & $<$ & $<$ & $<$ & $<$ \\
\hline 1,2-Dichloroethane & $<$ & $<$ & $<$ & $<$ & $<$ & $<$ & $<$ & $<$ & $<$ \\
\hline 1,1-Dichloroethane & $<$ & $<$ & $<$ & $<$ & $<$ & $<$ & $<$ & $<$ & $<$ \\
\hline Chloroethane & $<$ & $<$ & $<$ & $<$ & $<$ & $<$ & $<$ & $<$ & $<$ \\
\hline Chloromethanes ( $\mu \mathrm{g} / \mathrm{L})$ & & & & & & & & & \\
\hline Carbon tetrachloride & $<$ & $<$ & $<$ & $<$ & $<$ & $<$ & $<$ & $<$ & $<$ \\
\hline Chloroform & $<$ & $<$ & $<$ & $<$ & $<$ & $<$ & $<$ & $<$ & $<$ \\
\hline Methylene chloride & $<$ & $<$ & $<$ & $<$ & $<$ & $<$ & $<$ & $<$ & $<$ \\
\hline Chloromethane & $<$ & $<$ & $<$ & $<$ & $<$ & $<$ & $<$ & $<$ & $<$ \\
\hline Petrol. Hydrocarb. ( $\mu \mathrm{g} / \mathrm{L})$ & & & & & & & & & \\
\hline Benzene & $<$ & $<$ & $<$ & $<$ & $<$ & $<$ & $<$ & $<$ & $<$ \\
\hline Dimethylbenzene & $<$ & $<$ & $<$ & $<$ & $<$ & $<$ & $<$ & $<$ & $<$ \\
\hline Ethylbenzene & $<$ & $<$ & $<$ & $<$ & $<$ & $<$ & $<$ & $<$ & $<$ \\
\hline Toluene & $<$ & $<$ & $<$ & $<$ & $<$ & $<$ & $<$ & $<$ & $<$ \\
\hline Miscellaneous ( $\mu \mathrm{g} / \mathrm{L})$ & & & & & & & & & \\
\hline 1,2-Dichloropropane & $<$ & $<$ & $<$ & $<$ & $<$ & $<$ & $<$ & $<$ & $<$ \\
\hline 1,4-Dichlorobenzene & $<$ & $<$ & $<$ & $<$ & $<$ & & & & \\
\hline 2-Butanone & $<$ & $<$ & $<$ & $<$ & $<$ & $<$ & $<$ & $<$ & $<$ \\
\hline Acetone & $<$ & $<$ & $<$ & $<$ & $<$ & $<$ & $<$ & $<$ & $<$ \\
\hline Bromoform & $<$ & $<$ & $<$ & $<$ & $<$ & $<$ & $<$ & $<$ & $<$ \\
\hline Chlorobenzene & $<$ & $<$ & $<$ & $<$ & $<$ & $<$ & $<$ & $<$ & $<$ \\
\hline Dichlorodifluoromethane & $<$ & $<$ & $<$ & $<$ & $<$ & & & & \\
\hline Trichlorofluoromethane & $<$ & $<$ & $<$ & $<$ & $<$ & & & & \\
\hline
\end{tabular}


APPENDIX D.3

RADIOLOGICAL ANALYTES 
APPENDIX D.3: CY 2002 MONITORING DATA FOR THE BEAR CREEK HYDROGEOLOGIC REGIME Radiological Analytes: Gross Alpha and Gross Beta Activity

\begin{tabular}{|c|c|c|c|c|c|c|c|c|c|}
\hline \multirow{2}{*}{$\begin{array}{c}\text { Sampling } \\
\text { Point }\end{array}$} & \multirow{2}{*}{ Location } & \multirow{2}{*}{$\begin{array}{c}\text { Date } \\
\text { Sampled }\end{array}$} & \multirow{2}{*}{ Program } & \multicolumn{3}{|c|}{ Gross Alpha (pCi/L) } & \multicolumn{3}{|c|}{ Gross Beta (pCi/L) } \\
\hline & & & & Activity & Error & MDA & Activity & Error & MDA \\
\hline BCK-00.63 & EXP-SW & 01/08/02 & GWPP & 9 & 3.5 & 3.2 & 15 & 6.1 & 8.9 \\
\hline BCK-00.63 & EXP-SW & 07/10/02 & GWPP & 5.7 & 3.1 & 3.5 & 10 & 5.5 & 8.2 \\
\hline BCK-03.30 & EXP-SW & $03 / 12 / 02$ & BJC & . & . & . & 15.96 & 1.78 & 1.57 \\
\hline BCK-03.30 & EXP-SW & 09/09/02 & BJC & . & . & . & 6.94 & 1.51 & 1.81 \\
\hline BCK-04.55 & EXP-SW & 01/09/02 & GWPP & 21 & 5.1 & 3.3 & 32 & 6.6 & 7.7 \\
\hline BCK-04.55 & EXP-SW & 07/10/02 & GWPP & 5.3 & 3 & 3.5 & 11 & 4 & 5.4 \\
\hline BCK-07.87 & EXP-SW & 01/08/02 & GWPP & 62 & 8.7 & 2.7 & 110 & 11 & 8.8 \\
\hline BCK-07.87 & EXP-SW & 07/10/02 & GWPP & 31 & 6 & 2.6 & 33 & 6.4 & 7.2 \\
\hline BCK-09.20 & EXP-SW & 03/11/02 & BJC & . & . & . & 50.04 & 2.86 & 1.78 \\
\hline BCK-09.20 & EXP-SW & 09/09/02 & BJC & . & . & . & 48.28 & 3.23 & 2.24 \\
\hline BCK-09.40 & EXP-SW & 01/09/02 & GWPP & 80 & 9.8 & 0.82 & 120 & 11 & 7.4 \\
\hline BCK-09.40 & EXP-SW & 07/11/02 & GWPP & 68 & 8.9 & 3.9 & 23 & 5.8 & 7.2 \\
\hline BCK-09.40 Dup & EXP-SW & 07/11/02 & GWPP & 67 & 8.8 & 2.6 & 35 & 6.7 & 7.5 \\
\hline BCK-09.47 & EXP-SW & 03/11/02 & BJC & . & . & . & 76.09 & 3.81 & 2.14 \\
\hline BCK-11.54 & EXP-SW & 03/11/02 & BJC & . & . & . & 158.71 & 4.68 & 2.14 \\
\hline BCK-11.54 & EXP-SW & 09/09/02 & BJC & . & . & . & 4.3 & 1.39 & 1.83 \\
\hline BCK-11.84 & EXP-SW & 03/11/02 & BJC & . & . & . & 222.91 & 5.31 & 2.33 \\
\hline BCK-11.97 & EXP-SW & 01/09/02 & GWPP & 89 & 12 & 4.8 & 410 & 21 & 11 \\
\hline BCK-11.97 & EXP-SW & 07/11/02 & GWPP & 83 & 19 & 12 & 780 & 25 & 6.9 \\
\hline GW-008 & OLF & 01/07/02 & BJC & $<\mathrm{MDA}$ & . & 1.09 & $<\mathrm{MDA}$ & . & 1.88 \\
\hline GW-008 & OLF & $07 / 02 / 02$ & BJC & $<M D A$ & . & 1.23 & 2.52 & 1.06 & 1.45 \\
\hline GW-014 & $B G$ & 03/14/02 & GWPP & $<\mathrm{MDA}$ & . & 3.3 & $<\mathrm{MDA}$ & . & 7.3 \\
\hline GW-014 Dup & $B G$ & $03 / 14 / 02$ & GWPP & 1.3 & 1.3 & 0.85 & $<\mathrm{MDA}$ & . & 8.4 \\
\hline GW-014 & BG & $08 / 12 / 02$ & GWPP & 2.3 & 1.8 & 2 & $<\mathrm{MDA}$ & . & 10 \\
\hline GW-046 & $B G$ & 01/07/02 & BJC & 1.84 & 0.99 & 1.23 & 5.38 & 1.48 & 1.92 \\
\hline GW-046 & BG & $07 / 02 / 02$ & BJC & 0.98 & 0.72 & 0.97 & 3.71 & 1.09 & 1.39 \\
\hline GW-061 & BG & 03/12/02 & GWPP & 37 & 6.9 & 0.89 & 63 & 7.5 & 6.3 \\
\hline GW-061 & $B G$ & 08/05/02 & GWPP & 42 & 8.7 & 3.4 & 73 & 9.3 & 8.9 \\
\hline GW-066 & OLF & 03/25/02 & GWPP & 7.4 & 3.6 & 4 & $<\mathrm{MDA}$ & . & 9.3 \\
\hline GW-066 & OLF & 08/13/02 & GWPP & 6.7 & 3.5 & 3.8 & $<\mathrm{MDA}$ & . & 7.9 \\
\hline GW-066 Dup & OLF & 08/13/02 & GWPP & 12 & 4.2 & 3 & 10 & 5.2 & 7.7 \\
\hline GW-069 & BG & $03 / 11 / 02$ & GWPP & $<\mathrm{MDA}$ & $\cdot$ & 0.95 & 7.6 & 4 & 5.8 \\
\hline GW-069 & $B G$ & 08/05/02 & GWPP & $<\mathrm{MDA}$ & . & 2.7 & 11 & 5.9 & 8.9 \\
\hline GW-071 & $B G$ & 03/12/02 & GWPP & $<M D A$ & . & 24 & $<M D A$ & . & 72 \\
\hline GW-071 & $B G$ & 08/06/02 & GWPP & $<\mathrm{MDA}$ & . & 35 & $<\mathrm{MDA}$ & . & 90 \\
\hline GW-071 Dup & $B G$ & 08/06/02 & GWPP & $<\mathrm{MDA}$ & . & 35 & $<\mathrm{MDA}$ & . & 69 \\
\hline GW-072 & $B G$ & $03 / 11 / 02$ & GWPP & $<\mathrm{MDA}$ & . & 0.98 & $<\mathrm{MDA}$ & . & 7.5 \\
\hline GW-072 & $B G$ & 08/06/02 & GWPP & $<\mathrm{MDA}$ & . & 4.7 & $<M D A$ & . & 8.2 \\
\hline GW-076 & OLF & 03/19/02 & GWPP & $<\mathrm{MDA}$ & . & 2.3 & $<\mathrm{MDA}$ & . & 9.9 \\
\hline GW-077 & $B G$ & 02/26/02 & BJC & $<\mathrm{MDA}$ & . & 1.75 & $<M D A$ & . & 2 \\
\hline GW-077 & $B G$ & 08/08/02 & BJC & $<M D A$ & . & 1.83 & 3.19 & 1.3 & 1.77 \\
\hline GW-078 & $B G$ & 02/26/02 & BJC & $<\mathrm{MDA}$ & . & 1.71 & $<\mathrm{MDA}$ & . & 1.99 \\
\hline GW-078 & $B G$ & 08/08/02 & BJC & $<\mathrm{MDA}$ & . & 0.6 & $<M D A$ & . & 0.99 \\
\hline GW-079 & $B G$ & 02/26/02 & BJC & $<M D A$ & . & 1.29 & $<M D A$ & . & 1.89 \\
\hline GW-079 & $B G$ & 08/07/02 & BJC & $<\mathrm{MDA}$ & . & 1.72 & 2.63 & 1.31 & 1.86 \\
\hline GW-080 & $B G$ & 02/26/02 & BJC & $<\mathrm{MDA}$ & . & 1.31 & 3.51 & 1.38 & 1.89 \\
\hline GW-080 Dup & $B G$ & 02/26/02 & BJC & $<M D A$ & . & 1.29 & $<M D A$ & . & 1.89 \\
\hline GW-080 & $B G$ & 08/07/02 & BJC & $<\mathrm{MDA}$ & . & 0.9 & 1.61 & 1.1 & 1.6 \\
\hline GW-080 Dup & $B G$ & 08/07/02 & BJC & $<M D A$ & . & 1.14 & $<$ MDA & . & 1.91 \\
\hline
\end{tabular}


APPENDIX D.3: CY 2002 MONITORING DATA FOR THE BEAR CREEK HYDROGEOLOGIC REGIME Radiological Analytes: Gross Alpha and Gross Beta Activity

\begin{tabular}{|c|c|c|c|c|c|c|c|c|c|}
\hline \multirow{2}{*}{$\begin{array}{c}\text { Sampling } \\
\text { Point }\end{array}$} & \multirow{2}{*}{ Location } & \multirow{2}{*}{$\begin{array}{c}\text { Date } \\
\text { Sampled }\end{array}$} & \multirow{2}{*}{ Program } & \multicolumn{3}{|c|}{ Gross Alpha (pCi/L) } & \multicolumn{3}{|c|}{ Gross Beta (pCi/L) } \\
\hline & & & & Activity & Error & MDA & Activity & Error & MDA \\
\hline GW-082 & BG & $02 / 12 / 02$ & GWPP & $<\mathrm{MDA}$ & & 5.3 & $<\mathrm{MDA}$ & & 8.8 \\
\hline GW-082 & BG & 07/29/02 & GWPP & $<\mathrm{MDA}$ & & 4.8 & $<\mathrm{MDA}$ & & 8.9 \\
\hline GW-085 & OLF & 02/18/02 & GWPP & $<\mathrm{MDA}$ & & 8.4 & 240 & 16 & 11 \\
\hline GW-085 & OLF & 07/31/02 & GWPP & $<\mathrm{MDA}$ & . & 6.4 & 220 & 15 & 9.6 \\
\hline GW-087 & OLF & $03 / 25 / 02$ & GWPP & 20 & 5 & 2.3 & 15 & 6.8 & 10 \\
\hline GW-091 & BG & $03 / 14 / 02$ & GWPP & 2 & 1.5 & 0.77 & $<\mathrm{MDA}$ & . & 10 \\
\hline GW-091 & BG & 08/08/02 & GWPP & 8.3 & 3.2 & 1.9 & $<\mathrm{MDA}$ & & 8.2 \\
\hline GW-115 & S3 & $01 / 07 / 02$ & BJC & $<\mathrm{MDA}$ & & 1.61 & 2.29 & 1.76 & 2.08 \\
\hline GW-115 & s3 & 07/08/02 & BJC & $<\mathrm{MDA}$ & . & 3.47 & 6.54 & 1.95 & 2.14 \\
\hline GW-225 & OLF & 02/19/02 & GWPP & $<M D A$ & & 5.3 & 19 & 5.4 & 6.8 \\
\hline GW-225 & OLF & 08/01/02 & GWPP & $<M D A$ & . & 3.6 & 19 & 5 & 6.1 \\
\hline GW-226 & OLF & 02/19/02 & GWPP & 8.4 & 4.4 & 5.3 & 17 & 5.2 & 6.8 \\
\hline GW-226 & OLF & 08/01/02 & GWPP & 4.5 & 2.6 & 2.4 & 17 & 4.8 & 5.9 \\
\hline GW-229 & OLF & 03/19/02 & GWPP & 110 & 14 & 1.2 & 84 & 10 & 9.8 \\
\hline GW-229 Dup & OLF & 03/19/02 & GWPP & 260 & 22 & 3.7 & 130 & 13 & 12 \\
\hline GW-229 & OLF & 08/12/02 & GWPP & 73 & 30 & 24 & 87 & 48 & 73 \\
\hline GW-243 & S3 & 03/21/02 & GWPP & 540 & 190 & 44 & 13,000 & 520 & 220 \\
\hline GW-243 & S3 & 08/13/02 & GWPP & $<\mathrm{MDA}$ & & 740 & 17,000 & 1,300 & 840 \\
\hline GW-276 & S3 & $01 / 07 / 02$ & BJC & 238.65 & 12.17 & 3.47 & 357.54 & 8.47 & 3.63 \\
\hline GW-276 & s3 & 07/08/02 & BJC & 210.7 & 11.06 & 4.23 & 391.23 & 7.68 & 2.46 \\
\hline GW-288 & BG & 03/13/02 & GWPP & $<M D A$ & & 2.5 & $<M D A$ & . & 7.6 \\
\hline GW-288 & BG & 08/07/02 & GWPP & $<M D A$ & & 2.1 & $<M D A$ & & 7.8 \\
\hline GW-289 & $B G$ & 03/13/02 & GWPP & $<M D A$ & & 0.74 & $<M D A$ & & 6.5 \\
\hline GW-289 & $B G$ & 08/08/02 & GWPP & $<M D A$ & & 2.1 & $<M D A$ & . & 8.5 \\
\hline GW-291 & BG & $03 / 14 / 02$ & GWPP & $<\mathrm{MDA}$ & & 2.6 & $<\mathrm{MDA}$ & . & 7.3 \\
\hline GW-291 & BG & 08/07/02 & GWPP & $<M D A$ & & 3.2 & $<\mathrm{MDA}$ & . & 6.8 \\
\hline GW-311 & RS & $02 / 13 / 02$ & GWPP & 4.6 & 3.1 & 4.2 & $<\mathrm{MDA}$ & . & 8.7 \\
\hline GW-311 Dup & RS & 02/13/02 & GWPP & $<\mathrm{MDA}$ & . & 3.6 & $<\mathrm{MDA}$ & . & 8.8 \\
\hline GW-311 & RS & 07/30/02 & GWPP & $<M D A$ & & 2.7 & $<M D A$ & & 7 \\
\hline GW-315 & SPI & 02/13/02 & GWPP & $<\mathrm{MDA}$ & . & 5.5 & 27 & 8.3 & 11 \\
\hline GW-315 & SPI & 07/30/02 & GWPP & $<\mathrm{MDA}$ & & 2.4 & 38 & 6.7 & 6.9 \\
\hline GW-526 & S3 & 02/26/02 & BJC & $<M D A$ & & 40.2 & $<M D A$ & & 43.58 \\
\hline GW-526 & S3 & 08/08/02 & BJC & $<M D A$ & . & 27.04 & $<M D A$ & . & 41.82 \\
\hline GW-537 & OLF & 02/18/02 & GWPP & $<M D A$ & & 20 & 540 & 34 & 22 \\
\hline GW-537 & OLF & 07/31/02 & GWPP & $<M D A$ & & 14 & 640 & 51 & 36 \\
\hline GW-537 Dup & OLF & 07/31/02 & GWPP & $<\mathrm{MDA}$ & . & 15 & 660 & 46 & 25 \\
\hline GW-626 & BG & $02 / 11 / 02$ & GWPP & $<\mathrm{MDA}$ & . & 3 & $<\mathrm{MDA}$ & . & 9.9 \\
\hline GW-626 & BG & $07 / 25 / 02$ & GWPP & $<M D A$ & & 4.3 & $<\mathrm{MDA}$ & & 7.4 \\
\hline GW-627 & BG & 02/11/02 & GWPP & $<\mathrm{MDA}$ & . & 22 & $<\mathrm{MDA}$ & . & 11 \\
\hline GW-627 & BG & 07/29/02 & GWPP & $19 Q$ & 9.9 & 10 & $<\mathrm{MDA}$ & . & 18 \\
\hline GW-653 & BG & $02 / 12 / 02$ & GWPP & $<M D A$ & & 3.6 & $<M D A$ & & 8.4 \\
\hline GW-653 & BG & $07 / 25 / 02$ & GWPP & $<M D A$ & & 2.5 & $<\mathrm{MDA}$ & . & 9.6 \\
\hline GW-683 & EXP-A & $01 / 14 / 02$ & GWPP & 20 & 4.9 & 0.81 & 46 & 7.3 & 7.4 \\
\hline GW-683 & EXP-A & 07/09/02 & GWPP & 15 & 4.2 & 0.78 & 20 & 6 & 7.7 \\
\hline GW-684 & EXP-A & 01/14/02 & GWPP & 23 & 5.6 & 3.4 & 49 & 9.4 & 11 \\
\hline GW-684 & EXP-A & 07/09/02 & GWPP & 17 & 4.9 & 4.1 & 31 & 6.4 & 7.3 \\
\hline GW-684 Dup & EXP-A & 07/09/02 & GWPP & 16 & 4.4 & 2.6 & 31 & 6.2 & 6.7 \\
\hline GW-694 & EXP-B & 01/29/02 & GWPP & 22 & 5.1 & 3 & 14 & 5.9 & 8.5 \\
\hline GW-694 & EXP-B & 07/17/02 & GWPP & 15 & 4.5 & 3.6 & 20 & 5.7 & 7.2 \\
\hline
\end{tabular}


APPENDIX D.3: CY 2002 MONITORING DATA FOR THE BEAR CREEK HYDROGEOLOGIC REGIME Radiological Analytes: Gross Alpha and Gross Beta Activity

\begin{tabular}{|c|c|c|c|c|c|c|c|c|c|}
\hline \multirow{2}{*}{$\begin{array}{l}\text { Sampling } \\
\text { Point }\end{array}$} & \multirow{2}{*}{ Location } & \multirow{2}{*}{$\begin{array}{c}\text { Date } \\
\text { Sampled }\end{array}$} & \multirow{2}{*}{ Program } & \multicolumn{3}{|c|}{ Gross Alpha (pCi/L) } & \multicolumn{3}{|c|}{ Gross Beta (pCi/L) } \\
\hline & & & & Activity & Error & MDA & Activity & Error & MDA \\
\hline GW-695 & EXP-B & 01/15/02 & GWPP & 5.7 & 2.8 & 2.7 & 50 & 8 & 8.5 \\
\hline GW-695 & EXP-B & 07/15/02 & GWPP & 7.4 & 3.4 & 3.7 & 42 & 7.3 & 8 \\
\hline GW-703 & EXP-B & 01/15/02 & GWPP & 7.8 & 3.5 & 3.5 & 62 & 8.9 & 9.3 \\
\hline GW-703 & EXP-B & 07/15/02 & GWPP & 4.1 & 2.5 & 2.5 & 62 & 8.2 & 7.3 \\
\hline GW-704 & EXP-B & 01/16/02 & GWPP & 6.2 & 3.2 & 3.5 & 26 & 5.7 & 6.7 \\
\hline GW-704 Dup & EXP-B & 01/16/02 & GWPP & 5.2 & 3.3 & 4.3 & 27 & 5.5 & 5.9 \\
\hline GW-704 & EXP-B & 07/16/02 & GWPP & 6.2 & 2.9 & 2 & 41 & 8.1 & 8.8 \\
\hline GW-706 & EXP-B & 01/16/02 & GWPP & 58 & 9 & 3.9 & 140 & 11 & 6.8 \\
\hline GW-706 & EXP-B & 07/16/02 & GWPP & 45 & 7.8 & 2.1 & 72 & 8.5 & 6.9 \\
\hline GW-712 & EXP-W & 01/03/02 & BJC & $<\mathrm{MDA}$ & & 1.98 & 2.3 & 1.23 & 1.74 \\
\hline GW-712 & EXP-W & 07/01/02 & BJC & 2.13 & 1.68 & 1.96 & 5.73 & 2.17 & 2.45 \\
\hline GW-713 & EXP-W & 01/03/02 & BJC & 1.66 & 1.1 & 1.22 & 7.73 & 1.57 & 1.79 \\
\hline GW-713 & EXP-W & 07/01/02 & BJC & 15.75 & 2.77 & 2.09 & 39.75 & 3.06 & 2.48 \\
\hline GW-714 & EXP-W & $01 / 02 / 02$ & BJC & 2.2 & 1.36 & 1.75 & 6.1 & 1.54 & 1.94 \\
\hline GW-714 & EXP-W & 07/01/02 & BJC & 3 & 1.61 & 1.76 & 4.38 & 2.09 & 2.41 \\
\hline GW-715 & EXP-W & $01 / 02 / 02$ & BJC & 5.7 & 2.14 & 2.26 & 5.33 & 1.53 & 1.99 \\
\hline GW-715 Dup & EXP-W & $01 / 02 / 02$ & BJC & 5.24 & 1.61 & 1.51 & 4.91 & 1.84 & 2.09 \\
\hline GW-715 & EXP-W & 07/01/02 & BJC & 17.96 & 2.5 & 1.67 & 39.92 & 2.97 & 2.38 \\
\hline GW-715 Dup & EXP-W & 07/01/02 & BJC & 16.02 & 2.45 & 1.72 & 22.57 & 2.58 & 2.39 \\
\hline GW-723 & EXP-C & 03/05/02 & GWPP & 1.9 & 1.4 & 0.74 & $<\mathrm{MDA}$ & & 6.5 \\
\hline GW-723 & EXP-C & $07 / 23 / 02$ & GWPP & 6.3 & 3.2 & 3.7 & $<\mathrm{MDA}$ & & 9.3 \\
\hline GW-724 & EXP-C & 01/30/02 & GWPP & $<\mathrm{MDA}$ & & 3.5 & 38 & 8.7 & 11 \\
\hline GW-724 & EXP-C & 07/23/02 & GWPP & $<\mathrm{MDA}$ & . & 3.4 & $<\mathrm{MDA}$ & & 9.7 \\
\hline GW-725 & EXP-C & 01/31/02 & GWPP & $<\mathrm{MDA}$ & & 4.6 & 32 & 7.5 & 9.2 \\
\hline GW-725 & EXP-C & 07/18/02 & GWPP & 8.4 & 3.9 & 3.1 & 29 & 5.8 & 6.3 \\
\hline GW-736 & EXP-C & 03/06/02 & GWPP & 24 & 7.6 & 5.7 & 68 & 9.2 & 7.9 \\
\hline GW-736 & EXP-C & 07/17/02 & GWPP & $<\mathrm{MDA}$ & . & 4.3 & $<\mathrm{MDA}$ & . & 9 \\
\hline GW-737 & EXP-C & 03/06/02 & GWPP & 9.6 & 3.8 & 1 & 32 & 7.3 & 9 \\
\hline GW-737 & EXP-C & 07/18/02 & GWPP & 5.9 & 3.8 & 4.5 & 29 & 5.7 & 5.9 \\
\hline GW-738 & EXP-C & 01/30/02 & GWPP & $<M D A$ & & 2.6 & 57 & 8.5 & 9.1 \\
\hline GW-738 & EXP-C & 07/23/02 & GWPP & 7.5 & 3.7 & 3.7 & 55 & 9.1 & 10 \\
\hline GW-739 & EXP-C & 03/05/02 & GWPP & $<\mathrm{MDA}$ & . & 2.6 & $<\mathrm{MDA}$ & . & 6.1 \\
\hline GW-739 & EXP-C & 07/22/02 & GWPP & $<\mathrm{MDA}$ & . & 2.6 & $<\mathrm{MDA}$ & . & 9.3 \\
\hline GW-740 & EXP-C & 01/29/02 & GWPP & $<\mathrm{MDA}$ & & 2.2 & $<\mathrm{MDA}$ & & 9.4 \\
\hline GW-740 & EXP-C & $07 / 22 / 02$ & GWPP & $<\mathrm{MDA}$ & & 4.2 & $<\mathrm{MDA}$ & & 12 \\
\hline NT-01 & EXP-SW & 01/09/02 & GWPP & 20 & 7.6 & 6.5 & 970 & 33 & 12 \\
\hline NT-01 & EXP-SW & 07/11/02 & GWPP & 150 & 48 & 29 & 6000 & 250 & 88 \\
\hline SS-1 & EXP-SW & $01 / 09 / 02$ & GWPP & 21 & 6.4 & 5.2 & 60 & 8.2 & 7.4 \\
\hline SS-1 & EXP-SW & 07/15/02 & GWPP & 29 & 7.4 & 4 & 51 & 7.6 & 7.2 \\
\hline SS-4 & EXP-SW & $01 / 08 / 02$ & GWPP & 73 & 9.9 & 4.5 & 180 & 13 & 8.2 \\
\hline SS-4 & EXP-SW & 07/10/02 & GWPP & 43 & 7.6 & 2.5 & 53 & 7.1 & 6.3 \\
\hline SS-5 & EXP-SW & 01/08/02 & GWPP & 48 & 7.8 & 2.8 & 81 & 8.4 & 6.7 \\
\hline SS-5 Dup & EXP-SW & 01/08/02 & GWPP & 55 & 8.3 & 2.4 & 75 & 8.1 & 6.4 \\
\hline SS-5 & EXP-SW & 07/10/02 & GWPP & 31 & 6 & 2.3 & 37 & 7 & 8 \\
\hline SS-7 & EXP-SW & $03 / 12 / 02$ & BJC & . & . & . & 9.29 & 1.68 & 1.69 \\
\hline SS-8 & EXP-SW & $03 / 12 / 02$ & BJC & & & & 2.71 & 1.14 & 1.54 \\
\hline SS-8 & EXP-SW & 09/09/02 & BJC & & . & . & $<\mathrm{MDA}$ & & 1.8 \\
\hline SS-8 Dup & EXP-SW & 09/09/02 & BJC & . & . & . & $<M D A$ & . & 1.79 \\
\hline
\end{tabular}




$$
\text { D. 3-4 }
$$


APPENDIX D.3: CY 2002 MONITORING DATA FOR THE BEAR CREEK HYDROGEOLOGIC REGIME

Radiological Analytes: Isotope Activity

\begin{tabular}{|c|c|c|c|c|c|c|c|c|c|}
\hline Sampling Point & \multicolumn{6}{|c|}{ BCK-00.63 } & \multicolumn{3}{|c|}{ BCK-03.30 } \\
\hline Location & \multicolumn{6}{|c|}{ EXP-SW } & \multicolumn{3}{|c|}{ EXP-SW } \\
\hline Date Sampled & \multicolumn{3}{|c|}{$01 / 08 / 02$} & \multicolumn{3}{|c|}{$07 / 10 / 02$} & \multicolumn{3}{|c|}{$03 / 12 / 02$} \\
\hline Program & \multicolumn{3}{|c|}{ GWPP } & \multicolumn{3}{|c|}{ GWPP } & \multicolumn{3}{|c|}{ BJC } \\
\hline \multicolumn{10}{|l|}{ Sample Type } \\
\hline Result (pCi/L) & Activity & Error & MDA & Activity & Error & MDA & Activity & Error & MDA \\
\hline Gross Alpha & 9 & 3.5 & 3.2 & 5.7 & 3.1 & 3.5 & & & \\
\hline Gross Beta & 15 & 6.1 & 8.9 & 10 & 5.5 & 8.2 & 15.96 & 1.78 & 1.57 \\
\hline Americium-241 & & & & & & & & & \\
\hline Neptunium-237 & & & & & & & & & \\
\hline Plutonium-238 & & & & & & & & & \\
\hline Radium-223/224/226 & & & & & & & & & \\
\hline Strontium-89/90 & $<\mathrm{MDA}$ & 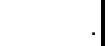 & 4.4 & $<\mathrm{MDA}$ & & 2.3 & & & \\
\hline Technetium-99 & 18 & 8 & 13 & $<\mathrm{MDA}$ & & 13 & 19.54 & 3.92 & 6.07 \\
\hline Thorium-228 & & & & & & & & & \\
\hline Thorium-230 & & & & & & & & & \\
\hline Thorium-232 & & & & & & & & & \\
\hline Thorium-234 & & & & & & & & & \\
\hline Tritium & & & & & & & & & \\
\hline Uranium-234 & 3.7 & 0.7 & 0.16 & 1.2 & 0.37 & 0.13 & 3.64 & 0.98 & 0.1 \\
\hline Uranium-235 & $<\mathrm{MDA}$ & & 0.25 & $<\mathrm{MDA}$ & & 0.14 & 0.18 & 0.18 & 0.12 \\
\hline Uranium-236 & & & & & & & $<\mathrm{MDA}$ & & 0.18 \\
\hline Uranium-238 & 7.2 & 1.1 & 0.16 & 2.9 & 0.6 & 0.12 & 8.87 & 1.98 & 0.23 \\
\hline
\end{tabular}

\begin{tabular}{|c|c|c|c|c|c|c|c|c|c|}
\hline Sampling Point & \multicolumn{3}{|c|}{ BCK-03.30 } & \multicolumn{6}{|c|}{ BCK-04.55 } \\
\hline Location & \multicolumn{3}{|c|}{ EXP-SW } & \multicolumn{6}{|c|}{ EXP-SW } \\
\hline Date Sampled & \multicolumn{3}{|c|}{$09 / 09 / 02$} & \multicolumn{3}{|c|}{$01 / 09 / 02$} & \multicolumn{3}{|c|}{$07 / 10 / 02$} \\
\hline Program & \multicolumn{3}{|c|}{ BJC } & \multicolumn{3}{|c|}{ GWPP } & \multicolumn{3}{|c|}{ GWPP } \\
\hline \multicolumn{10}{|l|}{ Sample Type } \\
\hline Result (pCi/L) & Activity & Error & MDA & Activity & Error & MDA & Activity & Error & MDA \\
\hline Gross Alpha & & & & 21 & 5.1 & 3.3 & 5.3 & 3 & 3.5 \\
\hline Gross Beta & 6.94 & 1.51 & 1.81 & 32 & 6.6 & 7.7 & 11 & 4 & 5.4 \\
\hline Americium-241 & & & & & & & & & \\
\hline Neptunium-237 & & & & & & & & & \\
\hline Plutonium-238 & & & & & & & & & \\
\hline Radium-223/224/226 & & & & & & & & & \\
\hline Strontium-89/90 & & & & $<\mathrm{MDA}$ & & 4.2 & $<M D A$ & & 2.9 \\
\hline Technetium-99 & $<\mathrm{MDA}$ & & 6.08 & 38 & 8.3 & 13 & $<$ MDA & & 13 \\
\hline Thorium-228 & & & & & & & & & \\
\hline Thorium-230 & & & & & & & & & \\
\hline Thorium-232 & & & & & & & & & \\
\hline Thorium-234 & & & & & & & & & \\
\hline Tritium & & & & & & & & & \\
\hline Uranium-234 & 3.76 & 1.46 & 0.76 & 7.5 & 1.1 & 0.064 & 1.6 & 0.41 & 0.19 \\
\hline Uranium-235 & $<\mathrm{MDA}$ & & 0.78 & 0.47 & 0.24 & 0.08 & $<$ MDA & & 0.14 \\
\hline Uranium-236 & $<$ MDA & & 0.35 & & & & & & \\
\hline Uranium-238 & 4.09 & 1.53 & 0.76 & 17 & 2.1 & 0.064 & 2.5 & 0.53 & 0.15 \\
\hline
\end{tabular}


APPENDIX D.3: CY 2002 MONITORING DATA FOR THE BEAR CREEK HYDROGEOLOGIC REGIME

Radiological Analytes: Isotope Activity

\begin{tabular}{|c|c|c|c|c|c|c|c|c|c|}
\hline Sampling Point & \multicolumn{6}{|c|}{ BCK-07.87 } & \multicolumn{3}{|c|}{ BCK-09.20 } \\
\hline Location & \multicolumn{6}{|c|}{ EXP-SW } & \multicolumn{3}{|c|}{ EXP-SW } \\
\hline Date Sampled & \multicolumn{3}{|c|}{$01 / 08 / 02$} & \multicolumn{3}{|c|}{$07 / 10 / 02$} & \multicolumn{3}{|c|}{$03 / 11 / 02$} \\
\hline Program & \multicolumn{3}{|c|}{ BJC } & \multicolumn{3}{|c|}{ BJC } & \multicolumn{3}{|c|}{ BJC } \\
\hline \multicolumn{10}{|l|}{ Sample Type } \\
\hline Result (pCi/L) & Activity & Error & MDA & Activity & Error & MDA & Activity & Error & MDA \\
\hline Gross Alpha & 62 & 8.7 & 2.7 & 31 & 6 & 2.6 & & & \\
\hline Gross Beta & 110 & 11 & 8.8 & 33 & 6.4 & 7.2 & 50.04 & 2.86 & 1.78 \\
\hline Americium-241 & & & & & & & & & \\
\hline Neptunium-237 & & & & & & & & & \\
\hline Plutonium-238 & & & & & & & & & \\
\hline Radium-223/224/226 & & & & & & & & & \\
\hline Strontium-89/90 & & & & & & & & & \\
\hline Technetium-99 & 74.44 & 5.75 & 7.85 & 21.34 & 4.68 & 7.28 & 69.84 & 4.6 & 5.82 \\
\hline Thorium-228 & & & & & & & & & \\
\hline Thorium-230 & & & & & & & & & \\
\hline Thorium-232 & & & & & & & & & \\
\hline Thorium-234 & & & & & & & & & \\
\hline Tritium & & & & & & & & & \\
\hline Uranium-234 & 18.4 & 3.81 & 0.19 & 12.52 & 4.09 & 1.88 & 14.19 & 3.14 & 0.18 \\
\hline Uranium-235 & 0.78 & 0.44 & 0.38 & $<\mathrm{MDA}$ & & 1.48 & 0.79 & 0.41 & 0.22 \\
\hline Uranium-236 & 0.58 & 0.35 & 0.28 & $<\mathrm{MDA}$ & & 1.14 & 0.41 & 0.28 & 0.19 \\
\hline Uranium-238 & 44.05 & 8.53 & 0.23 & 21.56 & 5.92 & 1.8 & 30.29 & 6.3 & 0.17 \\
\hline
\end{tabular}

\begin{tabular}{|c|c|c|c|c|c|c|c|c|c|}
\hline Sampling Point & \multicolumn{3}{|c|}{ BCK-09.20 } & \multicolumn{3}{|c|}{ BCK-09.47 } & \multicolumn{3}{|c|}{ BCK-11.54 } \\
\hline Location & \multicolumn{3}{|c|}{ EXP-SW } & \multicolumn{3}{|c|}{ EXP-SW } & \multicolumn{3}{|c|}{ EXP-SW } \\
\hline Date Sampled & \multicolumn{3}{|c|}{ 09/09/02 } & \multicolumn{3}{|c|}{$03 / 11 / 02$} & \multicolumn{3}{|c|}{$03 / 11 / 02$} \\
\hline Program & \multicolumn{3}{|c|}{ BJC } & \multicolumn{3}{|c|}{ BJC } & \multicolumn{3}{|c|}{ BJC } \\
\hline \multicolumn{10}{|l|}{ Sample Type } \\
\hline Result (pCi/L) & Activity & Error & MDA & Activity & Error & MDA & Activity & Error & MDA \\
\hline Gross Alpha & & & & & & & & & \\
\hline Gross Beta & 48.28 & 3.23 & 2.24 & 76.09 & 3.81 & 2.14 & 158.71 & 4.68 & 2.14 \\
\hline Americium-241 & & & & & & & & & \\
\hline Neptunium-237 & & & & & & & & & \\
\hline Plutonium-238 & & & & & & & & & \\
\hline Radium-223/224/226 & & & & & & & & & \\
\hline Strontium-89/90 & & & & & & & & & \\
\hline Technetium-99 & 54.47 & 4.43 & 5.93 & 118.07 & 6.05 & 7.07 & 235.57 & 6.58 & 5.78 \\
\hline Thorium-228 & & & & & & & & & \\
\hline Thorium-230 & & & & & & & & & \\
\hline Thorium-232 & & & & & & & & & \\
\hline Thorium-234 & & & & & & & & & \\
\hline Tritium & & & & & & & & & \\
\hline Uranium-234 & 11.26 & 3.25 & 1.16 & 16.76 & 4.1 & 0.55 & 18.07 & 3.78 & 0.23 \\
\hline Uranium-235 & 1.13 & 0.97 & 0.89 & 0.81 & 0.54 & 0.47 & 0.57 & 0.35 & 0.13 \\
\hline Uranium-236 & $<$ MDA & & 1.13 & 0.63 & 0.44 & 0.19 & 0.64 & 0.35 & 0.12 \\
\hline Uranium-238 & 19.12 & 4.67 & 0.72 & 44.46 & 9.99 & 0.17 & 30.72 & 6.15 & 0.23 \\
\hline
\end{tabular}


APPENDIX D.3: CY 2002 MONITORING DATA FOR THE BEAR CREEK HYDROGEOLOGIC REGIME Radiological Analytes: Isotope Activity

\begin{tabular}{|c|c|c|c|c|c|c|c|c|c|}
\hline Sampling Point & \multicolumn{3}{|c|}{ BCK-11.54 } & \multicolumn{3}{|c|}{ BCK-11.84 } & \multicolumn{3}{|c|}{ BCK-11.97 } \\
\hline Location & \multicolumn{3}{|c|}{ EXP-SW } & \multicolumn{3}{|c|}{ EXP-SW } & \multicolumn{3}{|c|}{ EXP-SW } \\
\hline Date Sampled & \multicolumn{3}{|c|}{$09 / 09 / 02$} & \multicolumn{3}{|c|}{$03 / 11 / 02$} & \multicolumn{3}{|c|}{$01 / 09 / 02$} \\
\hline Program & \multicolumn{3}{|c|}{ BJC } & \multicolumn{3}{|c|}{ BJC } & \multicolumn{3}{|c|}{ GWPP } \\
\hline \multicolumn{10}{|l|}{ Sample Type } \\
\hline Result (pCi/L) & Activity & Error & MDA & Activity & Error & MDA & Activity & Error & MDA \\
\hline Gross Alpha & & & & & & & 89 & 12 & 4.8 \\
\hline Gross Beta & 4.3 & 1.39 & 1.83 & 222.91 & 5.31 & 2.33 & 410 & 21 & 11 \\
\hline Americium-241 & & & & & & & $<\mathrm{MDA}$ & & 0.29 \\
\hline Neptunium-237 & & & & & & & 2.1 & 0.53 & 0.086 \\
\hline Plutonium-238 & & & & & & & $<\mathrm{MDA}$ & & 0.22 \\
\hline Radium-223/224/226 & & & & & & & $<\mathrm{MDA}$ & & 1.1 \\
\hline Strontium-89/90 & & & & & & & 5.3 & 2.9 & 4.4 \\
\hline Technetium-99 & $<\mathrm{MDA}$ & & 6.08 & 403.11 & 8.16 & 5.83 & 400 & 12 & 13 \\
\hline Thorium-228 & & & & & & & $<\mathrm{MDA}$ & & 0.34 \\
\hline Thorium-230 & & & & & & & 0.16 & 0.14 & 0.087 \\
\hline Thorium-232 & & & & & & & $<\mathrm{MDA}$ & & 0.086 \\
\hline Thorium-234 & & & & & & & 46 & 4.5 & 0.12 \\
\hline Tritium & & & & & & & & & \\
\hline Uranium-234 & 2.38 & 1.17 & 0.81 & 19.38 & 4.18 & 0.34 & 26 & 2.7 & 0.12 \\
\hline Uranium-235 & $<\mathrm{MDA}$ & & 0.83 & 1.36 & 0.63 & 0.33 & 0.88 & 0.3 & 0.15 \\
\hline Uranium-236 & $<M D A$ & & 0.74 & 1.11 & 0.54 & 0.33 & &. & \\
\hline Uranium-238 & 3.66 & 1.46 & 0.57 & 30.11 & 6.23 & 0.26 & 46 & 4.5 & 0.12 \\
\hline
\end{tabular}

\begin{tabular}{|c|c|c|c|c|c|c|c|c|c|}
\hline Sampling Point & \multicolumn{3}{|c|}{ BCK-11.97 } & \multicolumn{6}{|c|}{ GW-008 } \\
\hline Location & \multicolumn{3}{|c|}{ EXP-SW } & \multicolumn{6}{|c|}{ OLF } \\
\hline Date Sampled & \multicolumn{3}{|c|}{$07 / 11 / 02$} & \multicolumn{3}{|c|}{$01 / 07 / 02$} & \multicolumn{3}{|c|}{$07 / 02 / 02$} \\
\hline Program & \multicolumn{3}{|c|}{ GWPP } & \multicolumn{3}{|c|}{ BJC } & \multicolumn{3}{|c|}{ BJC } \\
\hline \multicolumn{10}{|l|}{ Sample Type } \\
\hline Result (pCi/L) & Activity & Error & MDA & Activity & Error & MDA & Activity & Error & MDA \\
\hline Gross Alpha & 83 & 19 & 12 & $<\mathrm{MDA}$ & & 1.09 & $<$ MDA & & 1.23 \\
\hline Gross Beta & 780 & 25 & 6.9 & $<M D A$ & & 1.88 & 2.52 & 1.06 & 1.45 \\
\hline Americium-241 & & & & $<$ MDA & & 1.57 & $<$ MDA & & 0.16 \\
\hline Neptunium-237 & 0.53 & 0.28 & 0.2 & $0.21 \mathrm{R}$ & 0.22 & 0.14 & $<$ MDA & & 0.16 \\
\hline Plutonium-238 & $<$ MDA & & 0.14 & & & & & & \\
\hline Radium-223/224/226 & $<M D A$ & & 2.5 & 1.73 & 0.81 & 0.85 & $<M D A$ & & 0.46 \\
\hline Strontium-89/90 & 4.4 & 1.8 & 2.5 & $<M D A$ & & 1.64 & $<M D A$ & & 1.59 \\
\hline Technetium-99 & 1500 & 23 & 13 & $<\mathrm{MDA}$ & & 11.18 & $<M D A$ & & 7.45 \\
\hline Thorium-228 & $<$ MDA & & 0.15 & & & & & & \\
\hline Thorium-230 & 0.26 & 0.23 & 0.17 & & & & & & \\
\hline Thorium-232 & $<$ MDA & & 0.099 & & & & & & \\
\hline Thorium-234 & 43 & 4.1 & 0.066 & & & & & & \\
\hline Tritium & & & & & & & & & \\
\hline Uranium-234 & 25 & 2.6 & 0.097 & $<\mathrm{MDA}$ & & 0.23 & $<M D A$ & & 0.95 \\
\hline Uranium-235 & 1.1 & 0.31 & 0.083 & $<$ MDA & & 0.19 & $<$ MDA & & 0.43 \\
\hline Uranium-236 & & & & $<$ MDA & & 0.24 & $<$ MDA & & 0.65 \\
\hline Uranium-238 & 43 & 4.1 & 0.066 & $<\mathrm{MDA}$ & & 0.27 & $<$ MDA & & 0.69 \\
\hline
\end{tabular}


APPENDIX D.3: CY 2002 MONITORING DATA FOR THE BEAR CREEK HYDROGEOLOGIC REGIME

Radiological Analytes: Isotope Activity

\begin{tabular}{|c|c|c|c|c|c|c|c|c|c|}
\hline Sampling Point & \multicolumn{6}{|c|}{ GW-046 } & \multicolumn{3}{|c|}{ GW-077 } \\
\hline Location & \multicolumn{6}{|c|}{ BG } & \multicolumn{3}{|c|}{ BG } \\
\hline Date Sampled & \multicolumn{3}{|c|}{$01 / 07 / 02$} & \multicolumn{3}{|c|}{$07 / 02 / 02$} & \multicolumn{3}{|c|}{$02 / 26 / 02$} \\
\hline Program & \multicolumn{3}{|c|}{ BJC } & \multicolumn{3}{|c|}{ BJC } & \multicolumn{3}{|c|}{ BJC } \\
\hline \multicolumn{10}{|l|}{ Sample Type } \\
\hline Result (pCi/L) & Activity & Error & MDA & Activity & Error & MDA & Activity & Error & MDA \\
\hline Gross Alpha & 1.84 & 0.99 & 1.23 & 0.98 & 0.72 & 0.97 & $<\mathrm{MDA}$ & & 1.75 \\
\hline Gross Beta & 5.38 & 1.48 & 1.92 & 3.71 & 1.09 & 1.39 & $<\mathrm{MDA}$ & & 2 \\
\hline Americium-241 & $<\mathrm{MDA}$ & & 1.83 & $<\mathrm{MDA}$ & & 0.2 & & & \\
\hline Neptunium-237 & 0.23 & 0.21 & 0.22 & $<\mathrm{MDA}$ & & 0.27 & & & \\
\hline Plutonium-238 & & & & & & & & & \\
\hline Radium-223/224/226 & 3.53 & 1.08 & 0.8 & $<\mathrm{MDA}$ & & 0.45 & & & \\
\hline Strontium-89/90 & $<\mathrm{MDA}$ & & 1.32 & $<\mathrm{MDA}$ & & 1.51 & & & \\
\hline Technetium-99 & $<\mathrm{MDA}$ & & 10.79 & $<\mathrm{MDA}$ & & 14.27 & $<\mathrm{MDA}$ & & 6.53 \\
\hline Thorium-228 & & & & & & & & & \\
\hline Thorium-230 & & & & & & & & & \\
\hline Thorium-232 & & & & & & & & & \\
\hline Thorium-234 & & & & & & & & & \\
\hline Tritium & & & & & & & & & \\
\hline Uranium-234 & $<\mathrm{MDA}$ & & 0.41 & $<\mathrm{MDA}$ & & 0.32 & $<M D A$ & & 0.39 \\
\hline Uranium-235 & $<\mathrm{MDA}$ & & 0.32 & $<\mathrm{MDA}$ & & 0.4 & $<\mathrm{MDA}$ & & 0.14 \\
\hline Uranium-236 & $<\mathrm{MDA}$ & & 0.31 & $<\mathrm{MDA}$ & & 0.36 & $<\mathrm{MDA}$ & & 0.28 \\
\hline Uranium-238 & $<\mathrm{MDA}$ & & 0.32 & $<\mathrm{MDA}$ & & 0.32 & $<\mathrm{MDA}$ & & 0.36 \\
\hline
\end{tabular}

\begin{tabular}{|c|c|c|c|c|c|c|c|c|c|}
\hline Sampling Point & \multicolumn{3}{|c|}{ GW-077 } & \multicolumn{6}{|c|}{ GW-078 } \\
\hline Location & \multicolumn{3}{|c|}{ BG } & \multicolumn{6}{|c|}{ BG } \\
\hline Date Sampled & \multicolumn{3}{|c|}{$08 / 08 / 02$} & \multicolumn{3}{|c|}{$02 / 26 / 02$} & \multicolumn{3}{|c|}{$08 / 08 / 02$} \\
\hline Program & \multicolumn{3}{|c|}{ BJC } & \multicolumn{3}{|c|}{ BJC } & \multicolumn{3}{|c|}{ BJC } \\
\hline \multicolumn{10}{|l|}{ Sample Type } \\
\hline Result (pCi/L) & Activity & Error & MDA & Activity & Error & MDA & Activity & Error & MDA \\
\hline Gross Alpha & $<$ MDA & & 1.83 & $<\mathrm{MDA}$ & & 1.71 & $<$ MDA & & $\overline{0.6}$ \\
\hline Gross Beta & 3.19 & 1.3 & 1.77 & $<$ MDA & & 1.99 & $<$ MDA & & 0.99 \\
\hline Americium-241 & & & & & & & & & \\
\hline Neptunium-237 & & & & & & & & & \\
\hline Plutonium-238 & & & & & & & & & \\
\hline Radium-223/224/226 & & & & & & & & & \\
\hline Strontium-89/90 & & & & & & & & & \\
\hline Technetium-99 & $<\mathrm{MDA}$ & & 5.75 & $<$ MDA & & 6.59 & $<$ MDA & & 5.85 \\
\hline Thorium-228 & & & & & & & & & \\
\hline Thorium-230 & & & & & & & & & \\
\hline Thorium-232 & & & & & & & & & \\
\hline Thorium-234 & & & & & & & & & \\
\hline Tritium & & & & & & & & & \\
\hline Uranium-234 & 0.29 & 0.2 & 0.2 & 0.38 & 0.28 & 0.31 & 0.36 & 0.2 & 0.15 \\
\hline Uranium-235 & $<\mathrm{MDA}$ & & 0.1 & $<$ MDA & & 0.14 & $<$ MDA & & 0.14 \\
\hline Uranium-236 & $<\mathrm{MDA}$ & & 0.15 & $<$ MDA & & 0.21 & $<$ MDA & & 0.07 \\
\hline Uranium-238 & $<\mathrm{MDA}$ & & 0.19 & $<\mathrm{MDA}$ & & 0.19 & 0.18 & 0.14 & 0.15 \\
\hline
\end{tabular}


APPENDIX D.3: CY 2002 MONITORING DATA FOR THE BEAR CREEK HYDROGEOLOGIC REGIME

Radiological Analytes: Isotope Activity

\begin{tabular}{|c|c|c|c|c|c|c|c|c|c|}
\hline Sampling Point & \multicolumn{6}{|c|}{ GW-079 } & \multicolumn{3}{|c|}{ GW-080 } \\
\hline Location & \multicolumn{6}{|c|}{ BG } & \multicolumn{3}{|c|}{ BG } \\
\hline Date Sampled & \multicolumn{3}{|c|}{$02 / 26 / 02$} & \multicolumn{3}{|c|}{$08 / 07 / 02$} & \multicolumn{3}{|c|}{$02 / 26 / 02$} \\
\hline Program & \multicolumn{3}{|c|}{ BJC } & \multicolumn{3}{|c|}{ BJC } & \multicolumn{3}{|c|}{$\mathrm{BJC}$} \\
\hline \multicolumn{10}{|l|}{ Sample Type } \\
\hline Result (pCi/L) & Activity & Error & MDA & Activity & Error & MDA & Activity & Error & MDA \\
\hline Gross Alpha & $<$ MDA & & 1.29 & $<\mathrm{MDA}$ & & 1.72 & $<\mathrm{MDA}$ & & 1.31 \\
\hline Gross Beta & $<\mathrm{MDA}$ & & 1.89 & 2.63 & 1.31 & 1.86 & 3.51 & 1.38 & 1.89 \\
\hline Americium-241 & & & & & & & & & \\
\hline Neptunium-237 & & & & & & & & & \\
\hline Plutonium-238 & & & & & & & & & \\
\hline Radium-223/224/226 & & & & & & & & & \\
\hline Strontium-89/90 & & & & & & & & & \\
\hline Technetium-99 & $<\mathrm{MDA}$ & & 7.28 & $<\mathrm{MDA}$ & & 6.04 & $<\mathrm{MDA}$ & & 6.64 \\
\hline Thorium-228 & & & & & & & & & \\
\hline Thorium-230 & & & & & & & & & \\
\hline Thorium-232 & & & & & & & & & \\
\hline Thorium-234 & & & & & & & & & \\
\hline Tritium & & & & & & & & & \\
\hline Uranium-234 & 0.25 & 0.2 & 0.1 & 0.21 & 0.17 & 0.21 & $<\mathrm{MDA}$ & & 0.26 \\
\hline Uranium-235 & $<\mathrm{MDA}$ & & 0.12 & $0.11 \mathrm{R}$ & 0.13 & 0.1 & $<\mathrm{MDA}$ & & 0.23 \\
\hline Uranium-236 & $<\mathrm{MDA}$ & & 0.24 & $<\mathrm{MDA}$ & & 0.18 & $<\mathrm{MDA}$ & & 0.18 \\
\hline Uranium-238 & $<\mathrm{MDA}$ & & 0.19 & $<\mathrm{MDA}$ & & 0.16 & $<\mathrm{MDA}$ & & 0.23 \\
\hline
\end{tabular}

\begin{tabular}{|c|c|c|c|c|c|c|c|c|c|}
\hline Sampling Point & \multicolumn{9}{|c|}{ GW-080 } \\
\hline Location & \multicolumn{9}{|c|}{ BG } \\
\hline Date Sampled & \multicolumn{3}{|c|}{$02 / 26 / 02$} & \multicolumn{6}{|c|}{$08 / 07 / 02$} \\
\hline Program & \multicolumn{3}{|c|}{ BJC } & \multicolumn{6}{|c|}{ BJC } \\
\hline Sample Type & \multicolumn{3}{|c|}{ DUP } & & & & \multicolumn{3}{|c|}{ DUP } \\
\hline Result (pCi/L) & Activity & Error & MDA & Activity & Error & MDA & Activity & Error & MDA \\
\hline Gross Alpha & $<$ MDA & & 1.29 & $<\mathrm{MDA}$ & & 0.9 & $<$ MDA & & 1.14 \\
\hline Gross Beta & $<\mathrm{MDA}$ & & 1.89 & 1.61 & 1.1 & 1.6 & $<$ MDA & & 1.91 \\
\hline Americium-241 & & & & & & & & & \\
\hline Neptunium-237 & & & & & & & & & \\
\hline Plutonium-238 & & & & & & & & & \\
\hline Radium-223/224/226 & & & & & & & & & \\
\hline Strontium-89/90 & & & & & & & & & \\
\hline Technetium-99 & $<\mathrm{MDA}$ & & 6.6 & $<$ MDA & & 5.97 & $<$ MDA & & 6.04 \\
\hline Thorium-228 & & & & & & & & & \\
\hline Thorium-230 & & & & & & & & & \\
\hline Thorium-232 & & & & & & & & & \\
\hline Thorium-234 & & & & & & & & & \\
\hline Tritium & & & & & & & & & \\
\hline Uranium-234 & 0.4 & 0.26 & 0.23 & 0.24 & 0.17 & 0.17 & 0.55 & 0.27 & 0.24 \\
\hline Uranium-235 & $<$ MDA & & 0.24 & $<\mathrm{MDA}$ & & 0.09 & $<$ MDA & & 0.21 \\
\hline Uranium-236 & $<\mathrm{MDA}$ & & 0.24 & $<$ MDA & & 0.08 & $<$ MDA & & 0.14 \\
\hline Uranium-238 & $<\mathrm{MDA}$ & & 0.21 & $<\mathrm{MDA}$ & & 0.14 & $<$ MDA & & 0.28 \\
\hline
\end{tabular}


APPENDIX D.3: CY 2002 MONITORING DATA FOR THE BEAR CREEK HYDROGEOLOGIC REGIME

Radiological Analytes: Isotope Activity

\begin{tabular}{|c|c|c|c|c|c|c|c|c|c|}
\hline Sampling Point & \multicolumn{6}{|c|}{ GW-115 } & \multicolumn{3}{|c|}{ GW-243 } \\
\hline Location & \multicolumn{6}{|c|}{ S3 } & \multicolumn{3}{|c|}{ S3 } \\
\hline Date Sampled & \multicolumn{3}{|c|}{$01 / 07 / 02$} & \multicolumn{3}{|c|}{$07 / 08 / 02$} & \multicolumn{3}{|c|}{$03 / 21 / 02$} \\
\hline Program & \multicolumn{3}{|c|}{ BJC } & \multicolumn{3}{|c|}{ BJC } & \multicolumn{3}{|c|}{ GWPP } \\
\hline \multicolumn{10}{|l|}{ Sample Type } \\
\hline Result (pCi/L) & Activity & Error & MDA & Activity & Error & MDA & Activity & Error & MDA \\
\hline Gross Alpha & $<\mathrm{MDA}$ & & 1.61 & $<\mathrm{MDA}$ & & 3.47 & 540 & 190 & 44 \\
\hline Gross Beta & 2.29 & 1.76 & 2.08 & 6.54 & 1.95 & 2.14 & 13,000 & 520 & 220 \\
\hline Americium-241 & $4.36 \mathrm{Q}$ & 1.91 & 1.03 & $<M D A$ & & 0.09 & $<M D A$ & & 0.6 \\
\hline Neptunium-237 & $<M D A$ & & 0.3 & $<M D A$ & & 0.31 & 30 & 2.7 & 0.29 \\
\hline Plutonium-238 & & & & & & & $<\mathrm{MDA}$ & & 3.5 \\
\hline Radium-223/224/226 & 3.31 & 1.08 & 0.79 & 1.87 & 0.81 & 0.7 & 39 & 11 & 5.7 \\
\hline Strontium-89/90 & $<\mathrm{MDA}$ & & 1.42 & $<\mathrm{MDA}$ & & 1.3 & 4.1 & 2.5 & 3.9 \\
\hline Technetium-99 & $<\mathrm{MDA}$ & & 11.08 & $<\mathrm{MDA}$ & & 7.47 & 8,300 & 52 & 13 \\
\hline Thorium-228 & & & & & & & 0.89 & 0.39 & 0.24 \\
\hline Thorium-230 & & & & & & & 5.6 & 1.2 & 0.099 \\
\hline Thorium-232 & & & & & & & 0.48 & 0.28 & 0.24 \\
\hline Thorium-234 & & & & & & & 270 & 27 & 0.13 \\
\hline Tritium & & & & & & & & & \\
\hline Uranium-234 & $<\mathrm{MDA}$ & & 0.44 & $<$ MDA & & 0.23 & 110 & 12 & 0.31 \\
\hline Uranium-235 & 0.28 & 0.24 & 0.22 & $<\mathrm{MDA}$ & & 0.41 & 5.9 & 1.3 & 0.39 \\
\hline Uranium-236 & $<M D A$ & & 0.26 & $<M D A$ & & 0.26 & & . & \\
\hline Uranium-238 & $<\mathrm{MDA}$ & & 0.25 & $<\mathrm{MDA}$ & & 0.27 & 270 & 27 & 0.13 \\
\hline
\end{tabular}

\begin{tabular}{|c|c|c|c|c|c|c|c|c|c|}
\hline Sampling Point & \multicolumn{3}{|c|}{ GW-243 } & \multicolumn{6}{|c|}{ GW-276 } \\
\hline Location & \multicolumn{3}{|c|}{ S3 } & \multicolumn{6}{|c|}{ S3 } \\
\hline Date Sampled & \multicolumn{3}{|c|}{$08 / 13 / 02$} & \multicolumn{3}{|c|}{$01 / 07 / 02$} & \multicolumn{3}{|c|}{$07 / 08 / 02$} \\
\hline Program & \multicolumn{3}{|c|}{ GWPP } & \multicolumn{3}{|c|}{ BJC } & \multicolumn{3}{|c|}{ BJC } \\
\hline \multicolumn{10}{|l|}{ Sample Type } \\
\hline Result (pCi/L) & Activity & Error & MDA & Activity & Error & MDA & Activity & Error & MDA \\
\hline Gross Alpha & $<$ MDA & & 740 & 238.65 & \begin{tabular}{l|}
12.17 \\
\end{tabular} & \begin{tabular}{l|l}
3.47 \\
\end{tabular} & 210.7 & 11.06 & 4.23 \\
\hline Gross Beta & 17,000 & 1,300 & 840 & 357.54 & 8.47 & 3.63 & 391.23 & 7.68 & 2.46 \\
\hline Americium-241 & $<\mathrm{MDA}$ & & 0.26 & $<\mathrm{MDA}$ & & 0.42 & $<$ MDA & & 0.19 \\
\hline Neptunium-237 & 32 & 2.2 & 0.15 & 15.67 & 3.31 & 0.3 & 9.64 & 1.55 & 0.32 \\
\hline Plutonium-238 & 0.35 & 0.21 & 0.11 & & & & & & \\
\hline Radium-223/224/226 & 53 & 17 & 13 & 4.4 & 1.06 & 0.64 & 2.46 & 0.94 & 0.73 \\
\hline Strontium-89/90 & 32 & 7.4 & 8.5 & $<\mathrm{MDA}$ & & 2.29 & 2.71 & 0.21 & 1.28 \\
\hline Technetium-99 & 14,000 & 70 & 40 & 501.55 & 11.3 & 11.29 & 501.01 & 10.36 & 7.61 \\
\hline Thorium-228 & 0.75 & 0.48 & 0.42 & & & & & & \\
\hline Thorium-230 & 2.9 & 1.1 & 0.31 & & & & & & \\
\hline Thorium-232 & $<M D A$ & & 0.29 & & & & & & \\
\hline Thorium-234 & 270 & 29 & 0.18 & & & & & & \\
\hline \multicolumn{10}{|l|}{ Tritium } \\
\hline Uranium-234 & 110 & 12 & 0.17 & 80.45 & 31.68 & 3.78 & 73.7 & 20.37 & 2.77 \\
\hline Uranium-235 & 5.7 & 1.1 & 0.18 & 3.44 & 3.42 & 3.3 & 8.4 & 4.79 & 2.62 \\
\hline Uranium-236 & & & & 5.01 & 4.04 & 2.96 & 2.46 & 2.35 & 2.36 \\
\hline Uranium-238 & 270 & 29 & 0.18 & 182.3 & 67.91 & 4.26 & 193.8 & 47.78 & 2.12 \\
\hline
\end{tabular}


APPENDIX D.3: CY 2002 MONITORING DATA FOR THE BEAR CREEK HYDROGEOLOGIC REGIME

Radiological Analytes: Isotope Activity

\begin{tabular}{|c|c|c|c|c|c|c|c|c|c|}
\hline Sampling Point & \multicolumn{6}{|c|}{ GW-363 } & \multicolumn{3}{|c|}{ GW-526 } \\
\hline Location & \multicolumn{6}{|c|}{ EMWMF } & \multirow{2}{*}{\multicolumn{3}{|c|}{$\begin{array}{c}\text { S3 } \\
02 / 26 / 02\end{array}$}} \\
\hline Date Sampled & \multicolumn{3}{|c|}{$08 / 14 / 02$} & \multicolumn{3}{|c|}{$12 / 04 / 02$} & \multicolumn{2}{|c|}{$02 / 26 / 02$} & \\
\hline Program & \multicolumn{3}{|c|}{ BJC } & \multicolumn{3}{|c|}{ BJC } & \multicolumn{3}{|c|}{ BJC } \\
\hline \multicolumn{10}{|l|}{ Sample Type } \\
\hline Result (pCi/L) & Activity & Error & MDA & Activity & Error & MDA & Activity & Error & MDA \\
\hline Gross Alpha & & & & & & & $<\mathrm{MDA}$ & & 40.2 \\
\hline Gross Beta & & & & & & & $<\mathrm{MDA}$ & & 43.58 \\
\hline Americium-241 & $<M D A$ & & 0.12 & $<M D A$ & & 0.107 & & & \\
\hline Neptunium-237 & $<\mathrm{MDA}$ & & 0.122 & $<\mathrm{MDA}$ & & 0.105 & & & \\
\hline Plutonium-238 & $<\mathrm{MDA}$ & & 0.102 & $<\mathrm{MDA}$ & & 0.22 & & & \\
\hline \multicolumn{10}{|l|}{ Radium-223/224/226 } \\
\hline Strontium-89/90 & $<\mathrm{MDA}$ & & 0.53 & $<\mathrm{MDA}$ & & 1.11 & & & \\
\hline Technetium-99 & $<\mathrm{MDA}$ & & 7.04 & 13.3 & 3.4 & 7.42 & & & \\
\hline Thorium-228 & & & & & & & & & \\
\hline Thorium-230 & & & & & & & & & \\
\hline Thorium-232 & & & & & & & & & \\
\hline Thorium-234 & & & & & & & & & \\
\hline Tritium & & & & & & & & & \\
\hline Uranium-234 & 0.0562 & 0.037 & 0.0169 & 0.159 & 0.097 & 0.0978 & 0.29 & 0.22 & 0.23 \\
\hline Uranium-235 & $<\mathrm{MDA}$ & & 0.0285 & $<\mathrm{MDA}$ & & 0.0913 & $<\mathrm{MDA}$ & & 0.22 \\
\hline Uranium-236 & & & & & & & $<\mathrm{MDA}$ & & 0.11 \\
\hline Uranium-238 & $<\mathrm{MDA}$ & & 0.0333 & $<\mathrm{MDA}$ & & 0.0839 & $<\mathrm{MDA}$ & & 0.27 \\
\hline
\end{tabular}

\begin{tabular}{|c|c|c|c|c|c|c|c|c|c|}
\hline Sampling Point & \multicolumn{3}{|c|}{ GW-526 } & \multicolumn{6}{|c|}{ GW-639 } \\
\hline Location & \multicolumn{3}{|c|}{ S3 } & \multicolumn{6}{|c|}{ EMWMF } \\
\hline Date Sampled & \multicolumn{3}{|c|}{$08 / 08 / 02$} & \multicolumn{3}{|c|}{$08 / 14 / 02$} & \multicolumn{3}{|c|}{$12 / 04 / 02$} \\
\hline Program & \multicolumn{3}{|c|}{ BJC } & \multicolumn{3}{|c|}{ BJC } & \multicolumn{3}{|c|}{ BJC } \\
\hline \multicolumn{10}{|l|}{ Sample Type } \\
\hline Result (pCi/L) & Activity & Error & MDA & Activity & Error & MDA & Activity & Error & MDA \\
\hline Gross Alpha & $<$ MDA & & 27.04 & & & & & & \\
\hline Gross Beta & $<\mathrm{MDA}$ & & 41.82 & & & & & & \\
\hline Americium-241 & & & & $<\mathrm{MDA}$ & & 0.115 & 0.225 & 0.2 & 0.122 \\
\hline Neptunium-237 & & & & $<$ MDA & & 0.114 & $<$ MDA & & 0.126 \\
\hline Plutonium-238 & & & & $<$ MDA & & 0.175 & $<M D A$ & & 0.123 \\
\hline \multicolumn{10}{|l|}{ Radium-223/224/226 } \\
\hline Strontium-89/90 & & & & $<\mathrm{MDA}$ & & 0.663 & $<M D A$ & & 1.04 \\
\hline Technetium-99 & & & & $<$ MDA & & 7.02 & $<$ MDA & & 7.35 \\
\hline Thorium-228 & & & & & & & & & \\
\hline Thorium-230 & & & & & & & & & \\
\hline Thorium-232 & & & & & & & & & \\
\hline Thorium-234 & & & & & & & & & \\
\hline Tritium & & & & & & & & & \\
\hline Uranium-234 & 0.35 & 0.2 & 0.13 & 0.327 & 0.092 & 0.0174 & 0.238 & 0.12 & 0.0723 \\
\hline Uranium-235 & $<$ MDA & & 0.15 & $<\mathrm{MDA}$ & & 0.0174 & $<$ MDA & & 0.0409 \\
\hline Uranium-236 & $<\mathrm{MDA}$ & & 0.14 & & & & & & \\
\hline Uranium-238 & $<\mathrm{MDA}$ & & 0.12 & 0.0577 & 0.038 & 0.0174 & 0.106 & 0.08 & 0.0409 \\
\hline
\end{tabular}


APPENDIX D.3: CY 2002 MONITORING DATA FOR THE BEAR CREEK HYDROGEOLOGIC REGIME

Radiological Analytes: Isotope Activity

\begin{tabular}{|c|c|c|c|c|c|c|c|c|c|}
\hline Sampling Point & \multicolumn{6}{|c|}{ GW-694 } & \multicolumn{3}{|c|}{ GW-695 } \\
\hline Location & \multicolumn{6}{|c|}{ EXP-B } & \multicolumn{3}{|c|}{ EXP-B } \\
\hline Date Sampled & \multicolumn{3}{|c|}{$01 / 29 / 02$} & \multicolumn{3}{|c|}{$07 / 17 / 02$} & \multicolumn{3}{|c|}{$01 / 15 / 02$} \\
\hline Program & \multicolumn{3}{|c|}{ GWPP } & \multicolumn{3}{|c|}{ GWPP } & \multicolumn{3}{|c|}{ GWPP } \\
\hline \multicolumn{10}{|l|}{ Sample Type } \\
\hline Result (pCi/L) & Activity & Error & MDA & Activity & Error & MDA & Activity & Error & MDA \\
\hline Gross Alpha & 22 & 5.1 & 3 & 15 & 4.5 & 3.6 & 5.7 & 2.8 & 2.7 \\
\hline Gross Beta & 14 & 5.9 & 8.5 & 20 & 5.7 & 7.2 & 50 & 8 & 8.5 \\
\hline Americium-241 & & & & & & & & & \\
\hline Neptunium-237 & & & & & & & & & \\
\hline Plutonium-238 & & & & & & & & & \\
\hline Radium-223/224/226 & & & & & & & & & \\
\hline Strontium-89/90 & & & & & & & & & \\
\hline Technetium-99 & $<M D A$ & & 13 & $<\mathrm{MDA}$ & & 13 & 43 & 8.2 & 13 \\
\hline Thorium-228 & & & & & & & & & \\
\hline Thorium-230 & & & & & & & & & \\
\hline Thorium-232 & & & & & & & & & \\
\hline Thorium-234 & & & & & & & & & \\
\hline Tritium & & & & & & & & & \\
\hline Uranium-234 & & & & & & & & & \\
\hline Uranium-235 & & & & & & & & & \\
\hline Uranium-236 & & & & & & & & & \\
\hline Uranium-238 & & & & & & & & & \\
\hline
\end{tabular}

\begin{tabular}{|c|c|c|c|c|c|c|c|c|c|}
\hline Sampling Point & \multicolumn{3}{|c|}{ GW-695 } & \multicolumn{6}{|c|}{ GW-703 } \\
\hline Location & \multicolumn{3}{|c|}{ EXP-B } & \multicolumn{6}{|c|}{ EXP-B } \\
\hline Date Sampled & \multicolumn{3}{|c|}{$07 / 15 / 02$} & \multicolumn{3}{|c|}{$01 / 15 / 02$} & \multicolumn{3}{|c|}{$07 / 15 / 02$} \\
\hline Program & \multicolumn{3}{|c|}{ GWPP } & \multicolumn{3}{|c|}{ GWPP } & \multicolumn{3}{|c|}{ GWPP } \\
\hline \multicolumn{10}{|l|}{ Sample Type } \\
\hline Result (pCi/L) & Activity & Error & MDA & Activity & Error & MDA & Activity & Error & MDA \\
\hline Gross Alpha & 7.4 & 3.4 & 3.7 & 7.8 & 3.5 & 3.5 & 4.1 & 2.5 & 2.5 \\
\hline Gross Beta & 42 & 7.3 & 8 & 62 & 8.9 & 9.3 & 62 & 8.2 & 7.3 \\
\hline Americium-241 & & & & & & & & & \\
\hline Neptunium-237 & & & & & & & & & \\
\hline Plutonium-238 & & & & & & & & & \\
\hline Radium-223/224/226 & & & & & & & & & \\
\hline Strontium-89/90 & & & & & & & & & \\
\hline Technetium-99 & 45 & 8.7 & 13 & 66 & 8.6 & 13 & 62 & 8.7 & 13 \\
\hline Thorium-228 & & & & & & & & & \\
\hline Thorium-230 & & & & & & & & & \\
\hline Thorium-232 & & & & & & & & & \\
\hline Thorium-234 & & & & & & & & & \\
\hline Tritium & & & & & & & & & \\
\hline Uranium-234 & & & & & & & & & \\
\hline Uranium-235 & & & & & & & & & \\
\hline Uranium-236 & & & & & & & & & \\
\hline Uranium-238 & & & & & & & & & \\
\hline
\end{tabular}


APPENDIX D.3: CY 2002 MONITORING DATA FOR THE BEAR CREEK HYDROGEOLOGIC REGIME

Radiological Analytes: Isotope Activity

\begin{tabular}{|c|c|c|c|c|c|c|c|c|c|}
\hline Sampling Point & \multicolumn{9}{|c|}{ GW-704 } \\
\hline Location & \multicolumn{9}{|c|}{ EXP-B } \\
\hline Date Sampled & \multicolumn{9}{|c|}{$01 / 16 / 02$} \\
\hline Program & \multicolumn{6}{|c|}{ GWPP } & \multicolumn{3}{|c|}{ BJC } \\
\hline Sample Type & \multicolumn{6}{|c|}{ DUP } & & & \\
\hline Result (pCi/L) & Activity & Error & MDA & Activity & Error & MDA & Activity & Error & MDA \\
\hline Gross Alpha & 6.2 & 3.2 & 3.5 & 5.2 & 3.3 & 4.3 & & & \\
\hline Gross Beta & 26 & 5.7 & 6.7 & 27 & 5.5 & 5.9 & & & \\
\hline Americium-241 & & & & & & & & & \\
\hline Neptunium-237 & & & & & & & & & \\
\hline Plutonium-238 & & & & & & & & & \\
\hline Radium-223/224/226 & & & & & & & & & \\
\hline Strontium-89/90 & & & & & & & & & \\
\hline Technetium-99 & 38 & 8.2 & 13 & 32 & 8.2 & 13 & & & \\
\hline Thorium-228 & & & & & & & & & \\
\hline Thorium-230 & & & & & & & & & \\
\hline Thorium-232 & & & & & & & & & \\
\hline Thorium-234 & & & & & & & & & \\
\hline Tritium & & & & & & & & & \\
\hline Uranium-234 & & & & & & & 2.89 & 0.89 & 0.32 \\
\hline Uranium-235 & & & & & & & $<\mathrm{MDA}$ & & 0.32 \\
\hline Uranium-236 & & & & & & & $<\mathrm{MDA}$ & & 0.22 \\
\hline Uranium-238 & & & & & & & 4.63 & 1.25 & 0.53 \\
\hline
\end{tabular}

\begin{tabular}{|c|c|c|c|c|c|c|c|c|c|}
\hline Sampling Point & \multicolumn{6}{|c|}{ GW-704 } & \multicolumn{3}{|c|}{ GW-706 } \\
\hline Location & \multicolumn{6}{|c|}{ EXP-B } & \multicolumn{3}{|c|}{ EXP-B } \\
\hline Date Sampled & \multicolumn{6}{|c|}{$07 / 16 / 02$} & \multicolumn{3}{|c|}{$01 / 16 / 02$} \\
\hline Program & \multicolumn{3}{|c|}{ GWPP } & \multicolumn{3}{|c|}{ BJC } & \multicolumn{3}{|c|}{ GWPP } \\
\hline \multicolumn{10}{|l|}{ Sample Type } \\
\hline Result (pCi/L) & Activity & Error & MDA & Activity & Error & MDA & Activity & Error & MDA \\
\hline Gross Alpha & 6.2 & 2.9 & 2 & & & & 58 & 9 & 3.9 \\
\hline Gross Beta & 41 & 8.1 & 8.8 & & & & 140 & 11 & 6.8 \\
\hline Americium-241 & & & & & & & & & \\
\hline Neptunium-237 & & & & & & & & & \\
\hline Plutonium-238 & & & & & & & & & \\
\hline Radium-223/224/226 & & & & & & & & & \\
\hline Strontium-89/90 & & & & & & & & & \\
\hline Technetium-99 & 30 & 8.3 & 13 & & & & 160 & 10 & 13 \\
\hline Thorium-228 & & & & & & & & & \\
\hline Thorium-230 & & & & & & & & & \\
\hline Thorium-232 & & & & & & & & & \\
\hline Thorium-234 & & & & & & & & & \\
\hline Tritium & & & & & & & & & \\
\hline Uranium-234 & & & & 3.07 & 0.99 & 0.28 & & & \\
\hline Uranium-235 & & & & $<$ MDA & & 0.45 & & & \\
\hline Uranium-236 & & & & $<$ MDA & & 0.18 & & & \\
\hline Uranium-238 & & & & 5.34 & 1.41 & 0.28 & & & \\
\hline
\end{tabular}


APPENDIX D.3: CY 2002 MONITORING DATA FOR THE BEAR CREEK HYDROGEOLOGIC REGIME

Radiological Analytes: Isotope Activity

\begin{tabular}{|c|c|c|c|c|c|c|c|c|c|}
\hline Sampling Point & \multicolumn{9}{|c|}{ GW-706 } \\
\hline Location & \multicolumn{9}{|c|}{ EXP-B } \\
\hline Date Sampled & \multicolumn{3}{|c|}{$01 / 16 / 02$} & \multicolumn{6}{|c|}{$07 / 16 / 02$} \\
\hline Program & \multicolumn{3}{|c|}{ BJC } & \multicolumn{3}{|c|}{ GWPP } & \multicolumn{3}{|c|}{ BJC } \\
\hline \multicolumn{10}{|l|}{ Sample Type } \\
\hline Result (pCi/L) & Activity & Error & MDA & Activity & Error & MDA & Activity & Error & MDA \\
\hline Gross Alpha & & & & 45 & 7.8 & 2.1 & & & \\
\hline Gross Beta & & & & 72 & 8.5 & 6.9 & & & \\
\hline Americium-241 & & & & & & & & & \\
\hline Neptunium-237 & & & & & & & & & \\
\hline Plutonium-238 & & & & & & & & & \\
\hline Radium-223/224/226 & & & & & & & & & \\
\hline Strontium-89/90 & & & & & & & & & \\
\hline Technetium-99 & & & & 57 & 8 & 13 & & & \\
\hline Thorium-228 & & & & & & & & & \\
\hline Thorium-230 & & & & & & & & & \\
\hline Thorium-232 & & & & & & & & & \\
\hline Thorium-234 & & & & & & & & & \\
\hline Tritium & & & & & & & & & \\
\hline Uranium-234 & 21.89 & 4.74 & 0.34 & & & & 19.78 & 4.72 & 0.8 \\
\hline Uranium-235 & 1.79 & 0.79 & 0.32 & & & & 1.45 & 1.05 & 0.49 \\
\hline Uranium-236 & 0.67 & 0.43 & 0.29 & & & & $0.49 \mathrm{R}$ & 0.57 & 0.44 \\
\hline Uranium-238 & 45.9 & 9.36 & 0.37 & & & & 32.99 & 6.99 & 0.88 \\
\hline
\end{tabular}

\begin{tabular}{|c|c|c|c|c|c|c|c|c|c|}
\hline Sampling Point & \multicolumn{6}{|c|}{ GW-712 } & \multicolumn{3}{|c|}{ GW-713 } \\
\hline Location & \multicolumn{6}{|c|}{ EXP-W } & \multicolumn{3}{|c|}{ EXP-W } \\
\hline Date Sampled & \multicolumn{3}{|c|}{$01 / 03 / 02$} & \multicolumn{3}{|c|}{$07 / 01 / 02$} & \multicolumn{3}{|c|}{$01 / 03 / 02$} \\
\hline Program & \multicolumn{3}{|c|}{ BJC } & \multicolumn{3}{|c|}{ BJC } & \multicolumn{3}{|c|}{ BJC } \\
\hline \multicolumn{10}{|l|}{ Sample Type } \\
\hline Result (pCi/L) & Activity & Error & MDA & Activity & Error & MDA & Activity & Error & MDA \\
\hline Gross Alpha & $<$ MDA & & 1.98 & 2.13 & 1.68 & 1.96 & 1.66 & 1.1 & 1.22 \\
\hline Gross Beta & 2.3 & 1.23 & 1.74 & 5.73 & 2.17 & 2.45 & 7.73 & 1.57 & 1.79 \\
\hline Americium-241 & $<$ MDA & & 1.7 & $<$ MDA & & 0.04 & $<$ MDA & & 1.4 \\
\hline Neptunium-237 & $<\mathrm{MDA}$ & & 0.44 & $<$ MDA & & 0.1 & $<$ MDA & & 0.28 \\
\hline Plutonium-238 & & & & & & & & & \\
\hline Radium-223/224/226 & 0.98 & 0.62 & 0.76 & $<M D A$ & & 0.36 & 3.73 & 1.14 & 0.84 \\
\hline Strontium-89/90 & $<M D A$ & & 1.33 & $<\mathrm{MDA}$ & & 1.38 & $<M D A$ & & 1.41 \\
\hline Technetium-99 & $<\mathrm{MDA}$ & & 13.75 & $<$ MDA & & 7.74 & 15.59 & 8.21 & 13.48 \\
\hline Thorium-228 & & & & & & & & & \\
\hline Thorium-230 & & & & & & & & & \\
\hline Thorium-232 & & & & & & & & & \\
\hline Thorium-234 & & & & & & & & & \\
\hline Tritium & & & & & & & & & \\
\hline Uranium-234 & $<\mathrm{MDA}$ & & 1.07 & 0.4 & 0.27 & 0.25 & $<M D A$ & & 0.36 \\
\hline Uranium-235 & $<$ MDA & & 0.96 & $<\mathrm{MDA}$ & & 0.26 & $<$ MDA & & 0.41 \\
\hline Uranium-236 & $<\mathrm{MDA}$ & & 0.43 & $<$ MDA & & 0.23 & $<$ MDA & & 0.37 \\
\hline Uranium-238 & $<\mathrm{MDA}$ & & 1.34 & $<\mathrm{MDA}$ & & 0.18 & $<$ MDA & & 0.44 \\
\hline
\end{tabular}


APPENDIX D.3: CY 2002 MONITORING DATA FOR THE BEAR CREEK HYDROGEOLOGIC REGIME Radiological Analytes: Isotope Activity

\begin{tabular}{|c|c|c|c|c|c|c|c|c|c|}
\hline Sampling Point & \multicolumn{3}{|c|}{ GW-713 } & \multicolumn{6}{|c|}{ GW-714 } \\
\hline Location & \multicolumn{3}{|c|}{ EXP-W } & \multicolumn{6}{|c|}{ EXP-W } \\
\hline Date Sampled & \multicolumn{3}{|c|}{$07 / 01 / 02$} & \multicolumn{3}{|c|}{$01 / 02 / 02$} & \multicolumn{3}{|c|}{$07 / 01 / 02$} \\
\hline Program & \multicolumn{3}{|c|}{ BJC } & \multicolumn{3}{|c|}{ BJC } & \multicolumn{3}{|c|}{ BJC } \\
\hline \multicolumn{10}{|l|}{ Sample Type } \\
\hline Result (pCi/L) & Activity & Error & MDA & Activity & Error & MDA & Activity & Error & MDA \\
\hline Gross Alpha & 15.75 & 2.77 & 2.09 & 2.2 & 1.36 & 1.75 & 3 & 1.61 & 1.76 \\
\hline Gross Beta & 39.75 & 3.06 & 2.48 & 6.1 & 1.54 & 1.94 & 4.38 & 2.09 & 2.41 \\
\hline Americium-241 & $<$ MDA & & 0.07 & $<\mathrm{MDA}$ & & 1.61 & $<$ MDA & & 0.11 \\
\hline Neptunium-237 & $<\mathrm{MDA}$ & & 0.24 & 0.43 & 0.34 & 0.42 & $<M D A$ & & 0.17 \\
\hline Plutonium-238 & & & & & & & & & \\
\hline Radium-223/224/226 & $<M D A$ & & 0.3 & 3.78 & 1.02 & 0.63 & $<M D A$ & & 0.59 \\
\hline Strontium-89/90 & $<M D A$ & & 1.35 & $<M D A$ & & 1.47 & $<M D A$ & & 1.66 \\
\hline Technetium-99 & $<\mathrm{MDA}$ & & 7.87 & 27.34 & 9.9 & 15.91 & $<M D A$ & & 7.9 \\
\hline Thorium-228 & & & & & & & & & \\
\hline Thorium-230 & & & & & & & & & \\
\hline Thorium-232 & & & & & & & & & \\
\hline Thorium-234 & & & & & & & & & \\
\hline Tritium & & & & & & & & & \\
\hline Uranium-234 & $<M D A$ & & 0.32 & 1.34 & 0.51 & 0.23 & 0.8 & 0.36 & 0.15 \\
\hline Uranium-235 & $<M D A$ & & 0.12 & $<\mathrm{MDA}$ & & 0.24 & $<\mathrm{MDA}$ & & 0.11 \\
\hline Uranium-236 & $<M D A$ & & 0.27 & $<\mathrm{MDA}$ & & 0.24 & $<\mathrm{MDA}$ & & 0.1 \\
\hline Uranium-238 & $<\mathrm{MDA}$ & & 0.28 & 0.67 & 0.33 & 0.19 & 0.61 & 0.31 & 0.2 \\
\hline
\end{tabular}

\begin{tabular}{|c|c|c|c|c|c|c|c|c|c|}
\hline Sampling Point & \multicolumn{9}{|c|}{ GW-715 } \\
\hline Location & \multicolumn{9}{|c|}{ EXP-W } \\
\hline Date Sampled & \multicolumn{6}{|c|}{$01 / 02 / 02$} & \multicolumn{3}{|c|}{$07 / 01 / 02$} \\
\hline Program & \multicolumn{6}{|c|}{ BJC } & \multicolumn{3}{|c|}{ BJC } \\
\hline Sample Type & \multicolumn{6}{|c|}{ DUP } & & & \\
\hline Result (pCi/L) & Activity & Error & MDA & Activity & Error & MDA & Activity & Error & MDA \\
\hline Gross Alpha & 5.7 & 2.14 & 2.26 & 5.24 & 1.61 & \begin{tabular}{l|l}
1.51 \\
\end{tabular} & 17.96 & 2.5 & 1.67 \\
\hline Gross Beta & 5.33 & 1.53 & 1.99 & 4.91 & 1.84 & 2.09 & 39.92 & 2.97 & 2.38 \\
\hline Americium-241 & $<$ MDA & & 2.03 & 5.03 & 2.06 & 1.57 & $<$ MDA & & 0.04 \\
\hline Neptunium-237 & $<$ MDA & & 0.38 & $<$ MDA & & 1.32 & $<$ MDA & & 0.18 \\
\hline Plutonium-238 & & & & & & & & & \\
\hline Radium-223/224/226 & 6.01 & 1.29 & 0.69 & 4.27 & 1.05 & 0.6 & $<M D A$ & & 0.42 \\
\hline Strontium-89/90 & $<\mathrm{MDA}$ & & 1.5 & $<\mathrm{MDA}$ & & 1.46 & $<M D A$ & & 1.34 \\
\hline Technetium-99 & 19.4 & 8.75 & 14.23 & 20.96 & 8.39 & 13.57 & 17.33 & 5.06 & 8.08 \\
\hline Thorium-228 & & & & & & & & & \\
\hline Thorium-230 & & & & & & & & & \\
\hline Thorium-232 & & & & & & & & & \\
\hline Thorium-234 & & & & & & & & & \\
\hline Tritium & & & & & & & & & \\
\hline Uranium-234 & 1.9 & 0.62 & 0.22 & 2.63 & 0.81 & 0.18 & 4.77 & 2.01 & 1.01 \\
\hline Uranium-235 & $<\mathrm{MDA}$ & & 0.21 & $<$ MDA & & 0.13 & $<$ MDA & & 0.96 \\
\hline Uranium-236 & $0.12 \mathrm{R}$ & 0.14 & 0.11 & $<$ MDA & & 0.12 & $<$ MDA & & 0.86 \\
\hline Uranium-238 & 3.79 & 0.98 & 0.25 & 2.84 & 0.85 & 0.24 & 10.15 & 3.2 & 0.77 \\
\hline
\end{tabular}


APPENDIX D.3: CY 2002 MONITORING DATA FOR THE BEAR CREEK HYDROGEOLOGIC REGIME Radiological Analytes: Isotope Activity

\begin{tabular}{|c|c|c|c|c|c|c|c|c|c|}
\hline Sampling Point & \multicolumn{3}{|c|}{ GW-715 } & \multicolumn{6}{|c|}{ GW-835 } \\
\hline Location & \multicolumn{3}{|c|}{ EXP-W } & \multicolumn{6}{|c|}{ S3 } \\
\hline Date Sampled & \multicolumn{3}{|c|}{$07 / 01 / 02$} & \multicolumn{3}{|c|}{$03 / 04 / 02$} & \multicolumn{3}{|c|}{$05 / 20 / 02$} \\
\hline Program & \multicolumn{3}{|c|}{ BJC } & \multicolumn{3}{|c|}{ BJC } & \multicolumn{3}{|c|}{ BJC } \\
\hline Sample Type & \multicolumn{3}{|c|}{ DUP } & & & & & & \\
\hline Result (pCi/L) & Activity & Error & MDA & Activity & Error & MDA & Activity & Error & MDA \\
\hline Gross Alpha & 16.02 & 2.45 & 1.72 & & & & & & \\
\hline Gross Beta & 22.57 & 2.58 & 2.39 & & & & & & \\
\hline Americium-241 & 0.1 & 0.08 & 0.04 & & & & & & \\
\hline Neptunium-237 & $<\mathrm{MDA}$ & & 0.1 & & & & & & \\
\hline Plutonium-238 & & & & & & & & & \\
\hline Radium-223/224/226 & $<M D A$ & & 0.4 & & & & & & \\
\hline Strontium-89/90 & $<\mathrm{MDA}$ & & 1.55 & & & & & & \\
\hline Technetium-99 & 16.45 & 4.81 & 7.67 & & & & & & \\
\hline Thorium-228 & & & & & & & & & \\
\hline Thorium-230 & & & & & & & & & \\
\hline Thorium-232 & & & & & & & & & \\
\hline Thorium-234 & & & & & & & & & \\
\hline Tritium & & & & & & & & & \\
\hline Uranium-234 & 3.78 & 1.84 & 1.41 & 161.5 & 45.02 & 2.77 & 146.8 & 36.91 & 5.94 \\
\hline Uranium-235 & $<M D A$ & & 1.35 & 5.91 & 4.43 & 2 & 12.69 & 7.59 & 4.58 \\
\hline Uranium-236 & $<M D A$ & & 0.93 & 9.28 & 5.48 & 1.8 & $<\mathrm{MDA}$ & & 4.82 \\
\hline Uranium-238 & 8.79 & 2.97 & 0.98 & 399.9 & 104.9 & 1.62 & 407.5 & 90.3 & 5.23 \\
\hline
\end{tabular}

\begin{tabular}{|c|c|c|c|c|c|c|c|c|c|}
\hline Sampling Point & \multicolumn{6}{|c|}{ GW-835 } & \multicolumn{3}{|c|}{ GW-916 } \\
\hline Location & \multicolumn{6}{|c|}{ S3 } & \multicolumn{3}{|c|}{ EMWMF } \\
\hline Date Sampled & \multicolumn{3}{|c|}{$08 / 22 / 02$} & \multicolumn{3}{|c|}{$11 / 19 / 02$} & \multicolumn{3}{|c|}{$08 / 15 / 02$} \\
\hline Program & \multicolumn{3}{|c|}{ BJC } & \multicolumn{3}{|c|}{ BJC } & \multicolumn{3}{|c|}{ BJC } \\
\hline \multicolumn{10}{|l|}{ Sample Type } \\
\hline Result (pCi/L) & Activity & Error & MDA & Activity & Error & MDA & Activity & Error & MDA \\
\hline \multicolumn{10}{|l|}{ Gross Alpha } \\
\hline Gross Beta & & & & & & & & & \\
\hline Americium-241 & & & & & & & $<M D A$ & & 0.227 \\
\hline Neptunium-237 & & & & & & & $<$ MDA & & 0.129 \\
\hline Plutonium-238 & & & & & & & $<M D A$ & & 0.206 \\
\hline Radium-223/224/226 & & & & & & & & & \\
\hline Strontium-89/90 & & & & & & & $<M D A$ & & 0.597 \\
\hline Technetium-99 & & & & & & & $<$ MDA & & 6.85 \\
\hline Thorium-228 & & & & & & & & & \\
\hline Thorium-230 & & & & & & & & & \\
\hline Thorium-232 & & & & & & & & & \\
\hline Thorium-234 & & & & & & & & & \\
\hline Tritium & & & & & & & & & \\
\hline Uranium-234 & 173.8 & 52.54 & 6.38 & 157.4 & 38.34 & 3.59 & 0.202 & 0.078 & 0.0345 \\
\hline Uranium-235 & 10.64 & 7.03 & 2.62 & 11.42 & 8.83 & 4.42 & $<$ MDA & & 0.0345 \\
\hline Uranium-236 & 12.01 & 7.27 & 4.02 & 7.33 & 6.66 & 3.97 & & & \\
\hline Uranium-238 & 407.7 & 115.9 & 5.46 & 348.6 & 70.44 & 7.14 & 0.121 & 0.06 & 0.0204 \\
\hline
\end{tabular}


APPENDIX D.3: CY 2002 MONITORING DATA FOR THE BEAR CREEK HYDROGEOLOGIC REGIME Radiological Analytes: Isotope Activity

\begin{tabular}{|c|c|c|c|c|c|c|c|c|c|}
\hline Sampling Point & \multicolumn{3}{|c|}{ GW-916 } & \multicolumn{6}{|c|}{ GW-917 } \\
\hline Location & \multicolumn{3}{|c|}{ EMWMF } & \multicolumn{6}{|c|}{ EMWMF } \\
\hline Date Sampled & \multicolumn{3}{|c|}{$12 / 10 / 02$} & \multicolumn{3}{|c|}{$08 / 12 / 02$} & \multicolumn{3}{|c|}{$12 / 05 / 02$} \\
\hline Program & \multicolumn{3}{|c|}{ BJC } & \multicolumn{3}{|c|}{ BJC } & \multicolumn{3}{|c|}{ BJC } \\
\hline \multicolumn{10}{|l|}{ Sample Type } \\
\hline Result (pCi/L) & Activity & Error & MDA & Activity & Error & MDA & Activity & Error & MDA \\
\hline \multicolumn{10}{|l|}{ Gross Alpha } \\
\hline Gross Beta & & & & & & & & & \\
\hline Americium-241 & $<M D A$ & & 0.627 & $<M D A$ & & 0.26 & $<M D A$ & & 0.112 \\
\hline Neptunium-237 & $<$ MDA & & 0.349 & $<$ MDA & & 0.143 & $<M D A$ & & 0.282 \\
\hline Plutonium-238 & $<\mathrm{MDA}$ & & 0.135 & $<$ MDA & & 0.191 & $<M D A$ & & 0.126 \\
\hline \multicolumn{10}{|l|}{ Radium-223/224/226 } \\
\hline Strontium-89/90 & $<\mathrm{MDA}$ & & 1.28 & $<M D A$ & & 0.779 & $<M D A$ & & 1.03 \\
\hline Technetium-99 & $<M D A$ & & 7.1 & $<M D A$ & & 6.91 & $<M D A$ & & 7.22 \\
\hline Thorium-228 & & & & & & & & & \\
\hline Thorium-230 & & & & & & & & & \\
\hline Thorium-232 & & & & & & & & & \\
\hline Thorium-234 & & & & & & & & & \\
\hline Tritium & & & & & & & & & \\
\hline Uranium-234 & 0.122 & 0.086 & 0.0805 & 0.0373 & 0.031 & 0.0292 & $<\mathrm{MDA}$ & . & 0.0668 \\
\hline Uranium-235 & $<\mathrm{MDA}$ & & 0.0682 & $<\mathrm{MDA}$ & & 0.0292 & $<M D A$ & & 0.0378 \\
\hline Uranium-236 & & & & & & & & & \\
\hline Uranium-238 & 0.114 & 0.081 & 0.0386 & $<\mathrm{MDA}$ & & 0.0464 & $0.0418 \mathrm{R}$ & 0.048 & 0.0378 \\
\hline
\end{tabular}

\begin{tabular}{|c|c|c|c|c|c|c|c|c|c|}
\hline Sampling Point & \multicolumn{6}{|c|}{ GW-918 } & \multicolumn{3}{|c|}{ GW-919 } \\
\hline Location & \multicolumn{6}{|c|}{ EMWMF } & \multicolumn{3}{|c|}{ EMWMF } \\
\hline Date Sampled & \multicolumn{3}{|c|}{$08 / 15 / 02$} & \multicolumn{3}{|c|}{$12 / 10 / 02$} & \multicolumn{3}{|c|}{$08 / 14 / 02$} \\
\hline Program & \multicolumn{3}{|c|}{ BJC } & \multicolumn{3}{|c|}{ BJC } & \multicolumn{3}{|c|}{ BJC } \\
\hline \multicolumn{10}{|l|}{ Sample Type } \\
\hline Result (pCi/L) & Activity & Error & MDA & Activity & Error & MDA & Activity & Error & MDA \\
\hline \\
\hline Gross Beta & & & & & & & & & \\
\hline Americium-241 & $0.129 \mathrm{R}$ & 0.15 & 0.117 & $<$ MDA & & 0.113 & $<\mathrm{MDA}$ & & 0.12 \\
\hline Neptunium-237 & $<\mathrm{MDA}$ & & 0.13 & $<M D A$ & & 0.165 & $<M D A$ & & 0.108 \\
\hline Plutonium-238 & $<M D A$ & & 0.191 & $<\mathrm{MDA}$ & & 0.328 & $<M D A$ & & 0.105 \\
\hline \multicolumn{10}{|l|}{ Radium-223/224/226 } \\
\hline Strontium-89/90 & $<$ MDA & & 0.538 & $<$ MDA & & 1.34 & $<M D A$ & & 0.66 \\
\hline Technetium-99 & $<M D A$ & & 6.9 & $<M D A$ & & 7.03 & $<M D A$ & & 6.93 \\
\hline Thorium-228 & & & & & & & & & \\
\hline Thorium-230 & & & & & & & & & \\
\hline Thorium-232 & & & & & & & & & \\
\hline Thorium-234 & & & & & & & & & \\
\hline Tritium & & & & & & & & & \\
\hline Uranium-234 & 0.0491 & 0.036 & 0.0286 & 0.193 & 0.11 & 0.0722 & 0.12 & 0.065 & 0.0697 \\
\hline Uranium-235 & $<\mathrm{MDA}$ & & 0.0286 & $<$ MDA & & 0.0409 & $<$ MDA & & 0.0203 \\
\hline Uranium-236 & & & & & & & & & \\
\hline Uranium-238 & $<\mathrm{MDA}$ & & 0.0455 & 0.0905 & 0.074 & 0.0409 & $<$ MDA & & 0.0424 \\
\hline
\end{tabular}


APPENDIX D.3: CY 2002 MONITORING DATA FOR THE BEAR CREEK HYDROGEOLOGIC REGIME Radiological Analytes: Isotope Activity

\begin{tabular}{|c|c|c|c|c|c|c|c|c|c|}
\hline Sampling Point & \multicolumn{3}{|c|}{ GW-919 } & \multicolumn{6}{|c|}{ GW-920 } \\
\hline Location & \multicolumn{3}{|c|}{ EMWMF } & \multicolumn{6}{|c|}{ EMWMF } \\
\hline Date Sampled & \multicolumn{3}{|c|}{$12 / 09 / 02$} & \multicolumn{3}{|c|}{$08 / 13 / 02$} & \multicolumn{3}{|c|}{$12 / 05 / 02$} \\
\hline Program & \multicolumn{3}{|c|}{ BJC } & \multicolumn{3}{|c|}{ BJC } & \multicolumn{3}{|c|}{ BJC } \\
\hline \multicolumn{10}{|l|}{ Sample Type } \\
\hline Result (pCi/L) & Activity & Error & MDA & Activity & Error & MDA & Activity & Error & MDA \\
\hline \multirow{2}{*}{\multicolumn{10}{|c|}{$\begin{array}{r}\text { Gross Alpha } \\
\text { Gross Beta }\end{array}$}} \\
\hline & & & & & & & & & \\
\hline Americium-241 & $<\mathrm{MDA}$ & & 0.119 & $<\mathrm{MDA}$ & & 0.106 & $<\mathrm{MDA}$ & & 0.181 \\
\hline Neptunium-237 & $<M D A$ & & 0.0618 & $<\mathrm{MDA}$ & & 0.116 & $<M D A$ & & 0.208 \\
\hline Plutonium-238 & $<M D A$ & & 0.229 & $<M D A$ & & 0.161 & $<M D A$ & & 0.107 \\
\hline \multicolumn{10}{|l|}{ Radium-223/224/226 } \\
\hline Strontium-89/90 & $<\mathrm{MDA}$ & & 0.991 & $<\mathrm{MDA}$ & & 0.66 & $<\mathrm{MDA}$ & & 1.01 \\
\hline Technetium-99 & $<M D A$ & & 7.26 & 7.42 & 3.1 & 7 & $<M D A$ & & 7.27 \\
\hline Thorium-228 & & & & & & & & & \\
\hline Thorium-230 & & & & & & & & & \\
\hline Thorium-232 & & & & & & & & & \\
\hline Thorium-234 & & & & & & & & & \\
\hline Tritium & & & & & & & & & \\
\hline Uranium-234 & 0.108 & 0.087 & 0.0923 & 0.0253 & 0.025 & 0.0171 & $<\mathrm{MDA}$ & & 0.0764 \\
\hline Uranium-235 & $<M D A$ & & 0.0782 & $<M D A$ & & 0.0171 & $<M D A$ & & 0.0432 \\
\hline Uranium-236 & & & & & & & & & \\
\hline Uranium-238 & 0.111 & 0.087 & 0.0782 & $<\mathrm{MDA}$ & & 0.0171 & $<\mathrm{MDA}$ & & 0.0432 \\
\hline
\end{tabular}

\begin{tabular}{|c|c|c|c|c|c|c|c|c|c|}
\hline Sampling Point & \multicolumn{6}{|c|}{ GW-921 } & \multicolumn{3}{|c|}{ GW-922 } \\
\hline Location & \multicolumn{6}{|c|}{ EMWMF } & \multicolumn{3}{|c|}{ EMWMF } \\
\hline Date Sampled & \multicolumn{3}{|c|}{$08 / 12 / 02$} & \multicolumn{3}{|c|}{$12 / 04 / 02$} & \\
\hline Program & \multicolumn{3}{|c|}{ BJC } & \multicolumn{3}{|c|}{ BJC } & \multicolumn{3}{|c|}{ BJC } \\
\hline \multicolumn{10}{|l|}{ Sample Type } \\
\hline Result (pCi/L) & Activity & Error & MDA & Activity & Error & MDA & Activity & Error & MDA \\
\hline \multirow{2}{*}{\multicolumn{10}{|c|}{$\begin{array}{r}\text { Gross Alpha } \\
\text { Gross Beta }\end{array}$}} \\
\hline & & & & & & & & & \\
\hline Americium-241 & $<$ MDA & & 0.209 & $<\mathrm{MDA}$ & & 0.116 & $<\mathrm{MDA}$ & & 0.172 \\
\hline Neptunium-237 & $<$ MDA & & 0.124 & $<\mathrm{MDA}$ & & 0.122 & $<$ MDA & & 0.127 \\
\hline Plutonium-238 & $<M D A$ & & 0.11 & $<\mathrm{MDA}$ & & 0.211 & $<M D A$ & & 0.129 \\
\hline \multicolumn{10}{|l|}{ Radium-223/224/226 } \\
\hline Strontium-89/90 & $<M D A$ & & 0.76 & $<\mathrm{MDA}$ & & 0.994 & $<M D A$ & & 0.482 \\
\hline Technetium-99 & $<\mathrm{MDA}$ & & 7.03 & 8.27 & 3.3 & 7.38 & $<$ MDA & & 7.02 \\
\hline Thorium-228 & & & & & & & & & \\
\hline Thorium-230 & & & & & & & & & \\
\hline Thorium-232 & & & & & & & & & \\
\hline Thorium-234 & & & & & & & & & \\
\hline Tritium & & & & & & & & & \\
\hline Uranium-234 & $0.0202 \mathrm{R}$ & 0.023 & 0.0183 & $<\mathrm{MDA}$ & & 0.0844 & 0.0786 & 0.046 & 0.0303 \\
\hline Uranium-235 & $<\mathrm{MDA}$ & & 0.0381 & $<\mathrm{MDA}$ & & 0.0588 & $<\mathrm{MDA}$ & & 0.0303 \\
\hline Uranium-236 & & & & & & & & & \\
\hline Uranium-238 & $<\mathrm{MDA}$ & & 0.0381 & $<\mathrm{MDA}$ & & 0.0844 & $<$ MDA & & 0.0482 \\
\hline
\end{tabular}


APPENDIX D.3: CY 2002 MONITORING DATA FOR THE BEAR CREEK HYDROGEOLOGIC REGIME Radiological Analytes: Isotope Activity

\begin{tabular}{|c|c|c|c|c|c|c|c|c|c|}
\hline Sampling Point & \multicolumn{3}{|c|}{ GW-922 } & \multicolumn{6}{|c|}{ GW-923 } \\
\hline Location & \multicolumn{3}{|c|}{ EMWMF } & \multicolumn{6}{|c|}{ EMWMF } \\
\hline Date Sampled & \multicolumn{3}{|c|}{$12 / 09 / 02$} & \multicolumn{3}{|c|}{$08 / 14 / 02$} & \multicolumn{3}{|c|}{$12 / 09 / 02$} \\
\hline Program & \multicolumn{3}{|c|}{ BJC } & \multicolumn{3}{|c|}{ BJC } & \multicolumn{3}{|c|}{ BJC } \\
\hline \multicolumn{10}{|l|}{ Sample Type } \\
\hline Result (pCi/L) & Activity & Error & MDA & Activity & Error & MDA & Activity & Error & MDA \\
\hline \multicolumn{10}{|l|}{ Gross Alpha } \\
\hline Gross Beta & & & & & & & & & \\
\hline Americium-241 & $<M D A$ & & 0.121 & $<M D A$ & & 0.131 & $<\mathrm{MDA}$ & & 0.128 \\
\hline Neptunium-237 & $<$ MDA & & 0.135 & $<$ MDA & & 0.174 & $<\mathrm{MDA}$ & & 0.207 \\
\hline Plutonium-238 & $<\mathrm{MDA}$ & & 0.127 & $<$ MDA & & 0.112 & $<M D A$ & & 0.125 \\
\hline \multicolumn{10}{|l|}{ Radium-223/224/226 } \\
\hline Strontium-89/90 & $<\mathrm{MDA}$ & & 1.02 & $<M D A$ & & 0.752 & $<\mathrm{MDA}$ & & 0.931 \\
\hline Technetium-99 & $<M D A$ & & 7.23 & $<M D A$ & & 7.04 & $<\mathrm{MDA}$ & & 7.36 \\
\hline Thorium-228 & & & & & & & & & \\
\hline Thorium-230 & & & & & & & & & \\
\hline Thorium-232 & & & & & & & & & \\
\hline Thorium-234 & & & & & & & & & \\
\hline Tritium & & & & & & & & & \\
\hline Uranium-234 & 0.172 & 0.11 & 0.14 & 0.394 & 0.11 & 0.034 & 0.315 & 0.15 & 0.116 \\
\hline Uranium-235 & $<\mathrm{MDA}$ & & 0.107 & 0.0372 & 0.033 & 0.0202 & $<\mathrm{MDA}$ & & 0.108 \\
\hline Uranium-236 & & & & & & & & & \\
\hline Uranium-238 & $<\mathrm{MDA}$ & & 0.125 & 0.219 & 0.082 & 0.0479 & 0.195 & 0.11 & 0.0995 \\
\hline
\end{tabular}

\begin{tabular}{|c|c|c|c|c|c|c|c|c|c|}
\hline Sampling Point & \multicolumn{9}{|c|}{ GW-924 } \\
\hline Location & \multicolumn{9}{|c|}{ EMWMF } \\
\hline Date Sampled & \multicolumn{6}{|c|}{$08 / 13 / 02$} & \multicolumn{3}{|c|}{$12 / 10 / 02$} \\
\hline Program & \multicolumn{6}{|c|}{ BJC } & \multicolumn{3}{|c|}{ BJC } \\
\hline Sample Type & \multicolumn{6}{|c|}{ DUP } & & & \\
\hline Result (pCi/L) & Activity & Error & MDA & Activity & Error & MDA & Activity & Error & MDA \\
\hline \multicolumn{10}{|l|}{$\begin{array}{r}\text { Gross Alpha } \\
\text { Gross Beta }\end{array}$} \\
\hline Americium-241 & $<$ MDA & & 0.243 & $<M D A$ & & 0.126 & $<M D A$ & & 0.125 \\
\hline Neptunium-237 & $<$ MDA & & 0.118 & $<\mathrm{MDA}$ & & 0.197 & $<$ MDA & & 0.285 \\
\hline Plutonium-238 & $<M D A$ & & 0.172 & $<\mathrm{MDA}$ & & 0.0956 & $<M D A$ & & 0.385 \\
\hline \multicolumn{10}{|l|}{ Radium-223/224/226 } \\
\hline Strontium-89/90 & $<\mathrm{MDA}$ & & 1.29 & $<\mathrm{MDA}$ & & 0.919 & $<M D A$ & & 1.1 \\
\hline Technetium-99 & $<\mathrm{MDA}$ & & 7.08 & $<\mathrm{MDA}$ & & 7.07 & $<$ MDA & & 7.17 \\
\hline Thorium-228 & & & & & & & & & \\
\hline Thorium-230 & & & & & & & & & \\
\hline Thorium-232 & & & & & & & & & \\
\hline Thorium-234 & & & & & & & & & \\
\hline Tritium & & & & & & & & & \\
\hline Uranium-234 & 0.139 & 0.062 & 0.0188 & 0.0759 & 0.051 & 0.0228 & $<M D A$ & & 0.0634 \\
\hline Uranium-235 & $<$ MDA & & 0.0392 & $<$ MDA & & 0.0228 & $<$ MDA & & 0.0358 \\
\hline Uranium-236 & & & & & & & & & \\
\hline Uranium-238 & $<\mathrm{MDA}$ & & 0.0392 & 0.0422 & 0.038 & 0.0228 & $0.0397 \mathrm{R}$ & 0.046 & 0.0358 \\
\hline
\end{tabular}


APPENDIX D.3: CY 2002 MONITORING DATA FOR THE BEAR CREEK HYDROGEOLOGIC REGIME Radiological Analytes: Isotope Activity

\begin{tabular}{|c|c|c|c|c|c|c|c|c|c|}
\hline Sampling Point & \multicolumn{3}{|c|}{ GW-924 } & \multicolumn{6}{|c|}{ GW-925 } \\
\hline Location & \multicolumn{3}{|c|}{ EMWMF } & \multicolumn{6}{|c|}{ EMWMF } \\
\hline Date Sampled & \multicolumn{3}{|c|}{$12 / 10 / 02$} & \multicolumn{3}{|c|}{$08 / 12 / 02$} & \multicolumn{3}{|c|}{$12 / 04 / 02$} \\
\hline Program & \multicolumn{3}{|c|}{ BJC } & \multicolumn{3}{|c|}{ BJC } & \multicolumn{3}{|c|}{ BJC } \\
\hline Sample Type & \multicolumn{3}{|c|}{ DUP } & & & & & & \\
\hline Result (pCi/L) & Activity & Error & MDA & Activity & Error & MDA & Activity & Error & MDA \\
\hline \\
\hline Gross Beta & & & & & & & & & \\
\hline Americium-241 & $<M D A$ & & 0.104 & $<M D A$ & & 0.112 & $<\mathrm{MDA}$ & & 0.154 \\
\hline Neptunium-237 & $<M D A$ & & 0.164 & $<\mathrm{MDA}$ & & 0.204 & $<\mathrm{MDA}$ & & 0.213 \\
\hline Plutonium-238 & $<\mathrm{MDA}$ & & 0.263 & $<$ MDA & & 0.0949 & $<M D A$ & & 0.132 \\
\hline \multicolumn{10}{|l|}{ Radium-223/224/226 } \\
\hline Strontium-89/90 & $<\mathrm{MDA}$ & & 1.23 & $<M D A$ & & 0.69 & $<\mathrm{MDA}$ & & 1.07 \\
\hline Technetium-99 & $<M D A$ & & 7.1 & $<M D A$ & & 7.02 & $<\mathrm{MDA}$ & & 7.44 \\
\hline Thorium-228 & & & & & & & & & \\
\hline Thorium-230 & & & & & & & & & \\
\hline Thorium-232 & & & & & & & & & \\
\hline Thorium-234 & & & & & & & & & \\
\hline Tritium & & & & & & & & & \\
\hline Uranium-234 & 0.136 & 0.091 & 0.041 & 0.439 & 0.14 & 0.029 & 0.693 & 0.2 & 0.0774 \\
\hline Uranium-235 & $<\mathrm{MDA}$ & & 0.041 & $<\mathrm{MDA}$ & & 0.029 & $<\mathrm{MDA}$ & & 0.0656 \\
\hline Uranium-236 & & & & & & & & & \\
\hline Uranium-238 & $0.0605 \mathrm{R}$ & 0.061 & 0.041 & 0.171 & 0.086 & 0.029 & 0.246 & 0.12 & 0.0371 \\
\hline
\end{tabular}

\begin{tabular}{|c|c|c|c|c|c|c|c|c|c|}
\hline Sampling Point & \multicolumn{6}{|c|}{ GW-926 } & \multicolumn{3}{|c|}{ GW-927 } \\
\hline Location & \multicolumn{6}{|c|}{ EMWMF } & \multicolumn{3}{|c|}{ EMWMF } \\
\hline Date Sampled & \multicolumn{3}{|c|}{$08 / 13 / 02$} & \multicolumn{3}{|c|}{$12 / 10 / 02$} & \\
\hline Program & \multicolumn{3}{|c|}{ BJC } & \multicolumn{3}{|c|}{ BJC } & \multicolumn{3}{|c|}{ BJC } \\
\hline \multicolumn{10}{|l|}{ Sample Type } \\
\hline Result (pCi/L) & Activity & Error & MDA & Activity & Error & MDA & Activity & Error & MDA \\
\hline \multicolumn{10}{|l|}{$\begin{array}{r}\text { Gross Alpha } \\
\text { Gross Beta }\end{array}$} \\
\hline Americium-241 & $<$ MDA & & 0.115 & $<M D A$ & & 0.118 & $0.126 \mathrm{R}$ & 0.15 & 0.113 \\
\hline Neptunium-237 & $<$ MDA & & 0.134 & $<\mathrm{MDA}$ & & 0.175 & $<$ MDA & & 0.196 \\
\hline Plutonium-238 & $<M D A$ & & 0.107 & $<\mathrm{MDA}$ & & 0.0987 & $<M D A$ & & 0.106 \\
\hline \multicolumn{10}{|l|}{ Radium-223/224/226 } \\
\hline Strontium-89/90 & $<\mathrm{MDA}$ & & 1.37 & $<\mathrm{MDA}$ & & 1.22 & $<M D A$ & & 0.809 \\
\hline Technetium-99 & 11.6 & 3.3 & 7.07 & $<M D A$ & & 7 & $<$ MDA & & 7.05 \\
\hline Thorium-228 & & & & & & & & & \\
\hline Thorium-230 & & & & & & & & & \\
\hline Thorium-232 & & & & & & & & & \\
\hline Thorium-234 & & & & & & & & & \\
\hline Tritium & & & & & & & & & \\
\hline Uranium-234 & 0.0775 & 0.057 & 0.0687 & $<\mathrm{MDA}$ & & 0.034 & $<M D A$ & & 0.0658 \\
\hline Uranium-235 & $<\mathrm{MDA}$ & & 0.0498 & $<\mathrm{MDA}$ & & 0.034 & $<$ MDA & & 0.0476 \\
\hline Uranium-236 & & & & & & & & & \\
\hline Uranium-238 & $<\mathrm{MDA}$ & & 0.0605 & $<\mathrm{MDA}$ & & 0.0601 & $<$ MDA & & 0.0579 \\
\hline
\end{tabular}


APPENDIX D.3: CY 2002 MONITORING DATA FOR THE BEAR CREEK HYDROGEOLOGIC REGIME Radiological Analytes: Isotope Activity

\begin{tabular}{|c|c|c|c|c|c|c|c|c|c|}
\hline Sampling Point & \multicolumn{3}{|c|}{ GW-927 } & \multicolumn{3}{|c|}{ NT-03 } & \multicolumn{3}{|c|}{ NT-07 } \\
\hline Location & \multicolumn{3}{|c|}{ EMWMF } & \multicolumn{3}{|c|}{ EXP-SW } & \multicolumn{3}{|c|}{ EXP-SW } \\
\hline Date Sampled & \multicolumn{3}{|c|}{$12 / 05 / 02$} & \multicolumn{3}{|c|}{$03 / 11 / 02$} & \multicolumn{3}{|c|}{$03 / 13 / 02$} \\
\hline Program & \multicolumn{3}{|c|}{ BJC } & \multicolumn{3}{|c|}{ BJC } & \multicolumn{3}{|c|}{ BJC } \\
\hline \multicolumn{10}{|l|}{ Sample Type } \\
\hline Result (pCi/L) & Activity & Error & MDA & Activity & Error & MDA & Activity & Error & MDA \\
\hline \multicolumn{10}{|l|}{ Gross Alpha } \\
\hline Gross Beta & & & & & & & & & \\
\hline Americium-241 & $<M D A$ & & 0.115 & & & & & & \\
\hline Neptunium-237 & $<M D A$ & & 0.124 & & & & & & \\
\hline Plutonium-238 & $<\mathrm{MDA}$ & & 0.13 & & & & & & \\
\hline \multicolumn{10}{|l|}{ Radium-223/224/226 } \\
\hline Strontium-89/90 & $<\mathrm{MDA}$ & & 1.11 & & & & & & \\
\hline Technetium-99 & $<\mathrm{MDA}$ & & 7.29 & 30.16 & 3.96 & 5.81 & & & \\
\hline Thorium-228 & & & & & & & & & \\
\hline Thorium-230 & & & & & & & & & \\
\hline Thorium-232 & & & & & & & & & \\
\hline Thorium-234 & & & & & & & & & \\
\hline Tritium & & & & & & & & & \\
\hline Uranium-234 & $<M D A$ & & 0.0342 & & & & 2.56 & 0.83 & 0.31 \\
\hline Uranium-235 & $<\mathrm{MDA}$ & & 0.0342 & & & & $0.15 \mathrm{R}$ & 0.17 & 0.13 \\
\hline Uranium-236 & & & & & & & $<M D A$ & & 0.21 \\
\hline Uranium-238 & $<\mathrm{MDA}$ & & 0.0342 & & & & 4.27 & 1.19 & 0.22 \\
\hline
\end{tabular}

\begin{tabular}{|c|c|c|c|c|c|c|c|c|c|}
\hline Sampling Point & \multicolumn{3}{|c|}{ NT-08 } & \multicolumn{3}{|c|}{ NT-8-E } & \multicolumn{3}{|c|}{ NT-8-W } \\
\hline Location & \multicolumn{3}{|c|}{ EXP-SW } & \multicolumn{3}{|c|}{ EXP-SW } & \multicolumn{3}{|c|}{ EXP-SW } \\
\hline Date Sampled & \multicolumn{3}{|c|}{$03 / 13 / 02$} & \multicolumn{3}{|c|}{$03 / 13 / 02$} & \multicolumn{3}{|c|}{$03 / 13 / 02$} \\
\hline Program & \multicolumn{3}{|c|}{ BJC } & \multicolumn{3}{|c|}{ BJC } & \multicolumn{3}{|c|}{ BJC } \\
\hline \multicolumn{10}{|l|}{ Sample Type } \\
\hline Result (pCi/L) & Activity & Error & MDA & Activity & Error & MDA & Activity & Error & MDA \\
\hline \multicolumn{10}{|l|}{ Gross Alpha } \\
\hline \multicolumn{10}{|l|}{ Gross Beta } \\
\hline \multicolumn{10}{|l|}{ Americium-241 } \\
\hline \multicolumn{10}{|l|}{ Neptunium-237 } \\
\hline \multicolumn{10}{|l|}{ Plutonium-238 } \\
\hline \multicolumn{10}{|l|}{ Radium-223/224/226 } \\
\hline \multicolumn{10}{|l|}{ Strontium-89/90 } \\
\hline \multicolumn{10}{|l|}{ Technetium-99 } \\
\hline \multicolumn{10}{|l|}{ Thorium-228 } \\
\hline \multicolumn{10}{|l|}{ Thorium-230 } \\
\hline \multicolumn{10}{|l|}{ Thorium-232 } \\
\hline \multicolumn{10}{|l|}{ Thorium-234 } \\
\hline \multicolumn{10}{|l|}{ Tritium } \\
\hline Uranium-234 & 17.83 & 5.18 & 1.39 & $50.25 \mathrm{Q}$ & 22.02 & 5.97 & 1.3 & 0.52 & 0.28 \\
\hline Uranium-235 & 1.23 & 1.05 & 1.14 & 9.27 & 7.79 & 6.28 & $<\mathrm{MDA}$ & & 0.13 \\
\hline Uranium-236 & $<$ MDA & & 1.03 & 8.33 & 7 & 5.64 & $<\mathrm{MDA}$ & & 0.24 \\
\hline Uranium-238 & 90.59 & 21.46 & 0.92 & $223.2 \mathrm{Q}$ & 78.37 & 5.94 & 4.03 & 1.12 & 0.56 \\
\hline
\end{tabular}


APPENDIX D.3: CY 2002 MONITORING DATA FOR THE BEAR CREEK HYDROGEOLOGIC REGIME

Radiological Analytes: Isotope Activity

\begin{tabular}{|c|c|c|c|c|c|c|c|c|c|}
\hline Sampling Point & \multicolumn{3}{|c|}{ S07 } & \multicolumn{6}{|c|}{ SS-4 } \\
\hline Location & \multicolumn{3}{|c|}{ EXP-SW } & \multicolumn{6}{|c|}{ EXP-SW } \\
\hline Date Sampled & \multicolumn{3}{|c|}{$03 / 11 / 02$} & \multicolumn{6}{|c|}{$01 / 08 / 02$} \\
\hline Program & \multicolumn{3}{|c|}{ BJC } & \multicolumn{3}{|c|}{ GWPP } & \multicolumn{3}{|c|}{ BJC } \\
\hline \multicolumn{10}{|l|}{ Sample Type } \\
\hline Result (pCi/L) & Activity & Error & MDA & Activity & Error & MDA & Activity & Error & MDA \\
\hline Gross Alpha & & & & 73 & 9.9 & 4.5 & & & \\
\hline Gross Beta & & & & 180 & 13 & 8.2 & & & \\
\hline Americium-241 & & & & & & & & & \\
\hline Neptunium-237 & & & & & & & & & \\
\hline Plutonium-238 & & & & & & & & & \\
\hline Radium-223/224/226 & & & & & & & & & \\
\hline Strontium-89/90 & & & & & & & & & \\
\hline Technetium-99 & 94.14 & 4.92 & 5.78 & 180 & 10 & 13 & & & \\
\hline Thorium-228 & & & & & & & & & \\
\hline Thorium-230 & & & & & & & & & \\
\hline Thorium-232 & & & & & & & & & \\
\hline Thorium-234 & & & & & & & & & \\
\hline Tritium & & & & & & & & & \\
\hline Uranium-234 & 0.36 & 0.26 & 0.25 & & & & 28.11 & 5.81 & 0.22 \\
\hline Uranium-235 & $<M D A$ & & 0.26 & & & & 0.98 & 0.52 & 0.32 \\
\hline Uranium-236 & $<M D A$ & & 0.12 & & & & 0.76 & 0.43 & 0.32 \\
\hline Uranium-238 & 0.36 & 0.25 & 0.25 & & & & 53.34 & 10.61 & 0.37 \\
\hline
\end{tabular}

\begin{tabular}{|c|c|c|c|c|c|c|c|c|c|}
\hline Sampling Point & \multicolumn{9}{|c|}{ SS-4 } \\
\hline Location & \multicolumn{9}{|c|}{ EXP-SW } \\
\hline Date Sampled & \multicolumn{3}{|c|}{$01 / 08 / 02$} & \multicolumn{6}{|c|}{$07 / 10 / 02$} \\
\hline Program & \multicolumn{3}{|c|}{ BJC } & \multicolumn{3}{|c|}{ GWPP } & \multicolumn{3}{|c|}{ BJC } \\
\hline Sample Type & \multicolumn{3}{|c|}{ DUP } & & & & & & \\
\hline Result (pCi/L) & Activity & Error & MDA & Activity & Error & MDA & Activity & Error & MDA \\
\hline Gross Alpha & & & & 43 & 7.6 & 2.5 & & & \\
\hline Gross Beta & & & & 53 & 7.1 & 6.3 & & & \\
\hline Americium-241 & & & & & & & & & \\
\hline Neptunium-237 & & & & & & & & & \\
\hline Plutonium-238 & & & & & & & & & \\
\hline Radium-223/224/226 & & & & & & & & & \\
\hline Strontium-89/90 & & & & & & & & & \\
\hline Technetium-99 & & & & 34 & 9.8 & 13 & & & \\
\hline Thorium-228 & & & & & & & & & \\
\hline Thorium-230 & & & & & & & & & \\
\hline Thorium-232 & & & & & & & & & \\
\hline Thorium-234 & & & & & & & & & \\
\hline Tritium & & & & & & & & & \\
\hline Uranium-234 & 31.58 & 7.01 & 0.31 & & & & 18.65 & 5.96 & 2.04 \\
\hline Uranium-235 & 1.11 & 0.6 & 0.32 & & & & $<$ MDA & & 0.97 \\
\hline Uranium-236 & 1.19 & 0.6 & 0.17 & & & & $<$ MDA & & 1.94 \\
\hline Uranium-238 & 63.6 & 13.59 & 0.26 & & & & 31.07 & 8.59 & 1.9 \\
\hline
\end{tabular}


APPENDIX D.3: CY 2002 MONITORING DATA FOR THE BEAR CREEK HYDROGEOLOGIC REGIME Radiological Analytes: Isotope Activity

\begin{tabular}{|c|c|c|c|c|c|c|c|c|c|}
\hline Sampling Point & \multicolumn{3}{|c|}{ SS-4 } & \multicolumn{6}{|c|}{ SS-5 } \\
\hline Location & \multicolumn{3}{|c|}{ EXP-SW } & \multicolumn{6}{|c|}{ EXP-SW } \\
\hline Date Sampled & \multicolumn{3}{|c|}{$07 / 10 / 02$} & \multicolumn{3}{|c|}{$01 / 08 / 02$} & \multicolumn{3}{|c|}{$07 / 10 / 02$} \\
\hline Program & \multicolumn{3}{|c|}{ BJC } & \multicolumn{3}{|c|}{ BJC } & \multicolumn{3}{|c|}{ BJC } \\
\hline Sample Type & \multicolumn{3}{|c|}{ DUP } & & & & & & \\
\hline Result (pCi/L) & Activity & Error & MDA & Activity & Error & MDA & Activity & Error & MDA \\
\hline Gross Alpha & & & & 48 & 7.8 & 2.8 & 31 & 6 & 2.3 \\
\hline Gross Beta & & & & 81 & 8.4 & 6.7 & 37 & 7 & 8 \\
\hline Americium-241 & & & & & & & & & \\
\hline Neptunium-237 & & & & & & & & & \\
\hline Plutonium-238 & & & & & & & & & \\
\hline Radium-223/224/226 & & & & & & & & & \\
\hline Strontium-89/90 & & & & & & & & & \\
\hline Technetium-99 & & & & 78.57 & 5.76 & 7.77 & 16.17 & 4.63 & 7.36 \\
\hline Thorium-228 & & & & & & & & & \\
\hline Thorium-230 & & & & & & & & & \\
\hline Thorium-232 & & & & & & & & & \\
\hline Thorium-234 & & & & & & & & & \\
\hline Tritium & & & & & & & & & \\
\hline Uranium-234 & 14.42 & 4.7 & 1.51 & 20.69 & 4.03 & 0.24 & 8.54 & 3.46 & 1.24 \\
\hline Uranium-235 & 1.55 & 1.41 & 0.84 & 0.53 & 0.33 & 0.13 & $<\mathrm{MDA}$ & & 1.8 \\
\hline Uranium-236 & $0.83 \mathrm{R}$ & 0.98 & 0.75 & 0.51 & 0.31 & 0.2 & $<M D A$ & & 1.38 \\
\hline Uranium-238 & 33.59 & 8.6 & 1.51 & 36.72 & 6.82 & 0.23 & 15.18 & 4.99 & 1.24 \\
\hline
\end{tabular}

\begin{tabular}{|c|c|c|c|c|c|c|c|c|c|}
\hline Sampling Point & \multicolumn{6}{|c|}{ SS-6 } & \multicolumn{3}{|c|}{$\overline{\text { SS-7 }}$} \\
\hline Location & \multicolumn{6}{|c|}{ EXP-SW } & \multicolumn{3}{|c|}{ EXP-SW } \\
\hline Date Sampled & \multicolumn{3}{|c|}{$01 / 08 / 02$} & \multicolumn{3}{|c|}{$07 / 10 / 02$} & \multicolumn{3}{|c|}{$03 / 12 / 02$} \\
\hline Program & \multicolumn{3}{|c|}{ BJC } & \multicolumn{3}{|c|}{ BJC } & \multicolumn{3}{|c|}{ BJC } \\
\hline \multicolumn{10}{|l|}{ Sample Type } \\
\hline Result (pCi/L) & Activity & Error & MDA & Activity & Error & MDA & Activity & Error & MDA \\
\hline Gross Alpha & & & & & & & & & \\
\hline Gross Beta & & & & & & & 9.29 & 1.68 & 1.69 \\
\hline Americium-241 & & & & & & & & & \\
\hline Neptunium-237 & & & & & & & & & \\
\hline Plutonium-238 & & & & & & & & & \\
\hline Radium-223/224/226 & & & & & & & & & \\
\hline Strontium-89/90 & & & & & & & & & \\
\hline Technetium-99 & $<\mathrm{MDA}$ & & 7.91 & $<$ MDA & & 7.23 & 6.23 & 3.53 & 5.81 \\
\hline Thorium-228 & & & & & & & & & \\
\hline Thorium-230 & & & & & & & & & \\
\hline Thorium-232 & & & & & & & & & \\
\hline Thorium-234 & & & & & & & & & \\
\hline Tritium & & & & & & & & & \\
\hline Uranium-234 & 2.71 & 0.81 & 0.29 & 1.57 & 0.82 & 0.65 & 1.59 & 0.54 & 0.2 \\
\hline Uranium-235 & 0.19 & 0.19 & 0.13 & $<$ MDA & & 0.29 & $<$ MDA & & 0.19 \\
\hline Uranium-236 & $<$ MDA & & 0.23 & $<$ MDA & & 0.45 & $<$ MDA & & 0.17 \\
\hline Uranium-238 & 5.14 & 1.29 & 0.23 & 3.28 & 1.23 & 0.47 & 3.77 & 0.97 & 0.26 \\
\hline
\end{tabular}


APPENDIX D.3: CY 2002 MONITORING DATA FOR THE BEAR CREEK HYDROGEOLOGIC REGIME Radiological Analytes: Isotope Activity

\begin{tabular}{|c|c|c|c|c|c|c|c|c|c|}
\hline Sampling Point & \multicolumn{9}{|c|}{ SS-8 } \\
\hline Location & \multicolumn{9}{|c|}{ EXP-SW } \\
\hline Date Sampled & \multicolumn{3}{|c|}{$03 / 12 / 02$} & \multicolumn{6}{|c|}{$09 / 09 / 02$} \\
\hline Program & \multicolumn{3}{|c|}{ BJC } & \multicolumn{6}{|c|}{ BJC } \\
\hline Sample Type & & & & & & & \multicolumn{3}{|c|}{ DUP } \\
\hline Result (pCi/L) & Activity & Error & MDA & Activity & Error & MDA & Activity & Error & MDA \\
\hline Gross Alpha & & & & & & & & & \\
\hline Gross Beta & 2.71 & 1.14 & 1.54 & $<\mathrm{MDA}$ & & 1.8 & $<\mathrm{MDA}$ & & 1.79 \\
\hline Americium-241 & & & & & & & & & \\
\hline Neptunium-237 & & & & & & & & & \\
\hline Plutonium-238 & & & & & & & & & \\
\hline Radium-223/224/226 & & & & & & & & & \\
\hline Strontium-89/90 & & & & & & & & & \\
\hline Technetium-99 & $<\mathrm{MDA}$ & & 6.19 & $<\mathrm{MDA}$ & & 5.98 & $<\mathrm{MDA}$ & & 6.01 \\
\hline Thorium-228 & & & & & & & & & \\
\hline Thorium-230 & & & & & & & & & \\
\hline Thorium-232 & & & & & & & & & \\
\hline Thorium-234 & & & & & & & & & \\
\hline Tritium & & & & & & & & & \\
\hline Uranium-234 & 0.52 & 0.27 & 0.19 & 0.27 & 0.24 & 0.26 & 0.35 & 0.29 & 0.16 \\
\hline Uranium-235 & $<\mathrm{MDA}$ & & 0.11 & $<\mathrm{MDA}$ & & 0.32 & $<\mathrm{MDA}$ & & 0.19 \\
\hline Uranium-236 & $<\mathrm{MDA}$ & & 0.16 & $<\mathrm{MDA}$ & & 0.14 & $<\mathrm{MDA}$ & & 0.17 \\
\hline Uranium-238 & $<\mathrm{MDA}$ & & 0.17 & $<\mathrm{MDA}$ & & 0.22 & $<\mathrm{MDA}$ & & 0.31 \\
\hline
\end{tabular}


APPENDIX E

CY 2002 MONITORING DATA FOR THE

UPPER EAST FORK POPLAR CREEK HYDROGEOLOGIC REGIME 


\title{
EXPLANATION
}

\section{Sampling Point:}

\author{
GHK - Gum Hollow Branch Kilometer (surface water sampling location) \\ GW - Groundwater Monitoring Well; Westbay well GW-722 \\ LRSPW - Exit point of the New Hope Pond Distribution Channel underdrain \\ (surface water sampling location) \\ NPR - North of Pine Ridge near the Scarboro Community (surface water sampling location) \\ $\mathrm{OF}$ - Storm drain outfall (surface water sampling location) \\ SCR - Spring sampling location in Union Valley \\ STATION - Surface water sampling location in Upper East Fork Poplar Creek
}

\section{Location:}

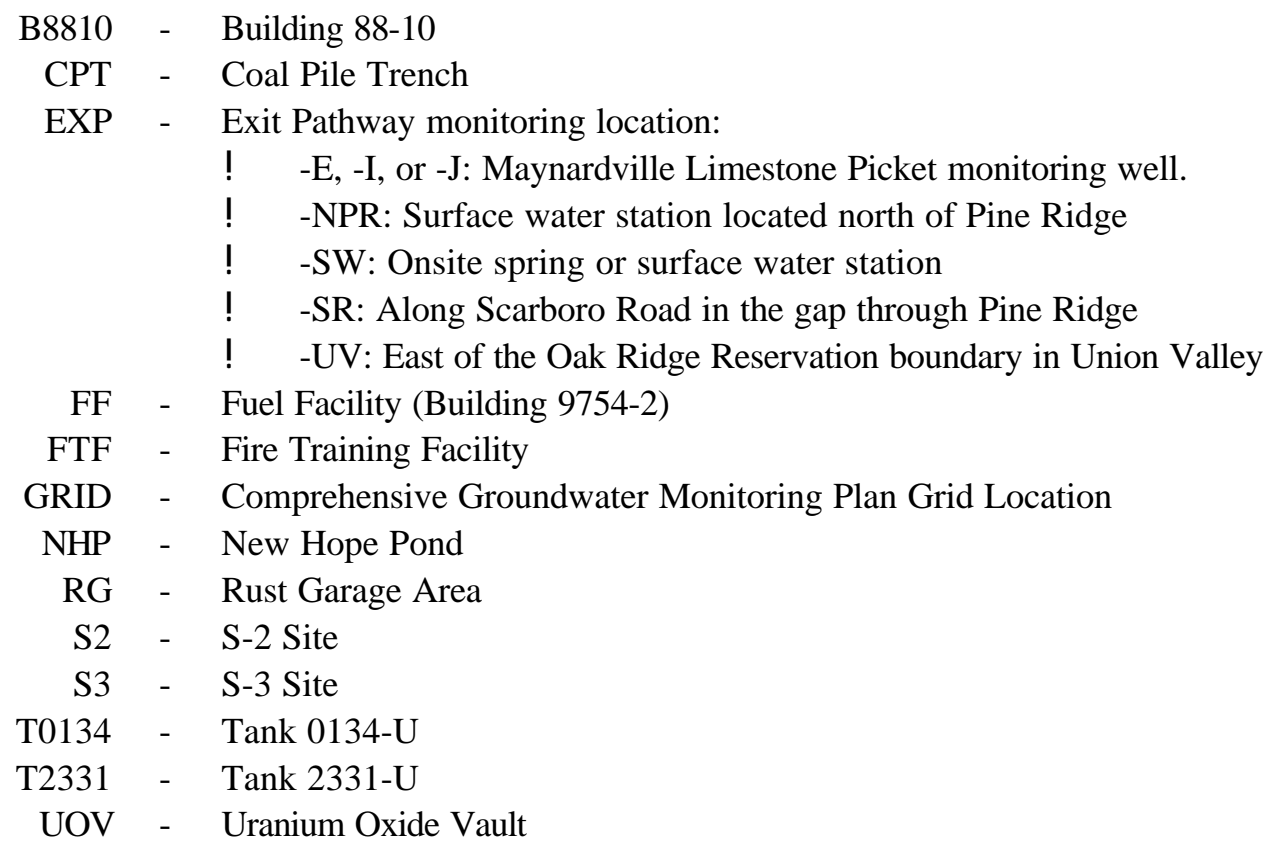

\section{Monitoring Program:}

BJC - managed by Bechtel Jacobs Company LLC

GWPP - managed by the Y-12 Groundwater Protection Program

\section{Sample Type:}

Dup - Field Duplicate Sample

\section{Units:}

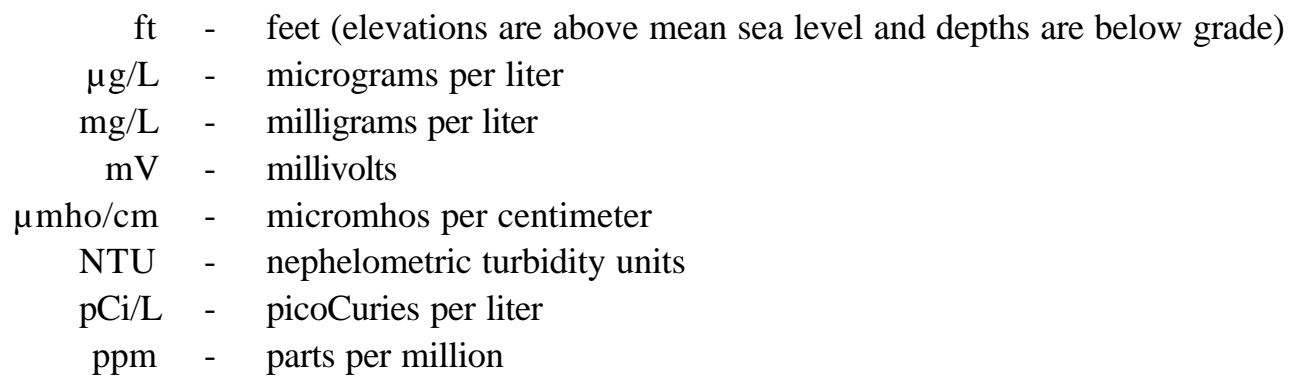




\section{EXPLANATION (continued)}

Only analytes detected above reporting limits in at least one sample are included in this appendix. Additionally, results that are below the reporting limits are replaced with missing values (e.g., " < ") to emphasize the detected results. The following sections describe the analytes, reporting limits, and data qualifiers for each subappendix. A comprehensive list of the GWPP analytes, analytical methods, and reporting limits is provided in Appendix B, Table B.5.

\section{E.1 Field Measurements, Miscellaneous Analytes, Major Ions, and Trace Metals:}

Results for all of the field measurements, miscellaneous analytes, and major ions are included in this appendix. The reporting limits for the major ions are shown in the following summary.

\begin{tabular}{|c|c|c|c|c|c|}
\hline \multirow{2}{*}{ Analyte } & \multicolumn{2}{|c|}{ Reporting Limit (mg/L) } & \multirow{2}{*}{ Analyte } & \multicolumn{2}{|c|}{ Reporting Limit (mg/L) } \\
\hline & GWPP & BJC & & GWPP & BJC \\
\hline $\begin{array}{r}\text { Calcium } \\
\text { Magnesium } \\
\text { Potassium } \\
\text { Sodium }\end{array}$ & $\begin{array}{l}0.2 \\
0.2 \\
2.0 \\
0.2\end{array}$ & $\begin{array}{l}0.25 \\
0.05 \\
0.25 \\
0.25\end{array}$ & $\begin{array}{l}\text { Anions } \\
\text { Alkalinity - HCO3 } \\
\text { Alkalinity - CO3 } \\
\text { Chloride } \\
\text { Fluoride } \\
\text { Nitrate (as Nitrogen) } \\
\text { Sulfate }\end{array}$ & $\begin{array}{c}1.0 \\
1.0 \\
0.2 \\
0.1 \\
0.028 \\
0.25\end{array}$ & $\begin{array}{c}\text { NA } \\
\text { NA } \\
0.1 \\
0.05 \\
0.1 \\
0.1\end{array}$ \\
\hline
\end{tabular}

The major ion results for the following samples are qualitative because the ion charge balance (relative percent difference [RPD]) exceeds $20 \%$.

\begin{tabular}{|c|c|c|c|}
\hline $\begin{array}{c}\text { Sampling } \\
\text { Point }\end{array}$ & $\begin{array}{c}\text { Date } \\
\text { Sampled }\end{array}$ & $\begin{array}{c}\text { Ion Charge } \\
\text { Balance RPD }\end{array}$ & Suspected Source of Error \\
\hline GW-170 & $02 / 04 / 02$ & -36.3 & High $\mathrm{pH}$ \\
GW-170 & $05 / 14 / 02$ & -56.0 & High $\mathrm{pH}$ \\
GW-170 & $11 / 11 / 02$ & -53.5 & High $\mathrm{pH}$ \\
GW-232 & $11 / 11 / 02$ & -24.9 & High alkalinity \\
GW-253 & $11 / 07 / 02$ & -67.9 & Very high nitrate \\
GW-618 & $05 / 09 / 02$ & 35.0 & Low bicarbonate \\
GW-618 & $11 / 07 / 02$ & -93.9 & Extremely high nitrate \\
GW-620 & $04 / 23 / 02$ & -51.6 & High pH \\
GW-689 & $11 / 14 / 02$ & -51.3 & Low pH \\
\hline
\end{tabular}




\section{EXPLANATION (continued)}

The Y-12 GWPP SAP (BWXT 2001a) specifies reporting limits for trace metals that are appropriate for DOE Order 5400.1 monitoring. The laboratories subcontracted for the monitoring programs managed by BJC may use reporting limits (sometimes reporting estimated values) that are lower than the GWPP reporting limits for the metals. To retain the highest quality data for DOE Order 5400.1 monitoring purposes and to standardize reporting limits for trace metal results obtained from all sources, the GWPP reporting limits were given precedence over the BJC reporting limits (BJC 2001 and 2002a) shown below. The trace metals shown in bold typeface below were detected in at least one sample collected during CY 2002 and are presented in Appendix E.1.

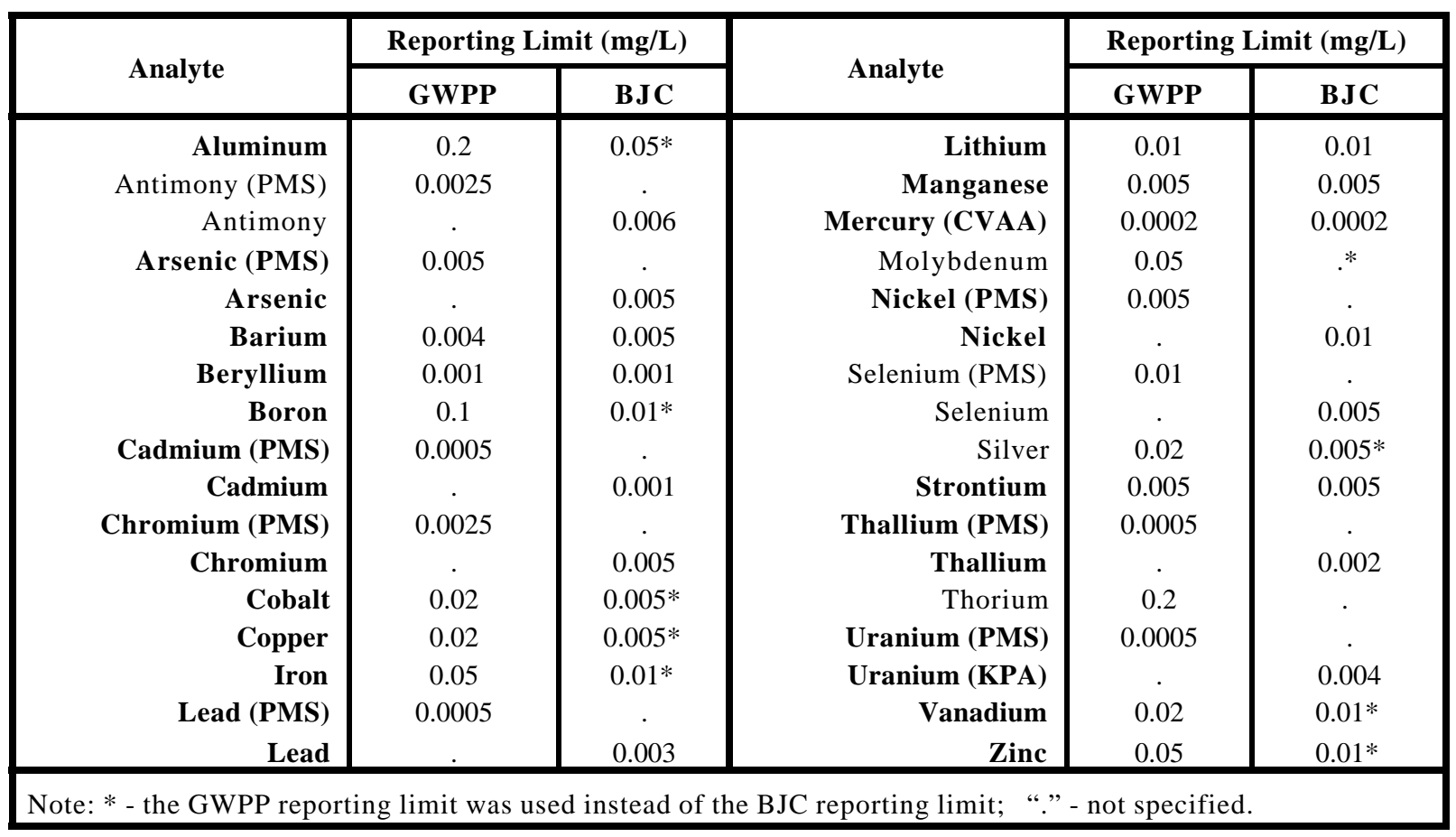

All metals analyses were performed using the inductively coupled plasma (ICP) spectroscopy method (SW846-6010B) unless otherwise noted.

$$
\begin{array}{rll}
\text { CVAA } & - & \text { Cold Vapor Atomic Absorption (EPA-7470) } \\
\text { KPA } & - & \text { Kinetic Phosphorescent Analysis (ASTM-D5174-M) } \\
\text { PMS } & - & \text { Plasma Mass Spectroscopy (EPA-200.8) }
\end{array}
$$

Groundwater samples collected the following wells for metals analysis by the ICP method during CY 2002 were diluted before analysis to obtain an optimum matrix. The detected results are valid, but some metals may be present at concentrations below the elevated reporting limits.

\begin{tabular}{|c|c|c|c|c|c|}
\hline Sampling Location & $\begin{array}{c}\text { Date } \\
\text { Sampled }\end{array}$ & Dilution Factor & $\begin{array}{c}\text { Sampling } \\
\text { Location }\end{array}$ & $\begin{array}{c}\text { Date } \\
\text { Sampled }\end{array}$ & $\begin{array}{c}\text { Dilution } \\
\text { Factor }\end{array}$ \\
\hline GW-108 & $01 / 08 / 02$ & $5 \mathrm{X}$ & $\mathrm{GW}-633$ & $04 / 23 / 02$ & $10 \mathrm{X}$ \\
GW-108 & $07 / 09 / 02$ & $5 \mathrm{X}$ & $\mathrm{GW}-633$ & $10 / 17 / 02$ & $10 \mathrm{X}$ \\
GW-253 & $05 / 07 / 02$ & $4 \mathrm{X}$ & $\mathrm{GW}-658$ & $04 / 24 / 02$ & $5 \mathrm{X}$ \\
& & & $\mathrm{GW}-658$ & $10 / 17 / 02$ & $10 \mathrm{X}$ \\
& & & $\mathrm{GW}-698$ & $11 / 14 / 02$ & $8 \mathrm{X}$ \\
\hline
\end{tabular}




\section{EXPLANATION (continued)}

The following symbols and qualifiers are used in Appendix E.1:

- $\quad$ Not analyzed or not applicable

$<\quad-\quad$ Analyzed but not detected at the project reporting level

[ ] - Calculated value for total uranium (GW-108); high calcium concentration interferes with the KPA method, so uranium mass is calculated from isotopic activity by the laboratory.

Q - Result is inconsistent with historical measurements.

$\mathrm{R}$ - Result does not meet data quality objectives (charge balances and duplicate metal results that differ by at least an order of magnitude).

\section{E.2 Volatile Organic Compounds:}

The Y-12 GWPP reporting limits for volatile organic compounds (Table B.5) and those used for monitoring programs managed by BJC are contract-required quantitation limits. Results below the quantitation limit and above the instrument detection limit are reported as estimated quantities. Therefore, non-detected results are assumed to equal zero for all compounds.

As summarized below, 28 compounds were detected (of the 53 compounds requested) in the CY 2002 groundwater samples collected in the East Fork Regime. Results for these compounds are grouped by similar chemical composition (e.g., chloroethenes) in Appendix E.2.

\begin{tabular}{|c|c|c|c|c|c|}
\hline Compound & $\begin{array}{c}\text { No. } \\
\text { Detected }\end{array}$ & $\begin{array}{c}\text { Maximum } \\
(\mu \mathrm{g} / \mathrm{L})\end{array}$ & Compound & $\begin{array}{c}\text { No. } \\
\text { Detected }\end{array}$ & $\begin{array}{c}\text { Maximum } \\
(\mu \mathrm{g} / \mathrm{L})\end{array}$ \\
\hline Tetrachloroethene & 86 & 2,400 & 1,1-Dichloroethane & 8 & 180 \\
\hline Chloroform & 78 & 620 & Bromodichloromethane & 8 & 2 \\
\hline Trichloroethene & 71 & 610 & Bromoform & 8 & 5 \\
\hline Carbon tetrachloride & 58 & 1,500 & Methylene chloride & 6 & 69 \\
\hline cis-1,2-Dichloroethene & 52 & 260 & Carbon disulfide & 3 & 9 \\
\hline Toluene & 18 & 4,900 & Acetone & 2 & 18 \\
\hline Ethylbenzene & 15 & 1,400 & Chlorodibromomethane & 2 & 1 \\
\hline Vinyl chloride & 14 & 63 & Chloroethane & 2 & 12 \\
\hline Benzene & 12 & 8,700 & Trichlorofluoromethane & 2 & 3 \\
\hline 1,1-Dichloroethene & 11 & 60 & 1,2-Dibromoethane & 1 & 77 \\
\hline Dimethylbenzene & 10 & 8,800 & 1,2-Dichloroethane & 1 & 570 \\
\hline trans-1,2-Dichloroethene & 9 & 2 & 4-Methyl-2-pentanone & 1 & 200 \\
\hline Styrene & 9 & 4 & Bromomethane & 1 & 14 \\
\hline 1,1,1-Trichloroethane & 8 & 9 & Chlorobenzene & 1 & 1 \\
\hline
\end{tabular}

The WRRP also requested analyses of volatile organic gases (ethane, ethylene, and methane) as natural attenuation indicators for groundwater samples collected from seven wells in the East Fork Regime during CY 2002. Because ethane was not detected in any of these samples, only ethylene and methane results are shown in the appendix. 


\section{EXPLANATION (continued)}

The following symbols and data qualifiers are used in Appendix E.2.

. $\quad$ Not analyzed or not applicable

$<\quad$ - Analyzed but not detected (also false-positive results for data provided by BJC)

J - Positively identified, estimated concentration below the contract-required quantitation limit; one result that exceeds instrument calibration (tetrachloroethene at well GW-383)

Q - Inconsistent with historical measurements for the location (e.g., carbon disulfide at well GW253)

$\mathrm{R}$ - Result does not meet data quality objectives; duplicate results that differ by at least an order of magnitude (e.g., benzene and chloroform at well GW-170)

\section{E.3 Radiological Analytes:}

Reporting limits for radiological analytes are sample-specific and analyte-specific minimum detectable activities that are reported with each result. The following summary shows the radiological analytes reported for at least one groundwater sample collected during CY 2002 in the East Fork Regime.

\begin{tabular}{|r|c|c|r|c|c|}
\hline Analyte & $\begin{array}{c}\text { No. of } \\
\text { Results }\end{array}$ & $\begin{array}{c}\text { No. } \\
\text { Detected }\end{array}$ & Analyte & $\begin{array}{c}\text { No. of } \\
\text { Results }\end{array}$ & $\begin{array}{c}\text { No. } \\
\text { Detected }\end{array}$ \\
\hline Gross Alpha & 187 & 66 & Uranium-233/234 & 18 & 16 \\
Gross Beta & 187 & 96 & Uranium-234 & 10 & 10 \\
Technetium-99 & 15 & 2 & Uranium-235 & 28 & 13 \\
& & & Uranium-236 & 17 & 4 \\
& & & Uranium-238 & 28 & 26 \\
\hline
\end{tabular}

Results for gross alpha and gross beta are presented in the first three pages of Appendix E.3, followed by the results for isotopes.

The following notes apply to Appendix E.3:

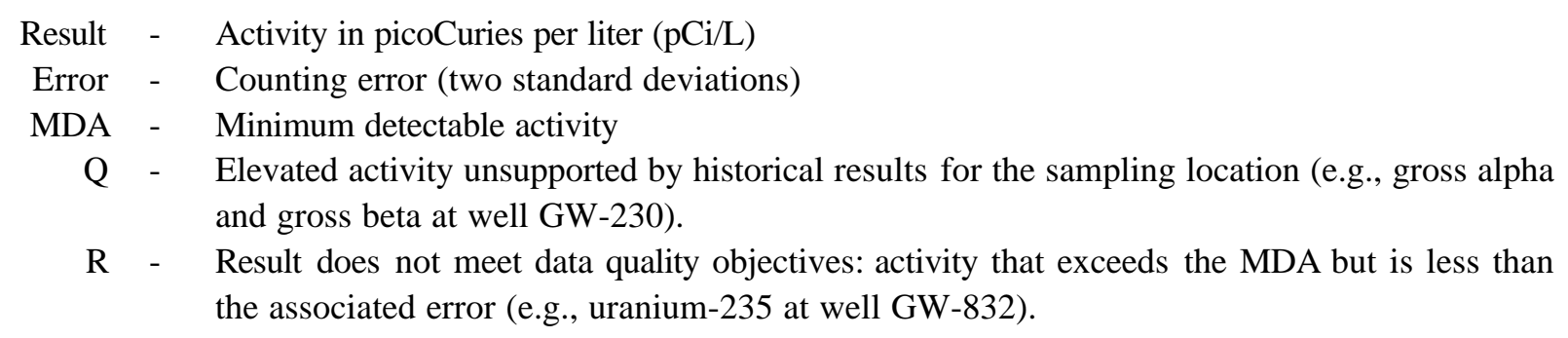


APPENDIX E.1

FIELD MEASUREMENTS, MISCELLANEOUS ANALYTES, MAJOR IONS, AND TRACE METALS 
APPENDIX E.1: CY 2002 MONITORING DATA FOR THE UPPER EAST FORK POPLAR CREEK HYDROGEOLOGIC REGIME

Field Measurements, Miscellaneous Analytes, Major Ions, and Trace Metals

\begin{tabular}{|c|c|c|c|c|c|c|c|c|}
\hline Sampling Point & \multicolumn{3}{|c|}{ 55-1B } & \multicolumn{2}{|c|}{ GHK2.51ESW } & \multicolumn{3}{|c|}{ GHK2.51WSW } \\
\hline Location & \multicolumn{3}{|c|}{ GRIDB2 } & \multicolumn{2}{|c|}{ EXP-NPR } & \multicolumn{3}{|c|}{ EXP-NPR } \\
\hline Date Sampled & \multicolumn{2}{|c|}{ 04/17/02 } & \multirow{2}{*}{$\begin{array}{l}10 / 14 / 02 \\
\text { GWPP }\end{array}$} & \multirow{2}{*}{$\begin{array}{l}\text { 05/15/02 } \\
\text { GWPP }\end{array}$} & \multirow{2}{*}{$\begin{array}{l}10 / 29 / 02 \\
\text { GWPP }\end{array}$} & \multirow{2}{*}{$\begin{array}{l}\text { 05/15/02 } \\
\text { GWPP }\end{array}$} & \multicolumn{2}{|c|}{$10 / 29 / 02$} \\
\hline Program & GWPP & GWPP & & & & & GWPP & GWPP \\
\hline Sample Type & & Dup & & & & & & Dup \\
\hline \multicolumn{9}{|l|}{ Field Measurements } \\
\hline Time Sampled & $9: 30$ & $9: 30$ & $9: 10$ & $10: 20$ & $8: 50$ & $10: 30$ & 9:00 & 9:00 \\
\hline Measuring Point Elev. (ft) & 986.26 & 986.26 & 986.26 & & & & & \\
\hline Depth to Water (ft) & 11.18 & 11.18 & 10.90 & & & & & \\
\hline Groundwater Elevation (ft) & 975.08 & 975.08 & 975.36 & & & & & \\
\hline Conductivity $(\mu \mathrm{mho} / \mathrm{cm})$ & 1,440 & 1,440 & 1,395 & 351 & 545 & 142.6 & 154 & 154 \\
\hline Dissolved Oxygen (ppm) & 0.63 & 0.63 & 0.63 & 4.51 & 2.05 & 5.64 & 3.85 & 3.85 \\
\hline Oxidation/Reduction (mV) & -38 & -38 & 145 & 151 & 222 & 139 & 198 & 198 \\
\hline Temperature (degrees C) & 17.8 & 17.8 & 16.1 & 12.8 & 16.3 & 13 & 16.1 & 16.1 \\
\hline Turbidity (NTU) & & & & & & & & \\
\hline Iron ++ (mg/L) & & & & & & & & \\
\hline Manganese $++(\mathrm{mg} / \mathrm{L})$ & & & & & & & & \\
\hline $\mathrm{pH}$ & 7.5 & 7.5 & 7.6 & 7.14 & 6.9 & 7.34 & 6.86 & 6.86 \\
\hline Miscellaneous Analytes & & & & & & & & \\
\hline Conductivity $(\mu \mathrm{mho} / \mathrm{cm})$ & 1,346 & 1,364 & 1,289 & 313 & 332 & 121.9 & 115.8 & 116.1 \\
\hline Dissolved Solids (mg/L) & 730 & 749 & 771 & 213 & 199 & 95 & 88 & 81 \\
\hline Suspended Solids (mg/L) & $<$ & $<$ & $<$ & 34.4 & $<$ & 2.8 & 8 & 8 \\
\hline Turbidity (NTU) & 14.9 & 15.9 & 5.74 & 5.85 & 12.9 & 6.77 & 17.6 & 17.5 \\
\hline $\mathrm{pH}$ & 7.36 & 7.43 & 7.41 & 7.56 & 7.15 & 7.62 & 7.27 & 7.27 \\
\hline Major lons (mg/L) & & & & & & & & \\
\hline Calcium & 182 & 187 & 178 & 60 & 63 & 14.4 & 13.5 & 13.7 \\
\hline Magnesium & 25.4 & 25.4 & 22.8 & 4 & 3 & 4.11 & 3.69 & 3.73 \\
\hline Potassium & 3.32 & 3.36 & 3.09 & $<$ & 2.07 & 2.17 & 2.63 & 2.79 \\
\hline Sodium & 28 & 27.9 & 26.5 & 0.678 & 0.799 & 1.32 & 1.2 & 1.21 \\
\hline Alkalinity as $\mathrm{CO} 3$ & $<$ & $<$ & $<$ & $<$ & $<$ & $<$ & $<$ & $<$ \\
\hline Alkalinity as $\mathrm{HCO} 3$ & 122 & 121 & 160 & 160 & 157 & 45 & 39.6 & 39.6 \\
\hline Chloride & 341 & 323 & 278 & 1.01 & 1.04 & 1.16 & 1.01 & 1.02 \\
\hline Fluoride & $<$ & $<$ & $<$ & $<$ & $<$ & $<$ & $<$ & $<$ \\
\hline Nitrate as $\mathrm{N}$ & 0.159 & 0.117 & 0.0847 & 0.15 & 0.0581 & 0.0693 & 0.0549 & 0.0583 \\
\hline Sulfate & 48.6 & 48.1 & 42.9 & 5.52 & 13.5 & 12.1 & 12.6 & 12.7 \\
\hline Charge balance & -2.4 & 0.7 & 0.2 & -0.9 & 0.3 & -0.8 & 0.5 & 1.2 \\
\hline Trace Metals (mg/L) & & & & & & & & \\
\hline Aluminum & $<$ & $<$ & $<$ & 0.757 & 1.28 & 0.574 & 1.74 & 1.69 \\
\hline Arsenic (PMS) & $<$ & $<$ & $<$ & $<$ & $<$ & $<$ & $<$ & $<$ \\
\hline Arsenic & & & & & & & & \\
\hline Barium & 0.24 & 0.239 & 0.254 & 0.0293 & 0.0367 & 0.0411 & 0.0499 & 0.0503 \\
\hline Beryllium & $<$ & $<$ & $<$ & $<$ & $<$ & $<$ & $<$ & $<$ \\
\hline Boron & $<$ & $<$ & $<$ & $<$ & $<$ & $<$ & $<$ & $<$ \\
\hline Cadmium (PMS) & 0.000787 & $<$ & $<$ & $<$ & $<$ & $<$ & $<$ & $<$ \\
\hline Cadmium & & & & & & & & \\
\hline Chromium (PMS) & 0.381 & 0.351 & 0.141 & 0.00286 & $<$ & 0.00271 & 0.00274 & 0.00284 \\
\hline Chromium & & & & & & & & \\
\hline Cobalt & $<$ & $<$ & $<$ & $<$ & $<$ & $<$ & $<$ & $<$ \\
\hline Copper & $<$ & $<$ & $<$ & $<$ & $<$ & $<$ & $<$ & $<$ \\
\hline Iron & 1.86 & 1.87 & 0.575 & 0.581 & 0.672 & 0.421 & 1 & 1 \\
\hline Lead (PMS) & 0.000653 & 0.00567 & $<$ & 0.000902 & $<$ & 0.00064 & $<$ & $<$ \\
\hline Lead & & & & & & & & \\
\hline Lithium & 0.0313 & 0.0321 & 0.0292 & $<$ & $<$ & $<$ & $<$ & $<$ \\
\hline Manganese & 0.131 & 0.13 & 0.363 & 0.0829 & 0.017 & 0.0355 & 0.047 & 0.0471 \\
\hline Mercury (CVAA) & & $<$ & $<$ & $<$ & $<$ & $<$ & $<$ & $<$ \\
\hline Nickel (PMS) & 0.705 & 0.786 & 0.301 & $<$ & $<$ & $<$ & $<$ & $<$ \\
\hline Nickel & & & & & & & & \\
\hline Strontium & 0.816 & 0.815 & 0.736 & 0.0838 & 0.0949 & 0.0416 & 0.0372 & 0.0377 \\
\hline Thallium (PMS) & $<$ & $<$ & $<$ & $<$ & $<$ & $<$ & $<$ & $<$ \\
\hline Thallium & & & & & & & & \\
\hline Uranium (PMS) & 0.000742 & 0.000703 & 0.000914 & $<$ & $<$ & $<$ & $<$ & $<$ \\
\hline Uranium (KPA) & & & & & & & & \\
\hline Vanadium & $<$ & $<$ & $<$ & $<$ & $<$ & $<$ & $<$ & $<$ \\
\hline Zinc & $<$ & $<$ & $<$ & $<$ & $<$ & $<$ & $<$ & $<$ \\
\hline
\end{tabular}


APPENDIX E.1: CY 2002 MONITORING DATA FOR THE UPPER EAST FORK POPLAR CREEK HYDROGEOLOGIC REGIME

Field Measurements, Miscellaneous Analytes, Major Ions, and Trace Metals

\begin{tabular}{|c|c|c|c|c|c|c|c|c|}
\hline Sampling Point & \multicolumn{2}{|c|}{ GW-108 } & \multicolumn{2}{|c|}{ GW-151 } & \multicolumn{2}{|c|}{ GW-153 } & \multicolumn{2}{|c|}{ GW-154 } \\
\hline Location & \multicolumn{2}{|c|}{ S3 } & \multicolumn{2}{|c|}{ NHP } & \multicolumn{2}{|c|}{ NHP } & \multicolumn{2}{|c|}{ NHP } \\
\hline Date Sampled & $01 / 08 / 02$ & 07/09/02 & $01 / 30 / 02$ & $08 / 05 / 02$ & $04 / 25 / 02$ & $10 / 21 / 02$ & $01 / 30 / 02$ & $08 / 05 / 02$ \\
\hline Program & BJC & BJC & BJC & BJC & GWPP & GWPP & BJC & BJC \\
\hline \multicolumn{9}{|l|}{ Sample Type } \\
\hline \multicolumn{9}{|l|}{ Field Measurements } \\
\hline Time Sampled & $15: 05$ & $13: 10$ & $10: 10$ & $14: 30$ & $8: 45$ & $8: 55$ & $14: 17$ & $14: 29$ \\
\hline Measuring Point Elev. (ft) & 999.00 & 999.00 & 916.17 & 916.17 & 921.68 & 921.68 & 911.70 & 911.70 \\
\hline Depth to Water (ft) & 8.05 & 7.91 & 14.93 & 16.04 & 20.43 & 20.63 & 8.16 & 9.97 \\
\hline Groundwater Elevation (ft) & 990.95 & 991.09 & 901.24 & 900.13 & 901.25 & 901.05 & 903.54 & 901.73 \\
\hline Conductivity ( $\mu \mathrm{mho} / \mathrm{cm})$ & 46,600 & 48,300 & 1,063 & 526 & 455 & 450 & 811 & 806 \\
\hline Dissolved Oxygen (ppm) & 3.41 & 1.94 & 0.23 & 1 & 2.41 & 1.55 & 10.38 & 4.45 \\
\hline Oxidation/Reduction (mV) & 355 & 238 & 246 & -20 & 176 & 177 & 223 & 203 \\
\hline Temperature (degrees C) & 15.6 & 21.8 & 15 & 19.4 & 14.3 & 15.7 & 14.3 & 24.8 \\
\hline Turbidity (NTU) & 28 & 10 & 16 & 21 & & & 39 & 46 \\
\hline Iron ++ (mg/L) & & & 0.02 & 0.01 & & & 0.02 & 0.28 \\
\hline Manganese ++ (mg/L) & & & 0.2 & 0.1 & & & 0.3 & 0.6 \\
\hline $\mathrm{pH}$ & 5.34 & 5.5 & 6.79 & 4.93 & 7.55 & 7.71 & 7.46 & 7.08 \\
\hline \multicolumn{9}{|l|}{ Miscellaneous Analytes } \\
\hline Conductivity $(\mu \mathrm{mho} / \mathrm{cm})$ & & & & & 396 & 395 & & \\
\hline Dissolved Solids (mg/L) & 57,700 & 69,200 & 285 & 323 & 220 & 232 & 495 & 568 \\
\hline Suspended Solids (mg/L) & 18.3 & 34.9 & $<$ & $<$ & $<$ & $<$ & $<$ & 8.5 \\
\hline Turbidity (NTU) & & & & & 3.04 & 2.37 & & \\
\hline $\mathrm{pH}$ & & & & & 7.8 & 7.77 & & \\
\hline \multicolumn{9}{|l|}{ Major lons (mg/L) } \\
\hline Calcium & 11,600 & 11,800 & 55.3 & 58.9 & 44.9 & 41.7 & 120 & 146 \\
\hline Magnesium & 999 & 1,160 & 25.4 & 27.6 & 19.1 & 18.5 & 21.2 & 22.5 \\
\hline Potassium & 23.9 & 30.5 & 2.45 & 2.77 & 2.06 & $<$ & 7.11 & 8.13 \\
\hline Sodium & 479 & 554 & 7.25 & 7.46 & 10 & 10.3 & 18.1 & 13.6 \\
\hline Alkalinity as $\mathrm{CO} 3$ & $<$ & $<$ & $<$ & $<$ & $<$ & $<$ & $<$ & $<$ \\
\hline Alkalinity as $\mathrm{HCO} 3$ & 280 & 689 & 230 & 218 & 169 & 159 & 294 & 360 \\
\hline Chloride & 193 & 77.9 & 16.2 & 17.1 & 15.7 & 16.6 & 22.9 & 7.5 \\
\hline Fluoride & $<$ & & $<$ & 0.13 & 0.163 & 0.179 & 0.18 & 0.34 \\
\hline Nitrate as $\mathrm{N}$ & 9,360 & 9,280 & 1.1 & 1.2 & 0.753 & 0.968 & 1.4 & 0.27 \\
\hline Sulfate & $<$ & $<$ & 18.3 & 18.2 & 13.8 & 15.8 & 101 & 84.4 \\
\hline Charge balance & 0.2 & 2.2 & -2.7 & 2.8 & 1.5 & 0.0 & -0.2 & 3.8 \\
\hline \multicolumn{9}{|l|}{ Trace Metals (mg/L) } \\
\hline Aluminum & $<$ & $<$ & $<$ & $<$ & $<$ & $<$ & $<$ & 0.368 \\
\hline Arsenic (PMS) & & & & & $<$ & $<$ & & \\
\hline Arsenic & $<$ & $<$ & $<$ & $<$ & & & $<$ & $<$ \\
\hline Barium & 86.9 & 86.8 & 0.178 & 0.184 & 0.0423 & 0.0413 & 0.0915 & 0.145 \\
\hline Beryllium & $<$ & $<$ & $<$ & $<$ & $<$ & $<$ & $<$ & $<$ \\
\hline Boron & $<$ & $<$ & $<$ & $<$ & $<$ & $<$ & $<$ & 0.131 \\
\hline Cadmium (PMS) & & & & & $<$ & $<$ & & \\
\hline Cadmium & $<$ & $<$ & $<$ & $<$ & & & $<$ & $<$ \\
\hline Chromium (PMS) & & & & & 0.00283 & 0.00375 & & \\
\hline Chromium & $<$ & $<$ & $<$ & $<$ & & & $<$ & $<$ \\
\hline Cobalt & 0.13 & 0.156 & $<$ & $<$ & $<$ & $<$ & $<$ & $<$ \\
\hline Copper & $<$ & $<$ & $<$ & $<$ & $<$ & $<$ & $<$ & $<$ \\
\hline Iron & $<$ & $<$ & $<$ & $<$ & $<$ & $<$ & 0.237 & 0.659 \\
\hline Lead (PMS) & & & 1 & & $<$ & $<$ & & \\
\hline Lead & $<$ & $<$ & $<$ & $<$ & & . & $<$ & 0.0049 \\
\hline Lithium & 0.553 & $<$ & $<$ & $<$ & $<$ & $<$ & $<$ & $<$ \\
\hline Manganese & 107 & 150 & $<$ & $<$ & $<$ & $<$ & 0.911 & 2.43 \\
\hline Mercury (CVAA) & $<$ & $<$ &. & & $<$ & $<$ & & \\
\hline Nickel (PMS) & & & & & $<$ & $<$ & & \\
\hline Nickel & 0.172 & 0.176 & $<$ & $<$ & & & $<$ & $<$ \\
\hline Strontium & 31 & 30.8 & 0.544 & 0.552 & 0.161 & 0.154 & 0.45 & 0.499 \\
\hline Thallium (PMS) & & & & & & $<$ & & \\
\hline Thallium & $<$ & $<$ & $<$ & $<$ & & & $<$ & $<$ \\
\hline Uranium (PMS) & & & & & 0.0013 & 0.00138 & & \\
\hline Uranium (KPA) & {$[0.0181]$} & {$[0.0157]$} & $<$ & $<$ & & & 0.853 & 0.884 \\
\hline Vanadium & $<$ & $<$ & $<$ & $<$ & $<$ & $<$ & $<$ & $<$ \\
\hline Zinc & $<$ & $<$ & $<$ & $<$ & $<$ & $<$ & $<$ & $<$ \\
\hline
\end{tabular}


APPENDIX E.1: CY 2002 MONITORING DATA FOR THE UPPER EAST FORK POPLAR CREEK HYDROGEOLOGIC REGIME

Field Measurements, Miscellaneous Analytes, Major lons, and Trace Metals

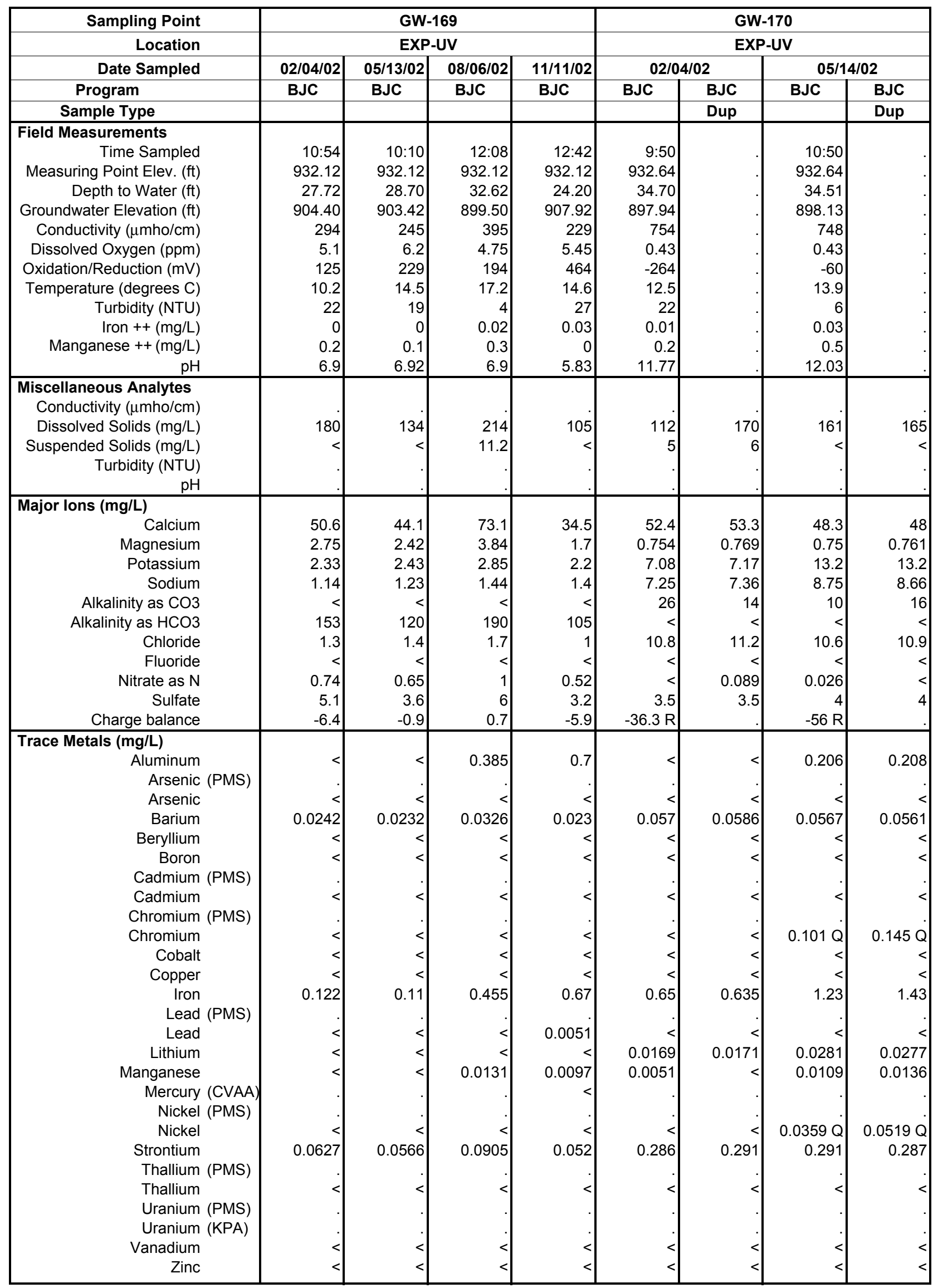


APPENDIX E.1: CY 2002 MONITORING DATA FOR THE UPPER EAST FORK POPLAR CREEK HYDROGEOLOGIC REGIME

Field Measurements, Miscellaneous Analytes, Major Ions, and Trace Metals

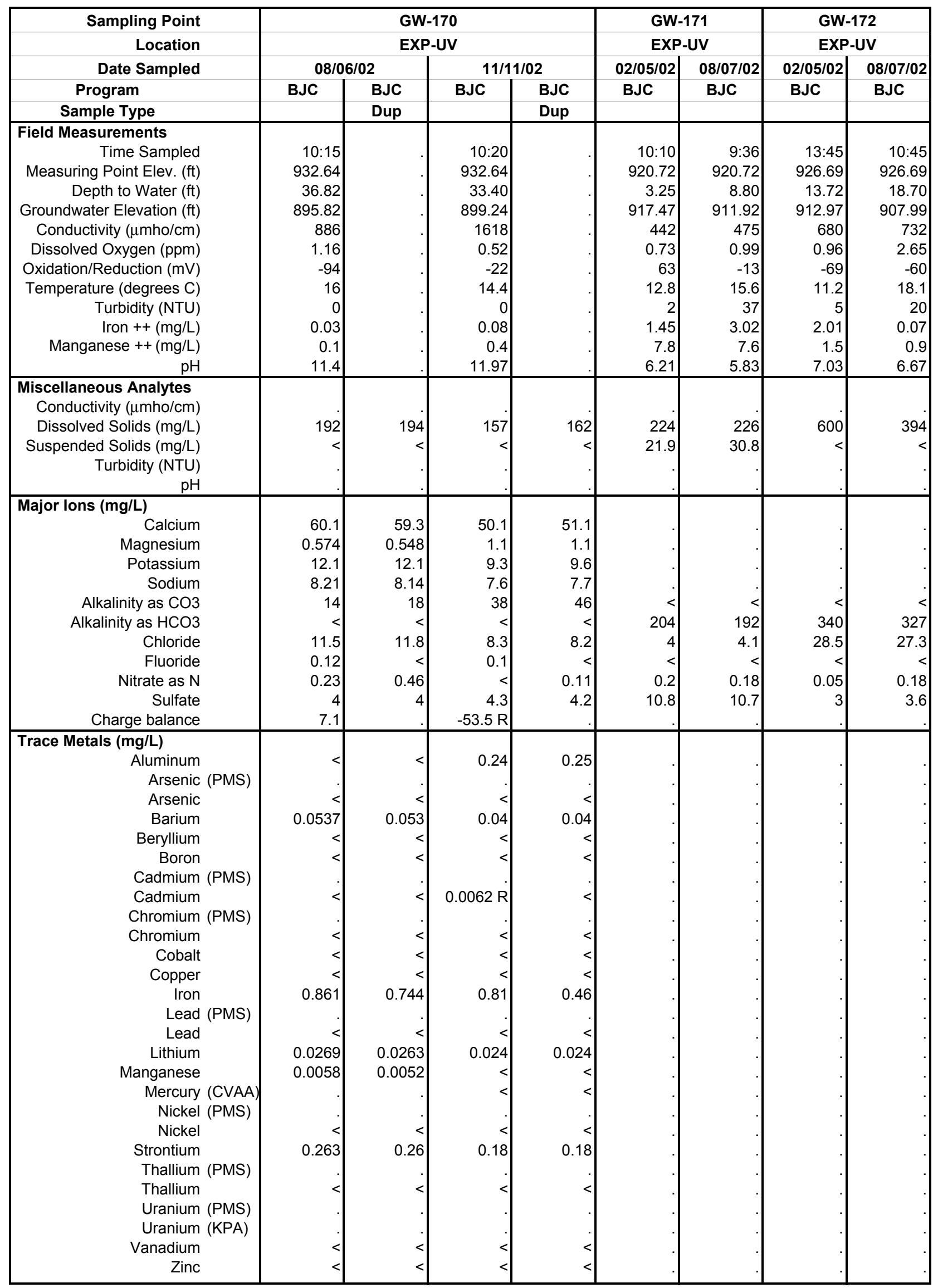


APPENDIX E.1: CY 2002 MONITORING DATA FOR THE UPPER EAST FORK POPLAR CREEK HYDROGEOLOGIC REGIME

Field Measurements, Miscellaneous Analytes, Major lons, and Trace Metals

\begin{tabular}{|c|c|c|c|c|c|c|c|c|}
\hline Sampling Point & \multicolumn{2}{|c|}{ GW-193 } & \multicolumn{2}{|c|}{ GW-204 } & \multicolumn{3}{|c|}{ GW-207 } & GW-208 \\
\hline Location & \multicolumn{2}{|c|}{ T2331 } & \multicolumn{2}{|c|}{ T0134 } & \multicolumn{3}{|c|}{ EXP-SR } & EXP-SR \\
\hline Date Sampled & 01/08/02 & $07 / 09 / 02$ & 05/02/02 & $11 / 06 / 02$ & $05 / 08 / 02$ & \multicolumn{2}{|c|}{$11 / 19 / 02$} & 05/08/02 \\
\hline Program & BJC & BJC & GWPP & GWPP & GWPP & GWPP & GWPP & GWPP \\
\hline Sample Type & & & & & & & Dup & \\
\hline \multicolumn{9}{|l|}{ Field Measurements } \\
\hline Time Sampled & $11: 20$ & $9: 40$ & $10: 25$ & $9: 30$ & $8: 35$ & $10: 55$ & $10: 55$ & $7: 45$ \\
\hline Measuring Point Elev. (ft) & 934.17 & 934.17 & 958.57 & 958.57 & 899.40 & 899.40 & 899.40 & 898.05 \\
\hline Depth to Water (ft) & 8.67 & 9.61 & 8.53 & 8.36 & 1.39 & 3.47 & 3.47 & 1.36 \\
\hline Groundwater Elevation (ft) & 925.50 & 924.56 & 950.04 & 950.21 & 898.01 & 895.93 & 895.93 & 896.69 \\
\hline Conductivity $(\mu \mathrm{mho} / \mathrm{cm})$ & 817 & 606 & 389 & 356 & 666 & 734 & 734 & 674 \\
\hline Dissolved Oxygen (ppm) & 2.09 & 1.03 & 0.79 & 0.82 & 2.39 & 1.63 & 1.63 & 2.79 \\
\hline Oxidation/Reduction (mV) & -214 & -186 & 19 & -221 & -65 & -69 & -69 & -161 \\
\hline Temperature (degrees $\mathrm{C}$ ) & 12.3 & 23.8 & 22.1 & 20.8 & 16.3 & 14.7 & 14.7 & 16 \\
\hline Turbidity (NTU) & 28 & 12 & & & & & & \\
\hline \multirow{2}{*}{\multicolumn{9}{|c|}{$\begin{array}{r}\text { Iron }++(\mathrm{mg} / \mathrm{L}) \\
\text { Manganese }++(\mathrm{mg} / \mathrm{L})\end{array}$}} \\
\hline & & & & & & & & \\
\hline $\mathrm{pH}$ & 7.75 & 7.04 & 7.58 & 7.21 & 7.27 & 7.29 & 7.29 & 7.79 \\
\hline \multicolumn{9}{|l|}{ Miscellaneous Analytes } \\
\hline Conductivity $(\mu \mathrm{mho} / \mathrm{cm})$ & & & 337 & 287 & 600 & 600 & 602 & 609 \\
\hline Dissolved Solids (mg/L) & 551 & 363 & 191 & 159 & 348 & 360 & 358 & 383 \\
\hline Suspended Solids (mg/L) & $<$ & $<$ & $<$ & 4 & $<$ & $<$ & $<$ & 3 \\
\hline Turbidity (NTU) & & & 0.434 & 3.58 & 5.6 & 3.28 & 3.33 & 14.1 \\
\hline $\mathrm{pH}$ & & & 7.77 & 7.65 & 7.78 & 7.82 & 7.81 & 8.03 \\
\hline \multicolumn{9}{|l|}{ Major lons (mg/L) } \\
\hline Calcium & 104 & 81.1 & 50.8 & 46.5 & 59.8 & 60.9 & 60.1 & 72.9 \\
\hline Magnesium & 34.1 & 22.7 & 8.86 & 6.55 & 39.1 & 40.6 & 40.3 & 26.3 \\
\hline Potassium & 8.9 & 8.07 & 2.33 & $<$ & 3.11 & 3.25 & 3.12 & 3.36 \\
\hline Sodium & 5.41 & 4.82 & 3.34 & 1.64 & 10.4 & 10.8 & 10.6 & 16.5 \\
\hline Alkalinity as $\mathrm{CO} 3$ & $<$ & $<$ & $<$ & $<$ & $<$ & $<$ & $<$ & $<$ \\
\hline Alkalinity as $\mathrm{HCO} 3$ & 181 & 236 & 135 & 142 & 278 & 288 & 280 & 214 \\
\hline Chloride & 3.7 & 4.5 & 2.41 & 0.937 & 1.38 & 1.33 & 1.31 & 1.94 \\
\hline Fluoride & 0.54 & 0.8 & 0.682 & 0.946 & 0.176 & 0.192 & 0.197 & 0.272 \\
\hline Nitrate as $\mathrm{N}$ & $<$ & $<$ & 0.577 & $<$ & & $<$ & $<$ & $<$ \\
\hline Sulfate & 243 & 61.5 & 32.7 & 4.22 & 56.8 & 54.5 & 54.7 & 116 \\
\hline Charge balance & -2.1 & 1.3 & -0.8 & -1.2 & -0.4 & 0.0 & 0.5 & -1.2 \\
\hline \multicolumn{9}{|l|}{ Trace Metals (mg/L) } \\
\hline Aluminum & $<$ & $<$ & $<$ & $<$ & $<$ & $<$ & $<$ & $<$ \\
\hline Arsenic (PMS) & & & $<$ & $<$ & $<$ & $<$ & $<$ & $<$ \\
\hline Arsenic & $<$ & $<$ & & & & & & \\
\hline Barium & 0.121 & 0.0854 & 0.0594 & 0.0713 & 0.0508 & 0.0542 & 0.0532 & 0.0386 \\
\hline Beryllium & $<$ & $<$ & $<$ & $<$ & & $<$ & $<$ & $<$ \\
\hline Boron & $<$ & $<$ & $<$ & $<$ & 0.119 & 0.119 & 0.118 & 0.233 \\
\hline Cadmium (PMS) & & & $<$ & $<$ & $<$ & $<$ & $<$ & $<$ \\
\hline Cadmium & $<$ & $<$ & & & & & & . \\
\hline Chromium (PMS) & & & $<$ & $<$ & $<$ & $<$ & $<$ & $<$ \\
\hline Chromium & $<$ & $<$ & . & &. & & & . \\
\hline Cobalt & $<$ & $<$ & $<$ & $<$ & $<$ & $<$ & $<$ & $<$ \\
\hline Copper & $<$ & $<$ & $<$ & $<$ & $<$ & $<$ & $<$ & $<$ \\
\hline Iron & $<$ & $<$ & $<$ & 0.752 & 0.767 & 0.524 & 0.465 & 1.31 \\
\hline Lead (PMS) & . &. & 0.000533 & $<$ & 0.00145 & 0.013 & 0.00698 & 0.00351 \\
\hline Lead & $<$ & $<$ & & & & & & \\
\hline Lithium & $<$ & $<$ & 0.121 & 0.052 & 0.0321 & 0.0334 & 0.0329 & 0.0332 \\
\hline Manganese & 0.213 & 0.292 & 0.0463 & 2.86 & 0.0132 & 0.0183 & 0.0171 & 0.0169 \\
\hline Mercury (CVAA) & $<$ & $<$ & $<$ & $<$ & $<$ & $<$ & $<$ & $<$ \\
\hline Nickel (PMS) & & & $<$ & $<$ & $<$ & $<$ & $<$ & $<$ \\
\hline Nickel & $<$ & $<$ & & & & & & \\
\hline Strontium & 0.629 & 0.454 & 0.13 & 0.119 & 0.545 & 0.57 & 0.56 & 1.28 \\
\hline Thallium (PMS) & & & $<$ & $<$ & $<$ & $<$ & $<$ & $<$ \\
\hline Thallium & $<$ & $<$ & & & & & & . \\
\hline Uranium (PMS) & & & 0.0439 & 0.0336 & $<$ & $<$ & $<$ & $<$ \\
\hline Uranium (KPA) & $<$ & $<$ & & & & & & \\
\hline Vanadium & $<$ & $<$ & $<$ & $<$ & $<$ & $<$ & $<$ & $<$ \\
\hline Zinc & $<$ & $<$ & $<$ & $<$ & $<$ & $<$ & $<$ & 1.73 \\
\hline
\end{tabular}


APPENDIX E.1: CY 2002 MONITORING DATA FOR THE UPPER EAST FORK POPLAR CREEK HYDROGEOLOGIC REGIME

Field Measurements, Miscellaneous Analytes, Major Ions, and Trace Metals

\begin{tabular}{|c|c|c|c|c|c|c|c|c|}
\hline Sampling Point & GW-208 & \multicolumn{3}{|c|}{ GW-219 } & \multicolumn{2}{|c|}{ GW-220 } & \multicolumn{2}{|c|}{ GW-223 } \\
\hline Location & EXP-SR & \multicolumn{3}{|c|}{ UOV } & \multicolumn{2}{|c|}{ NHP } & \multicolumn{2}{|c|}{ NHP } \\
\hline Date Sampled & $11 / 19 / 02$ & $05 / 06 / 02$ & \multicolumn{2}{|c|}{$11 / 07 / 02$} & $05 / 14 / 02$ & $11 / 21 / 02$ & $01 / 31 / 02$ & $08 / 05 / 02$ \\
\hline Program & GWPP & GWPP & GWPP & GWPP & GWPP & GWPP & BJC & BJC \\
\hline Sample Type & & & & Dup & & & & \\
\hline Field Measurements & & & & & & & & \\
\hline Time Sampled & $10: 10$ & 9:00 & $9: 55$ & $9: 55$ & $9: 55$ & $11: 05$ & $14: 10$ & $10: 35$ \\
\hline Measuring Point Elev. (ft) & 898.05 & 935.64 & 935.64 & 935.64 & 916.47 & 916.47 & 911.62 & 911.62 \\
\hline Depth to Water (ft) & 3.75 & 9.48 & 9.37 & 9.37 & 16.25 & 16.20 & 9.22 & 10.36 \\
\hline Groundwater Elevation (ft) & 894.30 & 926.16 & 926.27 & 926.27 & 900.22 & 900.27 & 902.40 & 901.26 \\
\hline Conductivity $(\mu \mathrm{mho} / \mathrm{cm})$ & 727 & 526 & 762 & 762 & 544 & 588 & 659 & 668 \\
\hline Dissolved Oxygen (ppm) & 0.7 & 3.13 & 1.6 & 1.6 & 0.44 & -0.03 & 9.67 & 1.08 \\
\hline Oxidation/Reduction (mV) & -147 & 108 & 95 & 95 & 56 & 81 & -2 & 2 \\
\hline Temperature (degrees C) & 15.2 & 16.6 & 18.2 & 18.2 & 15.5 & 15.6 & 17.3 & 20 \\
\hline Turbidity (NTU) & & & & & & & 1 & 26 \\
\hline Iron ++ (mg/L) & & & & & & & 0.32 & 0.11 \\
\hline Manganese ++ (mg/L) & & & & & & & 0.6 & 0.8 \\
\hline $\mathrm{pH}$ & 7.72 & 7.1 & 7.38 & 7.38 & 7.68 & 7.72 & 6.86 & 7.02 \\
\hline Miscellaneous Analytes & & & & & & & & \\
\hline Conductivity $(\mu \mathrm{mho} / \mathrm{cm})$ & 597 & 450 & 630 & 627 & 498 & 492 & & \\
\hline Dissolved Solids (mg/L) & 381 & 277 & 369 & 374 & 304 & 280 & 405 & 402 \\
\hline Suspended Solids (mg/L) & 2.8 & $<$ & $<$ & $<$ & $<$ & 10 & $<$ & $<$ \\
\hline Turbidity (NTU) & 11.3 & 1.62 & 0.794 & 0.797 & 1.54 & 8.08 & & \\
\hline $\mathrm{pH}$ & 8.06 & 7.27 & 7.16 & 7.23 & 7.77 & 7.78 & & \\
\hline Major lons (mg/L) & & & & & & & & \\
\hline Calcium & 76.2 & 71.5 & 107 & 105 & 59.3 & 59 & 102 & 104 \\
\hline Magnesium & 29.2 & 8.97 & 12 & 12.5 & 25.3 & 26.6 & 13 & 13.6 \\
\hline Potassium & 3.82 & 4.01 & 4.8 & 4.97 & 2.48 & 2.64 & 2.27 & 2.32 \\
\hline Sodium & 18 & 11.2 & 15.4 & 15.6 & 4.92 & 4.93 & 12.7 & 12.4 \\
\hline Alkalinity as $\mathrm{CO} 3$ & $<$ & $<$ & $<$ & $<$ & $<$ & $<$ & $<$ & $<$ \\
\hline Alkalinity as $\mathrm{HCO} 3$ & 202 & 218 & 298 & 294 & 230 & 232 & 306 & 271 \\
\hline Chloride & 1.65 & 3.76 & 3.13 & 3.89 & 13.7 & 12.9 & 27.8 & 26.8 \\
\hline Fluoride & 0.275 & $<$ & 0.12 & 0.117 & $<$ & $<$ & $<$ & 0.1 \\
\hline Nitrate as $\mathrm{N}$ & $<$ & $<$ & 0.426 & 0.425 & 0.852 & 0.744 & $<$ & 0.038 \\
\hline Sulfate & 114 & 21.1 & 37.7 & 37.4 & 16.1 & 14.4 & 40.3 & 35.7 \\
\hline Charge balance & 4.5 & -0.1 & 1.8 & 2.0 & -0.6 & 0.6 & -6.7 & -0.1 \\
\hline Trace Metals (mg/L) & & & & & & & & \\
\hline Aluminum & $<$ & $<$ & $<$ & $<$ & $<$ & 0.45 & $<$ & $<$ \\
\hline Arsenic (PMS) & $<$ & $<$ & $<$ & $<$ & $<$ & $<$ & & \\
\hline Arsenic & & & & & & & $<$ & $<$ \\
\hline Barium & 0.0409 & 0.0574 & 0.0904 & 0.0917 & 0.104 & 0.11 & 0.293 & 0.295 \\
\hline Beryllium & & $<$ & $<$ & $<$ & $<$ & $<$ & $<$ & $<$ \\
\hline Boron & 0.253 & $<$ & $<$ & $<$ & $<$ & $<$ & $<$ & $<$ \\
\hline Cadmium (PMS) & $<$ & $<$ & $<$ & $<$ & $<$ & $<$ & & \\
\hline Cadmium & & & & & & & $<$ & $<$ \\
\hline Chromium (PMS) & $<$ & 0.00411 & 0.00271 & 0.00279 & $<$ & $<$ & & \\
\hline Chromium & & & & & & & $<$ & $<$ \\
\hline Cobalt & $<$ & $<$ & $<$ & $<$ & $<$ & $<$ & $<$ & $<$ \\
\hline Copper & $<$ & $<$ & $<$ & $<$ & $<$ & $<$ & $<$ & $<$ \\
\hline Iron & 1.27 & $<$ & 0.053 & 0.0616 & 0.0955 & 0.31 & 0.382 & 0.396 \\
\hline Lead (PMS) & 0.0123 & $<$ & $0.000537 \mathrm{R}$ & $0.0139 \mathrm{R}$ & $<$ & 0.000753 & & \\
\hline Lead & & & & & & & $<$ & $<$ \\
\hline Lithium & 0.0364 & $<$ & $<$ & $<$ & $<$ & $<$ & $<$ & $<$ \\
\hline Manganese & 0.0184 & 0.00969 & 0.0117 & 0.00912 & $<$ & 0.012 & 0.704 & 0.71 \\
\hline Mercury (CVAA) & $<$ & $<$ & $<$ & $<$ & $<$ & $<$ & & \\
\hline Nickel (PMS) & $<$ & $<$ & 0.0707 & 0.0719 & $<$ & $<$ & & \\
\hline Nickel & & & & & & & $<$ & $<$ \\
\hline Strontium & 1.33 & 0.157 & 0.215 & 0.219 & 0.435 & 0.444 & 0.273 & 0.277 \\
\hline Thallium (PMS) & $<$ & $<$ & & $<$ & $<$ & $<$ & & \\
\hline Thallium & & & & & & & $<$ & $<$ \\
\hline Uranium (PMS) & $<$ & 0.359 & 0.594 & 0.592 & $<$ & $<$ & & \\
\hline Uranium (KPA) & & & & & & & 0.0343 & 0.0305 \\
\hline Vanadium & $<$ & $<$ & $<$ & $<$ & $<$ & $<$ & $<$ & $<$ \\
\hline Zinc & 1.77 & $<$ & $<$ & $<$ & $<$ & $<$ & $<$ & $<$ \\
\hline
\end{tabular}


APPENDIX E.1: CY 2002 MONITORING DATA FOR THE UPPER EAST FORK POPLAR CREEK HYDROGEOLOGIC REGIME

Field Measurements, Miscellaneous Analytes, Major Ions, and Trace Metals

\begin{tabular}{|c|c|c|c|c|c|c|c|c|}
\hline Sampling Point & \multicolumn{2}{|c|}{ GW-230 } & \multicolumn{4}{|c|}{ GW-232 } & \multicolumn{2}{|c|}{ GW-251 } \\
\hline Location & \multicolumn{2}{|c|}{ EXP-UV } & \multicolumn{4}{|c|}{ EXP-UV } & \multicolumn{2}{|c|}{ S2 } \\
\hline Date Sampled & $02 / 05 / 02$ & $08 / 07 / 02$ & $02 / 04 / 02$ & $05 / 13 / 02$ & 08/06/02 & $11 / 11 / 02$ & $04 / 18 / 02$ & $10 / 16 / 02$ \\
\hline Program & BJC & BJC & BJC & BJC & BJC & BJC & GWPP & GWPP \\
\hline \multicolumn{9}{|l|}{ Sample Type } \\
\hline Field Measurements & & & & & & & & \\
\hline Time Sampled & $12: 50$ & $13: 55$ & $13: 45$ & $13: 00$ & $13: 35$ & $14: 15$ & $8: 15$ & $9: 40$ \\
\hline Measuring Point Elev. (ft) & 923.11 & 923.11 & 931.39 & 931.39 & 931.39 & 931.39 & $1,003.80$ & $1,003.80$ \\
\hline Depth to Water (ft) & 12.20 & 16.07 & 34.35 & 34.71 & 36.27 & 33.11 & 16.10 & 16.06 \\
\hline Groundwater Elevation (ft) & 910.91 & 907.04 & 897.04 & 896.68 & 895.12 & 898.28 & 987.70 & 987.74 \\
\hline Conductivity ( $\mu \mathrm{mho} / \mathrm{cm})$ & 1,060 & 1,055 & 941 & 937 & 989 & 1,778 & 1,037 & 810 \\
\hline Dissolved Oxygen (ppm) & 0.5 & 1.38 & 0.63 & 1.12 & 0.51 & 0.45 & 1.08 & 0.04 \\
\hline Oxidation/Reduction (mV) & -94 & -122 & -214 & -110 & -162 & 4 & 268 & 268 \\
\hline Temperature (degrees C) & 12.5 & 19.5 & 8.9 & 15.5 & 20.2 & 15.4 & 15 & 15.2 \\
\hline Turbidity (NTU) & 26 & 122 & 22 & 3 & 0 & 4 & & \\
\hline Iron ++ (mg/L) & 3.83 & 1.42 & 0 & 0.02 & 0.01 & 0.02 & & \\
\hline Manganese ++ (mg/L) & 1 & 0.6 & 0.1 & 0 & 0.1 & 0.2 & & \\
\hline $\mathrm{pH}$ & 6.97 & 6.7 & 9.76 & 9.99 & 9.55 & 8.69 & 6.35 & 6.7 \\
\hline \multicolumn{9}{|l|}{ Miscellaneous Analytes } \\
\hline Conductivity $(\mu \mathrm{mho} / \mathrm{cm})$ & & & & & & & 857 & 733 \\
\hline Dissolved Solids (mg/L) & 407 & 641 & 556 & 507 & 595 & 505 & 514 & 482 \\
\hline Suspended Solids (mg/L) & $<$ & 22.4 & $<$ & $<$ & $<$ & $<$ & 2 & 5 \\
\hline Turbidity (NTU) & & & & & & & 3.44 & 10.8 \\
\hline $\mathrm{pH}$ & & & & & & & 6.53 & 6.79 \\
\hline \multicolumn{9}{|l|}{ Major lons (mg/L) } \\
\hline Calcium & & & 1.38 & 1.48 & 1.36 & 1.1 & 110 & 96.5 \\
\hline Magnesium & & & 0.951 & 0.953 & 0.92 & 0.69 & 14.6 & 13 \\
\hline Potassium & & & 6.05 & 5.8 & 5.67 & 5.7 & 3.18 & 2.62 \\
\hline Sodium & & & 188 & 222 & 222 & 184 & 14 & 10.1 \\
\hline Alkalinity as $\mathrm{CO} 3$ & $<$ & $<$ & 192 & 152 & 174 & $184 \mathrm{Q}$ & $<$ & $<$ \\
\hline Alkalinity as $\mathrm{HCO} 3$ & 427 & 405 & 258 & 295 & 458 & $479 Q$ & 164 & 177 \\
\hline Chloride & 107 & 112 & 11.9 & 12.1 & 12.6 & 9.7 & 8.05 & 5.58 \\
\hline Fluoride & & $<$ & 2 & 1.9 & 1.8 & 2.7 & 0.89 & 1.31 \\
\hline Nitrate as $\mathrm{N}$ & 0.024 & 0.2 & $<$ & $<$ & 0.34 & $<$ & 62.4 & 41.2 \\
\hline Sulfate & 1.3 & 1 & 5.3 & 4.6 & 4.1 & 3.2 & 13.6 & 10.9 \\
\hline Charge balance & & & -6.2 & 2.0 & -14.2 & $-24.9 R$ & -5.8 & -4.1 \\
\hline \multicolumn{9}{|l|}{ Trace Metals (mg/L) } \\
\hline Aluminum & & & $<$ & $<$ & $<$ & $<$ & 0.345 & 0.856 \\
\hline Arsenic (PMS) & & & & & & & & $<$ \\
\hline Arsenic & & & $<$ & $<$ & $<$ & $<$ & & \\
\hline Barium & & & 0.0149 & 0.0153 & 0.0151 & 0.013 & 0.112 & 0.0782 \\
\hline Beryllium & & & $<$ & & $<$ & $<$ & $<$ & $<$ \\
\hline Boron & & & 1.31 & 1.31 & 1.33 & 1.1 & & $<$ \\
\hline Cadmium (PMS) & & & & & & & 0.116 & 0.0746 \\
\hline Cadmium & & & $<$ & $<$ & $<$ & 0.0065 & & \\
\hline Chromium (PMS) & & & & & & & $<$ & 0.00282 \\
\hline Chromium & & & $<$ & 0.0729 & $<$ & $<$ & & \\
\hline Cobalt & & & $<$ & & $<$ & $<$ & $<$ & $<$ \\
\hline Copper & & & & & $<$ & $<$ & 0.235 & 0.155 \\
\hline Iron & & & 0.053 & 0.396 & $<$ & 0.44 & 0.221 & 0.571 \\
\hline Lead (PMS) & & & & & . & & 0.00116 & 0.00482 \\
\hline Lead & & & & $<$ & $<$ & $<$ & & \\
\hline Lithium & & & 0.201 & 0.18 & 0.182 & 0.19 & $<$ & $<$ \\
\hline Manganese & & & $<$ & 0.0061 & $<$ & $<$ & 3.6 & 1.7 \\
\hline Mercury (CVAA) & & & & & & $<$ & $<$ & $<$ \\
\hline Nickel (PMS) & & & & & & & 0.0312 & 0.015 \\
\hline Nickel & & & $<$ & 0.03 & $<$ & $<$ & & \\
\hline Strontium & & & 0.408 & 0.404 & 0.411 & 0.35 & 0.191 & 0.137 \\
\hline Thallium (PMS) & & & & & & & 0.00183 & 0.00149 \\
\hline Thallium & & & $<$ & $<$ & $<$ & $<$ & & \\
\hline Uranium (PMS) & & & & & & & 0.00395 & 0.00385 \\
\hline Uranium (KPA) & & & & & & & & \\
\hline Vanadium & & & $<$ & $<$ & $<$ & $<$ & $<$ & $<$ \\
\hline Zinc & & & $<$ & $<$ & $<$ & $<$ & 0.0576 & $<$ \\
\hline
\end{tabular}


APPENDIX E.1: CY 2002 MONITORING DATA FOR THE UPPER EAST FORK POPLAR CREEK HYDROGEOLOGIC REGIME

Field Measurements, Miscellaneous Analytes, Major lons, and Trace Metals

\begin{tabular}{|c|c|c|c|c|c|c|c|c|}
\hline Sampling Point & \multicolumn{2}{|c|}{ GW-253 } & \multicolumn{2}{|c|}{ GW-349 } & \multicolumn{3}{|c|}{ GW-350 } & GW-380 \\
\hline Location & \multicolumn{2}{|c|}{ S2 } & \multicolumn{2}{|c|}{ S2 } & \multicolumn{3}{|c|}{ S2 } & NHP \\
\hline Date Sampled & 05/07/02 & $11 / 07 / 02$ & $04 / 16 / 02$ & $10 / 14 / 02$ & $04 / 16 / 02$ & $10 / 15 / 02$ & $10 / 15 / 02$ & $02 / 04 / 02$ \\
\hline Program & BJC & BJC & GWPP & GWPP & GWPP & GWPP & GWPP & BJC \\
\hline Sample Type & & & & & & & Dup & \\
\hline Field Measurements & & & & & & & & \\
\hline Time Sampled & $9: 55$ & $13: 45$ & $10: 40$ & $10: 30$ & $9: 55$ & $9: 15$ & $9: 15$ & $11: 01$ \\
\hline Measuring Point Elev. (ft) & $1,004.24$ & $1,004.24$ & 993.50 & 993.50 & 993.51 & 993.51 & 993.51 & 913.75 \\
\hline Depth to Water (ft) & 4.42 & 2.98 & 4.44 & 4.50 & 4.35 & 4.50 & 4.50 & 9.92 \\
\hline Groundwater Elevation (ft) & 999.82 & $1,001.26$ & 989.06 & 989.00 & 989.16 & 989.01 & 989.01 & 903.83 \\
\hline Conductivity $(\mu \mathrm{mho} / \mathrm{cm})$ & 9,110 & 9,900 & 564 & 471 & 576 & 606 & 606 & 773 \\
\hline Dissolved Oxygen (ppm) & 7.18 & 0.45 & 1.28 & 0.03 & 1.97 & 0.03 & 0.03 & 13.87 \\
\hline Oxidation/Reduction (mV) & 232 & 285 & 27 & 15 & -11 & -15 & -15 & 223 \\
\hline Temperature (degrees $\mathrm{C}$ ) & 16.1 & 16.9 & 18.6 & 21.3 & 18.5 & 19.6 & 19.6 & 13.2 \\
\hline Turbidity (NTU) & 13 & 34 & & & & & & 47 \\
\hline Iron ++ (mg/L) & 0.07 & 0.2 & & & & & & \\
\hline Manganese ++ (mg/L) & 28 & 31 & & & & & & \\
\hline $\mathrm{pH}$ & 5.59 & 5.51 & 6.68 & 6.35 & 6.92 & 6.61 & 6.61 & 6.82 \\
\hline Miscellaneous Analytes & & & & & & & & \\
\hline Conductivity $(\mu \mathrm{mho} / \mathrm{cm})$ & & & 518 & 430 & 515 & 540 & 541 & \\
\hline Dissolved Solids (mg/L) & 3,990 & 4,990 & 289 & 241 & 302 & 306 & 303 & 415 \\
\hline Suspended Solids (mg/L) & $<$ & $<$ & $<$ & $<$ & $<$ & $<$ & $<$ & $<$ \\
\hline Turbidity (NTU) & & & 3.34 & 2.29 & 7.88 & 2.91 & 2.97 & \\
\hline $\mathrm{pH}$ & & & 6.83 & 6.66 & 7.06 & 7.01 & 7.05 & \\
\hline Major lons (mg/L) & & & & & & & & \\
\hline Calcium & 585 & 566 & 92 & 73.5 & 96.9 & 99 & 99.2 & 50.4 \\
\hline Magnesium & 133 & 101 & 10 & 7.22 & 7.24 & 7.48 & 7.25 & 21.2 \\
\hline Potassium & 9.05 & 14 & 2.68 & 2.72 & 2.99 & 2.98 & 2.99 & 1.56 \\
\hline Sodium & 147 & 150 & 4.83 & 4.26 & 3.95 & 4.05 & 4 & 74.4 \\
\hline Alkalinity as $\mathrm{CO} 3$ & $<$ & $<$ & $<$ & $<$ & $<$ & $<$ & $<$ & $<$ \\
\hline Alkalinity as $\mathrm{HCO} 3$ & 62.9 & 71 & 258 & 202 & 252 & 290 & 264 & 165 \\
\hline Chloride & 127 & 118 & 11.3 & 9.3 & 8.52 & 7.73 & 7.94 & 143 \\
\hline Fluoride & 4.7 & 0.9 & $<$ & $<$ & $<$ & $<$ & $<$ & $<$ \\
\hline Nitrate as $\mathrm{N}$ & 852 & $3,100 \mathrm{Q}$ & $<$ & $<$ & $<$ & $<$ & $<$ & 1.4 \\
\hline Sulfate & 76.5 & 65.3 & 9.02 & 13.1 & 14.1 & 14.7 & 14.9 & 13.1 \\
\hline Charge balance & -18.2 & $-67.9 \mathrm{R}$ & 0.3 & -0.6 & 1.0 & -4.2 & -0.1 & -1.1 \\
\hline Trace Metals (mg/L) & & & & & & & & \\
\hline Aluminum & 4.2 & 4 & $<$ & $<$ & $<$ & $<$ & $<$ & $<$ \\
\hline Arsenic (PMS) & & & $<$ & $<$ & $<$ & $<$ & $<$ & 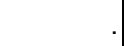 \\
\hline Arsenic & $<$ & 0.0062 & & & & & & $<$ \\
\hline Barium & 0.325 & 0.29 & 0.116 & 0.0855 & 0.0377 & 0.0406 & 0.0397 & 0.0396 \\
\hline Beryllium & 0.0122 & 0.011 & $<$ & $<$ & $<$ & $<$ & $<$ & $<$ \\
\hline Boron & 0.331 & 0.3 & $<$ & $<$ & $<$ & $<$ & $<$ & $<$ \\
\hline Cadmium (PMS) & & & 0.000753 & $<$ & 0.00126 & 0.00063 & 0.000609 & \\
\hline Cadmium & 4.35 & 4.8 & & & & & & $<$ \\
\hline Chromium (PMS) & & & $<$ & $<$ & $<$ & $<$ & $<$ & \\
\hline Chromium & & $<$ & . & & 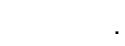 & 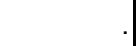 & & 0.405 \\
\hline Cobalt & 0.309 & 0.26 & $<$ & $<$ & $<$ & $<$ & $<$ & $<$ \\
\hline Copper & 68.7 & 77.4 & $<$ & $<$ & $<$ & $<$ & $<$ & $<$ \\
\hline Iron & $<$ & $<$ & 0.412 & 0.526 & 0.567 & 0.502 & 0.459 & 1.16 \\
\hline Lead (PMS) & & & 0.000782 & $<$ & 0.00205 & $0.0005 \mathrm{R}$ & $0.00908 \mathrm{R}$ & \\
\hline Lead & 0.031 & 0.13 & & & & & & $<$ \\
\hline Lithium & 0.0745 & 0.085 & $<$ & $<$ & $<$ & $<$ & $<$ & $<$ \\
\hline Manganese & 52.8 & 54 & 3.34 & 1.32 & 0.471 & 0.557 & 0.538 & 0.0482 \\
\hline Mercury (CVAA) & & 0.0091 & $<$ & $<$ & $<$ & $<$ & $<$ & \\
\hline Nickel (PMS) & & & $<$ & $<$ & $<$ & $<$ & $<$ & \\
\hline Nickel & 2.46 & 2.1 & & & & & & 0.153 \\
\hline Strontium & 1.1 & 1.1 & 0.131 & 0.102 & 0.113 & 0.12 & 0.117 & 0.0589 \\
\hline Thallium (PMS) & & & $<$ & $<$ & $<$ & $<$ & $<$ & \\
\hline Thallium & 0.0172 & 0.04 & ${ }^{\circ}$ & & & 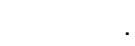 & & $<$ \\
\hline Uranium (PMS) & & & $<$ & $<$ & $<$ & $<$ & $<$ & \\
\hline Uranium (KPA) & $<$ & $<$ & & & & 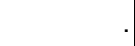 & & $<$ \\
\hline Vanadium & $<$ & $<$ & $<$ & $<$ & $<$ & $<$ & $<$ & $<$ \\
\hline Zinc & 6.29 & 7 & $<$ & $<$ & $<$ & $<$ & $<$ & $<$ \\
\hline
\end{tabular}


APPENDIX E.1: CY 2002 MONITORING DATA FOR THE UPPER EAST FORK POPLAR CREEK HYDROGEOLOGIC REGIME

Field Measurements, Miscellaneous Analytes, Major Ions, and Trace Metals

\begin{tabular}{|c|c|c|c|c|c|c|c|c|}
\hline Sampling Point & GW-380 & \multicolumn{3}{|c|}{ GW-381 } & \multicolumn{2}{|c|}{ GW-382 } & \multicolumn{2}{|c|}{ GW-383 } \\
\hline Location & NHP & \multicolumn{3}{|c|}{ NHP } & \multicolumn{2}{|c|}{ NHP } & \multicolumn{2}{|c|}{ NHP } \\
\hline Date Sampled & $08 / 05 / 02$ & $04 / 24 / 02$ & $06 / 12 / 02$ & $10 / 22 / 02$ & $02 / 04 / 02$ & $08 / 06 / 02$ & $04 / 25 / 02$ & $10 / 22 / 02$ \\
\hline Program & BJC & GWPP & GWPP & GWPP & BJC & BJC & GWPP & GWPP \\
\hline \multicolumn{9}{|l|}{ Sample Type } \\
\hline \multicolumn{9}{|l|}{ Field Measurements } \\
\hline Time Sampled & $9: 53$ & $10: 10$ & $9: 00$ & $10: 00$ & $13: 50$ & $9: 55$ & $10: 20$ & $8: 45$ \\
\hline Measuring Point Elev. (ft) & 913.75 & 913.36 & 913.36 & 913.36 & 913.17 & 913.17 & 908.77 & 908.77 \\
\hline Depth to Water (ft) & 11.19 & 10.64 & 10.53 & 10.60 & 11.38 & 11.36 & 9.34 & 9.53 \\
\hline Groundwater Elevation (ft) & 902.56 & 902.72 & 902.83 & 902.76 & 901.79 & 901.81 & 899.43 & 899.24 \\
\hline Conductivity $(\mu \mathrm{mho} / \mathrm{cm})$ & 469 & 744 & 821 & 886 & 657 & 670 & 677 & 708 \\
\hline Dissolved Oxygen (ppm) & 4.24 & 0.36 & 0.78 & 0.2 & 9.33 & 3.18 & 0.11 & 0 \\
\hline Oxidation/Reduction (mV) & 37 & -160 & -182 & -151 & 149 & -41 & -29 & -85 \\
\hline Temperature (degrees C) & 22.1 & 15.4 & 17.8 & 18.8 & 12.9 & 25.1 & 16 & 19.1 \\
\hline Turbidity (NTU) & 40 & & & & 31 & 26 & & \\
\hline Iron ++ (mg/L) & & & & & & & & \\
\hline Manganese $++(\mathrm{mg} / \mathrm{L})$ & & & & & & & & \\
\hline $\mathrm{pH}$ & 6.38 & 7.45 & 7.36 & 7.53 & 7.74 & 7.26 & 7.35 & 7.2 \\
\hline \multicolumn{9}{|l|}{ Miscellaneous Analytes } \\
\hline Conductivity $(\mu \mathrm{mho} / \mathrm{cm})$ & & 665 & & 764 & & & 624 & 625 \\
\hline Dissolved Solids (mg/L) & 242 & 346 & & 432 & 387 & 382 & 343 & 375 \\
\hline Suspended Solids (mg/L) & 13.6 & 9 & & 8 & 8.1 & 16.1 & $<$ & $<$ \\
\hline Turbidity (NTU) & & 79.3 & & 119 & & & 3.25 & 8.4 \\
\hline $\mathrm{pH}$ & & 7.6 & & 7.16 & & & 7.39 & 7.32 \\
\hline \multicolumn{9}{|l|}{ Major lons (mg/L) } \\
\hline Calcium & 35.5 & 81 & & 99.1 & 90.7 & 88.9 & 94.6 & 95.8 \\
\hline Magnesium & 13.9 & 23.2 & & 23.1 & 24.8 & 24.5 & 12.2 & 12.3 \\
\hline Potassium & 1.39 & 2.41 & & 2.76 & 5.26 & 5.04 & 2.64 & 2.79 \\
\hline Sodium & 33.7 & 21.4 & & 19.3 & 12 & 12.2 & 14.3 & 14.3 \\
\hline Alkalinity as $\mathrm{CO} 3$ & $<$ & $<$ & & $<$ & $<$ & $<$ & $<$ & $<$ \\
\hline Alkalinity as $\mathrm{HCO} 3$ & 149 & 232 & & 288 & 267 & 275 & 230 & 234 \\
\hline Chloride & 32.7 & 76.9 & & 68.7 & 53.6 & 58 & 44.8 & 44.6 \\
\hline Fluoride & 0.14 & $<$ & & $<$ & $<$ & 0.11 & $<$ & $<$ \\
\hline Nitrate as $\mathrm{N}$ & 1.6 & $<$ & & 0.0283 & 0.02 & $<$ & $<$ & $<$ \\
\hline Sulfate & 24.9 & 1.5 & & 5.79 & 3.8 & 3.5 & 18.9 & 18.9 \\
\hline Charge balance & -1.4 & 0.8 & & -0.4 & 2.1 & -0.7 & 1.3 & 1.2 \\
\hline \multicolumn{9}{|l|}{ Trace Metals (mg/L) } \\
\hline Aluminum & $<$ & $<$ & & $<$ & $<$ & $<$ & $<$ & $<$ \\
\hline Arsenic (PMS) & & $<$ & & $<$ & . & & $<$ & $<$ \\
\hline Arsenic & $<$ & & & & $<$ & $<$ & & \\
\hline Barium & 0.0304 & 0.182 & & 0.318 & 0.619 & 0.634 & 0.616 & 0.661 \\
\hline Beryllium & $<$ & $<$ & & $<$ & $<$ & $<$ & $<$ & $<$ \\
\hline Boron & $<$ & $<$ & & $<$ & $<$ & $<$ & $<$ & 0.103 \\
\hline Cadmium (PMS) & & $<$ & & $<$ & & & $<$ & $<$ \\
\hline Cadmium & $<$ & & & & $<$ & $<$ & & \\
\hline Chromium (PMS) & & $<$ & & $<$ & & & 0.0043 & 0.00458 \\
\hline Chromium & 0.186 & & & & $<$ & $<$ & & \\
\hline Cobalt & $<$ & $<$ & & $<$ & $<$ & $<$ & $<$ & $<$ \\
\hline Copper & $<$ & $<$ & & $<$ & $<$ & $<$ & $<$ & $<$ \\
\hline Iron & 1.87 & 5.87 & & 8.89 & 2.42 & 8.5 & 0.506 & 1.09 \\
\hline Lead (PMS) & & $<$ & & $<$ & & & $<$ & $<$ \\
\hline Lead & $<$ & & & & $<$ & $<$ & & \\
\hline Lithium & $<$ & $<$ & & $<$ & $<$ & $<$ & 0.0154 & 0.016 \\
\hline Manganese & 0.0829 & 0.243 & & 0.542 & 0.0395 & 0.0493 & 0.0948 & 0.118 \\
\hline Mercury (CVAA) & & $<$ & & $<$ & & & & $<$ \\
\hline Nickel (PMS) & & $<$ & & $<$ & & & 0.0239 & $<$ \\
\hline Nickel & 0.338 & & & & $<$ & $<$ & & \\
\hline Strontium & 0.0425 & 0.155 & & 0.203 & 0.312 & 0.307 & 0.445 & 0.455 \\
\hline Thallium (PMS) & & $<$ & & $<$ & & & $<$ & $<$ \\
\hline Thallium & $<$ & & & & $<$ & $<$ & & . \\
\hline Uranium (PMS) & & $<$ & & $<$ & . & & $<$ & $<$ \\
\hline Uranium (KPA) & $<$ & & & & $<$ & $<$ &. & . \\
\hline Vanadium & $<$ & $<$ & & $<$ & $<$ & $<$ & $<$ & $<$ \\
\hline Zinc & $<$ & $<$ & & $<$ & $<$ & $<$ & $<$ & $<$ \\
\hline
\end{tabular}


APPENDIX E.1: CY 2002 MONITORING DATA FOR THE UPPER EAST FORK POPLAR CREEK HYDROGEOLOGIC REGIME

Field Measurements, Miscellaneous Analytes, Major Ions, and Trace Metals

\begin{tabular}{|c|c|c|c|c|c|c|c|c|}
\hline Sampling Point & \multicolumn{4}{|c|}{ GW-605 } & \multicolumn{2}{|c|}{ GW-606 } & \multicolumn{2}{|c|}{ GW-618 } \\
\hline Location & \multicolumn{4}{|c|}{ EXP-I } & \multicolumn{2}{|c|}{ EXP-I } & \multicolumn{2}{|c|}{ EXP-E } \\
\hline Date Sampled & \multicolumn{2}{|c|}{$01 / 08 / 02$} & \multicolumn{2}{|c|}{$07 / 08 / 02$} & $01 / 08 / 02$ & 07/08/02 & 05/09/02 & $11 / 07 / 02$ \\
\hline Program & BJC & BJC & BJC & BJC & BJC & BJC & BJC & BJC \\
\hline Sample Type & & Dup & & Dup & & & & \\
\hline Field Measurements & & & & & & & & \\
\hline Time Sampled & $9: 35$ & & $12: 55$ & & $9: 55$ & $13: 15$ & $10: 20$ & $10: 35$ \\
\hline Measuring Point Elev. (ft) & 919.06 & & 919.06 & & 919.59 & 919.59 & 985.14 & 985.14 \\
\hline Depth to Water (ft) & 11.37 & & 11.25 & & 14.44 & 14.03 & 12.79 & 12.63 \\
\hline Groundwater Elevation (ft) & 907.69 & & 907.81 & & 905.15 & 905.56 & 972.35 & 972.51 \\
\hline Conductivity $(\mu \mathrm{mho} / \mathrm{cm})$ & 607 & & 579 & & 426 & 664 & 690 & 657 \\
\hline Dissolved Oxygen (ppm) & 1.33 & & 2.21 & & 1.43 & 3.23 & 0.67 & 0.38 \\
\hline Oxidation/Reduction (mV) & 188 & & 253 & & 216 & 142 & 31 & -15 \\
\hline Temperature (degrees C) & 16.1 & & 19.9 & & 9.7 & 21.9 & 18.1 & 17.4 \\
\hline Turbidity (NTU) & 16 & & 19 & & 9 & 4 & 3 & 16 \\
\hline Iron ++ (mg/L) & & & & & & & 0.24 & 0.14 \\
\hline Manganese $++(\mathrm{mg} / \mathrm{L})$ & & & & & & & 1.9 & \\
\hline $\mathrm{pH}$ & 7.38 & & 7.52 & & 10.61 & 7.96 & 6.59 & 6.66 \\
\hline $\begin{array}{l}\text { Miscellaneous Analytes } \\
\text { Conductivity }(\mu \mathrm{mho} / \mathrm{cm})\end{array}$ & & & & & & & & \\
\hline Dissolved Solids (mg/L) & 357 & 344 & 356 & 358 & 225 & 393 & 364 & 360 \\
\hline Suspended Solids (mg/L) & $<$ & $<$ & $<$ & $<$ & 9.9 & $<$ & $<$ & $<$ \\
\hline Turbidity (NTU) & & & & & & & & \\
\hline $\mathrm{pH}$ & & & & & & & & \\
\hline Major lons (mg/L) & & & & & & & & \\
\hline Calcium & 78.9 & 77.3 & 80.2 & 79.4 & 41.9 & 53.1 & 101 & 88.6 \\
\hline Magnesium & 17.8 & 17.4 & 18.9 & 18.6 & 6.16 & 38.3 & 8.72 & 7.5 \\
\hline Potassium & 2.44 & 2.39 & 2.89 & 2.82 & 12.1 & 4.27 & 4.38 & 4.1 \\
\hline Sodium & 10.9 & 10.7 & 13 & 12.8 & 6.94 & 8.46 & 16.4 & 16.7 \\
\hline Alkalinity as $\mathrm{CO} 3$ & $<$ & $<$ & $<$ & $<$ & $4 Q$ & $<$ & $<$ & $<$ \\
\hline Alkalinity as $\mathrm{HCO} 3$ & 216 & 220 & 244 & 240 & $2 Q$ & 198 & $119 Q$ & 333 \\
\hline Chloride & 21.8 & 22.5 & 21.9 & 21.4 & 36.3 & 35.1 & 12.1 & 10.9 \\
\hline Fluoride & $<$ & $<$ & 0.11 & $<$ & 0.14 & 0.1 & 0.21 & 0.3 \\
\hline Nitrate as $\mathrm{N}$ & 0.14 & 0.13 & 0.5 & 0.76 & 5.5 & 12 & 0.18 & $2,490 \mathrm{Q}$ \\
\hline Sulfate & 28.4 & 28.5 & 30.5 & 30.6 & 83.7 & 44.6 & 20.3 & 19 \\
\hline Charge balance & 3.5 & & 0.2 & & -6.5 & -3.5 & $35 \mathrm{R}$ & $-93.9 R$ \\
\hline Trace Metals (mg/L) & & & & & & & & \\
\hline Aluminum & $<$ & $<$ & $<$ & $<$ & $<$ & $<$ & $<$ & $<$ \\
\hline Arsenic (PMS) & & & & & & & & \\
\hline Arsenic & $<$ & $<$ & $<$ & $<$ & $<$ & $<$ & $<$ & $<$ \\
\hline Barium & 0.0447 & 0.0437 & 0.0485 & 0.0478 & 0.173 & 0.104 & 0.0532 & 0.05 \\
\hline Beryllium & $<$ & $<$ & $<$ & $<$ & $<$ & $<$ & $<$ & $<$ \\
\hline Boron & $<$ & $<$ & $<$ & $<$ & $<$ & $<$ & 0.105 & 0.12 \\
\hline Cadmium (PMS) & & & & & & & & \\
\hline Cadmium & $<$ & $<$ & $<$ & $<$ & $<$ & $<$ & 0.0046 & 0.0032 \\
\hline Chromium (PMS) & & & & & & & & \\
\hline Chromium & 0.0056 & $<$ & $<$ & $<$ & $<$ & $<$ & $0.144 \mathrm{Q}$ & $<$ \\
\hline Cobalt & $<$ & $<$ & $<$ & $<$ & $<$ & $<$ & $<$ & $<$ \\
\hline Copper & $<$ & $<$ & $<$ & $<$ & $<$ & $<$ & $<$ & $<$ \\
\hline Iron & 0.0989 & 0.0809 & 0.0986 & 0.103 & $<$ & $<$ & 0.775 & 0.14 \\
\hline Lead (PMS) & & & & & & & & . \\
\hline Lead & $<$ & $<$ & $<$ & $<$ & $<$ & $<$ & $<$ & $<$ \\
\hline Lithium & $<$ & $<$ & $<$ & $<$ & 0.0502 & 0.0136 & $<$ & $<$ \\
\hline Manganese & 0.306 & 0.271 & 0.198 & 0.184 & $<$ & 0.0057 & 1.2 & 0.9 \\
\hline Mercury (CVAA) & $<$ & $<$ & $<$ & $<$ & $<$ & $<$ & & $<$ \\
\hline Nickel (PMS) & & & & & & & & \\
\hline Nickel & $<$ & $<$ & $<$ & $<$ & $<$ & $<$ & $0.0621 \mathrm{Q}$ & $<$ \\
\hline Strontium & 0.153 & 0.15 & 0.157 & 0.156 & 1.52 & 0.615 & 0.198 & 0.18 \\
\hline Thallium (PMS) & & & & & & & & \\
\hline Thallium & $<$ & $<$ & $<$ & $<$ & $<$ & $<$ & $<$ & $<$ \\
\hline Uranium (PMS) & & & & & & & & \\
\hline Uranium (KPA) & 0.0983 & 0.108 & 0.114 & 0.107 & $<$ & $<$ & $<$ & $<$ \\
\hline Vanadium & $<$ & $<$ & $<$ & $<$ & $<$ & $<$ & $<$ & $<$ \\
\hline Zinc & $<$ & $<$ & $<$ & $<$ & $<$ & $<$ & $<$ & $<$ \\
\hline
\end{tabular}


APPENDIX E.1: CY 2002 MONITORING DATA FOR THE UPPER EAST FORK POPLAR CREEK HYDROGEOLOGIC REGIME

Field Measurements, Miscellaneous Analytes, Major lons, and Trace Metals

\begin{tabular}{|c|c|c|c|c|c|c|c|c|}
\hline Sampling Point & \multicolumn{2}{|c|}{ GW-620 } & \multicolumn{2}{|c|}{ GW-633 } & \multicolumn{2}{|c|}{ GW-658 } & GW-686 & GW-687 \\
\hline Location & \multicolumn{2}{|c|}{ FTF } & \multicolumn{2}{|c|}{ RG } & \multicolumn{2}{|c|}{ FF } & СРT & CPT \\
\hline Date Sampled & $04 / 23 / 02$ & $10 / 15 / 02$ & $04 / 23 / 02$ & $10 / 17 / 02$ & $04 / 24 / 02$ & $10 / 17 / 02$ & $11 / 14 / 02$ & $11 / 14 / 02$ \\
\hline Program & GWPP & GWPP & GWPP & GWPP & GWPP & GWPP & GWPP & GWPP \\
\hline \multicolumn{9}{|l|}{ Sample Type } \\
\hline \multicolumn{9}{|l|}{ Field Measurements } \\
\hline Time Sampled & $10: 35$ & $7: 45$ & 9:00 & $10: 45$ & $8: 30$ & $9: 45$ & $9: 56$ & $10: 58$ \\
\hline Measuring Point Elev. (ft) & $1,015.57$ & $1,015.57$ & 996.43 & 996.43 & 945.08 & 945.08 & 963.76 & 964.89 \\
\hline Depth to Water (ft) & 25.33 & 25.49 & 3.14 & 2.44 & 11.43 & 11.08 & 12.35 & 10.11 \\
\hline Groundwater Elevation (ft) & 990.24 & 990.08 & 993.29 & 993.99 & 933.65 & 934.00 & 951.41 & 954.78 \\
\hline Conductivity ( $\mu \mathrm{mho} / \mathrm{cm})$ & 1,475 & 1,335 & 10,820 & 6,000 & 519 & 426 & 1,173 & 4,310 \\
\hline Dissolved Oxygen (ppm) & 0.59 & 0.69 & 0.65 & 0.44 & -0.04 & -0.02 & 2.9 & 2.52 \\
\hline Oxidation/Reduction (mV) & -33 & -37 & 249 & 234 & -114 & -118 & -31 & 189 \\
\hline Temperature (degrees C) & 16.5 & 16 & 16.5 & 23.4 & 16.6 & 21.5 & 13 & 16.6 \\
\hline \multirow{3}{*}{\multicolumn{9}{|c|}{$\begin{array}{r}\text { Turbidity (NTU) } \\
\text { Iron }++(\mathrm{mg} / \mathrm{L}) \\
\text { Manganese }++(\mathrm{mg} / \mathrm{L})\end{array}$}} \\
\hline & & & & & & & & \\
\hline & & & & & & & & \\
\hline $\mathrm{pH}$ & 12.26 & 11.61 & 5.57 & 5.84 & 6.32 & 6.31 & 6.94 & 6.89 \\
\hline \multicolumn{9}{|l|}{ Miscellaneous Analytes } \\
\hline Conductivity ( $\mu \mathrm{mho} / \mathrm{cm})$ & 1,327 & 1,165 & 11,170 & 10,810 & 463 & 519 & 949 & 3,960 \\
\hline Dissolved Solids (mg/L) & 320 & 288 & 9,270 & 9,050 & 238 & 299 & 611 & 4,010 \\
\hline Suspended Solids (mg/L) & $<$ & $<$ & $<$ & 2 & 17 & 9 & 215 & 324 \\
\hline Turbidity (NTU) & 1.02 & 1.1 & 0.855 & 0.723 & 40.4 & 17.6 & 277 & 421 \\
\hline $\mathrm{pH}$ & 11.71 & 11.62 & 5.7 & 5.69 & 6.53 & 6.5 & 7.27 & 7.18 \\
\hline \multicolumn{9}{|l|}{ Major lons (mg/L) } \\
\hline Calcium & 113 & 95.7 & 2,170 & 1,980 & 44.4 & 50.1 & 170 & 600 \\
\hline Magnesium & $<$ & $<$ & 194 & 189 & 13.3 & 14.4 & 24.1 & 343 \\
\hline Potassium & 14.1 & 13.2 & $<$ & $<$ & $<$ & $<$ & 5.06 & 11.4 \\
\hline Sodium & 2.34 & 2.23 & 87 & 91.3 & 13.5 & 13.2 & 13.3 & 82.1 \\
\hline Alkalinity as $\mathrm{CO} 3$ & 32 & 32 & $<$ & $<$ & $<$ & $<$ & $<$ & $<$ \\
\hline Alkalinity as $\mathrm{HCO} 3$ & $<$ & $<$ & 298 & 314 & 204 & 236 & 340 & 520 \\
\hline Chloride & 2.12 & 1.82 & 52.2 & 51.4 & 17.1 & 14.6 & 14.7 & 23.5 \\
\hline Fluoride & 0.154 & 0.156 & $<$ & $<$ & $<$ & 0.109 & $<$ & $<$ \\
\hline Nitrate as $\mathrm{N}$ & 1.13 & 1.14 & 1,520 & 1,510 & $<$ & $<$ & $<$ & $<$ \\
\hline Sulfate & 6.1 & 5.61 & 3.15 & 5.74 & $<$ & $<$ & 169 & 2,290 \\
\hline Charge balance & $-51.6 \mathrm{R}$ & 2.3 & 4.9 & 1.1 & -7.8 & -9.3 & 2.0 & 2.7 \\
\hline \multicolumn{9}{|l|}{ Trace Metals (mg/L) } \\
\hline Aluminum & 1.26 & 1.02 & $<$ & $<$ & $<$ & $<$ & 13.6 & 18.8 \\
\hline Arsenic (PMS) & $<$ & $<$ & $<$ & $<$ & $<$ & 0.00712 & $<$ & 0.01 \\
\hline Arsenic & & & & & & & & \\
\hline Barium & 0.0333 & 0.0334 & 10 & 9.56 & 0.141 & 0.173 & 0.2 & 0.145 \\
\hline Beryllium & $<$ & $<$ & $<$ & $<$ & $<$ & $<$ & 0.00052 & 0.00116 \\
\hline Boron & $<$ & $<$ & $<$ & $<$ & $<$ & $<$ & $<$ & 0.15 \\
\hline Cadmium (PMS) & 0.000857 & $<$ & 0.00191 & 0.000757 & $<$ & $<$ & 0.00589 & 0.00656 \\
\hline Cadmium & & & & & & & & \\
\hline Chromium (PMS) & $<$ & $<$ & $<$ & $<$ & $<$ & $<$ & 0.0249 & 0.0236 \\
\hline Chromium & & & & & & & & \\
\hline Cobalt & $<$ & $<$ & $<$ & $<$ & $<$ & $<$ & $<$ & $<$ \\
\hline Copper & $<$ & $<$ & $<$ & $<$ & $<$ & $<$ & 0.0668 & 0.0343 \\
\hline Iron & $<$ & 0.135 & $<$ & $<$ & 15.9 & 20.4 & 19 & 20 \\
\hline Lead (PMS) & 0.00133 & $<$ & 0.000509 & 0.000548 & 0.00467 & $<$ & 0.0528 & 0.043 \\
\hline Lead & & & & & & & & \\
\hline Lithium & 0.0242 & 0.0257 & 0.177 & 0.15 & $<$ & $<$ & 0.0122 & 0.0551 \\
\hline Manganese & $<$ & $<$ & 4.13 & 5.95 & 17.1 & 20.4 & 3.98 & 5.62 \\
\hline Mercury (CVAA & $<$ & $<$ & $<$ & $<$ & $<$ & $<$ & $<$ & $<$ \\
\hline Nickel (PMS) & $<$ & $<$ & 0.297 & 0.293 & 0.00794 & 0.00519 & 0.0179 & 0.0425 \\
\hline Nickel & & & & & & & & \\
\hline Strontium & 0.391 & 0.385 & 5.37 & 4.98 & 0.14 & 0.15 & 0.389 & 1.34 \\
\hline Thallium (PMS) & $<$ & $<$ & $<$ & $<$ & $<$ & $<$ & & $<$ \\
\hline Thallium & & & & & & & & \\
\hline Uranium (PMS) & $<$ & $<$ & 0.00156 & 0.00191 & $<$ & $<$ & 0.00119 & 0.00773 \\
\hline Uranium (KPA) & & & & & & & & \\
\hline Vanadium & $<$ & $<$ & $<$ & $<$ & $<$ & $<$ & $<$ & 0.0248 \\
\hline Zinc & $<$ & $<$ & $<$ & $<$ & $<$ & $<$ & 0.0824 & 0.0998 \\
\hline
\end{tabular}


APPENDIX E.1: CY 2002 MONITORING DATA FOR THE UPPER EAST FORK POPLAR CREEK HYDROGEOLOGIC REGIME

Field Measurements, Miscellaneous Analytes, Major lons, and Trace Metals

\begin{tabular}{|c|c|c|c|c|c|c|c|c|}
\hline Sampling Point & GW-689 & \multicolumn{3}{|c|}{ GW-698 } & \multicolumn{4}{|c|}{ GW-722-06 } \\
\hline Location & CPT & \multicolumn{3}{|c|}{ B8110 } & \multicolumn{4}{|c|}{ EXP-J } \\
\hline Date Sampled & $11 / 14 / 02$ & \multicolumn{2}{|c|}{$05 / 02 / 02$} & $11 / 07 / 02$ & $02 / 04 / 02$ & $05 / 23 / 02$ & $07 / 15 / 02$ & $11 / 07 / 02$ \\
\hline Program & GWPP & GWPP & GWPP & GWPP & GWPP & BJC & GWPP & BJC \\
\hline Sample Type & & & Dup & & & & & \\
\hline Field Measurements & & & & & & & & \\
\hline Time Sampled & $12: 02$ & $8: 35$ & $8: 35$ & $8: 55$ & $18: 22$ & $9: 20$ & $9: 25$ & $12: 35$ \\
\hline Measuring Point Elev. (ft) & 967.00 & 970.29 & 970.29 & 970.29 & & & & \\
\hline Depth to Water (ft) & 8.76 & 35.80 & 35.80 & 27.48 & & & & \\
\hline Groundwater Elevation (ft) & 958.24 & 934.49 & 934.49 & 942.81 & & & & \\
\hline Conductivity $(\mu \mathrm{mho} / \mathrm{cm})$ & 8,600 & 1,971 & 1,971 & 1,158 & 919 & 948 & 922 & 1,240 \\
\hline Dissolved Oxygen (ppm) & 3.78 & 0.27 & 0.27 & 0.04 & 12 & 3.71 & 10.2 & 5.18 \\
\hline Oxidation/Reduction (mV) & 429 & 203 & 203 & 106 & & 44 & & -84 \\
\hline Temperature (degrees C) & 18.9 & 17.1 & 17.1 & 17.1 & 10.1 & 20.5 & 20.2 & 19.1 \\
\hline Turbidity (NTU) & & & & & & 8 & & 7 \\
\hline Iron ++ (mg/L) & & & & & & & & \\
\hline Manganese ++ (mg/L) & & & & & & & & \\
\hline $\mathrm{pH}$ & 2.76 & 6.84 & 6.84 & 7.35 & 7.8 & 6.35 & 7.5 & 8.32 \\
\hline Miscellaneous Analytes & & & & & & & & \\
\hline Conductivity $(\mu \mathrm{mho} / \mathrm{cm})$ & 7,150 & 1,820 & 1,810 & 1,008 & 1,005 & & 989 & \\
\hline Dissolved Solids (mg/L) & 10,200 & 1,280 & 1,300 & 623 & 557 & 556 & 592 & 510 \\
\hline Suspended Solids (mg/L) & 46 & 2 & 2 & $<$ & $<$ & $<$ & $<$ & $<$ \\
\hline Turbidity (NTU) & 47.6 & 4.25 & 5.33 & 2.07 & 1.94 & & 1.41 & \\
\hline $\mathrm{pH}$ & 2.5 & 7.06 & 7.12 & 7.1 & 8.13 & & 8.19 & \\
\hline Major lons (mg/L) & & & & & & & & \\
\hline Calcium & 490 & 229 & 222 & 142 & 18.3 & 18.4 & 17.2 & 14.5 \\
\hline Magnesium & 179 & 70.9 & 69.4 & 34.4 & 13.9 & 12.8 & 13.5 & 10.3 \\
\hline Potassium & $<$ & 3.56 & 3.52 & 2.53 & 5.11 & 6.71 & 4.7 & 6.8 \\
\hline Sodium & 93.9 & 25.6 & 25.1 & 17.4 & 176 & 164 & 167 & 152 \\
\hline Alkalinity as $\mathrm{CO} 3$ & $<$ & $<$ & $<$ & $<$ & $<$ & $<$ & $<$ & $<$ \\
\hline Alkalinity as $\mathrm{HCO} 3$ & $<$ & 268 & 286 & 260 & 252 & 248 & 254 & 255 \\
\hline Chloride & 31.4 & 20.6 & 20.2 & 16.3 & 130 & 135 & 122 & 115 \\
\hline Fluoride & $<$ & $<$ & $<$ & $<$ & 1.1 & 0.81 & 1.05 & 1.1 \\
\hline Nitrate as $\mathrm{N}$ & & 145 & 143 & 48 & $<$ & 0.071 & $<$ & 0.36 \\
\hline Sulfate & 6,660 & 68.3 & 68.2 & 55.2 & 53.5 & 50.9 & 53.9 & 53.3 \\
\hline Charge balance & $-51.3 R$ & 2.1 & 0.1 & 2.4 & -0.2 & -3.1 & -1.8 & -6.6 \\
\hline Trace Metals (mg/L) & & & & & & & & \\
\hline Aluminum & 262 & 0.205 & 0.212 & $<$ & $<$ & $<$ & $<$ & $<$ \\
\hline Arsenic (PMS) & 0.0434 & $<$ & $<$ & $<$ & $<$ & 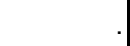 & $<$ & . \\
\hline Arsenic & & & & & & $<$ & & $<$ \\
\hline Barium & $<$ & 0.226 & 0.221 & 0.139 & 0.0341 & 0.0327 & 0.0338 & 0.03 \\
\hline Beryllium & 0.0711 & $<$ & $<$ & $<$ & $<$ & $<$ & $<$ & $<$ \\
\hline Boron & 1.22 & $<$ & $<$ & 0.1 & 0.703 & 0.672 & 0.686 & 0.61 \\
\hline Cadmium (PMS) & 0.0642 & $<$ & $<$ & $<$ & $<$ & & $<$ & \\
\hline Cadmium & & & & & & $<$ & & $<$ \\
\hline Chromium (PMS) & 0.0706 & $<$ & $<$ & $<$ & $<$ & & $<$ & \\
\hline Chromium & & & & & & $<$ & & $<$ \\
\hline Cobalt & 1.14 & $<$ & $<$ & $<$ & $<$ & $<$ & $<$ & $<$ \\
\hline Copper & 6.31 & $<$ & $<$ & $<$ & $<$ & $<$ & $<$ & $<$ \\
\hline Iron & 1190 & 0.183 & 0.175 & $<$ & 0.121 & 0.0806 & 0.101 & 0.066 \\
\hline Lead (PMS) & 0.0786 & $<$ & $<$ & $<$ & $<$ & & $<$ & \\
\hline Lead & & & & & & $<$ & & $<$ \\
\hline Lithium & 0.502 & $<$ & $<$ & $<$ & 0.128 & 0.154 & 0.122 & 0.17 \\
\hline Manganese & 39.5 & 0.106 & 0.103 & 0.0974 & 0.0068 & 0.0063 & 0.00627 & 0.0051 \\
\hline Mercury (CVAA) & $<$ & 0.000427 & 0.000368 & 0.0011 & $<$ & & $<$ & $<$ \\
\hline Nickel (PMS) & 1.97 & $<$ & & $<$ & $<$ & & $<$ & \\
\hline Nickel & & & & & & $<$ & & $<$ \\
\hline Strontium & 0.713 & 0.672 & 0.657 & 0.387 & 4.2 & 3.86 & 4 & 3.7 \\
\hline Thallium (PMS) & 0.00127 & $<$ & $<$ & $<$ & $<$ & & $<$ & \\
\hline Thallium & & & & & & $<$ & & $<$ \\
\hline Uranium (PMS) & 0.058 & 0.00141 & 0.00146 & 0.00182 & $<$ & . & $<$ & \\
\hline Uranium (KPA) & & & & & & $<$ & & $<$ \\
\hline Vanadium & $<$ & $<$ & $<$ & $<$ & $<$ & $<$ & $<$ & $<$ \\
\hline Zinc & 9.79 & $<$ & $<$ & $<$ & $<$ & 0.329 & $<$ & $<$ \\
\hline
\end{tabular}


APPENDIX E.1: CY 2002 MONITORING DATA FOR THE UPPER EAST FORK POPLAR CREEK HYDROGEOLOGIC REGIME

Field Measurements, Miscellaneous Analytes, Major lons, and Trace Metals

\begin{tabular}{|c|c|c|c|c|c|c|c|c|c|}
\hline Sampling Point & \multicolumn{5}{|c|}{ GW-722-10 } & \multicolumn{4}{|c|}{ GW-722-14 } \\
\hline Location & \multicolumn{5}{|c|}{ EXP-J } & \multicolumn{4}{|c|}{ EXP-J } \\
\hline Date Sampled & $02 / 06 / 02$ & $05 / 29 / 02$ & $07 / 16 / 02$ & $11 / 12 / 02$ & $12 / 12 / 02$ & $02 / 08 / 02$ & $05 / 30 / 02$ & $07 / 17 / 02$ & $11 / 13 / 02$ \\
\hline Program & GWPP & BJC & GWPP & BJC & BJC & GWPP & BJC & GWPP & BJC \\
\hline \multicolumn{10}{|l|}{ Sample Type } \\
\hline Field Measurements & & & & & & & & & \\
\hline Time Sampled & $17: 25$ & $13: 25$ & $10: 35$ & $11: 20$ & $10: 05$ & $11: 15$ & $13: 00$ & $14: 00$ & 9:00 \\
\hline Measuring Point Elev. (ft) & & & & & & & & & \\
\hline Depth to Water (ft) & & & & & & & & & \\
\hline Groundwater Elevation (ft) & & & & & & & & & \\
\hline Conductivity $(\mu \mathrm{mho} / \mathrm{cm})$ & 784 & 832 & 779 & 816 & 77.3 & 499 & 541 & 519 & 813 \\
\hline Dissolved Oxygen (ppm) & 13 & 4.59 & 10 & 7.51 & 4.81 & 11.8 & 7.02 & 10 & 5.33 \\
\hline Oxidation/Reduction (mV) & & 144 & & 47 & -66 & & 174 & & 205 \\
\hline Temperature (degrees C) & 12 & 25.1 & 17.8 & 17.3 & 12.1 & 11.5 & 23.1 & 18.9 & 15.7 \\
\hline Turbidity (NTU) & & 6 & & 5 & 11 & & 9 & & 9 \\
\hline Iron ++ (mg/L) & & & & & & & & & \\
\hline Manganese ++ (mg/L) & & & & & & & & & \\
\hline $\mathrm{pH}$ & 7.4 & 7.35 & 7.5 & 8.23 & 8.03 & 7.6 & 7.1 & 7.1 & 7.65 \\
\hline Miscellaneous Analytes & & & & & & & & & \\
\hline Conductivity $(\mu \mathrm{mho} / \mathrm{cm})$ & 865 & & 837 & & & 564 & & 555 & \\
\hline Dissolved Solids (mg/L) & 464 & 454 & 477 & 480 & & 297 & 268 & 301 & 336 \\
\hline Suspended Solids (mg/L) & $<$ & $<$ & $<$ & $<$ & & 4 & $<$ & $<$ & $<$ \\
\hline Turbidity (NTU) & 1.62 & & 0.733 & & & 3.27 & & 1.75 & \\
\hline $\mathrm{pH}$ & 8.05 & & 8 & & & 7.8 & & 7.73 & \\
\hline Major lons (mg/L) & & & & & & & & & \\
\hline Calcium & 30.8 & 30.1 & 27 & 27.5 & & 53.6 & 54.4 & 55.3 & $<$ \\
\hline Magnesium & 20.7 & 21 & 19.7 & $<$ & & 26.7 & 28.1 & 26.2 & $<$ \\
\hline Potassium & 3.79 & 5.36 & 3.67 & $<$ & & $<$ & 2.06 & $<$ & $<$ \\
\hline Sodium & 116 & 115 & 112 & 120 & & 23.7 & 20.9 & 22.2 & $<$ \\
\hline Alkalinity as $\mathrm{CO} 3$ & $<$ & $<$ & $<$ & $<$ & & $<$ & $<$ & $<$ & $<$ \\
\hline Alkalinity as $\mathrm{HCO} 3$ & 210 & 211 & 222 & 211 & & 242 & 243 & 244 & 250 \\
\hline Chloride & 109 & 109 & 93.8 & 98.5 & & 17.5 & 16.7 & 17 & 13.4 \\
\hline Fluoride & 0.943 & 0.67 & 0.861 & 0.7 & & 0.315 & 0.2 & 0.318 & 0.3 \\
\hline Nitrate as $\mathrm{N}$ & $<$ & $<$ & $<$ & 0.13 & & 0.553 & 0.56 & 0.444 & 0.76 \\
\hline Sulfate & 68.1 & 50.8 & 44.9 & 55.3 & & 20.3 & 18.7 & 20.6 & 17.6 \\
\hline Charge balance & -2.1 & -0.1 & -0.8 & 0.9 & & 0.8 & 1.9 & 0.4 & 1.7 \\
\hline Trace Metals (mg/L) & & & & & & & & & \\
\hline Aluminum & $<$ & $<$ & $<$ & $<$ & & $<$ & $<$ & $<$ & $<$ \\
\hline Arsenic (PMS) & $<$ & & $<$ & . & & $<$ & & $<$ & 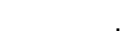 \\
\hline Arsenic & & & & $<$ & & & $<$ & & $<$ \\
\hline Barium & 0.0578 & 0.0594 & 0.0557 & $<$ & & 0.11 & 0.107 & 0.103 & $<$ \\
\hline Beryllium & $<$ & & & $<$ & & & $<$ & $<$ & $<$ \\
\hline Boron & 0.442 & 0.457 & 0.444 & 0.42 & & 0.143 & 0.139 & 0.147 & 0.13 \\
\hline Cadmium (PMS) & $<$ & & $<$ & & & & & $<$ & \\
\hline Cadmium & & $<$ & & $<$ & & & $<$ & & $<$ \\
\hline Chromium (PMS) & $<$ & & $<$ & & & 0.00295 & & $<$ & \\
\hline Chromium & & $<$ & & $<$ & & & $<$ & & $<$ \\
\hline Cobalt & $<$ & $<$ & $<$ & $<$ & & $<$ & $<$ & $<$ & $<$ \\
\hline Copper & $<$ & $<$ & $<$ & $<$ & & $<$ & $<$ & $<$ & $<$ \\
\hline Iron & 0.249 & 0.191 & 0.327 & 0.18 & & 0.0605 & $<$ & 0.0522 & $<$ \\
\hline Lead (PMS) & $<$ & & 0.000613 & & & $<$ & & 0.000582 & \\
\hline Lead & & $<$ & & $<$ & & & $<$ & & $<$ \\
\hline Lithium & 0.0904 & 0.123 & 0.0899 & 0.14 & & 0.0194 & 0.0234 & 0.0204 & $<$ \\
\hline Manganese & $<$ & $<$ & $<$ & $<$ & & $<$ & $<$ & $<$ & $<$ \\
\hline Mercury (CVAA) & $<$ & & $<$ & $<$ & & $<$ & & $<$ & $<$ \\
\hline Nickel (PMS) & $<$ & & $<$ & & & $<$ & & $<$ & . \\
\hline Nickel & & $<$ & & $<$ & & & $<1$ & & $<$ \\
\hline Strontium & 3.04 & 3.12 & 2.98 & $<$ & & 0.787 & 0.789 & 0.782 & $<$ \\
\hline Thallium (PMS) & $<$ & & 0.000577 & & & $<$ & & $<$ & \\
\hline Thallium & & $<$ & & $<$ & & . & $<$ & & $<$ \\
\hline Uranium (PMS) & $<$ &. & $<$ & & & $<$ & & 0.000536 & \\
\hline Uranium (KPA) & & $<$ & & $<$ & & & $<$ & & $<$ \\
\hline Vanadium & $<$ & $<$ & $<$ & $<$ & & $<$ & $<$ & $<$ & $<$ \\
\hline Zinc & $<$ & $<$ & 0.0737 & $<$ & & $<$ & $<$ & 0.106 & $<$ \\
\hline
\end{tabular}


APPENDIX E.1: CY 2002 MONITORING DATA FOR THE UPPER EAST FORK POPLAR CREEK HYDROGEOLOGIC REGIME

Field Measurements, Miscellaneous Analytes, Major lons, and Trace Metals

\begin{tabular}{|c|c|c|c|c|c|c|c|c|c|}
\hline Sampling Point & \multicolumn{4}{|c|}{ GW-722-17 } & \multicolumn{5}{|c|}{ GW-722-20 } \\
\hline Location & \multicolumn{4}{|c|}{ EXP-J } & \multicolumn{5}{|c|}{ EXP-J } \\
\hline Date Sampled & $02 / 08 / 02$ & $06 / 03 / 02$ & 07/17/02 & $11 / 13 / 02$ & $02 / 07 / 02$ & $05 / 30 / 02$ & \multicolumn{2}{|c|}{$07 / 17 / 02$} & $11 / 12 / 02$ \\
\hline Program & GWPP & BJC & GWPP & BJC & GWPP & BJC & GWPP & GWPP & BJC \\
\hline Sample Type & & & & & & & & Dup & \\
\hline Field Measurements & & & & & & & & & \\
\hline Time Sampled & $12: 42$ & $8: 55$ & 15:05 & $10: 00$ & $18: 05$ & $10: 00$ & $9: 30$ & $9: 30$ & $12: 40$ \\
\hline Measuring Point Elev. (ft) & & & & & & & & & \\
\hline Depth to Water (ft) & & & & & & & & & \\
\hline Groundwater Elevation (ft) & & & & & & & & & \\
\hline Conductivity $(\mu \mathrm{mho} / \mathrm{cm})$ & 515 & 572 & 523 & 691 & 488 & 525 & 500 & 500 & 538 \\
\hline Dissolved Oxygen (ppm) & 11.6 & 6.43 & 10.4 & 6.21 & 13.2 & 3.08 & 10.2 & 10.2 & 3.88 \\
\hline Oxidation/Reduction (mV) & & 187 & & 158 & & 77 & & & 123 \\
\hline Temperature (degrees C) & 12.2 & 25 & 18.2 & 15.8 & 11 & 22.8 & 16.9 & 16.9 & 18.6 \\
\hline Turbidity (NTU) & & 5 & & 8 & . & 8 & & & 8 \\
\hline Iron $++(\mathrm{mg} / \mathrm{L})$ & & & & & & & & & \\
\hline Manganese ++ $(\mathrm{mg} / \mathrm{L})$ & & & & & & & & & \\
\hline $\mathrm{pH}$ & 7.5 & 7.52 & 7.2 & 7.71 & 7.6 & 7.45 & 7.42 & 7.42 & 8.2 \\
\hline Miscellaneous Analytes & & & & & & & & & \\
\hline Conductivity $(\mu \mathrm{mho} / \mathrm{cm})$ & 575 & & 583 & & 532 & & 526 & 535 & \\
\hline Dissolved Solids (mg/L) & 303 & 305 & 321 & 328 & 283 & 261 & 311 & 306 & 297 \\
\hline Suspended Solids (mg/L) & 4 & $<$ & $<$ & $<$ & $<$ & $<$ & $<$ & $<$ & $<$ \\
\hline Turbidity (NTU) & 7.81 & & 2.97 & & 1.74 & & 2.71 & 2.61 & \\
\hline $\mathrm{pH}$ & 7.96 & & 7.91 & & 7.88 & & 8.03 & 7.96 & \\
\hline Major lons (mg/L) & & & & & & & & & \\
\hline Calcium & 49.5 & 48.3 & 50 & $<$ & 52.4 & 51.5 & 51.8 & 52.5 & 44.9 \\
\hline Magnesium & 27.9 & 29.2 & 26.8 & $<$ & 29 & 29.8 & 27.3 & 28.5 & $<$ \\
\hline Potassium & $<$ & 2.39 & $<$ & $<$ & $<$ & 1.89 & $<$ & $<$ & $<$ \\
\hline Sodium & 29.3 & 30.8 & 29.5 & $<$ & 17.8 & 16.8 & 17 & 17.6 & 17.5 \\
\hline Alkalinity as $\mathrm{CO} 3$ & $<$ & $<$ & $<$ & $<$ & $<$ & $<$ & $<$ & $<$ & $<$ \\
\hline Alkalinity as $\mathrm{HCO} 3$ & 220 & 231 & 210 & 228 & 222 & 219 & 228 & 230 & 222 \\
\hline Chloride & 33.7 & 31.6 & 32.9 & 26.7 & 20.3 & 20.3 & 18.5 & 21.8 & 17.6 \\
\hline Fluoride & 0.481 & 0.3 & 0.506 & 0.5 & 0.408 & 0.3 & 0.385 & 0.391 & 0.4 \\
\hline Nitrate as N & 0.523 & 0.52 & 0.312 & 0.44 & 1.18 & 1.2 & 0.925 & 1.24 & 1.2 \\
\hline Sulfate & 31.1 & 26.9 & 31.4 & 26.2 & 28.9 & 26.3 & 26 & 34.5 & 27.3 \\
\hline Charge balance & -0.2 & 0.7 & 1.3 & -7.2 & 0.5 & 1.8 & -1.2 & -2.6 & -3.0 \\
\hline Trace Metals (mg/L) & & & & & & & & & \\
\hline Aluminum & $<$ & $<$ & $<$ & $<$ & $<$ & $<$ & $<$ & $<$ & $<$ \\
\hline Arsenic (PMS) & $<$ & & $<$ & & $<$ &. & $<$ & $<$ & \\
\hline Arsenic & & $<$ & & $<$ & & $<$ & & & $<$ \\
\hline Barium & 0.0893 & 0.1 & 0.0841 & $<$ & 0.0688 & 0.0759 & 0.069 & 0.0713 & $<$ \\
\hline Beryllium & $<$ & $<$ & $<$ & $<$ & $<$ & $<$ & $<$ & $<$ & $<$ \\
\hline Boron & 0.136 & 0.138 & 0.143 & 0.32 & $<$ & $<$ & $<$ & $<$ & $<$ \\
\hline Cadmium (PMS) & $<$ & & $<$ & & $<$ &. & $<$ & $<$ & \\
\hline Cadmium & & $<$ & & $<$ & & $<$ & & & $<$ \\
\hline Chromium (PMS) & $<$ &. & $<$ & 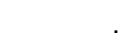 & $<$ &. & $<$ & $<$ & \\
\hline Chromium & & $<$ & & $<$ & & $<$ & & & 0.0057 \\
\hline Cobalt & $<$ & $<$ & $<$ & $<$ & $<$ & $<$ & $<$ & $<$ & $<$ \\
\hline Copper & $<$ & $<$ & $<$ & $<$ & $<$ & $<$ & $<$ & $<$ & $<$ \\
\hline Iron & $<$ & $<$ & 0.0684 & 0.05 & 0.167 & $<$ & 0.0948 & $<$ & 0.082 \\
\hline Lead (PMS) & $<$ & & 0.00427 & & $<$ & & $<$ & 0.000652 & \\
\hline Lead & & $<$ & & $<$ & & $<$ & & & $<$ \\
\hline Lithium & 0.0226 & 0.0313 & 0.0241 & $<$ & 0.0154 & 0.0185 & 0.0153 & 0.0156 & 0.022 \\
\hline Manganese & $<$ & $<$ & $<$ & $<$ & $<$ & $<$ & $<$ & $<$ & $<$ \\
\hline Mercury (CVAA) & $<$ & & $<$ & $<$ & $<$ & & $<$ & $<$ & $<$ \\
\hline Nickel (PMS) & $<$ & 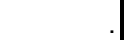 & $<$ & & $<$ &. & $<$ & $<$ & \\
\hline Nickel & & & & $<$ & & 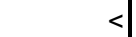 & & & $<$ \\
\hline Strontium & 0.909 & 0.965 & 0.916 & $<$ & 0.74 & 0.72 & 0.703 & 0.724 & $<$ \\
\hline Thallium (PMS) & $<$ & & $<$ & & $<$ & & $<$ & $<$ & \\
\hline Thallium & & $<$ & & $<$ & . & $<$ & & & $<$ \\
\hline Uranium (PMS) & $<$ &. & $<$ & & $<$ &. & $<$ & $<$ & \\
\hline Uranium (KPA) & & $<$ & & $<$ &. & $<$ & &. & $<$ \\
\hline Vanadium & $<$ & $<$ & $<$ & $<$ & $<$ & $<$ & $<$ & $<$ & $<$ \\
\hline Zinc & $<$ & $<$ & 0.157 & 1.9 & 0.132 & $<$ & 0.101 & $<$ & 0.071 \\
\hline
\end{tabular}


APPENDIX E.1: CY 2002 MONITORING DATA FOR THE UPPER EAST FORK POPLAR CREEK HYDROGEOLOGIC REGIME

Field Measurements, Miscellaneous Analytes, Major Ions, and Trace Metals

\begin{tabular}{|c|c|c|c|c|c|c|c|c|}
\hline Sampling Point & \multicolumn{5}{|c|}{ GW-722-22 } & \multicolumn{3}{|c|}{ GW-722-26 } \\
\hline Location & \multicolumn{5}{|c|}{ EXP-J } & \multicolumn{3}{|c|}{ EXP-J } \\
\hline Date Sampled & \multicolumn{2}{|c|}{ 02/07/02 } & $05 / 30 / 02$ & 07/16/02 & $11 / 12 / 02$ & $02 / 05 / 02$ & \multicolumn{2}{|c|}{$05 / 28 / 02$} \\
\hline Program & GWPP & GWPP & BJC & GWPP & BJC & GWPP & BJC & BJC \\
\hline Sample Type & & Dup & & & & & & Dup \\
\hline Field Measurements & & & & & & & & \\
\hline Time Sampled & $15: 35$ & $15: 35$ & $8: 55$ & $14: 25$ & $12: 06$ & $16: 00$ & $10: 00$ & \\
\hline Measuring Point Elev. (ft) & & & & & & & & \\
\hline Depth to Water (ft) & & & & & & & & \\
\hline Groundwater Elevation (ft) & & & & & & & & \\
\hline Conductivity $(\mu \mathrm{mho} / \mathrm{cm})$ & 480 & 480 & 741 & 480 & 538 & 396 & 423 & \\
\hline Dissolved Oxygen (ppm) & 12.2 & 12.2 & 5.01 & 10.1 & 6.4 & 8.4 & 2.68 & \\
\hline Oxidation/Reduction (mV) & & & 177 & & 16 & & -74 & \\
\hline Temperature (degrees C) & 12.2 & 12.2 & 21.8 & 18.8 & 16.9 & 11.6 & 19 & \\
\hline Turbidity (NTU) & & & 6 & & 9 & & 4 & \\
\hline Iron ++ (mg/L) & & & & & & & & \\
\hline Manganese ++ (mg/L) & & & & & & & & \\
\hline $\mathrm{pH}$ & 7.4 & 7.4 & 7.18 & 7.23 & 7.93 & 7.5 & 7.31 & \\
\hline Miscellaneous Analytes & & & & & & & & \\
\hline Conductivity $(\mu \mathrm{mho} / \mathrm{cm})$ & 519 & 518 & & 518 & & 423 & & \\
\hline Dissolved Solids (mg/L) & 272 & 275 & 262 & 286 & 303 & 230 & 221 & 223 \\
\hline Suspended Solids (mg/L) & $<$ & $<$ & $<$ & $<$ & $<$ & $<$ & $<$ & $<$ \\
\hline Turbidity (NTU) & 3.93 & 3.98 & & 1.26 & & 12 & & \\
\hline $\mathrm{pH}$ & 8.18 & 8.2 & & 7.73 & & 7.75 & & \\
\hline Major lons (mg/L) & & & & & & & & \\
\hline Calcium & 53.7 & 52.2 & 53.8 & 53.6 & 47 & 52.1 & 51.1 & 51.3 \\
\hline Magnesium & 26.6 & 26.7 & 28.3 & 26.2 & $<$ & 21.1 & 21.6 & 21.5 \\
\hline Potassium & $<$ & $<$ & 1.83 & $<$ & $<$ & 2.29 & 2.69 & 2.55 \\
\hline Sodium & 18.2 & 18.3 & 16.7 & 16.8 & 20.2 & 3.07 & 3.08 & 2.96 \\
\hline Alkalinity as $\mathrm{CO} 3$ & $<$ & $<$ & $<$ & $<$ & $<$ & $<$ & $<$ & $<$ \\
\hline Alkalinity as $\mathrm{HCO} 3$ & 238 & 240 & 242 & 246 & 247 & 220 & 213 & 217 \\
\hline Chloride & 10.9 & 11.1 & 13.5 & 7.72 & 7.6 & 3.7 & 3.9 & 3.8 \\
\hline Fluoride & 0.322 & 0.323 & 0.2 & 0.334 & 0.2 & 1.31 & 0.86 & 0.86 \\
\hline Nitrate as $\mathrm{N}$ & 0.544 & 0.575 & 0.041 & 0.369 & 0.7 & $<$ & $<$ & $<$ \\
\hline Sulfate & 20 & 19.5 & 18.7 & 15.5 & 18.3 & 0.28 & 0.31 & 0.29 \\
\hline Charge balance & 1.1 & 0.2 & 1.5 & 0.5 & -2.2 & -0.5 & 1.2 & \\
\hline Trace Metals (mg/L) & & & & & & & & \\
\hline Aluminum & $<$ & $<$ & $<$ & $<$ & $<$ & $<$ & $<$ & $<$ \\
\hline Arsenic (PMS) & $<$ & $<$ & 1 & $<$ & . & $<$ & 1 & \\
\hline Arsenic & & & $<$ & & $<$ & & $<$ & $<$ \\
\hline Barium & 0.0967 & 0.0953 & 0.0962 & 0.0912 & $<$ & 0.197 & 0.196 & 0.188 \\
\hline Beryllium & $<$ & & $<$ & $<$ & $<$ & $<$ & $<$ & $<$ \\
\hline Boron & 0.117 & 0.113 & 0.111 & 0.114 & 0.12 & $<$ & $<$ & $<$ \\
\hline Cadmium (PMS) & $<$ & $<$ & & $<$ & & $<$ & & \\
\hline Cadmium & & & $<$ & & $<$ & 1 & $<$ & $<$ \\
\hline Chromium (PMS) & $<$ & $<$ & & $<$ & & $<$ & & \\
\hline Chromium & & & $<$ & & $<$ &. & $<$ & $<$ \\
\hline Cobalt & $<$ & $<$ & $<$ & $<$ & $<$ & $<$ & $<$ & $<$ \\
\hline Copper & $<$ & $<$ & $<$ & $<$ & $<$ & $<$ & $<$ & $<$ \\
\hline Iron & 0.131 & 0.0799 & 0.0768 & $<$ & 0.17 & 0.916 & 0.529 & 0.271 \\
\hline Lead (PMS) & $<$ & $<$ & & $<$ & & $<$ & & \\
\hline Lead & & & $<$ & & $<$ & & $<$ & $<$ \\
\hline Lithium & 0.0149 & 0.0158 & 0.0186 & 0.0137 & 0.024 & $<$ & $<$ & $<$ \\
\hline Manganese & $<$ & $<$ & $<$ & $<$ & $<$ & 0.11 & 0.11 & 0.103 \\
\hline Mercury (CVAA) & $<$ & $<$ & . & $<$ & $<$ & $<$ & & \\
\hline Nickel (PMS) & $<$ & $<$ & & $<$ & & $<$ & & \\
\hline Nickel & & & $<$ & & $<$ & & $<$ & $<$ \\
\hline Strontium & 0.719 & 0.715 & 0.716 & 0.671 & $<$ & 1.53 & 1.55 & 1.48 \\
\hline Thallium (PMS) & $<$ & $<$ & & $<$ & & $<$ & & \\
\hline Thallium & & & $<$ & & $<$ & & $<$ & $<$ \\
\hline Uranium (PMS) & $<$ & $<$ & & $<$ & & $<$ & & \\
\hline Uranium (KPA) & & & $<$ & & $<$ & & $<$ & $<$ \\
\hline Vanadium & $<$ & $<$ & $<$ & $<$ & $<$ & $<$ & $<$ & $<$ \\
\hline Zinc & 0.0946 & $<$ & $<$ & $<$ & 0.056 & 0.126 & $<$ & $<$ \\
\hline
\end{tabular}


APPENDIX E.1: CY 2002 MONITORING DATA FOR THE UPPER EAST FORK POPLAR CREEK HYDROGEOLOGIC REGIME

Field Measurements, Miscellaneous Analytes, Major Ions, and Trace Metals

\begin{tabular}{|c|c|c|c|c|c|c|c|c|}
\hline Sampling Point & \multicolumn{5}{|c|}{ GW-722-26 } & \multicolumn{3}{|c|}{ GW-722-30 } \\
\hline Location & \multicolumn{5}{|c|}{ EXP-J } & \multicolumn{3}{|c|}{ EXP-J } \\
\hline Date Sampled & 07/15/02 & \multicolumn{2}{|c|}{$11 / 11 / 02$} & \multicolumn{2}{|c|}{$12 / 12 / 02$} & $02 / 05 / 02$ & $05 / 28 / 02$ & $07 / 15 / 02$ \\
\hline Program & GWPP & BJC & BJC & BJC & BJC & GWPP & BJC & GWPP \\
\hline Sample Type & & & Dup & & Dup & & & \\
\hline Field Measurements & & & & & & & & \\
\hline Time Sampled & $14: 05$ & $12: 50$ & & $9: 15$ & & $14: 20$ & $8: 55$ & $10: 25$ \\
\hline Measuring Point Elev. (ft) & & & & & & & & \\
\hline Depth to Water (ft) & & & & & & & & \\
\hline Groundwater Elevation (ft) & & & & & & & & \\
\hline Conductivity $(\mu \mathrm{mho} / \mathrm{cm})$ & 666 & 411 & & 628 & & 299.4 & 470 & 305 \\
\hline Dissolved Oxygen (ppm) & 5.3 & 5.24 & & 450 & & 9.1 & 2.9 & 7.8 \\
\hline Oxidation/Reduction (mV) & & -70 & & -79 & & & 158 & \\
\hline Temperature (degrees C) & 19.1 & 19.9 & & 12.6 & & 10.9 & 19.1 & 18.3 \\
\hline Turbidity (NTU) & & 8 & & 8 & & & 6 & \\
\hline Iron ++ (mg/L) & & & & & & & & \\
\hline Manganese $++(\mathrm{mg} / \mathrm{L})$ & & & & & & & & \\
\hline $\mathrm{pH}$ & 7 & 7.86 & & 7.47 & & 7.8 & 6.76 & 7.6 \\
\hline Miscellaneous Analytes & & & & & & & & \\
\hline Conductivity $(\mu \mathrm{mho} / \mathrm{cm})$ & 415 & & & & & 282 & & 304 \\
\hline Dissolved Solids (mg/L) & 225 & 158 & 120 & & & 163 & 163 & 177 \\
\hline Suspended Solids (mg/L) & $<$ & $<$ & $<$ & & & $<$ & $<$ & $<$ \\
\hline Turbidity (NTU) & 2.01 & & & & & 1.18 & & 0.651 \\
\hline $\mathrm{pH}$ & 7.91 & & & & & 7.92 & & 7.95 \\
\hline Major lons (mg/L) & & & & & & & & \\
\hline Calcium & 52.7 & 43.9 & 44.1 & & & 41.5 & 39.3 & 38.7 \\
\hline Magnesium & 20.1 & 18.2 & 18.2 & & & 15 & 14.8 & 14.5 \\
\hline Potassium & 2.27 & 2.1 & 2.1 & & & $<$ & 1.44 & $<$ \\
\hline Sodium & 2.83 & 3 & 3.2 & & & 0.742 & 0.84 & 0.723 \\
\hline Alkalinity as $\mathrm{CO} 3$ & $<$ & $<$ & $<$ & & & $<$ & $<$ & $<$ \\
\hline Alkalinity as $\mathrm{HCO} 3$ & 218 & 217 & 217 & & & 150 & 148 & 144 \\
\hline Chloride & 3.76 & 3.2 & 3.2 & & & 2.48 & 7 & 2.71 \\
\hline Fluoride & 1.19 & 1.2 & 1 & & & 0.179 & 0.14 & 0.181 \\
\hline Nitrate as $\mathrm{N}$ & & 0.17 & 0.2 & & & $<$ & 0.07 & 0.116 \\
\hline Sulfate & 0.258 & 0.24 & 0.32 & & & 9.49 & 10.4 & 9.94 \\
\hline Charge balance & -0.7 & -7.6 & & & & 0.9 & -2.0 & -0.4 \\
\hline Trace Metals (mg/L) & & & & & & & & \\
\hline Aluminum & $<$ & $<$ & $<$ & & & $<$ & $<$ & $<$ \\
\hline Arsenic (PMS) & $<$ & & & & & $<$ &. & $<$ \\
\hline Arsenic & & $<$ & $<$ & & & & $<$ & \\
\hline Barium & 0.172 & 0.17 & 0.18 & & & 0.0468 & 0.0487 & 0.0442 \\
\hline Beryllium & $<$ & $<$ & $<$ & & & $<$ & $<$ & $<$ \\
\hline Boron & $<$ & $<$ & $<$ & & & $<$ & $<$ & $<$ \\
\hline Cadmium (PMS) & $<$ & & & & & $<$ & & $<$ \\
\hline Cadmium & & $0.0031 \mathrm{R}$ & $<$ & & & & $<$ & . \\
\hline Chromium (PMS) & $<$ & & & & & $<$ & & $<$ \\
\hline Chromium & & $<$ & $<$ & & & 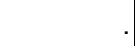 & $<$ & . \\
\hline Cobalt & $<$ & $<$ & $<$ & & & $<$ & $<$ & $<$ \\
\hline Copper & $<$ & $<$ & $<$ & & & $<$ & $<$ & $<$ \\
\hline Iron & 0.742 & 0.85 & 0.68 & & & 0.0698 & 0.0787 & $<$ \\
\hline Lead (PMS) & $<$ & & & & & $<$ & & $<$ \\
\hline Lead & & $<$ & $<$ & & & & $<$ & \\
\hline Lithium & $<$ & $<$ & $<$ & & & $<$ & $<$ & $<$ \\
\hline Manganese & 0.112 & 0.1 & 0.094 & & & 0.0056 & $<$ & $<$ \\
\hline Mercury (CVAA & $<$ & $<$ & $<$ & & & $<$ & . & $<$ \\
\hline Nickel (PMS) & $<$ & & & & & $<$ & & $<$ \\
\hline Nickel & & $<$ & $<$ & & & & $<$ & \\
\hline Strontium & 1.37 & 1.4 & 1.4 & & & 0.0817 & 0.0791 & 0.0894 \\
\hline Thallium (PMS) & $<$ & & & & & $<$ & & $<$ \\
\hline Thallium & & $<$ & $<$ & & & & $<$ & \\
\hline Uranium (PMS) & $<$ &. & & & & 0.000571 & . & 0.00057 \\
\hline Uranium (KPA) & & $<$ & $<$ & & & & $<$ & \\
\hline Vanadium & $<$ & $<$ & $<$ & & & $<$ & $<$ & $<$ \\
\hline Zinc & 0.177 & $<$ & $0.088 \mathrm{R}$ & & & $<$ & $<$ & 0.0958 \\
\hline
\end{tabular}


APPENDIX E.1: CY 2002 MONITORING DATA FOR THE UPPER EAST FORK POPLAR CREEK HYDROGEOLOGIC REGIME Field Measurements, Miscellaneous Analytes, Major Ions, and Trace Metals

\begin{tabular}{|c|c|c|c|c|c|c|c|}
\hline Sampling Point & \multicolumn{2}{|c|}{ GW-722-30 } & \multicolumn{5}{|c|}{ GW-722-32 } \\
\hline Location & \multicolumn{2}{|c|}{ EXP-J } & \multicolumn{5}{|c|}{ EXP-J } \\
\hline Date Sampled & $11 / 11 / 02$ & $12 / 12 / 02$ & $02 / 05 / 02$ & $05 / 28 / 02$ & 07/15/02 & $11 / 12 / 02$ & $12 / 12 / 02$ \\
\hline Program & BJC & BJC & GWPP & BJC & GWPP & BJC & BJC \\
\hline \multicolumn{8}{|l|}{ Sample Type } \\
\hline Field Measurements & & & & & & & \\
\hline Time Sampled & $11: 30$ & $12: 37$ & $17: 45$ & $13: 10$ & $8: 05$ & 9:00 & $13: 00$ \\
\hline Measuring Point Elev. (ft) & & & & & & & \\
\hline Depth to Water (ft) & & & & & & & \\
\hline Groundwater Elevation (ft) & & & & & & & \\
\hline Conductivity $(\mu \mathrm{mho} / \mathrm{cm})$ & 559 & 331 & 393 & 430 & 440 & 720 & 415 \\
\hline Dissolved Oxygen (ppm) & 4.75 & 2.95 & 9.4 & 4.51 & 8.8 & 6.81 & 5.89 \\
\hline Oxidation/Reduction (mV) & 143 & 217 & & 69 & & 181 & 141 \\
\hline Temperature (degrees $\mathrm{C}$ ) & 20.9 & 12 & 11.1 & 20.3 & 19.7 & 14.9 & 12.3 \\
\hline Turbidity (NTU) & 12 & 9 & & 7 & & 7 & 4 \\
\hline Iron ++ (mg/L) & & & & & & & \\
\hline Manganese $++(\mathrm{mg} / \mathrm{L})$ & & & & & & & \\
\hline $\mathrm{pH}$ & 8.08 & 7.68 & 7.4 & 6.98 & 7.3 & 7.5 & 7.3 \\
\hline Miscellaneous Analytes & & & & & & & \\
\hline Conductivity $(\mu \mathrm{mho} / \mathrm{cm})$ & & & 435 & & 456 & & \\
\hline Dissolved Solids (mg/L) & 82 & & 249 & 233 & 265 & 230 & \\
\hline Suspended Solids (mg/L) & $<$ & & 5 & $<$ & $<$ & $<$ & \\
\hline Turbidity (NTU) & & & 21.7 & & 2.42 & & \\
\hline $\mathrm{pH}$ & & & 7.46 & & 7.55 & & \\
\hline Major lons (mg/L) & & & & & & & \\
\hline Calcium & 31.9 & & 74.1 & 71 & 79.8 & 59.7 & \\
\hline Magnesium & 12.2 & & 10.9 & 11.1 & 12.9 & $<$ & \\
\hline Potassium & 0.98 & & 2.4 & 2.04 & $<$ & $<$ & \\
\hline Sodium & 1.2 & & 3.13 & 3.27 & 3.05 & 3.3 & \\
\hline Alkalinity as $\mathrm{CO} 3$ & $<$ & & $<$ & $<$ & $<$ & $<$ & \\
\hline Alkalinity as $\mathrm{HCO} 3$ & 148 & & 210 & 205 & 226 & 203 & \\
\hline Chloride & 2.1 & & 3.84 & 4.2 & 3.7 & 2.8 & \\
\hline Fluoride & 0.1 & & & $<$ & $<$ & $<$ & \\
\hline Nitrate as $\mathrm{N}$ & 0.33 & & 0.658 & 0.83 & 0.777 & 0.85 & \\
\hline Sulfate & 8.8 & & 10.5 & 10.6 & 10 & 9.3 & \\
\hline Charge balance & -9.4 & & 2.4 & 1.7 & 2.9 & -5.8 & \\
\hline Trace Metals (mg/L) & & & & & & & \\
\hline Aluminum & $<$ & & 2.02 & $<$ & 0.274 & $<$ & \\
\hline Arsenic (PMS) & & & & & $<$ &. & \\
\hline Arsenic & $<$ & & & $<$ & & $<$ & \\
\hline Barium & 0.041 & & 0.0359 & 0.0316 & 0.0346 & $<$ & \\
\hline Beryllium & $<$ & & $<$ & $<$ & $<$ & $<$ & \\
\hline Boron & $<$ & & $<$ & $<$ & $<$ & $<$ & \\
\hline Cadmium (PMS) & & & $<$ & & $<$ &. & \\
\hline Cadmium & 0.0024 & & & $<$ & & $<$ & \\
\hline Chromium (PMS) & & & 0.00554 & 1 & 0.00251 & . & \\
\hline Chromium & $<$ & & & $<$ & & $<$ & \\
\hline Cobalt & $<$ & & $<$ & $<$ & $<$ & $<$ & \\
\hline Copper & $<$ & & $<$ & $<$ & $<$ & $<$ & \\
\hline Iron & 0.28 & & 2 & $<$ & 0.406 & 0.052 & \\
\hline Lead (PMS) & & & 0.00297 & & 0.00127 & & \\
\hline Lead & $<$ & & & $<$ & & $<$ & \\
\hline Lithium & $<$ & & $<$ & $<$ & $<$ & $<$ & \\
\hline Manganese & $<$ & & 0.0314 & $<$ & 0.0104 & $<$ & \\
\hline Mercury (CVAA) & $<$ & & $<$ & & $<$ & $<$ & \\
\hline Nickel (PMS) & & & $<$ & & $<$ &. & \\
\hline Nickel & $<$ & & & $<$ & & $<$ & \\
\hline Strontium & 0.082 & & 0.072 & 0.0718 & 0.0804 & $<$ & \\
\hline Thallium (PMS) & & & $<$ & & $<$ &. & \\
\hline Thallium & $<$ & & & $<$ & & $<$ & \\
\hline Uranium (PMS) & & & $<$ & & $<$ &. & \\
\hline Uranium (KPA) & $<$ & & . & $<$ & & $<$ & \\
\hline Vanadium & $<$ & & $<$ & $<$ & $<$ & $<$ & \\
\hline Zinc & $<$ & & $<$ & $<$ & 0.143 & $<$ & \\
\hline
\end{tabular}


APPENDIX E.1: CY 2002 MONITORING DATA FOR THE UPPER EAST FORK POPLAR CREEK HYDROGEOLOGIC REGIME

Field Measurements, Miscellaneous Analytes, Major lons, and Trace Metals

\begin{tabular}{|c|c|c|c|c|c|c|c|c|c|}
\hline Sampling Point & \multicolumn{5}{|c|}{ GW-722-33 } & \multicolumn{2}{|c|}{ GW-733 } & \multicolumn{2}{|c|}{ GW-735 } \\
\hline Location & \multicolumn{5}{|c|}{ EXP-J } & \multicolumn{2}{|c|}{ EXP-J } & \multicolumn{2}{|c|}{ EXP-J } \\
\hline Date Sampled & $02 / 06 / 02$ & $05 / 29 / 02$ & $07 / 16 / 02$ & $11 / 12 / 02$ & $12 / 12 / 02$ & $01 / 08 / 02$ & $07 / 09 / 02$ & $05 / 14 / 02$ & $11 / 21 / 02$ \\
\hline Program & GWPP & BJC & GWPP & BJC & BJC & BJC & BJC & GWPP & GWPP \\
\hline \multicolumn{10}{|l|}{ Sample Type } \\
\hline \multicolumn{10}{|l|}{ Field Measurements } \\
\hline Time Sampled & $14: 40$ & $12: 40$ & $9: 05$ & $9: 50$ & $13: 15$ & $13: 45$ & $9: 25$ & $8: 15$ & 9:55 \\
\hline Measuring Point Elev. (ft) & & & & & & 959.84 & 959.84 & 924.46 & 924.46 \\
\hline Depth to Water (ft) & & & & & & 59.76 & 59.87 & 20.22 & 22.24 \\
\hline Groundwater Elevation (ft) & & & & & & 900.08 & 899.97 & 904.24 & 902.22 \\
\hline Conductivity $(\mu \mathrm{mho} / \mathrm{cm})$ & 376 & 572 & 443 & 476 & 419 & 338 & 309 & 788 & 829 \\
\hline Dissolved Oxygen (ppm) & 10.1 & 5.51 & 8.5 & 5.94 & 5.56 & 1.82 & 1.97 & 0.82 & 0.17 \\
\hline Oxidation/Reduction (mV) & & 157 & & 188 & 205 & 205 & 3 & 126 & 140 \\
\hline Temperature (degrees C) & 11.6 & 21.5 & 21 & 15 & 12.1 & 9.5 & 21.6 & 14.4 & 14.4 \\
\hline Turbidity (NTU) & & 5 & & 7 & 7 & 13 & 8 & & \\
\hline Iron ++ (mg/L) & & & & & & & & & \\
\hline Manganese ++ (mg/L) & & & & & & & & & \\
\hline $\mathrm{pH}$ & 6.9 & 6.62 & 7 & 7.53 & 7.39 & 7.87 & 7.98 & 7.03 & 6.93 \\
\hline \multicolumn{10}{|l|}{ Miscellaneous Analytes } \\
\hline Conductivity $(\mu \mathrm{mho} / \mathrm{cm})$ & 425 & & 484 & & & & & 716 & 701 \\
\hline Dissolved Solids (mg/L) & 231 & 246 & 274 & 237 & & 210 & 204 & 460 & 426 \\
\hline Suspended Solids (mg/L) & $<$ & $<$ & $<$ & $<$ & & $<$ & $<$ & $<$ & $<$ \\
\hline Turbidity (NTU) & 1.94 & & 0.401 & & & & & 0.75 & 0.553 \\
\hline $\mathrm{pH}$ & 7.39 & & 7.53 & & & & & 7.5 & 7.27 \\
\hline \multicolumn{10}{|l|}{ Major lons (mg/L) } \\
\hline Calcium & 72.3 & 69.6 & 77.5 & 61.7 & & 39.8 & 42.7 & 134 & 129 \\
\hline Magnesium & 10.2 & 10.7 & 14.4 & $<$ & & 16.3 & 17.3 & 9.79 & 9.88 \\
\hline Potassium & $<$ & 2.02 & 2.33 & $<$ & & 1.54 & 1.81 & 2.27 & 2.18 \\
\hline Sodium & 3.11 & 3.18 & 3.06 & 3.3 & & 2.28 & 2.54 & 8.63 & 6.24 \\
\hline Alkalinity as $\mathrm{CO} 3$ & $<$ & $<$ & $<$ & $<$ & & $<$ & $<$ & $<$ & $<$ \\
\hline Alkalinity as $\mathrm{HCO} 3$ & 208 & 212 & 232 & 213 & & 150 & 144 & 346 & 334 \\
\hline Chloride & 4.12 & 4.2 & 3.99 & 2.8 & & 10 & 12.7 & 15.7 & 14.9 \\
\hline Fluoride & & $<$ & $<$ & $<$ & & 0.18 & 0.22 & $<$ & $<$ \\
\hline Nitrate as $\mathrm{N}$ & 0.681 & 0.081 & 0.738 & 0.84 & & 0.46 & 0.41 & 0.551 & 0.323 \\
\hline Sulfate & 10.5 & 10.6 & 11.1 & 9.3 & & 8.5 & 8.4 & 35 & 27.3 \\
\hline Charge balance & 0.5 & -0.4 & 2.1 & -6.6 & & -0.5 & 3.6 & -1.2 & -0.7 \\
\hline \multicolumn{10}{|l|}{ Trace Metals (mg/L) } \\
\hline Aluminum & $<$ & $<$ & $<$ & $<$ & & $<$ & $<$ & $<$ & $<$ \\
\hline Arsenic (PMS) & $<$ & & $<$ & . & & & & $<$ & $<$ \\
\hline Arsenic & & $<$ & & $<$ & & $<$ & $<$ & & \\
\hline Barium & 0.0295 & 0.0301 & 0.0369 & $<$ & & 0.0231 & 0.0238 & 0.295 & 0.299 \\
\hline Beryllium & $<$ & $<$ & $<$ & $<$ & & $<$ & $<$ & $<$ & $<$ \\
\hline Boron & $<$ & $<$ & $<$ & $<$ & & $<$ & $<$ & $<$ & $<$ \\
\hline Cadmium (PMS) & $<$ & & $<$ & & & & & $<$ & $<$ \\
\hline Cadmium & & $<$ & & $<$ & & $<$ & $<$ & 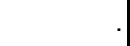 & . \\
\hline Chromium (PMS) & 0.00403 & & $<$ & & & & & $<$ & $<$ \\
\hline Chromium & & $<$ & & $<$ & & $<$ & $<$ & . & . \\
\hline Cobalt & $<$ & $<$ & $<$ & $<$ & & $<$ & $<$ & $<$ & $<$ \\
\hline Copper & $<$ & $<$ & $<$ & $<$ & & $<$ & $<$ & $<$ & $<$ \\
\hline Iron & 0.22 & $<$ & 0.0847 & $<$ & & $<$ & $<$ & $<$ & $<$ \\
\hline Lead (PMS) & $<$ & & $<$ & . & & $\cdot 1$ &. & $<$ & $<$ \\
\hline Lead & & $<$ & & $<$ & & $<$ & $<$ & & . \\
\hline Lithium & $<$ & $<$ & $<$ & $<$ & & $<$ & $<$ & $<$ & $<$ \\
\hline Manganese & 0.00508 & $<$ & $<$ & $<$ & & $<$ & 0.0054 & $<$ & $<$ \\
\hline Mercury (CVAA) & $<$ & & $<$ & $<$ & & $<$ & $<$ & $<$ & $<$ \\
\hline Nickel (PMS) & $<$ & & $<$ & & & & & $<$ & $<$ \\
\hline Nickel & & $<$ & & $<$ & & $<$ & 0.0109 & & \\
\hline Strontium & 0.0686 & 0.0676 & 0.0724 & $<$ & & 0.0956 & 0.0993 & 0.275 & 0.287 \\
\hline Thallium (PMS) & & & $<$ & . & & & & $<$ & $<$ \\
\hline Thallium & & $<$ & & $<$ & & $<$ & $<$ & & \\
\hline Uranium (PMS) & $<$ & & $<$ & . & & & & $<$ & $<$ \\
\hline Uranium (KPA) & & $<$ & & $<$ & & $<$ & $<$ & . & \\
\hline Vanadium & $<$ & $<$ & $<$ & $<$ & & $<$ & $<$ & $<$ & $<$ \\
\hline Zinc & 0.113 & $<$ & 0.0648 & $<$ & & $<$ & $<$ & $<$ & $<$ \\
\hline
\end{tabular}


APPENDIX E.1: CY 2002 MONITORING DATA FOR THE UPPER EAST FORK POPLAR CREEK HYDROGEOLOGIC REGIME

Field Measurements, Miscellaneous Analytes, Major Ions, and Trace Metals

\begin{tabular}{|c|c|c|c|c|c|c|c|c|}
\hline Sampling Point & \multicolumn{2}{|c|}{ GW-744 } & \multicolumn{2}{|c|}{ GW-747 } & \multicolumn{2}{|c|}{ GW-750 } & \multicolumn{2}{|c|}{ GW-762 } \\
\hline Location & \multicolumn{2}{|c|}{ GRIDK1 } & \multicolumn{2}{|c|}{ GRIDK2 } & \multicolumn{2}{|c|}{ EXP-J } & \multirow{2}{*}{\multicolumn{2}{|c|}{$\begin{array}{c}\text { GRIDJ3 } \\
01 / 31 / 02\end{array}$}} \\
\hline Date Sampled & $05 / 07 / 02$ & $11 / 18 / 02$ & 05/09/02 & $11 / 20 / 02$ & 05/05/02 & $11 / 20 / 02$ & & \\
\hline Program & GWPP & GWPP & GWPP & GWPP & GWPP & GWPP & BJC & BJC \\
\hline Sample Type & & & & & & & & Dup \\
\hline Field Measurements & & & & & & & & \\
\hline Time Sampled & $10: 25$ & $10: 45$ & $8: 50$ & $10: 20$ & $10: 00$ & $11: 10$ & $12: 55$ & \\
\hline Measuring Point Elev. (ft) & 907.60 & 907.60 & 921.14 & 921.14 & 919.03 & 919.03 & 915.34 & \\
\hline Depth to Water (ft) & 5.40 & 5.79 & 3.62 & 3.43 & 10.97 & 11.81 & 13.42 & \\
\hline Groundwater Elevation (ft) & 902.20 & 901.81 & 917.52 & 917.71 & 908.06 & 907.22 & 901.92 & \\
\hline Conductivity $(\mu \mathrm{mho} / \mathrm{cm})$ & 498 & 629 & 460 & 490 & 565 & 599 & 638 & \\
\hline Dissolved Oxygen (ppm) & 2.14 & 0.1 & 2.13 & -0.02 & 2.38 & 0.09 & 3.31 & \\
\hline Oxidation/Reduction (mV) & -234 & -204 & 58 & 6 & -43 & -34 & 180 & \\
\hline Temperature (degrees C) & 17.5 & 17.1 & 17 & 16.2 & 16.8 & 15 & 19 & \\
\hline Turbidity (NTU) & & & & & & & 12 & \\
\hline Iron ++ (mg/L) & & & & & & & 0.04 & \\
\hline Manganese ++ (mg/L) & & & & & & & 0.1 & \\
\hline $\mathrm{pH}$ & 7.76 & 7.75 & 7.09 & 7.57 & 6.62 & 7.5 & 6.86 & \\
\hline Miscellaneous Analytes & & & & & & & & \\
\hline Conductivity $(\mu \mathrm{mho} / \mathrm{cm})$ & 456 & 502 & 415 & 402 & 514 & 494 & & \\
\hline Dissolved Solids (mg/L) & 293 & 317 & 244 & 269 & 303 & 312 & 309 & 394 \\
\hline Suspended Solids (mg/L) & $<$ & $<$ & $<$ & $<$ & $<$ & $<$ & $<$ & $<$ \\
\hline Turbidity (NTU) & 0.436 & 2.51 & 0.166 & 0.219 & 1.58 & 1.19 & & \\
\hline $\mathrm{pH}$ & 7.57 & 7.54 & 7.95 & 7.92 & 7.67 & 7.77 & & \\
\hline Major lons (mg/L) & & & & & & & & \\
\hline Calcium & 45.1 & 57.5 & 46.7 & 45 & 83.5 & 84.5 & 81.4 & 81.2 \\
\hline Magnesium & 11.2 & 10.9 & 10.7 & 10.6 & 11.9 & 12.1 & 24 & 24 \\
\hline Potassium & 3.39 & 3.15 & $<$ & 2.05 & 4.49 & 4.76 & 3.92 & 3.88 \\
\hline Sodium & 35.7 & 34.2 & 27 & 26.8 & 6.04 & 6.36 & 10.4 & 10.4 \\
\hline Alkalinity as $\mathrm{CO} 3$ & $<$ & $<$ & $<$ & $<$ & $<$ & $<$ & $<$ & $<$ \\
\hline Alkalinity as $\mathrm{HCO} 3$ & 218 & 228 & 200 & 196 & 242 & 248 & 308 & 321 \\
\hline Chloride & 8.3 & 9.64 & 2.13 & 1.79 & 5.51 & 5.2 & 34.9 & 39.2 \\
\hline Fluoride & $<$ & $<$ & 0.137 & 0.164 & $<$ & $<$ & $<$ & $<$ \\
\hline Nitrate as $\mathrm{N}$ & $<$ & $<$ & $<$ & $<$ & $<$ & $<$ & 0.069 & 0.037 \\
\hline Sulfate & 13 & 20 & 17.1 & 15.8 & 20.9 & 18.1 & 15.5 & 14.8 \\
\hline Charge balance & -0.5 & 0.8 & -0.4 & 0.4 & 0.9 & 1.2 & -6.2 & \\
\hline Trace Metals (mg/L) & & & & & & & & \\
\hline Aluminum & $<$ & $<$ & $<$ & $<$ & $<$ & $<$ & $<$ & $<$ \\
\hline Arsenic (PMS) & $<$ & $<$ & $<$ & $<$ & $<$ & $<$ & 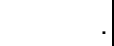 & 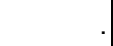 \\
\hline Arsenic & & & & & & & $<$ & $<$ \\
\hline Barium & 0.282 & 0.259 & 0.153 & 0.153 & 0.729 & 0.772 & 0.515 & 0.513 \\
\hline Beryllium & $<$ & $<$ & $<$ & $<$ & $<$ & $<$ & $<$ & $<$ \\
\hline Boron & $<$ & $<$ & $<$ & $<$ & $<$ & $<$ & $<$ & $<$ \\
\hline Cadmium (PMS) & $<$ & $<$ & $<$ & $<$ & $<$ & $<$ & & \\
\hline Cadmium & & & & & & & $<$ & $<$ \\
\hline Chromium (PMS) & $<$ & $<$ & $<$ & $<$ & $<$ & $<$ & & \\
\hline Chromium & & & & & & & $<$ & $<$ \\
\hline Cobalt & $<$ & $<$ & $<$ & $<$ & $<$ & $<$ & $<$ & $<$ \\
\hline Copper & $<$ & $<$ & $<$ & $<$ & $<$ & $<$ & $<$ & $<$ \\
\hline Iron & $<$ & 0.282 & $<$ & $<$ & 0.178 & 0.159 & $<$ & $<$ \\
\hline Lead (PMS) & $<$ & 0.0144 & $<$ & $<$ & $<$ & $<$ & . & . \\
\hline Lead & & & & & & & $<$ & $<$ \\
\hline Lithium & 0.0274 & 0.0272 & 0.015 & 0.0144 & $<$ & 0.0104 & 0.016 & 0.016 \\
\hline Manganese & 0.0395 & 0.132 & 0.0144 & 0.014 & 0.054 & 0.0555 & 0.057 & 0.057 \\
\hline Mercury (CVAA) & $<$ & $<$ & $<$ & $<$ & $<$ & $<$ & & \\
\hline Nickel (PMS) & $<$ & $<$ & $<$ & $<$ & $<$ & $<$ & & \\
\hline Nickel & & & & & & & $<$ & $<$ \\
\hline Strontium & 1.29 & 1.24 & 0.615 & 0.613 & 0.693 & 0.707 & 0.736 & 0.731 \\
\hline Thallium (PMS) & $<$ & $<$ & $<$ & $<$ & $<$ & $<$ & & \\
\hline Thallium & & & & & & & $<$ & $<$ \\
\hline Uranium (PMS) & $<$ & $<$ & $<$ & $<$ & $<$ & $<$ & & \\
\hline Uranium (KPA) & & & & & & & $<$ & $<$ \\
\hline Vanadium & $<$ & $<$ & $<$ & $<$ & $<$ & $<$ & $<$ & $<$ \\
\hline Zinc & $<$ & $<$ & $<$ & $<$ & $<$ & $<$ & $<$ & $<$ \\
\hline
\end{tabular}


APPENDIX E.1: CY 2002 MONITORING DATA FOR THE UPPER EAST FORK POPLAR CREEK HYDROGEOLOGIC REGIME

Field Measurements, Miscellaneous Analytes, Major lons, and Trace Metals

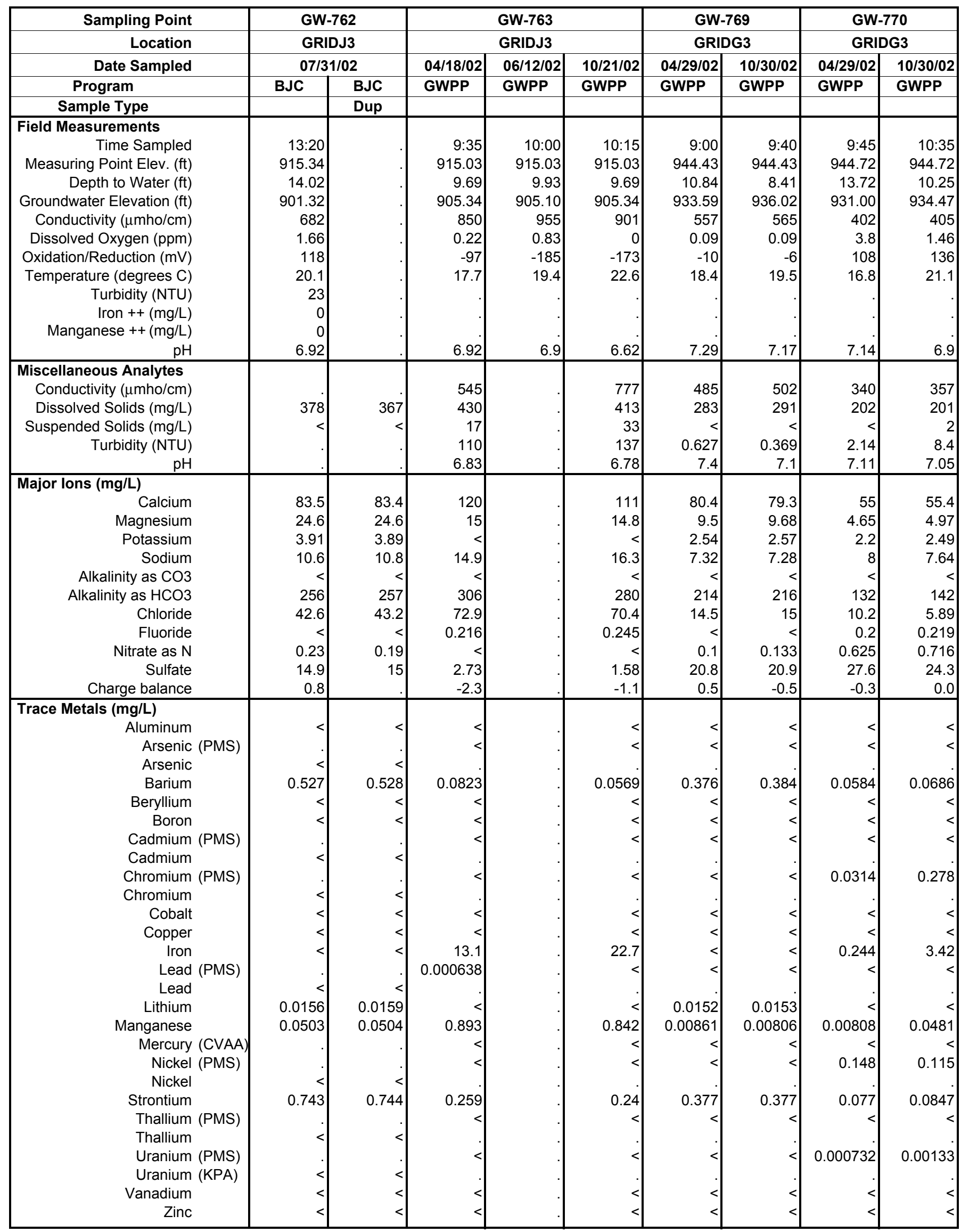


APPENDIX E.1: CY 2002 MONITORING DATA FOR THE UPPER EAST FORK POPLAR CREEK HYDROGEOLOGIC REGIME

Field Measurements, Miscellaneous Analytes, Major Ions, and Trace Metals

\begin{tabular}{|c|c|c|c|c|c|c|c|c|}
\hline Sampling Point & \multicolumn{2}{|c|}{ GW-775 } & \multicolumn{2}{|c|}{ GW-776 } & \multicolumn{2}{|c|}{ GW-782 } & \multicolumn{2}{|c|}{ GW-791 } \\
\hline Location & \multicolumn{2}{|c|}{ GRIDH3 } & \multicolumn{2}{|c|}{ GRIDH3 } & \multicolumn{2}{|c|}{ GRIDE3 } & \multicolumn{2}{|c|}{ GRIDD2 } \\
\hline Date Sampled & $04 / 30 / 02$ & $10 / 31 / 02$ & $04 / 30 / 02$ & $10 / 31 / 02$ & $05 / 01 / 02$ & $11 / 04 / 02$ & $05 / 01 / 02$ & $11 / 06 / 02$ \\
\hline Program & GWPP & GWPP & GWPP & GWPP & GWPP & GWPP & GWPP & GWPP \\
\hline \multicolumn{9}{|l|}{ Sample Type } \\
\hline \multicolumn{9}{|l|}{ Field Measurements } \\
\hline Time Sampled & $9: 00$ & $10: 00$ & $10: 00$ & $11: 00$ & $8: 40$ & $9: 00$ & $10: 20$ & $11: 00$ \\
\hline Measuring Point Elev. (ft) & 931.35 & 931.35 & 931.25 & 931.25 & 947.73 & 947.73 & 992.13 & 992.13 \\
\hline Depth to Water (ft) & 14.31 & 9.97 & 14.39 & 10.13 & 8.55 & 9.24 & 23.59 & 23.09 \\
\hline Groundwater Elevation (ft) & 917.04 & 921.38 & 916.86 & 921.12 & 939.18 & 938.49 & 968.54 & 969.04 \\
\hline Conductivity $(\mu \mathrm{mho} / \mathrm{cm})$ & 622 & 615 & 618 & 656 & 588 & 629 & 507 & 561 \\
\hline Dissolved Oxygen (ppm) & 0.2 & 0.55 & 2.25 & 1.95 & 0.03 & 0.01 & 1.02 & 5.09 \\
\hline Oxidation/Reduction (mV) & 49 & 119 & 48 & 38 & 28 & -44 & 2 & -41 \\
\hline Temperature (degrees C) & 18.3 & 18.7 & 18.1 & 18.9 & 17.1 & 18 & 19.3 & 17.7 \\
\hline \multirow{3}{*}{\multicolumn{9}{|c|}{$\begin{array}{r}\text { Turbidity (NTU) } \\
\text { Iron ++ }(\mathrm{mg} / \mathrm{L}) \\
\text { Manganese }++(\mathrm{mg} / \mathrm{L})\end{array}$}} \\
\hline & & & & & & & & \\
\hline & & & & & & & & \\
\hline $\mathrm{pH}$ & 7.44 & 6.9 & 7.3 & 7.07 & 7.04 & 7.33 & 7.17 & 7.12 \\
\hline \multicolumn{9}{|l|}{ Miscellaneous Analytes } \\
\hline Conductivity $(\mu \mathrm{mho} / \mathrm{cm})$ & 556 & 553 & 554 & 581 & 521 & 529 & 460 & 457 \\
\hline Dissolved Solids (mg/L) & 316 & 319 & 325 & 337 & 299 & 296 & 268 & 274 \\
\hline Suspended Solids (mg/L) & $<$ & $<$ & $<$ & $<$ & 2 & 2 & $<$ & $<$ \\
\hline Turbidity (NTU) & 0.292 & 0.316 & 8.31 & 2.01 & 6.91 & 7.58 & 0.157 & 0.324 \\
\hline $\mathrm{pH}$ & 7.35 & 7.44 & 7.27 & 7.17 & 7.48 & 7.43 & 7.7 & 7.53 \\
\hline \multicolumn{9}{|l|}{ Major lons (mg/L) } \\
\hline Calcium & 95.4 & 94.1 & 94 & 101 & 77.2 & 77.8 & 67 & 70.2 \\
\hline Magnesium & 8.04 & 7.85 & 5.3 & 4.74 & 17.2 & 16.7 & 14.5 & 13.9 \\
\hline Potassium & 2.59 & 2.41 & 3.59 & 2.79 & 5.28 & 5.46 & 2.01 & $<$ \\
\hline Sodium & 4.86 & 5.63 & 13.6 & 12.2 & 9.25 & 9.38 & 7.11 & 7.11 \\
\hline Alkalinity as $\mathrm{CO} 3$ & $<$ & $<$ & $<$ & $<$ & $<$ & $<$ & $<$ & $<$ \\
\hline Alkalinity as $\mathrm{HCO} 3$ & 206 & 190 & 195 & 206 & 242 & 242 & 222 & 216 \\
\hline Chloride & 30.6 & 30.8 & 32 & 30.7 & 12.8 & 11.6 & 7.6 & 7.73 \\
\hline Fluoride & & $<$ & $<$ & $<$ & $<$ & $<$ & $<$ & $<$ \\
\hline Nitrate as $\mathrm{N}$ & 0.383 & 0.657 & 2.91 & 1.3 & $<$ & $<$ & $<$ & $<$ \\
\hline Sulfate & 30.4 & 33.8 & 37.9 & 36.6 & 16.8 & 15.4 & 12.5 & 12.1 \\
\hline Charge balance & 0.5 & 2.1 & 0.1 & 1.6 & 2.3 & 2.8 & -0.1 & 1.7 \\
\hline Trace Metals (mg/L) & & & & & & & & \\
\hline Aluminum & $<$ & $<$ & $<$ & $<$ & $<$ & $<$ & $<$ & $<$ \\
\hline Arsenic (PMS) & $<$ & $<$ & $<$ & $<$ & $<$ & $<$ & $<$ & $<$ \\
\hline Arsenic & & & & & & & & \\
\hline Barium & 0.2 & 0.203 & 0.0814 & 0.0822 & 0.544 & 0.549 & 0.249 & 0.247 \\
\hline Beryllium & $<$ & $<$ & $<$ & $<$ & $<$ & $<$ & $<$ & $<$ \\
\hline Boron & $<$ & $<$ & $<$ & $<$ & 0.139 & 0.13 & $<$ & $<$ \\
\hline Cadmium (PMS) & $<$ & $<$ & $<$ & $<$ & $<$ & $<$ & $<$ & $<$ \\
\hline Cadmium & & & & & & & & \\
\hline Chromium (PMS) & $<$ & $<$ & 0.0952 & 0.0432 & $<$ & $<$ & $<$ & $<$ \\
\hline Chromium & & & & & & & & . \\
\hline Cobalt & $<$ & $<$ & $<$ & $<$ & $<$ & $<$ & $<$ & $<$ \\
\hline Copper & $<$ & $<$ & $<$ & $<$ & $<$ & $<$ & $<$ & $<$ \\
\hline Iron & $<$ & $<$ & 0.877 & 0.264 & 1.08 & 1.35 & $<$ & 0.063 \\
\hline Lead (PMS) & 0.000837 & 0.0105 & $<$ & $<$ & 0.00243 & $<$ & $<$ & 0.00151 \\
\hline Lead & & & & & & & & \\
\hline Lithium & 0.0114 & 0.0101 & $<$ & $<$ & 0.019 & 0.0201 & 0.0128 & 0.0132 \\
\hline Manganese & $<$ & $<$ & 0.00952 & 0.0107 & 0.0434 & 0.0482 & 0.0154 & 0.0186 \\
\hline Mercury (CVAA) & $<$ & $<$ & $<$ & $<$ & $<$ & $<$ & $<$ & $<$ \\
\hline Nickel (PMS) & $<$ & $<$ & 0.546 & 0.204 & $<$ & $<$ & $<$ & $<$ \\
\hline Nickel & & & & & & & & \\
\hline Strontium & 0.244 & 0.242 & 0.158 & 0.16 & 1.17 & 1.16 & 0.407 & 0.392 \\
\hline Thallium (PMS) & $<$ & $<$ & $<$ & $<$ & $<$ & $<$ & $<$ & $<$ \\
\hline Thallium & & & & & & & & \\
\hline Uranium (PMS) & $<$ & $<$ & $<$ & $<$ & 0.00169 & 0.00124 & $<$ & $<$ \\
\hline Uranium (KPA) & & & & & & & & \\
\hline Vanadium & $<$ & $<$ & $<$ & $<$ & $<$ & $<$ & $<$ & $<$ \\
\hline Zinc & $<$ & $<$ & $<$ & $<$ & $<$ & $<$ & $<$ & $<$ \\
\hline
\end{tabular}


APPENDIX E.1: CY 2002 MONITORING DATA FOR THE UPPER EAST FORK POPLAR CREEK HYDROGEOLOGIC REGIME

Field Measurements, Miscellaneous Analytes, Major Ions, and Trace Metals

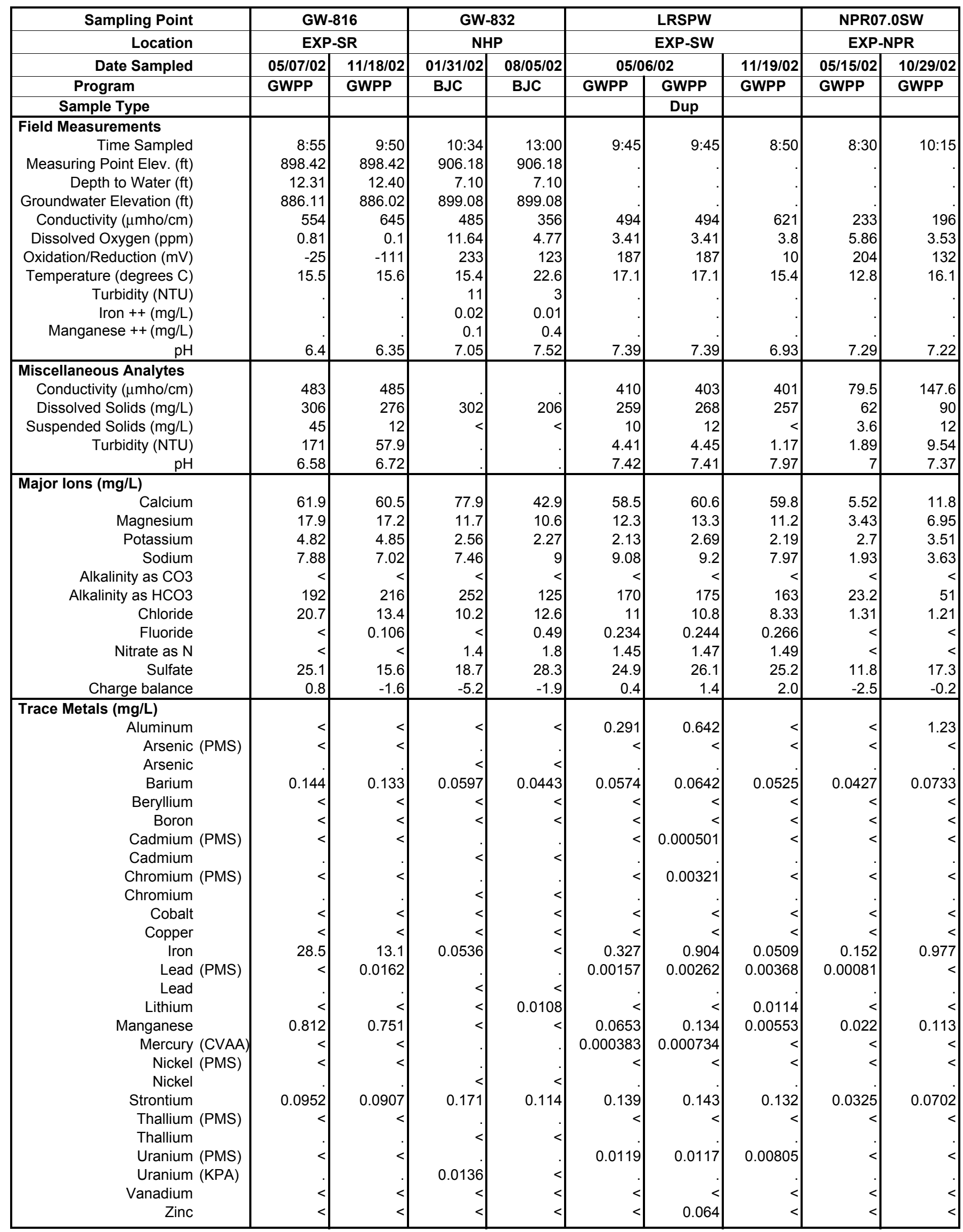


APPENDIX E.1: CY 2002 MONITORING DATA FOR THE UPPER EAST FORK POPLAR CREEK HYDROGEOLOGIC REGIME

Field Measurements, Miscellaneous Analytes, Major Ions, and Trace Metals

\begin{tabular}{|c|c|c|c|c|c|c|c|}
\hline Sampling Point & \multicolumn{3}{|c|}{ NPR12.0SW } & \multicolumn{2}{|c|}{ NPR23.0SW } & \multicolumn{2}{|c|}{ OF 51} \\
\hline Location & \multicolumn{3}{|c|}{ EXP-NPR } & \multicolumn{2}{|c|}{ EXP-NPR } & \multicolumn{2}{|c|}{ EXP-SW } \\
\hline Date Sampled & \multicolumn{2}{|c|}{$05 / 15 / 02$} & $10 / 29 / 02$ & $05 / 15 / 02$ & $10 / 29 / 02$ & $02 / 14 / 02$ & 09/03/02 \\
\hline Program & GWPP & GWPP & GWPP & GWPP & GWPP & BJC & BJC \\
\hline Sample Type & & Dup & & & & & \\
\hline Field Measurements & & & & & & & \\
\hline Time Sampled & 9:00 & 9:00 & $9: 55$ & 9:30 & $9: 30$ & $10: 31$ & $13: 45$ \\
\hline Measuring Point Elev. (ft) & & & & & & & \\
\hline Depth to Water (ft) & & & & & & & \\
\hline Groundwater Elevation (ft) & & & & & & & \\
\hline Conductivity $(\mu \mathrm{mho} / \mathrm{cm})$ & 98.1 & 98.1 & 186 & 166.4 & 124 & 242 & 223 \\
\hline Dissolved Oxygen (ppm) & 4.71 & 4.71 & 3.58 & 4.26 & 3.78 & 10.6 & 8.81 \\
\hline Oxidation/Reduction (mV) & 209 & 209 & 174 & 190 & 188 & 85 & 35 \\
\hline Temperature (degrees $\mathrm{C}$ ) & 13 & 13 & 16.1 & 13.6 & 16 & 14 & 19.3 \\
\hline Turbidity (NTU) & & & & & & 1 & 8 \\
\hline Iron ++ (mg/L) & & & & & & & \\
\hline Manganese ++ (mg/L) & & & & & & & \\
\hline $\mathrm{pH}$ & 6.86 & 6.86 & 7 & 7.03 & 6.88 & 8.1 & 7.76 \\
\hline Miscellaneous Analytes & & & & & & & \\
\hline Conductivity ( $\mu \mathrm{mho} / \mathrm{cm})$ & 82.4 & 81.6 & 140.4 & 71.9 & 124 & & \\
\hline Dissolved Solids (mg/L) & 64 & 79 & 90 & 68 & 83 & 139 & 257 \\
\hline Suspended Solids (mg/L) & 122 & 19.6 & 29 & 2.8 & 17 & $<$ & $<$ \\
\hline Turbidity (NTU) & 8.82 & 8.56 & 8.68 & 5.03 & 12.5 & & \\
\hline $\mathrm{pH}$ & 7.5 & 7.51 & 7.4 & 7.36 & 7.27 & & \\
\hline Major lons (mg/L) & & & & & & & \\
\hline Calcium & 7.02 & 6.46 & 12.1 & 5.72 & 10.5 & 56.6 & 54.2 \\
\hline Magnesium & 3.64 & 3.4 & 6.33 & 2.95 & 3.97 & 25.3 & 17.7 \\
\hline Potassium & 3.05 & 2.72 & 3.15 & 2.55 & 3.01 & 1.62 & 1.99 \\
\hline Sodium & 2.31 & 2.22 & 3.85 & 1.48 & 1.99 & 5.81 & 7.75 \\
\hline Alkalinity as $\mathrm{CO} 3$ & $<$ & $<$ & $<$ & $<$ & $<$ & $<$ & $<$ \\
\hline Alkalinity as $\mathrm{HCO} 3$ & 27.8 & 28.8 & 54.6 & 23.6 & 41 & 242 & 196 \\
\hline Chloride & 1.06 & 1.13 & 1.21 & 0.99 & 0.82 & 9.2 & 11 \\
\hline Fluoride & $<$ & $<$ & $<$ & $<$ & $<$ & 0.15 & 0.29 \\
\hline Nitrate as $\mathrm{N}$ & $<$ & $<$ & $<$ & $<$ & $<$ & 4.4 & 4.9 \\
\hline Sulfate & 9.88 & 9.92 & 12.9 & 9.7 & 10.6 & 17.1 & 25.6 \\
\hline Charge balance & 2.3 & -2.9 & -0.8 & -3.2 & -2.4 & -5.2 & -6.0 \\
\hline Trace Metals (mg/L) & & & & & & & \\
\hline Aluminum & 0.778 & 0.278 & 0.783 & 0.412 & 1.61 & $<$ & $<$ \\
\hline Arsenic (PMS) & & $<$ & $<$ & $<$ & $<$ & . & \\
\hline Arsenic & & & & & & $<$ & $<$ \\
\hline Barium & 0.066 & 0.048 & 0.0742 & 0.036 & 0.054 & 0.0804 & 0.0704 \\
\hline Beryllium & $<$ & $<$ & $<$ & $<$ & $<$ & $<$ & $<$ \\
\hline Boron & $<$ & $<$ & $<$ & $<$ & $<$ & $<$ & $<$ \\
\hline Cadmium (PMS) & $<$ & $<$ & $<$ & $<$ & $<$ & . & \\
\hline Cadmium & & & & & & $<$ & $<$ \\
\hline Chromium (PMS) & 0.00253 & $<$ & $<$ & 0.00278 & 0.00338 & . & \\
\hline Chromium & & & & & & $<$ & $<$ \\
\hline Cobalt & $<$ & $<$ & $<$ & $<$ & $<$ & $<$ & $<$ \\
\hline Copper & & $<$ & & $<$ & $<$ & 0.0209 & $<$ \\
\hline Iron & 0.952 & 0.356 & 0.723 & 0.279 & 1.03 & $<$ & 0.105 \\
\hline Lead (PMS) & 0.00136 & 0.000641 & 0.00156 & 0.00074 & $<$ & . & \\
\hline Lead & & & & & & $<$ & $<$ \\
\hline Lithium & $<$ & $<$ & $<$ & $<$ & $<$ & $<$ & 0.0114 \\
\hline Manganese & 0.127 & 0.0324 & 0.0854 & 0.0112 & 0.0356 & 0.0078 & 0.0123 \\
\hline Mercury (CVAA) & $<$ & $<$ & $<$ & $<$ & $<$ & & \\
\hline Nickel (PMS) & $<$ & $<$ & $<$ & $<$ & $<$ & . & \\
\hline Nickel & & & & & & $<$ & \\
\hline Strontium & 0.0356 & 0.0324 & 0.0626 & 0.023 & 0.031 & 0.0893 & 0.128 \\
\hline Thallium (PMS) & $<$ & $<$ & $<$ & $<$ & $<$ & & \\
\hline Thallium & & & & & & $<$ & $<$ \\
\hline Uranium (PMS) & $<$ & $<$ & $<$ & $<$ & $<$ & & \\
\hline Uranium (KPA) & & & & & & 0.00892 & 0.00881 \\
\hline Vanadium & $<$ & $<$ & $<$ & $<$ & $<$ & $<$ & $<$ \\
\hline Zinc & $<$ & $<$ & $<$ & $<$ & $<$ & $<$ & 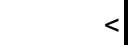 \\
\hline
\end{tabular}


APPENDIX E.1: CY 2002 MONITORING DATA FOR THE UPPER EAST FORK POPLAR CREEK HYDROGEOLOGIC REGIME

Field Measurements, Miscellaneous Analytes, Major Ions, and Trace Metals

\begin{tabular}{|c|c|c|c|c|c|c|c|c|}
\hline Sampling Point & \multicolumn{4}{|c|}{ OF 200} & \multicolumn{2}{|c|}{ SCR7.1SP } & \multicolumn{2}{|c|}{ SCR7.8SP } \\
\hline Location & \multicolumn{4}{|c|}{ EXP-SW } & \multicolumn{2}{|c|}{ EXP-UV } & \multicolumn{2}{|c|}{ EXP-UV } \\
\hline Date Sampled & $02 / 01 / 02$ & $02 / 14 / 02$ & $08 / 20 / 02$ & 09/03/02 & $02 / 11 / 02$ & 07/11/02 & $02 / 11 / 02$ & 07/11/02 \\
\hline Program & BJC & BJC & BJC & BJC & BJC & BJC & BJC & BJC \\
\hline \multicolumn{9}{|l|}{ Sample Type } \\
\hline \multicolumn{9}{|l|}{ Field Measurements } \\
\hline Time Sampled & $13: 13$ & $9: 54$ & $13: 18$ & 13:00 & $14: 05$ & $9: 49$ & $14: 18$ & $9: 30$ \\
\hline Measuring Point Elev. (ft) & & & & & & & & \\
\hline Depth to Water (ft) & & & & & & & & \\
\hline Groundwater Elevation (ft) & & & & & & & & \\
\hline Conductivity $(\mu \mathrm{mho} / \mathrm{cm})$ & 174 & 221 & 191 & 142 & 292 & 212 & 194 & 204 \\
\hline Dissolved Oxygen (ppm) & 9.82 & 9.3 & 7.3 & 6.16 & 11.79 & 10.59 & 11.82 & 11.2 \\
\hline Oxidation/Reduction (mV) & 175 & 115 & 55 & 40 & 20 & 95 & 45 & 85 \\
\hline Temperature (degrees C) & 17.6 & 15.9 & 24 & 25.4 & 11.7 & 16.7 & 12.5 & 17.1 \\
\hline Turbidity (NTU) & 7 & 4 & 4 & 33 & 35 & 22 & 3 & 45 \\
\hline Iron ++ (mg/L) & & & & & 0.02 & 0.03 & 0 & 0.01 \\
\hline Manganese ++ (mg/L) & & & & & 0 & 0.1 & 0.2 & 0.1 \\
\hline $\mathrm{pH}$ & 7.7 & 8.9 & 8 & 7.86 & 8.9 & 7.93 & 8.87 & 8.05 \\
\hline \multirow{2}{*}{\multicolumn{9}{|c|}{$\begin{array}{l}\text { Miscellaneous Analytes } \\
\text { Conductivity }(\mu \mathrm{mho} / \mathrm{cm})\end{array}$}} \\
\hline & & & & & & & & \\
\hline Dissolved Solids (mg/L) & 247 & 216 & 242 & 238 & 272 & 282 & 194 & 261 \\
\hline Suspended Solids (mg/L) & $<$ & $<$ & $<$ & $<$ & $<$ & 6.9 & $<$ & $<$ \\
\hline Turbidity (NTU) & & & & & & & & \\
\hline $\mathrm{pH}$ & & & & & & & & \\
\hline \multicolumn{9}{|l|}{ Major lons (mg/L) } \\
\hline Calcium & 53.4 & 52.7 & 49.7 & 45.4 & & & & \\
\hline Magnesium & 11.3 & 11.6 & 11.2 & 11 & & & & \\
\hline Potassium & 2.62 & 2.58 & 2.7 & 2.72 & & . & & \\
\hline Sodium & 9.97 & 10.8 & 9.65 & 9.16 & & & & \\
\hline Alkalinity as $\mathrm{CO} 3$ & $<$ & $<$ & $<$ & $<$ & $<$ & $<$ & $<$ & $<$ \\
\hline Alkalinity as $\mathrm{HCO} 3$ & 142 & 110 & 110 & 114 & 165 & 184 & 166 & 190 \\
\hline Chloride & 16.2 & 15.7 & 14.8 & 18.5 & 12.1 & 5.6 & 10.3 & 7.3 \\
\hline Fluoride & 0.61 & 0.87 & 1.1 & 1.1 & $<$ & $<$ & $<$ & $<$ \\
\hline Nitrate as $\mathrm{N}$ & 7.2 & 7.7 & 5.8 & 4.3 & 0.6 & 0.38 & 0.77 & 1.1 \\
\hline Sulfate & 32.5 & 36.2 & 35.2 & 33.2 & 27.4 & 19.8 & 12 & 9.9 \\
\hline Charge balance & -4.9 & 1.5 & 0.9 & -2.9 & & & & \\
\hline \multicolumn{9}{|l|}{ Trace Metals (mg/L) } \\
\hline Aluminum & $<$ & $<$ & $<$ & $<$ & & & & \\
\hline Arsenic (PMS) & & & & & & & & \\
\hline Arsenic & $<$ & $<$ & $<$ & $<$ & & & & \\
\hline Barium & 0.0572 & 0.0545 & 0.0558 & 0.0478 & & & & \\
\hline Beryllium & & $<$ & $<$ & $<$ & & & & \\
\hline Boron & 0.365 & $<$ & $<$ & $<$ & & & & \\
\hline Cadmium (PMS) & & & & & & & & \\
\hline Cadmium & $<$ & $<$ & $<$ & $<$ & & & & \\
\hline Chromium (PMS) & & & & & & & & \\
\hline Chromium & $<$ & $<$ & $<$ & $<$ & & & & \\
\hline Cobalt & $<$ & $<$ & $<$ & $<$ & & & & \\
\hline Copper & $<$ & $<$ & $<$ & $<$ & & & & \\
\hline Iron & 0.154 & 0.137 & 0.0656 & $<$ & & & & \\
\hline Lead (PMS) & & & & & & & & \\
\hline Lead & $<$ & $<$ & $<$ & $<$ & & & & \\
\hline Lithium & 0.126 & 0.0607 & 0.0279 & 0.0184 & & & & \\
\hline Manganese & 0.0594 & 0.0685 & 0.0388 & 0.0251 & & & & \\
\hline Mercury (CVAA) & & & & & & & & \\
\hline Nickel (PMS) & & & & & & & & \\
\hline Nickel & $<$ & & $<$ & $<$ & & & & \\
\hline Strontium & 0.154 & 0.155 & 0.146 & 0.135 & & & & \\
\hline Thallium (PMS) & & & & & & & & \\
\hline Thallium & $<$ & $<$ & $<$ & $<$ & & & & \\
\hline Uranium (PMS) & & & & & & & & \\
\hline Uranium (KPA) & 0.12 & 0.073 & 0.0344 & 0.00761 & & & & \\
\hline Vanadium & $<$ & $<$ & $<$ & $<$ & & & & \\
\hline Zinc & $<$ & $<$ & $<$ & $<$ & & & & \\
\hline
\end{tabular}


APPENDIX E.1: CY 2002 MONITORING DATA FOR THE UPPER EAST FORK POPLAR CREEK HYDROGEOLOGIC REGIME

Field Measurements, Miscellaneous Analytes, Major lons, and Trace Metals

\begin{tabular}{|c|c|c|c|c|c|c|c|c|}
\hline Sampling Point & \multicolumn{4}{|c|}{ STATION 17} & \multicolumn{4}{|c|}{ STATION 8} \\
\hline Location & \multicolumn{4}{|c|}{ EXP-SW } & \multicolumn{4}{|c|}{ EXP-SW } \\
\hline Date Sampled & $02 / 01 / 02$ & $02 / 14 / 02$ & $08 / 20 / 02$ & 09/03/02 & $02 / 01 / 02$ & $02 / 14 / 02$ & $08 / 20 / 02$ & 09/03/02 \\
\hline Program & BJC & BJC & BJC & BJC & BJC & BJC & BJC & BJC \\
\hline \multicolumn{9}{|l|}{ Sample Type } \\
\hline Field Measurements & & & & & & & & \\
\hline Time Sampled & $13: 56$ & $10: 53$ & $12: 37$ & $14: 00$ & $13: 31$ & $10: 18$ & $13: 57$ & $13: 30$ \\
\hline Measuring Point Elev. (ft) & & & & & & & & \\
\hline Depth to Water (ft) & & & & & & & & \\
\hline Groundwater Elevation (ft) & & & & & & & & \\
\hline Conductivity $(\mu \mathrm{mho} / \mathrm{cm})$ & 136 & 170 & 186 & 161 & 135 & 169 & 154 & 153 \\
\hline Dissolved Oxygen (ppm) & 11.45 & 12.2 & 6.95 & 7.35 & 11.84 & 11.12 & 7.8 & 9.09 \\
\hline Oxidation/Reduction (mV) & 155 & 75 & 35 & 30 & 180 & 70 & 45 & 25 \\
\hline Temperature (degrees C) & 15.4 & 11.5 & 24.8 & 23.7 & 14.5 & 12.4 & 22.8 & 21.3 \\
\hline Turbidity (NTU) & 31 & 3 & 27 & 34 & 10 & 6 & 10 & 56 \\
\hline Iron ++ (mg/L) & & & & & & & & \\
\hline Manganese $++(\mathrm{mg} / \mathrm{L})$ & & & & & & & & \\
\hline $\mathrm{pH}$ & 8.09 & 8.31 & 7.77 & 7.93 & 7.88 & 8.38 & 7.93 & 7.88 \\
\hline Miscellaneous Analytes & & & & & & & & \\
\hline Conductivity $(\mu \mathrm{mho} / \mathrm{cm})$ & & & & & & & & \\
\hline Dissolved Solids (mg/L) & 201 & 191 & 194 & 194 & 203 & 188 & 200 & 201 \\
\hline Suspended Solids (mg/L) & $<$ & $<$ & $<$ & $<$ & $<$ & $<$ & $<$ & $<$ \\
\hline Turbidity (NTU) & & & & & & & & \\
\hline $\mathrm{pH}$ & & & & & & & & \\
\hline Major lons (mg/L) & & & & & & & & \\
\hline Calcium & 44 & 42.5 & 40.7 & 38.6 & 41.2 & 42 & 39.5 & 38.3 \\
\hline Magnesium & 11.5 & 11.7 & 10.2 & 9.76 & 10.7 & 11.1 & 9.88 & 9.76 \\
\hline Potassium & 2.32 & 2.11 & 2.25 & 2.2 & 2.24 & 2.22 & 2.21 & 2.13 \\
\hline Sodium & 9.93 & 9.59 & 9.42 & 8.23 & 9.35 & 9.31 & 7.67 & 7.35 \\
\hline Alkalinity as $\mathrm{CO} 3$ & $<$ & $<$ & $<$ & $<$ & $<$ & $<$ & $<$ & $<$ \\
\hline Alkalinity as $\mathrm{HCO} 3$ & 135 & 123 & 108 & 105 & 136 & 125 & 105 & 105 \\
\hline Chloride & 12.9 & 11.6 & 12.8 & 11.7 & 11.6 & 11.6 & 10.2 & 10.4 \\
\hline Fluoride & 0.24 & 0.36 & 0.6 & 0.45 & 0.25 & 0.36 & 0.54 & 0.39 \\
\hline Nitrate as $\mathrm{N}$ & 2.5 & 1.5 & 2.1 & 1.4 & 2.7 & 2.5 & 2.1 & 1.4 \\
\hline Sulfate & 31.3 & 31.4 & 30.4 & 27.9 & 31.3 & 32.3 & 28.4 & 27.2 \\
\hline Charge balance & -3.6 & -0.2 & 0.0 & 0.1 & -6.9 & -3.2 & 0.3 & 0.1 \\
\hline Trace Metals (mg/L) & & & & & & & & \\
\hline Aluminum & 0.325 & $<$ & $<$ & 0.211 & $<$ & $<$ & $<$ & $<$ \\
\hline Arsenic (PMS) & & & & & & & & \\
\hline Arsenic & $<$ & $<$ & $<$ & $<$ & $<$ & $<$ & $<$ & $<$ \\
\hline Barium & 0.0484 & 0.0424 & 0.0438 & 0.0395 & 0.0423 & 0.0407 & 0.0422 & 0.039 \\
\hline Beryllium & $<$ & $<$ & $<$ & $<$ & $<$ & $<$ & $<$ & $<$ \\
\hline Boron & $<$ & $<$ & $<$ & $<$ & 0.124 & $<$ & $<$ & $<$ \\
\hline Cadmium (PMS) & & & & & & &. & . \\
\hline Cadmium & $<$ & $<$ & $<$ & $<$ & $<$ & $<$ & $<$ & $<$ \\
\hline Chromium (PMS) & & & & & & & & . \\
\hline Chromium & $<$ & $<$ & $<$ & $<$ & $<$ & $<$ & $<$ & $<$ \\
\hline Cobalt & $<$ & $<$ & $<$ & $<$ & $<$ & $<$ & $<$ & $<$ \\
\hline Copper & $<$ & $<$ & $<$ & $<$ & $<$ & $<$ & $<$ & $<$ \\
\hline Iron & 0.394 & 0.137 & 0.176 & 0.176 & 0.243 & 0.172 & 0.266 & 0.239 \\
\hline Lead (PMS) & & & & & & & & \\
\hline Lead & $<$ & $<$ & $<$ & $<$ & $<$ & $<$ & $<$ & $<$ \\
\hline Lithium & 0.0334 & 0.0311 & 0.0113 & $<$ & 0.0428 & 0.0208 & 0.0127 & $<$ \\
\hline Manganese & 0.0462 & 0.0338 & 0.0453 & 0.0487 & 0.0645 & 0.0566 & 0.0892 & 0.08 \\
\hline Mercury (CVAA) & & & & & & & & \\
\hline Nickel (PMS) & & & & & & & & \\
\hline Nickel & $<$ & $<$ & $<$ & $<$ & $<$ & $<$ & $<$ & $<$ \\
\hline Strontium & 0.131 & 0.124 & 0.119 & 0.113 & 0.134 & 0.13 & 0.117 & 0.114 \\
\hline Thallium (PMS) & & & & & & & & \\
\hline Thallium & $<$ & $<$ & $<$ & $<$ & $<$ & $<$ & $<$ & $<$ \\
\hline Uranium (PMS) & & & & & & & & \\
\hline Uranium (KPA) & 0.0281 & 0.0172 & 0.00877 & $<$ & 0.04 & 0.0185 & 0.00958 & $<$ \\
\hline Vanadium & $<$ & $<$ & $<$ & $<$ & $<$ & $<$ & $<$ & $<$ \\
\hline Zinc & $<$ & $<$ & $<$ & $<$ & $<$ & $<$ & $<$ & $<$ \\
\hline
\end{tabular}


APPENDIX E.2

VOLATILE ORGANIC COMPOUNDS 
APPENDIX E.2: CY 2002 MONITORING DATA FOR THE UPPER EAST FORK POPLAR CREEK HYDROGEOLOGIC REGIME Volatile Organic Compounds

\begin{tabular}{|c|c|c|c|c|c|c|c|c|c|c|}
\hline Sampling Point & \multicolumn{3}{|c|}{$55-1 B$} & \multicolumn{2}{|c|}{ GHK2.51ESW } & \multicolumn{3}{|c|}{ GHK2.51WSW } & \multicolumn{2}{|c|}{ GW-108 } \\
\hline Location & \multicolumn{3}{|c|}{ GRIDB2 } & \multicolumn{2}{|c|}{ EXP-NPR } & \multicolumn{3}{|c|}{ EXP-NPR } & \multicolumn{2}{|c|}{ S3 } \\
\hline Date Sampled & \multicolumn{2}{|c|}{$04 / 17 / 02$} & \multirow{2}{*}{\begin{tabular}{|c|}
$10 / 14 / 02$ \\
GWPP \\
\end{tabular}} & \multirow{2}{*}{\begin{tabular}{|c|} 
05/15/02 \\
GWPP \\
\end{tabular}} & \multirow{2}{*}{$\begin{array}{c}10 / 29 / 02 \\
\text { GWPP }\end{array}$} & \multirow{2}{*}{$\begin{array}{c}05 / 15 / 02 \\
\text { GWPP }\end{array}$} & \multicolumn{2}{|c|}{$10 / 29 / 02$} & \multirow{2}{*}{$\begin{array}{c}01 / 08 / 02 \\
\text { BJC }\end{array}$} & \multirow{2}{*}{\begin{tabular}{|c}
$07 / 09 / 02$ \\
BJC
\end{tabular}} \\
\hline Program & GWPP & GWPP & & & & & GWPP & GWPP & & \\
\hline Sample Type & & Dup & & & & & & Dup & & \\
\hline Chloroethenes ( $\mu \mathrm{g} / \mathrm{L}$ ) & & & & & & & & & & \\
\hline Tetrachloroethene & $<$ & $<$ & $<$ & $<$ & $<$ & $<$ & $<$ & $<$ & $1 \mathrm{~J}$ & $2 \mathrm{~J}$ \\
\hline Trichloroethene & $<$ & $<$ & $<$ & $<$ & $<$ & $<$ & $<$ & $<$ & $3 \mathrm{~J}$ & $3 \mathrm{~J}$ \\
\hline cis-1,2-Dichloroethene & $<$ & $<$ & $<$ & $<$ & $<$ & $<$ & $<$ & $<$ & $<$ & $<$ \\
\hline trans-1,2-Dichloroethene & $<$ & $<$ & $<$ & $<$ & $<$ & $<$ & $<$ & $<$ & $<$ & $<$ \\
\hline 1,1-Dichloroethene & $<$ & $<$ & $<$ & $<$ & $<$ & $<$ & $<$ & $<$ & $<$ & $<$ \\
\hline Vinyl chloride & $<$ & $<$ & $<$ & $<$ & $<$ & $<$ & $<$ & $<$ & $<$ & $<$ \\
\hline Chloroethanes $(\mu \mathrm{g} / \mathrm{L})$ & & & & & & & & & & \\
\hline 1,1,1-Trichloroethane & $<$ & $<$ & $<$ & $<$ & $<$ & $<$ & $<$ & $<$ & $<$ & $<$ \\
\hline 1,2-Dichloroethane & $<$ & $<$ & $<$ & $<$ & $<$ & $<$ & $<$ & $<$ & $<$ & $<$ \\
\hline 1,1-Dichloroethane & $<$ & $<$ & $<$ & $<$ & $<$ & $<$ & $<$ & $<$ & $<$ & $<$ \\
\hline Chloroethane & $<$ & $<$ & $<$ & $<$ & $<$ & $<$ & $<$ & $<$ & $<$ & $<$ \\
\hline Chloromethanes $(\mu \mathrm{g} / \mathrm{L})$ & & & & & & & & & & \\
\hline Carbon tetrachloride & $<$ & $<$ & $<$ & $<$ & $<$ & $<$ & $<$ & $<$ & $<$ & $<$ \\
\hline Chloroform & $<$ & $<$ & $<$ & $<$ & $<$ & $<$ & $<$ & $<$ & 29 & 32 \\
\hline Methylene chloride & $<$ & $<$ & $<$ & $<$ & $<$ & $<$ & $<$ & $<$ & $<$ & 69 \\
\hline Petrol. Hydrocarb. ( $\mu \mathrm{g} / \mathrm{L})$ & & & & & & & & & & \\
\hline Benzene & $<$ & $<$ & $<$ & $<$ & $<$ & $<$ & $<$ & $<$ & $<$ & $<$ \\
\hline Dimethylbenzene & $<$ & $<$ & $<$ & $<$ & $<$ & $<$ & $<$ & $<$ & $<$ & $<$ \\
\hline Ethylbenzene & $<$ & $<$ & $<$ & $<$ & $<$ & $<$ & $<$ & $<$ & $<$ & $<$ \\
\hline Toluene & $<$ & $<$ & $<$ & $<$ & $<$ & $<$ & $<$ & $<$ & $<$ & $<$ \\
\hline Styrene & $<$ & $<$ & $<$ & $<$ & $<$ & $<$ & $<$ & $<$ & $<$ & $<$ \\
\hline Miscellaneous ( $\mu \mathrm{g} / \mathrm{L})$ & & & & & & & & & & \\
\hline 1,2-Dibromoethane & $<$ & $<$ & $<$ & $<$ & $<$ & $<$ & $<$ & $<$ & & \\
\hline 4-Methyl-2-pentanone & $<$ & $<$ & $<$ & $<$ & $<$ & $<$ & $<$ & $<$ & $<$ & $<$ \\
\hline Acetone & $<$ & $<$ & $<$ & $<$ & $<$ & $<$ & $<$ & $<$ & $<$ & 18 \\
\hline Bromodichloromethane & $<$ & $<$ & $<$ & $<$ & $<$ & $<$ & $<$ & $<$ & $<$ & $<$ \\
\hline Bromoform & $<$ & $<$ & $<$ & $<$ & $<$ & $<$ & $<$ & $<$ & $4 \mathrm{~J}$ & $5 \mathrm{~J}$ \\
\hline Bromomethane & $<$ & $<$ & $<$ & $<$ & $<$ & $<$ & $<$ & $<$ & $<$ & 14 \\
\hline Carbon disulfide & $<$ & $<$ & $<$ & $<$ & $<$ & $<$ & $<$ & $<$ & $<$ & $<$ \\
\hline Chlorobenzene & $<$ & $<$ & $<$ & $<$ & $<$ & $<$ & $<$ & $<$ & $<$ & $<$ \\
\hline Chlorodibromomethane & $<$ & $<$ & $<$ & $<$ & $<$ & $<$ & $<$ & $<$ & $<$ & $<$ \\
\hline Trichlorofluoromethane & $<$ & $<$ & $<$ & $<$ & $<$ & $<$ & $<$ & $<$ & . & \\
\hline $\begin{array}{r}\text { Natural Attenuation }(\mu \mathrm{g} / \mathrm{L}) \\
\text { Ethylene } \\
\text { Methane }\end{array}$ & & & & & & & & & & \\
\hline
\end{tabular}


APPENDIX E.2: CY 2002 MONITORING DATA FOR THE UPPER EAST FORK POPLAR CREEK HYDROGEOLOGIC REGIME Volatile Organic Compounds

\begin{tabular}{|c|c|c|c|c|c|c|c|c|c|c|}
\hline Sampling Point & \multicolumn{2}{|c|}{ GW-151 } & \multicolumn{2}{|c|}{ GW-153 } & \multicolumn{2}{|c|}{ GW-154 } & \multicolumn{4}{|c|}{ GW-169 } \\
\hline Location & \multicolumn{2}{|c|}{ NHP } & \multicolumn{2}{|c|}{ NHP } & \multicolumn{2}{|c|}{ NHP } & \multicolumn{4}{|c|}{ EXP-UV } \\
\hline Date Sampled & $01 / 30 / 02$ & $08 / 05 / 02$ & $04 / 25 / 02$ & $10 / 21 / 02$ & $01 / 30 / 02$ & 08/05/02 & $02 / 04 / 02$ & $05 / 13 / 02$ & 08/06/02 & $11 / 11 / 02$ \\
\hline Program & BJC & BJC & GWPP & GWPP & BJC & BJC & BJC & BJC & BJC & BJC \\
\hline \multicolumn{11}{|l|}{ Sample Type } \\
\hline Chloroethenes ( $\mu \mathrm{g} / \mathrm{L}$ ) & & & & & & & & & & \\
\hline Tetrachloroethene & 240 & 500 & $2 \mathrm{~J}$ & $4 \mathrm{~J}$ & $<$ & $<$ & $2 \mathrm{~J}$ & $2 \mathrm{~J}$ & $2 \mathrm{~J}$ & 1 \\
\hline Trichloroethene & 120 & 120 & $<$ & $<$ & $<$ & $<$ & $<$ & $<$ & $<$ & $<$ \\
\hline cis-1,2-Dichloroethene & 32 & 46 & $<$ & $<$ & $<$ & $<$ & $<$ & $<$ & $<$ & $<$ \\
\hline trans-1,2-Dichloroethene & $<$ & $<$ & $<$ & $<$ & $<$ & $<$ & $<$ & $<$ & $<$ & $<$ \\
\hline 1,1-Dichloroethene & $<$ & $<$ & $<$ & $<$ & $<$ & $<$ & $<$ & $<$ & $<$ & $<$ \\
\hline Vinyl chloride & $<$ & $<$ & $<$ & $<$ & $<$ & $<$ & $<$ & $<$ & $<$ & $<$ \\
\hline \multicolumn{11}{|l|}{ Chloroethanes ( $\mu \mathrm{g} / \mathrm{L})$} \\
\hline 1,1,1-Trichloroethane & $<$ & $<$ & $<$ & $<$ & $<$ & $<$ & $<$ & $<$ & $<$ & $<$ \\
\hline 1,2-Dichloroethane & $<$ & $<$ & $<$ & $<$ & $<$ & $<$ & $<$ & $<$ & $<$ & $<$ \\
\hline 1,1-Dichloroethane & $<$ & $<$ & $<$ & $<$ & $<$ & $<$ & $<$ & $<$ & $<$ & $<$ \\
\hline Chloroethane & $<$ & $<$ & $<$ & $<$ & $<$ & $<$ & $<$ & $<$ & $<$ & $<$ \\
\hline \multicolumn{11}{|l|}{ Chloromethanes $(\mu \mathrm{g} / \mathrm{L})$} \\
\hline Carbon tetrachloride & 1,100 & 1,500 & 110 & 140 & $<$ & $<$ & $<$ & $<$ & $<$ & $<$ \\
\hline Chloroform & 77 & 91 & 7 & 7 & $<$ & $<$ & $<$ & $<$ & $<$ & $<$ \\
\hline Methylene chloride & $<$ & $<$ & $<$ & $<$ & $<$ & $<$ & $<$ & $<$ & $<$ & $<$ \\
\hline \multicolumn{11}{|l|}{ Petrol. Hydrocarb. ( $\mu \mathrm{g} / \mathrm{L})$} \\
\hline Benzene & $<$ & $<$ & $<$ & $<$ & $<$ & $<$ & $<$ & $<$ & $<$ & $<$ \\
\hline Dimethylbenzene & $<$ & $<$ & $<$ & $<$ & $<$ & $<$ & $<$ & $<$ & $<$ & $<$ \\
\hline Ethylbenzene & $<$ & $<$ & $<$ & $<$ & $<$ & $<$ & $<$ & $<$ & $<$ & $<$ \\
\hline Toluene & $<$ & $<$ & $<$ & $<$ & $<$ & $<$ & $<$ & $<$ & $<$ & $<$ \\
\hline Styrene & $<$ & $<$ & $<$ & $<$ & $<$ & $<$ & $<$ & $<$ & $<$ & $<$ \\
\hline \multicolumn{11}{|l|}{ Miscellaneous $(\mu \mathrm{g} / \mathrm{L})$} \\
\hline 4-Methyl-2-pentanone & $<$ & $<$ & $<$ & $<$ & $<$ & $<$ & $<$ & $<$ & $<$ & $<$ \\
\hline Acetone & $<$ & $<$ & $<$ & $<$ & $<$ & $<$ & $<$ & $<$ & $<$ & $<$ \\
\hline Bromodichloromethane & $<$ & $<$ & $<$ & $<$ & $<$ & $<$ & $<$ & $<$ & $<$ & $<$ \\
\hline Bromoform & $<$ & $<$ & $<$ & $<$ & $<$ & $<$ & $<$ & $<$ & $<$ & $<$ \\
\hline Bromomethane & $<$ & $<$ & $<$ & $<$ & $<$ & $<$ & $<$ & $<$ & $<$ & $<$ \\
\hline Carbon disulfide & $<$ & $63 \mathrm{Q}$ & $<$ & $<$ & $<$ & $<$ & $<$ & $<$ & $<$ & $<$ \\
\hline Chlorobenzene & $<$ & $<$ & $<$ & $<$ & $<$ & $<$ & $<$ & $<$ & $<$ & $<$ \\
\hline Chlorodibromomethane & $<$ & $<$ & $<$ & $<$ & $<$ & $<$ & $<$ & $<$ & $<$ & $<$ \\
\hline Trichlorofluoromethane & & & $<$ & $<$ &. & 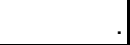 & & & & \\
\hline Natural Attenuation $(\mu \mathrm{g} / \mathrm{L})$ & & & & & & & & & & \\
\hline Ethylene & $<$ & $<$ & & & $<$ & $<$ & & & & \\
\hline Methane & 12 & 16 & & & 18 & 10 & & & & \\
\hline
\end{tabular}


APPENDIX E.2: CY 2002 MONITORING DATA FOR THE UPPER EAST FORK POPLAR CREEK HYDROGEOLOGIC REGIME Volatile Organic Compounds

\begin{tabular}{|c|c|c|c|c|c|c|c|c|c|c|}
\hline Sampling Point & \multicolumn{8}{|c|}{ GW-170 } & \multicolumn{2}{|c|}{ GW-171 } \\
\hline Location & \multicolumn{8}{|c|}{ EXP-UV } & \multicolumn{2}{|c|}{ EXP-UV } \\
\hline Date Sampled & \multicolumn{2}{|c|}{$02 / 04 / 02$} & \multicolumn{2}{|c|}{$05 / 14 / 02$} & \multicolumn{2}{|c|}{ 08/06/02 } & \multicolumn{2}{|c|}{$11 / 11 / 02$} & $02 / 05 / 02$ & $08 / 07 / 02$ \\
\hline Program & BJC & BJC & BJC & BJC & BJC & BJC & BJC & BJC & BJC & BJC \\
\hline Sample Type & & Dup & & Dup & & Dup & & Dup & & \\
\hline Chloroethenes ( $\mu \mathrm{g} / \mathrm{L}$ ) & & & & & & & & & & \\
\hline Tetrachloroethene & $2 \mathrm{~J}$ & $3 \mathrm{~J}$ & $3 \mathrm{~J}$ & $3 \mathrm{~J}$ & $2 \mathrm{~J}$ & $3 \mathrm{~J}$ & 3 & 0.6 & $<$ & $<$ \\
\hline Trichloroethene & $1 \mathrm{~J}$ & $2 \mathrm{~J}$ & $2 \mathrm{~J}$ & $2 \mathrm{~J}$ & $2 \mathrm{~J}$ & $2 \mathrm{~J}$ & 2 & $<$ & $<$ & $<$ \\
\hline cis-1,2-Dichloroethene & $<$ & $<$ & $<$ & $<$ & $<$ & $<$ & $0.3 \mathrm{~J}$ & $<$ & $<$ & $<$ \\
\hline trans-1,2-Dichloroethene & $<$ & $<$ & $<$ & $<$ & $<$ & $<$ & $<$ & $<$ & $<$ & $<$ \\
\hline 1,1-Dichloroethene & $<$ & $<$ & $<$ & $<$ & $<$ & $<$ & $<$ & $<$ & $<$ & $<$ \\
\hline Vinyl chloride & $<$ & $<$ & $<$ & $<$ & $<$ & $<$ & $<$ & $<$ & $<$ & $<$ \\
\hline Chloroethanes $(\mu \mathrm{g} / \mathrm{L})$ & & & & & & & & & & \\
\hline 1,1,1-Trichloroethane & $<$ & $<$ & $<$ & $<$ & $<$ & $<$ & $<$ & $<$ & $<$ & $<$ \\
\hline 1,2-Dichloroethane & $<$ & $<$ & $<$ & $<$ & $<$ & $<$ & $<$ & $<$ & $<$ & $<$ \\
\hline 1,1-Dichloroethane & $<$ & $<$ & $<$ & $<$ & $<$ & $<$ & $<$ & $<$ & $<$ & $<$ \\
\hline Chloroethane & $<$ & $<$ & $<$ & $<$ & $<$ & $<$ & $<$ & $<$ & $<$ & $<$ \\
\hline Chloromethanes ( $\mu \mathrm{g} / \mathrm{L})$ & & & & & & & & & & \\
\hline Carbon tetrachloride & $3 \mathrm{~J}$ & $3 \mathrm{~J}$ & $2 \mathrm{~J}$ & $2 \mathrm{~J}$ & $2 \mathrm{~J}$ & $2 \mathrm{~J}$ & 5 & 2 & $<$ & $<$ \\
\hline Chloroform & 8 & 8 & 8 & 8 & 7 & 7 & $6 \mathrm{R}$ & $0.2 \mathrm{R}$ & $<$ & $<$ \\
\hline Methylene chloride & $<$ & $<$ & $<$ & $<$ & $<$ & $<$ & $<$ & $<$ & $<$ & $<$ \\
\hline Petrol. Hydrocarb. ( $\mu \mathrm{g} / \mathrm{L})$ & & & & & & & & & & \\
\hline Benzene & $3 \mathrm{~J}$ & $3 \mathrm{~J}$ & $4 \mathrm{~J}$ & $4 \mathrm{~J}$ & $<$ & $<$ & $5 \mathrm{R}$ & $0.5 \mathrm{R}$ & $<$ & $<$ \\
\hline Dimethylbenzene & $<$ & $<$ & $<$ & $<$ & $<$ & $<$ & $<$ & $<$ & $<$ & $<$ \\
\hline Ethylbenzene & $<$ & $<$ & $<$ & $<$ & $<$ & $<$ & $<$ & $<$ & $<$ & $<$ \\
\hline Toluene & $<$ & $<$ & $<$ & $<$ & $<$ & $<$ & $0.4 \mathrm{~J}$ & $<$ & $<$ & $<$ \\
\hline Styrene & $<$ & $<$ & $<$ & $<$ & $<$ & $<$ & $<$ & $<$ & $<$ & $<$ \\
\hline $\begin{array}{c}\text { Miscellaneous }(\boldsymbol{\mu g} / \mathrm{L}) \\
1,2-\mathrm{Dibromoethane}\end{array}$ & & & & & & & & & & \\
\hline 4-Methyl-2-pentanone & $<$ & $<$ & $<$ & $<$ & $<$ & $<$ & $<$ & $<$ & $<$ & $<$ \\
\hline Acetone & $<$ & $<$ & $<$ & $<$ & $<$ & $<$ & $<$ & $<$ & $<$ & $<$ \\
\hline Bromodichloromethane & $<$ & $<$ & $<$ & $<$ & $<$ & $<$ & $<$ & $<$ & $<$ & $<$ \\
\hline Bromoform & $<$ & $<$ & $<$ & $<$ & $<$ & $<$ & $<$ & $<$ & $<$ & $<$ \\
\hline Bromomethane & $<$ & $<$ & $<$ & $<$ & $<$ & $<$ & $<$ & $<$ & $<$ & $<$ \\
\hline Carbon disulfide & $<$ & $<$ & $<$ & $<$ & $<$ & $<$ & $<$ & $<$ & $<$ & $<$ \\
\hline Chlorobenzene & $<$ & $<$ & $<$ & $<$ & $<$ & $<$ & $<$ & $<$ & $<$ & $<$ \\
\hline $\begin{array}{l}\text { Chlorodibromomethane } \\
\text { Trichlorofluoromethane }\end{array}$ & $<$ & $<$ & $<$ & $<$ & $<$ & $<$ & $<$ & $<$ & $<$ & $<$ \\
\hline Natural Attenuation $(\mu \mathrm{g} / \mathrm{L})$ & & & & & & & & & & \\
\hline Ethylene & & & & & & & & & & \\
\hline Methane & & & & & & & & & & \\
\hline
\end{tabular}


APPENDIX E.2: CY 2002 MONITORING DATA FOR THE UPPER EAST FORK POPLAR CREEK HYDROGEOLOGIC REGIME Volatile Organic Compounds

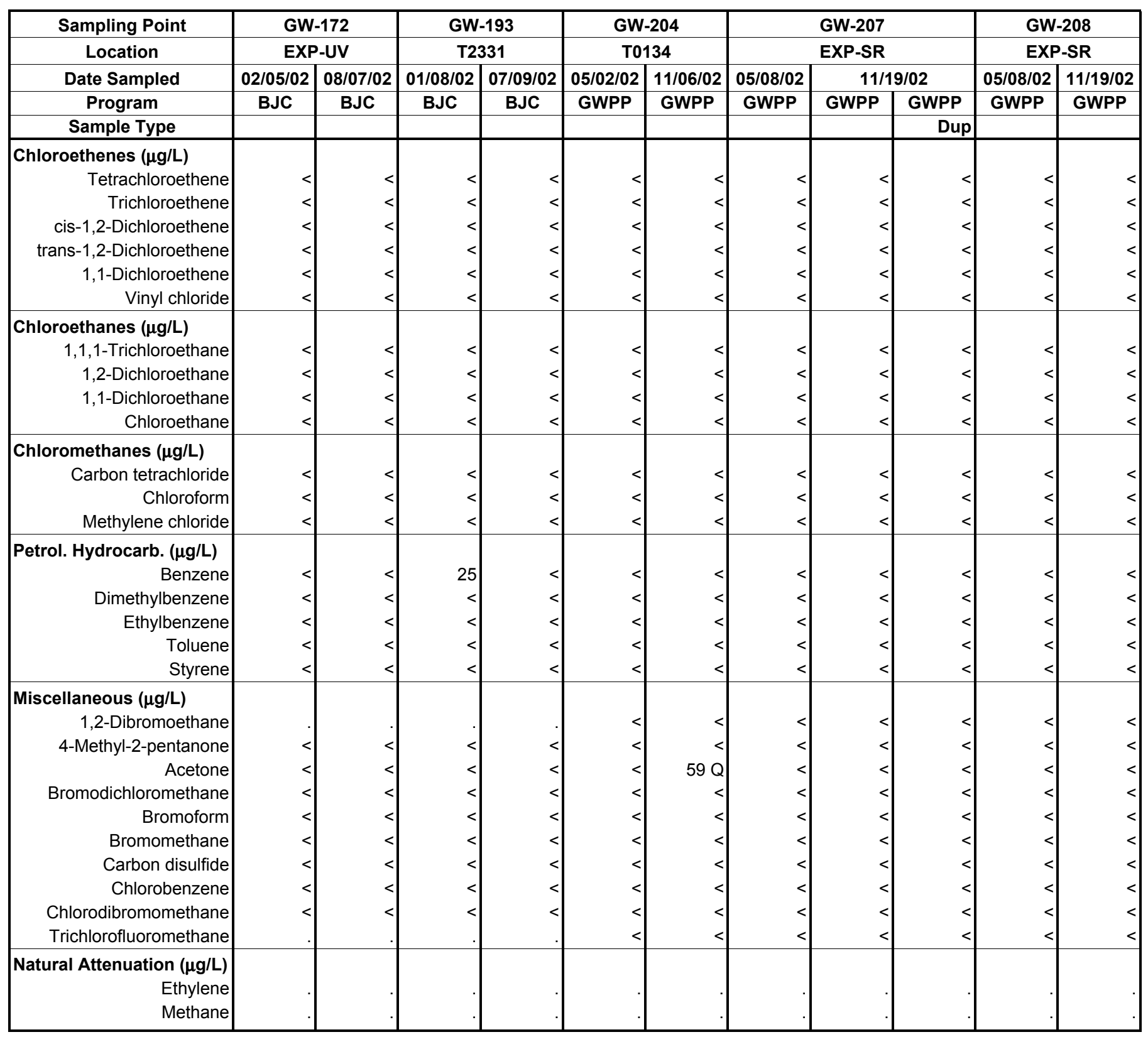


APPENDIX E.2: CY 2002 MONITORING DATA FOR THE UPPER EAST FORK POPLAR CREEK HYDROGEOLOGIC REGIME Volatile Organic Compounds

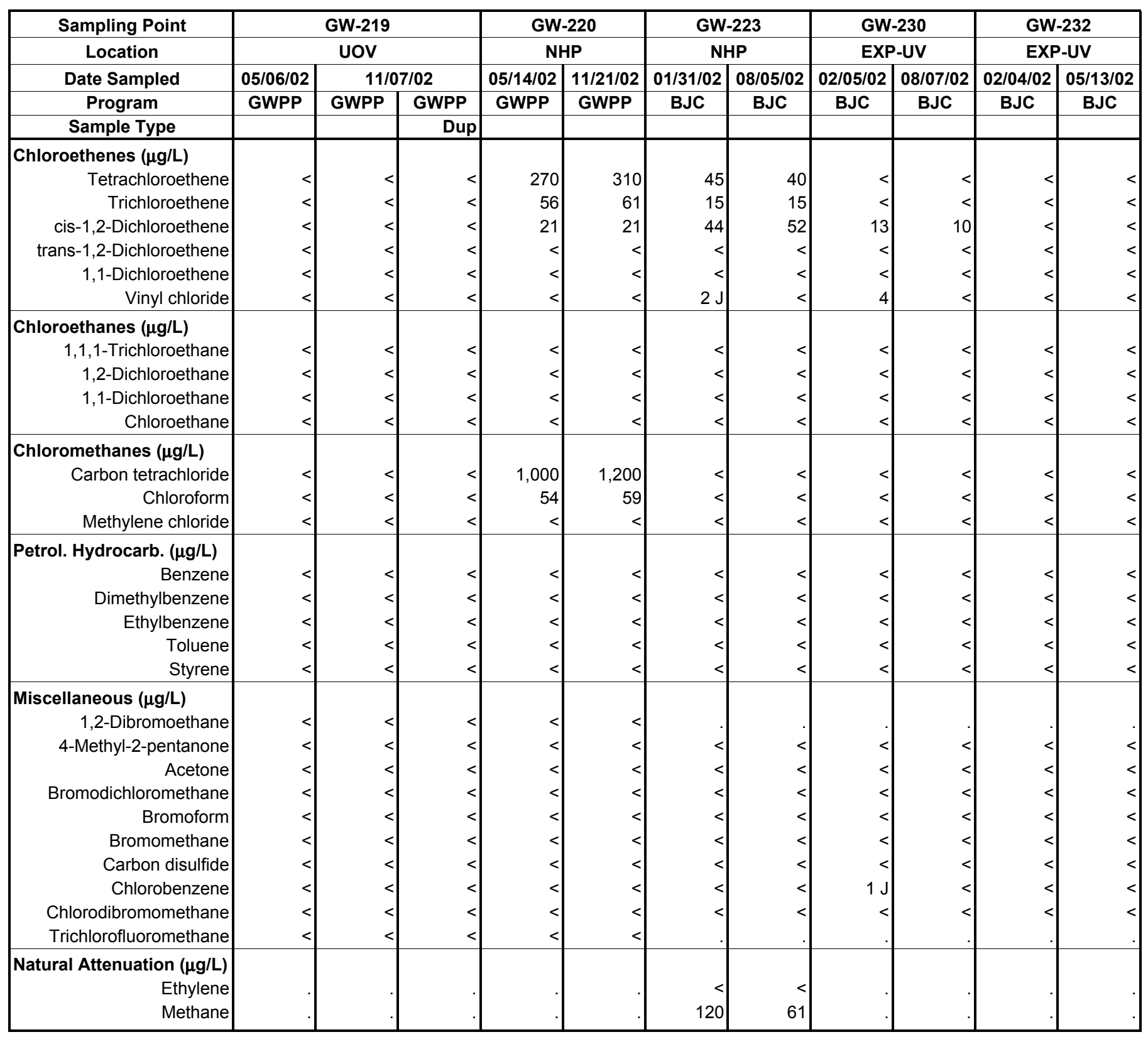


APPENDIX E.2: CY 2002 MONITORING DATA FOR THE UPPER EAST FORK POPLAR CREEK HYDROGEOLOGIC REGIME Volatile Organic Compounds

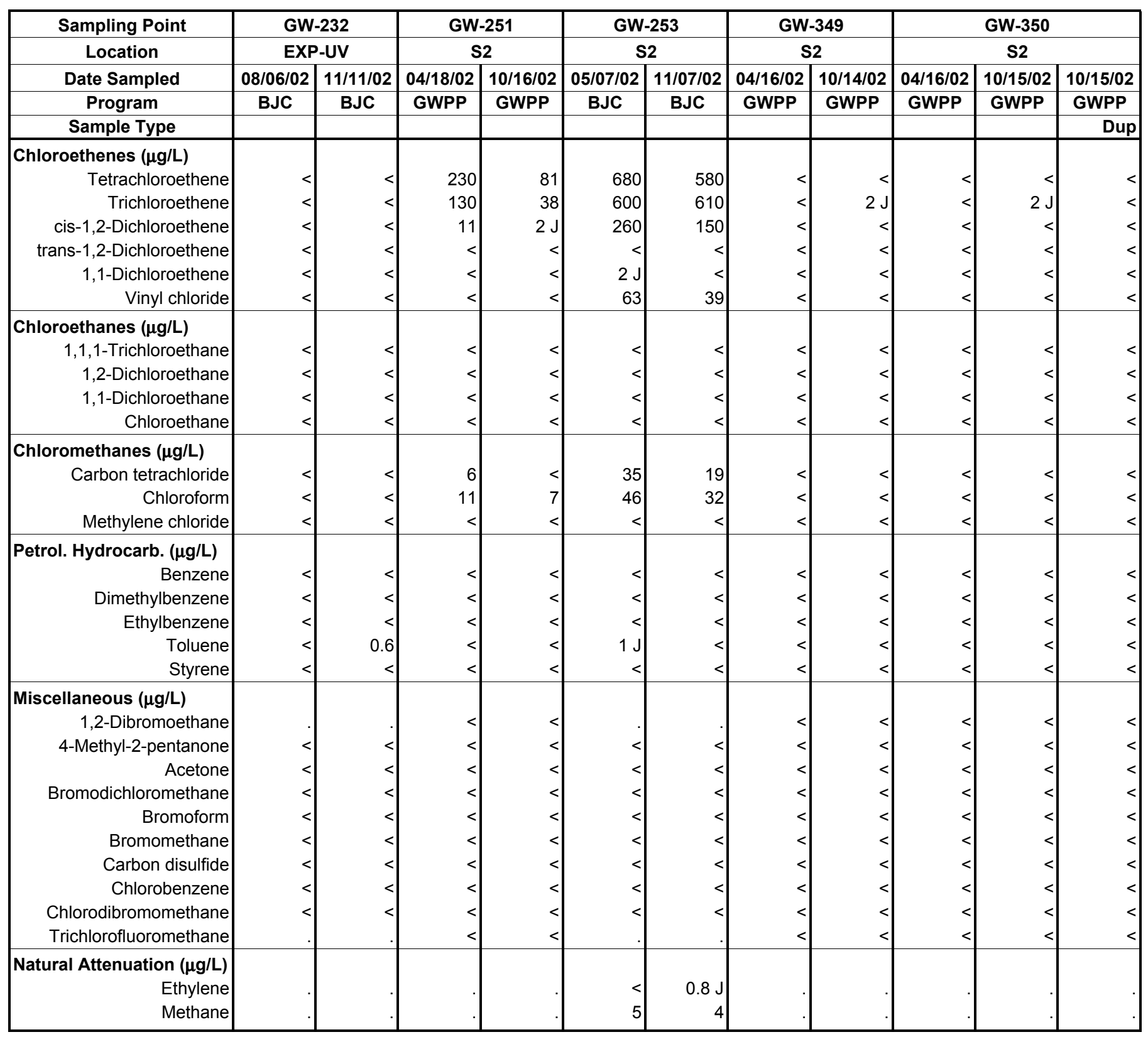


APPENDIX E.2: CY 2002 MONITORING DATA FOR THE UPPER EAST FORK POPLAR CREEK HYDROGEOLOGIC REGIME Volatile Organic Compounds

\begin{tabular}{|c|c|c|c|c|c|c|c|c|c|c|}
\hline Sampling Point & \multicolumn{2}{|c|}{ GW-380 } & \multicolumn{2}{|c|}{ GW-381 } & \multicolumn{2}{|c|}{ GW-382 } & \multicolumn{2}{|c|}{ GW-383 } & \multicolumn{2}{|c|}{ GW-605 } \\
\hline Location & \multicolumn{2}{|c|}{ NHP } & \multicolumn{2}{|c|}{ NHP } & \multicolumn{2}{|c|}{ NHP } & \multicolumn{2}{|c|}{ NHP } & \multirow{2}{*}{\multicolumn{2}{|c|}{$\begin{array}{c}\text { EXP-I } \\
01 / 08 / 02\end{array}$}} \\
\hline Date Sampled & 02/04/02 & 08/05/02 & $06 / 12 / 02$ & $10 / 22 / 02$ & $02 / 04 / 02$ & 08/06/02 & $04 / 25 / 02$ & $10 / 22 / 02$ & & \\
\hline Program & BJC & BJC & GWPP & GWPP & BJC & BJC & GWPP & GWPP & BJC & BJC \\
\hline Sample Type & & & & & & & & & & Dup \\
\hline Chloroethenes ( $\mu \mathrm{g} / \mathrm{L})$ & & & & & & & & & & \\
\hline Tetrachloroethene & $<$ & $<$ & $5 \mathrm{~J}$ & $<$ & 8 & $9 \mathrm{~J}$ & 400 & $380 \mathrm{~J}$ & 76 & 77 \\
\hline Trichloroethene & $<$ & $<$ & $<$ & $<$ & $2 \mathrm{~J}$ & $<$ & 170 & 200 & 82 & 85 \\
\hline cis-1,2-Dichloroethene & $<$ & $<$ & 6 & $2 \mathrm{~J}$ & $2 \mathrm{~J}$ & $<$ & 130 & 140 & 140 & 150 \\
\hline trans-1,2-Dichloroethene & $<$ & $<$ & $<$ & $<$ & $<$ & $<$ & $<$ & $<$ & $1 \mathrm{~J}$ & $1 \mathrm{~J}$ \\
\hline 1,1-Dichloroethene & $<$ & $<$ & $<$ & $<$ & $<$ & $<$ & $3 \mathrm{~J}$ & $3 \mathrm{~J}$ & $<$ & $<$ \\
\hline Vinyl chloride & $<$ & $<$ & $<$ & $<$ & $<$ & $<$ & $<$ & $2 \mathrm{~J}$ & $<$ & $<$ \\
\hline Chloroethanes $(\mu \mathrm{g} / \mathrm{L})$ & & & & & & & & & & \\
\hline 1,1,1-Trichloroethane & $<$ & $<$ & $<$ & $<$ & $<$ & $<$ & $<$ & $<$ & $<$ & $<$ \\
\hline 1,2-Dichloroethane & $<$ & $<$ & $<$ & $<$ & $<$ & $<$ & $<$ & $<$ & $<$ & $<$ \\
\hline 1,1-Dichloroethane & $<$ & $<$ & $<$ & $<$ & $<$ & $<$ & $<$ & $<$ & $<$ & $<$ \\
\hline Chloroethane & $<$ & $<$ & $<$ & $<$ & $<$ & $<$ & $<$ & $<$ & $<$ & $<$ \\
\hline Chloromethanes ( $\mu \mathrm{g} / \mathrm{L})$ & & & & & & & & & & \\
\hline Carbon tetrachloride & $<$ & $<$ & 380 & 160 & 510 & 230 & $<$ & $<$ & 53 & 54 \\
\hline Chloroform & $<$ & 5 & 620 & 19 & 380 & 400 & $<$ & $<$ & 11 & 11 \\
\hline Methylene chloride & $<$ & $<$ & $120 \mathrm{Q}$ & 11 & $<$ & 43 & $<$ & $<$ & $<$ & $<$ \\
\hline Petrol. Hydrocarb. ( $\mu \mathrm{g} / \mathrm{L})$ & & & & & & & & & & \\
\hline Benzene & $<$ & $<$ & $<$ & $<$ & $<$ & $<$ & $<$ & $<$ & $<$ & $<$ \\
\hline Dimethylbenzene & $<$ & $<$ & $<$ & $<$ & $<$ & $<$ & $<$ & $<$ & $<$ & $<$ \\
\hline Ethylbenzene & $<$ & $<$ & $<$ & $<$ & $<$ & $<$ & $<$ & $<$ & $<$ & $<$ \\
\hline Toluene & $<$ & $<$ & $<$ & $<$ & $<$ & $<$ & $<$ & $<$ & $<$ & $<$ \\
\hline Styrene & $<$ & $<$ & $<$ & $<$ & $<$ & $<$ & $<$ & $<$ & $<$ & $<$ \\
\hline Miscellaneous $(\mu \mathrm{g} / \mathrm{L})$ & & & & & & & & & & \\
\hline 1,2-Dibromoethane & & & $<$ & $<$ & & & $<$ & $<$ & & \\
\hline 4-Methyl-2-pentanone & $<$ & $<$ & $<$ & $<$ & $<$ & $<$ & $<$ & $<$ & $<$ & $<$ \\
\hline Acetone & $<$ & $<$ & $<$ & $<$ & $<$ & $<$ & $<$ & $<$ & $<$ & $<$ \\
\hline Bromodichloromethane & $<$ & $<$ & $<$ & $<$ & $<$ & $<$ & $<$ & $<$ & $<$ & $<$ \\
\hline Bromoform & $<$ & $<$ & $<$ & $<$ & $<$ & $<$ & $<$ & $<$ & $<$ & $<$ \\
\hline Bromomethane & $<$ & $<$ & $<$ & $<$ & $<$ & $<$ & $<$ & $<$ & $<$ & $<$ \\
\hline Carbon disulfide & $<$ & $<$ & $<$ & $<$ & $<$ & $9 \mathrm{~J}$ & $<$ & $<$ & $<$ & $<$ \\
\hline Chlorobenzene & $<$ & $<$ & $<$ & $<$ & $<$ & $<$ & $<$ & $<$ & $<$ & $<$ \\
\hline Chlorodibromomethane & $<$ & $<$ & $<$ & $<$ & $<$ & $<$ & $<$ & $<$ & $<$ & $<$ \\
\hline Trichlorofluoromethane & & & $<$ & $<$ & & & $<$ & $<$ & & \\
\hline Natural Attenuation $(\mu \mathrm{g} / \mathrm{L})$ & & & & & & & & & & \\
\hline Ethylene & & & & & & & & & & \\
\hline Methane & & & & & & & & & & \\
\hline
\end{tabular}


APPENDIX E.2: CY 2002 MONITORING DATA FOR THE UPPER EAST FORK POPLAR CREEK HYDROGEOLOGIC REGIME Volatile Organic Compounds

\begin{tabular}{|c|c|c|c|c|c|c|c|c|c|c|}
\hline Sampling Point & \multicolumn{2}{|c|}{ GW-605 } & \multicolumn{2}{|c|}{ GW-606 } & \multicolumn{2}{|c|}{ GW-618 } & \multicolumn{2}{|c|}{ GW-620 } & \multicolumn{2}{|c|}{ GW-633 } \\
\hline Location & \multicolumn{2}{|c|}{ EXP-I } & \multicolumn{2}{|c|}{ EXP-I } & \multicolumn{2}{|c|}{ EXP-E } & \multicolumn{2}{|c|}{ FTF } & \multicolumn{2}{|c|}{ RG } \\
\hline Date Sampled & \multicolumn{2}{|c|}{ 07/08/02 } & 01/08/02 & $07 / 08 / 02$ & 05/09/02 & $11 / 07 / 02$ & $04 / 23 / 02$ & $10 / 15 / 02$ & 04/23/02 & $10 / 17 / 02$ \\
\hline Program & BJC & BJC & BJC & BJC & BJC & BJC & GWPP & GWPP & GWPP & GWPP \\
\hline Sample Type & & Dup & & & & & & & & \\
\hline \multicolumn{11}{|l|}{ Chloroethenes ( $\mu \mathrm{g} / \mathrm{L})$} \\
\hline Tetrachloroethene & 33 & 30 & 5 & 7 & $4 \mathrm{~J}$ & 7 & 15 & 17 & 180 & 170 \\
\hline Trichloroethene & 33 & 32 & $<$ & $<$ & 11 & 16 & 7 & 7 & 8 & 6 \\
\hline cis-1,2-Dichloroethene & 50 & 50 & $<$ & $<$ & 17 & 31 & 17 & 13 & 10 & 10 \\
\hline trans-1,2-Dichloroethene & $<$ & $<$ & $<$ & $<$ & $<$ & 0.5 & $<$ & $<$ & $<$ & $<$ \\
\hline 1,1-Dichloroethene & $<$ & $<$ & $<$ & $<$ & $<$ & $<$ & $<$ & $<$ & $3 \mathrm{~J}$ & $<$ \\
\hline Vinyl chloride & $<$ & $<$ & $<$ & $<$ & $<$ & 3 & $<$ & $<$ & $<$ & $<$ \\
\hline \multicolumn{11}{|l|}{ Chloroethanes $(\mu \mathrm{g} / \mathrm{L})$} \\
\hline 1,1,1-Trichloroethane & $<$ & $<$ & $<$ & $<$ & $<$ & $<$ & $<$ & $<$ & $<$ & $<$ \\
\hline 1,2-Dichloroethane & $<$ & $<$ & $<$ & $<$ & $<$ & $<$ & $<$ & $<$ & $<$ & $<$ \\
\hline 1,1-Dichloroethane & $<$ & $<$ & $<$ & $<$ & $<$ & $<$ & $<$ & $<$ & $<$ & $<$ \\
\hline Chloroethane & $<$ & $<$ & $<$ & $<$ & $<$ & $<$ & $<$ & $<$ & $<$ & $<$ \\
\hline \multicolumn{11}{|l|}{ Chloromethanes ( $\mu \mathrm{g} / \mathrm{L})$} \\
\hline Carbon tetrachloride & 19 & 18 & 110 & 180 & $<$ & $<$ & $<$ & $<$ & $<$ & $<$ \\
\hline Chloroform & 12 & 12 & 230 & 140 & $<$ & $<$ & $<$ & $<$ & 20 & 17 \\
\hline Methylene chloride & $<$ & $<$ & $<$ & $<$ & $<$ & $<$ & $<$ & $<$ & 36 & 27 \\
\hline \multicolumn{11}{|l|}{ Petrol. Hydrocarb. ( $\mu \mathrm{g} / \mathrm{L})$} \\
\hline Benzene & $<$ & $<$ & $<$ & $<$ & $<$ & $<$ & $<$ & $<$ & 840 & 850 \\
\hline Dimethylbenzene & $<$ & $<$ & $<$ & $<$ & $<$ & $<$ & $<$ & $4 \mathrm{~J}$ & 110 & 95 \\
\hline Ethylbenzene & $<$ & $<$ & $<$ & $<$ & $<$ & $<$ & $<$ & $<$ & 20 & 8 \\
\hline Toluene & $<$ & $<$ & $<$ & $<$ & $<$ & $<$ & $1 \mathrm{~J}$ & $2 \mathrm{~J}$ & $4 \mathrm{~J}$ & $2 \mathrm{~J}$ \\
\hline Styrene & $<$ & $<$ & $<$ & $<$ & $<$ & $<$ & $<$ & $<$ & $<$ & $<$ \\
\hline \multicolumn{11}{|l|}{ Miscellaneous ( $\mu \mathrm{g} / \mathrm{L})$} \\
\hline 1,2-Dibromoethane & & & & & & & $<$ & $<$ & $<$ & $<$ \\
\hline 4-Methyl-2-pentanone & $<$ & $<$ & $<$ & $<$ & $<$ & $<$ & $<$ & $<$ & $<$ & $<$ \\
\hline Acetone & $<$ & $<$ & $<$ & $<$ & $<$ & $<$ & $<$ & $<$ & $<$ & $<$ \\
\hline Bromodichloromethane & $<$ & $<$ & $<$ & $<$ & $<$ & $<$ & $<$ & $<$ & $<$ & $<$ \\
\hline Bromoform & $<$ & $<$ & $<$ & $<$ & $<$ & $<$ & $<$ & $<$ & $4 \mathrm{~J}$ & $3 \mathrm{~J}$ \\
\hline Bromomethane & $<$ & $<$ & $<$ & $<$ & $<$ & $<$ & $<$ & $<$ & $<$ & $<$ \\
\hline Carbon disulfide & $<$ & $<$ & $<$ & $<$ & $<$ & $<$ & $<$ & $<$ & $<$ & $<$ \\
\hline Chlorobenzene & $<$ & $<$ & $<$ & $<$ & $<$ & $<$ & $<$ & $<$ & $<$ & $<$ \\
\hline Chlorodibromomethane & $<$ & $<$ & $<$ & $<$ & $<$ & $<$ & $<$ & $<$ & $<$ & $<$ \\
\hline Trichlorofluoromethane & & & & & & 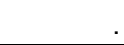 & $<$ & $<$ & $<$ & $<$ \\
\hline Natural Attenuation $(\mu \mathrm{g} / \mathrm{L})$ & & & & & & & & & & \\
\hline Ethylene & & & & & $<$ & $<$ & & & & \\
\hline Methane & & & & & 9 & 14 & & & & \\
\hline
\end{tabular}


APPENDIX E.2: CY 2002 MONITORING DATA FOR THE UPPER EAST FORK POPLAR CREEK HYDROGEOLOGIC REGIME Volatile Organic Compounds

\begin{tabular}{|c|c|c|c|c|c|c|c|c|c|c|c|c|}
\hline Sampling Point & \multicolumn{2}{|c|}{ GW-658 } & GW-686 & GW-687 & GW-689 & \multicolumn{3}{|c|}{ GW-698 } & \multicolumn{4}{|c|}{ GW-722-06 } \\
\hline Location & \multicolumn{2}{|c|}{ FF } & CPT & CPT & CPT & \multicolumn{3}{|c|}{ B8110 } & \multicolumn{4}{|c|}{ EXP-J } \\
\hline Date Sampled & $04 / 24 / 02$ & $10 / 17 / 02$ & $11 / 14 / 02$ & $11 / 14 / 02$ & $11 / 14 / 02$ & \multicolumn{2}{|c|}{$05 / 02 / 02$} & $11 / 07 / 02$ & $02 / 04 / 02$ & $05 / 23 / 02$ & $07 / 15 / 02$ & $11 / 07 / 02$ \\
\hline Program & GWPP & GWPP & GWPP & GWPP & GWPP & GWPP & GWPP & GWPP & GWPP & BJC & GWPP & BJC \\
\hline Sample Type & & & & & & & Dup & & & & & \\
\hline Chloroethenes ( $\mu \mathrm{g} / \mathrm{L}$ ) & & & & & & & & & & & & \\
\hline Tetrachloroethene & $<$ & $<$ & $<$ & $<$ & $<$ & 150 & 130 & 130 & $<$ & $<$ & $<$ & $<$ \\
\hline trans-1,2-Dichloroethene & $<$ & $<$ & $<$ & $<$ & $<$ & $<$ & $<$ & $<$ & $<$ & $<$ & $<$ & $<$ \\
\hline 1,1-Dichloroethene & $<$ & $<$ & $<$ & $<$ & $<$ & $<$ & $<$ & $<$ & $<$ & $<$ & $<$ & $<$ \\
\hline Vinyl chloride & $<$ & $<$ & 11 & $<$ & $<$ & $<$ & $<$ & $<$ & $<$ & $<$ & $<$ & $<$ \\
\hline Chloroethanes ( $\mu \mathrm{g} / \mathrm{L})$ & & & & & & & & & & & & \\
\hline 1,1,1-Trichloroethane & $<$ & $<$ & $<$ & $<$ & $<$ & $<$ & $<$ & $<$ & $<$ & $<$ & $<$ & $<$ \\
\hline Carbon tetrachloride & $<$ & $<$ & $<$ & $<$ & $<$ & 11 & 10 & $3 \mathrm{~J}$ & $<$ & $<$ & $<$ & $<$ \\
\hline Chloroform & $<$ & $<$ & $<$ & $<$ & $<$ & 16 & 17 & 5 & $<$ & $<$ & $<$ & $<$ \\
\hline Methylene chloride & $<$ & $<$ & $<$ & $<$ & $<$ & $<$ & $<$ & $<$ & $<$ & $<$ & $<$ & $<$ \\
\hline Petrol. Hydrocarb. ( $\mu \mathrm{g} / \mathrm{L})$ & & & & & & & & & & & & \\
\hline Benzene & 7,800 & 8,700 & $<$ & $<$ & $<$ & $<$ & $<$ & $<$ & $<$ & $<$ & $<$ & $0.2 \mathrm{~J}$ \\
\hline Dimethylbenzene & 8,800 & 8,800 & $<$ & $<$ & $<$ & $<$ & $<$ & $<$ & $<$ & $<$ & $<$ & $<$ \\
\hline Ethylbenzene & 920 & 1,400 & $<$ & $<$ & $<$ & $<$ & $<$ & $<$ & $<$ & $<$ & $<$ & $<$ \\
\hline Toluene & 4,800 & 4,900 & $<$ & $<$ & $<$ & $<$ & $<$ & $<$ & $<$ & $<$ & $<$ & $<$ \\
\hline Styrene & $<$ & $<$ & $<$ & $<$ & $<$ & $<$ & $<$ & $<$ & $<$ & $<$ & $<$ & $<$ \\
\hline Miscellaneous ( $\mu \mathrm{g} / \mathrm{L})$ & & & & & & & & & & & & \\
\hline 1,2-Dibromoethane & $<$ & 77 & $<$ & $<$ & $<$ & $<$ & $<$ & $<$ & $<$ & 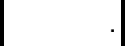 & $<$ & \\
\hline Trichlorofluoromethane & $<$ & $<$ & $<$ & $<$ & $<$ & $<$ & $<$ & $<$ & $<$ &. & $<$ & \\
\hline $\begin{array}{r}\text { Natural Attenuation }(\mu \mathrm{g} / \mathrm{L}) \\
\text { Ethylene } \\
\text { Methane }\end{array}$ & & & & & & & & & & & & \\
\hline
\end{tabular}


APPENDIX E.2: CY 2002 MONITORING DATA FOR THE UPPER EAST FORK POPLAR CREEK HYDROGEOLOGIC REGIME Volatile Organic Compounds

\begin{tabular}{|c|c|c|c|c|c|c|c|c|c|}
\hline Sampling Point & \multicolumn{5}{|c|}{ GW-722-10 } & \multicolumn{4}{|c|}{ GW-722-14 } \\
\hline Location & \multicolumn{5}{|c|}{ EXP-J } & \multicolumn{4}{|c|}{ EXP-J } \\
\hline Date Sampled & 02/06/02 & 05/29/02 & 07/16/02 & $11 / 12 / 02$ & $12 / 12 / 02$ & $02 / 08 / 02$ & 05/30/02 & 07/17/02 & $11 / 13 / 02$ \\
\hline Program & GWPP & BJC & GWPP & BJC & BJC & GWPP & BJC & GWPP & BJC \\
\hline \multicolumn{10}{|l|}{ Sample Type } \\
\hline Chloroethenes ( $\mu \mathrm{g} / \mathrm{L}$ ) & & & & & & & & & \\
\hline Tetrachloroethene & $<$ & $3 \mathrm{~J}$ & $<$ & 2 & 3 & 10 & 6 & $4 \mathrm{~J}$ & $0.2 \mathrm{~J}$ \\
\hline Trichloroethene & $<$ & $<$ & $<$ & 0.5 & 0.8 & $2 \mathrm{~J}$ & $2 \mathrm{~J}$ & $<$ & $<$ \\
\hline cis-1,2-Dichloroethene & $<$ & $<$ & $<$ & $0.3 \mathrm{~J}$ & $<$ & $<$ & $<$ & $<$ & $0.3 \mathrm{~J}$ \\
\hline trans-1,2-Dichloroethene & $<$ & $<$ & $<$ & $<$ & $<$ & $<$ & $<$ & $<$ & $<$ \\
\hline 1,1-Dichloroethene & $<$ & $<$ & $<$ & $<$ & $<$ & $<$ & $<$ & $<$ & $<$ \\
\hline Vinyl chloride & $<$ & $<$ & $<$ & $<$ & $<$ & $<$ & $<$ & $<$ & $<$ \\
\hline \multicolumn{10}{|l|}{ Chloroethanes ( $\mu \mathrm{g} / \mathrm{L})$} \\
\hline 1,1,1-Trichloroethane & $<$ & $<$ & $<$ & $<$ & $<$ & $<$ & $<$ & $<$ & $<$ \\
\hline 1,2-Dichloroethane & $<$ & $<$ & $<$ & $<$ & $<$ & $<$ & $<$ & $<$ & $<$ \\
\hline 1,1-Dichloroethane & $<$ & $<$ & $<$ & $<$ & $<$ & $<$ & $<$ & $<$ & $<$ \\
\hline Chloroethane & $<$ & $<$ & $<$ & $<$ & $<$ & $<$ & $<$ & $<$ & $<$ \\
\hline \multicolumn{10}{|l|}{ Chloromethanes $(\mu \mathrm{g} / \mathrm{L})$} \\
\hline Carbon tetrachloride & $<$ & $<$ & $<$ & $0.4 \mathrm{~J}$ & $<$ & 160 & 65 & 55 & $7 Q$ \\
\hline Chloroform & $<$ & $<$ & $<$ & $<$ & $0.4 \mathrm{~J}$ & 11 & 12 & 7 & $<$ \\
\hline Methylene chloride & $<$ & $<$ & $<$ & $<$ & $<$ & $<$ & $<$ & $<$ & $<$ \\
\hline \multicolumn{10}{|l|}{ Petrol. Hydrocarb. ( $\mu \mathrm{g} / \mathrm{L})$} \\
\hline Benzene & $<$ & $<$ & $<$ & $<$ & $<$ & $<$ & $<$ & $<$ & $<$ \\
\hline Dimethylbenzene & $<$ & $1 \mathrm{~J}$ & $<$ & $<$ & $<$ & $<$ & $<$ & $<$ & $<$ \\
\hline Ethylbenzene & $<$ & $1 \mathrm{~J}$ & $<$ & $<$ & 2 & $<$ & $<$ & $<$ & $<$ \\
\hline Toluene & $<$ & $<$ & $<$ & $<$ & $0.4 \mathrm{~J}$ & $<$ & $<$ & $<$ & $<$ \\
\hline Styrene & $<$ & $<$ & $<$ & $<$ & $0.3 \mathrm{~J}$ & $<$ & $<$ & $<$ & $<$ \\
\hline \multicolumn{10}{|l|}{ Miscellaneous ( $\mu \mathrm{g} / \mathrm{L})$} \\
\hline 1,2-Dibromoethane & $<$ & & $<$ & & & $<$ & & $<$ & \\
\hline 4-Methyl-2-pentanone & $<$ & $<$ & $<$ & $<$ & $<$ & $<$ & $<$ & $<$ & $<$ \\
\hline Acetone & $<$ & $<$ & $<$ & $<$ & $<$ & $5 \mathrm{~J}$ & $<$ & $<$ & $<$ \\
\hline Bromodichloromethane & $<$ & $<$ & $<$ & $<$ & $<$ & $<$ & $<$ & $<$ & $<$ \\
\hline Bromoform & $<$ & $<$ & $<$ & $<$ & $<$ & $<$ & $<$ & $<$ & $<$ \\
\hline Bromomethane & $<$ & $<$ & $<$ & $<$ & $<$ & $<$ & $<$ & $<$ & $<$ \\
\hline Carbon disulfide & $<$ & $<$ & $<$ & $<$ & $<$ & $<$ & $<$ & $<$ & $<$ \\
\hline Chlorobenzene & $<$ & $<$ & $<$ & $<$ & $<$ & $<$ & $<$ & $<$ & $<$ \\
\hline Chlorodibromomethane & $<$ & $<$ & $<$ & $<$ & $<$ & $<$ & $<$ & $<$ & $<$ \\
\hline Trichlorofluoromethane & $<$ & & $<$ & & & $<$ & . & $<$ & \\
\hline \multicolumn{10}{|l|}{ Natural Attenuation $(\mu \mathrm{g} / \mathrm{L})$} \\
\hline Ethylene & & & & & & & & & \\
\hline Methane & & & & & & & & & \\
\hline
\end{tabular}


APPENDIX E.2: CY 2002 MONITORING DATA FOR THE UPPER EAST FORK POPLAR CREEK HYDROGEOLOGIC REGIME Volatile Organic Compounds

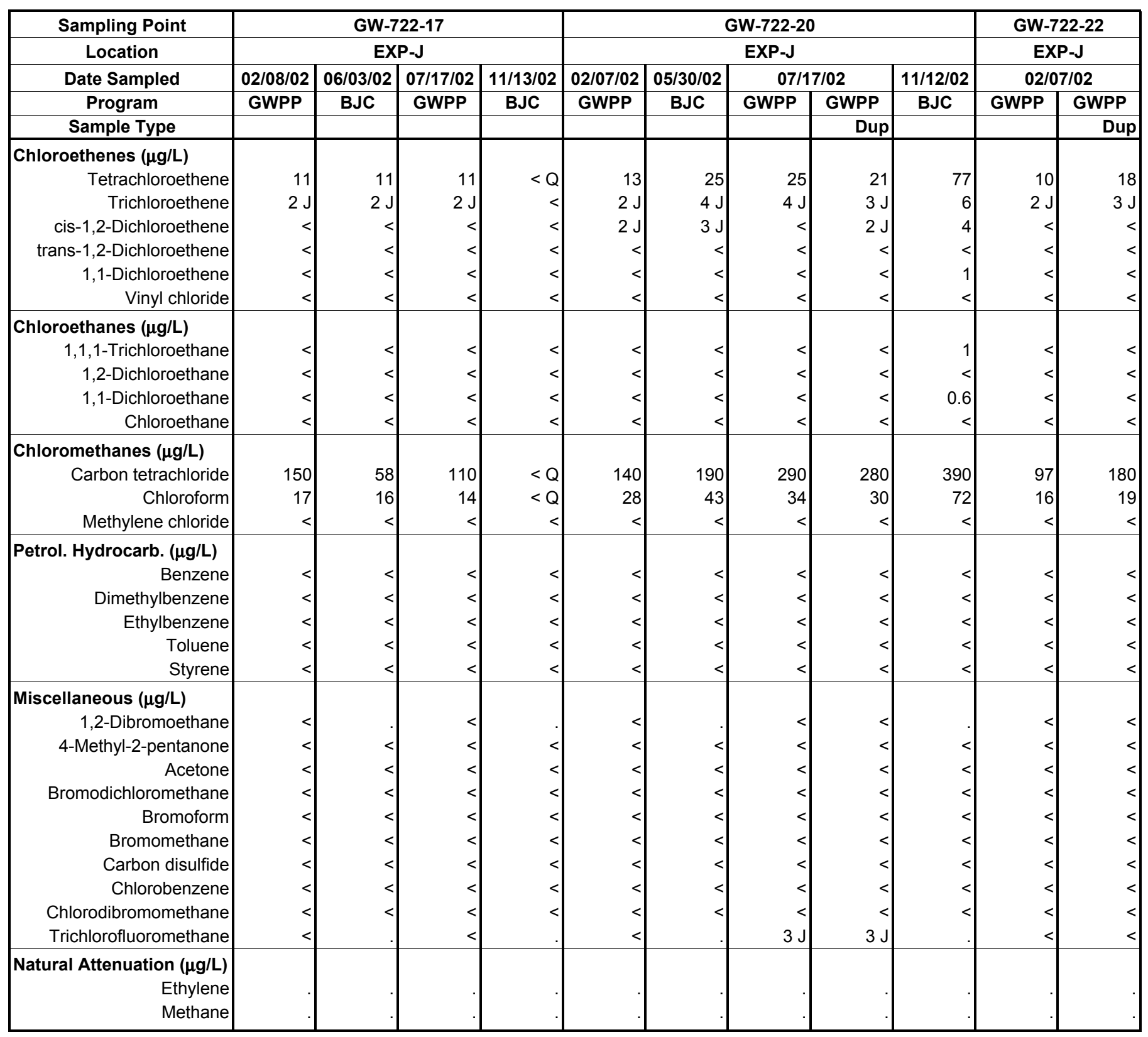


APPENDIX E.2: CY 2002 MONITORING DATA FOR THE UPPER EAST FORK POPLAR CREEK HYDROGEOLOGIC REGIME Volatile Organic Compounds

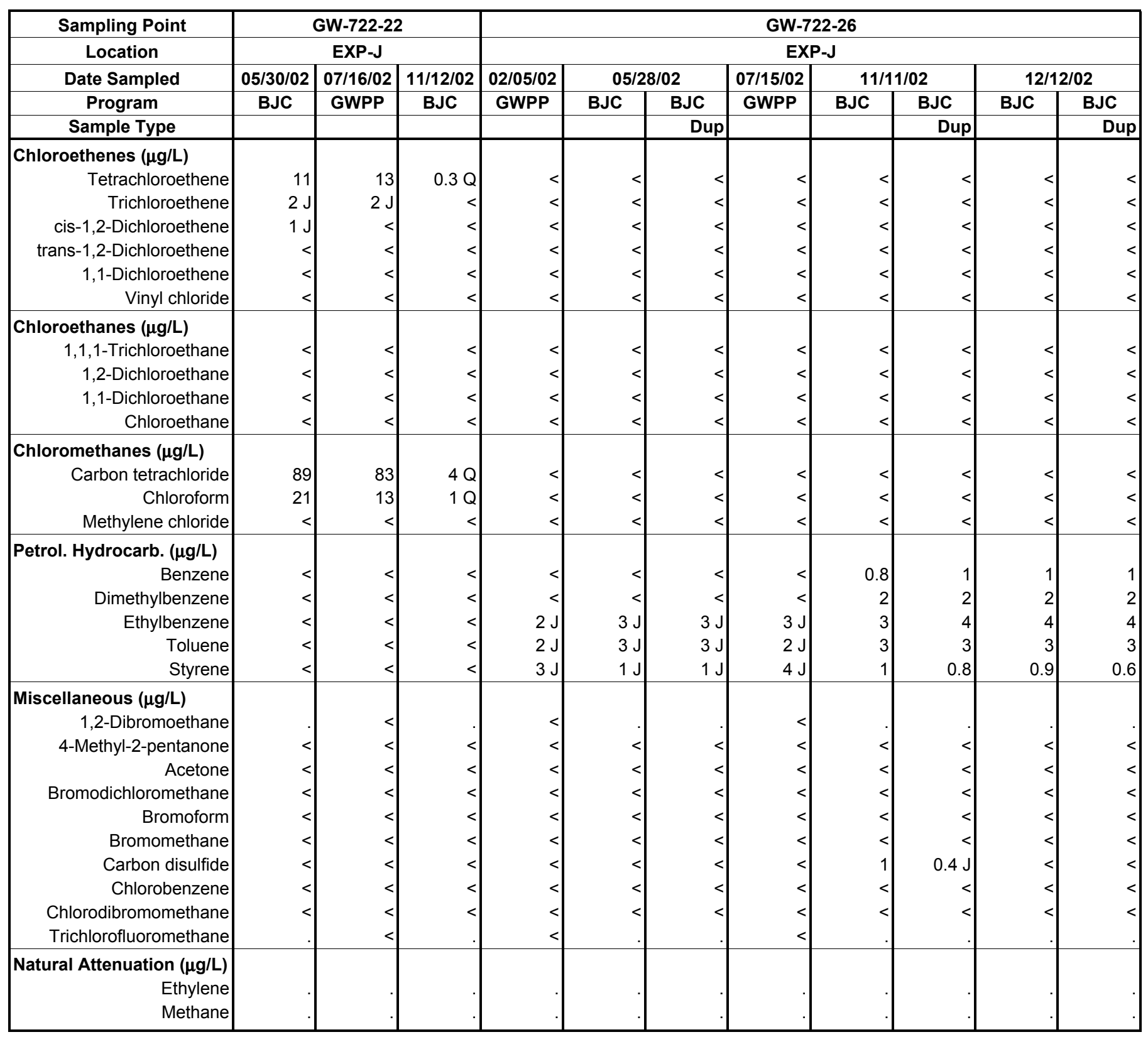


APPENDIX E.2: CY 2002 MONITORING DATA FOR THE UPPER EAST FORK POPLAR CREEK HYDROGEOLOGIC REGIME Volatile Organic Compounds

\begin{tabular}{|c|c|c|c|c|c|c|c|c|c|c|}
\hline Sampling Point & \multicolumn{5}{|c|}{ GW-722-30 } & \multicolumn{5}{|c|}{ GW-722-32 } \\
\hline Location & \multicolumn{5}{|c|}{ EXP-J } & \multicolumn{5}{|c|}{ EXP-J } \\
\hline Date Sampled & $02 / 05 / 02$ & 05/28/02 & 07/15/02 & $11 / 11 / 02$ & $12 / 12 / 02$ & $02 / 05 / 02$ & $05 / 28 / 02$ & $07 / 15 / 02$ & $11 / 12 / 02$ & $12 / 12 / 02$ \\
\hline Program & GWPP & BJC & GWPP & BJC & BJC & GWPP & BJC & GWPP & BJC & BJC \\
\hline \multicolumn{11}{|l|}{ Sample Type } \\
\hline Chloroethenes ( $\mu \mathrm{g} / \mathrm{L}$ ) & & & & & & & & & & \\
\hline Tetrachloroethene & $<$ & $<$ & $<$ & $<$ & $<$ & $<$ & $<$ & $<$ & $<$ & $<$ \\
\hline Trichloroethene & $<$ & $<$ & $<$ & $<$ & $<$ & $<$ & $<$ & $<$ & $<$ & $<$ \\
\hline cis-1,2-Dichloroethene & $<$ & $<$ & $<$ & $<$ & $<$ & $<$ & $<$ & $<$ & $<$ & $<$ \\
\hline trans-1,2-Dichloroethene & $<$ & $<$ & $<$ & $<$ & $<$ & $<$ & $<$ & $<$ & $<$ & $<$ \\
\hline 1,1-Dichloroethene & $<$ & $<$ & $<$ & $<$ & $<$ & $<$ & $<$ & $<$ & $<$ & $<$ \\
\hline Vinyl chloride & $<$ & $<$ & $<$ & $<$ & $<$ & $<$ & $<$ & $<$ & $<$ & $<$ \\
\hline \multicolumn{11}{|l|}{ Chloroethanes ( $\mu \mathrm{g} / \mathrm{L})$} \\
\hline 1,1,1-Trichloroethane & $<$ & $<$ & $<$ & $<$ & $<$ & $<$ & $<$ & $<$ & $<$ & $<$ \\
\hline 1,2-Dichloroethane & $<$ & $<$ & $<$ & $<$ & $<$ & $<$ & $<$ & $<$ & $<$ & $<$ \\
\hline 1,1-Dichloroethane & $<$ & $<$ & $<$ & $<$ & $<$ & $<$ & $<$ & $<$ & $<$ & $<$ \\
\hline Chloroethane & $<$ & $<$ & $<$ & $<$ & $<$ & $<$ & $<$ & $<$ & $<$ & $<$ \\
\hline \multicolumn{11}{|l|}{ Chloromethanes ( $\mu \mathrm{g} / \mathrm{L})$} \\
\hline Carbon tetrachloride & $<$ & $<$ & $<$ & $<$ & $<$ & $<$ & $<$ & $<$ & $<$ & $<$ \\
\hline Chloroform & $<$ & $<$ & $<$ & $<$ & $<$ & $<$ & $<$ & $<$ & 0.9 & 1 \\
\hline Methylene chloride & $<$ & $<$ & $<$ & $<$ & $<$ & $<$ & $<$ & $<$ & $<$ & $<$ \\
\hline \multicolumn{11}{|l|}{ Petrol. Hydrocarb. ( $\mu \mathrm{g} / \mathrm{L})$} \\
\hline Benzene & $<$ & $<$ & $<$ & $<$ & $<$ & $<$ & $<$ & $<$ & $<$ & $<$ \\
\hline Dimethylbenzene & $<$ & $<$ & $<$ & $<$ & $<$ & $<$ & $<$ & $<$ & $<$ & $<$ \\
\hline Ethylbenzene & $<$ & $<$ & $<$ & $<$ & $0.3 \mathrm{~J}$ & $<$ & $<$ & $<$ & $<$ & $<$ \\
\hline Toluene & $<$ & $<$ & $<$ & $<$ & $<$ & $<$ & $<$ & $<$ & $<$ & $<$ \\
\hline Styrene & $<$ & $<$ & $<$ & $<$ & $<$ & $<$ & $<$ & $<$ & $<$ & $<$ \\
\hline \multicolumn{11}{|l|}{ Miscellaneous ( $\mu \mathrm{g} / \mathrm{L})$} \\
\hline 1,2-Dibromoethane & $<$ & & $<$ & & & $<$ & & $<$ & & \\
\hline 4-Methyl-2-pentanone & $<$ & $<$ & $<$ & $<$ & $<$ & $<$ & $<$ & $<$ & $<$ & $<$ \\
\hline Acetone & $<$ & $<$ & $<$ & $<$ & $<$ & $<$ & $<$ & $<$ & $<$ & $<$ \\
\hline Bromodichloromethane & $<$ & $<$ & $<$ & $<$ & $<$ & $<$ & $<$ & $<$ & $<$ & $<$ \\
\hline Bromoform & $<$ & $<$ & $<$ & $<$ & $<$ & $<$ & $<$ & $<$ & $<$ & $<$ \\
\hline Bromomethane & $<$ & $<$ & $<$ & $<$ & $<$ & $<$ & $<$ & $<$ & $<$ & $<$ \\
\hline Carbon disulfide & $<$ & $<$ & $<$ & $<$ & $<$ & $<$ & $<$ & $<$ & $<$ & $<$ \\
\hline Chlorobenzene & $<$ & $<$ & $<$ & $<$ & $<$ & $<$ & $<$ & $<$ & $<$ & $<$ \\
\hline Chlorodibromomethane & $<$ & $<$ & $<$ & $<$ & $<$ & $<$ & $<$ & $<$ & $<$ & $<$ \\
\hline Trichlorofluoromethane & $<$ & & $<$ & & 1 & $<$ & & $<$ & & \\
\hline \multicolumn{11}{|l|}{ Natural Attenuation $(\mu \mathrm{g} / \mathrm{L})$} \\
\hline Ethylene & & & & & & & & & & \\
\hline Methane & & & & & & & & & & \\
\hline
\end{tabular}


APPENDIX E.2: CY 2002 MONITORING DATA FOR THE UPPER EAST FORK POPLAR CREEK HYDROGEOLOGIC REGIME Volatile Organic Compounds

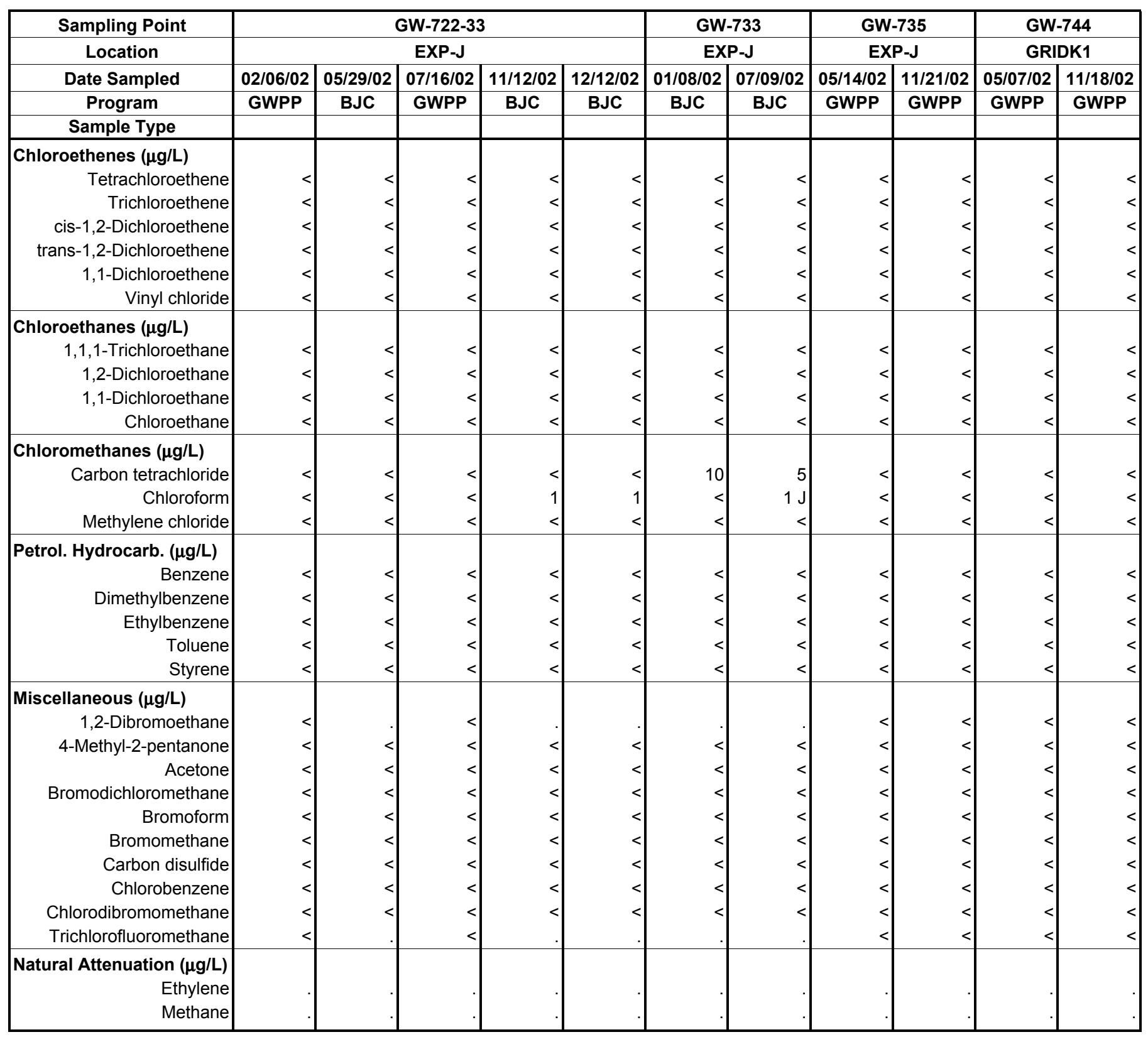


APPENDIX E.2: CY 2002 MONITORING DATA FOR THE UPPER EAST FORK POPLAR CREEK HYDROGEOLOGIC REGIME Volatile Organic Compounds

\begin{tabular}{|c|c|c|c|c|c|c|c|c|c|c|}
\hline Sampling Point & \multicolumn{2}{|c|}{ GW-747 } & \multicolumn{2}{|c|}{ GW-750 } & \multicolumn{4}{|c|}{ GW-762 } & \multicolumn{2}{|c|}{ GW-763 } \\
\hline Location & \multicolumn{2}{|c|}{ GRIDK2 } & \multicolumn{2}{|c|}{ EXP-J } & \multicolumn{4}{|c|}{ GRIDJ3 } & \multicolumn{2}{|c|}{ GRIDJ3 } \\
\hline Date Sampled & \multirow{2}{*}{\begin{tabular}{c|}
$05 / 09 / 02$ \\
GWPP
\end{tabular}} & \multirow{2}{*}{\begin{tabular}{|c|}
$11 / 20 / 02$ \\
GWPP \\
\end{tabular}} & \multirow{2}{*}{$\begin{array}{c}\text { 05/05/02 } \\
\text { GWPP }\end{array}$} & \multirow{2}{*}{$\begin{array}{c}11 / 20 / 02 \\
\text { GWPP }\end{array}$} & \multicolumn{2}{|c|}{$01 / 31 / 02$} & \multicolumn{2}{|c|}{ 07/31/02 } & \multirow{2}{*}{\begin{tabular}{|c|}
$06 / 12 / 02$ \\
GWPP
\end{tabular}} & \multirow{2}{*}{$\begin{array}{c}10 / 21 / 02 \\
\text { GWPP }\end{array}$} \\
\hline Program & & & & & BJC & BJC & BJC & BJC & & \\
\hline Sample Type & & & & & & Dup & & Dup & & \\
\hline Chloroethenes ( $\mu \mathrm{g} / \mathrm{L})$ & & & & & & & & & & \\
\hline Tetrachloroethene & $<$ & $<$ & $<$ & $<$ & 2,200 & 2,100 & 2,400 & 2,300 & 32 & 43 \\
\hline Trichloroethene & $<$ & $<$ & $<$ & $<$ & $110 \mathrm{~J}$ & 190 & 150 & 170 & 8 & 10 \\
\hline cis-1,2-Dichloroethene & $<$ & $<$ & $<$ & $<$ & 70 & 67 & 50 & 58 & 79 & 130 \\
\hline trans-1,2-Dichloroethene & $<$ & $<$ & $<$ & $<$ & $2 \mathrm{~J}$ & $2 \mathrm{~J}$ & $2 \mathrm{~J}$ & $2 \mathrm{~J}$ & $<$ & $<$ \\
\hline 1,1-Dichloroethene & $<$ & $<$ & $<$ & $<$ & $<$ & $<$ & 44 & 60 & $3 \mathrm{~J}$ & $4 \mathrm{~J}$ \\
\hline Vinyl chloride & $<$ & $<$ & $<$ & $<$ & 4 & 5 & 4 & 4 & 5 & 10 \\
\hline Chloroethanes $(\mu \mathrm{g} / \mathrm{L})$ & & & & & & & & & & \\
\hline 1,1,1-Trichloroethane & $<$ & $<$ & $<$ & $<$ & 6 & 5 & $4 \mathrm{~J}$ & $4 \mathrm{~J}$ & $<$ & $<$ \\
\hline 1,2-Dichloroethane & $<$ & $<$ & $<$ & $<$ & $<$ & $<$ & $<$ & $<$ & $<$ & $<$ \\
\hline 1,1-Dichloroethane & $<$ & $<$ & $<$ & $<$ & 15 & 13 & 10 & 12 & $<$ & $<$ \\
\hline Chloroethane & $<$ & $<$ & $<$ & $<$ & $<$ & $<$ & $<$ & $<$ & $<$ & $<$ \\
\hline Chloromethanes ( $\mu \mathrm{g} / \mathrm{L})$ & & & & & & & & & & \\
\hline Carbon tetrachloride & $<$ & $<$ & $<$ & $<$ & $<$ & $<$ & $<$ & $<$ & $<$ & $<$ \\
\hline Chloroform & $<$ & $<$ & $<$ & $<$ & $<$ & $<$ & $<$ & $<$ & $<$ & $<$ \\
\hline Methylene chloride & $<$ & $<$ & $<$ & $<$ & $<$ & $<$ & $<$ & $<$ & $<$ & $<$ \\
\hline Petrol. Hydrocarb. ( $\mu \mathrm{g} / \mathrm{L})$ & & & & & & & & & & \\
\hline Benzene & $<$ & $<$ & $<$ & $<$ & $<$ & $<$ & $<$ & $<$ & $<$ & $<$ \\
\hline Dimethylbenzene & $<$ & $<$ & $<$ & $<$ & $<$ & $<$ & $<$ & $<$ & $<$ & $<$ \\
\hline Ethylbenzene & $<$ & $<$ & $<$ & $<$ & $<$ & $<$ & $<$ & $<$ & $<$ & $<$ \\
\hline Toluene & $<$ & $<$ & $<$ & $<$ & $<$ & $<$ & $<$ & $<$ & $<$ & $<$ \\
\hline Styrene & $<$ & $<$ & $<$ & $<$ & $<$ & $<$ & $<$ & $<$ & $<$ & $<$ \\
\hline Miscellaneous ( $\mu \mathrm{g} / \mathrm{L})$ & & & & & & & & & & \\
\hline 1,2-Dibromoethane & $<$ & $<$ & $<$ & $<$ & . & 0 & & & $<$ & $<$ \\
\hline 4-Methyl-2-pentanone & $<$ & $<$ & $<$ & $<$ & $<$ & $<$ & $<$ & $<$ & $<$ & $<$ \\
\hline Acetone & $<$ & $<$ & $<$ & $<$ & $<$ & $<$ & $<$ & $<$ & $<$ & $<$ \\
\hline Bromodichloromethane & $<$ & $<$ & $<$ & $<$ & $<$ & $<$ & $<$ & $<$ & $<$ & $<$ \\
\hline Bromoform & $<$ & $<$ & $<$ & $<$ & $<$ & $<$ & $<$ & $<$ & $<$ & $<$ \\
\hline Bromomethane & $<$ & $<$ & $<$ & $<$ & $<$ & $<$ & $<$ & $<$ & $<$ & $<$ \\
\hline Carbon disulfide & $<$ & $<$ & $<$ & $<$ & $<$ & $<$ & $<$ & $<$ & $<$ & $<$ \\
\hline Chlorobenzene & $<$ & $<$ & $<$ & $<$ & $<$ & $<$ & $<$ & $<$ & $<$ & $<$ \\
\hline Chlorodibromomethane & $<$ & $<$ & $<$ & $<$ & $<$ & $<$ & $<$ & $<$ & $<$ & $<$ \\
\hline Trichlorofluoromethane & $<$ & $<$ & $<$ & $<$ & . &. &. & 1 & $<$ & $<$ \\
\hline Natural Attenuation $(\mu \mathrm{g} / \mathrm{L})$ & & & & & & & & & & \\
\hline Ethylene & & & & & $8 \mathrm{~J}$ & $<$ & $<$ & 8 & & \\
\hline Methane & & & & & 46 & 39 & 46 & 55 & & \\
\hline
\end{tabular}


APPENDIX E.2: CY 2002 MONITORING DATA FOR THE UPPER EAST FORK POPLAR CREEK HYDROGEOLOGIC REGIME Volatile Organic Compounds

\begin{tabular}{|c|c|c|c|c|c|c|c|c|c|c|}
\hline Sampling Point & \multicolumn{2}{|c|}{ GW-769 } & \multicolumn{2}{|c|}{ GW-770 } & \multicolumn{2}{|c|}{ GW-775 } & \multicolumn{2}{|c|}{ GW-776 } & \multicolumn{2}{|c|}{ GW-782 } \\
\hline Location & \multicolumn{2}{|c|}{ GRIDG3 } & \multicolumn{2}{|c|}{ GRIDG3 } & \multicolumn{2}{|c|}{ GRIDH3 } & \multicolumn{2}{|c|}{ GRIDH3 } & \multicolumn{2}{|c|}{ GRIDE3 } \\
\hline Date Sampled & $04 / 29 / 02$ & $10 / 30 / 02$ & $04 / 29 / 02$ & $10 / 30 / 02$ & $04 / 30 / 02$ & $10 / 31 / 02$ & $04 / 30 / 02$ & $10 / 31 / 02$ & 05/01/02 & $11 / 04 / 02$ \\
\hline Program & GWPP & GWPP & GWPP & GWPP & GWPP & GWPP & GWPP & GWPP & GWPP & GWPP \\
\hline \multicolumn{11}{|l|}{ Sample Type } \\
\hline Chloroethenes ( $\mu \mathrm{g} / \mathrm{L}$ ) & & & & & & & & & & \\
\hline Tetrachloroethene & 11 & 22 & $<$ & $<$ & $<$ & $<$ & $<$ & $<$ & 170 & 160 \\
\hline Trichloroethene & $3 \mathrm{~J}$ & 6 & $<$ & $<$ & $4 \mathrm{~J}$ & 6 & $<$ & $2 \mathrm{~J}$ & 60 & 56 \\
\hline cis-1,2-Dichloroethene & $3 \mathrm{~J}$ & 6 & $<$ & $<$ & $<$ & $<$ & $<$ & $<$ & 13 & 13 \\
\hline trans-1,2-Dichloroethene & $<$ & $<$ & $<$ & $<$ & $<$ & $<$ & $<$ & $<$ & $2 \mathrm{~J}$ & $2 \mathrm{~J}$ \\
\hline 1,1-Dichloroethene & $<$ & $<$ & $<$ & $<$ & $<$ & $<$ & $<$ & $<$ & 48 & 32 \\
\hline Vinyl chloride & $<$ & $<$ & $<$ & $<$ & $<$ & $<$ & $<$ & $<$ & $<$ & 2 \\
\hline \multicolumn{11}{|l|}{ Chloroethanes ( $\mu \mathrm{g} / \mathrm{L})$} \\
\hline 1,1,1-Trichloroethane & $<$ & $<$ & $<$ & $<$ & $<$ & $<$ & $<$ & $<$ & 9 & $3 \mathrm{~J}$ \\
\hline 1,2-Dichloroethane & $<$ & $<$ & $<$ & $<$ & $<$ & $<$ & $<$ & $<$ & $<$ & $<$ \\
\hline 1,1-Dichloroethane & $<$ & $<$ & $<$ & $<$ & $<$ & $<$ & $<$ & $<$ & 180 & 130 \\
\hline Chloroethane & $<$ & $<$ & $<$ & $<$ & $<$ & $<$ & $<$ & $<$ & 12 & 7 \\
\hline \multicolumn{11}{|l|}{ Chloromethanes ( $\mu \mathrm{g} / \mathrm{L})$} \\
\hline Carbon tetrachloride & 58 & 160 & $3 \mathrm{~J}$ & 7 & $<$ & $<$ & $<$ & $<$ & $<$ & $<$ \\
\hline Chloroform & $3 \mathrm{~J}$ & 6 & $5 \mathrm{~J}$ & $3 \mathrm{~J}$ & $<$ & $<$ & $<$ & $<$ & $<$ & $<$ \\
\hline Methylene chloride & $<$ & 7 & $<$ & $<$ & $<$ & $<$ & $<$ & $<$ & $<$ & $<$ \\
\hline \multicolumn{11}{|l|}{ Petrol. Hydrocarb. ( $\mu \mathrm{g} / \mathrm{L})$} \\
\hline Benzene & $<$ & $<$ & $<$ & $<$ & $<$ & $<$ & $<$ & $<$ & $<$ & $<$ \\
\hline Dimethylbenzene & $<$ & $<$ & $<$ & $<$ & $<$ & $<$ & $<$ & $<$ & $<$ & $<$ \\
\hline Ethylbenzene & $<$ & $<$ & $<$ & $<$ & $<$ & $<$ & $<$ & $<$ & $<$ & $<$ \\
\hline Toluene & $<$ & $<$ & $<$ & $<$ & $<$ & $<$ & $<$ & $<$ & $<$ & $<$ \\
\hline Styrene & $<$ & $<$ & $<$ & $<$ & $<$ & $<$ & $<$ & $<$ & $<$ & $<$ \\
\hline \multicolumn{11}{|l|}{ Miscellaneous $(\mu \mathrm{g} / \mathrm{L})$} \\
\hline 1,2-Dibromoethane & $<$ & $<$ & $<$ & $<$ & $<$ & $<$ & $<$ & $<$ & $<$ & $<$ \\
\hline 4-Methyl-2-pentanone & $<$ & $<$ & $<$ & $<$ & $<$ & $<$ & $<$ & $<$ & $<$ & $<$ \\
\hline Acetone & $<$ & $<$ & $<$ & $<$ & $<$ & $<$ & $<$ & $<$ & $<$ & $<$ \\
\hline Bromodichloromethane & $<$ & $<$ & $<$ & $<$ & $<$ & $<$ & $<$ & $<$ & $<$ & $<$ \\
\hline Bromoform & $<$ & $<$ & $<$ & $<$ & $<$ & $<$ & $<$ & $<$ & $<$ & $<$ \\
\hline Bromomethane & $<$ & $<$ & $<$ & $<$ & $<$ & $<$ & $<$ & $<$ & $<$ & $<$ \\
\hline Carbon disulfide & $<$ & $<$ & $<$ & $<$ & $<$ & $<$ & $<$ & $<$ & $<$ & $<$ \\
\hline Chlorobenzene & $<$ & $<$ & $<$ & $<$ & $<$ & $<$ & $<$ & $<$ & $<$ & $<$ \\
\hline Chlorodibromomethane & $<$ & $<$ & $<$ & $<$ & $<$ & $<$ & $<$ & $<$ & $<$ & $<$ \\
\hline Trichlorofluoromethane & $<$ & $<$ & $<$ & $<$ & $<$ & $<$ & $<$ & $<$ & $<$ & $<$ \\
\hline Natural Attenuation $(\mu \mathrm{g} / \mathrm{L})$ & & & & & & & & & & \\
\hline Ethylene & & & & & & & & & & \\
\hline Methane & & & & & & & & & & \\
\hline
\end{tabular}


APPENDIX E.2: CY 2002 MONITORING DATA FOR THE UPPER EAST FORK POPLAR CREEK HYDROGEOLOGIC REGIME Volatile Organic Compounds

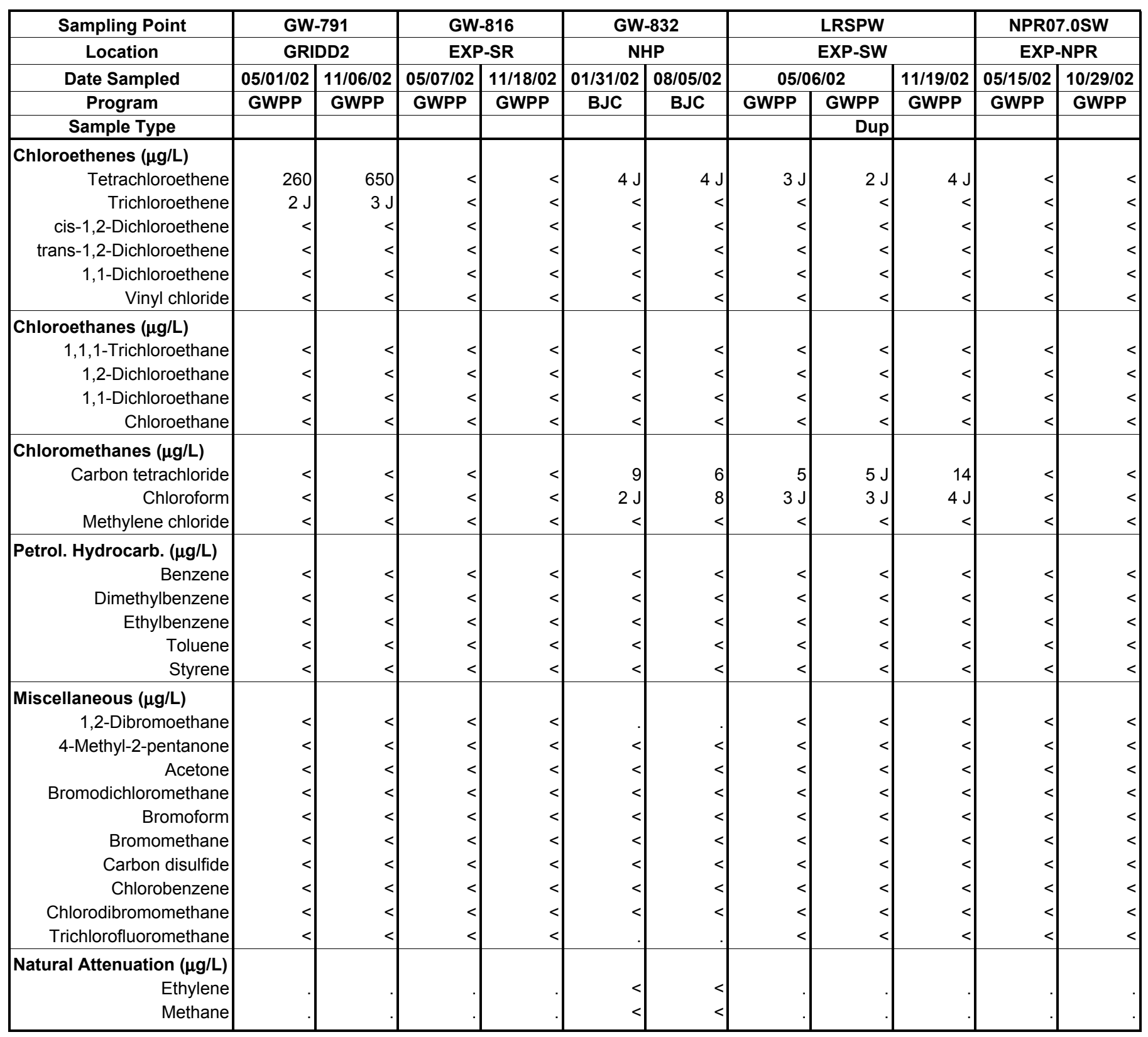


APPENDIX E.2: CY 2002 MONITORING DATA FOR THE UPPER EAST FORK POPLAR CREEK HYDROGEOLOGIC REGIME Volatile Organic Compounds

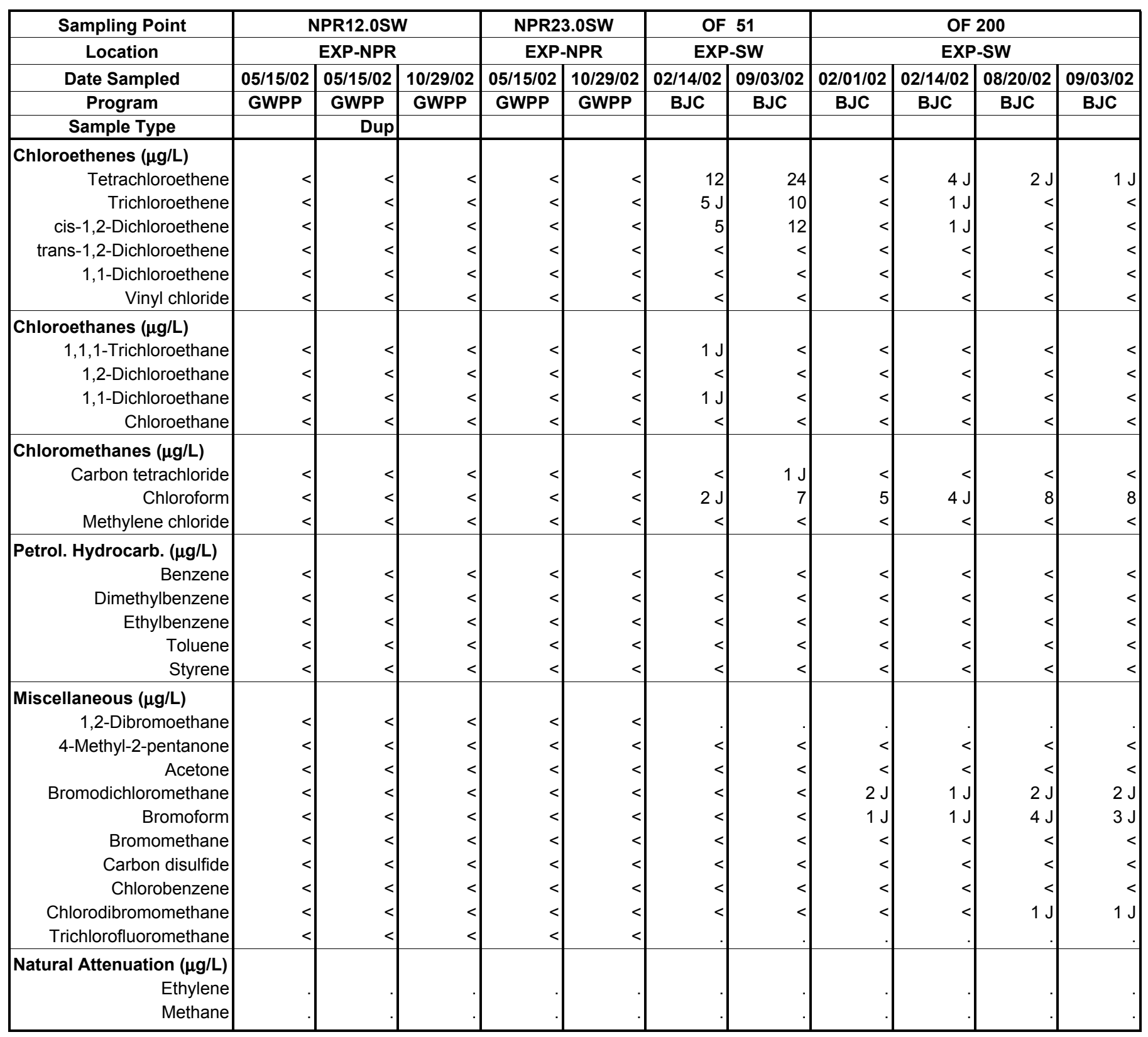


APPENDIX E.2: CY 2002 MONITORING DATA FOR THE UPPER EAST FORK POPLAR CREEK HYDROGEOLOGIC REGIME Volatile Organic Compounds

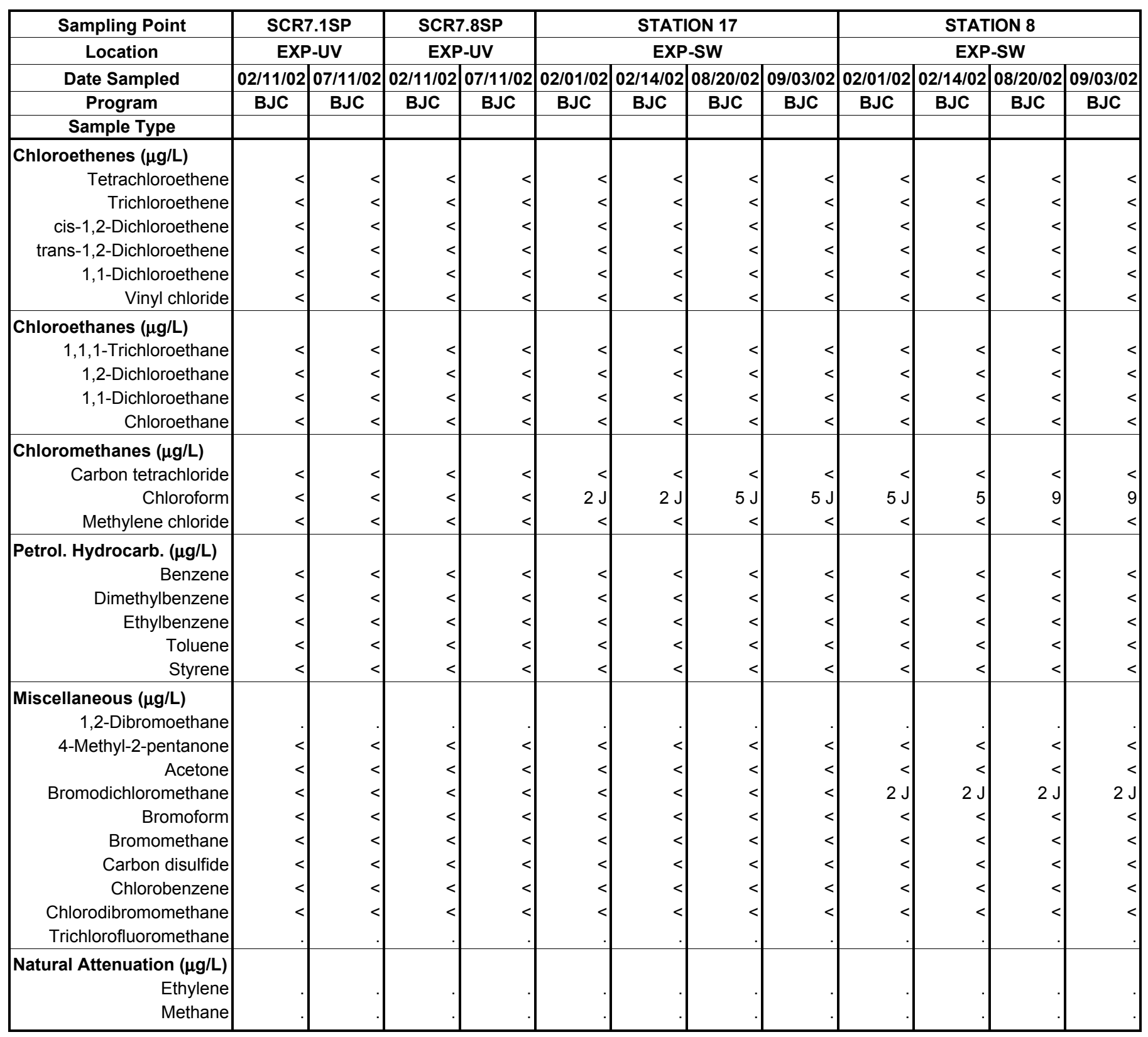


APPENDIX E.3

RADIOLOGICAL ANALYTES 
APPENDIX E.3: CY 2002 MONITORING DATA FOR THE UPPER EAST FORK POPLAR CREEK HYDROGEOLOGIC REGIME Radiological Analytes: Gross Alpha and Gross Beta Activity

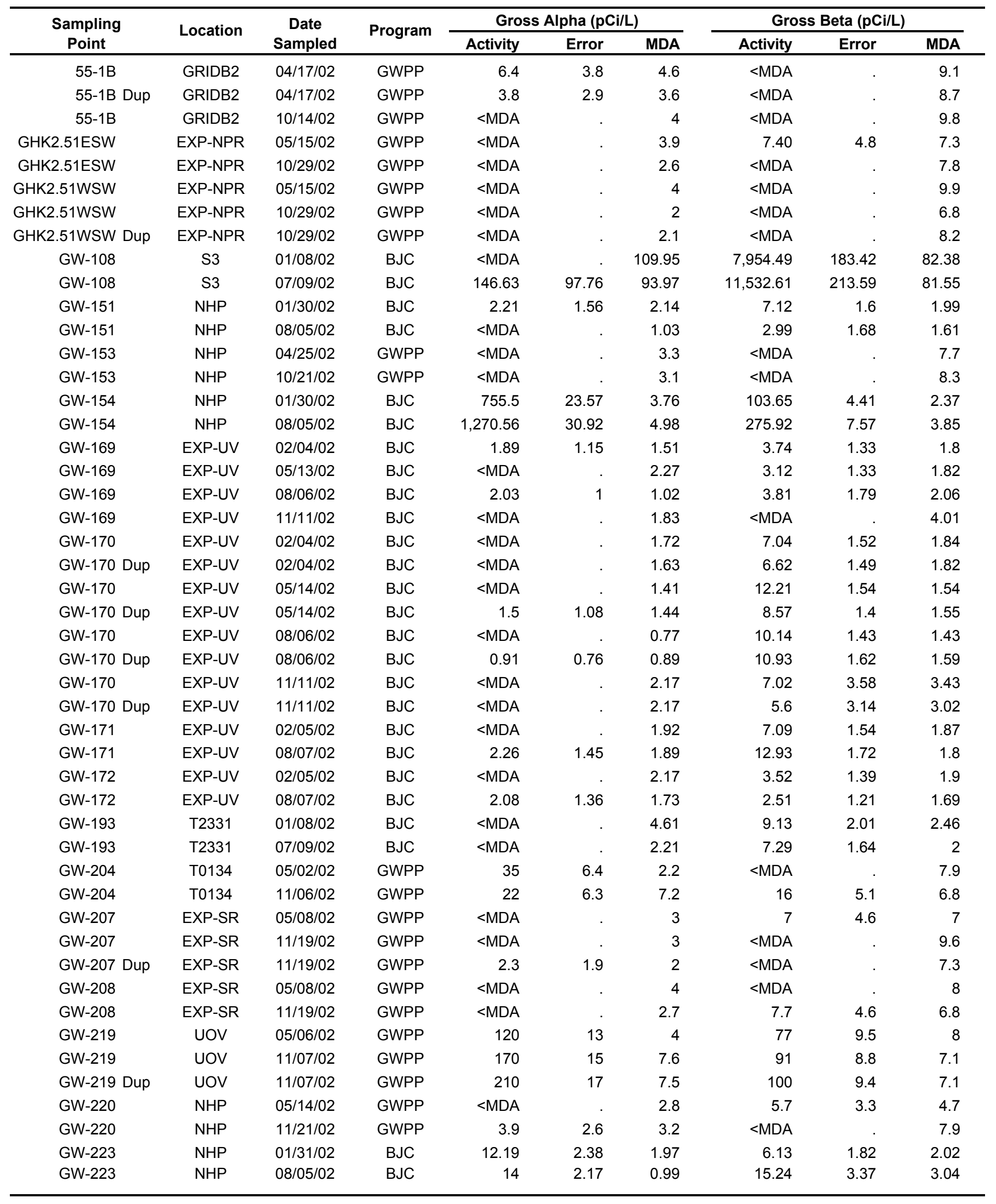


APPENDIX E.3: CY 2002 MONITORING DATA FOR THE UPPER EAST FORK POPLAR CREEK HYDROGEOLOGIC REGIME Radiological Analytes: Gross Alpha and Gross Beta Activity

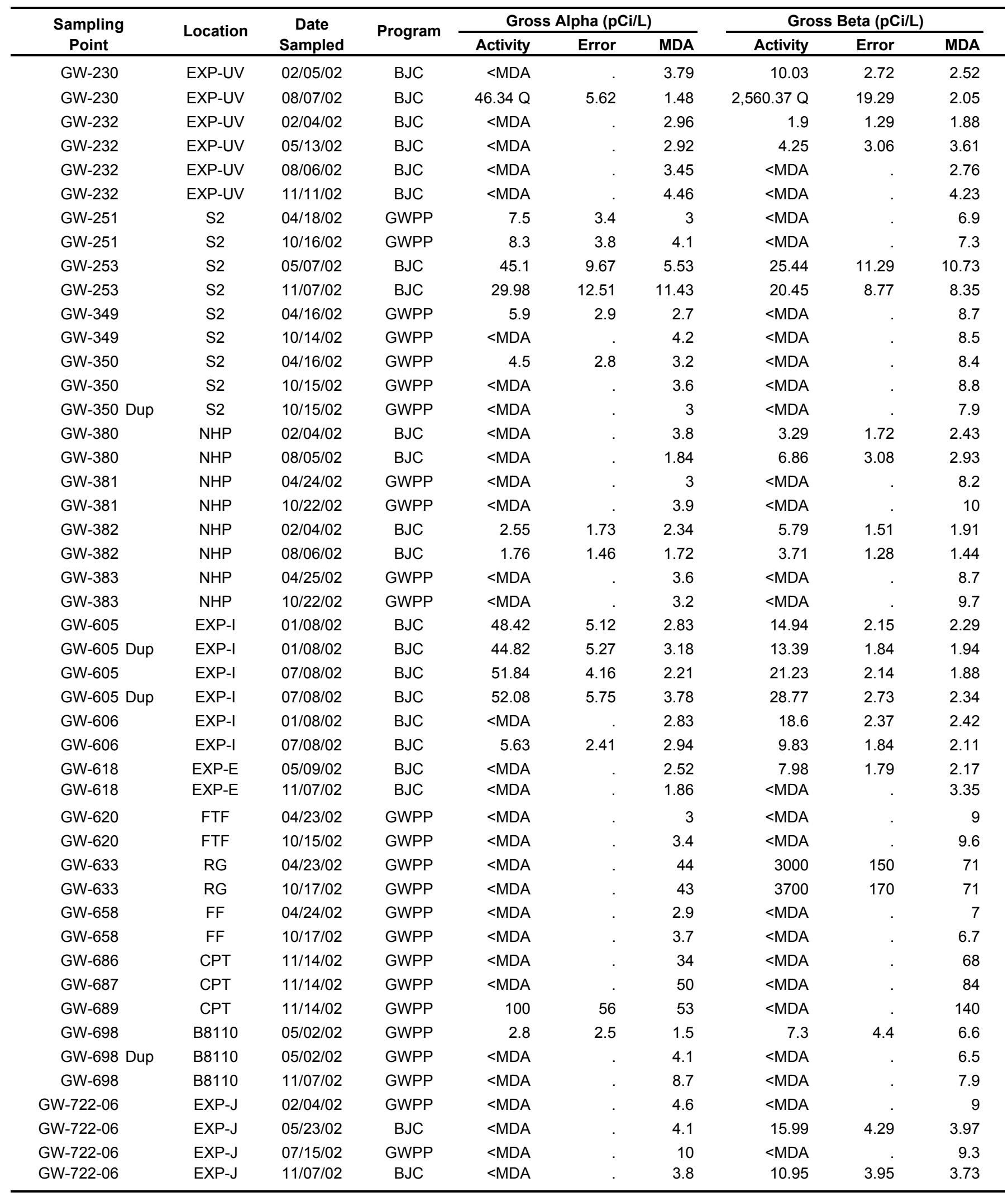


APPENDIX E.3: CY 2002 MONITORING DATA FOR THE UPPER EAST FORK POPLAR CREEK HYDROGEOLOGIC REGIME Radiological Analytes: Gross Alpha and Gross Beta Activity

\begin{tabular}{|c|c|c|c|c|c|c|c|c|c|}
\hline \multirow{2}{*}{$\begin{array}{c}\text { Sampling } \\
\text { Point }\end{array}$} & \multirow{2}{*}{ Location } & \multirow{2}{*}{$\begin{array}{c}\text { Date } \\
\text { Sampled }\end{array}$} & \multirow{2}{*}{ Program } & \multicolumn{3}{|c|}{ Gross Alpha (pCi/L) } & \multicolumn{3}{|c|}{ Gross Beta (pCi/L) } \\
\hline & & & & Activity & Error & MDA & Activity & Error & MDA \\
\hline GW-722-10 & EXP-J & 02/06/02 & GWPP & $<M D A$ & . & 3.2 & $<M D A$ & & 7.4 \\
\hline GW-722-10 & EXP-J & $05 / 29 / 02$ & BJC & $<M D A$ & . & 3.74 & 7.79 & 6.39 & 6.22 \\
\hline GW-722-10 & EXP-J & 07/16/02 & GWPP & $<M D A$ & . & 7 & $<M D A$ & . & 9.2 \\
\hline GW-722-10 & EXP-J & $11 / 12 / 02$ & BJC & $<M D A$ & . & 2.55 & 3.56 & 2.84 & 2.77 \\
\hline GW-722-14 & EXP-J & 02/08/02 & GWPP & $<M D A$ & . & 2.7 & $<M D A$ & . & 8.4 \\
\hline GW-722-14 & EXP-J & 05/30/02 & BJC & $<M D A$ & . & 2.53 & $<M D A$ & . & 3.51 \\
\hline GW-722-14 & EXP-J & $07 / 17 / 02$ & GWPP & $<M D A$ & . & 3.6 & $<M D A$ & . & 7.4 \\
\hline GW-722-14 & EXP-J & $11 / 13 / 02$ & BJC & 9.58 & 2.72 & 2.29 & 13.22 & 3.86 & 3.59 \\
\hline GW-722-17 & EXP-J & 02/08/02 & GWPP & 2.6 & 2 & 2.3 & $<M D A$ & . & 8.5 \\
\hline GW-722-17 & EXP-J & 06/03/02 & BJC & $<M D A$ & . & 2.06 & $<M D A$ & . & 3.11 \\
\hline GW-722-17 & EXP-J & $07 / 17 / 02$ & GWPP & $<$ MDA & . & 4.1 & $<\mathrm{MDA}$ & . & 8.3 \\
\hline GW-722-17 & EXP-J & $11 / 13 / 02$ & BJC & $<M D A$ & . & 2.37 & 3.93 & 3.7 & 3.61 \\
\hline GW-722-20 & EXP-J & 02/07/02 & GWPP & $<M D A$ & . & 3.1 & $<M D A$ & . & 7.5 \\
\hline GW-722-20 & EXP-J & $05 / 30 / 02$ & BJC & $<M D A$ & . & 1.49 & $<\mathrm{MDA}$ & . & 3.38 \\
\hline GW-722-20 & EXP-J & 07/17/02 & GWPP & $<M D A$ & . & 2.9 & $<M D A$ & . & 6.5 \\
\hline GW-722-20 Dup & EXP-J & 07/17/02 & GWPP & $<M D A$ & . & 2.9 & $<M D A$ & . & 8.1 \\
\hline GW-722-20 & EXP-J & $11 / 12 / 02$ & BJC & $<M D A$ & . & 1.97 & $<M D A$ & . & 2.44 \\
\hline GW-722-22 & EXP-J & 02/07/02 & GWPP & $<M D A$ & . & 2.7 & $<M D A$ & . & 6.8 \\
\hline GW-722-22 Dup & EXP-J & 02/07/02 & GWPP & $<$ MDA & . & 2.6 & $<M D A$ & . & 8.2 \\
\hline GW-722-22 & EXP-J & 05/30/02 & BJC & 2.62 & 1.49 & 1.39 & $<\mathrm{MDA}$ & . & 3.17 \\
\hline GW-722-22 & EXP-J & 07/16/02 & GWPP & $<M D A$ & . & 4.1 & $<M D A$ & . & 5.8 \\
\hline GW-722-22 & EXP-J & $11 / 12 / 02$ & BJC & $<$ MDA & . & 1.94 & 8.02 & 2.24 & 2.44 \\
\hline GW-722-26 & EXP-J & 02/05/02 & GWPP & 1.9 & 1.5 & 0.74 & $<M D A$ & . & 8.3 \\
\hline GW-722-26 & EXP-J & 05/28/02 & BJC & $<M D A$ & . & 2.09 & 9.48 & 3.45 & 3.25 \\
\hline GW-722-26 Dup & EXP-J & $05 / 28 / 02$ & BJC & 4.59 & 2.15 & 1.98 & $<\mathrm{MDA}$ & . & 3.84 \\
\hline GW-722-26 & EXP-J & 07/15/02 & GWPP & $<M D A$ & . & 2.6 & $<M D A$ & . & 9.1 \\
\hline GW-722-26 & EXP-J & $11 / 11 / 02$ & BJC & $<\mathrm{MDA}$ & . & 1.83 & 8.81 & 2.74 & 2.53 \\
\hline GW-722-26 Dup & EXP-J & $11 / 11 / 02$ & BJC & $<M D A$ & . & 2.27 & 3.74 & 3.1 & 3.02 \\
\hline GW-722-30 & EXP-J & 02/05/02 & GWPP & $<M D A$ & . & 3.1 & $<M D A$ & & 11 \\
\hline GW-722-30 & EXP-J & 05/28/02 & BJC & 1.96 & 1.83 & 1.82 & 11.22 & 3.59 & 3.36 \\
\hline GW-722-30 & EXP-J & 07/15/02 & GWPP & $<$ MDA & . & 2.6 & $<\mathrm{MDA}$ & $\cdot$ & 7.4 \\
\hline GW-722-30 & EXP-J & $11 / 11 / 02$ & BJC & $<$ MDA & . & 1.73 & 28.4 & 3.79 & 3.24 \\
\hline GW-722-32 & EXP-J & 02/05/02 & GWPP & 8.1 & 3.1 & 0.81 & $<M D A$ & 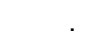 & 9.6 \\
\hline GW-722-32 & EXP-J & 05/28/02 & BJC & $<M D A$ & . & 1.82 & $<M D A$ & . & 3.8 \\
\hline GW-722-32 & EXP-J & 07/15/02 & GWPP & $<M D A$ & . & 2.3 & $<M D A$ & & 7 \\
\hline GW-722-32 & EXP-J & $11 / 12 / 02$ & BJC & $44.16 \mathrm{Q}$ & 4.28 & 2.29 & 5.43 & 3.68 & 3.57 \\
\hline GW-722-33 & EXP-J & 02/06/02 & GWPP & $<M D A$ & . & 2.2 & $<M D A$ & . & 6.4 \\
\hline GW-722-33 & EXP-J & 05/29/02 & BJC & $<M D A$ & . & 6.61 & $<M D A$ & 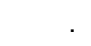 & 8.88 \\
\hline GW-722-33 & EXP-J & 07/16/02 & GWPP & $<M D A$ & . & 3.9 & $<M D A$ & & 7.5 \\
\hline GW-722-33 & EXP-J & $11 / 12 / 02$ & BJC & 3.24 & 1.65 & 1.78 & 19.73 & 2.55 & 2.4 \\
\hline GW-733 & EXP-J & 01/08/02 & BJC & $<M D A$ & . & 1.84 & 3.41 & 1.33 & 1.82 \\
\hline GW-733 & EXP-J & 07/09/02 & BJC & $<M D A$ & & 1.94 & $<\mathrm{MDA}$ & 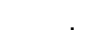 & 3.57 \\
\hline GW-735 & EXP-J & $05 / 14 / 02$ & GWPP & $<M D A$ & . & 4.9 & $<M D A$ & . & 6.4 \\
\hline GW-735 & EXP-J & $11 / 21 / 02$ & GWPP & $<M D A$ & . & 2.8 & $<M D A$ & . & 6.8 \\
\hline GW-744 & GRIDK1 & 05/07/02 & GWPP & 4.9 & 2.9 & 3.2 & 6.3 & 4.1 & 6.2 \\
\hline GW-744 & GRIDK1 & $11 / 18 / 02$ & GWPP & $<M D A$ & . & 2.1 & $<M D A$ & . & 7.7 \\
\hline GW-747 & GRIDK2 & 05/09/02 & GWPP & 1.2 & 1.2 & 0.83 & $<M D A$ & . & 7.5 \\
\hline GW-747 & GRIDK2 & $11 / 20 / 02$ & GWPP & $<M D A$ & . & 3.3 & $<M D A$ & . & 5.7 \\
\hline
\end{tabular}


APPENDIX E.3: CY 2002 MONITORING DATA FOR THE UPPER EAST FORK POPLAR CREEK HYDROGEOLOGIC REGIME Radiological Analytes: Gross Alpha and Gross Beta Activity

\begin{tabular}{|c|c|c|c|c|c|c|c|c|c|}
\hline \multirow{2}{*}{$\begin{array}{l}\text { Sampling } \\
\text { Point }\end{array}$} & \multirow{2}{*}{ Location } & \multirow{2}{*}{$\begin{array}{c}\text { Date } \\
\text { Sampled }\end{array}$} & \multirow{2}{*}{ Program } & \multicolumn{3}{|c|}{ Gross Alpha (pCi/L) } & \multicolumn{3}{|c|}{ Gross Beta (pCi/L) } \\
\hline & & & & Activity & Error & MDA & Activity & Error & MDA \\
\hline GW-750 & EXP-J & 05/05/02 & GWPP & $<\mathrm{MDA}$ & . & 2.4 & $<\mathrm{MDA}$ & . & 7.4 \\
\hline GW-762 & GRIDJ3 & $01 / 31 / 02$ & BJC & $<M D A$ & . & 2.06 & 5.4 & 1.42 & 1.74 \\
\hline GW-762 Dup & GRIDJ3 & $01 / 31 / 02$ & BJC & $<$ MDA & . & 1.54 & 5.28 & 1.44 & 1.55 \\
\hline GW-762 & GRIDJ3 & $07 / 31 / 02$ & BJC & $<M D A$ & . & 1.23 & 3.84 & 1.61 & 1.53 \\
\hline GW-763 & GRIDJ3 & 04/18/02 & GWPP & $<\mathrm{MDA}$ & . & 4.2 & $<\mathrm{MDA}$ & . & 7.1 \\
\hline GW-763 & GRIDJ3 & $10 / 21 / 02$ & GWPP & $<$ MDA & . & 4 & $<\mathrm{MDA}$ & . & 9.2 \\
\hline GW-769 & GRIDG3 & $04 / 29 / 02$ & GWPP & 2.9 & 2.2 & 2.8 & $<\mathrm{MDA}$ & . & 8.9 \\
\hline GW-769 & GRIDG3 & 10/30/02 & GWPP & $<M D A$ & . & 7.3 & $<M D A$ & . & 8.6 \\
\hline GW-770 & GRIDG3 & 04/29/02 & GWPP & $<M D A$ & . & 3.7 & $<\mathrm{MDA}$ & . & 7.9 \\
\hline GW-776 & GRIDH3 & 04/30/02 & GWPP & $<M D A$ & . & 2.4 & 7.5 & 4.6 & 7 \\
\hline GW-776 & GRIDH3 & $10 / 31 / 02$ & GWPP & $<M D A$ & . & 7.8 & $<\mathrm{MDA}$ & . & 8.4 \\
\hline GW-782 & GRIDE3 & 05/01/02 & GWPP & 45 & 7.7 & 2.5 & 9 & 4.6 & 6.7 \\
\hline GW-782 & GRIDE3 & $11 / 04 / 02$ & GWPP & 38 & 7.8 & 7.3 & 11 & 6.3 & 9.7 \\
\hline GW-791 & GRIDD2 & 05/01/02 & GWPP & $<M D A$ & . & 2.7 & $<\mathrm{MDA}$ & . & 8.7 \\
\hline GW-791 & GRIDD2 & $11 / 06 / 02$ & GWPP & $<M D A$ & . & 7 & $<\mathrm{MDA}$ & . & 9.2 \\
\hline GW-816 & EXP-SR & 05/07/02 & GWPP & $<\mathrm{MDA}$ & . & 3.5 & 8.7 & 5.5 & 8.5 \\
\hline GW-816 & EXP-SR & 11/18/02 & GWPP & $<\mathrm{MDA}$ & . & 2.3 & 7.2 & 4.6 & 6.9 \\
\hline GW-832 & NHP & $01 / 31 / 02$ & BJC & 4.67 & 1.82 & 2.08 & 5.15 & 1.51 & 1.98 \\
\hline GW-832 & $\mathrm{NHP}$ & 08/05/02 & BJC & 2.72 & 1.06 & 0.93 & 4.23 & 1.93 & 1.85 \\
\hline NPR12.0SW & EXP-NPR & $10 / 29 / 02$ & GWPP & $<M D A$ & . & 2.5 & 6.9 & 3.8 & 5.5 \\
\hline NPR23.0SW & EXP-NPR & 05/15/02 & GWPP & $<M D A$ & . & 3.1 & $<\mathrm{MDA}$ & . & 7 \\
\hline NPR23.0SW & EXP-NPR & 10/29/02 & GWPP & 3.7 & 2.5 & 3 & $<\mathrm{MDA}$ & . & 9.7 \\
\hline OF 51 & EXP-SW & $02 / 14 / 02$ & BJC & 4.27 & 1.39 & 1.18 & 4.67 & 1.3 & 1.68 \\
\hline OF 51 & EXP-SW & 09/03/02 & BJC & 3.74 & 1.3 & 1.16 & 4.89 & 1.17 & 1.41 \\
\hline SCR7.1SP & EXP-UV & $02 / 11 / 02$ & BJC & 1.85 & 1.03 & 1.2 & 3.39 & 1.3 & 1.78 \\
\hline SCR7.1SP & EXP-UV & 07/11/02 & BJC & $<M D A$ & . & 1.9 & 2.53 & 1.21 & 1.7 \\
\hline SCR7.8SP & EXP-UV & $02 / 11 / 02$ & BJC & $<\mathrm{MDA}$ & . & 1.27 & 2.39 & 1.3 & 1.85 \\
\hline SCR7.8SP & EXP-UV & 07/11/02 & BJC & $<\mathrm{MDA}$ & . & 1.15 & 3.53 & 1.08 & 1.39 \\
\hline STATION 8 & EXP-SW & 02/01/02 & BJC & 16.72 & 2.73 & 1.92 & 8.96 & 1.66 & 1.95 \\
\hline STATION 8 & EXP-SW & $02 / 14 / 02$ & BJC & 7.6 & 2.16 & 2.14 & 7.31 & 1.41 & 1.64 \\
\hline STATION 8 & EXP-SW & 08/20/02 & BJC & 3.56 & 1.25 & 1.2 & 5.43 & 1.42 & 1.77 \\
\hline STATION 8 & EXP-SW & 09/03/02 & BJC & 2.35 & 1.08 & 1.18 & 4.28 & 1.26 & 1.64 \\
\hline
\end{tabular}


APPENDIX E.3: CY 2002 MONITORING DATA FOR THE UPPER EAST FORK POPLAR CREEK HYDROGEOLOGIC REGIME Radiological Analytes: Isotope Activity

\begin{tabular}{|c|c|c|c|c|c|c|c|c|c|}
\hline Sampling Point & \multicolumn{6}{|c|}{ GW-108 } & \multicolumn{3}{|c|}{ GW-151 } \\
\hline Location & \multicolumn{6}{|c|}{ S3 } & \multicolumn{3}{|c|}{ NHP } \\
\hline Date Sampled & \multicolumn{3}{|c|}{$01 / 08 / 02$} & \multicolumn{3}{|c|}{$07 / 09 / 02$} & \multicolumn{3}{|c|}{$01 / 30 / 02$} \\
\hline Program & \multicolumn{3}{|c|}{ BJC } & \multicolumn{3}{|c|}{ BJC } & \multicolumn{3}{|c|}{ BJC } \\
\hline \multicolumn{10}{|l|}{ Sample Type } \\
\hline Result (pCi/L) & Activity & Error & MDA & Activity & Error & MDA & Activity & Error & MDA \\
\hline Gross Alpha & $<\mathrm{MDA}$ & & 109.95 & 146.63 & 97.76 & 93.97 & 2.21 & 1.56 & 2.14 \\
\hline Gross Beta & $7,954.49$ & 183.42 & 82.38 & $11,532.61$ & 213.59 & 81.55 & 7.12 & 1.6 & 1.99 \\
\hline Technetium-99 & $29,525.35$ & 201.6 & 31 & $26,872.71$ & 65.54 & 6.77 & & & \\
\hline Uranium-234 & 15 & 3.58 & 0.47 & 12.91 & 2.63 & 0.14 & 0.48 & 0.39 & 0.47 \\
\hline Uranium-235 & $<\mathrm{MDA}$ & & 0.64 & 0.38 & 0.32 & 0.17 & $<\mathrm{MDA}$ & & 0.45 \\
\hline Uranium-236 & & & & $<M D A$ & & 0.15 & $<\mathrm{MDA}$ & & 0.34 \\
\hline Uranium-238 & 6.04 & 1.82 & 0.46 & 5.23 & 1.33 & 0.14 & $<\mathrm{MDA}$ & & 0.44 \\
\hline
\end{tabular}

\begin{tabular}{|c|c|c|c|c|c|c|c|c|c|}
\hline Sampling Point & \multicolumn{3}{|c|}{ GW-151 } & \multicolumn{6}{|c|}{ GW-154 } \\
\hline Location & \multicolumn{3}{|c|}{ NHP } & \multicolumn{6}{|c|}{ NHP } \\
\hline Date Sampled & \multicolumn{3}{|c|}{$08 / 05 / 02$} & \multicolumn{3}{|c|}{$01 / 30 / 02$} & \multicolumn{3}{|c|}{$08 / 05 / 02$} \\
\hline Program & \multicolumn{3}{|c|}{ BJC } & \multicolumn{3}{|c|}{ BJC } & \multicolumn{3}{|c|}{ BJC } \\
\hline \multicolumn{10}{|l|}{ Sample Type } \\
\hline Result (pCi/L) & Activity & Error & MDA & Activity & Error & MDA & Activity & Error & MDA \\
\hline Gross Alpha & $<\mathrm{MDA}$ & & 1.03 & 755.5 & 23.57 & 3.76 & $1,270.56$ & 30.92 & 4.98 \\
\hline Gross Beta & 2.99 & 1.68 & 1.61 & 103.65 & 4.41 & 2.37 & 275.92 & 7.57 & 3.85 \\
\hline \multicolumn{10}{|l|}{ Technetium-99 } \\
\hline Uranium-234 & $<\mathrm{MDA}$ & & 0.88 & 392.7 & 139.6 & 3.83 & 295.3 & 54.14 & 6.69 \\
\hline Uranium-235 & $<\mathrm{MDA}$ & & 0.83 & 14.9 & 7.72 & 2.92 & 10.99 & 6.2 & 3.68 \\
\hline Uranium-236 & $<\mathrm{MDA}$ & & 0.44 & 9.1 & 5.39 & 3.77 & 11.42 & 5.99 & 1.93 \\
\hline Uranium-238 & $<\mathrm{MDA}$ & & 0.88 & 251 & 90.13 & 2.85 & 248 & 46.52 & 3.87 \\
\hline
\end{tabular}

\begin{tabular}{|c|c|c|c|c|c|c|c|c|c|}
\hline Sampling Point & \multicolumn{6}{|c|}{ GW-193 } & \multicolumn{3}{|c|}{ GW-204 } \\
\hline Location & \multicolumn{6}{|c|}{ T2331 } & \multicolumn{3}{|c|}{ T0134 } \\
\hline Date Sampled & \multicolumn{3}{|c|}{$01 / 08 / 02$} & \multicolumn{3}{|c|}{$07 / 09 / 02$} & \multicolumn{3}{|c|}{$05 / 02 / 02$} \\
\hline Program & \multicolumn{3}{|c|}{ BJC } & \multicolumn{3}{|c|}{ BJC } & \multicolumn{3}{|c|}{ GWPP } \\
\hline \multicolumn{10}{|l|}{ Sample Type } \\
\hline Result (pCi/L) & Activity & Error & MDA & Activity & Error & MDA & Activity & Error & MDA \\
\hline Gross Alpha & $<\mathrm{MDA}$ & & 4.61 & $<\mathrm{MDA}$ & & 2.21 & 35 & 6.4 & 2.2 \\
\hline Gross Beta & 9.13 & 2.01 & 2.46 & 7.29 & 1.64 & 2 & $<$ MDA & & 7.9 \\
\hline Technetium-99 & $<\mathrm{MDA}$ & & 32.92 & $<M D A$ & & 7.2 & & & \\
\hline Uranium-234 & & & & & & & 21 & 2.6 & 0.11 \\
\hline Uranium-235 & & & & & & & 0.84 & 0.35 & 0.14 \\
\hline Uranium-236 & & & & & & & & & \\
\hline Uranium-238 & & & & & & & 16 & 2 & 0.14 \\
\hline
\end{tabular}

\begin{tabular}{|c|c|c|c|c|c|c|c|c|c|}
\hline Sampling Point & \multicolumn{3}{|c|}{ GW-204 } & \multicolumn{6}{|c|}{ GW-219 } \\
\hline Location & \multicolumn{3}{|c|}{ T0134 } & \multicolumn{6}{|c|}{ UOV } \\
\hline Date Sampled & \multicolumn{3}{|c|}{$11 / 06 / 02$} & \multicolumn{3}{|c|}{$05 / 06 / 02$} & \multicolumn{3}{|c|}{$11 / 07 / 02$} \\
\hline Program & \multicolumn{3}{|c|}{ GWPP } & \multicolumn{3}{|c|}{ GWPP } & \multicolumn{3}{|c|}{ GWPP } \\
\hline \multicolumn{10}{|l|}{ Sample Type } \\
\hline Result (pCi/L) & Activity & Error & MDA & Activity & Error & MDA & Activity & Error & MDA \\
\hline Gross Alpha & 22 & 6.3 & 7.2 & 120 & 13 & 4 & 170 & 15 & $\overline{7.6}$ \\
\hline Gross Beta & 16 & 5.1 & 6.8 & 77 & 9.5 & 8 & 91 & 8.8 & 7.1 \\
\hline \multicolumn{10}{|l|}{ Technetium-99 } \\
\hline Uranium-234 & 13 & 1.7 & 0.14 & 18 & 2.1 & 0.14 & 27 & 3 & 0.17 \\
\hline Uranium-235 & 0.45 & 0.25 & 0.16 & 1.4 & 0.41 & 0.11 & 2.8 & 0.6 & 0.17 \\
\hline Uranium-236 & & & & & & & & & \\
\hline Uranium-238 & 10 & 1.4 & 0.14 & 120 & 12 & 0.16 & 190 & 19 & 0.14 \\
\hline
\end{tabular}


APPENDIX E.3: CY 2002 MONITORING DATA FOR THE UPPER EAST FORK POPLAR CREEK HYDROGEOLOGIC REGIME Radiological Analytes: Isotope Activity

\begin{tabular}{|c|c|c|c|c|c|c|c|c|c|}
\hline Sampling Point & \multicolumn{3}{|c|}{ GW-219 } & \multicolumn{6}{|c|}{ GW-223 } \\
\hline Location & \multicolumn{3}{|c|}{ UOV } & \multicolumn{6}{|c|}{ NHP } \\
\hline Date Sampled & \multicolumn{3}{|c|}{$11 / 07 / 02$} & \multicolumn{3}{|c|}{$01 / 31 / 02$} & \multicolumn{3}{|c|}{$08 / 05 / 02$} \\
\hline Program & \multicolumn{3}{|c|}{ GWPP } & \multicolumn{3}{|c|}{ BJC } & \multicolumn{3}{|c|}{ BJC } \\
\hline Sample Type & \multicolumn{3}{|c|}{ Dup } & & & & & & \\
\hline Result (pCi/L) & Activity & Error & MDA & Activity & Error & MDA & Activity & Error & MDA \\
\hline Gross Alpha & 210 & 17 & 7.5 & 12.19 & 2.38 & 1.97 & 14 & 2.17 & 0.99 \\
\hline Gross Beta & 100 & 9.4 & 7.1 & 6.13 & 1.82 & 2.02 & 15.24 & 3.37 & 3.04 \\
\hline Technetium-99 & & & & & & & & & \\
\hline Uranium-234 & 30 & 3.4 & 0.14 & 3.88 & 1.29 & 0.72 & 5.02 & 1.72 & 0.54 \\
\hline Uranium-235 & 2.5 & 0.6 & 0.14 & 0.66 & 0.49 & 0.39 & $<\mathrm{MDA}$ & & 0.67 \\
\hline Uranium-236 & & & & $<M D A$ & & 0.45 & $<$ MDA & & 0.6 \\
\hline Uranium-238 & 200 & 20 & 0.12 & 9.17 & 2.35 & 0.55 & 9.2 & 2.52 & 0.54 \\
\hline
\end{tabular}

\begin{tabular}{|c|c|c|c|c|c|c|c|c|c|}
\hline Sampling Point & \multicolumn{3}{|c|}{ GW-253 } & \multicolumn{6}{|c|}{ GW-605 } \\
\hline Location & \multicolumn{3}{|c|}{ S2 } & \multicolumn{6}{|c|}{ EXP-I } \\
\hline Date Sampled & \multicolumn{3}{|c|}{$05 / 07 / 02$} & \multicolumn{6}{|c|}{$01 / 08 / 02$} \\
\hline Program & \multicolumn{3}{|c|}{ BJC } & \multicolumn{6}{|c|}{ BJC } \\
\hline Sample Type & & & & & & & \multicolumn{3}{|c|}{ Dup } \\
\hline Result (pCi/L) & Activity & Error & MDA & Activity & Error & MDA & Activity & Error & MDA \\
\hline Gross Alpha & 45.1 & 9.67 & \begin{tabular}{l|l|}
5.53 \\
\end{tabular} & 48.42 & 5.12 & 2.83 & 44.82 & 5.27 & 3.18 \\
\hline Gross Beta & 25.44 & 11.29 & 10.73 & 14.94 & 2.15 & 2.29 & 13.39 & 1.84 & 1.94 \\
\hline Technetium-99 & & & & $<\mathrm{MDA}$ & & 32.06 & $<\mathrm{MDA}$ & & 32.1 \\
\hline Uranium-234 & 7.15 & 1.93 & 0.44 & & & & & & \\
\hline Uranium-235 & $<\mathrm{MDA}$ & & 0.36 & & & & & & \\
\hline Uranium-236 & $<M D A$ & & 0.16 & & & & & & \\
\hline Uranium-238 & 1.36 & 0.62 & 0.35 & & & & & & \\
\hline
\end{tabular}

\begin{tabular}{|c|c|c|c|c|c|c|c|c|c|}
\hline Sampling Point & \multicolumn{6}{|c|}{ GW-605 } & \multicolumn{3}{|c|}{ GW-606 } \\
\hline Location & \multicolumn{6}{|c|}{ EXP-I } & \multicolumn{3}{|c|}{ EXP-I } \\
\hline Date Sampled & \multicolumn{6}{|c|}{$07 / 08 / 02$} & \multicolumn{3}{|c|}{$01 / 08 / 02$} \\
\hline Program & \multicolumn{6}{|c|}{ BJC } & \multicolumn{3}{|c|}{ BJC } \\
\hline Sample Type & & & & \multicolumn{3}{|c|}{ Dup } & & & \\
\hline Result (pCi/L) & Activity & Error & MDA & Activity & Error & MDA & Activity & Error & MDA \\
\hline Gross Alpha & 51.84 & 4.16 & 2.21 & 52.08 & 5.75 & 3.78 & $<$ MDA & & 2.83 \\
\hline Gross Beta & 21.23 & 2.14 & 1.88 & 28.77 & 2.73 & 2.34 & 18.6 & 2.37 & 2.42 \\
\hline Technetium-99 & $<\mathrm{MDA}$ & & 7.49 & $<\mathrm{MDA}$ & & 7.42 & $<M D A$ & & 33.17 \\
\hline Uranium-234 & & & & & & & & & \\
\hline Uranium-235 & & & & & & & & & \\
\hline Uranium-236 & & & & & & & & & \\
\hline Uranium-238 & & & & & & & & & \\
\hline
\end{tabular}

\begin{tabular}{|c|c|c|c|c|c|c|c|c|c|}
\hline Sampling Point & \multicolumn{3}{|c|}{ GW-606 } & \multicolumn{3}{|c|}{ GW-618 } & \multicolumn{3}{|c|}{ GW-686 } \\
\hline Location & \multicolumn{3}{|c|}{ EXP-I } & \multicolumn{3}{|c|}{ EXP-E } & \multicolumn{3}{|c|}{ CPT } \\
\hline Date Sampled & \multicolumn{3}{|c|}{$07 / 08 / 02$} & \multicolumn{3}{|c|}{$05 / 09 / 02$} & \multicolumn{3}{|c|}{$11 / 14 / 02$} \\
\hline Program & \multicolumn{3}{|c|}{ BJC } & \multicolumn{3}{|c|}{ BJC } & \multicolumn{3}{|c|}{ GWPP } \\
\hline \multicolumn{10}{|l|}{ Sample Type } \\
\hline Result (pCi/L) & Activity & Error & MDA & Activity & Error & MDA & Activity & Error & MDA \\
\hline Gross Alpha & 5.63 & 2.41 & 2.94 & $<\mathrm{MDA}$ & & 2.52 & $<\mathrm{MDA}$ & & 34 \\
\hline Gross Beta & 9.83 & 1.84 & 2.11 & 7.98 & 1.79 & 2.17 & $<\mathrm{MDA}$ & & 68 \\
\hline Technetium-99 & $<\mathrm{MDA}$ & & 7.6 & & & & $<\mathrm{MDA}$ & & 13 \\
\hline Uranium-234 & & & & $<M D A$ & & 0.4 & & & \\
\hline Uranium-235 & & & & $<\mathrm{MDA}$ & & 0.28 & & & \\
\hline Uranium-236 & & & & $<M D A$ & & 0.23 & & & \\
\hline Uranium-238 & & & & 0.28 & 0.22 & 0.25 & & & \\
\hline
\end{tabular}


APPENDIX E.3: CY 2002 MONITORING DATA FOR THE UPPER EAST FORK POPLAR CREEK HYDROGEOLOGIC REGIME Radiological Analytes: Isotope Activity

\begin{tabular}{|c|c|c|c|c|c|c|c|c|c|}
\hline Sampling Point & \multicolumn{3}{|c|}{ GW-687 } & \multicolumn{3}{|c|}{ GW-689 } & \multicolumn{3}{|c|}{ GW-698 } \\
\hline Location & \multicolumn{3}{|c|}{ CPT } & \multicolumn{3}{|c|}{ CPT } & \multicolumn{3}{|c|}{ B8110 } \\
\hline Date Sampled & \multicolumn{3}{|c|}{$11 / 14 / 02$} & \multicolumn{3}{|c|}{$11 / 14 / 02$} & \multicolumn{3}{|c|}{$05 / 02 / 02$} \\
\hline Program & \multicolumn{3}{|c|}{ GWPP } & \multicolumn{3}{|c|}{ GWPP } & \multicolumn{3}{|c|}{ GWPP } \\
\hline \multicolumn{10}{|l|}{ Sample Type } \\
\hline Result (pCi/L) & Activity & Error & MDA & Activity & Error & MDA & Activity & Error & MDA \\
\hline Gross Alpha & $<\mathrm{MDA}$ & & 50 & 100 & 56 & 53 & 2.8 & 2.5 & 1.5 \\
\hline Gross Betal & $<\mathrm{MDA}$ & & 84 & $<\mathrm{MDA}$ & & 140 & 7.3 & 4.4 & 6.6 \\
\hline Technetium-99 & $<M D A$ & & 13 & $<\mathrm{MDA}$ & & 13 & & & \\
\hline Uranium-234 & & & & & & & 1.2 & 0.39 & 0.17 \\
\hline Uranium-235 & & & & & & & $<$ MDA & & 0.17 \\
\hline Uranium-236 & & & & & & & & & \\
\hline Uranium-238 & & & & & & & 0.34 & 0.19 & 0.11 \\
\hline
\end{tabular}

\begin{tabular}{|c|c|c|c|c|c|c|c|c|c|}
\hline Sampling Point & \multicolumn{6}{|c|}{ GW-698 } & \multicolumn{3}{|c|}{ GW-733 } \\
\hline Location & \multicolumn{6}{|c|}{ B8110 } & \multicolumn{3}{|c|}{ EXP-J } \\
\hline Date Sampled & \multicolumn{3}{|c|}{$05 / 02 / 02$} & \multicolumn{3}{|c|}{$11 / 07 / 02$} & \\
\hline Program & \multicolumn{3}{|c|}{ GWPP } & \multicolumn{3}{|c|}{ GWPP } & \multicolumn{3}{|c|}{ BJC } \\
\hline Sample Type & \multicolumn{3}{|c|}{ Dup } & & & & & & \\
\hline Result (pCi/L) & Activity & Error & MDA & Activity & Error & MDA & Activity & Error & MDA \\
\hline Gross Alpha & $<$ MDA & & 4.1 & $<\mathrm{MDA}$ & & 8.7 & $<\mathrm{MDA}$ & & 1.84 \\
\hline Gross Beta & $<M D A$ & & 6.5 & $<\mathrm{MDA}$ & & 7.9 & 3.41 & 1.33 & 1.82 \\
\hline Technetium-99 & & & & & & & $<\mathrm{MDA}$ & & 30.22 \\
\hline Uranium-234 & 0.92 & 0.33 & 0.17 & 0.82 & 0.29 & 0.12 & & & \\
\hline Uranium-235 & $<\mathrm{MDA}$ & & 0.19 & $<\mathrm{MDA}$ & & 0.12 & & & \\
\hline Uranium-236 & & & & & & & & & \\
\hline Uranium-238 & 0.43 & 0.22 & 0.11 & 0.72 & 0.28 & 0.13 & & & \\
\hline
\end{tabular}

\begin{tabular}{|c|c|c|c|c|c|c|c|c|c|}
\hline Sampling Point & \multicolumn{3}{|c|}{ GW-733 } & \multicolumn{6}{|c|}{ GW-782 } \\
\hline Location & \multicolumn{3}{|c|}{ EXP-J } & \multicolumn{6}{|c|}{ GRIDE3 } \\
\hline Date Sampled & \multicolumn{3}{|c|}{$07 / 09 / 02$} & \multicolumn{3}{|c|}{$05 / 01 / 02$} & \multicolumn{3}{|c|}{$11 / 04 / 02$} \\
\hline Program & \multicolumn{3}{|c|}{ BJC } & \multicolumn{3}{|c|}{ GWPP } & \multicolumn{3}{|c|}{ GWPP } \\
\hline \multicolumn{10}{|l|}{ Sample Type } \\
\hline Result (pCi/L) & Activity & Error & MDA & Activity & Error & MDA & Activity & Error & MDA \\
\hline Gross Alpha & $<\mathrm{MDA}$ & & 1.94 & 45 & 7.7 & 2.5 & 38 & 7.8 & 7.3 \\
\hline Gross Beta & $<M D A$ & & 3.57 & 9 & 4.6 & 6.7 & 11 & 6.3 & 9.7 \\
\hline Technetium-99 & $<\mathrm{MDA}$ & & 7.19 & & & & & & \\
\hline Uranium-234 & & & & 49 & 5 & 0.13 & 44 & 4.9 & 0.16 \\
\hline Uranium-235 & & & & 0.33 & 0.2 & 0.11 & 0.16 & 0.14 & 0.15 \\
\hline Uranium-236 & & & & & & & & & \\
\hline Uranium-238 & & & & 0.47 & 0.21 & 0.13 & 0.53 & 0.25 & 0.14 \\
\hline
\end{tabular}

\begin{tabular}{|c|c|c|c|c|c|c|c|c|c|}
\hline Sampling Point & \multicolumn{6}{|c|}{ GW-832 } & \multicolumn{3}{|c|}{ OF 51} \\
\hline Location & \multicolumn{6}{|c|}{ NHP } & \multicolumn{3}{|c|}{ EXP-SW } \\
\hline Date Sampled & \multicolumn{3}{|c|}{$01 / 31 / 02$} & \multicolumn{3}{|c|}{$08 / 05 / 02$} & \multicolumn{3}{|c|}{$02 / 14 / 02$} \\
\hline Program & \multicolumn{3}{|c|}{ BJC } & \multicolumn{3}{|c|}{ BJC } & \multicolumn{3}{|c|}{ BJC } \\
\hline \multicolumn{10}{|l|}{ Sample Type } \\
\hline Result (pCi/L) & Activity & Error & MDA & Activity & Error & MDA & Activity & Error & MDA \\
\hline Gross Alpha & 4.67 & 1.82 & 2.08 & 2.72 & 1.06 & 0.93 & 4.27 & 1.39 & 1.18 \\
\hline Gross Beta & 5.15 & 1.51 & 1.98 & 4.23 & 1.93 & 1.85 & 4.67 & 1.3 & 1.68 \\
\hline \multicolumn{10}{|l|}{ Technetium-99 } \\
\hline Uranium-234 & 2.74 & 0.97 & 0.28 & 0.68 & 0.37 & 0.31 & 1.66 & 0.61 & 0.11 \\
\hline Uranium-235 & $0.3 \mathrm{R}$ & 0.31 & 0.2 & $<M D A$ & & 0.15 & $0.2 \mathrm{R}$ & 0.21 & 0.14 \\
\hline Uranium-236 & $<\mathrm{MDA}$ & & 0.31 & $<\mathrm{MDA}$ & & 0.23 & $<\mathrm{MDA}$ & & 0.21 \\
\hline Uranium-238 & 3.39 & 1.13 & 0.58 & 1.66 & 0.6 & 0.29 & 2.21 & 0.73 & 0.25 \\
\hline
\end{tabular}


APPENDIX E.3: CY 2002 MONITORING DATA FOR THE UPPER EAST FORK POPLAR CREEK HYDROGEOLOGIC REGIME Radiological Analytes: Isotope Activity

\begin{tabular}{|c|c|c|c|c|c|c|c|c|c|}
\hline Sampling Point & \multicolumn{3}{|c|}{ OF 51} & \multicolumn{6}{|c|}{ STATION 8} \\
\hline Location & \multicolumn{3}{|c|}{ EXP-SW } & \multicolumn{6}{|c|}{ EXP-SW } \\
\hline Date Sampled & \multicolumn{3}{|c|}{$09 / 03 / 02$} & \multicolumn{3}{|c|}{$02 / 01 / 02$} & \multicolumn{3}{|c|}{$02 / 14 / 02$} \\
\hline Program & \multicolumn{3}{|c|}{ BJC } & \multicolumn{3}{|c|}{ BJC } & \multicolumn{3}{|c|}{ BJC } \\
\hline \multicolumn{10}{|l|}{ Sample Type } \\
\hline Result (pCi/L) & Activity & Error & MDA & Activity & Error & MDA & Activity & Error & MDA \\
\hline Gross Alpha & 3.74 & 1.3 & 1.16 & 16.72 & 2.73 & 1.92 & 7.6 & 2.16 & 2.14 \\
\hline Gross Beta & 4.89 & 1.17 & 1.41 & 8.96 & 1.66 & 1.95 & 7.31 & 1.41 & 1.64 \\
\hline \multicolumn{10}{|l|}{ Technetium-99 } \\
\hline Uranium-234 & 1.9 & 0.56 & 0.17 & 3.49 & 1.02 & 0.32 & 1.17 & 0.47 & 0.23 \\
\hline Uranium-235 & $<\mathrm{MDA}$ & & 0.17 & 0.33 & 0.28 & 0.31 & $<\mathrm{MDA}$ & & 0.2 \\
\hline Uranium-236 & $<\mathrm{MDA}$ & & 0.19 & $<\mathrm{MDA}$ & & 0.25 & 0.19 & 0.18 & 0.18 \\
\hline Uranium-238 & 2.49 & 0.67 & 0.17 & 8.97 & 2.12 & 0.55 & 4.44 & 1.16 & 0.26 \\
\hline
\end{tabular}

\begin{tabular}{|c|c|c|c|c|c|c|}
\hline Sampling Point & \multicolumn{6}{|c|}{ STATION 8} \\
\hline Location & \multicolumn{6}{|c|}{ EXP-SW } \\
\hline Date Sampled & \multicolumn{3}{|c|}{$08 / 20 / 02$} & \multicolumn{3}{|c|}{$09 / 03 / 02$} \\
\hline Program & \multicolumn{3}{|c|}{ BJC } & \multicolumn{3}{|c|}{ BJC } \\
\hline \multicolumn{7}{|l|}{ Sample Type } \\
\hline Result (pCi/L) & Activity & Error & MDA & Activity & Error & MDA \\
\hline Gross Alpha & 3.56 & 1.25 & 1.2 & 2.35 & 1.08 & 1.18 \\
\hline Gross Beta & 5.43 & 1.42 & 1.77 & 4.28 & 1.26 & 1.64 \\
\hline \multicolumn{7}{|l|}{ Technetium-99 } \\
\hline Uranium-234 & 1.43 & 0.6 & 0.14 & 1.37 & 0.74 & 0.63 \\
\hline Uranium-235 & $<\mathrm{MDA}$ & & 0.3 & 0.96 & 0.64 & 0.26 \\
\hline Uranium-236 & $<\mathrm{MDA}$ & & 0.27 & 0.5 & 0.43 & 0.4 \\
\hline Uranium-238 & 2.54 & 0.84 & 0.14 & 1.79 & 0.85 & 0.54 \\
\hline
\end{tabular}




\section{APPENDIX F \\ CY 2002 MONITORING DATA FOR THE CHESTNUT RIDGE HYDROGEOLOGIC REGIME}




\title{
EXPLANATION
}

\section{Sampling Point:}

GW - Groundwater monitoring well (also 1090)

MCK - McCoy Branch Kilometer

SCR - South Chestnut Ridge (tributary prefix for spring and surface water sampling locations)

\section{Location:}

\author{
CDLVI - Construction/Demolition Landfill VI \\ CDLVII - Construction/Demolition Landfill VII \\ CRBAWP - Chestnut Ridge Borrow Area Waste Pile \\ CRSDB - Chestnut Ridge Sediment Disposal Basin \\ CRSP - Chestnut Ridge Security Pits \\ EXP-SW - Exit Pathway (spring or surface water sampling location) \\ FCAP - Filled Coal Ash Pond \\ KHQ - Kerr Hollow Quarry \\ LII - Industrial Landfill II \\ LIV - Industrial Landfill IV \\ LV - Industrial Landfill V \\ UNCS - United Nuclear Corporation Site
}

\section{Monitoring Program:}

BJC - monitoring program managed by Bechtel Jacobs Company LLC

GWPP - managed by the Y-12 Groundwater Protection Program

\section{Sample Type:}

Dup - Field Duplicate Sample

Units:

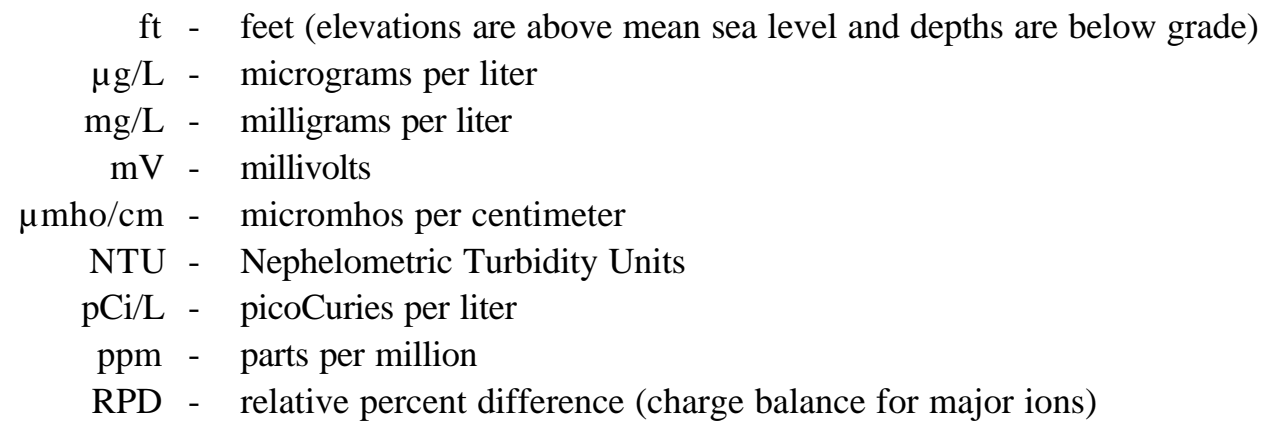




\section{EXPLANATION (continued)}

Only analytes detected above the program reporting limits in at least one sample are included in this appendix. Additionally, results that are below the reporting limits are replaced with values (e.g., "<") to emphasize the detected results. The following sections describe the reporting limits and data qualifiers for each subsection of the appendix. A comprehensive list of the Y-12 GWPP analytes, analytical methods, and reporting limits is provided in Appendix B, Table B.5.

\section{F.1 Field Measurements, Miscellaneous Analytes, Major Ions, and Trace Metals}

The Y-12 GWPP SAP (BWXT 2001a) specifies reporting limits for trace metals that are appropriate for DOE Order 5400.1 monitoring. Some of the laboratories used for the monitoring programs managed by BJC report metals results (often as estimated values) that are much lower than the GWPP reporting limits for the metals using the same analytical method. To retain the highest quality data for DOE Order 5400.1 monitoring purposes and to standardize reporting limits for trace metal results obtained from all sources, the GWPP reporting limits were given precedence over the BJC reporting limits (BJC 2001 and 2002a) shown below. Results for the trace metals shown in bold typeface below are presented in Appendix F.1 because the metal was detected at a level above the associated reporting limit in at least one groundwater or surface water sample.

\begin{tabular}{|c|c|c|c|c|c|}
\hline \multirow{2}{*}{ Analyte } & \multicolumn{2}{|c|}{ Reporting Limit (mg/L) } & \multirow{2}{*}{ Analyte } & \multicolumn{2}{|c|}{ Reporting Limit (mg/L) } \\
\hline & GWPP & BJC & & GWPP & BJC \\
\hline $\begin{array}{r}\text { Calcium } \\
\text { Magnesium } \\
\text { Potassium } \\
\text { Sodium }\end{array}$ & $\begin{array}{l}0.2 \\
0.2 \\
2.0 \\
0.2\end{array}$ & $\begin{array}{l}0.25 \\
0.05 \\
0.25 \\
0.25\end{array}$ & $\begin{array}{r}\text { Anions } \\
\text { Alkalinity - HCO3 } \\
\text { Alkalinity - CO3 } \\
\text { Chloride } \\
\text { Fluoride } \\
\text { Nitrate (as Nitrogen) } \\
\text { Sulfate }\end{array}$ & $\begin{array}{c}1.0 \\
1.0 \\
0.2 \\
0.1 \\
0.028 \\
0.25 \\
\end{array}$ & $\begin{array}{c}\text { NA } \\
\text { NA } \\
0.1 \\
0.05 \\
0.1 \\
0.1 \\
\end{array}$ \\
\hline Trace Metals & & & Trace Metals (continued) & & \\
\hline Aluminum & 0.2 & $0.05^{*}$ & Lithium & 0.01 & 0.01 \\
\hline Antimony (PMS) & 0.0025 & . & Manganese & 0.005 & 0.005 \\
\hline Antimony & & 0.006 & Mercury (CVAA) & 0.0002 & 0.0002 \\
\hline Arsenic (PMS) & 0.005 & . & Molybdenum & 0.05 &.$^{*}$ \\
\hline Arsenic & 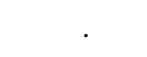 & 0.005 & Nickel (PMS) & 0.005 & \\
\hline Barium & 0.004 & 0.005 & Nickel & & 0.01 \\
\hline Beryllium & 0.001 & 0.001 & Selenium (PMS) & 0.01 & \\
\hline Boron & 0.1 & $0.01 *$ & Selenium & . & 0.005 \\
\hline Cadmium (PMS) & 0.0005 & . & Silver & 0.02 & $0.005^{*}$ \\
\hline Cadmium & . & 0.001 & Strontium & 0.005 & 0.005 \\
\hline Chromium (PMS) & 0.0025 & . & Thallium (PMS) & 0.0005 & \\
\hline Chromium & . & 0.005 & Thallium & . & 0.002 \\
\hline Cobalt & 0.02 & $0.005^{*}$ & Thorium & 0.2 & . \\
\hline Copper & 0.02 & $0.005^{*}$ & Uranium (PMS) & 0.0005 & \\
\hline Iron & 0.05 & $0.01 *$ & Uranium (KPA) & . & 0.004 \\
\hline Lead (PMS) & 0.0005 & & Vanadium & 0.02 & $0.01 *$ \\
\hline Lead & & 0.003 & Zinc & 0.05 & $0.01 *$ \\
\hline
\end{tabular}




\section{EXPLANATION (continued)}

Metals analyses were performed using the inductively coupled plasma spectroscopy method (SW846-6010B) unless otherwise noted:

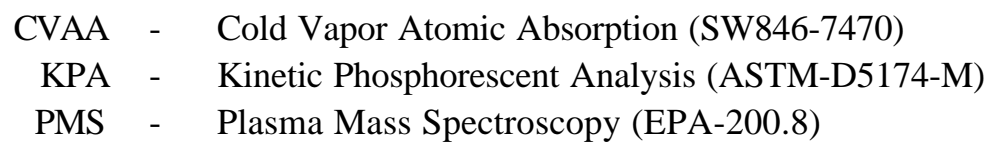

The following symbols and data qualifiers are used in Appendix F.1:

- $\quad$ Not analyzed or not applicable

$<\quad$ - Analyzed but not detected at the project reporting level

$\mathrm{J}$ - Positively identified; estimated concentration (BJC data for landfills)

$\mathrm{Q}$ - Inconsistent with historical measurements for a sampling location (low bicarbonate alkalinity at MCK 2.0)

$\mathrm{R}$ - Unusable result that does not meet data quality objectives: duplicate results differ by an order of magnitude (dissolved solids and boron at GW-156); charge balance out of acceptable range (MCK 2.0).

\section{F.2 Volatile Organic Compounds}

The reporting limits for volatile organic compounds shown in Table B.5 and those used for monitoring programs managed by BJC are contract-required quantitation limits. Results below the quantitation limit and above the instrument detection limit are reported as estimated quantities. Therefore, non-detected results are assumed to equal zero for all compounds.

The following symbols and data qualifiers are used in Appendix F.2.

- $\quad$ Not analyzed or not applicable

$<\quad$ - Analyzed but not detected at the project reporting level (also false-positive results for data provided by BJC)

J - Positively identified; estimated concentration below the contract-required quantitation limit.

Only 15 compounds were detected (of the 53 compounds requested) in the CY 2002 groundwater samples collected in the Chestnut Ridge. Results for the following eight compounds were detected in at least two samples.

\begin{tabular}{|r|c|c|r|c|c|}
\hline Compound & $\begin{array}{c}\text { No. } \\
\text { Detected }\end{array}$ & $\begin{array}{c}\text { Maximum } \\
(\boldsymbol{\mu} \mathbf{g} / \mathbf{L})\end{array}$ & $\begin{array}{c}\text { No. } \\
\text { Detected }\end{array}$ & $\begin{array}{c}\text { Maximum } \\
(\boldsymbol{\mu} \mathbf{g} / \mathbf{L})\end{array}$ \\
\hline 1,1,1-Trichloroethane & 12 & 22 & cis-1,2-Dichloroethene & 4 & 7 \\
1,1-Dichloroethane & 10 & 25 & Chloroform & 4 & 2.4 \\
1,1-Dichloroethene & 9 & 8 & Trichloroethene & 2 & $0.78 \mathrm{~J}$ \\
Tetrachloroethene & 6 & 7.1 & Trichlorofluoromethane & 2 & 11 \\
\hline
\end{tabular}




\section{EXPLANATION (continued)}

Because seven compounds were detected in only one sample at very low (estimated) concentrations during CY 2002, results for these compounds are shown below and are not presented in Appendix F.2.

\begin{tabular}{|c|c|c|c|c|}
\hline Well Number & Location & Date Sampled & Compound & Result $(\boldsymbol{\mu g} / \mathbf{L})$ \\
\hline GW-522 & LIV & $07 / 15 / 02$ & $1,1,2,2$-Tetrachloroethane & $0.57 \mathrm{~J}$ \\
GW-143 & KHQ & $12 / 02 / 02$ & Acetone & $1 \mathrm{~J}$ \\
GW-543 & CDLVI & $01 / 15 / 02$ & Bromoform & $0.48 \mathrm{~J}$ \\
SCR4.3SP & EXP-SW & $01 / 14 / 02$ & Carbon disulfide & $0.39 \mathrm{~J}$ \\
GW-557 & LV & $01 / 10 / 02$ & Chloromethane & $0.42 \mathrm{~J}$ \\
GW-539 & LII & $01 / 14 / 02$ & Ethanol & $280 \mathrm{Q}$ \\
GW-142 & KHQ & $12 / 02 / 02$ & Methylene chloride & $0.2 \mathrm{~J}$ \\
GW-798 & CDLVII & $07 / 16 / 02$ & trans-1,2-Dichloroethene & $0.39 \mathrm{~J}$ \\
\hline
\end{tabular}

\section{F.3 Radiological Analytes}

Reporting limits for radiological analytes are sample-specific and analyte-specific minimum detectable activities that are reported with each result. The following summary shows the radiological analytes reported for at least one groundwater sample collected during CY 2002 in the Chestnut Ridge Regime.

\begin{tabular}{|r|c|c|r|c|c|}
\hline Analyte & $\begin{array}{c}\text { No. of } \\
\text { Results }\end{array}$ & $\begin{array}{c}\text { No. } \\
\text { Detected }\end{array}$ & Analyte & $\begin{array}{c}\text { No. of } \\
\text { Results }\end{array}$ & $\begin{array}{c}\text { No. } \\
\text { Detected }\end{array}$ \\
\hline Gross Alpha & 110 & 26 & Technetium-99 & 2 & 1 \\
Gross Beta & 110 & 58 & Uranium-233/234 & 14 & 11 \\
Cesium-137 & 2 & 0 & Uranium-235 & 14 & 0 \\
Cobalt-60 & 2 & 0 & Uranium-236 & 14 & 0 \\
Potassium-40 & 2 & 2 & Uranium-238 & 14 & 6 \\
Strontium-90 & 14 & 1 & & & \\
\hline
\end{tabular}

Only the results for gross alpha and gross beta are presented in Appendix F.3, and the following notes apply to this appendix:

$$
\begin{array}{rll}
\text { Result } & - & \text { Activity in picoCuries per liter }(\mathrm{pCi} / \mathrm{L}) \\
\text { Error } & - & \text { Counting error (two standard deviations) } \\
\text { MDA } & - & \text { Minimum detectable activity }
\end{array}
$$




\section{EXPLANATION (continued)}

The CY 2002 isotopic results that exceed MDAs, all for wells at the UNCS, are shown below:

\begin{tabular}{|c|c|c|c|c|c|}
\hline \multirow{2}{*}{ Well } & Date & & \multicolumn{3}{|c|}{ Activity (pCi/L) } \\
Number & Sampled & Isotope & Result & Error & MDA \\
\cline { 4 - 6 } 1090 & $01 / 30 / 02$ & Uranium-238 & 0.29 & 0.22 & 0.25 \\
1090 & $07 / 30 / 02$ & Uranium-233/234 & 0.5 & 0.26 & 0.2 \\
GW-203 & $01 / 29 / 02$ & Uranium-238 & 0.25 & 0.2 & 0.24 \\
GW-203 & $07 / 30 / 02$ & Uranium-233/234 & 0.46 & 0.25 & 0.21 \\
GW-203 & $07 / 30 / 02$ & Uranium-238 & 0.14 & 0.14 & 0.14 \\
GW-205 & $01 / 30 / 02$ & Potassium-40 & 52.22 & 49.65 & 43.79 \\
GW-205 & $01 / 30 / 02$ & Technetium-99 & 10.59 & 6.14 & 10.17 \\
GW-205 & $01 / 30 / 02$ & Uranium-233/234 & 0.54 & 0.3 & 0.27 \\
GW-205 & $01 / 30 / 02$ & Uranium-238 & 0.22 & 0.19 & 0.22 \\
GW-205 & $07 / 30 / 02$ & Potassium-40 & 89.36 & 52.76 & 46.7 \\
GW-205 & $07 / 30 / 02$ & Uranium-233/234 & 0.22 & 0.17 & 0.18 \\
GW-221 & $01 / 29 / 02$ & Uranium-233/234 & 0.34 & 0.24 & 0.27 \\
GW-302 & $01 / 31 / 02$ & Strontium-90 & $1.66 \mathrm{Q}$ & 0.07 & 1.56 \\
GW-302 & $01 / 31 / 02$ & Uranium-233/234 & 0.41 & 0.27 & 0.31 \\
GW-302 & $07 / 31 / 02$ & Uranium-233/234 & 0.64 & 0.42 & 0.3 \\
GW-302 & $07 / 31 / 02$ & Uranium-238 & 0.26 & 0.26 & 0.17 \\
GW-339 & $01 / 29 / 02$ & Uranium-233/234 & 0.58 & 0.34 & 0.35 \\
GW-339D & $01 / 29 / 02$ & Uranium-233/234 & 0.45 & 0.27 & 0.2 \\
GW-339 & $07 / 30 / 02$ & Uranium-233/234 & 0.38 & 0.23 & 0.18 \\
GW-339D & $07 / 30 / 02$ & Uranium-233/234 & 0.43 & 0.24 & 0.2 \\
GW-339 & $07 / 30 / 02$ & Uranium-238 & 0.12 & 0.12 & 0.08 \\
\hline Note: Q - inconsistent with historical measurements for the location; D - field duplicate; \\
Uranium-233/234 is functionally equivalent to Uranium-234 analysis. & \\
\hline
\end{tabular}


APPENDIX F.1

FIELD MEASUREMENTS, MISCELLANEOUS ANALYTES, MAJOR IONS, AND TRACE METALS 
APPENDIX F.1: CY 2002 MONITORING DATA FOR THE CHESTNUT RIDGE HYDROGEOLOGIC REGIME

Field Measurements, Miscellaneous Analytes, Major Ions, and Trace Metals

\begin{tabular}{|c|c|c|c|c|c|c|c|c|c|}
\hline Station & \multicolumn{2}{|c|}{1090} & \multicolumn{2}{|c|}{ GW-141 } & \multicolumn{3}{|c|}{ GW-142 } & \multicolumn{2}{|c|}{ GW-143 } \\
\hline Site & \multicolumn{2}{|c|}{ UNCS } & \multicolumn{2}{|c|}{ LIV } & \multicolumn{3}{|c|}{ KHQ } & \multicolumn{2}{|c|}{ KHQ } \\
\hline Date & $01 / 30 / 02$ & 07/30/02 & $01 / 23 / 02$ & 07/25/02 & 04/10/02 & $10 / 16 / 02$ & $12 / 02 / 02$ & 04/09/02 & $10 / 16 / 02$ \\
\hline Program & BJC & BJC & BJC & BJC & BJC & BJC & BJC & BJC & BJC \\
\hline \multicolumn{10}{|l|}{ Sample Type } \\
\hline Field Measurements & & & & & & & & & \\
\hline Time Sampled & $9: 55$ & $9: 10$ & $9: 55$ & $9: 55$ & $9: 40$ & $12: 41$ & $10: 50$ & $9: 45$ & $14: 30$ \\
\hline Measuring Point Elev. (ft) & 1104.48 & 1104.48 & 1186.23 & 1186.23 & 971.15 & 971.15 & 971.15 & 913.98 & 913.98 \\
\hline Depth to Water (ft) & 40.95 & 58.23 & 90.18 & 97.36 & 133.98 & 138.40 & 135.81 & 78.37 & 81.45 \\
\hline Groundwater Elevation (ft) & 1063.53 & 1046.25 & 1096.05 & 1088.87 & 837.17 & 832.75 & 835.34 & 835.61 & 832.53 \\
\hline Conductivity ( $\mu \mathrm{mho} / \mathrm{cm})$ & 550 & 572 & 408 & 402 & 358 & 365 & 686 & 494 & 470 \\
\hline Dissolved Oxygen (ppm) & 4.03 & 2.08 & 9.9 & 6.75 & 4.77 & 1.2 & 1.1 & 3.88 & 2.7 \\
\hline Oxidation/Reduction (mV) & 209 & 151 & 267 & 183 & 173 & 47 & -123 & 133 & -79 \\
\hline Temperature (degrees C) & 15.3 & 17 & 12.4 & 18.8 & 15.9 & 18.8 & 10.1 & 14.9 & 16.9 \\
\hline Turbidity (NTU) & 18 & 15 & 29 & 10 & 31 & 89 & 76 & 9 & 12 \\
\hline $\mathrm{pH}$ & 7.04 & 6.63 & 6.23 & 6.59 & 7.4 & 8.02 & 8.21 & 7.58 & 8.22 \\
\hline \multicolumn{10}{|l|}{\begin{tabular}{|l|} 
Miscellaneous Analytes \\
\end{tabular}} \\
\hline Conductivity ( $\mu \mathrm{mho} / \mathrm{cm})$ & & & $332 \mathrm{~J}$ & $354 \mathrm{~J}$ & & & & & \\
\hline Dissolved Solids (mg/L) & 341 & 285 & 214 & 202 & 197 & 171 & & 276 & 264 \\
\hline Suspended Solids (mg/L) & $<$ & $<$ & $<$ & $<$ & 8.3 & 10 & & $<$ & $<$ \\
\hline Turbidity (NTU) & & & 4.5 & 1.4 & & & & & \\
\hline $\mathrm{pH}$ & & & 7.3 & 7.4 & & & & & \\
\hline \multicolumn{10}{|l|}{ Major Ions (mg/L) } \\
\hline Calcium & 54.7 & 58.7 & 40.7 & 42.1 & 29.4 & 27.7 & & 30.1 & 28.6 \\
\hline Magnesium & 32.4 & 34.2 & 25 & 26.4 & 29.9 & 29.1 & & 25.4 & 23.8 \\
\hline Potassium & 0.999 & 1.04 & $<$ & $<$ & 4.17 & 4.4 & & 17.9 & 18.6 \\
\hline Sodium & 8.82 & 9.33 & $<$ & $<$ & 0.973 & 1.3 & & 20.8 & 23.2 \\
\hline Alkalinity as $\mathrm{CO} 3$ & $<$ & $<$ & $<$ & $<$ & & & & & \\
\hline Alkalinity as $\mathrm{HCO} 3$ & 384 & 259 & 207 & 215 & & & & & \\
\hline Chloride & 18.5 & 21.9 & $2 \mathrm{~J}$ & $2.3 \mathrm{~J}$ & & & & & \\
\hline Fluoride & $<$ & $<$ & $<$ & $0.16 \mathrm{~J}$ & & & & & \\
\hline Nitrate as $\mathrm{N}$ & 0.72 & 0.97 & $0.44 \mathrm{~J}$ & $0.43 \mathrm{~J}$ & & & & & \\
\hline Sulfate & 3.5 & 3.4 & $2.3 \mathrm{~J}$ & $2.5 \mathrm{~J}$ & & & & & \\
\hline Charge Balance (RPD) & -17.8 & 2 & -2 & -1.9 & & & & & \\
\hline \multicolumn{10}{|l|}{ Trace Metals (mg/L) } \\
\hline Aluminum & $<$ & $<$ & $<$ & $<$ & $<$ & $<$ & & $<$ & $<$ \\
\hline Arsenic & $<$ & $<$ & $<$ & $<$ & $<$ & $<$ & & $<$ & $<$ \\
\hline Barium & 0.029 & 0.0292 & 0.017 & 0.014 & 0.397 & 0.41 & & 0.0462 & 0.045 \\
\hline Boron & $<$ & $<$ & $<$ & $<$ & $<$ & $<$ & & 0.866 & 0.84 \\
\hline Cadmium (PMS) & & & & & & & & & \\
\hline Chromium & $<$ & $<$ & $<$ & 0.0056 & $<$ & $<$ & & $<$ & $<$ \\
\hline Iron & $<$ & $<$ & $<$ & $<$ & 3.81 & 5.6 & & 0.552 & 0.74 \\
\hline Lead (PMS) & & & & & & & & & \\
\hline Lithium & $<$ & $<$ & ${ }^{\circ}$ & ${ }^{\circ}$ & 0.0262 & 0.032 & & 0.311 & 0.4 \\
\hline Manganese & $<$ & $<$ & $<$ & $<$ & 0.0497 & 0.054 & & 0.0111 & 0.01 \\
\hline Nickel & $<$ & $<$ & $<$ & $<$ & $<$ & $<$ & & $<$ & $<$ \\
\hline Strontium & 0.025 & 0.0257 & 0.014 & 0.016 & 0.315 & 0.31 & & 3.05 & 3 \\
\hline Thallium (PMS) & & & & & & & & & \\
\hline Uranium (PMS) & & & & & & & & & \\
\hline Uranium (KPA) & $<$ & 0.0127 & $<$ & $<$ & $<$ & $<$ & & $<$ & $<$ \\
\hline
\end{tabular}


APPENDIX F.1: CY 2002 MONITORING DATA FOR THE CHESTNUT RIDGE HYDROGEOLOGIC REGIME

Field Measurements, Miscellaneous Analytes, Major Ions, and Trace Metals

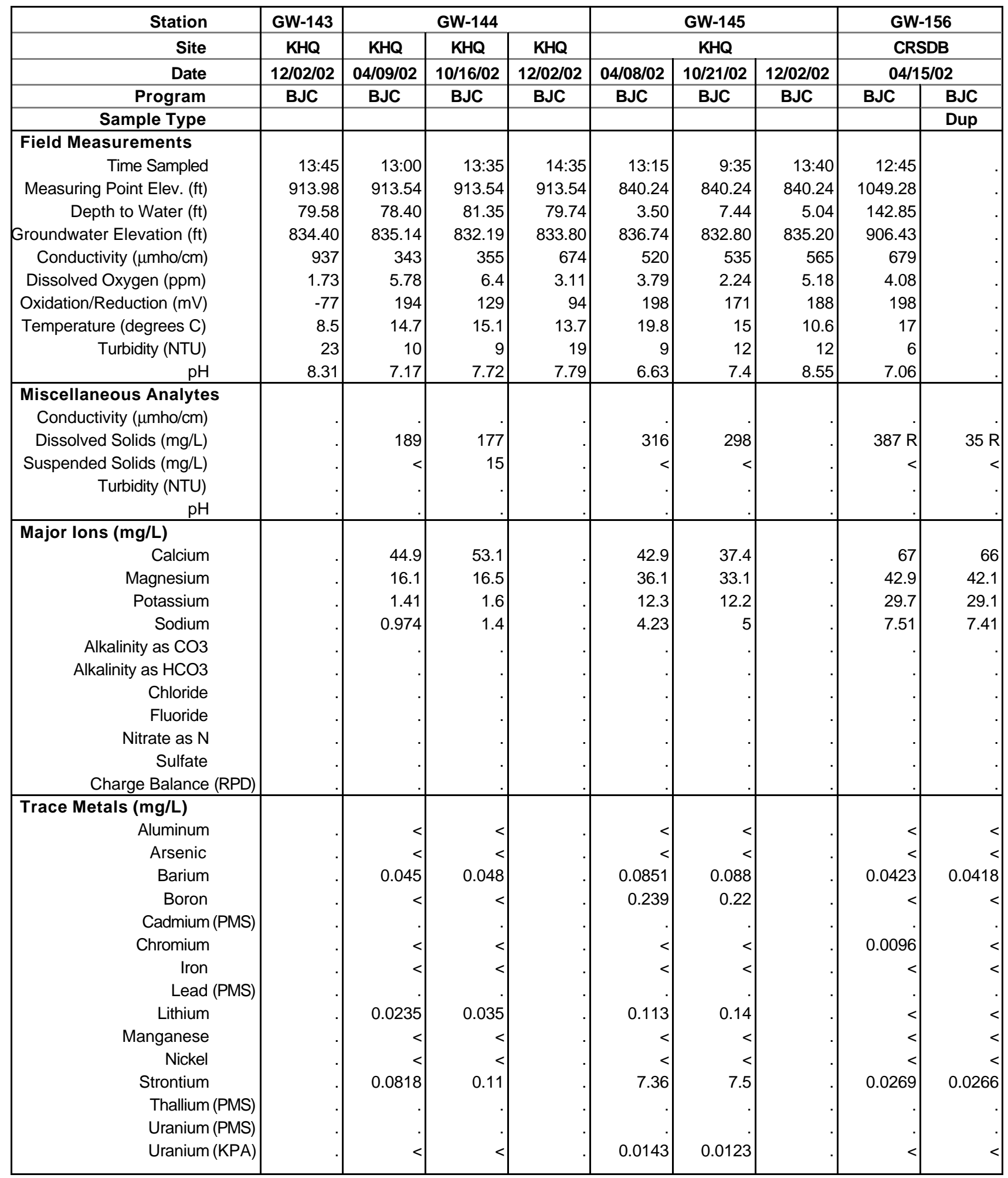


APPENDIX F.1: CY 2002 MONITORING DATA FOR THE CHESTNUT RIDGE HYDROGEOLOGIC REGIME

Field Measurements, Miscellaneous Analytes, Major Ions, and Trace Metals

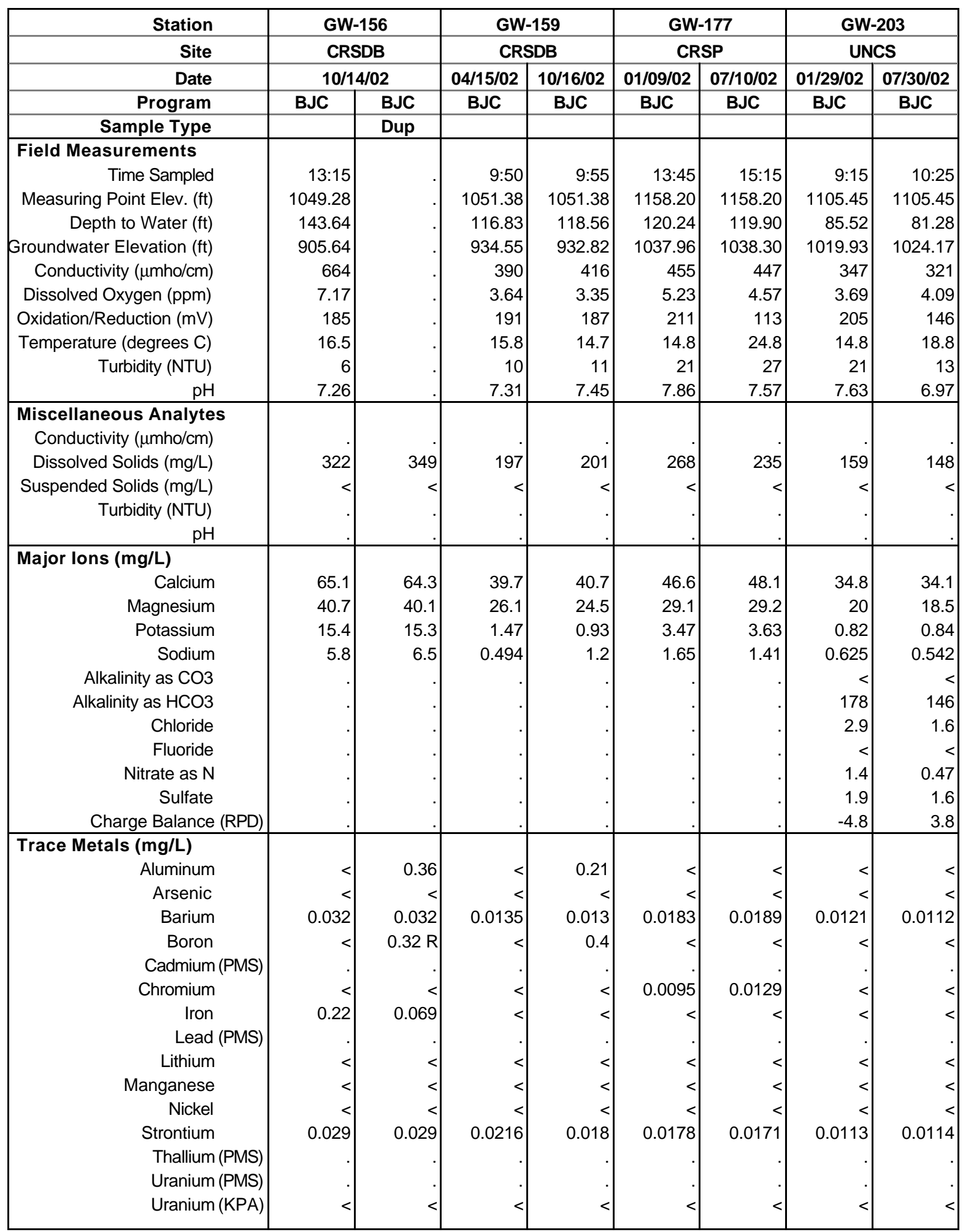


APPENDIX F.1: CY 2002 MONITORING DATA FOR THE CHESTNUT RIDGE HYDROGEOLOGIC REGIME

Field Measurements, Miscellaneous Analytes, Major Ions, and Trace Metals

\begin{tabular}{|c|c|c|c|c|c|c|c|c|c|}
\hline Station & \multicolumn{2}{|c|}{ GW-205 } & \multicolumn{3}{|c|}{ GW-217 } & \multicolumn{2}{|c|}{ GW-221 } & \multirow{3}{*}{\multicolumn{2}{|c|}{$\begin{array}{c}\text { GW-231 } \\
\text { KHQ } \\
04 / 08 / 02\end{array}$}} \\
\hline Site & \multicolumn{2}{|c|}{ UNCS } & \multicolumn{3}{|c|}{ LIV } & \multicolumn{2}{|c|}{ UNCS } & & \\
\hline Date & $01 / 30 / 02$ & 07/30/02 & $01 / 22 / 02$ & $01 / 28 / 02$ & 07/22/02 & $01 / 29 / 02$ & 07/30/02 & & \\
\hline Program & BJC & BJC & BJC & BJC & BJC & BJC & BJC & BJC & BJC \\
\hline Sample Type & & & & & & & & & Dup \\
\hline Field Measurements & & & & & & & & & \\
\hline Time Sampled & $14: 20$ & $9: 15$ & $11: 30$ & $9: 30$ & $9: 25$ & $10: 45$ & $13: 20$ & $9: 50$ & \\
\hline Measuring Point Elev. (ft) & 1104.14 & 1104.14 & 1177.03 & 1177.03 & 1177.03 & 1106.16 & 1106.16 & 849.67 & \\
\hline Depth to Water (ft) & 80.55 & 78.74 & 112.15 & 104.01 & 114.99 & 88.15 & 82.70 & 12.23 & \\
\hline Groundwater Elevation (ft) & 1023.59 & 1025.40 & 1064.88 & 1073.02 & 1062.04 & 1018.01 & 1023.46 & 837.44 & \\
\hline Conductivity ( $\mu \mathrm{mho} / \mathrm{cm})$ & 416 & 365 & 361 & 365 & 363 & 289 & 306 & 293 & \\
\hline Dissolved Oxygen (ppm) & 3.35 & 3.08 & 8.96 & 7.84 & 4.89 & 3.82 & 3.77 & 2.27 & \\
\hline Oxidation/Reduction (mV) & 120 & 97 & 213 & 214 & 166 & 197 & 143 & 182 & \\
\hline Temperature (degrees C) & 18.8 & 21.6 & 14.2 & 11.1 & 21.2 & 15.1 & 17.3 & 12 & \\
\hline Turbidity (NTU) & 16 & 9 & 20 & 27 & 14 & 22 & 7 & 15 & \\
\hline $\mathrm{pH}$ & 10.18 & 9.83 & 8 & 7.87 & 8.06 & 7.72 & 7.44 & 6.75 & \\
\hline \begin{tabular}{|l|} 
Miscellaneous Analytes \\
\end{tabular} & & & & & & & & & \\
\hline Conductivity $(\mu \mathrm{mho} / \mathrm{cm})$ & & & $315 \mathrm{~J}$ & & $332 \mathrm{~J}$ & & & & \\
\hline Dissolved Solids (mg/L) & 232 & 235 & 197 & & $178 \mathrm{~J}$ & 138 & 144 & 144 & 140 \\
\hline Suspended Solids (mg/L) & $<$ & $<$ & $2.2 \mathrm{~J}$ & & $<$ & $<$ & $<$ & $<$ & 11.1 \\
\hline Turbidity (NTU) & & & $<$ & & 0.12 & & & & \\
\hline $\mathrm{pH}$ & & & 8.1 & & 8 & & & & \\
\hline Major Ions (mg/L) & & & & & & & & & \\
\hline Calcium & 9.28 & 1.36 & 28.7 & & 33.7 & 30 & 33.1 & 29.3 & 29.2 \\
\hline Magnesium & 11.2 & 10.3 & 17.6 & & 21.1 & 18 & 19.1 & 14.9 & 14.9 \\
\hline Potassium & 64.8 & 78 & $<$ & & $<$ & 0.885 & 1.07 & 1.03 & 1.03 \\
\hline Sodium & 10 & 12.4 & 5.4 & & 5.7 & 0.456 & 0.543 & 0.679 & 0.669 \\
\hline Alkalinity as $\mathrm{CO} 3$ & 128 & 95.6 & $<$ & & $<$ & $<$ & $<$ & $\cdot$ & 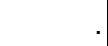 \\
\hline Alkalinity as $\mathrm{HCO} 3$ & 52 & 66.6 & 185 & & 175 & 141 & 143 & . & . \\
\hline Chloride & 2.3 & 2.5 & $2.5 \mathrm{~J}$ & & $4 \mathrm{~J}$ & 1.1 & 1.2 & & . \\
\hline Fluoride & $<$ & & $<$ & & $<$ & $<$ & $<$ & $\cdot 1$ & . \\
\hline Nitrate as $\mathrm{N}$ & 0.12 & 0.058 & $0.44 \mathrm{~J}$ & & $0.45 \mathrm{~J}$ & 0.59 & 0.9 &. & . \\
\hline Sulfate & 2.6 & 3.1 & 7.7 & & 6.4 & 1.3 & 1.3 &. & . \\
\hline Charge Balance (RPD) & -5.4 & 0 & -11.5 & & -0.9 & 1.7 & 4.6 & & \\
\hline Trace Metals (mg/L) & & & & & & & & & \\
\hline Aluminum & $<$ & $<$ & $<$ & & $<$ & $<$ & $<$ & $<$ & $<$ \\
\hline Arsenic & $<$ & $<$ & $<$ & & $<$ & $<$ & $<$ & $<$ & $<$ \\
\hline Barium & 0.005 & $<$ & 0.03 & & 0.033 & 0.0073 & 0.0082 & 0.0506 & 0.0506 \\
\hline Boron & $<$ & $<$ & $<$ & & $<$ & $<$ & $<$ & $<$ & $<$ \\
\hline Cadmium (PMS) & & & & & & & & & \\
\hline Chromium & $<$ & $<$ & $<$ & & $<$ & $<$ & $<$ & $<$ & $<$ \\
\hline Iron & $<$ & $<$ & $<$ & & $<$ & $<$ & $<$ & $<$ & $<$ \\
\hline Lead (PMS) & & & & & & & & & 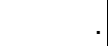 \\
\hline Lithium & 0.114 & 0.154 & & & & $<$ & $<$ & $<$ & $<$ \\
\hline Manganese & $<$ & $<$ & $<$ & & $<$ & $<$ & $<$ & $<$ & $<$ \\
\hline Nickel & $<$ & $<$ & $<$ & & $<$ & $<$ & $<$ & $<$ & $<$ \\
\hline Strontium & 0.0057 & $<$ & 0.016 & & 0.017 & 0.0091 & 0.01 & 0.0299 & 0.0297 \\
\hline Thallium (PMS) & & & & & & & & & \\
\hline Uranium (PMS) & & & & & & & & . & \\
\hline Uranium (KPA) & $<$ & $<$ & $<$ & & $<$ & $<$ & $<$ & $<$ & $<$ \\
\hline
\end{tabular}


APPENDIX F.1: CY 2002 MONITORING DATA FOR THE CHESTNUT RIDGE HYDROGEOLOGIC REGIME

Field Measurements, Miscellaneous Analytes, Major Ions, and Trace Metals

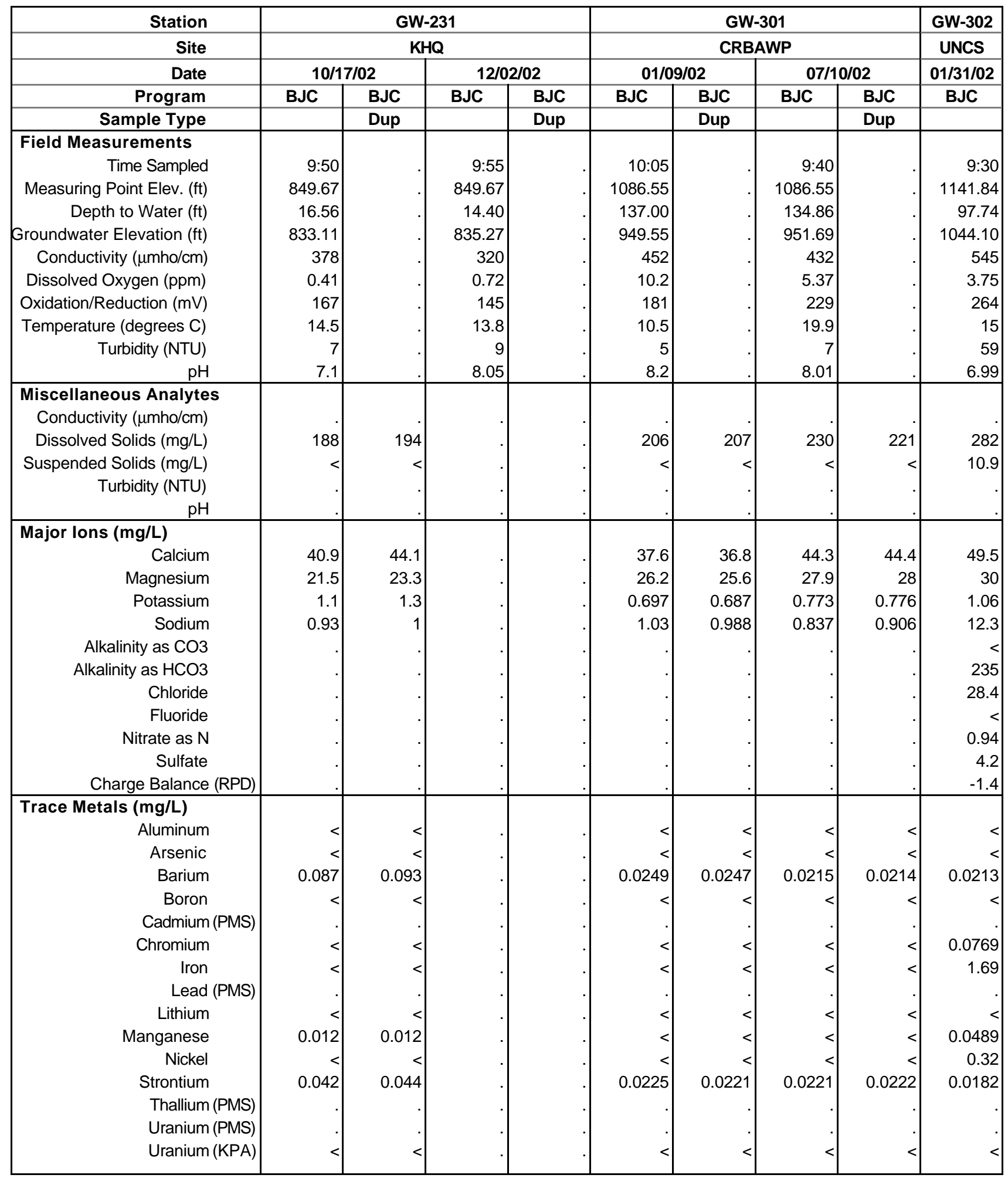


APPENDIX F.1: CY 2002 MONITORING DATA FOR THE CHESTNUT RIDGE HYDROGEOLOGIC REGIME

Field Measurements, Miscellaneous Analytes, Major Ions, and Trace Metals

\begin{tabular}{|c|c|c|c|c|c|c|c|c|}
\hline Station & GW-302 & \multicolumn{5}{|c|}{ GW-305 } & \multirow{3}{*}{\multicolumn{2}{|c|}{$\begin{array}{c}\text { GW-339 } \\
\text { UNCS } \\
01 / 29 / 02\end{array}$}} \\
\hline Site & UNCS & \multicolumn{5}{|c|}{ LIV } & & \\
\hline Date & $07 / 31 / 02$ & $01 / 22 / 02$ & $01 / 28 / 02$ & 05/02/02 & 07/15/02 & $11 / 21 / 02$ & & \\
\hline Program & BJC & BJC & BJC & BJC & BJC & BJC & BJC & BJC \\
\hline Sample Type & & & & & & & & Dup \\
\hline Field Measurements & & & & & & & & \\
\hline Time Sampled & $10: 00$ & $10: 40$ & $10: 25$ & $10: 05$ & $11: 55$ & $10: 20$ & 13:35 & \\
\hline Measuring Point Elev. (ft) & 1141.84 & 1183.72 & 1183.72 & 1183.72 & 1183.72 & 1183.72 & 1124.83 & \\
\hline Depth to Water (ft) & 104.82 & 123.89 & 115.93 & 121.89 & 125.40 & 121.10 & 63.15 & \\
\hline Groundwater Elevation (ft) & 1037.02 & 1059.83 & 1067.79 & 1061.83 & 1058.32 & 1062.62 & 1061.68 & \\
\hline Conductivity ( $\mu \mathrm{mho} / \mathrm{cm})$ & 579 & 323 & 315 & 394 & 413 & 381 & 587 & \\
\hline Dissolved Oxygen (ppm) & 2.31 & 5.75 & 4.99 & 4.39 & 2.55 & 3.82 & 4.74 & \\
\hline Oxidation/Reduction (mV) & 147 & 218 & 231 & 233 & 139 & 238 & 165 & \\
\hline Temperature (degrees C) & 18.2 & 12.2 & 13.4 & 18.8 & 18.9 & 14 & 18 & \\
\hline Turbidity (NTU) & 20 & 12 & 13 & 20 & 10 & 9 & 25 & \\
\hline $\mathrm{pH}$ & 6.71 & 7.43 & 7.56 & 7.48 & 7.26 & 8.14 & 7.4 & \\
\hline Miscellaneous Analytes & & & & & & & & \\
\hline Conductivity ( $\mu \mathrm{mho} / \mathrm{cm})$ & & $273 \mathrm{~J}$ & & $410 \mathrm{~J}$ & $277 \mathrm{~J}$ & $310 \mathrm{~J}$ & & \\
\hline Dissolved Solids (mg/L) & 282 & 174 & & 190 & $167 \mathrm{~J}$ & 150 & 301 & 300 \\
\hline Suspended Solids (mg/L) & $<$ & $<$ & & $<$ & $<$ & $<$ & $<$ & $<$ \\
\hline Turbidity (NTU) & & 0.1 & & 1.3 & 0.19 & 0.14 & & \\
\hline $\mathrm{pH}$ & & 8.2 & & 7.8 & 8 & 8 & & \\
\hline Major lons (mg/L) & & & & & & & & \\
\hline Calcium & 51.7 & 26.9 & & $<$ & 26.9 & 32 & 57.5 & 58 \\
\hline Magnesium & 31.6 & 17.6 & & 20.3 & 18.9 & 20 & 34.6 & 34.8 \\
\hline Potassium & 1.02 & $<$ & & $<$ & $<$ & $<$ & 1.51 & 1.49 \\
\hline Sodium & 11.6 & $<$ & & $<$ & $<$ & $<$ & 9.85 & 9.9 \\
\hline Alkalinity as $\mathrm{CO} 3$ & $<$ & $<$ & & $<$ & $<$ & $<$ & $<$ & $<$ \\
\hline Alkalinity as $\mathrm{HCO} 3$ & 237 & 169 & & 184 & 158 & 160 & 288 & 261 \\
\hline Chloride & 26.2 & 3.1 & & 5.4 & $3.9 \mathrm{~J}$ & $5 \mathrm{~J}$ & 18.8 & 17.9 \\
\hline Fluoride & $<$ & $<$ & & $<$ & $<$ & $0.11 \mathrm{~J}$ & $<$ & $<$ \\
\hline Nitrate as $\mathrm{N}$ & 1.2 & $0.45 \mathrm{~J}$ & & 0.58 & $0.43 \mathrm{~J}$ & $0.41 \mathrm{~J}$ & 0.7 & 0.71 \\
\hline Sulfate & 3.6 & $1.3 \mathrm{~J}$ & & $1.7 \mathrm{~J}$ & $1.6 \mathrm{~J}$ & $1.9 \mathrm{~J}$ & 3.7 & 3.6 \\
\hline Charge Balance (RPD) & 0.7 & -11.6 & & -5 & -4.7 & -1.3 & -1.8 & \\
\hline Trace Metals (mg/L) & & & & & & & & \\
\hline Aluminum & $<$ & $<$ & & $<$ & $<$ & $<$ & $<$ & - \\
\hline Arsenic & $<$ & $<$ & & $<$ & $<$ & $<$ & $<$ & $<$ \\
\hline Barium & 0.0223 & 0.01 & & 0.014 & $<$ & 0.01 & 0.0225 & 0.0214 \\
\hline Boron & $<$ & $<$ & & $<$ & $<$ & $<$ & $<$ & $<$ \\
\hline Cadmium (PMS) & & & & & & & & \\
\hline Chromium & 0.0425 & $<$ & & $<$ & $<$ & $<$ & $<$ & $<$ \\
\hline Iron & 0.383 & $<$ & & $<$ & $<$ & $<$ & 0.205 & 0.218 \\
\hline Lead (PMS) & & & & & & & & \\
\hline Lithium & $<$ & & & & & & $<$ & . \\
\hline Manganese & 0.0079 & $<$ & & 0.012 & $<$ & $<$ & 0.0054 & 0.0071 \\
\hline Nickel & 0.0548 & 0.25 & & 0.76 & 0.31 & 0.39 & 0.161 & 0.162 \\
\hline Strontium & 0.019 & 0.014 & & 0.024 & 0.015 & 0.017 & 0.0247 & 0.0247 \\
\hline Thallium (PMS) & & & & & & & & \\
\hline Uranium (PMS) & & & & & & & & \\
\hline Uranium (KPA) & $<$ & $<$ & & $<$ & $<$ & $<$ & $<$ & $<$ \\
\hline
\end{tabular}


APPENDIX F.1: CY 2002 MONITORING DATA FOR THE CHESTNUT RIDGE HYDROGEOLOGIC REGIME

Field Measurements, Miscellaneous Analytes, Major Ions, and Trace Metals

\begin{tabular}{|c|c|c|c|c|c|c|c|c|c|}
\hline Station & \multicolumn{2}{|c|}{ GW-339 } & \multicolumn{2}{|c|}{ GW-521 } & \multicolumn{3}{|c|}{ GW-522 } & \multicolumn{2}{|c|}{ GW-539 } \\
\hline Site & \multicolumn{2}{|c|}{ UNCS } & \multicolumn{2}{|c|}{ LIV } & \multicolumn{3}{|c|}{ LIV } & \multicolumn{2}{|c|}{ LII } \\
\hline Date & \multicolumn{2}{|c|}{ 07/30/02 } & 01/10/02 & 07/15/02 & $01 / 22 / 02$ & $01 / 28 / 02$ & 07/15/02 & $01 / 14 / 02$ & 07/17/02 \\
\hline Program & BJC & BJC & BJC & BJC & BJC & BJC & BJC & BJC & BJC \\
\hline Sample Type & & Dup & & & & & & & \\
\hline Field Measurements & & & & & & & & & \\
\hline Time Sampled & $13: 35$ & & $10: 45$ & $10: 25$ & $12: 10$ & $11: 25$ & $11: 45$ & $9: 59$ & $10: 00$ \\
\hline Measuring Point Elev. (ft) & 1124.83 & & 1182.88 & 1182.88 & 1175.48 & 1175.48 & 1175.48 & 1093.20 & 1093.20 \\
\hline Depth to Water (ft) & 78.48 & & 91.54 & 85.19 & 109.30 & 94.65 & 107.52 & 113.58 & 107.60 \\
\hline Groundwater Elevation (ft) & 1046.35 & & 1091.34 & 1097.69 & 1066.18 & 1080.83 & 1067.96 & 979.62 & 985.60 \\
\hline Conductivity ( $\mu \mathrm{mho} / \mathrm{cm})$ & 579 & & 306 & 301 & 292 & 358 & 358 & 339 & 351 \\
\hline Dissolved Oxygen (ppm) & 0.69 & & 5.73 & 4.49 & 5.97 & 6.22 & 5.21 & 6.47 & 4.89 \\
\hline Oxidation/Reduction (mV) & 174 & & 247 & 165 & 229 & 208 & 177 & 246 & 201 \\
\hline Temperature (degrees C) & 21.4 & & 13.6 & 18.1 & 14.6 & 13.8 & 17.3 & 9.3 & 21.5 \\
\hline Turbidity (NTU) & 11 & & 14 & 13 & 8 & 9 & 10 & 10 & 9 \\
\hline $\mathrm{pH}$ & 7.47 & & 7.65 & 8.45 & 7.79 & 7.34 & 8.18 & 7.79 & 8.29 \\
\hline Miscellaneous Analytes & & & & & & & & & \\
\hline Conductivity ( $\mu \mathrm{mho} / \mathrm{cm})$ & & & $234 \mathrm{~J}$ & $254 \mathrm{~J}$ & $258 \mathrm{~J}$ & & $276 \mathrm{~J}$ & $303 \mathrm{~J}$ & $280 \mathrm{~J}$ \\
\hline Dissolved Solids (mg/L) & 311 & 313 & 163 & $157 \mathrm{~J}$ & 169 & & $169 \mathrm{~J}$ & 178 & 164 \\
\hline Suspended Solids (mg/L) & $<$ & $<$ & $<$ & $<$ & $<$ & & $<$ & $<$ & $<$ \\
\hline Turbidity (NTU) & & & 0.15 & 1.7 & 0.31 & & $0.08 \mathrm{~J}$ & $0.06 \mathrm{~J}$ & 0.24 \\
\hline $\mathrm{pH}$ & & & 7.5 & 8.2 & 7.9 & & 7.8 & 8.1 & 8 \\
\hline Major lons (mg/L) & & & & & & & & & \\
\hline Calcium & 64.6 & 64.5 & $<$ & 27.9 & 28.1 & & 32.3 & 33.4 & 34.7 \\
\hline Magnesium & 37 & 37 & 21.2 & 20.1 & 17.3 & & 20.1 & 20.7 & 21.2 \\
\hline Potassium & 2.49 & 2.56 & $<$ & $<$ & $<$ & & $<$ & $<$ & $<$ \\
\hline Sodium & 10.6 & 10.8 & $<$ & $<$ & $<$ & & $<$ & $<$ & $<$ \\
\hline Alkalinity as $\mathrm{CO} 3$ & $<$ & $<$ & $<$ & $<$ & $<$ & & $<$ & $<$ & $<$ \\
\hline Alkalinity as $\mathrm{HCO} 3$ & 280 & 279 & 160 & 153 & 160 & & 163 & 168 & 164 \\
\hline Chloride & 18.8 & 19.9 & $1.9 \mathrm{~J}$ & $1.9 \mathrm{~J}$ & $2 \mathrm{~J}$ & & $2.4 \mathrm{~J}$ & 4.2 & 4.1 \\
\hline Fluoride & $<$ & $<$ & $<$ & $<$ & $<$ & & $0.14 \mathrm{~J}$ & $<$ & $<$ \\
\hline Nitrate as $\mathrm{N}$ & 0.49 & 0.51 & $0.35 \mathrm{~J}$ & $0.3 \mathrm{~J}$ & 0.5 & & 0.53 & 0.52 & 0.51 \\
\hline Sulfate & 3.7 & 3.6 & $2 \mathrm{~J}$ & $1.8 \mathrm{~J}$ & $1.7 \mathrm{~J}$ & & $3.1 \mathrm{~J}$ & 6.3 & 7.1 \\
\hline Charge Balance (RPD) & 4.3 & & 0.7 & -0.2 & -8.1 & & -1 & -3.9 & 0.9 \\
\hline Trace Metals (mg/L) & & & & & & & & & \\
\hline Aluminum & $<$ & $<$ & $<$ & $<$ & $<$ & & $<$ & $<$ & $<$ \\
\hline Arsenic & $<$ & $<$ & $<$ & $<$ & $<$ & & $<$ & $<$ & $<$ \\
\hline Barium & 0.0218 & 0.0226 & $<$ & $<$ & $<$ & & $<$ & $<$ & $<$ \\
\hline Boron & $<$ & $<$ & $<$ & $<$ & $<$ & & $<$ & $<$ & $<$ \\
\hline Cadmium (PM & & & & & & & & & \\
\hline Chromium & 0.0117 & 0.0122 & $<$ & $<$ & $<$ & & $<$ & 0.0069 & 0.0057 \\
\hline Iron & $<$ & 0.053 & $<$ & $<$ & $<$ & & $<$ & $<$ & $<$ \\
\hline Lead (PMS) & & & & & & & & & \\
\hline Lithium & $<$ & $<$ & & & & & & & \\
\hline Manganese & $<$ & $<$ & $<$ & $<$ & $<$ & & $<$ & $<$ & $<$ \\
\hline Nickel & 0.2 & 0.198 & $<$ & $<$ & $<$ & & $<$ & 0.022 & 0.022 \\
\hline Strontium & 0.0269 & 0.0275 & 0.011 & 0.011 & 0.011 & & 0.014 & 0.019 & 0.019 \\
\hline Thallium (PMS) & & & & & & & & & \\
\hline Uranium (PMS) & & & & & & & & & \\
\hline Uranium (KPA) & $<$ & $<$ & $<$ & $<$ & $<$ & & $<$ & $<$ & $<$ \\
\hline
\end{tabular}


APPENDIX F.1: CY 2002 MONITORING DATA FOR THE CHESTNUT RIDGE HYDROGEOLOGIC REGIME

Field Measurements, Miscellaneous Analytes, Major Ions, and Trace Metals

\begin{tabular}{|c|c|c|c|c|c|c|c|c|c|c|}
\hline Station & \multicolumn{2}{|c|}{ GW-540 } & \multicolumn{2}{|c|}{ GW-542 } & \multicolumn{2}{|c|}{ GW-543 } & \multicolumn{2}{|c|}{ GW-544 } & \multicolumn{2}{|c|}{ GW-557 } \\
\hline Site & \multicolumn{2}{|c|}{ LII } & \multicolumn{2}{|c|}{ CDLVI } & \multicolumn{2}{|c|}{ CDLVI } & \multicolumn{2}{|c|}{ CDLVI } & \multicolumn{2}{|c|}{ LV } \\
\hline Date & $01 / 16 / 02$ & 07/18/02 & $01 / 16 / 02$ & 07/18/02 & $01 / 15 / 02$ & 07/18/02 & $01 / 15 / 02$ & 07/22/02 & \multicolumn{2}{|c|}{$01 / 10 / 02$} \\
\hline Program & BJC & BJC & BJC & BJC & BJC & BJC & BJC & BJC & BJC & BJC \\
\hline Sample Type & & & & & & & & & & Dup \\
\hline \multicolumn{11}{|l|}{ Field Measurements } \\
\hline Measuring Point Elev. (ft) & 1072.31 & 1072.31 & 1051.81 & 1051.81 & 1024.01 & 1024.01 & 1045.19 & 1045.19 & 1081.36 & \\
\hline Depth to Water (ft) & 88.98 & 83.75 & 72.14 & 70.08 & 65.77 & 65.37 & 65.22 & 61.14 & 125.20 & \\
\hline Groundwater Elevation (ft) & 983.33 & 988.56 & 979.67 & 981.73 & 958.24 & 958.64 & 979.97 & 984.05 & 956.16 & \\
\hline Conductivity $(\mu \mathrm{mho} / \mathrm{cm})$ & 415 & 435 & 229 & 171 & 528 & 508 & 507 & 545 & 358 & \\
\hline Dissolved Oxygen (ppm) & 0.77 & 6.13 & 4.77 & 8.13 & 6.37 & 4.37 & 2.51 & 3.82 & 7.01 & \\
\hline Oxidation/Reduction (mV) & 175 & 142 & 248 & 239 & 221 & 219 & 239 & 183 & 214 & \\
\hline Temperature (degrees C) & 13.1 & 19.8 & 13.2 & 17.3 & 11.8 & 16.8 & 9.4 & 19.3 & 14.1 & \\
\hline Turbidity (NTU) & 11 & 11 & 27 & 12 & 6 & 9 & 5 & 15 & 14 & \\
\hline $\mathrm{pH}$ & 8.04 & 7.48 & 6.66 & 6.38 & 6.83 & 6.73 & 7.19 & 7.32 & 7.55 & \\
\hline \multicolumn{11}{|l|}{ Miscellaneous Analytes } \\
\hline Conductivity $(\mu \mathrm{mho} / \mathrm{cm})$ & $345 \mathrm{~J}$ & $375 \mathrm{~J}$ & $188 \mathrm{~J}$ & $153 \mathrm{~J}$ & $440 \mathrm{~J}$ & $444 \mathrm{~J}$ & $420 \mathrm{~J}$ & $480 \mathrm{~J}$ & $256 \mathrm{~J}$ & $263 \mathrm{~J}$ \\
\hline Dissolved Solids (mg/L) & 220 & $221 \mathrm{~J}$ & 137 & $104 \mathrm{~J}$ & 286 & $259 \mathrm{~J}$ & 273 & $274 \mathrm{~J}$ & 179 & 172 \\
\hline Suspended Solids (mg/L) & 8.2 & $2 \mathrm{~J}$ & 7.2 & $<$ & $<$ & $<$ & $<$ & $<$ & $<$ & $<$ \\
\hline Turbidity (NTU) & $0.08 \mathrm{~J}$ & 2.5 & 2.9 & 0.65 & $0.09 \mathrm{~J}$ & $<$ & $0.06 \mathrm{~J}$ & $0.07 \mathrm{~J}$ & 0.26 & 0.33 \\
\hline $\mathrm{pH}$ & 7.9 & 7.8 & 7.2 & 6.9 & 7.4 & 7.3 & 7.9 & 7.8 & 7.6 & 7.6 \\
\hline \multicolumn{11}{|l|}{ Major lons (mg/L) } \\
\hline Calcium & 36.1 & 38.5 & 25.6 & 21.5 & 58.3 & 53.5 & 52.8 & 53.9 & $<$ & $<$ \\
\hline Magnesium & 25.7 & 27.3 & 13.6 & 8 & 34.1 & 31.5 & 30.8 & 31.6 & 21.3 & 21.3 \\
\hline Potassium & $<$ & $<$ & $<$ & $<$ & $<$ & $<$ & $<$ & $<$ & $<$ & $<$ \\
\hline Sodium & 14.1 & 14.7 & $<$ & $<$ & $<$ & $<$ & 5.6 & 5.8 & $<$ & $<$ \\
\hline Alkalinity as $\mathrm{CO} 3$ & $<$ & $<$ & $<$ & $<$ & $<$ & $<$ & $<$ & $<$ & $<$ & $<$ \\
\hline Alkalinity as $\mathrm{HCO} 3$ & 217 & 218 & 117 & 90.1 & 265 & 244 & 261 & 237 & 170 & 168 \\
\hline Chloride & $2.2 \mathrm{~J}$ & 6.3 & $1.7 \mathrm{~J}$ & $1.8 \mathrm{~J}$ & $2.8 \mathrm{~J}$ & 5.4 & 9.4 & $9.7 \mathrm{~J}$ & $2.1 \mathrm{~J}$ & $2.2 \mathrm{~J}$ \\
\hline Fluoride & $0.19 \mathrm{~J}$ & $<$ & $0.19 \mathrm{~J}$ & $0.16 \mathrm{~J}$ & $<$ & $<$ & $<$ & $<$ & $0.19 \mathrm{~J}$ & $<$ \\
\hline Nitrate as $\mathrm{N}$ & $0.17 \mathrm{~J}$ & $<$ & $0.4 \mathrm{~J}$ & $0.37 \mathrm{~J}$ & $0.41 \mathrm{~J}$ & $0.37 \mathrm{~J}$ & 0.63 & 0.6 & 0.73 & 0.73 \\
\hline Sulfate & 9.3 & 8.3 & $2.9 \mathrm{~J}$ & $2.3 \mathrm{~J}$ & 18.2 & 15.1 & 19 & 25.2 & $2 \mathrm{~J}$ & $2.1 \mathrm{~J}$ \\
\hline Charge Balance (RPD) & 0 & 1.6 & 1.2 & -1.9 & 0.3 & 0.8 & -4.5 & 0 & 0.2 & \\
\hline \multicolumn{11}{|l|}{ Trace Metals $(\mathrm{mg} / \mathrm{L})$} \\
\hline Aluminum & $<$ & $<$ & $<$ & $<$ & $<$ & $<$ & $<$ & $<$ & $<$ & $<$ \\
\hline Arsenic & $<$ & $<$ & $<$ & $<$ & $<$ & $<$ & $<$ & $<$ & $<$ & $<$ \\
\hline Barium & 0.012 & 0.012 & 0.016 & 0.015 & 0.013 & 0.014 & 0.014 & 0.015 & 0.011 & 0.011 \\
\hline Boron & $<$ & $<$ & $<$ & $<$ & $<$ & $<$ & $<$ & $<$ & $<$ & $<$ \\
\hline Cadmium (PMS) & & & & & & & & & & \\
\hline Chromium & $<$ & $<$ & $<$ & $<$ & $<$ & $<$ & $<$ & $<$ & $<$ & $<$ \\
\hline Iron & $<$ & $<$ & $<$ & $<$ & $<$ & $<$ & $<$ & $<$ & $<$ & $<$ \\
\hline Lead (PMS) & & & & & & & & & & \\
\hline Lithium & & & & & & & & & & \\
\hline Manganese & $<$ & $<$ & $<$ & $<$ & $<$ & $<$ & $<$ & $<$ & $<$ & $<$ \\
\hline Nickel & $<$ & $<$ & $<$ & $<$ & $<$ & $<$ & $<$ & $<$ & $<$ & 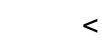 \\
\hline Strontium & 0.027 & 0.03 & 0.019 & 0.02 & 0.031 & 0.037 & 0.028 & 0.031 & 0.018 & 0.018 \\
\hline Thallium (PMS) & & & & & & & & & & \\
\hline Uranium (PMS) & & & & & & & & & & \\
\hline Uranium (KPA) & $<$ & $<$ & $<$ & $<$ & $<$ & $<$ & $<$ & $<$ & $<$ & $<$ \\
\hline
\end{tabular}


APPENDIX F.1: CY 2002 MONITORING DATA FOR THE CHESTNUT RIDGE HYDROGEOLOGIC REGIME

Field Measurements, Miscellaneous Analytes, Major Ions, and Trace Metals

\begin{tabular}{|c|c|c|c|c|c|c|c|c|c|}
\hline Station & \multicolumn{2}{|c|}{ GW-557 } & \multicolumn{2}{|c|}{ GW-560 } & \multicolumn{5}{|c|}{ GW-562 } \\
\hline Site & \multicolumn{2}{|c|}{ LV } & \multicolumn{2}{|c|}{ CDLVII } & \multicolumn{5}{|c|}{ CDLVII } \\
\hline Date & \multicolumn{2}{|c|}{ 07/11/02 } & $01 / 17 / 02$ & 07/16/02 & $01 / 09 / 02$ & $01 / 22 / 02$ & $01 / 28 / 02$ & 07/10/02 & 07/16/02 \\
\hline Program & BJC & BJC & BJC & BJC & BJC & BJC & BJC & BJC & BJC \\
\hline Sample Type & & Dup & & & & & & & \\
\hline Field Measurements & & & & & & & & & \\
\hline Time Sampled & $11: 15$ & & $10: 55$ & $11: 23$ & $14: 15$ & $9: 50$ & $13: 35$ & $9: 35$ & $9: 43$ \\
\hline Measuring Point Elev. (ft) & 1081.36 & & 949.05 & 949.05 & 934.69 & 934.69 & 934.69 & 934.69 & 934.69 \\
\hline Depth to Water (ft) & 122.78 & & 48.99 & 42.13 & 13.85 & 12.90 & 10.15 & 8.64 & 8.78 \\
\hline Groundwater Elevation (ft) & 958.58 & & 900.06 & 906.92 & 920.84 & 921.79 & 924.54 & 926.05 & 925.91 \\
\hline Conductivity ( $\mu \mathrm{mho} / \mathrm{cm})$ & 344 & & 332 & 330 & 385 & 370 & 366 & 405 & 390 \\
\hline Dissolved Oxygen (ppm) & 7.5 & & 8.6 & 6.43 & 6.89 & 8.89 & 3.41 & 4.7 & 6.2 \\
\hline Oxidation/Reduction (mV) & 176 & & 249 & 206 & 182 & 205 & 176 & 161 & 142 \\
\hline Temperature (degrees C) & 17.1 & & 13.7 & 17 & 13.7 & 13 & 13.8 & 18.7 & 17.2 \\
\hline Turbidity (NTU) & 4 & & 16 & 9 & 11 & 16 & 11 & 12 & 11 \\
\hline $\mathrm{pH}$ & 7.15 & & 7.54 & 7.16 & 7.72 & 7.6 & 7.22 & 7.19 & 7.18 \\
\hline Miscellaneous Analytes & & & & & & & & & \\
\hline Conductivity $(\mu \mathrm{mho} / \mathrm{cm})$ & $308 \mathrm{~J}$ & $308 \mathrm{~J}$ & $264 \mathrm{~J}$ & $278 \mathrm{~J}$ & & $308 \mathrm{~J}$ & & & $319 \mathrm{~J}$ \\
\hline Dissolved Solids (mg/L) & $165 \mathrm{~J}$ & $179 \mathrm{~J}$ & 181 & 157 & 211 & 209 & & 214 & 171 \\
\hline Suspended Solids (mg/L) & $<$ & $<$ & $<$ & $<$ & $<$ & $<$ & & $<$ & 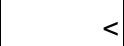 \\
\hline Turbidity (NTU) & 0.2 & 0.21 & 0.17 & 0.21 & & 5.2 & & & 3.2 \\
\hline $\mathrm{pH}$ & 7.8 & 7.8 & 7.7 & 7.7 & & 7.4 & & & 7.7 \\
\hline Major lons (mg/L) & & & & & & & & & \\
\hline Calcium & 36.5 & 37.3 & 37.7 & 37.4 & 39.6 & 29.9 & & 41.4 & 38.5 \\
\hline Magnesium & 21 & 21.4 & 16.2 & 16 & 23.6 & 17.2 & & 24 & 22.7 \\
\hline Potassium & $<$ & $<$ & $<$ & $<$ & 0.609 & $<$ & & 0.665 & $<$ \\
\hline Sodium & $<$ & $<$ & $<$ & $<$ & 2.81 & $<$ & & 2.58 & $<$ \\
\hline Alkalinity as $\mathrm{CO} 3$ & $<$ & $<$ & $<$ & $<$ & & $<$ & & & - \\
\hline Alkalinity as $\mathrm{HCO} 3$ & 172 & 173 & 169 & 162 & & 196 & & & 192 \\
\hline Chloride & 3.2 & $2.1 \mathrm{~J}$ & $2.2 \mathrm{~J}$ & $2 \mathrm{~J}$ & & $2.3 \mathrm{~J}$ & & & $2.5 \mathrm{~J}$ \\
\hline Fluoride & $0.13 \mathrm{~J}$ & $0.13 \mathrm{~J}$ & $0.21 \mathrm{~J}$ & $0.15 \mathrm{~J}$ & & $<$ & & & $0.14 \mathrm{~J}$ \\
\hline Nitrate as $\mathrm{N}$ & 0.69 & 0.7 & $0.34 \mathrm{~J}$ & $0.29 \mathrm{~J}$ & & $0.36 \mathrm{~J}$ & & & $0.34 \mathrm{~J}$ \\
\hline Sulfate & $3.8 \mathrm{~J}$ & $2.2 \mathrm{~J}$ & $3.1 \mathrm{~J}$ & $2.8 \mathrm{~J}$ & & $1.9 \mathrm{~J}$ & & & $2.2 \mathrm{~J}$ \\
\hline Charge Balance (RPD) & -1.2 & & -4.5 & -2.6 & & -16.4 & & & -0.8 \\
\hline Trace Metals (mg/L) & & & & & & & & & \\
\hline Aluminum & $<$ & $<$ & $<$ & $<$ & 0.311 & $<$ & & 0.332 & $<$ \\
\hline Arsenic & $<$ & $<$ & $<$ & $<$ & $<$ & $<$ & & $<$ & . \\
\hline Barium & 0.013 & 0.011 & 0.23 & 0.25 & 0.0123 & $<$ & & 0.013 & 0.011 \\
\hline Boron & $<$ & $<$ & $<$ & $<$ & & $<$ & & $<$ & $<$ \\
\hline Cadmium (PN & & & & & & & & & \\
\hline Chromium & $<$ & $<$ & $<$ & $<$ & $<$ & $<$ & & $<$ & $<$ \\
\hline Iron & $<$ & $<$ & $<$ & $<$ & 0.285 & $<$ & & 0.41 & $<$ \\
\hline Lead (PMS) & & & & & & & & & \\
\hline Lithium & & & & & $<$ & & & $<$ & \\
\hline Manganese & $<$ & $<$ & $<$ & $<$ & 0.0055 & $<$ & & 0.0087 & $<$ \\
\hline Nickel & $<$ & $<$ & $<$ & $<$ & $<$ & $<$ & & $<$ & $<$ \\
\hline Strontium & 0.018 & 0.018 & 0.025 & 0.027 & 0.0206 & 0.017 & & 0.0209 & 0.022 \\
\hline Thallium (PMS) & & & & & & & & & \\
\hline Uranium (PMS) & & & & & & & & & \\
\hline Uranium (KPA) & $<$ & $<$ & $<$ & $<$ & $<$ & $<$ & & $<$ & $<$ \\
\hline
\end{tabular}


APPENDIX F.1: CY 2002 MONITORING DATA FOR THE CHESTNUT RIDGE HYDROGEOLOGIC REGIME

Field Measurements, Miscellaneous Analytes, Major Ions, and Trace Metals

\begin{tabular}{|c|c|c|c|c|c|c|c|c|c|c|}
\hline Station & \multicolumn{4}{|c|}{ GW-564 } & \multicolumn{2}{|c|}{ GW-709 } & \multicolumn{2}{|c|}{ GW-731 } & \multicolumn{2}{|c|}{ GW-732 } \\
\hline Site & \multicolumn{4}{|c|}{ CDLVII } & \multicolumn{2}{|c|}{ LII } & \multicolumn{2}{|c|}{ CRSDB } & \multicolumn{2}{|c|}{ CRSDB } \\
\hline Date & \multicolumn{2}{|c|}{ 01/16/02 } & \multicolumn{2}{|c|}{ 07/16/02 } & $01 / 15 / 02$ & 07/17/02 & $04 / 16 / 02$ & $10 / 15 / 02$ & 04/16/02 & $10 / 15 / 02$ \\
\hline Program & BJC & BJC & BJC & BJC & BJC & BJC & BJC & BJC & BJC & BJC \\
\hline Sample Type & & Dup & & Dup & & & & & & \\
\hline $\begin{array}{r}\text { Field Measurements } \\
\text { Time Sampled }\end{array}$ & $10: 10$ & & 9:20 & & $9: 40$ & $11: 35$ & $12: 30$ & $14: 48$ & $13: 40$ & $10: 07$ \\
\hline Measuring Point Elev. (ft) & 938.07 & & 938.07 & & 906.81 & 906.81 & 1049.29 & 1049.29 & 1064.29 & 1064.29 \\
\hline Depth to Water (ft) & 13.17 & & 11.47 & & 30.02 & 29.79 & 124.70 & 126.08 & 157.50 & 157.90 \\
\hline Groundwater Elevation (ft) & 924.90 & & 926.60 & & 876.79 & 877.02 & 924.59 & 923.21 & 906.79 & 906.39 \\
\hline Conductivity $(\mu \mathrm{mho} / \mathrm{cm})$ & 277 & & 274 & & 304 & 283 & 261 & 246 & 269 & 313 \\
\hline Dissolved Oxygen (ppm) & 5.74 & & 5.48 & & 1.32 & 2.8 & 5.31 & 6.72 & 5.13 & 7.21 \\
\hline Oxidation/Reduction (mV) & 234 & & 195 & & 190 & 154 & 172 & 169 & 180 & 199 \\
\hline Temperature (degrees C) & 14.2 & & 16 & & 7.5 & 23.2 & 18.2 & 16.4 & 17 & 15.6 \\
\hline Turbidity (NTU) & 11 & & 9 & & 15 & 6 & 12 & 17 & 14 & 10 \\
\hline $\mathrm{pH}$ & 6.49 & & 7.16 & & 8.66 & 8.68 & 7.87 & 7.6 & 8.41 & 7.64 \\
\hline Miscellaneous Analytes & & & & & & & & & & \\
\hline Conductivity ( $\mu \mathrm{mho} / \mathrm{cm})$ & $223 \mathrm{~J}$ & $226 \mathrm{~J}$ & $239 \mathrm{~J}$ & $233 \mathrm{~J}$ & $241 \mathrm{~J}$ & $244 \mathrm{~J}$ & & & & \\
\hline Dissolved Solids (mg/L) & 159 & 157 & 131 & 142 & 150 & 133 & 138 & 111 & 156 & 118 \\
\hline Suspended Solids (mg/L) & $<$ & 6 & $<$ & $<$ & 6.2 & $<$ & $<$ & 4 & $<$ & $<$ \\
\hline Turbidity (NTU) & 0.59 & 0.41 & 0.6 & 0.56 & 0.32 & 0.19 & & & & \\
\hline $\mathrm{pH}$ & 6.8 & 6.9 & 7.2 & 7.2 & 7.8 & 8.4 & & & & \\
\hline Major lons (mg/L) & & & & & & & & & & \\
\hline Calcium & 32.2 & 34 & 32.4 & 32.5 & 18.7 & 20.1 & 28.4 & 25.3 & 26.8 & 29.6 \\
\hline Magnesium & 11.4 & 11.7 & 14 & 14 & 23 & 24.5 & 17.9 & 14.3 & 18.3 & 15.9 \\
\hline Potassium & $<$ & $<$ & $<$ & $<$ & $<$ & $<$ & 1.09 & 2.6 & 1.26 & 5.2 \\
\hline Sodium & $<$ & $<$ & $<$ & $<$ & $<$ & $<$ & 0.652 & 1.7 & 0.667 & 2.5 \\
\hline Alkalinity as $\mathrm{CO} 3$ & $<$ & $<$ & $<$ & $<$ & 85.7 & 6.5 & & & & \\
\hline Alkalinity as $\mathrm{HCO} 3$ & 107 & 109 & 132 & 130 & 26.7 & 140 & & & & \\
\hline Chloride & $4.4 \mathrm{~J}$ & $4.2 \mathrm{~J}$ & $2.5 \mathrm{~J}$ & $2.5 \mathrm{~J}$ & $2.7 \mathrm{~J}$ & $2.1 \mathrm{~J}$ & & & & \\
\hline Fluoride & $0.19 \mathrm{~J}$ & $0.19 \mathrm{~J}$ & $0.13 \mathrm{~J}$ & $0.14 \mathrm{~J}$ & $0.2 \mathrm{~J}$ & $<$ & & & & \\
\hline Nitrate as $\mathrm{N}$ & 1.6 & 1.5 & 0.53 & 0.53 & $<$ & $<$ & & & & \\
\hline Sulfate & 16 & 15.9 & 6.4 & 6.4 & $4.9 \mathrm{~J}$ & 5.3 & & & & \\
\hline Charge Balance (RPD) & -0.4 & & -1.5 & & 11.3 & 1.2 & & & & \\
\hline Trace Metals (mg/L) & & & & & & & & & & \\
\hline Aluminum & $<$ & $<$ & $<$ & $<$ & $<$ & $<$ & $<$ & 0.3 & $<$ & 0.36 \\
\hline Arsenic & $<$ & $<$ & $<$ & $<$ & $<$ & $<$ & $<$ & $<$ & $<$ & $<$ \\
\hline Barium & 0.029 & 0.034 & 0.016 & 0.016 & 0.47 & 0.46 & 0.0078 & 0.01 & 0.0101 & 0.021 \\
\hline Boron & $<$ & $<$ & $<$ & $<$ & $<$ & $<$ & $<$ & $<$ & $<$ & 0.36 \\
\hline Cadmium (PMS) & & & & & & & & & & \\
\hline Chromium & $<$ & $<$ & $<$ & $<$ & $<$ & $<$ & $<$ & $<$ & $<$ & 0.0056 \\
\hline Iron & $<$ & $<$ & $<$ & $<$ & $<$ & $<$ & $<$ & 0.3 & 0.131 & 0.14 \\
\hline Lead (PMS) & & & & & & & & & & \\
\hline Lithium & & & & & & & $<$ & & $<$ & 0.017 \\
\hline Manganese & $<$ & $<$ & $<$ & $<$ & $<$ & $<$ & $<$ & 0.015 & $<$ & $<$ \\
\hline Nickel & $<$ & $<$ & $<$ & $<$ & $<$ & $<$ & $<$ & $<$ & $<$ & $<$ \\
\hline Strontium & 0.072 & 0.077 & 0.035 & 0.035 & 0.034 & 0.035 & 0.0147 & 0.033 & 0.015 & 0.033 \\
\hline Thallium (PMS) & & & & & & & & & & \\
\hline Uranium (PMS) & & & & & & & & & & \\
\hline Uranium (KPA) & $<$ & $<$ & $<$ & $<$ & $<$ & $<$ & $<$ & $<$ & $<$ & $<$ \\
\hline
\end{tabular}


APPENDIX F.1: CY 2002 MONITORING DATA FOR THE CHESTNUT RIDGE HYDROGEOLOGIC REGIME

Field Measurements, Miscellaneous Analytes, Major lons, and Trace Metals

\begin{tabular}{|c|c|c|c|c|c|c|c|c|c|c|}
\hline Station & \multicolumn{2}{|c|}{ GW-757 } & \multicolumn{2}{|c|}{ GW-796 } & \multicolumn{2}{|c|}{ GW-797 } & \multicolumn{4}{|c|}{ GW-798 } \\
\hline Site & \multicolumn{2}{|c|}{ LII } & \multicolumn{2}{|c|}{ LV } & \multicolumn{2}{|c|}{ LV } & \multicolumn{4}{|c|}{ CDLVII } \\
\hline Date & $01 / 15 / 02$ & $07 / 17 / 02$ & $01 / 14 / 02$ & $07 / 11 / 02$ & $01 / 14 / 02$ & $07 / 11 / 02$ & $01 / 09 / 02$ & $01 / 16 / 02$ & 07/10/02 & 07/16/02 \\
\hline Program & BJC & BJC & BJC & BJC & BJC & BJC & BJC & BJC & BJC & BJC \\
\hline \multicolumn{11}{|l|}{ Sample Type } \\
\hline $\begin{array}{r}\text { Field Measurements } \\
\text { Time Sampled }\end{array}$ & $11: 15$ & $10: 30$ & $9: 30$ & $11: 20$ & $10: 50$ & $9: 35$ & $12: 55$ & $11: 15$ & $10: 45$ & $10: 30$ \\
\hline Measuring Point Elev. (ft) & 961.64 & 961.64 & 1052.62 & 1052.62 & 1060.00 & 1060.00 & 1006.00 & 1006.00 & 1006.00 & 1006.00 \\
\hline Depth to Water (ft) & 84.96 & 84.80 & 88.05 & 80.53 & 78.40 & 75.60 & 85.78 & 86.27 & 80.16 & 80.50 \\
\hline Groundwater Elevation (ft) & 876.68 & 876.84 & 964.57 & 972.09 & 981.60 & 984.40 & 920.22 & 919.73 & 925.84 & 925.50 \\
\hline Conductivity $(\mu \mathrm{mho} / \mathrm{cm})$ & 304 & 311 & 250 & 264 & 390 & 417 & 276 & 274 & 280 & 271 \\
\hline Dissolved Oxygen (ppm) & 1.16 & 2.06 & 8.09 & 6.07 & 4.73 & 6.7 & 10.41 & 8.62 & 4.81 & 5.41 \\
\hline Oxidation/Reduction (mV) & 42 & 135 & 213 & 230 & 205 & 179 & 166 & 214 & 135 & 165 \\
\hline Temperature (degrees C) & 9.1 & 21.9 & 13.9 & 16.7 & 11.4 & 20.5 & 14.9 & 14.6 & 18.4 & 17.7 \\
\hline Turbidity (NTU) & 12 & 16 & 17 & 11 & 19 & 7 & 8 & 15 & 8 & 5 \\
\hline $\mathrm{pH}$ & 10.11 & 9.47 & 8.35 & 8.3 & 8.04 & 7.71 & 7.88 & 7.84 & 8.09 & 8.06 \\
\hline \multicolumn{11}{|l|}{ Miscellaneous Analytes } \\
\hline Conductivity $(\mu \mathrm{mho} / \mathrm{cm})$ & $228 \mathrm{~J}$ & $254 \mathrm{~J}$ & $201 \mathrm{~J}$ & $217 \mathrm{~J}$ & $326 \mathrm{~J}$ & $369 \mathrm{~J}$ & & $232 \mathrm{~J}$ & & $246 \mathrm{~J}$ \\
\hline Dissolved Solids (mg/L) & 157 & 141 & 119 & $133 \mathrm{~J}$ & 200 & $215 \mathrm{~J}$ & 168 & 147 & 155 & 128 \\
\hline Suspended Solids (mg/L) & $<$ & $2.8 \mathrm{~J}$ & $2 \mathrm{~J}$ & $<$ & $<$ & $<$ & $<$ & $2.6 \mathrm{~J}$ & $<$ & $<$ \\
\hline Turbidity (NTU) & 1.7 & 1.5 & 5.3 & 3 & 0.13 & 0.22 & & $0.06 \mathrm{~J}$ & & $<$ \\
\hline $\mathrm{pH}$ & 9.8 & 10 & 7.6 & 8.1 & 7.9 & 7.9 & & 7.6 & & 7.8 \\
\hline \multicolumn{11}{|l|}{ Major lons (mg/L) } \\
\hline Calcium & 3.2 & 2.9 & 22.1 & 25.8 & 36.3 & 41.4 & 28.8 & 28.5 & 29.5 & 27.5 \\
\hline Magnesium & 3.7 & 3.3 & 13.7 & 15.2 & 21.5 & 24.1 & 16.5 & 16.2 & 16.5 & 15.7 \\
\hline Potassium & 17.7 & 17.1 & $<$ & $<$ & $<$ & $<$ & 1.71 & $<$ & 1.35 & $<$ \\
\hline Sodium & 41 & 41.3 & $<$ & $<$ & $<$ & $<$ & 0.479 & $<$ & 0.461 & $<$ \\
\hline Alkalinity as $\mathrm{CO} 3$ & $<$ & 70.7 & $<$ & $<$ & $<$ & $<$ & & $<$ & & $<$ \\
\hline Alkalinity as $\mathrm{HCO} 3$ & 139 & 43.6 & 125 & 123 & 177 & 173 & & 135 & & 134 \\
\hline Chloride & $2.1 \mathrm{~J}$ & $1.8 \mathrm{~J}$ & $1.4 \mathrm{~J}$ & $2.2 \mathrm{~J}$ & 3.5 & 5.3 & & $1.6 \mathrm{~J}$ & & $1.7 \mathrm{~J}$ \\
\hline Fluoride & 1.7 & 1.7 & $<$ & $0.13 \mathrm{~J}$ & $<$ & $0.15 \mathrm{~J}$ & & $0.19 \mathrm{~J}$ & & $0.13 \mathrm{~J}$ \\
\hline Nitrate as $\mathrm{N}$ & $0.31 \mathrm{~J}$ & $0.33 \mathrm{~J}$ & $0.21 \mathrm{~J}$ & $0.19 \mathrm{~J}$ & 0.63 & 0.74 & & 0.76 & & 0.74 \\
\hline Sulfate & 14.3 & 14.6 & $1.2 \mathrm{~J}$ & $1.3 \mathrm{~J}$ & 20.8 & 25.2 & & $3.1 \mathrm{~J}$ & & $2.8 \mathrm{~J}$ \\
\hline Charge Balance (RPD) & -9.9 & -2.4 & -6.3 & -0.2 & -4.1 & 0.8 & & -1.2 & & -2.8 \\
\hline \multicolumn{11}{|l|}{ Trace Metals $(\mathrm{mg} / \mathrm{L})$} \\
\hline Aluminum & $<$ & $<$ & $<$ & $<$ & $<$ & $<$ & $<$ & $<$ & $<$ & $<$ \\
\hline Arsenic & $<$ & $<$ & $<$ & $<$ & $<$ & $<$ & $<$ & $<$ & $<$ & $<$ \\
\hline Barium & 0.071 & 0.069 & $<$ & $<$ & $<$ & $<$ & 0.011 & 0.013 & 0.0105 & 0.01 \\
\hline Boron & $<$ & $<$ & $<$ & $<$ & $<$ & $<$ & $<$ & $<$ & $<$ & $<$ \\
\hline Cadmium (PMS) & & & & & & & & & & \\
\hline Chromium & $<$ & $<$ & $<$ & $<$ & $<$ & 0.0053 & $<$ & $<$ & $<$ & $<$ \\
\hline Iron & $<$ & $<$ & $<$ & $<$ & $<$ & & $<$ & $<$ & $<$ & $<$ \\
\hline Lead (PMS) & & & & & & & & & & \\
\hline Lithium & & & & & & & $<$ & & $<$ & \\
\hline Manganese & $<$ & $<$ & $<$ & $<$ & $<$ & $<$ & $<$ & $<$ & $<$ & 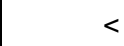 \\
\hline Nickel & $<$ & $<$ & $<$ & $<$ & $<$ & $<$ & $<$ & $<$ & $<$ & r \\
\hline Strontium & 0.18 & 0.18 & 0.013 & 0.014 & 0.025 & 0.026 & 0.0176 & 0.019 & 0.0166 & 0.018 \\
\hline Thallium (PMS) & & & & & & & & & & \\
\hline Uranium (PMS) & & & & & & & & & & \\
\hline Uranium (KPA) & $<$ & 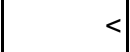 & 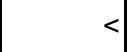 & $<$ & $<$ & 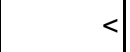 & $<$ & $<$ & $<$ & $<$ \\
\hline
\end{tabular}


APPENDIX F.1: CY 2002 MONITORING DATA FOR THE CHESTNUT RIDGE HYDROGEOLOGIC REGIME

Field Measurements, Miscellaneous Analytes, Major Ions, and Trace Metals

\begin{tabular}{|c|c|c|c|c|c|c|c|c|c|}
\hline Station & \multicolumn{2}{|c|}{ GW-799 } & \multicolumn{2}{|c|}{ GW-801 } & \multicolumn{2}{|c|}{ GW-827 } & \multicolumn{2}{|c|}{ GW-831 } & MCK 2.0 \\
\hline Site & \multicolumn{2}{|c|}{ LV } & \multicolumn{2}{|c|}{ LV } & \multicolumn{2}{|c|}{ CDLVI } & \multicolumn{2}{|c|}{ FCAP } & EXP-SW \\
\hline Date & $01 / 10 / 02$ & 07/11/02 & $01 / 14 / 02$ & 07/15/02 & 01/17/02 & 07/18/02 & 01/09/02 & 07/09/02 & $02 / 21 / 02$ \\
\hline Program & BJC & BJC & BJC & BJC & BJC & BJC & BJC & BJC & BJC \\
\hline \multicolumn{10}{|l|}{ Sample Type } \\
\hline \multicolumn{10}{|l|}{ Field Measurements } \\
\hline Time Sampled & $11: 50$ & $9: 50$ & $12: 10$ & $10: 20$ & $9: 30$ & $10: 35$ & $10: 05$ & $13: 40$ & $14: 20$ \\
\hline Measuring Point Elev. (ft) & 981.29 & 981.29 & 1097.16 & 1097.16 & 1051.60 & 1051.60 & 1091.29 & 1091.29 & \\
\hline Depth to Water (ft) & 21.55 & 18.40 & 121.24 & 114.85 & 47.81 & 43.66 & 134.00 & 129.28 & \\
\hline Groundwater Elevation (ft) & 959.74 & 962.89 & 975.92 & 982.31 & 1003.79 & 1007.94 & 957.29 & 962.01 & \\
\hline Conductivity ( $\mu \mathrm{mho} / \mathrm{cm})$ & 291 & 291 & 285 & 297 & 316 & 298 & 392 & 335 & 194 \\
\hline Dissolved Oxygen (ppm) & 3.19 & 2.17 & 8.07 & 6.03 & 5.29 & 6.65 & 2.14 & 2.52 & 10.58 \\
\hline Oxidation/Reduction (mV) & 226 & 152 & 209 & 133 & 210 & 268 & 241 & 4 & 115 \\
\hline Temperature (degrees C) & 13.4 & 16.8 & 12.7 & 15.2 & 13.2 & 16.3 & 8.5 & 24.3 & 14.8 \\
\hline Turbidity (NTU) & 12 & 11 & 9 & 12 & 11 & 12 & 12 & 17 & 2 \\
\hline $\mathrm{pH}$ & 8.2 & 8.46 & 7.69 & 7.47 & 7.75 & 7.78 & 8.51 & 8.6 & 6.12 \\
\hline \multicolumn{10}{|l|}{\begin{tabular}{|l|} 
Miscellaneous Analytes \\
\end{tabular}} \\
\hline Conductivity $(\mu \mathrm{mho} / \mathrm{cm})$ & $229 \mathrm{~J}$ & $260 \mathrm{~J}$ & $249 \mathrm{~J}$ & $247 \mathrm{~J}$ & $247 \mathrm{~J}$ & $261 \mathrm{~J}$ & & & \\
\hline Dissolved Solids (mg/L) & 143 & $155 \mathrm{~J}$ & 148 & $139 \mathrm{~J}$ & 161 & $146 \mathrm{~J}$ & 215 & 210 & 236 \\
\hline Suspended Solids (mg/L) & $3 \mathrm{~J}$ & $<$ & $<$ & $<$ & $<$ & $<$ & $<$ & $<$ & $<$ \\
\hline Turbidity (NTU) & 4.6 & 0.41 & 0.78 & 0.29 & 2.6 & 0.46 & & & \\
\hline $\mathrm{pH}$ & 8.2 & 8.3 & 7.6 & 7.9 & 7.4 & 7.8 & & & \\
\hline \multicolumn{10}{|l|}{ Major Ions (mg/L) } \\
\hline Calcium & $<$ & 32.5 & 29.1 & 28.4 & 31.3 & 31.5 & 38.9 & 40.6 & 51.1 \\
\hline Magnesium & 17.3 & 17.3 & 17 & 16.7 & 18 & 18.7 & 25.2 & 26.4 & 16 \\
\hline Potassium & $<$ & $<$ & $<$ & $<$ & $<$ & $<$ & 1.52 & 1.66 & 4.61 \\
\hline Sodium & $<$ & $<$ & $<$ & $<$ & $<$ & $<$ & 0.526 & 0.549 & 2.23 \\
\hline Alkalinity as $\mathrm{CO} 3$ & $<$ & $3.4 \mathrm{~J}$ & $<$ & $<$ & $<$ & $<$ & & & $<$ \\
\hline Alkalinity as $\mathrm{HCO} 3$ & 141 & 138 & 147 & 148 & 157 & 165 & & & 164 \\
\hline Chloride & $1.8 \mathrm{~J}$ & $1.8 \mathrm{~J}$ & $1.3 \mathrm{~J}$ & $2 \mathrm{~J}$ & $1.6 \mathrm{~J}$ & $1.7 \mathrm{~J}$ & & & 1.8 \\
\hline Fluoride & $0.23 \mathrm{~J}$ & $0.18 \mathrm{~J}$ & $<$ & $0.13 \mathrm{~J}$ & $0.19 \mathrm{~J}$ & $0.17 \mathrm{~J}$ & & & $<$ \\
\hline Nitrate as $\mathrm{N}$ & 1.1 & 1 & $0.21 \mathrm{~J}$ & $0.19 \mathrm{~J}$ & $0.2 \mathrm{~J}$ & $0.19 \mathrm{~J}$ & & & \\
\hline Sulfate & $3.7 \mathrm{~J}$ & $3.5 \mathrm{~J}$ & $2.8 \mathrm{~J}$ & $3.3 \mathrm{~J}$ & $2 \mathrm{~J}$ & $2 \mathrm{~J}$ & & & 42 \\
\hline Charge Balance (RPD) & -0.4 & 0.5 & -3 & -5 & -1.5 & -4.1 & & & -1.5 \\
\hline \multicolumn{10}{|l|}{ Trace Metals (mg/L) } \\
\hline Aluminum & $<$ & $<$ & $<$ & $<$ & $<$ & $<$ & $<$ & $<$ & $<$ \\
\hline Arsenic & $<$ & $<$ & $<$ & $<$ & $<$ & $<$ & $<$ & $<$ & 0.0111 \\
\hline Barium & $<$ & $<$ & $<$ & $<$ & $<$ & $<$ & 0.0211 & 0.0199 & 0.0817 \\
\hline Boron & $<$ & $<$ & $<$ & $<$ & $<$ & $<$ & $<$ & $<$ & 0.251 \\
\hline Cadmium (PMS) & & & & & & & & & \\
\hline Chromium & 0.061 & 0.051 & $<$ & $<$ & $<$ & $<$ & $<$ & $<$ & $<$ \\
\hline Iron & $<$ & $<$ & $<$ & $<$ & $<$ & $<$ & 0.0785 & 0.0932 & 0.0609 \\
\hline Lead (PMS) & & & & & & & & & \\
\hline Lithium & & & & 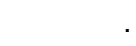 & & & $<$ & $<$ & 0.0908 \\
\hline Manganese & $<$ & $<$ & $<$ & $<$ & $<$ & $<$ & 0.0295 & 0.0259 & 0.0308 \\
\hline Nickel & $<$ & $<$ & $<$ & $<$ & $<$ & $<$ & $<$ & $<$ & $<$ \\
\hline Strontium & 0.022 & 0.021 & 0.016 & 0.016 & 0.018 & 0.018 & 0.0263 & 0.0258 & 0.994 \\
\hline Thallium (PMS) & & & & & & & & & \\
\hline Uranium (PMS) & & & & & & & & & \\
\hline Uranium (KPA) & $<$ & $<$ & $<$ & $<$ & $<$ & $<$ & $<$ & $<$ & $<$ \\
\hline
\end{tabular}


APPENDIX F.1: CY 2002 MONITORING DATA FOR THE CHESTNUT RIDGE HYDROGEOLOGIC REGIME

Field Measurements, Miscellaneous Analytes, Major Ions, and Trace Metals

\begin{tabular}{|c|c|c|c|c|c|c|c|}
\hline Station & \multirow{3}{*}{$\begin{array}{c}\text { MCK } 2.0 \\
\text { EXP-SW } \\
07 / 09 / 02\end{array}$} & \multicolumn{4}{|c|}{ MCK 2.05} & \multicolumn{2}{|c|}{ SCR1.25SP } \\
\hline Site & & \multicolumn{4}{|c|}{ EXP-SW } & \multicolumn{2}{|c|}{ EXP-SW } \\
\hline Date & & \multicolumn{2}{|c|}{$02 / 21 / 02$} & \multicolumn{2}{|c|}{ 07/09/02 } & \multirow{2}{*}{$\begin{array}{c}02 / 21 / 02 \\
\text { BJC }\end{array}$} & \multirow{2}{*}{$\begin{array}{c}\text { 07/10/02 } \\
\text { BJC }\end{array}$} \\
\hline Program & BJC & BJC & BJC & BJC & BJC & & \\
\hline Sample Type & & & Dup & & Dup & & \\
\hline \multicolumn{8}{|l|}{\begin{tabular}{|l|} 
Field Measurements \\
\end{tabular}} \\
\hline Time Sampled & $13: 55$ & $14: 33$ & & $14: 10$ & & $14: 07$ & $14: 30$ \\
\hline \multicolumn{8}{|l|}{$\begin{array}{l}\text { Measuring Point Elev. }(\mathrm{ft}) \\
\text { Depth to Water (ft) }\end{array}$} \\
\hline Depth to Water (ft) & & & & & & & \\
\hline \multicolumn{8}{|l|}{ Groundwater Elevation (ft) } \\
\hline Conductivity $(\mu \mathrm{mho} / \mathrm{cm})$ & 211 & 198 & & 197 & & 127 & 163 \\
\hline Dissolved Oxygen (ppm) & 9.48 & 10.35 & & 12 & & 11.54 & 9.85 \\
\hline Oxidation/Reduction (mV) & 120 & -10 & & 20 & & 180 & 110 \\
\hline Temperature (degrees C) & 19.1 & 13.9 & & 16.7 & & 13.3 & 18.4 \\
\hline Turbidity (NTU) & 51 & 4 & & 12 & & 4 & 45 \\
\hline $\mathrm{pH}$ & 7.76 & 6.35 & & 7.85 & & 5.6 & 7.91 \\
\hline \multicolumn{8}{|l|}{ Miscellaneous Analytes } \\
\hline Dissolved Solids (mg/L) & 477 & 262 & 249 & 274 & 253 & 132 & 188 \\
\hline Suspended Solids (mg/L) & $<$ & $<$ & $<$ & $<$ & $<$ & $<$ & 59.9 \\
\hline Turbidity (NTU) & & & & & & & \\
\hline $\mathrm{pH}$ & & & & & & & \\
\hline \multicolumn{8}{|l|}{ Major Ions (mg/L) } \\
\hline Calcium & 50.6 & 51.5 & 49.7 & 51.9 & 50.8 & 30.3 & 41.2 \\
\hline Magnesium & 16.8 & 16.5 & 16 & 17.6 & 17.3 & 12.4 & 18.3 \\
\hline Potassium & 4.46 & 4.64 & 4.55 & 4.61 & 4.61 & 0.843 & 1.21 \\
\hline Sodium & 2.48 & 2.28 & 2.23 & 2.45 & 2.43 & 1.36 & 1.42 \\
\hline Alkalinity as $\mathrm{CO} 3$ & $<$ & $<$ & $<$ & $<$ & $<$ & $<$ & $<$ \\
\hline Alkalinity as $\mathrm{HCO} 3$ & $<Q$ & 162 & 161 & 170 & 160 & 114 & 151 \\
\hline Chloride & 1.9 & 1.9 & 1.8 & 2.1 & 2.2 & 2.7 & 2.8 \\
\hline Fluoride & 0.11 & $<$ & $<$ & $<$ & 0.12 & $<$ & $<$ \\
\hline \multicolumn{8}{|l|}{ Nitrate as $\mathrm{N}$} \\
\hline Sulfate & 33.5 & 41.8 & 41.8 & 33.9 & 34.1 & 7.7 & 4.7 \\
\hline Charge Balance (RPD) & $69 \mathrm{R}$ & -0.2 & & 1.2 & & 2 & 6.7 \\
\hline \multicolumn{8}{|l|}{ Trace Metals (mg/L) } \\
\hline Aluminum & $<$ & $<$ & $<$ & $<$ & $<$ & $<$ & 0.331 \\
\hline Arsenic & 0.0144 & 0.0559 & 0.0551 & 0.0504 & 0.0498 & $<$ & $<$ \\
\hline Barium & 0.0741 & 0.104 & 0.103 & 0.103 & 0.102 & 0.0451 & 0.0729 \\
\hline Boron & 0.215 & 0.251 & 0.246 & 0.224 & 0.224 & $<$ & $<$ \\
\hline Cadmium (PMS) & & & & & & & \\
\hline Chromium & & $<$ & $<$ & $<$ & $<$ & $<$ & $<$ \\
\hline Iron & 0.229 & 1.46 & 1.48 & 1.24 & 1.16 & 0.108 & 0.464 \\
\hline Lead (PMS) & & & & & & & \\
\hline Lithium & 0.0798 & 0.0893 & 0.0886 & 0.0801 & 0.08 & $<$ & $<$ \\
\hline Manganese & 0.123 & 1.49 & 1.49 & 1.5 & 1.47 & 0.0129 & 0.0677 \\
\hline Nickel & & $<$ & & 0.0175 & & $<$ & $<$ \\
\hline Strontium & 0.955 & 1 & 0.995 & 0.983 & 0.967 & 0.036 & 0.0455 \\
\hline Thallium (PMS) & & & & & & & \\
\hline Uranium (PMS) & & & & & & & \\
\hline Uranium (KPA) & $<$ & $<$ & $<$ & $<$ & $<$ & $<$ & $<$ \\
\hline
\end{tabular}


APPENDIX F.1: CY 2002 MONITORING DATA FOR THE CHESTNUT RIDGE HYDROGEOLOGIC REGIME

Field Measurements, Miscellaneous Analytes, Major Ions, and Trace Metals

\begin{tabular}{|c|c|c|c|c|c|c|c|}
\hline Station & \multicolumn{3}{|c|}{ SCR1.5SW } & \multicolumn{2}{|c|}{ SCR2.2SW } & \multicolumn{2}{|c|}{ SCR3.5SP } \\
\hline Site & \multicolumn{3}{|c|}{ EXP-SW } & \multicolumn{2}{|c|}{ EXP-SW } & \multicolumn{2}{|c|}{ EXP-SW } \\
\hline Date & \multicolumn{2}{|c|}{ 02/21/02 } & 08/19/02 & 03/21/02 & 09/23/02 & 02/21/02 & 07/10/02 \\
\hline Program & GWPP & GWPP & GWPP & GWPP & GWPP & BJC & BJC \\
\hline Sample Type & & Dup & & & & & \\
\hline Field Measurements & & & & & & & \\
\hline Time Sampled & $9: 30$ & $9: 30$ & $8: 20$ & $7: 45$ & $9: 15$ & $14: 50$ & $13: 40$ \\
\hline Measuring Point Elev. (ft) & & & & & & & \\
\hline Depth to Water (ft) & & & & & & & \\
\hline Groundwater Elevation (ft) & & & & & & & \\
\hline Conductivity ( $\mu \mathrm{mho} / \mathrm{cm})$ & 309 & 309 & 597 & 243 & 339 & 163 & 196 \\
\hline Dissolved Oxygen (ppm) & 5.23 & 5.23 & 3.1 & 5.54 & 4.21 & 11.73 & 8.54 \\
\hline Oxidation/Reduction (mV) & 232 & 232 & 199 & 221 & 208 & 40 & 190 \\
\hline Temperature (degrees C) & 9.4 & 9.4 & 21.1 & 11.6 & 16.7 & 13.2 & 20.9 \\
\hline Turbidity (NTU) & & & & & & 54 & 51 \\
\hline $\mathrm{pH}$ & 7.07 & 7.07 & 7.49 & 7.24 & 7.06 & 6.86 & 8.41 \\
\hline Miscellaneous Analytes & & & & & & & \\
\hline Conductivity $(\mu \mathrm{mho} / \mathrm{cm})$ & 275 & 276 & 339 & 166.3 & 259 & & \\
\hline Dissolved Solids (mg/L) & 139 & 136 & 183 & 91 & 154 & 185 & 232 \\
\hline Suspended Solids (mg/L) & $<$ & $<$ & $<$ & $<$ & $<$ & $<$ & 8.1 \\
\hline Turbidity (NTU) & 2.65 & 2.72 & 10 & 5.63 & 7.15 & & \\
\hline $\mathrm{pH}$ & 8.16 & 8.17 & 7.95 & 7.8 & 8.1 & & \\
\hline Major lons (mg/L) & & & & & & & \\
\hline Calcium & 34.8 & 34.8 & 39.8 & 19.1 & 34.6 & 46.6 & 50.5 \\
\hline Magnesium & 14 & 14.3 & 18.7 & 6.61 & 10.2 & 14.7 & 16.6 \\
\hline Potassium & $<$ & $<$ & $<$ & $<$ & $<$ & 2.09 & 2.46 \\
\hline Sodium & 1.57 & 1.59 & 1.23 & 2.04 & 2.92 & 1.43 & 1.75 \\
\hline Alkalinity as $\mathrm{CO} 3$ & $<$ & $<$ & $<$ & $<$ & $<$ & $<$ & $<$ \\
\hline Alkalinity as $\mathrm{HCO} 3$ & 133 & 135 & 179 & 71.2 & 110 & 149 & 178 \\
\hline Chloride & 2.85 & 2.87 & 1.99 & 3.5 & 4.59 & 1.6 & 2.1 \\
\hline Fluoride & $<$ & $<$ & $<$ & $<$ & $<$ & $<$ & $<$ \\
\hline Nitrate as $\mathrm{N}$ & 0.0715 & 0.0719 & 0.123 & 0.141 & 0.231 & & \\
\hline Sulfate & 7.37 & 7.17 & 5.65 & 6.65 & 12.1 & 16.9 & 14.6 \\
\hline Charge Balance (RPD) & 1 & 0.8 & -2.5 & -2.6 & 1.8 & 3.9 & 1.3 \\
\hline Trace Metals (mg/L) & & & & & & & \\
\hline Aluminum & $<$ & 0.217 & 0.96 & 0.306 & 0.397 & $<$ & $<$ \\
\hline Arsenic & & & & & & $<$ & $<$ \\
\hline Barium & 0.0423 & 0.0436 & 0.0646 & 0.0207 & 0.0309 & 0.0723 & 0.0922 \\
\hline Boron & $<$ & $<$ & & $<$ & $<$ & $<$ & $<$ \\
\hline Cadmium (PMS) & $<$ & $<$ & 0.00089 & $<$ & $<$ & & \\
\hline Chromium & & & & & & $<$ & $<$ \\
\hline Iron & 0.0794 & 0.119 & 0.59 & 0.134 & 0.568 & 0.196 & 0.337 \\
\hline Lead (PMS) & $<$ & $<$ & 0.000688 & 0.0028 & $<$ & & \\
\hline Lithium & $<$ & $<$ & & $<$ & $<$ & 0.0207 & 0.0232 \\
\hline Manganese & $<$ & 0.00628 & 0.04 & $<$ & 0.00608 & 0.0334 & 0.0523 \\
\hline Nickel & & & & & & $<$ & $<$ \\
\hline Strontium & 0.0446 & 0.0454 & 0.0606 & 0.0298 & 0.0509 & 0.303 & 0.339 \\
\hline Thallium (PMS) & & $<$ & 0.000799 & $<$ & $<$ & & \\
\hline Uranium (PMS) & 0.00157 & 0.00149 & 0.00161 & 0.00585 & 0.00678 & & \\
\hline Uranium (KPA) & & & & & & $<$ & $<$ \\
\hline
\end{tabular}


APPENDIX F.1: CY 2002 MONITORING DATA FOR THE CHESTNUT RIDGE HYDROGEOLOGIC REGIME

Field Measurements, Miscellaneous Analytes, Major Ions, and Trace Metals

\begin{tabular}{|c|c|c|c|c|c|c|c|}
\hline Station & \multicolumn{2}{|c|}{ SCR4.3SP } & \multicolumn{3}{|c|}{ SCR4.4SW } & \multicolumn{2}{|c|}{ SCR5.2SP } \\
\hline Site & \multicolumn{2}{|c|}{ EXP-SW } & \multicolumn{3}{|c|}{ EXP-SW } & \multicolumn{2}{|c|}{ EXP-SW } \\
\hline Date & $01 / 14 / 02$ & 07/15/02 & 02/21/02 & \multicolumn{2}{|c|}{$09 / 23 / 02$} & 02/21/02 & 08/19/02 \\
\hline Program & BJC & BJC & GWPP & GWPP & GWPP & GWPP & GWPP \\
\hline Sample Type & & & & & Dup & & \\
\hline Field Measurements & & & & & & & \\
\hline Time Sampled & $13: 32$ & $9: 24$ & $8: 45$ & $8: 50$ & $8: 50$ & $8: 15$ & 9:00 \\
\hline Measuring Point Elev. (ft) & & & & & & & \\
\hline Depth to Water (ft) & & & & & & & \\
\hline Groundwater Elevation (ft) & & & & & & & \\
\hline Conductivity ( $\mu \mathrm{mho} / \mathrm{cm})$ & 370 & 563 & 312 & 361 & 361 & 521 & 624 \\
\hline Dissolved Oxygen (ppm) & 4.07 & 4.26 & 6.48 & 4.28 & 4.28 & 4.64 & 4.8 \\
\hline Oxidation/Reduction (mV) & 208 & 101 & 220 & 213 & 213 & 238 & 160 \\
\hline Temperature (degrees C) & 11.5 & 17.7 & 6.1 & 18.7 & 18.7 & 12.9 & 16.4 \\
\hline Turbidity (NTU) & & 40 & & & & & \\
\hline $\mathrm{pH}$ & 6.96 & 6.37 & 7.63 & 6.89 & 6.89 & 7.05 & 7.2 \\
\hline Miscellaneous Analytes & & & & & & & \\
\hline Conductivity $(\mu \mathrm{mho} / \mathrm{cm})$ & $293 \mathrm{~J}$ & $263 \mathrm{~J}$ & 280 & 213 & 213 & 434 & 465 \\
\hline Dissolved Solids (mg/L) & 195 & $178 \mathrm{~J}$ & 150 & 234 & 235 & 224 & 257 \\
\hline Suspended Solids (mg/L) & $<$ & 7.2 & $<$ & 12 & 11 & $<$ & $<$ \\
\hline Turbidity (NTU) & 2.8 & 28.1 & 11.9 & 148 & 149 & 0.941 & 1.38 \\
\hline $\mathrm{pH}$ & 7.1 & 7 & 7.93 & 7.78 & 7.72 & 7.48 & 7.41 \\
\hline Major lons (mg/L) & & & & & & & \\
\hline Calcium & 39.7 & 34.5 & 34.9 & 27.9 & 27.5 & 60.4 & 63.8 \\
\hline Magnesium & 16.3 & 10.7 & 13 & 6.53 & 6.32 & 17.3 & 19.6 \\
\hline Potassium & $<$ & $<$ & $<$ & 3.12 & 2.82 & $<$ & $<$ \\
\hline Sodium & $<$ & $<$ & 1.31 & 1 & 0.968 & 1.2 & 1.46 \\
\hline Alkalinity as $\mathrm{CO} 3$ & $<$ & $<$ & $<$ & $<$ & $<$ & $<$ & $<$ \\
\hline Alkalinity as $\mathrm{HCO} 3$ & 158 & 115 & 123 & 89 & 87.4 & 199 & 226 \\
\hline Chloride & $2.9 \mathrm{~J}$ & $3.3 \mathrm{~J}$ & 2.76 & 1.88 & 1.87 & 2.27 & 1.89 \\
\hline Fluoride & $0.15 \mathrm{~J}$ & $0.22 \mathrm{~J}$ & $<$ & 0.122 & 0.12 & 0.131 & 0.147 \\
\hline Nitrate as $\mathrm{N}$ & 1.7 & 0.84 & 1.24 & 0.415 & 0.449 & 5.53 & 3.16 \\
\hline Sulfate & 10.6 & 25.6 & 9.98 & 29.8 & 29.6 & 9.49 & 9.1 \\
\hline Charge Balance (RPD) & -3.2 & -4.4 & 0.6 & -9.6 & -10 & -1.7 & -1.4 \\
\hline Trace Metals (mg/L) & & & & & & & \\
\hline Aluminum & $<$ & 0.62 & 0.888 & 7.78 & 5.92 & $<$ & $<$ \\
\hline Arsenic & $<$ & $<$ & & & & & \\
\hline Barium & 0.15 & 0.12 & 0.0801 & 0.0501 & 0.0484 & 0.046 & 0.0623 \\
\hline Boron & $<$ & $<$ & $<$ & $<$ & $<$ & $<$ & $<$ \\
\hline Cadmium (PMS) & & & $<$ & $<$ & $<$ & $<$ & $<$ \\
\hline Chromium & $<$ & $<$ & & & & & \\
\hline Iron & $<$ & 0.67 & 0.374 & 4.22 & 4.09 & $<$ & $<$ \\
\hline Lead (PMS) & & & $<$ & 0.0019 & 0.00794 & $<$ & 0.00186 \\
\hline Lithium & & & $<$ & & $<$ & $<$ & \\
\hline Manganese & $<$ & $<$ & $<$ & 0.0549 & 0.0544 & $<$ & 0.00568 \\
\hline Nickel & $<$ & $<$ & & & & & \\
\hline Strontium & 0.11 & 0.13 & 0.0944 & 0.0984 & 0.0962 & 0.0673 & 0.0902 \\
\hline Thallium (PMS) & & & & $<$ & $<$ & $<$ & \\
\hline Uranium (PMS) & & & $<$ & $<$ & $<$ & 0.00055 & 0.000738 \\
\hline Uranium (KPA) & $<$ & $<$ & & & & & \\
\hline
\end{tabular}


APPENDIX F.2

\section{VOLATILE ORGANIC COMPOUNDS}


APPENDIX F.2: CY 2002 MONITORING DATA FOR THE CHESTNUT RIDGE HYDROGEOLOGIC REGIME

Volatile Organic Compounds

\begin{tabular}{|c|c|c|c|c|c|c|c|c|c|}
\hline Station & \multicolumn{2}{|c|}{ GW-141 } & \multicolumn{3}{|c|}{ GW-142 } & \multicolumn{3}{|c|}{ GW-143 } & GW-144 \\
\hline Site & \multicolumn{2}{|c|}{ LIV } & \multicolumn{3}{|c|}{ KHQ } & \multicolumn{3}{|c|}{ KHQ } & KHQ \\
\hline Date & $01 / 23 / 02$ & $07 / 25 / 02$ & $04 / 10 / 02$ & $10 / 16 / 02$ & $12 / 02 / 02$ & $04 / 09 / 02$ & $10 / 16 / 02$ & $12 / 02 / 02$ & 04/09/02 \\
\hline Program & BJC & BJC & BJC & BJC & BJC & BJC & BJC & BJC & BJC \\
\hline \multicolumn{10}{|l|}{ Sample Type } \\
\hline \multicolumn{10}{|l|}{\begin{tabular}{|l|} 
Chloroethanes $(\mu \mathrm{g} / \mathrm{L})$ \\
\end{tabular}} \\
\hline 1,1,1-Trichloroethane & $<$ & $<$ & $<$ & $<$ & $<$ & $<$ & $<$ & $<$ & $<$ \\
\hline 1,1-Dichloroethane & $<$ & $<$ & $<$ & $<$ & $<$ & $<$ & $<$ & $<$ & $<$ \\
\hline \multicolumn{10}{|l|}{ Chloroethenes ( $\mu \mathrm{g} / \mathrm{L})$} \\
\hline Tetrachloroethene & $<$ & $<$ & $<$ & $<$ & $<$ & $<$ & $<$ & $<$ & $<$ \\
\hline Trichloroethene & $<$ & $<$ & $<$ & $<$ & $<$ & $<$ & $<$ & $<$ & $<$ \\
\hline cis-1,2-Dichloroethene & $<$ & $<$ & $<$ & $<$ & $<$ & $<$ & $<$ & $<$ & $<$ \\
\hline 1,1-Dichloroethene & $<$ & $<$ & $<$ & $<$ & $<$ & $<$ & $<$ & $<$ & $<$ \\
\hline Miscellaneous ( $\mu \mathrm{g} / \mathrm{L})$ & & & & & & & & & \\
\hline Chloroform & $<$ & $<$ & $<$ & $<$ & $<$ & $<$ & $<$ & $<$ & $<$ \\
\hline Trichlorofluoromethane & $<$ & $<$ & & & & & & & \\
\hline
\end{tabular}

\begin{tabular}{|c|c|c|c|c|c|c|c|c|c|}
\hline Station & \multicolumn{2}{|c|}{ GW-144 } & \multicolumn{3}{|c|}{ GW-145 } & \multicolumn{2}{|c|}{ GW-177 } & \multicolumn{2}{|c|}{ GW-217 } \\
\hline Site & \multicolumn{2}{|c|}{ KHQ } & \multicolumn{3}{|c|}{ KHQ } & \multicolumn{2}{|c|}{ CRSP } & \multicolumn{2}{|c|}{ LIV } \\
\hline Date & $10 / 16 / 02$ & $12 / 02 / 02$ & $04 / 08 / 02$ & $10 / 21 / 02$ & $12 / 02 / 02$ & $01 / 09 / 02$ & $07 / 10 / 02$ & $01 / 28 / 02$ & $07 / 22 / 02$ \\
\hline Program & BJC & BJC & BJC & BJC & BJC & BJC & BJC & BJC & BJC \\
\hline Sample Type & & & & & & & & & \\
\hline Chloroethanes $(\mu \mathrm{g} / \mathrm{L})$ & & & & & & & & & \\
\hline 1,1,1-Trichloroethane & $<$ & $<$ & $<$ & $<$ & $<$ & 16 & 12 & $<$ & $<$ \\
\hline 1,1-Dichloroethane & $<$ & $<$ & $<$ & $<$ & $<$ & 25 & 19 & $<$ & $<$ \\
\hline Chloroethenes ( $\mu \mathrm{g} / \mathrm{L})$ & & & & & & & & & \\
\hline Tetrachloroethene & $<$ & $0.4 \mathrm{~J}$ & $<$ & $<$ & $<$ & $<$ & $<$ & $<$ & $<$ \\
\hline Trichloroethene & $<$ & $<$ & $<$ & $<$ & $<$ & $<$ & $<$ & $<$ & $<$ \\
\hline cis-1,2-Dichloroethene & $<$ & $<$ & $<$ & $<$ & $<$ & $<$ & $<$ & $<$ & $<$ \\
\hline 1,1-Dichloroethene & $<$ & $<$ & $<$ & $<$ & $<$ & 8 & 5 & $<$ & $<$ \\
\hline $\begin{array}{r}\text { Miscellaneous }(\mu \mathrm{g} / \mathrm{L}) \\
\text { Chloroform } \\
\text { Trichlorofluoromethane }\end{array}$ & $<$ & $<$ & $<$ & $<$ & $<$ & $<$ & $<$ & $<<$ & $\begin{array}{l}< \\
<\end{array}$ \\
\hline
\end{tabular}

\begin{tabular}{|c|c|c|c|c|c|c|c|c|c|}
\hline Station & \multicolumn{6}{|c|}{ GW-231 } & \multicolumn{3}{|c|}{ GW-301 } \\
\hline Site & \multicolumn{6}{|c|}{ KHQ } & \multicolumn{3}{|c|}{ CRBAWP } \\
\hline Date & \multicolumn{2}{|c|}{ 04/08/02 } & \multicolumn{2}{|c|}{$10 / 17 / 02$} & \multicolumn{2}{|c|}{$12 / 02 / 02$} & \multicolumn{2}{|c|}{ 01/09/02 } & 07/10/02 \\
\hline Program & BJC & BJC & BJC & BJC & BJC & BJC & BJC & BJC & BJC \\
\hline Sample Type & & Dup & & Dup & & Dup & & Dup & \\
\hline Chloroethanes $(\mu \mathrm{g} / \mathrm{L})$ & & & & & & & & & \\
\hline 1,1,1-Trichloroethane & $<$ & $<$ & $<$ & $<$ & $<$ & $<$ & $<$ & $<$ & $<$ \\
\hline 1,1-Dichloroethane & $<$ & $<$ & $<$ & $<$ & $<$ & $<$ & $<$ & $<$ & $<$ \\
\hline Chloroethenes ( $\mu \mathrm{g} / \mathrm{L})$ & & & & & & & & & \\
\hline Tetrachloroethene & $<$ & $<$ & $<$ & $<$ & $<$ & $<$ & $<$ & $<$ & $<$ \\
\hline Trichloroethene & $<$ & $<$ & $<$ & $<$ & $<$ & $<$ & $<$ & $<$ & $<$ \\
\hline cis-1,2-Dichloroethene & $<$ & $<$ & $<$ & $<$ & $<$ & $<$ & $<$ & $<$ & $<$ \\
\hline 1,1-Dichloroethene & $<$ & $<$ & $<$ & $<$ & $<$ & $<$ & $<$ & $<$ & $<$ \\
\hline Miscellaneous $(\mu \mathrm{g} / \mathrm{L})$ & & & & & & & & & \\
\hline $\begin{array}{r}\text { Chloroform } \\
\text { Trichlorofluoromethane }\end{array}$ & $<$ & $<$ & $<$ & $<$ & $<$ & $<$ & $<$ & $<$ & $<$ \\
\hline
\end{tabular}


APPENDIX F.2: CY 2002 MONITORING DATA FOR THE CHESTNUT RIDGE HYDROGEOLOGIC REGIME

Volatile Organic Compounds

\begin{tabular}{|c|c|c|c|c|c|c|c|c|c|}
\hline Station & GW-301 & \multicolumn{4}{|c|}{ GW-305 } & \multicolumn{2}{|c|}{ GW-521 } & \multicolumn{2}{|c|}{ GW-522 } \\
\hline Site & CRBAWP & \multicolumn{4}{|c|}{ LIV } & \multicolumn{2}{|c|}{ LIV } & \multicolumn{2}{|c|}{ LIV } \\
\hline Date & 07/10/02 & $01 / 28 / 02$ & $05 / 02 / 02$ & $07 / 15 / 02$ & $11 / 21 / 02$ & $01 / 10 / 02$ & $07 / 15 / 02$ & $01 / 28 / 02$ & $07 / 15 / 02$ \\
\hline Program & BJC & BJC & BJC & BJC & BJC & BJC & BJC & BJC & BJC \\
\hline Sample Type & Dup & & & & & & & & \\
\hline \multicolumn{10}{|l|}{ Chloroethanes ( $\mu \mathrm{g} / \mathrm{L}$ ) } \\
\hline 1,1,1-Trichloroethane & $<$ & 22 & 17 & 18 & 18 & $<$ & $<$ & $<$ & $<$ \\
\hline 1,1-Dichloroethane & $<$ & 15 & 12 & 13 & 13 & $<$ & $<$ & $<$ & $<$ \\
\hline \multicolumn{10}{|l|}{ Chloroethenes $(\mu \mathrm{g} / \mathrm{L})$} \\
\hline Tetrachloroethene & $<$ & $<$ & $<$ & $<$ & $<$ & $<$ & $<$ & $<$ & $0.43 \mathrm{~J}$ \\
\hline Trichloroethene & $<$ & $<$ & $<$ & $<$ & $<$ & $<$ & $<$ & $<$ & $<$ \\
\hline cis-1,2-Dichloroethene & $<$ & $<$ & $<$ & $<$ & $<$ & $<$ & $<$ & $<$ & $<$ \\
\hline 1,1-Dichloroethene & $<$ & $<$ & 4.1 & 3.7 & 4.3 & $<$ & $<$ & $<$ & $<$ \\
\hline \multicolumn{10}{|l|}{ Miscellaneous ( $\mu \mathrm{g} / \mathrm{L})$} \\
\hline Chloroform & $<$ & $<$ & $<$ & $<$ & $<$ & $<$ & $<$ & $<$ & $<$ \\
\hline Trichlorofluoromethane & & $<$ & $<$ & $<$ & $<$ & $<$ & $<$ & $<$ & $<$ \\
\hline
\end{tabular}

\begin{tabular}{|c|c|c|c|c|c|c|c|c|c|}
\hline Station & \multicolumn{2}{|c|}{ GW-539 } & \multicolumn{2}{|c|}{ GW-540 } & \multicolumn{2}{|c|}{ GW-542 } & \multicolumn{2}{|c|}{ GW-543 } & GW-544 \\
\hline Site & \multicolumn{2}{|c|}{ LII } & \multicolumn{2}{|c|}{ LII } & \multicolumn{2}{|c|}{ CDLVI } & \multicolumn{2}{|c|}{ CDLVI } & CDLVI \\
\hline Date & 01/14/02 & 07/17/02 & $01 / 16 / 02$ & $07 / 18 / 02$ & $01 / 16 / 02$ & $07 / 18 / 02$ & 01/15/02 & $07 / 18 / 02$ & $01 / 15 / 02$ \\
\hline Program & BJC & BJC & BJC & BJC & BJC & BJC & BJC & BJC & BJC \\
\hline \multicolumn{10}{|l|}{ Sample Type } \\
\hline \multicolumn{10}{|l|}{ Chloroethanes $(\mu \mathrm{g} / \mathrm{L})$} \\
\hline 1,1,1-Trichloroethane & $<$ & $<$ & $<$ & $<$ & $<$ & $<$ & $<$ & $<$ & $<$ \\
\hline 1,1-Dichloroethane & $<$ & $<$ & $<$ & $<$ & $<$ & $<$ & $<$ & $<$ & $<$ \\
\hline \multicolumn{10}{|l|}{ Chloroethenes ( $\mu \mathrm{g} / \mathrm{L})$} \\
\hline Tetrachloroethene & $<$ & $<$ & $<$ & $<$ & $<$ & $<$ & $<$ & $<$ & $<$ \\
\hline Trichloroethene & $<$ & $<$ & $<$ & $<$ & $<$ & $<$ & $<$ & $<$ & $<$ \\
\hline cis-1,2-Dichloroethene & $<$ & $<$ & $<$ & $<$ & $<$ & $<$ & $<$ & $<$ & $<$ \\
\hline 1,1-Dichloroethene & $<$ & $0.37 \mathrm{~J}$ & $<$ & $<$ & $<$ & $<$ & $<$ & $<$ & $<$ \\
\hline \multicolumn{10}{|l|}{ Miscellaneous ( $\mu \mathrm{g} / \mathrm{L}$ ) } \\
\hline Chloroform & $<$ & $<$ & $<$ & $<$ & $<$ & $<$ & $<$ & $<$ & 1.4 \\
\hline Trichlorofluoromethane & $<$ & $<$ & $<$ & $<$ & $<$ & $<$ & $<$ & $<$ & $<$ \\
\hline
\end{tabular}

\begin{tabular}{|c|c|c|c|c|c|c|c|c|c|}
\hline Station & GW-544 & \multicolumn{4}{|c|}{ GW-557 } & \multicolumn{2}{|c|}{ GW-560 } & \multicolumn{2}{|c|}{ GW-562 } \\
\hline Site & CDLVI & \multicolumn{4}{|c|}{ LV } & \multicolumn{2}{|c|}{ CDLVII } & \multicolumn{2}{|c|}{ CDLVII } \\
\hline Date & $07 / 22 / 02$ & \multicolumn{2}{|c|}{$01 / 10 / 02$} & \multicolumn{2}{|c|}{$07 / 11 / 02$} & $01 / 17 / 02$ & 07/16/02 & $01 / 09 / 02$ & $01 / 28 / 02$ \\
\hline Program & BJC & BJC & BJC & BJC & BJC & BJC & BJC & BJC & BJC \\
\hline Sample Type & & & Dup & & Dup & & & & \\
\hline \multicolumn{10}{|l|}{\begin{tabular}{|l|} 
Chloroethanes $(\mu \mathrm{g} / \mathrm{L})$ \\
\end{tabular}} \\
\hline 1,1,1-Trichloroethane & $<$ & $<$ & $<$ & $<$ & $<$ & $<$ & $<$ & $<$ & $<$ \\
\hline 1,1-Dichloroethane & $<$ & $<$ & $<$ & $<$ & $<$ & $<$ & $<$ & $<$ & $<$ \\
\hline \multicolumn{10}{|l|}{ Chloroethenes $(\mu \mathrm{g} / \mathrm{L})$} \\
\hline Tetrachloroethene & $<$ & $<$ & $<$ & $<$ & $<$ & $<$ & $<$ & $<$ & $<$ \\
\hline Trichloroethene & $<$ & $<$ & $<$ & $<$ & $<$ & $<$ & $<$ & $<$ & $<$ \\
\hline cis-1,2-Dichloroethene & $<$ & $<$ & $<$ & $<$ & $<$ & $<$ & $<$ & $<$ & $<$ \\
\hline 1,1-Dichloroethene & $<$ & $<$ & $<$ & $<$ & $<$ & $<$ & $<$ & $<$ & $<$ \\
\hline \multicolumn{10}{|l|}{ Miscellaneous ( $\mu \mathrm{g} / \mathrm{L})$} \\
\hline Chloroform & 2.4 & $<$ & $<$ & $<$ & $<$ & $<$ & $<$ & $<$ & $<$ \\
\hline Trichlorofluoromethane & $<$ & $<$ & $<$ & $<$ & $<$ & $<$ & $<$ & & $<$ \\
\hline
\end{tabular}


APPENDIX F.2: CY 2002 MONITORING DATA FOR THE CHESTNUT RIDGE HYDROGEOLOGIC REGIME

Volatile Organic Compounds

\begin{tabular}{|c|c|c|c|c|c|c|c|c|c|}
\hline Station & \multicolumn{2}{|c|}{ GW-562 } & \multicolumn{4}{|c|}{ GW-564 } & \multicolumn{2}{|c|}{ GW-709 } & GW-757 \\
\hline Site & \multicolumn{2}{|c|}{ CDLVII } & \multicolumn{4}{|c|}{ CDLVII } & \multicolumn{2}{|c|}{ LII } & LII \\
\hline Date & $07 / 10 / 02$ & $07 / 16 / 02$ & \multicolumn{2}{|c|}{$01 / 16 / 02$} & \multicolumn{2}{|c|}{$07 / 16 / 02$} & $01 / 15 / 02$ & 07/17/02 & $01 / 15 / 02$ \\
\hline Program & BJC & BJC & BJC & BJC & BJC & BJC & BJC & BJC & BJC \\
\hline Sample Type & & & & Dup & & Dup & & & \\
\hline \multicolumn{10}{|l|}{ Chloroethanes ( $\mu \mathrm{g} / \mathrm{L})$} \\
\hline 1,1,1-Trichloroethane & $<$ & $<$ & $<$ & $<$ & $<$ & $<$ & $<$ & $<$ & $<$ \\
\hline 1,1-Dichloroethane & $<$ & $<$ & $<$ & $<$ & $<$ & $<$ & $<$ & $<$ & $<$ \\
\hline \multicolumn{10}{|l|}{ Chloroethenes $(\mu \mathrm{g} / \mathrm{L})$} \\
\hline Tetrachloroethene & $<$ & $<$ & $<$ & $<$ & $<$ & $<$ & $<$ & $<$ & $<$ \\
\hline Trichloroethene & $<$ & $<$ & $<$ & $<$ & $<$ & $<$ & $<$ & $<$ & $<$ \\
\hline cis-1,2-Dichloroethene & $<$ & $<$ & $<$ & $<$ & $<$ & $<$ & $<$ & $<$ & $<$ \\
\hline 1,1-Dichloroethene & $<$ & $<$ & $<$ & $<$ & $<$ & $<$ & $<$ & $<$ & $<$ \\
\hline \multicolumn{10}{|l|}{ Miscellaneous ( $\mu \mathrm{g} / \mathrm{L})$} \\
\hline Chloroform & $<$ & $<$ & $0.3 \mathrm{~J}$ & $0.3 \mathrm{~J}$ & $<$ & $<$ & $<$ & $<$ & $<$ \\
\hline Trichlorofluoromethane & & $<$ & $<$ & $<$ & $<$ & $<$ & $<$ & $<$ & $<$ \\
\hline
\end{tabular}

\begin{tabular}{|c|c|c|c|c|c|c|c|c|c|}
\hline Station & GW-757 & \multicolumn{2}{|c|}{ GW-796 } & \multicolumn{2}{|c|}{ GW-797 } & \multicolumn{4}{|c|}{ GW-798 } \\
\hline Site & LII & \multicolumn{2}{|c|}{ LV } & \multicolumn{2}{|c|}{ LV } & \multicolumn{4}{|c|}{ CDLVII } \\
\hline Date & $07 / 17 / 02$ & $01 / 14 / 02$ & 07/11/02 & $01 / 14 / 02$ & 07/11/02 & 01/09/02 & 01/16/02 & $07 / 10 / 02$ & $07 / 16 / 02$ \\
\hline Program & BJC & BJC & BJC & BJC & BJC & BJC & BJC & BJC & BJC \\
\hline \multicolumn{10}{|l|}{ Sample Type } \\
\hline Chloroethanes ( $\mu \mathrm{g} / \mathrm{L})$ & & & & & & & & & \\
\hline 1,1,1-Trichloroethane & $<$ & $0.8 \mathrm{~J}$ & $0.64 \mathrm{~J}$ & $<$ & $<$ & $3 \mathrm{~J}$ & 2.6 & $4 \mathrm{~J}$ & 3.2 \\
\hline 1,1-Dichloroethane & $<$ & $<$ & $<$ & $<$ & $<$ & $2 \mathrm{~J}$ & 2.3 & $3 \mathrm{~J}$ & 2.6 \\
\hline \multicolumn{10}{|l|}{ Chloroethenes ( $\mu \mathrm{g} / \mathrm{L})$} \\
\hline Tetrachloroethene & $<$ & $<$ & $<$ & $<$ & $<$ & $5 \mathrm{~J}$ & 5.1 & 7 & 7.1 \\
\hline Trichloroethene & $<$ & $<$ & $<$ & $<$ & $<$ & $<$ & $0.55 \mathrm{~J}$ & $<$ & $0.78 \mathrm{~J}$ \\
\hline cis-1,2-Dichloroethene & $<$ & $<$ & $<$ & $<$ & $<$ & $3 \mathrm{~J}$ & 5.1 & 7 & 6 \\
\hline 1,1-Dichloroethene & $<$ & $<$ & $<$ & $<$ & $<$ & $<$ & 2.5 & 5 & 2.3 \\
\hline \multicolumn{10}{|l|}{ Miscellaneous ( $\mu \mathrm{g} / \mathrm{L}$ ) } \\
\hline Chloroform & $<$ & $<$ & $<$ & $<$ & $<$ & $<$ & $<$ & $<$ & $<$ \\
\hline Trichlorofluoromethane & $<$ & $<$ & $<$ & $<$ & $<$ & & 11 & & 10 \\
\hline
\end{tabular}

\begin{tabular}{|c|c|c|c|c|c|c|c|c|}
\hline Station & \multicolumn{2}{|c|}{ GW-799 } & \multicolumn{2}{|c|}{ GW-801 } & \multicolumn{2}{|c|}{ GW-827 } & \multicolumn{2}{|c|}{ GW-831 } \\
\hline Site & \multicolumn{2}{|c|}{ LV } & \multicolumn{2}{|c|}{ LV } & \multicolumn{2}{|c|}{ CDLVI } & \multicolumn{2}{|c|}{ FCAP } \\
\hline Date & $01 / 10 / 02$ & $07 / 11 / 02$ & $01 / 14 / 02$ & $07 / 15 / 02$ & $01 / 17 / 02$ & $07 / 18 / 02$ & $01 / 09 / 02$ & 07/09/02 \\
\hline Program & BJC & BJC & BJC & BJC & BJC & BJC & BJC & BJC \\
\hline \multicolumn{9}{|l|}{ Sample Type } \\
\hline \multicolumn{9}{|l|}{ Chloroethanes $(\mu \mathrm{g} / \mathrm{L})$} \\
\hline 1,1,1-Trichloroethane & $<$ & $<$ & $<$ & $<$ & $<$ & $<$ & $<$ & $<$ \\
\hline 1,1-Dichloroethane & $<$ & $<$ & $<$ & $<$ & $<$ & $<$ & $<$ & $<$ \\
\hline \multicolumn{9}{|l|}{ Chloroethenes ( $\mu \mathrm{g} / \mathrm{L})$} \\
\hline Tetrachloroethene & $<$ & $<$ & $<$ & $<$ & $<$ & $<$ & $<$ & $<$ \\
\hline Trichloroethene & $<$ & $<$ & $<$ & $<$ & $<$ & $<$ & $<$ & $<$ \\
\hline cis-1,2-Dichloroethene & $<$ & $<$ & $<$ & $<$ & $<$ & $<$ & $<$ & $<$ \\
\hline 1,1-Dichloroethene & $<$ & $<$ & $<$ & $<$ & $<$ & $<$ & $<$ & $<$ \\
\hline \multicolumn{9}{|l|}{ Miscellaneous ( $\mu \mathrm{g} / \mathrm{L}$ ) } \\
\hline Chloroform & $<$ & $<$ & $<$ & $<$ & $<$ & $<$ & $<$ & $<$ \\
\hline Trichlorofluoromethane & $<$ & $<$ & $<$ & $<$ & $<$ & $<$ & & \\
\hline
\end{tabular}


APPENDIX F.2: CY 2002 MONITORING DATA FOR THE CHESTNUT RIDGE HYDROGEOLOGIC REGIME

Volatile Organic Compounds

\begin{tabular}{|c|c|c|c|c|c|c|c|c|c|}
\hline Station & \multicolumn{2}{|c|}{ SCR1.25SP } & \multicolumn{3}{|c|}{ SCR1.5SW } & \multicolumn{2}{|c|}{ SCR2.2SW } & \multicolumn{2}{|c|}{ SCR3.5SP } \\
\hline Site & \multicolumn{2}{|c|}{ EXP-SW } & \multicolumn{3}{|c|}{ EXP-SW } & \multicolumn{2}{|c|}{ EXP-SW } & \multicolumn{2}{|c|}{ EXP-SW } \\
\hline Date & $02 / 21 / 02$ & 07/10/02 & \multicolumn{2}{|c|}{$02 / 21 / 02$} & 08/19/02 & $03 / 21 / 02$ & $09 / 23 / 02$ & $02 / 21 / 02$ & 07/10/02 \\
\hline Program & BJC & BJC & GWPP & GWPP & GWPP & GWPP & GWPP & BJC & BJC \\
\hline Sample Type & & & & Dup & & & & & \\
\hline \multicolumn{10}{|l|}{ Chloroethanes $(\mu \mathrm{g} / \mathrm{L})$} \\
\hline 1,1,1-Trichloroethane & $<$ & $<$ & $<$ & $<$ & $<$ & $<$ & $<$ & $<$ & $<$ \\
\hline 1,1-Dichloroethane & $<$ & $<$ & $<$ & $<$ & $<$ & $<$ & $<$ & $<$ & $<$ \\
\hline \multicolumn{10}{|l|}{ Chloroethenes $(\mu \mathrm{g} / \mathrm{L})$} \\
\hline Tetrachloroethene & $<$ & $<$ & $<$ & $<$ & $<$ & $<$ & $<$ & $<$ & $<$ \\
\hline Trichloroethene & $<$ & $<$ & $<$ & $<$ & $<$ & $<$ & $<$ & $<$ & $<$ \\
\hline cis-1,2-Dichloroethene & $<$ & $<$ & $<$ & $<$ & $<$ & $<$ & $<$ & $<$ & $<$ \\
\hline 1,1-Dichloroethene & $<$ & $<$ & $<$ & $<$ & $<$ & $<$ & $<$ & $<$ & $<$ \\
\hline Miscellaneous $(\mu \mathrm{g} / \mathrm{L})$ & & & & & & & & & \\
\hline Chloroform & $<$ & $<$ & $<$ & $<$ & $<$ & $<$ & $<$ & $<$ & $<$ \\
\hline Trichlorofluoromethane & & & $<$ & $<$ & $<$ & $<$ & $<$ & & \\
\hline
\end{tabular}

\begin{tabular}{|c|c|c|c|c|c|c|c|}
\hline \multirow{3}{*}{\begin{tabular}{|r|} 
Station \\
Site \\
Date
\end{tabular}} & \multicolumn{2}{|c|}{ SCR4.3SP } & \multirow{2}{*}{\multicolumn{3}{|c|}{$\begin{array}{c}\text { SCR4.4SW } \\
\text { EXP-SW }\end{array}$}} & \multirow{2}{*}{\multicolumn{2}{|c|}{$\begin{array}{c}\text { SCR5.2SP } \\
\text { EXP-SW }\end{array}$}} \\
\hline & \multirow{2}{*}{\begin{tabular}{|l|} 
EXP-SW \\
$01 / 14 / 02$
\end{tabular}} & \multirow{2}{*}{$\begin{array}{c}\text { EXP-SW } \\
07 / 15 / 02\end{array}$} & & & & & \\
\hline & & & $02 / 21 / 02$ & \multicolumn{2}{|c|}{$09 / 23 / 02$} & $02 / 21 / 02$ & 08/19/02 \\
\hline Program & BJC & BJC & GWPP & GWPP & GWPP & GWPP & GWPP \\
\hline Sample Type & & & & & Dup & & \\
\hline \multicolumn{8}{|l|}{ Chloroethanes $(\mu \mathrm{g} / \mathrm{L})$} \\
\hline 1,1,1-Trichloroethane & $<$ & $<$ & $<$ & $<$ & $<$ & $<$ & $<$ \\
\hline 1,1-Dichloroethane & $<$ & $<$ & $<$ & $<$ & $<$ & $<$ & $<$ \\
\hline \multicolumn{8}{|l|}{ Chloroethenes ( $\mu \mathrm{g} / \mathrm{L})$} \\
\hline Tetrachloroethene & $<$ & $<$ & $<$ & $<$ & $<$ & $<$ & $<$ \\
\hline Trichloroethene & $<$ & $<$ & $<$ & $<$ & $<$ & $<$ & $<$ \\
\hline cis-1,2-Dichloroethene & $<$ & $<$ & $<$ & $<$ & $<$ & $<$ & $<$ \\
\hline 1,1-Dichloroethene & $<$ & $<$ & $<$ & $<$ & $<$ & $<$ & $<$ \\
\hline \multicolumn{8}{|l|}{ Miscellaneous $(\mu \mathrm{g} / \mathrm{L})$} \\
\hline Chloroform & $<$ & $<$ & $<$ & $<$ & $<$ & $<$ & $<$ \\
\hline Trichlorofluoromethane & $<$ & $<$ & $<$ & $<$ & $<$ & $<$ & $<$ \\
\hline
\end{tabular}


APPENDIX F.3

RADIOLOGICAL ANALYTES 
APPENDIX F.3: CY 2002 MONITORING DATA FOR THE CHESTNUT RIDGE HYDROGEOLOGIC REGIME

Radiological Analytes: Gross Alpha and Gross Beta Activity

\begin{tabular}{|c|c|c|c|c|c|c|c|c|c|}
\hline \multirow{2}{*}{$\begin{array}{c}\text { Sampling } \\
\text { Point }\end{array}$} & \multirow{2}{*}{ Location } & \multirow{2}{*}{$\begin{array}{c}\text { Date } \\
\text { Sampled }\end{array}$} & \multirow{2}{*}{ Program } & \multicolumn{3}{|c|}{ Gross Alpha (pCi/L) } & \multicolumn{3}{|c|}{ Gross Beta (pCi/L) } \\
\hline & & & & Result & Error & MDA & Result & Error & MDA \\
\hline 1090 & UNCS & 01/30/02 & BJC & $<\mathrm{MDA}$ & . & 1.86 & 4.21 & 1.29 & 1.64 \\
\hline 1090 & UNCS & 07/30/02 & BJC & $<\mathrm{MDA}$ & . & 1.57 & 5.35 & 1.1 & 1.15 \\
\hline GW-141 & LIV & $01 / 23 / 02$ & BJC & $<M D A$ & . & 2.7 & $<\mathrm{MDA}$ & . & 2.2 \\
\hline GW-141 & LIV & 07/25/02 & BJC & $<M D A$ & . & 4 & $<M D A$ & . & 2.5 \\
\hline GW-142 & $\mathrm{KHQ}$ & 04/10/02 & BJC & $<M D A$ & & 1.22 & 5.8 & 1.22 & 1.41 \\
\hline GW-142 & $\mathrm{KHQ}$ & 10/16/02 & BJC & $<M D A$ & & 3.2 & $<\mathrm{MDA}$ & & 4.19 \\
\hline GW-143 & $\mathrm{KHQ}$ & 04/09/02 & BJC & 3.65 & 1.37 & 1.01 & 17.37 & 1.83 & 1.72 \\
\hline GW-143 & $\mathrm{KHQ}$ & 10/16/02 & BJC & $<\mathrm{MDA}$ & & 2.19 & 15.73 & 2.62 & 2.27 \\
\hline GW-144 & $\mathrm{KHQ}$ & 04/09/02 & BJC & 2.26 & 0.96 & 0.9 & 1.7 & 1.04 & 1.5 \\
\hline GW-144 & $\mathrm{KHQ}$ & 10/16/02 & BJC & 2.64 & 1.9 & 1.84 & 3.28 & 2.14 & 2.07 \\
\hline GW-145 & $\mathrm{KHQ}$ & $04 / 08 / 02$ & BJC & 8.82 & 2.36 & 2.31 & 11.31 & 1.76 & 1.95 \\
\hline GW-145 & $\mathrm{KHQ}$ & 10/21/02 & BJC & 9.69 & 1.93 & 1.4 & 13.54 & 2.33 & 2.38 \\
\hline GW-177 & CRSP & 01/09/02 & BJC & 4.56 & 1.57 & 1.55 & 6.66 & 1.43 & 1.72 \\
\hline GW-177 & CRSP & 07/10/02 & BJC & $<M D A$ & & 1.82 & 4.52 & 1.41 & 1.86 \\
\hline GW-203 & UNCS & 01/29/02 & BJC & 2.92 & 1.22 & 1.41 & 4.95 & 1.2 & 1.47 \\
\hline GW-203 & UNCS & 07/30/02 & BJC & 3.77 & 0.84 & 0.6 & 7.96 & 1.12 & 1.09 \\
\hline GW-205 & UNCS & 01/30/02 & BJC & 2.47 & 1.64 & 2.17 & 47.23 & 3.06 & 1.88 \\
\hline GW-205 & UNCS & 07/30/02 & BJC & 2.86 & 1.35 & 1.41 & 84.85 & 2.56 & 1.32 \\
\hline GW-217 & LIV & 01/22/02 & BJC & $<M D A$ & & 2.7 & $<\mathrm{MDA}$ & . & 2.2 \\
\hline GW-217 & LIV & 07/22/02 & BJC & $<\mathrm{MDA}$ & & 3.1 & 2.3 & 1.4 & 2.2 \\
\hline GW-221 & UNCS & $01 / 29 / 02$ & BJC & 2.89 & 1.09 & 1.07 & 3.26 & 1.18 & 1.59 \\
\hline GW-221 & UNCS & 07/30/02 & BJC & 0.95 & 0.66 & 0.76 & 3.08 & 1.05 & 1.18 \\
\hline GW-231 & $\mathrm{KHQ}$ & 04/08/02 & BJC & $<M D A$ & & 1.43 & $<\mathrm{MDA}$ & . & 1.79 \\
\hline GW-231 Dup & $\mathrm{KHQ}$ & 04/08/02 & BJC & $<\mathrm{MDA}$ & & 1.48 & 1.82 & 1.24 & 1.8 \\
\hline GW-231 & $\mathrm{KHQ}$ & 10/17/02 & BJC & $<\mathrm{MDA}$ & & 1 & 2.19 & 1.48 & 1.73 \\
\hline GW-231 Dup & $\mathrm{KHQ}$ & 10/17/02 & BJC & $<\mathrm{MDA}$ & & 1.28 & 2.36 & 1.59 & 1.87 \\
\hline GW-301 & CRBAWP & 01/09/02 & BJC & $<M D A$ & & 1.68 & 2.55 & 1.27 & 1.81 \\
\hline GW-301 Dup & CRBAWP & 01/09/02 & BJC & 3.43 & 1.19 & 1.07 & 5.58 & 1.31 & 1.61 \\
\hline GW-301 & CRBAWP & 07/10/02 & BJC & $<\mathrm{MDA}$ & . & 1.61 & $<\mathrm{MDA}$ & . & 1.82 \\
\hline GW-301 Dup & CRBAWP & 07/10/02 & BJC & $<M D A$ & & 1.61 & $<\mathrm{MDA}$ & & 1.82 \\
\hline GW-302 & UNCS & 01/31/02 & BJC & 2.68 & 1.36 & 1.47 & 4.22 & 1.55 & 1.73 \\
\hline GW-302 & UNCS & 07/31/02 & BJC & 10.77 & 1.37 & 0.82 & 6.26 & 1.56 & 1.45 \\
\hline GW-305 & LIV & $01 / 22 / 02$ & BJC & $<\mathrm{MDA}$ & & 2.5 & $<\mathrm{MDA}$ & . & 1.9 \\
\hline GW-305 & LIV & 05/02/02 & BJC & $<M D A$ & & 2.6 & $<\mathrm{MDA}$ & . & 2 \\
\hline GW-305 & LIV & 07/15/02 & BJC & $<M D A$ & & 3 & $<\mathrm{MDA}$ & . & 3 \\
\hline GW-305 & LIV & $11 / 21 / 02$ & BJC & $<\mathrm{MDA}$ & & 2.1 & $<\mathrm{MDA}$ & . & 1.9 \\
\hline GW-339 & UNCS & 01/29/02 & BJC & 2.19 & 1.14 & 1.28 & 2.59 & 1.07 & 1.46 \\
\hline GW-339 Dup & UNCS & 01/29/02 & BJC & $<\mathrm{MDA}$ & & 1.12 & 2.04 & 1.3 & 1.89 \\
\hline GW-339 & UNCS & 07/30/02 & BJC & $<M D A$ & & 1.47 & $<\mathrm{MDA}$ & . & 1.81 \\
\hline GW-339 Dup & UNCS & 07/30/02 & BJC & 2.07 & 1.16 & 1.26 & 5.29 & 1.36 & 1.48 \\
\hline GW-521 & LIV & 01/10/02 & BJC & $<\mathrm{MDA}$ & & 2.4 & $<\mathrm{MDA}$ & . & 2 \\
\hline GW-521 & LIV & 07/15/02 & BJC & $<M D A$ & & 3.1 & $<\mathrm{MDA}$ & . & 3 \\
\hline GW-522 & LIV & 01/22/02 & BJC & $<\mathrm{MDA}$ & & 2.3 & 2.4 & 1.2 & 1.8 \\
\hline GW-522 & LIV & 07/15/02 & BJC & $<M D A$ & & 3 & $<\mathrm{MDA}$ & . & 2.9 \\
\hline GW-539 & LII & $01 / 14 / 02$ & BJC & $<M D A$ & & 2.6 & $<\mathrm{MDA}$ & . & 2 \\
\hline GW-539 & LII & 07/17/02 & BJC & $<M D A$ & & 3.6 & $<\mathrm{MDA}$ & . & 3.3 \\
\hline GW-540 & LII & 01/16/02 & BJC & $<\mathrm{MDA}$ & & 2.9 & 3.2 & 1.3 & 2 \\
\hline GW-540 & LII & 07/18/02 & BJC & $<M D A$ & & 4.5 & $<\mathrm{MDA}$ & . & 3.2 \\
\hline GW-542 & CDLVI & 01/16/02 & BJC & $<\mathrm{MDA}$ & & 1.6 & 2 & 1.2 & 1.9 \\
\hline GW-542 & CDLVI & 07/18/02 & BJC & $<M D A$ & & 1.9 & 2.1 & 1.2 & 1.9 \\
\hline GW-543 & CDLVI & 01/15/02 & BJC & $<M D A$ & & 1.6 & 2.4 & 1.1 & 1.8 \\
\hline GW-543 & CDLVI & 07/18/02 & BJC & $<\mathrm{MDA}$ & & 4.4 & $<\mathrm{MDA}$ & . & 3.6 \\
\hline
\end{tabular}


APPENDIX F.3: CY 2002 MONITORING DATA FOR THE CHESTNUT RIDGE HYDROGEOLOGIC REGIME

Radiological Analytes: Gross Alpha and Gross Beta Activity

\begin{tabular}{|c|c|c|c|c|c|c|c|c|c|}
\hline \multirow{2}{*}{$\begin{array}{l}\text { Sampling } \\
\text { Point }\end{array}$} & \multirow{2}{*}{ Location } & \multirow{2}{*}{$\begin{array}{c}\text { Date } \\
\text { Sampled }\end{array}$} & \multirow{2}{*}{ Program } & \multicolumn{3}{|c|}{ Gross Alpha (pCi/L) } & \multicolumn{3}{|c|}{ Gross Beta (pCi/L) } \\
\hline & & & & Result & Error & MDA & Result & Error & MDA \\
\hline GW-544 & CDLVI & 01/15/02 & BJC & $<\mathrm{MDA}$ & & 2.5 & 3 & 1.3 & 2 \\
\hline GW-544 & CDLVI & 07/22/02 & BJC & $<\mathrm{MDA}$ & & 4.6 & 4.5 & 2.3 & 3.5 \\
\hline GW-557 & LV & 01/10/02 & BJC & $<\mathrm{MDA}$ & & 2.9 & $<\mathrm{MDA}$ & . & 4.2 \\
\hline GW-557 Dup & LV & 01/10/02 & BJC & $<\mathrm{MDA}$ & & 2.5 & $<\mathrm{MDA}$ & . & 1.9 \\
\hline GW-557 & LV & 07/11/02 & BJC & $<\mathrm{MDA}$ & & 30 & $<\mathrm{MDA}$ & . & 24 \\
\hline GW-557 Dup & LV & 07/11/02 & BJC & $<\mathrm{MDA}$ & & 2.4 & 2.1 & 1.3 & 2.1 \\
\hline GW-560 & CDLVII & 01/17/02 & BJC & $<\mathrm{MDA}$ & & 2.1 & 2.1 & 1.2 & 2 \\
\hline GW-560 & CDLVII & 07/16/02 & BJC & $<\mathrm{MDA}$ & & 3.6 & $<\mathrm{MDA}$ & . & 3.2 \\
\hline GW-562 & CDLVII & 01/09/02 & BJC & 1.25 & 0.78 & 0.96 & $<\mathrm{MDA}$ & . & 1.71 \\
\hline GW-562 & CDLVII & $01 / 22 / 02$ & BJC & $<\mathrm{MDA}$ & & 2 & $<\mathrm{MDA}$ & . & 1.9 \\
\hline GW-562 & CDLVII & 07/10/02 & BJC & $<\mathrm{MDA}$ & & 1.28 & 2.37 & 1.91 & 1.86 \\
\hline GW-562 & CDLVII & 07/16/02 & BJC & $<\mathrm{MDA}$ & & 4.2 & $<\mathrm{MDA}$ & . & 3.6 \\
\hline GW-564 & CDLVII & 01/16/02 & BJC & $<M D A$ & & 1.6 & $<\mathrm{MDA}$ & . & 1.9 \\
\hline GW-564 Dup & CDLVII & $01 / 16 / 02$ & BJC & $<M D A$ & & 2 & $<\mathrm{MDA}$ & & 2 \\
\hline GW-564 & CDLVII & 07/16/02 & BJC & $<M D A$ & & 3.4 & $<\mathrm{MDA}$ & . & 2.6 \\
\hline GW-564 Dup & CDLVII & 07/16/02 & BJC & $<\mathrm{MDA}$ & . & 3 & $<\mathrm{MDA}$ & . & 2.1 \\
\hline GW-709 & LII & 01/15/02 & BJC & $<\mathrm{MDA}$ & & 1.7 & 2 & 1.2 & 1.9 \\
\hline GW-709 & LII & 07/17/02 & BJC & $<\mathrm{MDA}$ & & 2.1 & $<\mathrm{MDA}$ & & 2 \\
\hline GW-757 & LII & $01 / 15 / 02$ & BJC & 2.1 & 1.3 & 1.8 & 15.4 & 2.3 & 1.9 \\
\hline GW-757 & LII & 07/17/02 & BJC & 4.1 & 1.8 & 2.2 & 17.8 & 2.6 & 2.1 \\
\hline GW-796 & LV & $01 / 14 / 02$ & BJC & $<M D A$ & & 2 & $<\mathrm{MDA}$ & & 1.9 \\
\hline GW-796 & LV & 07/11/02 & BJC & 3.3 & 1.5 & 1.8 & $<\mathrm{MDA}$ & . & 1.9 \\
\hline GW-797 & LV & $01 / 14 / 02$ & BJC & $<\mathrm{MDA}$ & & 2.2 & $<\mathrm{MDA}$ & . & 2.2 \\
\hline GW-797 & LV & 07/11/02 & BJC & $<\mathrm{MDA}$ & & 31 & $<\mathrm{MDA}$ & . & 27 \\
\hline GW-798 & CDLVII & 01/09/02 & BJC & $<\mathrm{MDA}$ & . & 1.52 & 3.59 & 1.12 & 1.46 \\
\hline GW-798 & CDLVII & 01/16/02 & BJC & $<\mathrm{MDA}$ & & 1.8 & 2.7 & 1.2 & 1.8 \\
\hline GW-798 & CDLVII & 07/10/02 & BJC & $<M D A$ & & 1.34 & 1.96 & 1.22 & 1.76 \\
\hline GW-798 & CDLVII & 07/16/02 & BJC & $<\mathrm{MDA}$ & . & 2.7 & $<\mathrm{MDA}$ & . & 2.5 \\
\hline GW-799 & LV & 01/10/02 & BJC & $<\mathrm{MDA}$ & & 1.6 & $<\mathrm{MDA}$ & & 1.9 \\
\hline GW-799 & LV & 07/11/02 & BJC & $<\mathrm{MDA}$ & . & 26 & $<\mathrm{MDA}$ & . & 21 \\
\hline GW-801 & LV & $01 / 14 / 02$ & BJC & $<\mathrm{MDA}$ & & 2.5 & $<\mathrm{MDA}$ & . & 1.9 \\
\hline GW-801 & LV & 07/15/02 & BJC & $<\mathrm{MDA}$ & & 2.4 & $<\mathrm{MDA}$ & . & 2.7 \\
\hline GW-827 & CDLVI & $01 / 17 / 02$ & BJC & $<\mathrm{MDA}$ & . & 1.8 & 4.4 & 1.6 & 2.5 \\
\hline GW-827 & CDLVI & 07/18/02 & BJC & $<M D A$ & 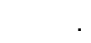 & 3 & $<\mathrm{MDA}$ & . & 2.3 \\
\hline GW-831 & FCAP & 01/09/02 & BJC & $<\mathrm{MDA}$ & & 1.71 & 2.69 & 1.29 & 1.82 \\
\hline GW-831 & FCAP & 07/09/02 & BJC & $<\mathrm{MDA}$ & & 1.37 & 2.15 & 1.25 & 1.79 \\
\hline MCK 2.0 & EXP-SW & $02 / 21 / 02$ & BJC & $<\mathrm{MDA}$ & . & 1.57 & 5.26 & 1.55 & 1.91 \\
\hline MCK 2.0 & EXP-SW & 07/09/02 & BJC & $<\mathrm{MDA}$ & 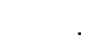 & 2.14 & 4.57 & 1.59 & 2.16 \\
\hline MCK 2.05 & EXP-SW & $02 / 21 / 02$ & BJC & $<\mathrm{MDA}$ & & 1.89 & 4.76 & 1.72 & 2.18 \\
\hline MCK 2.05 Dup & EXP-SW & $02 / 21 / 02$ & BJC & $<\mathrm{MDA}$ & & 1.63 & 6.21 & 1.44 & 1.72 \\
\hline MCK 2.05 & EXP-SW & 07/09/02 & BJC & $<M D A$ & & 2.23 & 5.98 & 1.6 & 2.08 \\
\hline MCK 2.05 Dup & EXP-SW & 07/09/02 & BJC & $<\mathrm{MDA}$ & & 2.31 & 5.03 & 1.81 & 2.46 \\
\hline SCR1.25SP & EXP-SW & 02/21/02 & BJC & 1.5 & 0.9 & 1.11 & 1.97 & 1.2 & 1.61 \\
\hline SCR1.25SP & EXP-SW & 07/10/02 & BJC & 2.91 & 1.12 & 1.06 & 2.72 & 1.19 & 1.63 \\
\hline SCR1.5SW & EXP-SW & $02 / 21 / 02$ & GWPP & $<\mathrm{MDA}$ & . & 3.9 & $<\mathrm{MDA}$ & . & 7.2 \\
\hline SCR1.5SW Dup & EXP-SW & $02 / 21 / 02$ & GWPP & $<\mathrm{MDA}$ & . & 3.6 & $<\mathrm{MDA}$ & . & 8.5 \\
\hline SCR1.5SW & EXP-SW & 08/19/02 & GWPP & 3.7 & 2.1 & 1.7 & $<\mathrm{MDA}$ & . & 9.3 \\
\hline SCR2.2SW & EXP-SW & 03/21/02 & GWPP & 3.7 & 2.2 & 2.1 & $<\mathrm{MDA}$ & . & 7.9 \\
\hline SCR2.2SW & EXP-SW & 09/23/02 & GWPP & 11 & 3.6 & 2.9 & $<\mathrm{MDA}$ & . & 7.7 \\
\hline SCR3.5SP & EXP-SW & $02 / 21 / 02$ & BJC & $<M D A$ & & 1.42 & $<\mathrm{MDA}$ & & 2.07 \\
\hline SCR3.5SP & EXP-SW & 07/10/02 & BJC & $<\mathrm{MDA}$ & & 1.33 & 4.33 & 1.19 & 1.52 \\
\hline
\end{tabular}


APPENDIX F.3: CY 2002 MONITORING DATA FOR THE CHESTNUT RIDGE HYDROGEOLOGIC REGIME Radiological Analytes: Gross Alpha and Gross Beta Activity

\begin{tabular}{|c|c|c|c|c|c|c|c|c|c|}
\hline \multirow{2}{*}{$\begin{array}{c}\text { Sampling } \\
\text { Point }\end{array}$} & \multirow{2}{*}{ Location } & \multirow{2}{*}{$\begin{array}{c}\text { Date } \\
\text { Sampled }\end{array}$} & \multirow{2}{*}{ Program } & \multicolumn{3}{|c|}{ Gross Alpha (pCi/L) } & \multicolumn{3}{|c|}{ Gross Beta (pCi/L) } \\
\hline & & & & Result & Error & MDA & Result & Error & MDA \\
\hline SCR4.3SP & EXP-SW & $01 / 14 / 02$ & BJC & $<\mathrm{MDA}$ &. & 1.8 & 3.2 & 1.3 & 1.9 \\
\hline SCR4.3SP & EXP-SW & 07/15/02 & BJC & $<\mathrm{MDA}$ & . & 2.9 & $<$ MDA & . & 2.6 \\
\hline SCR4.4SW & EXP-SW & $02 / 21 / 02$ & GWPP & $<\mathrm{MDA}$ & . & 4.2 & $<\mathrm{MDA}$ & . & 7.7 \\
\hline SCR4.4SW & EXP-SW & 09/23/02 & GWPP & $<\mathrm{MDA}$ & . & 3 & 7.2 & 4.7 & 7.2 \\
\hline SCR4.4SW Dup & EXP-SW & $09 / 23 / 02$ & GWPP & $<\mathrm{MDA}$ & . & 2.6 & $<M D A$ & . & 8.4 \\
\hline SCR5.2SP & EXP-SW & $02 / 21 / 02$ & GWPP & $<\mathrm{MDA}$ & . & 4 & $<M D A$ & . & 8.6 \\
\hline SCR5.2SP & EXP-SW & 08/19/02 & GWPP & $<\mathrm{MDA}$ & . & 2.2 & $<M D A$ & . & 8.6 \\
\hline
\end{tabular}


APPENDIX G

CY 2002 QUALITY ASSURANCE/QUALITY CONTROL DATA 


\section{EXPLANATION}

\section{Sampling Point:}

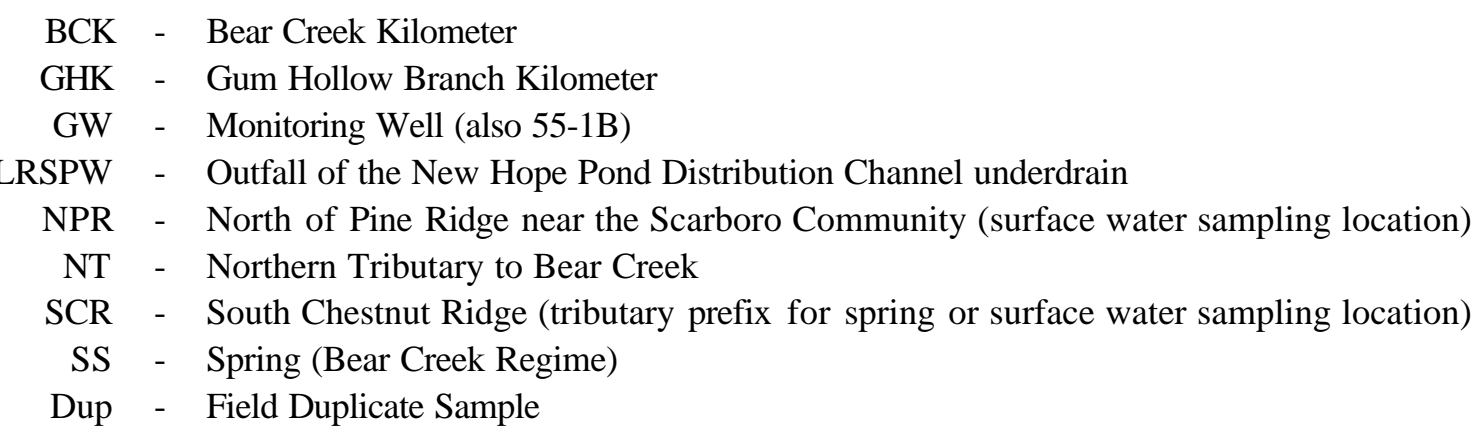

\section{Hydrogeologic Regime:}
BC - Bear Creek Hydrogeologic Regime
CR - Chestnut Ridge Hydrogeologic Regime
EF - Upper East Fork Poplar Creek Hydrogeologic Regime

\section{Notes:}

Appendix $\mathrm{G}$ shows the method (laboratory) blank and trip blank samples associated with each groundwater and surface water sample collected under management of the GWPP during CY 2002. Each method and trip blank was analyzed for volatile organic compounds (VOCs). As summarized below, VOCs were only detected in one method blank and one trip blank sample. Note that no groundwater or surface water sample results were screened as false positive.

\begin{tabular}{|c|c|c|c|c|c|}
\hline $\begin{array}{c}\text { Sample } \\
\text { Type }\end{array}$ & Sample Number & Compound & $\begin{array}{c}\text { Result } \\
(\boldsymbol{\mu g} / \mathbf{L})\end{array}$ & $\begin{array}{c}\text { Associated } \\
\text { Locations }\end{array}$ & $\begin{array}{c}\text { Date } \\
\text { Sampled }\end{array}$ \\
\hline $\begin{array}{c}\text { Method Blank } \\
\text { Trip Blank }\end{array}$ & $\begin{array}{c}\text { Q023170043 } \\
\text { A020420208 }\end{array}$ & $\begin{array}{c}\text { Tetrachloroethene } \\
\text { Acetone }\end{array}$ & $\begin{array}{c}2 \mathrm{~J} \\
19\end{array}$ & $\begin{array}{c}\text { GW-219, GW-689 } \\
\text { Rinsate }(\mathrm{GW}-722-17)\end{array}$ & $\begin{array}{c}11 / 07 / 02 \\
02 / 08 / 02\end{array}$ \\
\hline
\end{tabular}

A field blank sample was collected once during each calendar year quarter at the following wells in the Bear Creek and East Fork Regimes.

\begin{tabular}{|c|c|c|c|c|c|}
\hline \multicolumn{3}{|c|}{ Bear Creek Regime } & \multicolumn{3}{c|}{ East Fork Regime } \\
\hline $\begin{array}{c}\text { Sample } \\
\text { Number }\end{array}$ & $\begin{array}{c}\text { Monitoring } \\
\text { Well }\end{array}$ & $\begin{array}{c}\text { Date } \\
\text { Sampled }\end{array}$ & $\begin{array}{c}\text { Sample } \\
\text { Number }\end{array}$ & $\begin{array}{c}\text { Monitoring } \\
\text { Well }\end{array}$ & $\begin{array}{c}\text { Date } \\
\text { Sampled }\end{array}$ \\
\hline A020150121 & GW-226 & $03 / 01 / 02$ & A020930116 & GW-383 & $05 / 08 / 02$ \\
A021820123 & GW-225 & $08 / 12 / 02$ & A022730012 & GW-383 & $10 / 31 / 02$ \\
\hline
\end{tabular}

The field blanks were analyzed for VOCs and none were detected in the samples. 


\section{EXPLANATION (continued)}

A total of three equipment rinsate samples were collected during CY 2002: two were collected at well GW-722 (first and third quarter samples) and one at well GW-689 (fourth quarter sample) in the East Fork Regime. The rinsate samples were analyzed for the standard suite of analytes (see Section 3.3), but only the constituents shown below were detected.

\begin{tabular}{|c|c|c|c|c|c|}
\hline Well/Port & $\begin{array}{l}\text { Sample } \\
\text { Number }\end{array}$ & $\begin{array}{c}\text { Date } \\
\text { Sampled }\end{array}$ & Analyte & Result & Units \\
\hline GW-722-17 & A020080109 & $02 / 08 / 02$ & $\begin{array}{c}\text { Acetone } \\
\text { Alkalinity as HCO3 } \\
\text { Conductivity } \\
\text { Sodium } \\
\text { Turbidity } \\
\text { pH }\end{array}$ & $\begin{array}{c}46 \\
2.46 \\
0.9 \\
0.517 \\
0.505 \\
7.61\end{array}$ & $\begin{array}{c}\mu \mathrm{g} / \mathrm{L} \\
\mathrm{mg} / \mathrm{L} \\
\mu \mathrm{mho} / \mathrm{cm} \\
\mathrm{mg} / \mathrm{L} \\
\mathrm{NTU}\end{array}$ \\
\hline GW-722-17 & A021820166 & $07 / 17 / 02$ & $\begin{array}{c}\text { Alkalinity as } \mathrm{HCO} 3 \\
\text { Conductivity } \\
\text { Lead } \\
\text { Turbidity } \\
\text { pH }\end{array}$ & $\begin{array}{c}2.32 \\
0.9 \\
0.0013 \\
0.278 \\
7.92\end{array}$ & $\begin{array}{c}\mathrm{mg} / \mathrm{L} \\
\mu \mathrm{mho} / \mathrm{cm} \\
\mathrm{mg} / \mathrm{L} \\
\mathrm{NTU}\end{array}$ \\
\hline GW-689 & A023100003 & $11 / 14 / 02$ & $\begin{array}{c}\text { Conductivity } \\
\text { Dissolved Solids } \\
\text { Turbidity } \\
\text { pH } \\
\text { Barium } \\
\text { Iron } \\
\text { Sodium } \\
\text { Chloride } \\
\text { Sulfate }\end{array}$ & $\begin{array}{c}7.6 \\
15 \\
0.525 \\
4.75 \\
0.0073 \\
0.135 \\
0.205 \\
0.0569 \\
3.07\end{array}$ & $\begin{array}{c}\mu \mathrm{mho} / \mathrm{cm} \\
\mathrm{mg} / \mathrm{L} \\
\mathrm{NTU} \\
\\
\mathrm{mg} / \mathrm{L} \\
\mathrm{mg} / \mathrm{L} \\
\mathrm{mg} / \mathrm{L} \\
\mathrm{mg} / \mathrm{L} \\
\mathrm{mg} / \mathrm{L}\end{array}$ \\
\hline
\end{tabular}


APPENDIX G: CY 2001 QUALITY ASSURANCE/QUALITY CONTROL DATA

Correlation with Associated Groundwater and Surface Water Samples

\begin{tabular}{|c|c|c|c|c|c|}
\hline $\begin{array}{l}\text { Sampling } \\
\text { Point }\end{array}$ & $\begin{array}{c}\text { Hydrogeologic } \\
\text { Regime }\end{array}$ & $\begin{array}{c}\text { Date } \\
\text { Sampled }\end{array}$ & $\begin{array}{l}\text { Sample } \\
\text { Number }\end{array}$ & $\begin{array}{c}\text { Trip Blank } \\
\text { Sample Number }\end{array}$ & $\begin{array}{l}\text { Method Blank } \\
\text { Sample Number }\end{array}$ \\
\hline $55-1 \mathrm{~B}$ & EF & $04 / 17 / 02$ & A020930104 & A020930099 & Q021300058 \\
\hline 55-1B Dup & $\mathrm{EF}$ & $04 / 17 / 02$ & A020930105 & A020930099 & Q021300058 \\
\hline $55-1 \mathrm{~B}$ & $\mathrm{EF}$ & $10 / 14 / 02$ & A022730000 & A022730072 & Q022950185 \\
\hline BCK-00.63 & $\mathrm{BC}$ & 01/08/02 & A020080000 & A020080029 & Q020160446 \\
\hline BCK-00.63 & $\mathrm{BC}$ & 07/10/02 & A021820124 & A021890276 & Q021990086 \\
\hline BCK-04.55 & $\mathrm{BC}$ & 01/09/02 & A020080001 & A020080030 & Q020160446 \\
\hline BCK-04.55 & $\mathrm{BC}$ & 07/10/02 & A021820125 & A021890276 & Q021990086 \\
\hline BCK-07.87 & $\mathrm{BC}$ & 01/08/02 & A020080002 & A020080029 & Q020160446 \\
\hline BCK-07.87 & $\mathrm{BC}$ & 07/10/02 & A021820126 & A021890276 & Q021990086 \\
\hline BCK-09.40 & $\mathrm{BC}$ & 01/09/02 & A020080005 & A020080030 & Q020160446 \\
\hline BCK-09.40 & $\mathrm{BC}$ & $07 / 11 / 02$ & A021820128 & A021890277 & Q021990085 \\
\hline BCK-09.40 Dup & $\mathrm{BC}$ & $07 / 11 / 02$ & A021820129 & A021890277 & Q021990085 \\
\hline BCK-11.97 & $\mathrm{BC}$ & 01/09/02 & A020080009 & A020080030 & Q020220075 \\
\hline BCK-11.97 & $\mathrm{BC}$ & $07 / 11 / 02$ & A021820133 & A021890277 & Q021990085 \\
\hline GHK2.51ESW & $\mathrm{EF}$ & 05/15/02 & A020930122 & A020930117 & Q021510035 \\
\hline GHK2.51ESW & EF & $10 / 29 / 02$ & A022730164 & A022760214 & Q023110022 \\
\hline GHK2.51WSW & $\mathrm{EF}$ & 05/15/02 & A020930123 & A020930117 & Q021510035 \\
\hline GHK2.51WSW & $\mathrm{EF}$ & $10 / 29 / 02$ & A022730165 & A022760214 & Q023110022 \\
\hline GHK2.51WSW Dup & $\mathrm{EF}$ & $10 / 29 / 02$ & A022730166 & A022760214 & Q023110022 \\
\hline GW-014 & $\mathrm{BC}$ & $03 / 14 / 02$ & A020080050 & A020170239 & Q020950030 \\
\hline GW-014 Dup & $\mathrm{BC}$ & $03 / 14 / 02$ & A020080051 & A020170239 & Q020950030 \\
\hline GW-014 & $\mathrm{BC}$ & 08/12/02 & A021820143 & A022050189 & Q022330016 \\
\hline GW-061 & $\mathrm{BC}$ & $03 / 12 / 02$ & A020080043 & A020170237 & Q020950026 \\
\hline GW-061 & $\mathrm{BC}$ & $08 / 05 / 02$ & A021820135 & A022050185 & Q022330017 \\
\hline GW-066 & $\mathrm{BC}$ & $03 / 25 / 02$ & A020080055 & A020170244 & Q020940174 \\
\hline GW-066 & $\mathrm{BC}$ & 08/13/02 & A021820146 & A022050190 & Q022330364 \\
\hline GW-066 Dup & $\mathrm{BC}$ & $08 / 13 / 02$ & A021820147 & A022050190 & Q022330364 \\
\hline GW-069 & $\mathrm{BC}$ & 03/11/02 & A020080042 & A020170236 & Q020950052 \\
\hline GW-069 & $\mathrm{BC}$ & 08/05/02 & A021820134 & A022050185 & Q022330017 \\
\hline GW-071 & $\mathrm{BC}$ & $03 / 12 / 02$ & A020080045 & A020170237 & Q020950026 \\
\hline GW-071 & $\mathrm{BC}$ & $08 / 06 / 02$ & A021820137 & A022050186 & Q022330017 \\
\hline GW-071 Dup & $\mathrm{BC}$ & $08 / 06 / 02$ & A021820138 & A022050186 & Q022330017 \\
\hline GW-072 & $\mathrm{BC}$ & 03/11/02 & A020080044 & A020170236 & Q020950052 \\
\hline GW-072 & $\mathrm{BC}$ & 08/06/02 & A021820136 & A022050186 & Q022330017 \\
\hline GW-076 & $\mathrm{BC}$ & 03/19/02 & A020080052 & A020170242 & Q020950073 \\
\hline GW-082 & $\mathrm{BC}$ & $02 / 12 / 02$ & A020080034 & A020170231 & Q020500049 \\
\hline GW-082 & $\mathrm{BC}$ & $07 / 29 / 02$ & A021820114 & A022050134 & Q022320143 \\
\hline GW-085 & $\mathrm{BC}$ & $02 / 18 / 02$ & A020080038 & A020170233 & Q020710001 \\
\hline GW-085 & $\mathrm{BC}$ & $07 / 31 / 02$ & A021820117 & A022050136 & Q022320201 \\
\hline GW-087 & $\mathrm{BC}$ & $03 / 25 / 02$ & A020080056 & A020170244 & Q020940174 \\
\hline GW-091 & $\mathrm{BC}$ & 03/14/02 & A020080049 & A020170239 & Q020950026 \\
\hline GW-091 & $\mathrm{BC}$ & 08/08/02 & A021820142 & A022050188 & Q022330016 \\
\hline GW-153 & $\mathrm{EF}$ & $04 / 25 / 02$ & A020930114 & A020930103 & Q021300334 \\
\hline GW-153 & $\mathrm{EF}$ & $10 / 21 / 02$ & A022730010 & A022730076 & Q023100049 \\
\hline GW-204 & $\mathrm{EF}$ & 05/02/02 & A020930141 & A020930127 & Q021500035 \\
\hline GW-204 & $\mathrm{EF}$ & $11 / 06 / 02$ & A022730139 & A022760178 & Q023120054 \\
\hline GW-207 & $\mathrm{EF}$ & 05/08/02 & A021010110 & A021010103 & Q021400174 \\
\hline GW-207 & $\mathrm{EF}$ & $11 / 19 / 02$ & A022730153 & A022760210 & Q023400005 \\
\hline GW-207 Dup & $\mathrm{EF}$ & $11 / 19 / 02$ & A022730154 & A022760210 & Q023400005 \\
\hline
\end{tabular}


APPENDIX G: CY 2001 QUALITY ASSURANCE/QUALITY CONTROL DATA

Correlation with Associated Groundwater and Surface Water Samples

\begin{tabular}{|c|c|c|c|c|c|}
\hline $\begin{array}{l}\text { Sampling } \\
\text { Point }\end{array}$ & $\begin{array}{l}\text { Hydrogeologic } \\
\text { Regime }\end{array}$ & $\begin{array}{c}\text { Date } \\
\text { Sampled }\end{array}$ & $\begin{array}{l}\text { Sample } \\
\text { Number }\end{array}$ & $\begin{array}{c}\text { Trip Blank } \\
\text { Sample Number }\end{array}$ & $\begin{array}{l}\text { Method Blank } \\
\text { Sample Number }\end{array}$ \\
\hline GW-208 & $\mathrm{EF}$ & 05/08/02 & A021010109 & A021010103 & Q021400174 \\
\hline GW-208 & $\mathrm{EF}$ & $11 / 19 / 02$ & $\mathrm{~A} 022730152$ & A022760210 & Q023400005 \\
\hline GW-219 & $\mathrm{EF}$ & 05/06/02 & A020930142 & A020930128 & Q021510042 \\
\hline GW-219 & $\mathrm{EF}$ & $11 / 07 / 02$ & $\mathrm{~A} 022730140$ & A022760179 & Q023170043 \\
\hline GW-219 Dup & $\mathrm{EF}$ & $11 / 07 / 02$ & $\mathrm{~A} 022730141$ & A022760179 & Q023170043 \\
\hline GW-220 & $\mathrm{EF}$ & $05 / 14 / 02$ & $\mathrm{~A} 021010116$ & A021010105 & Q021500036 \\
\hline GW-220 & EF & $11 / 21 / 02$ & A022730160 & A022760212 & Q023390011 \\
\hline GW-225 & $B C$ & 02/19/02 & A020080041 & A020170234 & Q020670116 \\
\hline GW-225 & $\mathrm{BC}$ & $08 / 01 / 02$ & A021820121 & A022050137 & Q022320201 \\
\hline GW-226 & $\mathrm{BC}$ & 02/19/02 & A020080040 & A020170234 & Q020670116 \\
\hline GW-226 & $B C$ & $08 / 01 / 02$ & A021820120 & A022050137 & Q022320201 \\
\hline GW-229 & $\mathrm{BC}$ & 03/19/02 & A020080053 & A020170242 & Q020950073 \\
\hline GW-229 Dup & $\mathrm{BC}$ & 03/19/02 & A020080054 & A020170242 & Q020950073 \\
\hline GW-229 & $\mathrm{BC}$ & $08 / 12 / 02$ & A021820145 & A022050189 & Q022330364 \\
\hline GW-243 & $\mathrm{BC}$ & $03 / 21 / 02$ & A020080092 & A020170243 & Q020950073 \\
\hline GW-243 & $\mathrm{BC}$ & $08 / 13 / 02$ & $\mathrm{~A} 021820149$ & A022050190 & Q022330364 \\
\hline GW-251 & $\mathrm{EF}$ & $04 / 18 / 02$ & A020930109 & A020930100 & Q021300058 \\
\hline GW-251 & $\mathrm{EF}$ & 10/16/02 & A022730005 & A022730074 & Q022960100 \\
\hline GW-288 & $\mathrm{BC}$ & $03 / 13 / 02$ & A020080047 & A020170238 & Q020950026 \\
\hline GW-288 & $\mathrm{BC}$ & $08 / 07 / 02$ & A021820140 & A022050187 & Q022330017 \\
\hline GW-289 & $\mathrm{BC}$ & $03 / 13 / 02$ & A020080048 & A020170238 & Q020950026 \\
\hline GW-289 & $\mathrm{BC}$ & $08 / 08 / 02$ & $\mathrm{~A} 021820141$ & A022050188 & Q022330016 \\
\hline GW-291 & $\mathrm{BC}$ & $03 / 14 / 02$ & A020080046 & A020170239 & Q020950026 \\
\hline GW-291 & $\mathrm{BC}$ & $08 / 07 / 02$ & A021820139 & A022050187 & Q022330017 \\
\hline GW-311 & $\mathrm{BC}$ & $02 / 13 / 02$ & A020080036 & A020170232 & Q020500055 \\
\hline GW-311 Dup & $\mathrm{BC}$ & $02 / 13 / 02$ & $\mathrm{~A} 020080037$ & A020170232 & Q020500055 \\
\hline GW-311 & $\mathrm{BC}$ & $07 / 30 / 02$ & A021820116 & A022050135 & Q022320143 \\
\hline GW-315 & $\mathrm{BC}$ & $02 / 13 / 02$ & A020080035 & A020170232 & Q020500055 \\
\hline GW-315 & $\mathrm{BC}$ & $07 / 30 / 02$ & A021820115 & A022050135 & Q022320143 \\
\hline GW-349 & EF & 04/16/02 & A020930106 & A020930098 & Q021300058 \\
\hline GW-349 & $\mathrm{EF}$ & $10 / 14 / 02$ & $\mathrm{~A} 022730001$ & A022730072 & Q022950185 \\
\hline GW-350 & $\mathrm{EF}$ & $04 / 16 / 02$ & A020930107 & A020930098 & Q021300058 \\
\hline GW-350 & $\mathrm{EF}$ & $10 / 15 / 02$ & $\mathrm{~A} 022730002$ & A022730073 & Q022960100 \\
\hline GW-350 Dup & $\mathrm{EF}$ & $10 / 15 / 02$ & A022730003 & A022730073 & Q022960100 \\
\hline GW-381 & $\mathrm{EF}$ & $04 / 24 / 02$ & A020930113 & A020930102 & Q021300334 \\
\hline GW-381 & EF & $06 / 12 / 02$ & A021620086 & A021620088 & Q021650007 \\
\hline GW-381 & $E F$ & $10 / 22 / 02$ & A022730009 & A022730077 & Q023100049 \\
\hline GW-383 & $\mathrm{EF}$ & $04 / 25 / 02$ & A020930115 & A020930103 & Q021300334 \\
\hline GW-383 & $\mathrm{EF}$ & $10 / 22 / 02$ & $\mathrm{~A} 022730011$ & A022730077 & Q023100049 \\
\hline GW-537 & $\mathrm{BC}$ & $02 / 18 / 02$ & A020080039 & A020170233 & Q020710001 \\
\hline GW-537 & $\mathrm{BC}$ & $07 / 31 / 02$ & A021820118 & A022050136 & Q022320201 \\
\hline GW-537 Dup & $\mathrm{BC}$ & 07/31/02 & A021820119 & A022050136 & Q022320201 \\
\hline GW-620 & $\mathrm{EF}$ & $04 / 23 / 02$ & A020930108 & A020930101 & Q021300062 \\
\hline GW-620 & $\mathrm{EF}$ & $10 / 15 / 02$ & $\mathrm{~A} 022730004$ & A022730073 & Q022960100 \\
\hline GW-626 & $B C$ & $02 / 11 / 02$ & A020080033 & A020170230 & Q020500028 \\
\hline GW-626 & $\mathrm{BC}$ & $07 / 25 / 02$ & A021820113 & A022050133 & Q022320143 \\
\hline GW-627 & $\mathrm{BC}$ & $02 / 11 / 02$ & A020080032 & A020170230 & Q020500049 \\
\hline GW-627 & $\mathrm{BC}$ & 07/29/02 & A021820112 & A022050134 & Q022320143 \\
\hline GW-633 & $\mathrm{EF}$ & $04 / 23 / 02$ & A020930110 & A020930101 & Q021300062 \\
\hline
\end{tabular}


APPENDIX G: CY 2001 QUALITY ASSURANCE/QUALITY CONTROL DATA

Correlation with Associated Groundwater and Surface Water Samples

\begin{tabular}{|c|c|c|c|c|c|}
\hline $\begin{array}{l}\text { Sampling } \\
\text { Point }\end{array}$ & $\begin{array}{c}\text { Hydrogeologic } \\
\text { Regime }\end{array}$ & $\begin{array}{c}\text { Date } \\
\text { Sampled }\end{array}$ & $\begin{array}{l}\text { Sample } \\
\text { Number }\end{array}$ & $\begin{array}{c}\text { Trip Blank } \\
\text { Sample Number }\end{array}$ & $\begin{array}{l}\text { Method Blank } \\
\text { Sample Number }\end{array}$ \\
\hline GW-633 & EF & 10/17/02 & A022730006 & A022730075 & Q023100089 \\
\hline GW-653 & $\mathrm{BC}$ & $02 / 12 / 02$ & A020080031 & A020170231 & Q020500049 \\
\hline GW-653 & $\mathrm{BC}$ & $07 / 25 / 02$ & A021820111 & A022050133 & Q022190138 \\
\hline GW-658 & $\mathrm{EF}$ & $04 / 24 / 02$ & A020930111 & A020930102 & Q021300334 \\
\hline GW-658 & $\mathrm{EF}$ & 10/17/02 & A022730007 & A022730075 & Q023100089 \\
\hline GW-683 & $\mathrm{BC}$ & 01/14/02 & A020070179 & A020070171 & Q020280008 \\
\hline GW-683 & $\mathrm{BC}$ & 07/09/02 & A021820086 & A021890264 & Q021990086 \\
\hline GW-684 & $\mathrm{BC}$ & 01/14/02 & A020070180 & A020070171 & Q020280008 \\
\hline GW-684 & $\mathrm{BC}$ & 07/09/02 & A021820084 & A021890264 & Q021990086 \\
\hline GW-684 Dup & $\mathrm{BC}$ & 07/09/02 & A021820085 & A021890264 & Q021990086 \\
\hline GW-686 & $\mathrm{EF}$ & $11 / 14 / 02$ & A023100001 & A023170059 & Q023240142 \\
\hline GW-687 & $\mathrm{EF}$ & $11 / 14 / 02$ & A023100002 & A023170059 & Q023240142 \\
\hline GW-689 & $\mathrm{EF}$ & $11 / 14 / 02$ & A023100004 & A023170059 & Q023240142 \\
\hline GW-694 & $\mathrm{BC}$ & 01/29/02 & A020080016 & A020070174 & Q020500012 \\
\hline GW-694 & $\mathrm{BC}$ & 07/17/02 & A021820099 & A021890267 & Q022190132 \\
\hline GW-695 & $\mathrm{BC}$ & 01/15/02 & A020080011 & A020070172 & Q020280008 \\
\hline GW-695 & $\mathrm{BC}$ & 07/15/02 & A021820095 & A021890265 & Q022030237 \\
\hline GW-698 & $\mathrm{EF}$ & $05 / 02 / 02$ & A020930139 & A020930127 & Q021500035 \\
\hline GW-698 Dup & $\mathrm{EF}$ & 05/02/02 & A020930140 & A020930127 & Q021500035 \\
\hline GW-698 & EF & $11 / 07 / 02$ & A022730138 & A022760179 & Q023170043 \\
\hline GW-703 & $\mathrm{BC}$ & 01/15/02 & A020080012 & A020070172 & Q020280008 \\
\hline GW-703 & $\mathrm{BC}$ & 07/15/02 & A021820096 & A021890265 & Q022030237 \\
\hline GW-704 & $\mathrm{BC}$ & 01/16/02 & A020080013 & $\mathrm{A} 020070173$ & Q020280008 \\
\hline GW-704 Dup & $\mathrm{BC}$ & 01/16/02 & A020080014 & A020070173 & Q020280008 \\
\hline GW-704 & $\mathrm{BC}$ & 07/16/02 & A021820097 & A021890266 & Q022030237 \\
\hline GW-706 & $\mathrm{BC}$ & 01/16/02 & A020080015 & A020070173 & Q020280008 \\
\hline GW-706 & $\mathrm{BC}$ & 07/16/02 & A021820098 & A021890266 & Q022030237 \\
\hline GW-722-06 & $\mathrm{EF}$ & $02 / 04 / 02$ & A020080098 & A020360070 & Q020500020 \\
\hline GW-722-06 & $\mathrm{EF}$ & 07/15/02 & A021820155 & A021960125 & Q022070046 \\
\hline GW-722-10 & $\mathrm{EF}$ & $02 / 06 / 02$ & A020080103 & A020380048 & Q020500024 \\
\hline GW-722-10 & $\mathrm{EF}$ & $07 / 16 / 02$ & A021820160 & A021970081 & Q022030237 \\
\hline GW-722-14 & $\mathrm{EF}$ & $02 / 08 / 02$ & A020080107 & A020390064 & Q020500028 \\
\hline GW-722-14 & $\mathrm{EF}$ & $07 / 17 / 02$ & A021820164 & A021990073 & Q022180314 \\
\hline GW-722-17 & $\mathrm{EF}$ & $02 / 08 / 02$ & A020080106 & A020390064 & Q020500028 \\
\hline GW-722-17 & $\mathrm{EF}$ & $07 / 17 / 02$ & A021820165 & A021990073 & Q022180314 \\
\hline GW-722-20 & $\mathrm{EF}$ & $02 / 07 / 02$ & A020080108 & A020390054 & Q020500028 \\
\hline GW-722-20 & $\mathrm{EF}$ & $07 / 17 / 02$ & A021820162 & A021980076 & Q022180314 \\
\hline GW-722-20 Dup & $\mathrm{EF}$ & $07 / 17 / 02$ & A021820163 & A021980076 & Q022180314 \\
\hline GW-722-22 & EF & $02 / 07 / 02$ & A020080104 & A020390054 & Q020500028 \\
\hline GW-722-22 Dup & $\mathrm{EF}$ & 02/07/02 & A020080105 & A020390054 & Q020500028 \\
\hline GW-722-22 & $\mathrm{EF}$ & $07 / 16 / 02$ & A021820161 & A021970127 & Q022030237 \\
\hline GW-722-26 & $\mathrm{EF}$ & $02 / 05 / 02$ & A020080100 & A020370037 & Q020500020 \\
\hline GW-722-26 & EF & $07 / 15 / 02$ & A021820157 & A021970003 & Q022070046 \\
\hline GW-722-30 & $\mathrm{EF}$ & 02/05/02 & A020080099 & A020370037 & Q020500020 \\
\hline GW-722-30 & $\mathrm{EF}$ & 07/15/02 & A021820156 & A021960125 & Q022070046 \\
\hline GW-722-32 & $\mathrm{EF}$ & 02/05/02 & A020080101 & A020370037 & Q020500020 \\
\hline GW-722-32 & $\mathrm{EF}$ & $07 / 15 / 02$ & A021820158 & A021970081 & Q022030237 \\
\hline GW-722-33 & $\mathrm{EF}$ & 02/06/02 & A020080102 & A020380048 & Q020500024 \\
\hline GW-722-33 & $\mathrm{EF}$ & $07 / 16 / 02$ & A021820159 & A021970081 & Q022030237 \\
\hline
\end{tabular}


APPENDIX G: CY 2001 QUALITY ASSURANCE/QUALITY CONTROL DATA

Correlation with Associated Groundwater and Surface Water Samples

\begin{tabular}{|c|c|c|c|c|c|}
\hline $\begin{array}{l}\text { Sampling } \\
\text { Point }\end{array}$ & $\begin{array}{c}\text { Hydrogeologic } \\
\text { Regime }\end{array}$ & $\begin{array}{c}\text { Date } \\
\text { Sampled }\end{array}$ & $\begin{array}{l}\text { Sample } \\
\text { Number }\end{array}$ & $\begin{array}{c}\text { Trip Blank } \\
\text { Sample Number }\end{array}$ & $\begin{array}{l}\text { Method Blank } \\
\text { Sample Number }\end{array}$ \\
\hline GW-723 & $\mathrm{BC}$ & 03/05/02 & A020070187 & A020070177 & Q020950052 \\
\hline GW-723 & $\mathrm{BC}$ & 07/23/02 & A021820087 & A021890270 & Q022190138 \\
\hline GW-724 & $\mathrm{BC}$ & 01/30/02 & A020070193 & A020070175 & Q020500012 \\
\hline GW-724 & $\mathrm{BC}$ & $07 / 23 / 02$ & A021820093 & A021890270 & Q022190138 \\
\hline GW-725 & $\mathrm{BC}$ & 01/31/02 & A020070194 & A020070176 & Q020500013 \\
\hline GW-725 & $\mathrm{BC}$ & 07/18/02 & A021820094 & A021890268 & Q022190136 \\
\hline GW-735 & $\mathrm{EF}$ & $05 / 14 / 02$ & A021010115 & A021010105 & Q021500036 \\
\hline GW-735 & $\mathrm{EF}$ & $11 / 21 / 02$ & A022730159 & A022760212 & Q023390011 \\
\hline GW-736 & $\mathrm{BC}$ & 03/06/02 & A020070188 & A020070178 & Q020950052 \\
\hline GW-736 & $\mathrm{BC}$ & 07/17/02 & A021820088 & A021890267 & Q022190132 \\
\hline GW-737 & $\mathrm{BC}$ & 03/06/02 & A020070189 & A020070178 & Q020950052 \\
\hline GW-737 & $\mathrm{BC}$ & 07/18/02 & A021820089 & A021890268 & Q022190136 \\
\hline GW-738 & $\mathrm{BC}$ & 01/30/02 & A020070192 & A020070175 & Q020500012 \\
\hline GW-738 & $\mathrm{BC}$ & $07 / 23 / 02$ & A021820092 & A021890270 & Q022190138 \\
\hline GW-739 & $\mathrm{BC}$ & 03/05/02 & A020070190 & A020070177 & Q020950052 \\
\hline GW-739 & $\mathrm{BC}$ & $07 / 22 / 02$ & A021820090 & A021890269 & Q022190136 \\
\hline GW-740 & $\mathrm{BC}$ & 01/29/02 & A020070191 & A020070174 & Q020500012 \\
\hline GW-740 & $\mathrm{BC}$ & $07 / 22 / 02$ & A021820091 & A021890269 & Q022190136 \\
\hline GW-744 & $\mathrm{EF}$ & 05/07/02 & A021010112 & A021010102 & Q021510042 \\
\hline GW-744 & EF & $11 / 18 / 02$ & A022730156 & A022760209 & Q023400005 \\
\hline GW-747 & $\mathrm{EF}$ & 05/09/02 & A021010113 & A021010104 & Q021500036 \\
\hline GW-747 & EF & $11 / 20 / 02$ & A022730157 & A022760211 & Q023390011 \\
\hline GW-750 & EF & 05/05/02 & A021010114 & A021010104 & Q021500036 \\
\hline GW-750 & $\mathrm{EF}$ & $11 / 20 / 02$ & A022730158 & A022760211 & Q023390011 \\
\hline GW-763 & $\mathrm{EF}$ & 04/18/02 & A020930112 & A020930100 & Q021300058 \\
\hline GW-763 & $\mathrm{EF}$ & $06 / 12 / 02$ & A021620087 & A021620088 & Q021650007 \\
\hline GW-763 & $\mathrm{EF}$ & $10 / 21 / 02$ & A022730008 & A022730076 & Q023100089 \\
\hline GW-769 & $\mathrm{EF}$ & 04/29/02 & A020930136 & A020930124 & Q021300333 \\
\hline GW-769 & $\mathrm{EF}$ & 10/30/02 & A022730135 & A022760175 & Q023110022 \\
\hline GW-770 & $\mathrm{EF}$ & 04/29/02 & A020930135 & A020930124 & Q021300333 \\
\hline GW-770 & $\mathrm{EF}$ & $10 / 30 / 02$ & A022730134 & A022760175 & Q023110022 \\
\hline GW-775 & $\mathrm{EF}$ & $04 / 30 / 02$ & A020930133 & A020930125 & Q021300333 \\
\hline GW-775 & $\mathrm{EF}$ & 10/31/02 & A022730132 & A022760176 & Q023110160 \\
\hline GW-776 & $\mathrm{EF}$ & $04 / 30 / 02$ & A020930134 & A020930125 & Q021300333 \\
\hline GW-776 & $\mathrm{EF}$ & $10 / 31 / 02$ & A022730133 & A022760176 & Q023110160 \\
\hline GW-782 & $\mathrm{EF}$ & $05 / 01 / 02$ & A020930138 & A020930126 & Q021490004 \\
\hline GW-782 & $\mathrm{EF}$ & $11 / 04 / 02$ & A022730137 & A022760177 & Q023110160 \\
\hline GW-791 & $\mathrm{EF}$ & $05 / 01 / 02$ & A020930137 & A020930126 & Q021490004 \\
\hline GW-791 & $\mathrm{EF}$ & $11 / 06 / 02$ & A022730136 & A022760178 & Q023120054 \\
\hline GW-816 & $\mathrm{EF}$ & 05/07/02 & A021010111 & A021010102 & Q021510042 \\
\hline GW-816 & $\mathrm{EF}$ & $11 / 18 / 02$ & A022730155 & A022760209 & Q023400005 \\
\hline LRSPW & $\mathrm{EF}$ & 05/06/02 & A021010107 & A020930128 & Q021510042 \\
\hline LRSPW Dup & $\mathrm{EF}$ & $05 / 06 / 02$ & A021010108 & A020930128 & Q021510042 \\
\hline LRSPW & $\mathrm{EF}$ & $11 / 19 / 02$ & A022730151 & A022760210 & Q023400005 \\
\hline NPR07.0SW & $\mathrm{EF}$ & $05 / 15 / 02$ & A020930118 & A020930117 & Q021510035 \\
\hline NPR07.0SW & $\mathrm{EF}$ & $10 / 29 / 02$ & A022730161 & A022760214 & Q023110022 \\
\hline NPR12.0SW & $\mathrm{EF}$ & $05 / 15 / 02$ & A020930119 & A020930117 & Q021510035 \\
\hline NPR12.0SW Dup & $\mathrm{EF}$ & 05/15/02 & A020930120 & A020930117 & Q021510035 \\
\hline NPR12.0SW & $\mathrm{EF}$ & $10 / 29 / 02$ & A022730162 & A022760214 & Q023110022 \\
\hline
\end{tabular}


APPENDIX G: CY 2001 QUALITY ASSURANCE/QUALITY CONTROL DATA

Correlation with Associated Groundwater and Surface Water Samples

\begin{tabular}{|c|c|c|c|c|c|}
\hline $\begin{array}{l}\text { Sampling } \\
\text { Point }\end{array}$ & $\begin{array}{l}\text { Hydrogeologic } \\
\text { Regime }\end{array}$ & $\begin{array}{c}\text { Date } \\
\text { Sampled }\end{array}$ & $\begin{array}{l}\text { Sample } \\
\text { Number }\end{array}$ & $\begin{array}{c}\text { Trip Blank } \\
\text { Sample Number }\end{array}$ & $\begin{array}{l}\text { Method Blank } \\
\text { Sample Number }\end{array}$ \\
\hline NPR23.0SW & $\mathrm{EF}$ & 05/15/02 & A020930121 & A020930117 & Q021510035 \\
\hline NPR23.0SW & EF & 10/29/02 & A022730163 & A022760214 & Q023110022 \\
\hline NT-01 & $\mathrm{BC}$ & 01/09/02 & A020080008 & A020080030 & Q020220075 \\
\hline NT-01 & $\mathrm{BC}$ & 07/11/02 & A021820131 & A021890277 & Q021990085 \\
\hline SCR1.5SW & $\mathrm{CR}$ & $02 / 21 / 02$ & A020080093 & A020170245 & Q020670116 \\
\hline SCR1.5SW Dup & $\mathrm{CR}$ & $02 / 21 / 02$ & A020080094 & A020170245 & Q020670116 \\
\hline SCR1.5SW & $\mathrm{CR}$ & 08/19/02 & A021820150 & A022050193 & Q022330365 \\
\hline SCR2.2SW & $\mathrm{CR}$ & $03 / 21 / 02$ & A020080095 & A020170243 & Q020950073 \\
\hline SCR2.2SW & $\mathrm{CR}$ & 09/23/02 & A021820151 & A022660111 & Q022760007 \\
\hline SCR4.4SW & $\mathrm{CR}$ & 02/21/02 & A020080096 & A020170245 & Q020670116 \\
\hline SCR4.4SW & $\mathrm{CR}$ & 09/23/02 & A021820152 & A022660111 & Q022760007 \\
\hline SCR4.4SW Dup & $\mathrm{CR}$ & 09/23/02 & A021820153 & A022660111 & Q022760007 \\
\hline SCR5.2SP & $\mathrm{CR}$ & $02 / 21 / 02$ & A020080097 & A020170245 & Q020670116 \\
\hline SCR5.2SP & CR & 08/19/02 & A021820154 & A022050193 & Q022330365 \\
\hline SS-1 & $\mathrm{BC}$ & 01/09/02 & A020080007 & A020080030 & Q020220075 \\
\hline SS-1 & $\mathrm{BC}$ & 07/15/02 & A021820130 & A021890265 & Q022030237 \\
\hline SS-4 & $\mathrm{BC}$ & 01/08/02 & A020080010 & A020080029 & Q020160446 \\
\hline SS-4 & $\mathrm{BC}$ & 07/10/02 & A021820132 & A021890276 & Q021990086 \\
\hline SS-5 & $\mathrm{BC}$ & 01/08/02 & A020080003 & A020080029 & Q020160446 \\
\hline SS-5 Dup & $\mathrm{BC}$ & 01/08/02 & A020080004 & A020080029 & Q020160446 \\
\hline SS-5 & $B C$ & 07/10/02 & A021820127 & A021890276 & Q021990086 \\
\hline
\end{tabular}




\section{DISTRIBUTION}

U.S. DEPARTMENT OF ENERGY

J.D. Darby, DOE-EM

J.P. Donnelly, DOE-NNSA

M.S. Ferre, DOE-EM

TENNESSEE DEPARTMENT OF

ENVIRONMENT AND CONSERVATION DOE-

ORR OVERSIGHT DIVISION

D. Gilmore (3)

BWXT Y-12, L.L.C.

ENVIRONMENTAL COMPLIANCE

DEPARTMENT

S.M. Field

S.B. Jones

C.C. Hill

J.E. Powell

E.R. Schultz

L.O. Vaughan

File-GWPP (2)

BWXT Y-12, L.L.C.

ANALYTICAL CHEMISTRY

ORGANIZATION

D.D. Altom

Y-12 Central Files-RC

Y-12 Records Services (Electronic copy- OSTI)
BECHTEL JACOBS COMPANY LLC

E.L. Berglund

J.F. Hensley

R.H. Ketelle

H.L. King

D.W. McCune

J.R. McNutt

L.M. Sims (2)

E. Trujillo

File-EMEF-DMC

SCIENCE APPLICATIONS

INTERNATIONAL CORPORATION

W.K. Jago

P.D. Moss

UT-BATTELLE, LLC

D.B. Watson

ELVADO ENVIRONMENTAL LLC.

T.R. Harrison

J.R. Walker 